Marco Henry V. Neumueller

\title{
Family Compliance: Der erfolgreiche Umgang mit einer Familienverfassung
}




\section{Wittener Schriften zu Familienunternehmen}

Band 27

Herausgegeben von

Tom A. Rüsen, Marcel Hülsbeck und Arist von Schlippe im Wittener Institut für Familienunternehmen (WIFU) an der Universität Witten/Herdecke 
Marco Henry V. Neumueller

\section{Family Compliance: Der erfolgreiche Umgang mit einer Familienverfassung}

Kontextuelle Einordnung und konkrete Handlungsempfehlungen für Unternehmerfamilien

Mit Geleitworten von Prof. Dr. Arist von Schlippe und Prof. Dr. Dr. h.c. mult. Brun-Hagen Hennerkes

Mit 14 Abbildungen

V\&R unipress 
Bibliografische Information der Deutschen Nationalbibliothek

Die Deutsche Nationalbibliothek verzeichnet diese Publikation in der Deutschen Nationalbibliografie; detaillierte bibliografische Daten sind im Internet über https://dnb.de abrufbar.

( 2020 , Vandenhoeck \& Ruprecht GmbH \& Co. KG, Theaterstraße 13, D-37073 Göttingen Dieses Werk ist als Open-Access-Publikation im Sinne der Creative-Commons-Lizenz BY International 4.0 (»Namensnennung«) unter dem DOI 10.14220/9783737011716 abzurufen. Um eine Kopie dieser Lizenz zu sehen, besuchen Sie https://creativecommons.org/licenses/by/4.0/. Jede Verwertung in anderen als den durch diese Lizenz zugelassenen Fällen bedarf der vorherigen schriftlichen Einwilligung des Verlages.

Vandenhoeck \& Ruprecht Verlage | www.vandenhoeck-ruprecht-verlage.com

ISSN 2198-526X

ISBN 978-3-7370-1171-6 


\section{Inhalt}

Geleitwort von Arist von Schlippe . . . . . . . . . . . . . . . . . 11

Geleitwort von Brun-Hagen Hennerkes . . . . . . . . . . . . . . . . 15

Vorwort und Danksagung . . . . . . . . . . . . . . 17

Abbildungsverzeichnis ... . . . . . . . . . . . . . . 19

Abkürzungsverzeichnis . . . . . . . . . . . . . . . 21

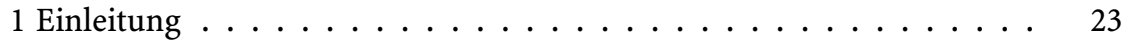

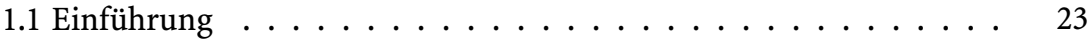

1.2 Zielsetzung und Forschungsaufbau . . . . . . . . . . . . . . 26

1.3 Gang der Untersuchung . . . . . . . . . . . . . . 28

2 Theoretische Grundlagen . . . . . . . . . . . . . . . 31

2.1 Das Familienunternehmen: eine besondere Unternehmensform mit Stärken und Schwächen . . . . . . . . . . . . . . . 31

2.1.1 Das Forschungsfeld Familienunternehmen . . . . . . . . . 32

2.1.2 Definition eines Familienunternehmens im Rahmen der Untersuchung ..................... 33

2.1.3 Familienunternehmen: eine unmögliche Kopplung sozialer Systeme?! . . . . . . . . . . . . . . . 34

2.1.4 Pragmatische Paradoxien: die Wurzel des Erfolgs?! . . . . . . 37

2.1.5 Konfliktpotenziale in Unternehmerfamilien . . . . . . . . . 43

2.2 Die Familienverfassung als Instrument der Family Governance . . 47

2.2.1 Die Familienverfassung: Versuch einer begrifflichen Rechtfertigung . . . . . . . . . . . . 47

2.2.2 Die Familienverfassung. Merkmale und Kontext . . . . . . . . 49

2.2.3 Aufgaben und konkrete Funktionen einer Familienverfassung $\quad 51$ 
2.2.4 Die Familienstrategie als Wegbereiter der Familienverfassung 52

2.2.4.1 Praktische Umsetzung einer Familienstrategie . . . . 55

2.2.4.1.1 Die »Inventur« der Familie . . . . . . . 56

2.2.4.1.2 Die Richtungsentscheidung . . . . . . 58

2.2.5 Die Familienverfassung und ihre Stellung im System der

"Family Governance» . . . . . . . . . . . . . 60

2.2.5.1 Von der Corporate Governance zur Family

Governance ................. 60

2.2.5.2 Family Governance: eine Begriffsdefinition . . . . . . 61

2.2.5.3 Eine erfolgreiche Family Governance . . . . . . . 62

2.3 Die Familienverfassung in der Gestaltungspraxis . . . . . . . . . . . . . 69

2.3.1 Aufbau einer Familienverfassung . . . . . . . . . . . . . . 69

2.3.1.1 Allgemeines . . . . . . . . . . . . 69

2.3.1.2 Typische Regelungsinhalte und der Bezug zur »Familiness" ............... . 70

2.3.1.3 Institutionen, Instrumente und Verfahrensregeln . . 77

2.3.1.3.1 Der Familientag . . . . . . . . . . . . . 77

2.3.1.3.2 Der Familienrat . . . . . . . . . . 80

2.3.1.3.3 Der Familienmanager . . . . . . . . 82

2.3.1.3.4 Family Education - die

Kompetenzförderung der Gesellschafter . . 82

2.3.1.3.5 Kommunikationsregeln und

Konfliktmanagement ......... . . 85

2.3.1.4 Geltungsdauer und Anpassungserfordernisse . . . . 87

2.4 Die Bindungswirkung der Familienverfassung . . . . . . . . . . . 89

2.4.1 Rechtliche oder moralische Bindung? Eine kursorische

Übersicht . . . . . . . . . . . . . . . . . . . . . . . 89

2.4.2 Die Familienverfassung im Verhältnis zum

Gesellschaftsvertrag ................. 92

2.5 Der Legitimationsgedanke der Familienverfassung im Lichte privater Normsetzung und moralphilosophischer Ansätze . . . . . 95

2.5.1 Private Normsetzung und Selbstregulierung . . . . . . . . . . . . 96

2.5.1.1 Regeln schaffen Ordnung . . . . . . . . . . . . 96

2.5.1.1.1 Die Ökonomik der Ordnung . . . . . . . 97

2.5.1.1.2 Der Begriffsstreit Norm versus Regel . . . . 97

2.5.1.1.3 Die Regelbeteiligten . . . . . . . . . . . . . 99

2.5.1.2 Der Begriff der Selbstregulierung . . . . . . . . . . . . . . . 99

2.5.1.3 Beurteilung der Selbstregulierung . . . . . . . . . . 102

2.5.1.3.1 Vorteile der Selbstregulierung . . . . . . 102

a) Die Flexibilität und Geschwindigkeit . . . . . . . 102 
b) Die Akzeptanz und Zustimmung . . . . . . . . . . 103

2.5.1.3.2 Nachteile der Selbstregulierung . . . . . . 104

a) Die Unverbindlichkeit der Regeln . . . . . . . . . . . . . 105

b) Das Durchsetzungsdefizit . . . . . . . . . . . . . 106

c) Die mangelnde Legitimation . . . . . . . . . . 107

2.5.1.3.3 Kritische Bewertung der Vor- und Nachteile . . . . . . . . . . . 108

2.5.1.4 Regelbefolgung durch höhere Akzeptanz . . . . . . 108

2.5.1.4.1 Sachnähe zur Förderung der Akzeptanz . . 109

2.5.1.4.2 Verbindlichkeit zur Förderung der Akzeptanz ............. 109

2.5.1.4.3 Durchsetzbarkeit zur Förderung der Akzeptanz .............. 110

a) Die Identifikation mit dem sozialen Umfeld . . . . 110

b) Die Internalisierung als Form der inneren Akzeptanz ................... 111

c) Die Sanktionsorientierung zur Vermeidung negativer Konsequenzen . . . . . . . . . 112

2.5.1.4.4 Kontrolle als Voraussetzung einer effektiven Durchsetzung . . . . . . . . . . . 112

2.5.1.5 Sanktionsmechanismen . . . . . . . . . . 113

2.5.1.5.1 Freiwilligkeit und Sanktionen . . . . . . . 113

2.5.1.5.2 Sanktionsmöglichkeiten privater Selbstregulierung .......... . 114

a) Die Wirksamkeit privatautonomer Regeldurchsetzung .............. . 114

b) Sanktionierung durch den Markt oder das Umfeld. 115

2.5.2 Soziologisch-moralphilosophische Annäherung an ein Chamäleon . . . . . . . . . . . . . . . . . . 115

2.5.2.1 Das Wesen der Moral . . . . . . . . . . . . 116

2.5.2.2 Vom moralischen Sollen und Müssen . . . . . . . . 118

2.5.2.3 Die Bedeutung von Sanktionen für die Normgeltung • 120

2.5.2.4 Soziale Sanktioniertheit und moralische Verpflichtung ... . . . . . . . . . 124

2.5.2.5 Moral: Interesse rechtfertigt, Sanktion motiviert . . . 125

2.5.2.6 Moralische Pflicht und praktische Gründe . . . . . . 127

2.5.2.7 Intersubjektive Sanktionen: Moralbegründung nach Adam Smith . . . . . . . . . . . . . . 130

2.5.2.8 Die Forderungstheorie als weiterer Erklärungsversuch moralischer Verpflichtungen . . . 132 
2.5.2.9 Ohne Ermächtigung keine Autorität . . . . . . . . . 134

3 Darstellung der Fallstudien . . . . . . . . . . . . . . . . 137

3.1 Fallstudie 1: Unternehmerfamilie Asbrecht (A) . . . . . . . . . . . 138

3.1.1 Entstehung und Erstellung der Familienverfassung . . . . . . 138

3.1.2 Inhalt, Institutionen und Alltag . . . . . . . . . . . . . 140

3.1.3 Regelbefolgung, -brüche und moralische Verbindlichkeit . . . 142

3.2 Fallstudie 2: Unternehmerfamilie Brunai (B) . . . . . . . . . . . . 143

3.2.1 Entstehung und Erstellung der Familienverfassung . . . . . . 143

3.2.2 Inhalt, Institutionen und Alltag . . . . . . . . . . . . . 145

3.2.3 Regelbefolgung, -brüche und moralische Verbindlichkeit . . . 148

3.3 Fallstudie 3: Unternehmerfamilie Calari (C) . . . . . . . . . . . . 152

3.3.1 Entstehung und Erstellung der Familienverfassung . . . . . . 152

3.3.2 Inhalt, Institutionen und Alltag . . . . . . . . . . . . . 154

3.3.3 Regelbefolgung, -brüche und moralische Verbindlichkeit . . . 155

3.4 Fallstudie 4: Unternehmerfamilie Deuther (D) . . . . . . . . . . 158

3.4.1 Entstehung und Erstellung der Familienverfassung . . . . . . 159

3.4.2 Inhalte, Institutionen und Alltag . . . . . . . . . . . 160

3.4.3 Regelbefolgung, -brüche und moralische Verbindlichkeit . . . 162

3.5 Fallstudie 5: Unternehmerfamilie Eichmyller und Erhardt (E) . . . 165

3.5.1 Entstehung und Erstellung der Familienverfassung . . . . . . 165

3.5.2 Inhalt, Institutionen und Alltag . . . . . . . . . . . . 167

3.5.3 Regelbefolgung, -brüche und moralische Verbindlichkeit . . . 170

3.6 Fallstudie 6: Unternehmerfamilie Feyner (F) . . . . . . . . . . . . 173

3.6.1 Entstehung und Erstellung der Familienverfassung . . . . . . 173

3.6.2 Inhalt, Institutionen und Alltag . . . . . . . . . . . . . 174

3.6.3 Regelbefolgung, -brüche und moralische Verbindlichkeit . . . 177

3.7 Fallstudie 7: Unternehmerfamilie Gönner (G) . . . . . . . . . . . . 180

3.7.1 Entstehung und Erstellung der Familienverfassung . . . . . . 180

3.7.2 Inhalt, Institutionen und Alltag . . . . . . . . . . . . 181

3.7.3 Regelbefolgung, -brüche und moralische Verbindlichkeit . . . 183

3.8 Fallstudie 8: Unternehmerfamilie Hirter $(\mathrm{H}) \ldots \ldots$. . . . . . . . . 185

3.8.1 Entstehung und Erstellung der Familienverfassung . . . . . . 185

3.8.2 Inhalt, Institutionen und Alltag . . . . . . . . . . . . 187

3.8.3 Regelbefolgung, -brüche und moralische Verbindlichkeit . . . 189

4 Analyse und Diskussion der Ergebnisse . . . . . . . . . . . . . . 193

4.1 Wesentliche Ergebnisse der fallübergreifenden Auswertung . . . . 193

4.1 .1 Allgemeine Vorbemerkungen . . . . . . . . . . . . . . . . . 193

4.1.2 Der Impetus für die Erstellung . . . . . . . . . . . . . 195 
4.1.3 Vorsorge ist besser als Nachsorge: die präventiven Maßnahmen zur Sicherung der Regelbefolgung . . . . . . . . 198

4.1.3.1 Die Ausgangslage entscheidet . . . . . . . . . . 199

4.1.3.2 Betroffene zu Beteiligten machen ......... 201

4.1.3.3 (Moralische) Verbindlichkeit fördert Akzeptanz . . . 203

4.1.3.4 Inkonsistenz vermeiden: die Anpassung des Gesellschaftsvertrags . . . . . . . . . . 206

4.1.3.5 Die Business-Fassung als Garant für Regelkonformität!! . . . . . . . . . . . . . . 207

4.1.3.6 Wissen schafft Vertrauen und fördert Akzeptanz . . . 208

4.1.3.7 Die Zwangswirkung des Gesellschafterdaseins relativieren ................. . . . 210

4.1.3.8 Vertrauen ist gut, Kontrolle ist besser . . . . . . . . 212

4.1.3.9 Blut ist dicker als Wasser: Stärkung innerfamiliärer Kohäsion . . . . . . . . . . . . . 213

4.1.3.9.1 Ein gemeinsamer Erstellungsprozess schweißt zusammen . . . . . . . . . . . 214

4.1.3.9.2 Die gemeinsame Geschichte verbindet . . . 216

4.1.3.9.3 Bindung durch Kommunikation: der Familientag . . . . . . . . . . 218

4.1.3.9.4 Kommunikationsregeln: das Was und Wie der Kommunikation . . . . . . . . . . 221

4.1.3.9.5 Die Koordinatoren des familiären Zusammenhalts: der Familienrat . . . . . . 223

4.1.3.10 Präventive Wirkung von Sanktionen . . . . . . . . . 226

4.1.4 Wo kein Maß ist, ist keine Vergeltung?! Die reaktiven Maßnahmen zur Sicherung der Regelbefolgung . . . . . . . . . 227

4.1.4.1 Allgemeine Erkenntnisse . . . . . . . . . . 227

4.1.4.2 Sanktionen innerhalb der Familie. Wirklich? Immer?. 229

4.1.4.3 Konkrete Maßnahmen zum Umgang mit Regelbrüchen ... . . . . . . . . 232

4.1.4.3.1 Die Thematisierung des Regelbruchs . . . . 232

4.1.4.3.2 Die Aufklärung des Sachverhalts als Chance .............. 233

4.1.4.3.3 Verwarnung und Bekanntgabe: die gelbe Karte ............... . 234

4.1.4.3.4 Konsequenzen bei wiederholtem Vergehen 234

4.1.4.3.4.1 Die familien-öffentliche Aussprache: die rote Karte . . . . . . . . . . . . . 235

4.1.4.3.4.2 Das Mediationsverfahren . . . . . . 235 
4.1.4.3.4.3 Der (temporäre) Ausschluss . . . . . . . 238

4.1.4.3.4.4 Der Gesellschafteraustritt als Ultima Ratio . 240

4.2 Das Maßnahmenraster zur Regelbefolgung . . . . . . . . . . . 241

5 Zusammenfassung und Ausblick . . . . . . . . . . . . . . . 243

5.1 Thesenartige Zusammenfassung der wichtigsten

Handlungsempfehlungen . . . . . . . . . . . . . . . . . 243

5.2 Limitationen . . . . . . . . . . . . . . . . . 244

6 Literatur . . . . . . . . . . . . . . . . . . . 247 


\section{Geleitwort von Arist von Schlippe}

Ein Kernthema der Forschung zu Familienunternehmen ist die Frage, wie das Verhältnis von Familie und Unternehmen langfristig konstruktiv gestaltet werden kann. Immer wieder stößt man dabei darauf, dass offenbar die Qualität der Family Governance als entscheidend dafür angesehen wird, wie und ob eine Familie über Generationen hinweg dauerhaft erfolgreich in der Umgebung ihres Unternehmens vorkommen kann. Die Frage erscheint zentral: Wie organisiert die Familie sich selbst, wie bewältigt sie die Aufgabe, ein Stück weit »Organisation « zu werden und zugleich (Unternehmer-)Familie bleiben zu können? Im Allgemeinen geht es hier um einen Prozess der bewussten Auseinandersetzung der Familie mit sich selbst. "Consciousness Raising", sich bewusst mit der Komplexität der eigenen Unternehmens- und Familienform auseinanderzusetzen, scheint der Schlüssel für Langfristigkeit zu sein, scheint zu gewährleisten, dass die Familienmitglieder die eigenen Interessen immer wieder gut mit den Interessen des Unternehmens ausbalancieren. Meist geht ein solcher Selbstauseinandersetzungsprozess mit der Erarbeitung eines Regelwerks einher, das dann als "Family Protocol« oder, in unserem Sprachraum, als »Familienverfassung « in eine verbindliche Form gebracht wird. Diese Verfassung, so sind sich alle Akteure, Betroffene wie Forscher, einig, sollte jedoch trotz dieses Namens keinen rechtsverbindlichen Charakter haben, da dadurch gerade ihr legitimatorischer Charakter als Ausformulierung des freien Familienwillens verlorenginge.

Die enge Verbindung der beiden Sozialsysteme Familie und Unternehmen bringt die Beteiligten immer wieder in sehr typische Dilemmata, denn als Kommunikationssysteme beziehen sich beide auf ganz widersprüchliche Logiken: Was in der einen Logik "richtig" ist, ist in der anderen Logik "falsch". Eine Familienverfassung dient dazu, die sich hier ergebenden Paradoxien zu entschärfen, indem für viele der Themen, die hier auftauchen, jeweils bewusst entschieden wird, welche Logik den Vorrang haben soll. Da dieses Regelsystem den Willen der Familie widerspiegelt, ist es eben - wie bereits gesagt - zugleich wichtig, dass eine Familienverfassung kein starr festgeschriebenes justiziables 
Konstrukt darstellt, sondern das Ergebnis eines intensiven Prozesses der Auseinandersetzung der Familie mit sich selbst.

Genau diese Grauzone des erarbeiteten Regelwerks zwischen unverbindlicher Absichtserklärung und juristischer Festschreibung bringt nun aber für das Familienmanagement neue Probleme mit sich. Denn es gibt keine unmittelbaren Sanktionsmöglichkeiten, wenn ein Familienmitglied sich nicht "verfassungsgemäß« verhält. Man kann mahnen, aber keine Geldstrafen verhängen, man kann das Verhalten eines Familienmitglieds unerträglich finden, aber es weder ins Gefängnis stecken noch aus der Familie ausschließen. Zugehörigkeit zur Familie ist ja ein unveräußerliches Recht. Wie kann aber ein Regelsystem seine Kraft behalten und durchgesetzt werden, wenn es keine Instrumente zur Durchsetzung gibt? Wie kann Family Compliance aufrechterhalten werden, wenn die »Toolbox" für die Akteure begrenzt ist, bzw. welche Werkzeuge stehen denn überhaupt zur Verfügung?

In der Praxis sehen sich viele Unternehmerfamilien gerade dem Konfliktfall, also dem Fall eines expliziten Regelverstoßes, auch dann recht hilflos ausgeliefert, wenn es eine Verfassung gibt, die den Verstoß eindeutig als solchen markiert. Obwohl es hier eindeutig Unterstützungsbedarf gibt, wurden diese Fragen von der Wissenschaft bislang nur am Rande behandelt, es fehlen wissenschaftlich gestützte Anregungen zur Handhabung derart schwieriger Fälle. Schon Themen wie Family Governance und Familienverfassung sind neue und bislang noch nicht erschöpfend beackerte Forschungsfelder, bei Fragen nach Family Compliance, also den Möglichkeiten, wie eine Familie sich selbst disziplinieren kann, liegen praktisch keine Erkenntnisse vor. Diese wichtige Forschungslücke hat Marco Henry V. Neumueller entdeckt, er ist hier in Neuland vorgestoßen und ist bedeutsame Schritte zur Aufarbeitung der sich hier ergebenden Fragen gegangen. Die vorliegende Arbeit fragt nach den Maßnahmen, durch die Unternehmerfamilien sicherstellen, dass die Regeln der Familienverfassung von den Mitgliedern befolgt werden.

Um sich seiner Forschungsfrage zu nähern, hat der Autor zunächst den Stand des Wissens zum Thema Familienverfassung ausführlich und geradezu handbuchartig dargestellt. Bereits dieser Teil ist ausgesprochen informativ und lesenswert. Anschließend bezieht er sich auf der Basis der eigenen empirischen Erkenntnisse auf die zentralen Fragen, welche Möglichkeiten auch innerhalb einer familiären Logik bestehen, sich mit kritischen Regelverstößen bzw. mit den entsprechenden Familienmitgliedern möglichst so konstruktiv auseinanderzusetzen, dass zukünftige Kooperation nicht unmöglich gemacht wird. In der Familie sind dabei Fragen danach, wie der Betroffene sein "Gesicht« wahren kann, sehr zentral, denn Familie kann man ja nicht einfach verlassen, nicht einfach "aufkündigen«. 
Bereits in den 1950er Jahren hatte der Systemtheoretiker W. Ross Ashby darauf hingewiesen, dass, wenn auf Komplexität mit vereinfachenden Handlungen reagiert wird, diese paradoxerweise immer schwerer steuerbar wird. Vereinfachung kann Komplexität massiv erhöhen, es können sich unsteuerbare Konflikte ergeben. Komplexität, so Ashby, muss mit Komplexität beantwortet werden (»law of requisite variety«), wenn man dies vermeiden will. Ich kann daher dieses grundlegende Buch jedem sehr empfehlen, der sich mit dem Thema beschäftigt, wie es einer Familie gelingen kann, sich langfristig als Unternehmerfamilie zu bewähren, und der daran interessiert ist, die Komplexität der Verbindung von Familie und Unternehmen nicht durch schlichte Vereinfachungen zu reduzieren, sondern mit angemessener Komplexität zu beantworten.

Witten, im Mai 2020

Arist v. Schlippe 
Open-Access-Publikation im Sinne der CC-Lizenz BY 4.0

(c) 2020, Vandenhoeck \& Ruprecht GmbH \& Co. KG, Göttingen ISBN Print: 9783847111719 - ISBN E-Lib: 9783737011716 


\section{Geleitwort von Brun-Hagen Hennerkes}

Die Dissertation von Marco Henry V. Neumueller bietet eine große Vielzahl an Erfahrungen aus dem Bereich des Familienunternehmens. Von besonderem Interesse sind seine Ausführungen zu dem Instrument der Familienverfassung.

Die Familienverfassung als Mittel der Streitvermeidung unter Familiengesellschaftern ist vergleichbar mit den Hausgesetzen des Adels. Sie ist juristisch zunächst eine reine Absichtserklärung ohne unmittelbare anspruchsbegründende rechtliche Wirkung. Sie beeinflusst allerdings den unter den Gesellschaftern vereinbarten Werte- und Verhaltenskodex und mittelbar auch die Auslegung des Gesellschaftsvertrags. Die Familienverfassung wird durch den Gesellschaftsvertrag in konkrete verbindliche Vertragsnormen umgesetzt. Sie ist das Gerüst, auf dem der Gesellschaftsvertrag und alle vertraglichen Vereinbarungen innerhalb der Unternehmerfamilie beruhen. Durch nichts kann dies besser verdeutlicht werden als durch das nachstehende Zitat aus der Familienverfassung einer bedeutenden deutschen Unternehmerfamilie:

"Wir haben großen Respekt vor der Arbeit und dem Engagement der uns vorangegangenen Generationen. Wir wollen deren ideelle Werte bewahren. Wir stellen uns dieser verantwortungsvollen Lebensaufgabe. Die Familienverfassung soll uns und den uns nachfolgenden Generationen helfen, die Verbundenheit gegenüber Unternehmen und Familie stets im Auge zu behalten. Wir wollen für die Zukunft eine gemeinsame unternehmerische Strategie verfolgen und unser Familienunternehmen auf Dauer erhalten. Hierzu werden wir mit Respekt und Fairness untereinander umgehen und geschlossen handeln. Unser unternehmerisches Werk kann nur dann erfolgreich fortgeführt werden, wenn sich die nachfolgenden Generationen - trotz Verschiedenheit in der Persönlichkeit - den in unserer Familienverfassung niedergelegten Regeln unserer Familie unterordnen."

Marco Henry V. Neumueller ist es gelungen, diese prägenden Gedanken einer erfolgreichen Unternehmerfamilie in seiner Dissertation zur Geltung zu bringen. 
Open-Access-Publikation im Sinne der CC-Lizenz BY 4.0

(c) 2020, Vandenhoeck \& Ruprecht GmbH \& Co. KG, Göttingen ISBN Print: 9783847111719 - ISBN E-Lib: 9783737011716 


\section{Vorwort und Danksagung}

Die vorliegende Publikation enthält eine gekürzte, inhaltlich leicht geänderte und damit praxisrelevantere Version meiner im Juli 2019 von der Universität Witten/ Herdecke angenommenen Dissertationsschrift. Sie befasst sich mit dem Instrument der Familienverfassung. Familienunternehmen fragen sich allgemein, wie sie das Verhältnis zwischen Familie und gemeinsamem Unternehmen langfristig konstruktiv gestalten können. Dies impliziert, dass die Überlebensfähigkeit des Familienunternehmens über Generationen verbessert und damit der langfristige Fortbestand des Unternehmens abgesichert werden soll.

Mit einer Familienverfassung wird der Unternehmerfamilie ein Instrument an die Hand gegeben werden, das helfen kann, destruktive Konflikte zu vermeiden oder beherrschbar zu machen, sehen sich doch viele Unternehmerfamilien gerade dem Konfliktfall - also dem Fall eines Regelverstoßes - recht hilflos gegenüber. Es herrscht meist Einigkeit darüber, dass dieses Regelsystem, trotz des möglicherweise irreführenden Namens, keinen rechtsverbindlichen Charakter haben soll. Vielmehr werden die Regeln als moralisch verbindlich verstanden. Umso mehr stellen sich Unternehmerfamilien berechtigterweise die Frage, von welchen Determinanten es abhängt, ob die in einer Familienverfassung festgelegten Spielregeln auch (möglichst) von allen Familienmitgliedern befolgt werden. Genau hier setzte die Untersuchung im Rahmen meines Dissertationsprojekts an. Ziel war es, Unternehmerfamilien dahingehend mit konkreten Vorschlägen zu präventiven und reaktiven Maßnahmen zu unterstützen.

Als externer Doktorand, neben Beruf und Familie, ein solches über mehrere Jahre dauerndes Forschungsvorhaben erfolgreich zu beenden, erfordert viel Idealismus, Durchhaltevermögen und insbesondere die vielfältige Unterstützung zahlreicher Personen, denen an dieser Stelle gedankt werden soll.

Zunächst möchte ich dem geschäftsführenden Direktor des Wittener Instituts für Familienunternehmen, Professor Dr. Tom A. Rüsen, danken, der mich auf die Forschungslücke aufmerksam machte und ermutigte, mich dieses praxisrelevanten Themas anzunehmen. Zu großem Dank bin ich Professor Dr. Arist von Schlippe und Professor Dr. Marcel Tyrell für die großartige persönliche und 
fachliche Zusammenarbeit sowie insbesondere für die Tatsache, dass sie auch in einer besonders schwierigen Situation zu mir standen, verpflichtet.

Ein ganz großes Dankeschön gebührt allen Teilnehmern der Fallstudien, ohne deren Unterstützung die Arbeit kaum hätte entstehen können. Sie alle haben mir mit erstaunlicher Offenheit und Geduld intime Einblicke gewährt, die man so kaum hätte erwarten können.

Dem Geschäftsführer der Stiftung Familienunternehmen, Stefan Heidbreder, sei an dieser Stelle sehr herzlich für die finanzielle Unterstützung gedankt. Der Geschäftsführerin der EQUA-Stiftung, Dr. Rena Haftlmeier-Seiffert, danke ich für die ideelle Förderung. Wenngleich ich lediglich die Teilnahme an einem Doktorandenkolloquium möglich machen konnte, so durfte ich mehrere spannende Menschen kennenlernen, mit denen ich noch heute in regelmäßigem Kontakt und Austausch stehe.

Schließlich gebührt mein allergrößter Dank meiner gesamten Familie. Ohne ihre bedingungslose Unterstützung und ihr grenzenloses Vertrauen wäre diese Arbeit vermutlich niemals fertig geworden.

Stuttgart, im Mai 2020

Marco Henry V. Neumueller

Mit Ihren Fragen, Anregungen und Kommentaren erreichen Sie den Autor unter: info@familienunternehmen.eu 


\section{Abbildungsverzeichnis}

Abbildung 1: Übersicht über den Aufbau der Arbeit 29

Abbildung 2: Das Drei-Kreis-Modell nach TAGIURI/DAVIS (1996) 37

Abbildung 3: Übersicht der unterschiedlichen Rollenbilder nach

TAGIURI/DAVIS (1996) 39

Abbildung 4: Gerechtigkeitslogiken von Familie, Unternehmen, Eigentum 44

Abbildung 5: Aufgaben und Funktionen einer Familienverfassung 51

Abbildung 6: Der Weg zur Familienstrategie $\quad 57$

$\begin{array}{ll}\text { Abbildung 7: } & \text { Die Family Governance Pyramide }\end{array}$

Abbildung 8: Die Family Business Governance $\quad 65$

Abbildung 9: $\quad$ Eigentümerthemen in der Evolution des

Abbildung 10: Gängige Umsetzungselemente einer Familienverfassung 71

Abbildung 11: Ü̈bersicht über gängige Inhalte einer Familienverfassung $\quad$ 74-76

Abbildung 12: Unterschiede zwischen Familienverfassung und

Gesellschaftsvertrag 93-94

Abbildung 13: Erweiterte Darstellung der Funktionen einer

Familienverfassung 198

Abbildung 14: Maßnahmenraster zur Regelbefolgung 242 
Open-Access-Publikation im Sinne der CC-Lizenz BY 4.0

(c) 2020, Vandenhoeck \& Ruprecht GmbH \& Co. KG, Göttingen ISBN Print: 9783847111719 - ISBN E-Lib: 9783737011716 


\section{Abkürzungsverzeichnis}

A. a.O. Am angegebenen Ort

Abs. Absatz

Abschn. Abschnitt

AG Aktiengesellschaft

AR Aufsichtsrat

Aufl. Auflage

bspw. beispielsweise

bzgl. bezüglich

bzw. beziehungsweise

CAQDAS Computer-Aided Qualitative Data Analysis Software

d. der/die/das

DCGK Deutscher Corporate Governance Kodex

d.h. das heißt

Ed. Edition

Ehem. Ehemalige(r)

Eingeh. Eingeheiratet

EQUA Eigentümer-Qualifizierungs-Akademie

et al. et alii (und andere)

etc. et cetera

evtl. eventuell

f. folgende Seite

ff. folgende Seiten

Gen. Generation

Geschäftsf. Geschäftsführer/in

ggf. gegebenenfalls

GKE Gesellschafterkompetenzentwicklung

GKFU Governance Kodex für Familienunternehmen

GL Geschäftsleitung

$\mathrm{GmbH} \quad$ Gesellschaft mit beschränkter Haftung

Hervorh. Hervorhebung(en)

Hrsg. Herausgeber

i.d.R. in der Regel 
i. S.d. im Sinne des

i.S.v. im Sinne von

Jg. Jahrgang

Kap. Kapitel

m.w.N. mit weiteren Nachweisen

Mio. Millionen

Mitgl. Mitglied

Mrd. Milliarden

Nr. Nummer

o.J. ohne Jahreszahl/-angabe

OHG Offene Handelsgesellschaft

PWC Das Unternehmen PricewaterhouseCoopers

Rn. Randnummer

Rz. Randziffer

ROI Return on Investment

s. siehe

S. $\quad$ Seite/n

sic sic erat scriptum (so stand es geschrieben)

sog. sogenannte

Tab. Tabelle

u. a. unter anderen

u. Ä. und Ähnliche

u.U. unter Umständen

usw. und so weiter

u.v.a. und viele andere

Verf. Verfasser

Vors. Vorsitzende(r)

vgl. vergleiche

vs. versus

WIFU Wittener Institut für Familienunternehmen

z.B. zum Beispiel

zit. zitiert 


\section{$1 \quad$ Einleitung}

\subsection{Einführung}

"Der Vater erstellt's, der Sohn erhält's, dem Enkel zerfällt's."

(Buddenbrooks) ${ }^{12}$

Ungefähr 90 Prozent der Unternehmen in Deutschland sind Familienunternehmen, welche etwa 50 Prozent zum Gesamtumsatz in Deutschland beitragen. ${ }^{3}$ So ist es schon eine traurige Erkenntnis, die im Laufe der Jahre in der wirtschaftswissenschaftlichen Forschung reifte: Ein Familienunternehmen scheitert letztlich in seltenen Fällen am Markt, sondern in den meisten Fällen an der

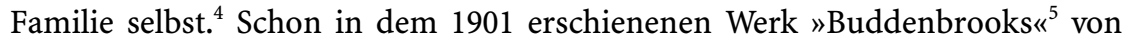
Thomas Mann findet sich die als Eingangszitat verwendete und im Volksmund häufig kolportierte Triade, deren Relevanz jeder für sich selbst in der Realität überprüfen mag. ${ }^{6}$ In den 1980er Jahren untersuchte WARD (2011) erstmals sys-

1 Mann (1901); Villalonga/Amit (2006) haben beispielsweise empirisch analysiert, dass die Werteentwicklung deutlich an Leistung einbüßt, sobald Unternehmen nicht mehr länger von ihren Gründern geführt oder mittels Aufsichtsrat/Beirat beeinflusst werden.

2 Der besseren Verständlichkeit und Lesefreundlichkeit wegen wird im Text das generische Maskulinum verwendet, das Frauen gleichermaßen einschließt. Wenn also beispielsweise von Gesellschafter, Unternehmer etc. die Rede ist, schließt diese Formulierung die weibliche Variante ebenfalls mit ein.

3 Die Zahlen variieren leicht, wenn man »familienkontrollierte Unternehmen« und »eigentümergeführte Unternehmen « unterscheidet. Da hier lediglich eine grobe Richtgröße gegeben werden soll, kann dieser relativ kleine Unterschied vernachlässigt werden. Vgl. http://www.fa milienunternehmen.de/de/daten-fakten-zahlen. Zuletzt geprüft am 01.03.2020.

4 Vgl. Miller et al. (2013), S. 40; Vernekohl (2007), S. 33.

5 Mann (1901).

6 Ähnliche Zitate finden sich auch in anderen Sprach- und Kulturkreisen. So ist beispielsweise in den USA der Ausspruch »shirt sleeves to shirt sleeves in three generations« weit verbreitet. Gemeint ist damit ein Drei-Generationen-Kreislauf à la »rags-to-riches-to-rags«; vgl. Ward (2005), S. 13. 
tematisch die Nachfolge in Familienunternehmen. ${ }^{7}$ Diese und weitere Studien gelangten zu der Erkenntnis, dass nicht einmal zwei Drittel der Familienunternehmen die zweite Generation überleben, wohingegen lediglich etwa ein Drittel die dritte Generation überdauern. Die vierte Generation erreichen je nach Studie lediglich zehn bis 15 Prozent. $^{8}$ Dem Wechselspiel der Kräfte von Macht, Geld und Liebe kann selbst die stärkste Familie erliegen. ${ }^{9}$ Vorrangiges Ziel bei einem Familienunternehmen ist fast immer der Erhalt und Verbleib des Unternehmens in der Familie - nicht zuletzt auch, um die Existenz der Eigentümerfamilie sowie im besten Fall zukünftiger Generationen zu sichern. ${ }^{10}$

Was könnte diese Existenz gefährden? »Der größte Wertvernichter in Familienunternehmen ist der Streit. ${ }^{11}$ Gründe für Streit finden sich ständig. Sei es, dass zwischen dem Sohn und der Tochter ein interner Konkurrenzkampf um die Unternehmensnachfolge und damit um die Gunst des Vaters ausbricht - wobei grundsätzlich zu klären wäre, ob einer der beiden überhaupt objektiv für die Nachfolge geeignet erscheint $-{ }^{12}$, oder dass ein Gesellschafter dem Unternehmen den Rücken kehrt und das Unternehmen durch die Auszahlung seiner Gesellschaftsanteile in eine existenziell bedrohliche Lage bringt.

Es gehört zu den nicht ganz einfachen täglichen Herausforderungen von Familienunternehmern, den Spagat zwischen Familie und Unternehmen zu meistern. Wo immer mehr Menschen mit teils unterschiedlichen Intentionen aufeinandertreffen, steigt die Komplexität zwangsläufig. Die angesprochene Komplexität umfasst im Wesentlichen drei Konstellationen: Unternehmen versus Familie, Gemeinschaft versus Individuum und ältere Generation versus jüngere Generation. ${ }^{13}$ Hier setzt die Familienverfassung an und will als »[g]roße Klammer ${ }^{14}$ zwischen den Generationen und dem Unternehmen gesehen werden. ${ }^{15}$ So

7 Vgl. Ward (2011), S. 2, wobei die Studie nicht näher spezifizierte »200 successful manufacturers" umfasst. Auch eine lokale Eingrenzung fehlt.

8 Vgl. Ward (2005), S. 1; Ward (2011), S. 2; Hennerkes (1998), S. 373. Laut Jaskiewicz et al. (2005) erreichen weniger als $15 \%$ die dritte Generation, vgl. Jaskiewicz et al. (2005), S. 180. Groth (2011) kommt zum Schluss, dass lediglich unter $5 \%$ der Familienunternehmen bis in die vierte Generation überleben, vgl. Groth (2011), S. 30.

9 Vgl. dazu auch Simon (2001), S. 333 ff.; Gläßer (2014), S. 237.

10 Vgl. Prym (2014), S. 15. So ist es kaum verwunderlich, dass "nicht in Quartalen, sondern in Generationen« gedacht wird, vgl. May (2008), S. 419.

11 Hennerkes/Kirchdörfer (2015), S. 62. Zu einem ähnlichen Schluss kommend Ward (2004), S. 6: »The most critical issues facing business-owning families are family-based issues more than they are business-based issues.« Vgl. dazu auch Rüsen (2017b); Levinson (1971), S. 90ff.; Davis/Harveston (2001), S. 14ff. Ein Phasenmodell vom Konflikt zum Untergang des Familienunternehmens zeigend Großmann (2014), S. 143.

12 Vgl. dazu auch Hauck (2012b).

13 Vgl. Born (2014), Rn. 49.

14 Koeberle-Schmid (22.03.2017).

15 Prigge/Braun/Kleefass (18.12.2014). 
ist dieser Spagat zwischen Familie und Unternehmen am wahrscheinlichsten mit von allen akzeptierten Spielregeln zu meistern. ${ }^{16}$ Eine Familienverfassung möchte Leitlinien - und meint damit Strategie, Struktur und Spielregeln - sowohl für die Familie als auch im Hinblick auf das gemeinsame Unternehmen aufstellen. Dabei schafft die Familienverfassung Transparenz, erhöht die Sicherheit für jeden einzelnen und professionalisiert die Zusammenarbeit. ${ }^{17}$ Sie möchte als Instrument zur Streitvermeidung verstanden werden, das einen "grundlegenden Wertekonsens in zentralen Fragen rund um das Verhältnis zwischen Familie und Unternehmen unter den Beteiligten herstellt. « ${ }^{18}$

Die Verbreitung der Familienverfassung in Deutschland steht allerdings erst am Anfang ${ }^{19}$. In Ermangelung fundierter empirischer Untersuchungen darüber, in wie vielen Familienunternehmen tatsächlich eine Familienverfassung existiert, lässt sich diese Frage nicht präzise genug beantworten. Eine im Jahre 2011 durchgeführte stichprobenartige Befragung von 148 Inhabern deutscher Familienunternehmen ergab zumindest eine erste Tendenz: Etwa ein Viertel der befragten Unternehmen haben eine Familienverfassung. Immerhin zirka 50 Prozent der Unternehmen, die zu diesem Zeitpunkt keine hatten, zogen ernsthaft in Erwägung, in naher Zukunft ein solches Regelwerk erstellen zu wollen. ${ }^{20}$ Der Mehrwert einer Familienverfassung im Sinne eines positiven Einflusses auf die Gesamtsituation innerhalb der Unternehmerfamilie soll in dieser vorliegenden Arbeit vorausgesetzt werden. Die vorgenannte Studie gibt auch dazu eine erste Indikation. Die Nutzer einer Familienverfassung sehen ihre Erwartungen an diese in der Praxis in hohem Maße erfüllt: sowohl in emotionaler als auch in ökonomischer Hinsicht. ${ }^{21}$ So wird auch in der Literatur die positive Wirkung einer Familienverfassung nicht in Frage gestellt. ${ }^{22}$

Wenngleich dieser Mehrwert einer Familienverfassung ganz grundsätzlich nicht in Frage gestellt wird, so lässt sich dennoch feststellen, dass die Grundlage für die Entfaltung ihrer positiven Eigenschaften häufig zu kontroversen Diskussionen führt, die nicht selten in Ausführungen zur rechtlichen Bindungs-

16 Vgl. Stöhlker/Müller Tiberini (2005), S. 11.

17 So im Ergebnis wohl auch Kormann (2018), S. 266.

18 Hennerkes/Kirchdörfer (2015), S. 65.

19 In den USA scheinen Familienverfassungen in den vergangenen zehn bis 20 Jahren zum Trend geworden zu sein; vgl. dazu McClain (2006), S. 837; Montemerlo/Ward (2011), S. xiii.

20 Vgl. Schween et al. (2011), S. 9; darauf bezugnehmend Kirchdörfer/Lorz (2011), S. 98. Für eine sicherlich nicht abschließende Aufzählung der Unternehmerfamilien mit bereits vorhandener Familienverfassung oder zumindest einer in der Entstehung begriffenen vgl. Hueck (2017), S. 15, 18.

21 Weitere Ausführungen dazu in Schween et al. (2011), S. 9 f.

22 Vgl. Kormann (2018), S. 181. So im Ergebnis wohl auch Kirchdörfer/Lorz (2011), S. 98; Gläßer (2014), S. 236. 
wirkung eines solchen Instruments münden. ${ }^{23}$ In diesem Zusammenhang mahnt GLÄSSER (2014) zu Recht an, die Betrachtung der "Leistungsfähigkeit» einer Familienverfassung nicht immer nur in Verbindung mit der materiellen Bindungswirkung der Inhalte $\mathrm{zu}$ sehen, sondern vielmehr deren "prozedurale Wirkung « näher zu untersuchen. ${ }^{24}$ Dies spricht bereits für die Relevanz dieser Arbeit. Familienverfassungen haben trotz ihrer praktischen Relevanz bislang noch keine vertiefte wissenschaftliche Betrachtung erfahren. ${ }^{25}$ Die Literatur scheint vielmehr durch die Beraterpraxis geprägt $\mathrm{zu}$ sein. ${ }^{26}$ Die spärlich vorhandene Literatur zu Familienverfassungen handelt zumeist lediglich von den Inhalten und der Herangehensweise zur Erstellung einer solchen. Das Fehlen einer wissenschaftlichen Untersuchung, die jene Maßnahmen beleuchtet, die die Wahrscheinlichkeit der Regelbefolgung durch die Familienmitglieder erhöhen, spricht für die Dringlichkeit einer solchen Forschungsarbeit und damit für deren wissenschaftliche Relevanz.

Es lässt sich festhalten, dass der Mehrwert einer Familienverfassung für die Familie und damit auch für den langfristigen Fortbestand des Unternehmens von besonderer Bedeutung ist und somit geeignete Maßnahmen ergriffen werden sollten, um sicherzustellen, dass die aufgestellten Regeln stets auch möglichst von allen befolgt werden ( $»$ Family Compliance $\left.{ }^{27}\right)$. Diese Maßnahmen zu definieren und den Unternehmerfamilien an die Hand zu geben ist die grundlegende Zielsetzung dieser Arbeit.

\subsection{Zielsetzung und Forschungsaufbau}

Die vier Ziele der vorliegenden Forschungsarbeit lassen sich wie folgt beschreiben:

1. Die Einordnung der Familienverfassung in den wissenschaftlichen Kontext des noch immer recht jungen Forschungsfelds Familienunternehmen.

2. Die Vorstellung des Forschungsgegenstandes Familienverfassung als besonderes Instrument der Family Governance zur Konfliktprävention sowie Sicherung von Frieden und Stabilität in der Unternehmerfamilie als Garant für

23 Vgl. nur Hueck (2017).

24 Vgl. Gläßer (2014), S. 236.

25 Vgl. Hueck (2017), S. 2.

26 Vgl. Gläßer (2014), S. 229; Uffmann (2015), S. 2446; Kormann (2018), S. 181.

27 Unter Family Compliance versteht man in Anlehnung an das betriebswirtschaftliche Begriffsverständnis die Bereitschaft zur Einhaltung eines freiwilligen Kodex (i.S.v. einer Familienverfassung), den sich eine Unternehmerfamilie gegeben hat; vgl. dazu Rüsen (2016); Schlippe/Groth/Rüsen (2017), S. 33. 
den Bestand des Familienunternehmens über Generationen - mit Hauptaugenmerk auf ihre Inhalte, Bindungswirkung und Legitimation.

3. Die qualitativ-empirische Untersuchung der Regelbefolgung von Familienverfassungen deutscher Unternehmerfamilien in der Praxis - unter Berücksichtigung theoretischer Vorüberlegungen.

4. Die Ableitung konkreter und in der Praxis anwendbarer präventiver und reaktiver Maßnahmen zur Einhaltung der Regelbefolgung einer Familienverfassung (»Family Compliance«) sowie Denkanstöße für weiterführende Forschungsarbeiten.

Aus den zuvor ausgeführten Untersuchungszielen lässt sich die folgende Forschungsfrage ableiten:

Welche Maßnahmen ergreifen Unternehmerfamilien, um sicherzustellen, dass die Regeln einer Familienverfassung von allen Familienmitgliedern befolgt werden?

Um dieser Fragestellung nachgehen zu können, werden folgende Unterfragen zu beantworten sein: Hat der Grund der Erstellung eine Auswirkung auf die Einhaltung der Regeln? Hat die Art der Erstellung einen Einfluss auf die Qualität der Regelbefolgung? Welche Institutionen der Familienverfassung helfen, die Regelbefolgung positiv zu beeinflussen? Welche weiteren präventiven Maßnahmen können die Wahrscheinlichkeit der Regeltreue erhöhen? Welche Reaktionen sind bei einem Regelbruch erforderlich?

Um diese Forschungsfragen beantworten zu können, bedarf es auf Grund der derzeit noch unzureichenden theoretischen Erkenntnisse über Familienverfassungen einer empirischen Untersuchung. Mithin wurde eine explorativ geprägte Vorgehensweise ${ }^{28}$ gewählt. Der Arbeit liegt ein induktives Forschungsdesign zugrunde, das sich durch ein qualitatives Vorgehen auszeichnet.

Im Kern wurden hierfür Fallstudien über acht deutsche Familienunternehmen und deren Erfahrungen mit Familienverfassungen erstellt und ausgewertet. Diese empirische Untersuchung hat Neuheitscharakter, da sie in diesem Umfang die einzige ihrer Art ist. Dies betrifft sowohl die Fragestellung und Zielsetzung als auch die empirische Forschungsarbeit. Hierdurch möchte die vorliegende Arbeit einen wichtigen Beitrag im Bereich der Anwendung von Familienverfassungen in der Familienunternehmensforschung leisten.

28 Vgl. Kaiser (2014), S. 29: In explorativen Forschungsdesigns wird Expertenwissen zur Gewinnung von Informationen über bisher wenig erforschte Fragestellungen genutzt. Explorative Studien werden unternommen, wenn zu einem wissenschaftlich relevanten Problembereich bisher keine oder kaum gesicherten theoretische Annahmen oder belastbare empirische Daten vorliegen. Bortz/Döring (2006), S. 360: Man verfolgt damit das Ziel, "Sachverhalte zu erkunden, zu erforschen oder ausfindig zu machen«. 


\subsection{Gang der Untersuchung}

Die vorliegende Forschungsarbeit ist gemäß der Forschungsfrage sowie der Zielsetzungen und dem Forschungsaufbau gestaltet und unterteilt sich in fünf Kapitel. Abbildung 1 illustriert den Gang der Untersuchung.

$\mathrm{Zu}$ Beginn werden in Kapitel 2 die theoretischen Grundlagen für diese Arbeit gelegt. Es wird zunächst das Familienunternehmen als eine Unternehmensform mit besonderen Stärken und Schwächen vorgestellt (Abschnitt 2.1). Es folgt eine ausführliche Darstellung der Familienverfassung als Instrument der Family Governance (Abschnitt 2.2). Nachdem die Gestaltungspraxis näher beleuchtet wurde (Abschnitt 2.3), wird die Bindungswirkung dieses Instruments erläutert (Abschnitt 2.4). Abschließend wird der Legitimationsgedanke der Familienverfassung im Lichte privatrechtlicher und moralphilosophischer Ansätze diskutiert (Abschnitt 2.5).

In Kapitel 3 folgt eine übersichtliche Darstellung aller acht Fallstudien.

Die wesentlichen Erkenntnisse der fallübergreifenden Auswertung werden in Kapitel 4 überblicksartig dargestellt. Diese Darstellung erfolgt unterteilt nach den Themen: Grund der Erstellung, präventive und reaktive Maßnahmen zur Regelbefolgung (Abschnitt 4.1) sowie ein davon abgeleitetes Maßnahmenraster (Abschnitt 4.2).

Die Arbeit schließt mit einer Schlussbetrachtung in Kapitel 5, in der konkrete Handlungsempfehlungen für den Umgang mit Familienverfassungen in der Praxis gegeben (Abschnitt 5.1) und die Begrenzung der vorliegenden Untersuchung sowie weiterführende Forschungsdesiderate aufgezeigt werden (Abschnitt 5.2). 
Kapitel 2

Theoretische Grundlagen

Familienunternehmen - Stärken und Schwächen

Familienverfassung als Instrument der Family Governance

Die Gestaltungspraxis

Die Bindungswirkung

Der Legitimationsgedanke

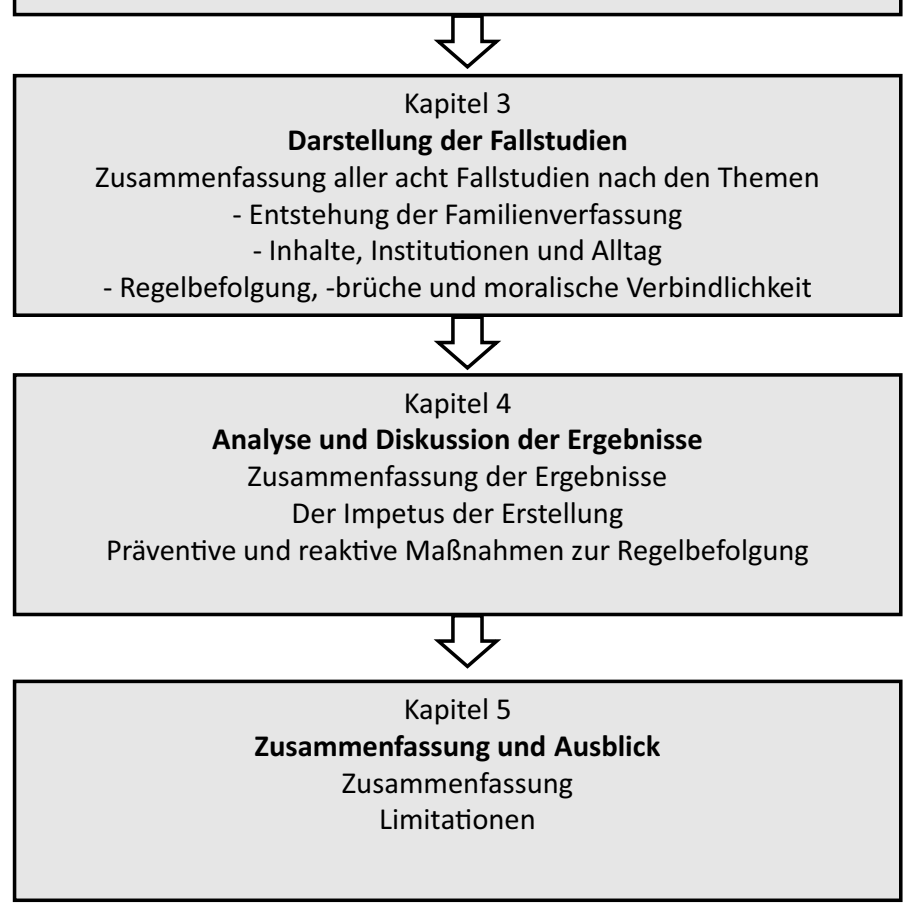

Abbildung 1: Übersicht über den Aufbau der Arbeit ${ }^{29}$

29 Eigene Darstellung. 
Open-Access-Publikation im Sinne der CC-Lizenz BY 4.0

(c) 2020, Vandenhoeck \& Ruprecht GmbH \& Co. KG, Göttingen ISBN Print: 9783847111719 - ISBN E-Lib: 9783737011716 


\title{
2 Theoretische Grundlagen
}

Einer ersten Einleitung in das Thema sowie der Darstellung der Zielsetzung und Relevanz dieser Arbeit folgend sollen in diesem Teil die theoretischen Grundlagen bzw. der "theoretische Bezugsrahmen ${ }^{30}$ gelegt werden, worauf sich die anschließende empirische Untersuchung beziehen wird.

\subsection{Das Familienunternehmen: eine besondere Unternehmensform mit Stärken und Schwächen}

\author{
»Wenn man eine große Sache beginnt, ist es nicht \\ gut, wenn man mit dem Schornstein und Dach anfängt, \\ sondern erst muss das Fundament da sein. Das Fundament \\ aber ist das Elternhaus, die Kinderstube, die Familie."
} $\left(\right.$ Friedrich »Fritz« Karl Henkel) ${ }^{31}$

30 Kirsch (1974), S. 26: Ein theoretischer Bezugsrahmen umfasst »eine Reihe theoretischer Begriffe, von denen angenommen wird, dass sie einmal Bestandteil von Modellen bzw. Theorien werden könnten. Darüber hinaus umfasst jedoch ein theoretischer Bezugsrahmen auch einige freilich sehr allgemeine Gesetzeshypothesen, die jedoch meist nur tendenzielle Zusammenhänge andeuten. Insofern ist ein theoretischer Bezugsrahmen nicht nur ein Vokabular, mit dem man über einen bestimmten Gegenstandsbereich sprechen kann. In erster Linie dient ein theoretischer Bezugsrahmen dazu, das Denken über komplexe reale Systeme zu ordnen und zu exploratorischen Beobachtungen anzuleiten, die mit der Zeit eine genügend große Zahl von Beobachtungsaussagen erbringen, um konkrete Modelle mit konkreten Gesetzeshypothesen formulieren zu können.»

31 Ein Zitat von Fritz Henkel, dem Gründer des Henkel Konzerns anlässlich des 50-jährigen Firmenjubiläums der deutschen Henkel Gruppe, Hennerkes/Berlin/Berlin (2007), S. 40. 


\subsubsection{Das Forschungsfeld Familienunternehmen}

Familienunternehmen leisten in Deutschland - aber auch in anderen Volkswirtschaften - einen nicht unerheblichen Anteil zum Gesamtumsatz ${ }^{32}$ und stellen global gesehen die vorherrschende Unternehmensform dar ${ }^{33}$; so werden sie daher auch gerne als das Rückgrat der Wirtschaft bezeichnet. ${ }^{34}$ Wurde diesem Organisationstypus lange Zeit keine besondere Beachtung geschenkt, so schlug sich die wachsende Wahrnehmung der ökonomischen Relevanz dieser Unternehmensform auch im zunehmendem Interesse der Wissenschaft an diesem Forschungsgebiet nieder. ${ }^{35}$ In den 1960er/ 1970er Jahren begann die Erforschung von Familienunternehmen als Organisationsphänomen - beginnend mit einer überschaubaren Anzahl an Veröffentlichungen von vornehmlich amerikanischen Autoren. ${ }^{36}$ Bis vor einigen Jahren befasste sich gerade einmal etwa ein Prozent der wirtschaftswissenschaftlichen Publikationen mit dem Forschungsgebiet der Familienunternehmen. ${ }^{37}$ Heutzutage kann diesem Forschungsfeld die wissenschaftliche Relevanz schon lange nicht mehr abgesprochen werden ${ }^{38}$; und dennoch befindet es sich noch immer im Entwicklungsstadium ${ }^{39}$, obwohl sich mittlerweile zahlreiche Disziplinen, wie die Psychologie, Rechtswissenschaft, Soziologie oder Ökonomie, der Erforschung der Charakteristika von Familienunternehmen angenommen haben. ${ }^{40}$

32 Vgl. Fn. 3; darüber hinaus auch Wiechers (2006), S. 11; Haunschild/Wolter (2010), S. 26.

33 Vgl. Sharma/Chrisman/Gersick (2012), S. 5; La Porta/Lopez-de-Silanes/Shleifer (1999), S. 498 f.

34 Vgl. Poutziouris/Smyrnios/Klein (2008), S. 1; auch darauf verweisend Binder (2014), S. 27.

35 Vgl. Binder (2014), S. 28. Plate et al. (2011), S. 12: »Die öffentliche Wertschätzung für Familienunternehmen war noch nie so hoch wie in heutiger Zeit. Das in Unternehmen gepflegte Langfristdenken sowie die Identifikation und auch die emotionale Bindung zwischen Familieneigentümern und der unternehmerischen Aufgabe sind als Werte für die Wirtschaft erkannt worden.«

36 Vgl. Gersick et al. (1997), S. 4f.; Heck/Hoy/Poutziouris (2008); Siebels/Knyphausen-Aufseß (2012), S. 280; vgl. zu frühen Werken bspw. u. a. Calder (1961); Donnelly (1964); Barry (1989).

37 Vgl. Binder (2014), S. 28, der auf die Ausführungen von Dyer (2003), S. 402 referenziert: Die Analyse bezieht sich auf 59 Artikel im "Academy of Management Journal» sowie auf 69 Artikel im »Academy of Management Review«, von welchen lediglich ein Artikel das Familienunternehmen als Variable berücksichtigt.

38 Vgl. Craig/Salvato (2012), S. 109; Craig et al. (2009), S. $282 \mathrm{ff.}$

39 Vgl. Chrisman et al. (2008), S. 931.

40 Vgl. Wiechers (2006), S. 11.; Hueck (2017), S. 22 Fn. 113 m. w. N. 


\subsubsection{Definition eines Familienunternehmens im Rahmen der Untersuchung}

Nachfolgend soll eine begriffliche Abgrenzung erfolgen, welche Unternehmen im Rahmen dieser Forschungsarbeit als Familienunternehmen zu betrachten und welche auszuschließen sind.

Bis heute scheint keine einheitliche Definition von Familienunternehmen zu existieren. ${ }^{41}$ Bereits Lansberg/Perrow/Rogolsky (1988) forderten im ersten Artikel des »Family Business Review» eine solche einheitliche Definition, mussten aber zeitgleich die damit verbundenen Schwierigkeiten einräumen. ${ }^{42}$ Pfannenschwarz (2006) fand allein in der deutschen Literatur mehr als 70 abweichende Definitionen. ${ }^{43}$ Die Dichotomisierung eines Unternehmens in Familienunternehmen und Nicht-Familienunternehmen erfolgt in aller Regel, je nach Forschungsfrage, unterschiedlich. So ist der Begriff des Familienunternehmens eher dem Volksmund entsprungen. Jedenfalls ist keine spezifische Rechtsform damit verbunden. ${ }^{44}$ Kurioserweise wissen trotzdem die meisten in etwa, was mit einem Familienunternehmen gemeint ist, wenngleich jedem eine exakte Begriffsdefinition sicherlich schwerfallen würde. ${ }^{45}$

Die meisten Definitionsansätze entstammen vornehmlich der ökonomischen und soziologischen Literatur. ${ }^{46}$ Die verschiedenen Ansätze sowie deren Spezifika zu diskutieren, würde den Rahmen dieser reduzierten Arbeit sprengen. ${ }^{47}$ Daher soll lediglich kurz der Ansatz vorgestellt werden, welcher für die vorliegende Forschungsarbeit am besten geeignet zu sein scheint. Auf Grund des gewählten qualitativen Forschungsansatzes eignet sich ein recht weit gefasstes Verständnis des Begriffs Familienunternehmen. Darüber hinaus sollte die verwendete Definition zeitgleich eine eindeutige Abgrenzung ermöglichen, um Familienunternehmen eindeutig von Nicht-Familienunternehmen abgrenzen zu können. Mit diesen beiden Anforderungen als Voraussetzung eignet sich die Definition des WIFU.

Demnach spricht man immer dann von einem Familienunternehmen, wenn sich ein Unternehmen

41 Vgl. Schlippe/Groth/Rüsen (2017), S. 27; Harms (2014); Astrachan/Klein/Smyrinos (2002); Litz (1995); Klein (2010), S. 12; Wiechers (2006), S. 48; Chrisman/Chua/Sharma (2003), S. 2; Zahra/Sharma (2004), S. 333; Schlippe (2011), S. 19.

42 Vgl. Lansberg (1999), S. $1 \mathrm{f}$.

43 Vgl. Pfannenschwarz (2006), S. 23. Vgl. dazu auch Tänzler (2014), S. 9 ff., der bereits die Reichweite des Begriffs »Familie« hinterfragt.

44 Vgl. Wimmer et al. (2018), S. 6.

45 Vgl. Wimmer et al. (2018), S. 6. Vgl. hierzu auch die Ausführungen von Hueck (2017), S. $22 \mathrm{ff}$.

46 Zur Übersicht Tänzler (2014), S. $13 \mathrm{f}$.

47 Für eine umfassende Übersicht der verschiedenen Definitionsansätze vgl. Redlefsen (2004), S. $5 \mathrm{ff}$; Klein (2010), S. $12 \mathrm{ff}$;; Mertens (2009), S. $423 \mathrm{ff}$; Wiechers (2006), S. $31 \mathrm{ff}$. 
- "ganz oder teilweise im Eigentum einer Familie oder mehrerer Familien bzw. Familienverbänden befindet und wenn diese aus einer unternehmerischen Verantwortung heraus die Entwicklung des Unternehmens maßgeblich bestimmen.

- Diese Verantwortung der Unternehmerfamilie(n) wird entweder aus einer Führungs- oder Aufsichtsfunktion bzw. aus beiden Funktionen heraus wahrgenommen. Dabei spielen die Rechtsform und Größe des Unternehmens keine Rolle.

- Bei einem Unternehmen kann [...] erst dann von einem Familienunternehmen gesprochen werden, wenn in der Familie geplant wird, das Unternehmen in die nächste Familiengeneration weiterzugeben.» ${ }^{48}$

\subsubsection{Familienunternehmen: eine unmögliche Kopplung sozialer Systeme?!}

Um die Wirkweise einer Familienverfassung besser einordnen zu können, bedarf es eines eingehenderen Verständnisses der verschiedenen, sich gegenseitig beeinflussenden Dimensionen innerhalb eines Familienunternehmens. Bis in die 1980er Jahre hinein gab es recht wenige theoretische Arbeiten über Familienunternehmen. Diese beschäftigen sich vornehmlich mit typischen Problemfeldern wie unprofessionelles Management, Rivalitäten zwischen Geschwistern, Generationenkonflikte oder auch Vetternwirtschaft. ${ }^{49}$ Darüber hinaus finden sich zahlreiche Publikationen zur Problematik bei der Nachfolge in Familienunternehmen. ${ }^{50}$ Mittlerweile existiert eine große Anzahl an Theorien, die sich mit dem komplexen Geschehen innerhalb von Familienunternehmen konzeptuell befassen. ${ }^{51}$ In der Forschung über Familienunternehmen kann man einen gewissen "Grundkonsens ${ }^{52}$ ausmachen, dass ein Systemblick (»a >systems approach $~{ }^{53}$ ) ein guter Ausgangspunkt ist, um die diverseren "Analyseebenen angemessen zu rekonstruieren. ${ }^{54}$ Dieser Konsens allein führte jedoch noch nicht zwingend dazu, dass eine einheitliche Theorie existiert. ${ }^{55}$ Das Gegenteil ist der Fall: Es werden in der Forschung verschiedene theoretische Ansätze diskutiert.

$48 \mathrm{https://www.wifu.de/forschung/definitionen/familienunternehmen;} \mathrm{zuletzt} \mathrm{geprüft} \mathrm{am} \mathrm{01.03.}$ 2020.

49 Vgl. Barnes/Hershon (1976).

50 Für den englischsprachigen Raum findet sich eine Übersicht bei Handler (1994), für den deutschsprachigen Raum bei Albach (1989), S. 23ff. oder auch bei Klett (2005), S. $35 \mathrm{ff}$.

51 Für einen aktuellen Überblick vgl. Melin/Nordqvist/Sharma (2014).

52 Schlippe/Groth/Rüsen (2017), S. 73.

53 Zahra/Klein/Astrachan (2008), S. 614.

54 Schlippe/Groth/Rüsen (2017), S. 73.

55 Vgl. Wiechers (2006), S. 11; Simon (2011b), S. 7f.; Simon/Wimmer/Groth (2017), S. 18; Schlippe/Frank (2013), S. 384. 
Neben Wirtschaftswissenschaftlern haben auch Psychologen, Soziologen und Juristen ein besonderes Interesse an diesem Unternehmenstypus entwickelt. Jede der genannten Disziplinen greift jedoch vornehmlich auf ihre eigenen Theorien und Modelle zurück; nicht zuletzt erschwert auch dieser Tatbestand die Formulierung einer einheitlichen und allgemein anerkannten Theorie über Familienunternehmen.

So greifen beispielsweise die Wirtschaftswissenschaftler auf die PrinzipalAgenten- und Stewardship-Theorien sowie auf den ressourcenbasierten Strategieansatz zurück, wenn sie die Unterschiede zwischen Familienunternehmen und Nicht-Familienunternehmen zu erklären versuchen. ${ }^{56}$ Diese rein betriebswirtschaftlichen Ansätze müssen dann versagen, wenn die gesamte Komplexität von Familienunternehmen erfasst werden soll. Insbesondere wenn die Wechselwirkung zwischen Unternehmerfamilie und Unternehmen in den Fokus der Betrachtung rückt, stoßen die vorgenannten betriebswirtschaftlichen Ansätze schnell an ihre Grenzen.

Vielversprechender ist ein ganzheitlich orientierter Ansatz: Die Systemtheorie nach LuHmann $(1984)^{57}$, welche »soziale Systeme aller Art « untersucht und »deshalb eine Theorie sowohl der Familie wie des Unternehmens als auch der Kopplung und Ko-Evolution beider Systeme im Laufe ihrer gemeinsamen Geschichte in einem sich ändernden gesellschaftlichen Kontext zur Verfügung“ stellen kann. ${ }^{58}$ Dieser soziologische Ansatz findet insbesondere in der deutschen Forschungslandschaft über Familienunternehmen Anwendung. ${ }^{59}$ Das WIFU entwickelt diesen theoretischen Ansatz beständig weiter und versucht ihn als festen Bestandteil in der Forschung über Familienunternehmen zu verankern.

"Die neuere soziologische Systemtheorie ist unseres Erachtens am besten geeignet, Erklärungsmodelle für die vielfältigen Situationen und Gefühle der Zerrissenheit von Familienmitgliedern zu liefern und die Komplexität der Unternehmerfamilien zu erfassen. ${ }^{60}$

Im Rahmen dieses theoretischen Ansatzes werden soziale Phänomene unter systemischen Gesichtspunkten betrachtet. Die Subsysteme der Familienunternehmen (Unternehmen, Familie, Eigentümer ${ }^{61}$ ) werden dabei als autonome Kommunikationssysteme verstanden, die sich auf eine besondere Art und Weise

56 Vgl. Wirsching (2017), S. 15ff., die sich der beiden theoretischen Ansätze bedient, um den Familieneinfluss auf den Unternehmenserfolg zu erläutern.

57 Vgl. Luhmann (1984).

58 Simon (2012), S. 10.

59 Vgl. Stamm (2013), S. 75.

60 Schlippe/Groth/Rüsen (2017), S. 73.

61 Vgl. Schlippe/Frank (2013), S. 384. 
entwickeln. ${ }^{62}$ Darunter versteht man, dass die sozialen Systeme ausschließlich aus Kommunikation bestehen. ${ }^{63}$ Diese Systeme definieren sich somit nicht über die zugehörigen Menschen und deren Interaktionen, sondern alleine über deren spezifische Kommunikationsmuster ${ }^{64}$ Legt man diesen systemtheoretischen Ansatz zu Grunde, wird recht schnell klar, dass es sich bei den angesprochenen Subsystemen Unternehmen, Familie und Eigentümer um Sozialsysteme handelt, die jeweils einer eigenen Kommunikationslogik folgen. ${ }^{65}$

»Vereinfacht gesprochen geht es sozusagen um Spiele, die durch spezifische Regeln bestimmt sind. ${ }^{66}$

Diese Logiken lassen sich bezogen auf die drei Subsysteme entsprechend zusammenfassen: Im Unternehmen dominiert die »Entscheidungs-kommunikation ", die "Bindungskommunikation" herrscht im Subsystem Familie vor und dem Eigentümerkreis wird die »Rechtskommunikation « zugeschrieben. ${ }^{67}$

Jedes System folgt damit eigenen "Spielregeln« ${ }^{68}$ SCHLIPPE/GROTH/RüSEN (2017) führen in diesem Kontext ein treffendes Beispiel an, das veranschaulicht, dass Systeme nicht (nur) aus Menschen bestehen. Sie bedienen sich dabei des Schachspiels. Man mag versucht sein, dieses allseits bekannte Spiel lediglich mit Figuren und einem Brett in Verbindung zu bringen. Dies wäre aber zu kurz gegriffen:

»Ein Schachspiel kann man mit Muscheln, Steinen, [...] spielen. Die Figuren sind notwendig, sind sozusagen ein notwendiger Kontext, ohne den das Spiel nur sehr schwer (etwa gedanklich) zu spielen ist, aber das Spiel besteht nicht aus den Figuren. Es besteht aus den Regeln, nach denen die Figuren sich bewegen. Das Spiel ist also eigentlich unsichtbar, ein komplexes Gebilde aus aufeinander bezogenen Dynamiken, die das Verhalten der einzelnen Figuren begrenzen (ohne es komplett vorherzusagen). « ${ }^{69}$

Bezogen auf soziale Systeme ist es vergleichbar. Nicht die spezifischen Menschen, die rein zufällig interagieren, machen ein soziales System aus, sondern vielmehr die zwischen ihnen stattfindende Kommunikation. Die Kommunikationsmuster sind dabei entscheidend. Diese gehen stets einher mit "spezifischen Erwartungen $\aleph^{70}$, die sich im Laufe der Zeit entwickelt haben:

62 Vgl. Luhmann (2010), S. 56; Schlippe/Groth/Rüsen (2017), S. 74; Kleve (2017), S. 227.

63 Vgl. Luhmann (1984), S. 60.

64 Vgl. Simon (2012), S. 12.

65 Vgl. Simon (2012), S. 12.

66 Schlippe/Groth/Rüsen (2017), S. 74.

67 Vgl. Simon (2012), S. 19ff.; Simon (2011a), S. 37f.; Schlippe/Groth/Plate (2011), S. $526 \mathrm{ff}$.

68 Simon (2012), S. 12.

69 Schlippe/Groth/Rüsen (2017), S. 74. Hervorh. im Original.

70 Schlippe/Groth/Rüsen (2017), S. 75. 
$»[\ldots]$ doch es geht nicht um eine Ansammlung von Menschen, sondern um die Art des Spiels und die jeweils zugrundeliegenden Spielregeln, die sich zwischen ihnen entwickelt haben. "1 $^{71}$

Auch die vorliegende Forschungsarbeit basiert auf diesem systemtheoretischen Ansatz und wird an verschiedenen Stellen bei Bedarf darauf zurückgreifen.

\subsubsection{Pragmatische Paradoxien: die Wurzel des Erfolgs?!}

Mit Hilfe des im Abschnitt 2.13 eingeführten systemischen Ansatzes können die zahlreichen intrapsychischen und interpersonellen Konflikte, die in Familienunternehmen sehr häufig auftreten ${ }^{72}$, einfacher erklärt werden. ${ }^{73}$ Hierfür lohnt es sich, das »Drei-Kreis-Modell ${ }^{74}$ - ein Konzept, das in der Familienforschung einen hohen Bekanntheitsgrad erreicht hat ${ }^{75}$ - näher zu betrachten.

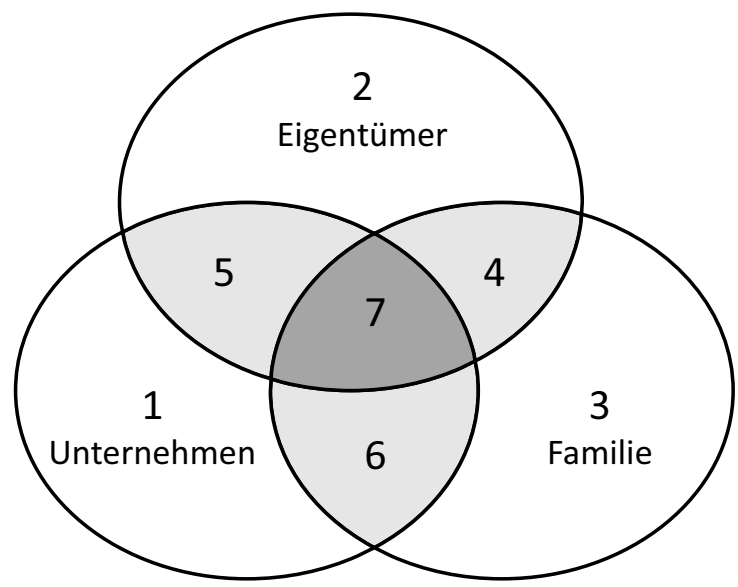

Abbildung 2: Das Drei-Kreis-Modell nach TAgIURI/DAvis (1996) ${ }^{76}$

71 Schlippe/Groth/Rüsen (2017), S. 75.

72 Vgl. Wiechers (2006), S. 20.

73 Vgl. dazu auch Wiechers (2006), S. 20. Ein hier gern verwendetes Beispiel ist die Figur des Vaters und Unternehmensleiters in Personalunion. Dieser kann in der Kommunikation mit seinem Sohn, als sein designierter Nachfolger, in verschiedene Rollen schlüpfen: je nachdem, ob er mit ihm als Vater oder Unternehmenslenker spricht. Die Verwechslung der jeweiligen Rolle dient dabei als Erklärungsmuster für die Entstehung entsprechender Konflikte, die in Familienunternehmen durchaus existenzgefährdend verlaufen können.

74 Vgl. Abbildung 2.

75 Vgl. nur Schlippe/Groth/Rüsen (2017), S. 25.

76 Eigene Darstellung; in Anlehnung an Simon (2012), S. 26. 
Dieses Modell ist eine grafische Darstellung der zuvor erläuterten Subsysteme Familie, Unternehmen und Eigentum in Form eines einfachen Venn-Diagramms (vgl. Abbildung 2). Diese Darstellungsform (drei Kreise und vier Schnittmengen) kann in einer simplen Auslegungsform dazu genutzt werden, die jeweiligen Personen eines Familienunternehmens im jeweiligen Kreis bzw. in der zutreffenden Schnittmenge zu verorten. ${ }^{77}$ Sehr häufig sind die Mitglieder eines Familienunternehmens nicht nur Teil eines Systems (wie die Personen in den Bereichen 1, 2 und 3), sondern parallel auch in einem zweiten System (wie in den Fällen 4, 5 und 6) oder gar in drei Systemen (Fall 7) einzuordnen. Innerhalb und zwischen den drei Dimensionen lassen sich also in Summe sieben generische Rollenbilder beschreiben (vgl. Abbildung 3). Diese Sichtweise blendet die Betrachtung als Kommunikationssysteme jedoch aus.

In der Praxis wird diese Darstellung häufig als rollenbasiertes Modell verstanden, denn es veranschaulicht auf einfache Weise die verschiedenen, teilweise auch gleichzeitigen Rollen (»overlapping memberships ${ }^{78}$ ), die ein Familienmitglied wahrnehmen kann. Es treffen damit nicht mehr »einzelne Individuen aufeinander, sondern Rollenträger in ihren jeweiligen Rollen. ${ }^{79}$

Das Modell skizziert Unternehmerfamilien als "hybride Sozialformen ${ }^{80}$, deren Mitglieder bis zu drei verschiedene Rollen gleichzeitig ausfüllen können: Sie sind Verwandte, Eigner (Gesellschafter) und Manager in einem und verfolgen in jeder dieser Rollen unterschiedliche Ziele. In der Rolle des Familienmitglieds liegt ihnen natürlich primär das Wohlergehen und die Einheit der Familie am Herzen, als Gesellschafter ist man vornehmlich an einem hohen $\mathrm{ROI}^{81}$ und dem Fortbestand des Unternehmens interessiert, wohingegen man als Unternehmenslenker primär am Wachstum interessiert ist und möglicherweise eigene Karriereziele verfolgt. ${ }^{82}$

»Die Zerrissenheit, die viele Gesellschafter in Entscheidungssituationen erfahren, ist [...] als Folge von widerstreitenden Rollenerwartungen zu sehen [...].. ${ }^{83}$

Die sieben unterschiedlichen Rollenbilder lassen sich entsprechend klassifizieren:

77 Vgl. Schlippe/Groth/Rüsen (2017), S. 76.

78 Tagiuri/Davis (1996), S. 201.

79 Schlippe/Groth/Rüsen (2017), S. 76.

80 Kleve (2017), S. 241.

81 Der ROI (Return on Investment) gibt an, welche Rendite das gesamte eingesetzte Kapitel in einem Unternehmen innerhalb einer definierten Periode gebracht hat; anders gesagt: wie hoch der prozentuale Anteil des Gewinns am Gesamtkapital ist.

82 Vgl. Tagiuri/Davis (1996), S. $199 \mathrm{ff}$.

83 Schlippe/Groth/Rüsen (2017), S. 77. 


\begin{tabular}{|l|l|}
\hline Nr. & Rollenbild \\
\hline 1 & $\begin{array}{l}\text { Ein Mitarbeiter, der weder Teil des Unternehmens ist noch Anteile hält. Beispiel: } \\
\text { Angestellter Fremdmanager. }\end{array}$ \\
\hline 2 & $\begin{array}{l}\text { Ein Anteilseigner, der weder zur Familie gehört noch im Unternehmen arbeitet. } \\
\text { Beispiel: dritter Investor, Eigenkapitalgeber. }\end{array}$ \\
\hline 3 & $\begin{array}{l}\text { Ein Familienmitglied, das weder Anteile am Unternehmen hält, noch im Unter- } \\
\text { nehmen arbeitet. Beispiel: Ehepartner. }\end{array}$ \\
\hline 4 & $\begin{array}{l}\text { Ein Familienmitglied, das am Unternehmen beteiligt ist, nicht jedoch in diesem } \\
\text { arbeitet. }\end{array}$ \\
\hline 5 & $\begin{array}{l}\text { Ein Mitarbeiter des Unternehmens, der am Unternehmen beteiligt ist, nicht jedoch } \\
\text { zur Familie zählt. Beispiel: minderheitsbeteiligter Fremdmanager. }\end{array}$ \\
\hline 6 & $\begin{array}{l}\text { Ein Familienmitglied, das im Unternehmen arbeitet, jedoch nicht über Anteile } \\
\text { verfügt. Beispiel: mitarbeitender Schwiegersohn. }\end{array}$ \\
\hline 7 & $\begin{array}{l}\text { Ein Familienmitglied, das sowohl Anteile am Unternehmen hält als auch im Un- } \\
\text { ternehmen tätig ist. Beispiel: klassische Figur des Familienunternehmers. }\end{array}$ \\
\hline
\end{tabular}

Abbildung 3: Übersicht der unterschiedlichen Rollenbilder nach TAGIURI/Davis (1996) ${ }^{84}$

Nach Wimmer/Domayer/Oswald/Vater (2018) besteht eine besondere Herausforderung darin, die Dimensionen Familie, Eigentum und Unternehmen so in Balance $\mathrm{zu}$ bringen und $\mathrm{zu}$ integrieren, dass sie sich gegenseitig stützen und stärken. ${ }^{85}$ Es ist für die Mitglieder von Unternehmerfamilien keineswegs immer einfach, diese verschiedenen Systeme klar voneinander zu trennen. Dies ist für jemanden, der keinem Familienunternehmen angehört, oftmals nicht einfach nachzuvollziehen, denn

$»[\ldots]$ in unserem westlichen Gesellschafts- und Wirtschaftssystem ist es im `Normalfall zu einer diachronen Dissoziation, d.h. zu einem zeitlichen Nacheinander zwischen Familie und Organisation, zwischen Privatleben und Arbeitsleben, gekommen. Das heißt, morgens verlässt man das Haus und geht zur Arbeit, übernimmt dort seine Rolle als profitorientierte Managerin, kundenorientierter Versicherungsvertreter oder angepasster Schüler usw., und wenn man abends wieder nach Hause kommt, gibt man diese Rolle an der Garderobe ab und wird zur altruistischen Mutter, zum strengen Vater und zum trotzigen Kind. ${ }^{86}$

Eine solch strikte Trennung gelingt Mitgliedern von Unternehmerfamilien meist nicht. $\mathrm{Zu}$ eng sind die drei Dimensionen zeitlich und räumlich miteinander verwoben, sodass die Beteiligten oftmals selbst größte Schwierigkeiten damit haben dürften, sich in der jeweils richtigen Rolle zu verorten. So kommt es häufig

84 Eigene Darstellung, in Anlehnung an Wiechers (2006), S. $19 \mathrm{f}$.

85 So im Ergebnis auch Wimmer et al. (2018), S. 189.

86 Simon (2011a), S. 39. 
dazu, dass die Grenzen zwischen den Dimensionen und die jeweils damit verbundenen Erwartungen gänzlich zu verschwimmen drohen. ${ }^{87}$

Dies führt auch zu der dritten Lesart dieses Modells, die die systemtheoretische Sicht eines Familienunternehmens verkörpert, wie sie insbesondere am WIFU entwickelt wurde und Anwendung findet. ${ }^{88}$ Danach stellen die drei Dimensionen Familie, Unternehmen und Gesellschafter eigenständige soziale Systeme dar, die jeweils nach eigenen Regeln, Erwartungen oder Voraussetzungen funktionieren: ${ }^{89}$

»Immerfort bringen juristische und finanzstrategische (Eigentum) oder ökonomische Logiken auf der einen Seite (Unternehmen) sowie Fragen von emotionaler Nähe und Distanz auf der anderen Seite (Familie) die Betroffenen in die Lage, nicht genau zu wissen, als wer, das heißt welche Person (also in welcher Logik), sie gerade sprechen und handeln bzw. gerade angesprochen werden. ${ }^{90}$

Allerdings sind sich die meisten Familienmitglieder dieser Problematik gar nicht bewusst. Somit stellt sich vielmehr die Frage, woran sich die Kommunikation orientiert?

»Woran erkennt eine Kommunikation überhaupt, dass sie in die Familie gehört und nicht in die Umwelt?»

So bietet es sich an, das Drei-Kreis-Modell aus kommunikationstheoretischer Sicht als ein $"$ Modell der Kontexturen ${ }^{91}{ }^{91}$ neu zu formulieren. In jedem der drei Kreise findet sich ein anderer Erwartungskontext. In der Familie wird beispielsweise etwas anderes erwartet als im Unternehmen. In jedem Kontext wird auch eine andere Kommunikationslogik verwendet - ganz ohne dass einem dies im jeweiligen Moment bewusst wäre. Da in einem Familienunternehmen »alle Kontexte gleichzeitig gegeben sind - VogD (2013) spricht in diesem Zusammenhang von "polykontexturalen Verhältnissen ${ }^{92}{ }^{92}$, sind die vorherrschenden

87 Vgl. Simon (2011a), S. 39ff. Kormann (2018) S. 257 spricht hier auch von sogenannten "tektonische[n] Verwerfungszonen«.

88 Vgl. Schlippe/Groth/Rüsen (2017), S. 77 m.w.N.

89 Viel früher wurde dies schon alleine für die beiden Systeme Unternehmen und Familie erkannt: Vgl. Simon (2011a), S. 17: „Familie und Unternehmen können heute, Anfang des 21. Jahrhunderts, als zwei unterschiedliche Typen sozialer Systeme verstanden werden, in denen unterschiedliche Verhaltens- und Kommunikationsregeln gelten.«; und Simon (2011a), S. 36: »Im Familienunternehmen haben wir es mit der Kopplung und Ko-Evolution der beiden sozialen Systeme Familie und Unternehmen zu tun. Dies ist meist mit spezifischen Konsequenzen für die Familiendynamik verbunden.«; sowie Ward (2004), S. 22 f.: "Family members must have emotional and other personal needs satisfied; meeting those needs within the business may be harmful to its success«; ebenso Fleming (2000), S. $21 \mathrm{ff}$.

90 Schlippe/Groth/Rüsen (2017), S. 78.

91 Schlippe/Groth/Rüsen (2017), S. 78.

92 Vogd (2013). Vgl. dazu auch Schlippe/Groth/Rüsen (2017), S. 77 ff. 
Logiken der Kommunikation nicht so leicht zu durchschauen. ${ }^{93}$ So treffen in Familienunternehmen Familien-, Unternehmens- und Eigentümererwartungen aufeinander. Die Herausforderung besteht darin, mit diesen einander widersprechenden, teilweise paradoxen Erwartungen im jeweiligen Kontext umzugehen.

Aber gerade das Verständnis dieser Paradoxien trägt zum besseren Verständnis von Familienunternehmen bei. ${ }^{94}$ Simon/Wimmer/Groth (2017) führen dazu aus, dass es

»der Blick auf Paradoxien ist [...], der [...] am ehesten ermöglicht, die Besonderheiten von Familienunternehmen [...] zu erfassen und ihre Risiken und Chancen zu analysieren. Es geht stets um die Bewältigung paradoxer Handlungsaufforderungen, um die Erledigung von Aufgaben, die nicht gleichzeitig dem Unternehmen und der Familien gerecht werden können, und um den Umgang mit Werten, die sich gegenseitig ausschließen und trotzdem realisiert werden müssen. ${ }^{95}$

Sogenannte pragmatische Paradoxien ${ }^{96}$ finden sich kontinuierlich im Umfeld von Familienunternehmen an ganz unterschiedlichen Stellen und in verschiedenen Ausprägungen. Diese entstehen durch "paradoxe Handlungsaufforderungen ${ }^{97}$, also durch sich "einander widersprechende Verhaltenserwartungen ${ }^{98}{ }^{99}$ Sie bilden "sowohl das organisationale Kernstück des Familienunternehmens als auch die Grundherausforderung der Unternehmerfamilie. ${ }^{100}$

Als wesentliche Paradoxie in diesem Kontext wird häufig das Dilemma verstanden, dass ein Mitglied einer Unternehmerfamilie nie ganz genau weiß, als welche Person man gerade spricht bzw. als wer man gerade angesprochen wird, insbesondere wenn man grundsätzlich Teil mehrerer Subsysteme des DreiKreis-Modells ist. Die daraus resultierende paradoxe, unerfüllbare Verhaltensaufforderung beschreiben SCHLIPPE/GRoTH/RüSEN (2017) so: »Kommuniziere gleichzeitig als Familienmitglied, als Unternehmensmitglied und/oder als Gesellschafter! «. ${ }^{101}$

Ist man auf der Suche nach einer vermeintlich einfachen Lösung dieses Problems, würde man diese sicherlich in einer kompletten Entkoppelung dieser drei Subsysteme finden. In der Realität würde dies so aussehen, dass das Unternehmen verkauft oder die Möglichkeit der unternehmerischen Einflussnah-

\footnotetext{
93 Schlippe/Groth/Rüsen (2017), S. 78.

94 Vgl. Plate/Groth (2009), S. 167.

95 Simon/Wimmer/Groth (2017), S. 23.

96 Vgl. Watzlawick/Beavin/Jackson (2011), S. $213 \mathrm{ff}$.

97 Watzlawick/Beavin/Jackson (2011), S. 213.

98 Schlippe/Groth/Rüsen (2017), S. 98.

99 Vgl. Kleve (2017), S. $228 \mathrm{f}$.

100 Plate/Groth (2009), S. 167.

101 Schlippe/Groth/Rüsen (2017), S. 91.
} 
men durch die Familie gänzlich beschränkt werden würde. ${ }^{102}$ Letzteres, so SıMON (2012), würde bedeuten, »die Familie zu entmachten. ${ }^{103}$ Doch gerade die enge Verzahnung von Familie und Unternehmen macht ein Familienunternehmen erst aus. Eine strikte Entkoppelung käme dem Untergang des Familienunternehmens gleich. ${ }^{104}$ Wäre eine Entkoppelung der Dimensionen die einzige mögliche Lösung, wäre kaum zu begründen, warum dennoch so viele erfolgreiche Familienunternehmen existieren.

"I used to think that paradoxes were the visible signs of an imperfect world, a world which would, one day, be better understood and better organized. There had to be the one right way to bring up children, I thought. There should be no reason for people to starve while others gorge. [...] We lacked only the knowledge and the will to resolve such paradoxes. [...] Paradox I now see to be inevitable, endemic, and perpetual. The more turbulent the times, the more complex the world, the more paradoxes there are. [...] Paradox has to be accepted, coped with, and made sense of, in life, in work, in the community, and among nations. ${ }^{105}$

Das Rezept erfolgreicher Familienunternehmen kann somit darin gesehen werden, dass es ihnen gelingt, die Paradoxie zu akzeptieren, vielleicht sogar bewusst aufrechtzuerhalten, und nicht ständig und dauerhaft zu versuchen, diesen Konflikt zu Gunsten der einen oder anderen Seite zu entscheiden. Indem sie diese »unterschiedlichen Rationalitäten von Familie und Unternehmen « ${ }^{106}$ akzeptieren, können sie diese sogar als »innovations- und kreativitätsfördernde Triebkraft « ${ }^{107}$ nutzen. Paradoxien dürfen somit keineswegs nur als Blockade verstanden werden, sondern sind im Kontext der Familienunternehmen gar die »Wurzel des Erfolgs $(!) \varkappa^{108}$.

Um die systemimmanenten Paradoxien in Familienunternehmen in erfolgversprechende Chancen zu wandeln, bedarf es eines effektiven »Paradoxiemanagements ${ }^{109}$

»Familienunternehmen wie Unternehmerfamilien sind diese Paradoxien sin die Wiege gelegtı, sie müssen lernen, die sich aus den widersprüchlichen Funktionslogiken ergebenden Spannungsfelder und Paradoxien zu managen. ${ }^{110}$

102 So auch Ammer (2017), S. 27.

103 Simon (2012), S. 40.

104 Vgl. Hennerkes (2004), S. 293; Kormann (2018), S. 30; Simon (2012), S. 40; Wiechers (2006), S. 160.

105 Schlippe/Groth/Rüsen (2017), S. 92 mit Verweis auf Handy (1995), S. $12 \mathrm{ff}$.

106 Schlippe/Groth/Rüsen (2017), S. 92 mit Verweis auf Simon (2012), S. $39 \mathrm{ff}$.

107 Simon/Wimmer/Groth (2017), S. 34.

108 Schlippe/Groth/Rüsen (2017), S. 92 mit Verweis auf Simon (2012), S. 39 ff.; Hervorh. im Original. Vgl. dazu auch Baecker (2003); Wimmer/Groth/Simon (2009), S. 148.

109 Plate (2009); Groth (2011); Schlippe (2009a); so auch Ammer (2017), S. 28.

110 Schlippe/Nischak/El Hachimi (2011b), S. 40. 
Eine zentrale Aufgabe besteht dabei darin, dass man selbst erkennt (»Consciousness Raising « ${ }^{111}$ ), wenn man in einer Paradoxie steckt:

»Wer sich bewusst wird, dass er in einer Paradoxie steckt, ist bereits nicht mehr ganz ihr Gefangener. ${ }^{112}$

Um dieses Bewusstsein zu schärfen, ist gutes und vor allem rechtzeitiges Familienmanagement essenziell. Die beste Möglichkeit, diesen Paradoxien in konstruktiver und nicht in destruktiver Weise zu begegnen, wird darin gesehen, frühzeitig Strukturen zu etablieren, in denen "gemeinsam gesprochen ${ }^{113}$ wird. ${ }^{114}$ Diese Kommunikationsstrukturen sollen den Beteiligten einen mutuellen Austausch über Rollenwahrnehmung, Erwartungen etc. ermöglichen. ${ }^{115}$ Ein solches Familienmanagement zur Bewältigung der Paradoxien kann durch eine gut ausformulierte Familienverfassung institutionalisiert werden. ${ }^{116}$ Dies allein kann jedoch nicht genügen. Die Einhaltung der darin niedergeschriebenen (Kommunikations-)Regeln muss gewährleistet werden.

\subsubsection{Konfliktpotenziale in Unternehmerfamilien}

»Durch Eintracht wächst das Kleine, durch Zwietracht zerfällt das Größte.»

(Sallust)

Systemtheoretisch betrachtet folgen die drei Dimensionen Unternehmen, Familie und Eigentum jeweils sehr unterschiedlichen Logiken. Sowohl Unternehmen als auch Familien bieten diese Logiken (vgl. dazu Abbildung 4) einen Nährboden für Konflikte. ${ }^{117}$

111 Schlippe/Nischak/El Hachimi (2011b), S. 29 mit Verweis auf Harvey/Evans (1994), S. 342.

112 Schlippe (2009a), S. 63.

113 Schlippe (2009a), S. 63.

114 Vgl. Wiechers/Klett (2005).

115 Ammer (2017), S. 28.

116 Vgl. Schlippe (2009a), S. 63.

117 Vgl. Ebel (2014), S. 171; Frank et al. (2011), S. $130 \mathrm{ff}$. Allgemein zu verschiedenen Ausprägungen von Konflikten in Unternehmerfamilien Fabis (2009), S. 4 ff. Allgemein zu verschiedenen Konflikttypen in Organisationen und Unternehmen Kreyenberg (2004), S. 25 f. 


\begin{tabular}{|c|c|c|c|}
\hline $\begin{array}{ll} & \text { System } \\
\text { Thema } & \end{array}$ & Familie & Unternehmen & Eigentum \\
\hline Zugang & Geburt, Heirat, Adoption & Eintritt, Einstellung & Eigentum \\
\hline Ausgang & Prinzipiell nicht möglich & $\begin{array}{l}\text { Prinzipiell jederzeit, } \\
\text { je nach Vertrag }\end{array}$ & $\begin{array}{l}\text { Prinzipiell möglich, durch } \\
\text { Verkauf }\end{array}$ \\
\hline Kommunikation & Bindungskommunikation & $\begin{array}{l}\text { Entscheidungskommunika } \\
\text { tion }\end{array}$ & $\begin{array}{l}\text { Geld als generalisiertes } \\
\text { Kommunikationsmedium }\end{array}$ \\
\hline Kommunikationswege & $\begin{array}{l}\text { Wenig formalisiert, } \\
\text { mündlich, wenig } \\
\text { hierarchisiert }\end{array}$ & $\begin{array}{l}\text { Formalisiert, schriftlich, } \\
\text { hierarchisiert }\end{array}$ & Formalisiert, schriftlich \\
\hline Entscheidung & $\begin{array}{l}\text { Viel } \\
\text { Verhandlungsspielraum, } \\
\text { eher über Einigung }\end{array}$ & $\begin{array}{l}\text { Weniger } \\
\text { Verhandlungsspielraum, } \\
\text { eher hierarchisch }\end{array}$ & Mehrheitsbeschlüsse \\
\hline Wichtig ist & $\begin{array}{l}\text { Die einzelne Person (nicht } \\
\text { austauschbar) }\end{array}$ & $\begin{array}{l}\text { Funktion, Kompetenz } \\
\text { (Person ist prinzipiell } \\
\text { austauschbar) }\end{array}$ & $\begin{array}{l}\text { Höhe der Anteile, } \\
\text { Funktion }\end{array}$ \\
\hline „Währung“ & $\begin{array}{l}\text { Liebe, Bindung, Treue, } \\
\text { Loyalität }\end{array}$ & Arbeitskraft, Karriere & $\begin{array}{l}\text { Anteile, Langfristigkeit der } \\
\text { Anteile }\end{array}$ \\
\hline Ausgleich & $\begin{array}{l}\text { Anerkennung, } \\
\text { Wertschätzung } \\
\text { langfristig (gegebenenfalls } \\
\text { Jahrzehnte) }\end{array}$ & $\begin{array}{l}\text { Geld, Gehalt } \\
\text { kurzfristig („zum 31.“) }\end{array}$ & $\begin{array}{l}\text { Regelmäßige } \\
\text { Ausschüttung; } \\
\text { Wertsteigerung }\end{array}$ \\
\hline Gerechtigkeitskriterien & $\begin{array}{l}\text { Gleichheit: Alle } \\
\text { bekommen das Gleiche }\end{array}$ & $\begin{array}{l}\text { Ungleichheit: Position und } \\
\text { Gehalt nach Leistung, } \\
\text { Fähigkeit, Einsatz } \\
\text { unterschiedlich }\end{array}$ & $\begin{array}{l}\text { A) Gleichheit auf Ebene } \\
\text { der Information } \\
\text { B) Ungleichheit auf Ebene } \\
\text { der Stimme: je nach } \\
\text { Anteilen }\end{array}$ \\
\hline
\end{tabular}

Abbildung 4: Gerechtigkeitslogiken von Familie, Unternehmen, Eigentum ${ }^{118}$

So gibt es natürlich durchaus »Aufgaben- und Prozesskonflikte«, die als hilfreich und förderlich gesehen werden. ${ }^{119}$ Meinungspluralität kann helfen, die beste Lösung für ein Problem zu finden. ${ }^{120}$ Problematisch wird es allerdings dann, wenn Konflikte innerhalb des Gesellschafter- und Familienkreises die Entscheidungsfähigkeit des Unternehmens negativ beeinflussen. ${ }^{121}$ Dies kann - in gar nicht so seltenen Fällen ${ }^{122}$ - in ein die Existenz des Unternehmens bedrohendes Szenario münden. ${ }^{123}$

118 Schlippe (2009b), S. 3.

119 So im Ergebnis auch Schlippe/Kellermanns (2017), S. 239; Lorz/Sarholz (2014), S. 3.

120 Vgl. Nosé et al. (2013), S. 56.

121 Vgl. Rüsen/Schlippe/Groth (2014), S. 102.

122 Ganz allgemein die Wechselwirkungen zwischen Konflikten in Familie und Unternehmen und dem Untergang von Familienunternehmen untersuchend Großmann (2014).

123 Vgl. Rüsen (2017b); Levinson (1971), S. 90 ff.; Davis/Harveston (2001), S. 14ff. Ein Phasenmodell vom Konflikt zum Untergang des Familienunternehmens zeigend Großmann (2014), S. 143. 
Gerade durch die Überlagerung der Interessengegensätze in den Systemen Familie und Unternehmen kann es zu diversen Konfliktfeldern kommen:

"Dabei handelt es sich zu einem großen Teil um Konfliktfelder, die aus der Überlagerung der natürlichen und notwendigen Interessengegensätze zwischen Unternehmen und Familie entstehen. Während in der Familie Entscheidungen aus persönlichen, emotionalen Motiven und im Hinblick auf die soziale Verbundenheit getroffen werden, treten in Unternehmen, auch in Familienunternehmen, rein rationale, überwiegend ökonomische Motive bei der Entscheidungsfindung in den Vordergrund. In einem Familienunternehmen treffen diese beiden Entscheidungsmuster schließlich ungebremst aufeinander. $\aleph^{124}$

Die Konfliktarten in Familienunternehmen lassen sich nach verschiedenen Perspektiven gliedern: die strukturorientierte, inhaltsorientierte oder personale Ebene. ${ }^{125}$ Unter Konflikten mit strukturellem Hintergrund versteht man Meinungsdifferenzen, die auf Grund unklarer Regelungen entstehen können. Als Beispiel können hier Informationsasymmetrien ${ }^{126}$ angeführt werden oder eine unklare Regelung, wie umfangreich und häufig Gesellschafter informiert werden. ${ }^{127}$ Bei Konflikten, die der inhaltsorientierten Ebene entstammen, treffen die beiden Systeme Familie und Unternehmen aufeinander. Hier steht beispielsweise die Privatheit der Familie der Öffentlichkeit des Unternehmens gegenüber oder das Unternehmen als gemeinhin formaler Raum der Familie als eher informaler Raum. ${ }^{128}$ Die Konflikte können auch einen kulturellen Hintergrund haben. Auch wenn klare Regelungen vorliegen (beispielsweise in Bezug auf Werte und Ziele des Unternehmens), kann eine unterschiedliche Bewertung der Situation Konfliktauslöser sein. ${ }^{129}$ Bei Konflikten auf der personalen Ebene handelt es sich beispielsweise um Nachfolge- oder Genderkonflikte, Konflikte beim Generationenwechsel ${ }^{130}$ oder klassische Geschwister ${ }^{131}$ und Stammesrivalitäten. ${ }^{132}$

FABIS (2009) hat die verschiedenen Ausprägungen der Unterschiede zwischen den beiden Dimensionen Familie und Unternehmen, die als Konfliktpotenziale gesehen werden können, analysiert und zusammengetragen. ${ }^{133}$ Dem interessierten Leser sei die ausführliche Lektüre jenes Werkes empfohlen.

124 Fabis (2009), S. 1.

125 Vgl. Großmann (2014), S. 101; Neuvians (2011), S. 62 ff.

126 Vgl. Großmann (2014), S. 101.

127 Vgl. Ebel (2014), S. 171.

128 Vgl. Großmann (2014), S. 101.

129 Vgl. Ebel (2014), S. 172.

130 Sich der besonderen Konfliktsituation beim Generationswechsel widmend Kirchdörfer/ Sarholz (2012).

131 Vgl. Ebel (2014), S. 174.

132 Vgl. Großmann (2014), S. 101.

133 Vgl. Fabis (2009), S. 4 ff. 
Bereits in der Überschrift des Abschnitts 2.1 kommt zum Ausdruck, dass Familienunternehmen eine besondere Unternehmensform mit Stärken und Schwächen sind. Eine Unternehmerfamilie kann somit eine bedeutende Ressource mit »stabilisierenden und stärkenden Eigenschaften ${ }^{134}$ für das Unternehmen sein. Aber genauso gut kann sie dessen Untergang auslösen. ${ }^{135}$ Astrachan/McMillan (2003) sind davon überzeugt, dass Familienunternehmen erfolgreicher sind, wenn die Familienmitglieder ihre Differenzen lösen und eine wohlwollende Kommunikationskultur etablieren. ${ }^{136}$ So argumentieren auch NosÉ/KorUnKA/FrANK/ Suchy (2013), dass ein »förderliches Familienklima die Konfliktdynamik günstig beeinflusst und dadurch die Unternehmensperformance und Zufriedenheit verbessert « wird. ${ }^{137}$

Die gängigen Instrumente zur Streitschlichtung lassen sich bei Familienunternehmen nur bedingt anwenden. ${ }^{138}$ Während sich sicherlich die Mediation als Instrument zur Beilegung von Konflikten in Familienunternehmen in den letzten Jahren bewährt hat ${ }^{139}$, sollten Konfliktherde möglichst im Voraus beseitigt werden. ${ }^{140}$ Die Familienverfassung mit ihren Institutionen ist eines der wirksamen Instrumente zur Konfliktprävention. ${ }^{141}$ Doch auch nachdem eine Familienverfassung etabliert ist, kann es durchaus zu konfliktären Situationen in Unternehmerfamilien kommen. In solchen Situationen werden oftmals »starke, bisher zurückgehaltene Emotionen und infolgedessen auch irrationale Verhaltensweisen und Handlungen freigesetzt «. ${ }^{142}$ Bei solchen irrationalen Handlungen kann es durchaus auch (beispielsweise im Affekt) zu ungewollten Verstößen gegen die Regeln einer Familienverfassung kommen. Insofern sollte jede Unternehmerfamilie auch im Rahmen ihrer Familienverfassung präventiv konfliktäre Situationen vermeiden. ${ }^{143}$

134 Nosé et al. (2013), S. 56.

135 Ausführlich zu destruktive familiäre Beziehungen in Familienunternehmen Eddleston/ Kellermanns (2007), S. $545 \mathrm{ff}$.

136 Vgl. Astrachan/McMillan (2003), S. 2: »When everyone is working together to improve communication skills and manage conflict, the chances of success are much greater, and the family becomes closer and more unified."

137 Nosé et al. (2013), S. 57.

138 Vgl. Fabis (2007), S. 364.

139 Ausführlich zu Mediation in Familienunternehmen Neuvians (2011).

140 Vgl. nur Ebel (2014), S. 176.

141 Vgl. Fabis (2009), S. $13 \mathrm{ff}$.

142 Gasteiger-Klicpera et al. (2008), S. 605.

143 Vgl. Abschnitt 4.1. 


\subsection{Die Familienverfassung als Instrument der Family Governance $^{144}$}

„Für Menschen wie für Nationen gibt es eine Zeit der Reife, die man abwarten muß, bevor man sie Gesetzen unterwerfen kann. ${ }^{145}$ (Jean-Jacques Rousseau)

\subsubsection{Die Familienverfassung: Versuch einer begrifflichen Rechtfertigung}

Den Begriff „Familienverfassung« wird man in einschlägigen Enzyklopädien vergeblich suchen. Eine offizielle Definition scheint bis heute nicht zu existieren. ${ }^{146}$ In der Beratungspraxis existieren unterschiedliche, synonym gebrauchte deutsche Terminologien ${ }^{147}$. Auch in der angloamerikanischen Literatur finden sich für die Familienverfassung eine Reihe von verschiedenen Namensgebungen, welche aber meist ähnliche Zielsetzungen verfolgen. ${ }^{148}$

Montemerlo/WARD (2011) untersuchten in einer Studie 80 Familienvereinbarungen (sog. »family agreements«) von Eigentümerfamilien in Nord- und Südamerika, Europa und Asien und unterteilten diese Vereinbarungen in vier Subtypen (family statements, family (business) protocol, owner's contracts/ shareholder's agreements und family constitutions). ${ }^{149}$ Eine deutsche Familienverfassung deckt klassischerweise die Inhalte der Family Statements sowie des Family Business Protocol ab. ${ }^{150}$

Schenkt man einer Suche bei Google Glauben, so ist der Begriff »Familienverfassung« mit $14.200^{151}$ Ergebnissen der mit Abstand am häufigsten verwen-

144 Unter »Family Governance« versteht man in Anlehnung an den Begriff »Corporate Governance» einen »Ordnungsrahmen für die Familie an der Schnittstelle zum Unternehmen«, Breyer (2015), S. 151.

145 Herb/Brandt (2012), S. 143.

146 So auch Holler (2018), S. 554.

147 Das Glossar der EQUA-Stiftung listet hierfür zahlreiche Synonyme auf: Familienmaximen, Familienkodex, Familienleitlinien/Familienrichtlinien, Familiensatzung, Family Governance. Vgl. http://www.equa-stiftung.de/glossary_detail.html?command=setViewAttribu tes\&id=735. Zuletzt geprüft am: 01.03.2020.

148 Vgl. Papesch (2010), S. 139; vgl. zu einer Übersicht der unterschiedlichen Begrifflichkeiten Neubauer/Lank (1998), S. 89.

149 Vgl. Montemerlo/Ward (2005); auf diese Subtypen bezugnehmend auch Corbetta/Salvato (2012), S. 155f.: Montemerlo/Ward (2011), S. 61 ff.; Kormann (2018), S. 180.

150 Kormann (2018), S. 180 hingegen sieht lediglich die Inhalte des »Family Business Protocol« mit dem vergleichbar, was hierzulande unter einer Familienverfassung verstanden wird.

151 Vgl. https://www.google.de/?gws_rd=ssl\#q=\%22Familienverfassung\%22. Zuletzt geprüft am: 01.03.2020. 
dete. Auf dem zweiten Platz mit 3.630 Treffern folgt die »Familiencharta « ${ }^{152}$. Alle anderen, äquivalent verwendeten Begriffe aus dem deutschen Sprachraum führen eher ein Schattendasein. ${ }^{153}$ Es scheint so, als habe sich der Begriff »Familienverfassung" hierzulande trotz seiner vermeintlichen Ambivalenz eingebürgert. ${ }^{154}$ So kommen KIRCHDöRfER/LoRZ (2011) zum Ergebnis, dass bei »strenger Betrachtung" die Begrifflichkeit "Familienverfassung" irreführend sei, zumal eine Verfassung im juristischen Sinne "ein durchaus verpflichtendes und allen anderen Rechtsnormen übergeordnetes Regelwerk « darstelle. ${ }^{155}$

Fraglich ist jedoch in diesem Kontext, ob es überhaupt eine allgemeingültige Definition des Begriffs "Verfassung" gibt, der dann in allen Anwendungsfällen auch etwas rechtlich Verbindliches meint. So führt Winterhoff (2007) aus, dass es deutlich leichter wäre, das Wesen einer Verfassung zu ermitteln, wenn sich eine "verbindliche positiv-rechtliche Verfassungsdefinition" finden ließe. Auf Grundlage der "Selbstdefinition einer positiven Verfassung läßt sich indes nicht nur [...] kein allgemeiner, sondern auch kein (rechtlich) allgemeinverbindlicher Verfassungsbegriff entwickeln. ${ }^{156}$ Insofern ist es also durchaus denkbar, dass das, was sich selbst eine »Verfassung« nennt, in Wirklichkeit gar keine Verfassung ist ${ }^{157}$, oder zumindest nicht das, was man gemeinhin darunter verstehen mag. ${ }^{158}$

Da dem Begriff "Verfassung " also keine universell gültige Definition zu Grunde liegt, mag dieser im Kontext Familienunternehmen durchaus passend sein. Beispielsweise dann, wenn man die Familienverfassung ganz nüchtern als Ordnungsrahmen einer Familie betrachtet. Nicht zuletzt zeigt die Häufigkeit der Verwendung, dass sich dieser Begriff offenkundig etabliert zu haben scheint. ${ }^{159}$ Aus diesen Gründen soll in dieser Forschungsarbeit der Begriff "Familienverfassung« stellvertretend für alle anderen, synonym verwendeten Begrifflichkeiten verwendet werden.

152 Vgl. https://www.google.de/?gws_rd=ssl\#q=\%22Familiencharta\%22. Zuletzt geprüft am: 01.03.2020.

153 Eine Google-Suche ergab beispielsweise für den Begriff „Familienmaximen « 115 Treffer, für »Familienleitlinien« bzw. »Familienrichtlinien« 535 bzw. 85 Treffer, für »Familiensatzung» 223 Treffer. Zuletzt geprüft am 01.03.2020.

154 So auch Kirchdörfer/Lorz (2011), S. 97.

155 Kirchdörfer et al. (2009), S. 97.

156 Winterhoff (2007), S. 53f.; Hervorh. im Original.

157 Winterhoff (2007), S. 54; vgl. zur weiteren Erläuterung auch Winterhoff (2007), Fn. 265.

158 Was man gemeinhin unter einer »Verfassung « versteht, leitet sich daher häufig an konkreten Beispielen und Erfahrungen ab. Winterhoff (2007), S. 55: „Was in einem abstrakt-generellen Sinne >Verfassung` ist, kann nach dem Ausgeführten nicht einer positiv-rechtlichen oder sonst rechtsverbindlichen Legaldefinition entnommen werden. Erkenntnisse über das Wesen der >Verfassung k können stattdessen nur durch die Anschauung einer Mehrzahl konkreter Objekte, d.h. solcher Normensysteme gewonnen werden, die sich selbst als ,Verfassung begreifen oder gemeinhin als solche charakterisiert werden."

159 So im Ergebnis auch Hueck (2017), S. 9. 


\subsubsection{Die Familienverfassung. Merkmale und Kontext}

Ein solcher Abschnitt kann kaum ohne eine Definition dieses für die vorliegende Forschungsarbeit zentralen Begriffs auskommen. Praktisch im selben Moment muss man jedoch die Hoffnung auf eine sehr konkrete Definition trüben, zumal Familienverfassungen inhaltlich immer speziell auf die besonderen Bedürfnisse einer Familie und ihr Unternehmen zugeschnitten sind. Die Regelungen variieren in der Praxis. ${ }^{160}$ Genauso wenig wie es das Familienunternehmen gibt, kann es auch die Familienverfassung nicht geben. ${ }^{161}$ Mit einer "terminologische[n] Unschärfe « ${ }^{162}$ wird man - gerade bei einem "neuen« Phänomen in der Anfangszeit - leben müssen. Dennoch lassen sich zumindest typische Themen von Familienverfassungen identifizieren, die immer wieder auf die jeweilige Situation zugeschnitten konkret ausgestaltet werden. ${ }^{163}$ Auf die einzelnen Themenfelder wird noch näher eingegangen werden. ${ }^{164}$

Montemerlo/WARD (2011) führen in wenigen Sätzen aus, was dabei den Kern einer Familienverfassung ausmacht:

»The family constitution, a comprehensive articulation of philosophy, principles, and policies for the future that balances and synthesizes the welfare of family, owners, and the business, is among the most important steps a business-owning family can take to secure and strengthen its business and, most preciously, its family. ${ }^{165}$

Sie ist damit nichts anderes als ein umfassendes Dokument mit der klaren Zielsetzung, die Werte, Ziele und Rollenverständnisse einer Unternehmerfamilie zusammenzufassen. ${ }^{166}$ Die Familienverfassung soll als Instrument verstanden werden, welches den Zusammenhalt der Familie stärkt und der zunehmenden Entfremdung der Familienmitglieder entgegenzutreten sucht. Gleichzeitig soll sie eine stärkere Bindung der Familie an das Unternehmen fördern, um dessen Fortbestand in den Händen der Familie sowie ihr Vermögen zu sichern. ${ }^{167}$ Eine

160 Vgl. Hennerkes/Kirchdörfer (2015), S. 67; Hueck (2017), S. 9 f.; Uffmann (2015), S. 2447; Born (2014), 48f. So im Ergebnis auch Reich/Bode (2018), S. 305.

161 Vgl. Fleischer (2016), S. 1515; Uffmann (2015), S. 2447 f.

162 Uffmann (2015), S. 2447.

$163 \mathrm{Zu}$ den typischen Themenfeldern vgl. Gläßer (2014), S. 232f.; Kirchdörfer/Breyer (2014), S. 13, 18; Kirchdörfer/Lorz (2011), S. 97, $101 \mathrm{ff} .$, Koeberle-Schmid/Fahrion/Witt (2012), S. 487 ff.; Redlefsen (2004), S. 83; Witt (2008), S. 15; Wedemann (2013), S. $95 \mathrm{ff}$.; Baus (2017), S. $110 \mathrm{ff}$.

164 Vgl. Abschn. 2.3.1.2.

165 Montemerlo/Ward (2011), S. 9.

166 Vgl. Holler (2018), S. 555; Reich/Bode (2018), S. 305.

167 Vgl. Hauck (2012a), S. 1; Hennerkes/Kirchdörfer (2015), S. 65; Kalss/Probst (2013a), S. 47; Spiegelberger (2008), S. 94f.; Kormann (2018), S. 180f.; Lange (2009), S. 148; Hueck (2017), S. 18; Kirchdörfer/Lorz (2011), S. 97 f.; Gläßer (2014), S. 228, 231 f.; Koeberle-Schmid/Fahrion/Witt (2012), S. $469 \mathrm{ff}$. 
Familienverfassung "schafft Planungssicherheit und stärkt [damit] den Zusammenhalt ${ }^{168}$. Unerlässlich für einen dauerhaften familiären Zusammenhalt ist es, dem zunehmenden Konfliktpotenzial ${ }^{169}$ entsprechend präventiv zu begegnen. Auch das ist eine zentrale Funktion der Familienverfassung.

Die Adressaten einer Familienverfassung sind die Mitglieder der Unternehmerfamilie $^{170}$. Für Gesellschafter ist sie insoweit relevant, als sie zum Kreise der Familie nach Maßgabe der Familienverfassung zählen. Der Sprachstil sollte so gewählt werden, dass sie einfach geschrieben und mithin für jeden verständlich sowie in sich schlüssig ist (d.h. saubere Definitionen sowie der Verzicht auf Umgangssprache, um Widersprüche zu vermeiden). Von elementarer Bedeutung ist auch, dass sie »im Konsens [von der Familie] beschlossen ${ }^{171}$ und (schon auf Grund der daraus resultierenden moralischen Bindungswirkung) als »affirmativer Akt ${ }^{172}$ von allen unterzeichnet wird. ${ }^{173}$

Führt man sich die jüngste Entwicklung im Bereich der Familienunternehmen vor Augen, wird man feststellen, dass «familienspezifische Werte und Zielvorstellungen ${ }^{174}$ (wieder) einen größeren Stellenwert einnehmen. Gerade bei größeren Familienunternehmen und in der sich damit auseinandersetzenden Literatur wird auf das Erfordernis, »dass die Mitglieder des Familienverbunds die sie verbindenden Werte, ihre Zielvorstellungen, die Leitlinien für die Entwicklung der gemeinsamen Unternehmung(en) sowie die Eckpunkte für die Gestaltung ihrer Beziehungen untereinander in schriftlicher Form festhalten ${ }^{175}$, hingewiesen. $^{176}$

Eine Familienverfassung ist also eine

schriftlich niedergelegte, langfristige Strategie einer Unternehmerfamilie, die die Interessen von Familie, Unternehmen und Gesellschaftern sorgfältig abwägt und mit klaren Strukturen den Geschäftsbetrieb und den familiären Zusammenhalt fördern und sichern soll. ${ }^{177}$

168 Baus (2017), S. 107.

169 Für weiterführende Ausführungen zu Gesellschafterkonflikten vgl. Wedemann (2013), S. $83 \mathrm{f}$.

170 Und dabei ist insbesondere zu beachten, wer nach Definition der jeweiligen Familienverfassung zur Familie zählt. Dies entscheidet jede Unternehmerfamilie selbst. Vgl. dazu auch Abschnitt 2.3.1.

171 Baus (2017), S. 108.

172 Fabis (2007), S. 362.

173 Vgl. Baus (2017), S. 108; Brockhoff et al. (2012), S. 479; Brückner et al. (2010), S. 248; Schlippe/ Groth/Rüsen (2017), S. 32.

174 Kirchdörfer/Lorz (2011), S. 97.

175 Kirchdörfer/Lorz (2011), S. 97.

176 Vgl. dazu Kirchdörfer/Lorz (2011), S. 97 ff.; Kormann (2018), S. 175 ff.; Schulz/Werz (2007a), S. $310 \mathrm{ff} . ;$ Schulz/Werz (2007b), S. $353 \mathrm{ff}$.

177 Eigene Definition, in Anlehnung an Schlippe/Groth/Rüsen (2017), S. 32; Felden/Hack (2014), S. 321; Le Breton-Miller/Miller (2009); Baus (2017), S. 107. 
Eine Familienverfassung sollte dabei nicht mit einem Gesellschaftsvertrag verglichen werden, sie wird vielmehr durch ihn »überlagert« und stellt somit eher ein »Wertegerüst « dar, auf welchem der Gesellschaftsvertrag fußt. ${ }^{178}$

An dieser Stelle soll vorsorglich bereits jetzt darauf hingewiesen werden, dass es sich bei einer Familienverfassung um ein Dokument handelt, welches lebt. Ist sie erst einmal geschrieben, muss sie in regelmäßigen Abständen überprüft und gegebenenfalls angepasst werden. ${ }^{179}$

\subsubsection{Aufgaben und konkrete Funktionen einer Familienverfassung}

Wenngleich die Familienverfassung keine Verfassung im klassischen Sinne sein möchte, kann man sich durchaus dieses Begriffs bedienen, um die Funktionen einer Familienverfassung zu erläutern. KormanN (2018) beispielsweise wählt diese Vorgehensweise. ${ }^{180}$ Eine Verfassung verkörpert zunächst einmal ganz allgemein ein staatliches Gestaltungs- und Ordnungsinstrument. ${ }^{181}$ Eine Familienverfassung sucht damit den Ordnungsrahmen für das Sozialgefüge der (Familien-)Gesellschafter und Familienmitglieder zu gestalten.

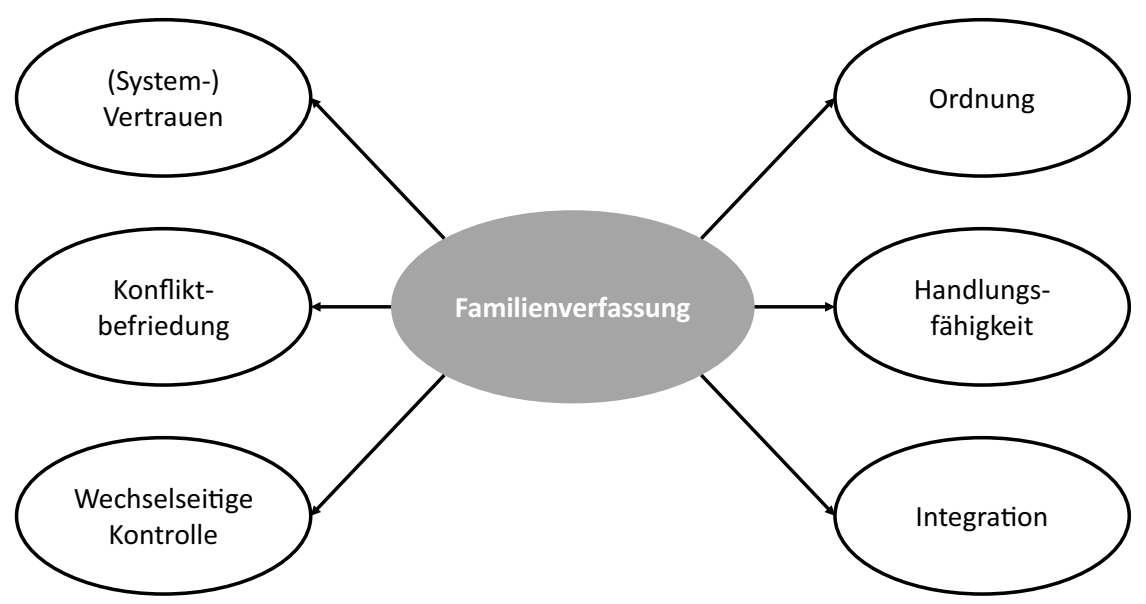

Abbildung 5: Aufgaben und Funktionen einer Familienverfassung ${ }^{182}$

178 Hennerkes/Berlin/Berlin (2007), S. 69. Die Familienverfassung in Abgrenzung zum Gesellschaftsvertrag vgl. Abschnitt 2.4.2.

179 Vgl. Abschn. 2.3.1.4.

180 Vgl. Kormann (2018), S. $176 \mathrm{ff}$.

181 Vgl. Aulehner (2011), S. 260; Calliess/Mahlmann (2002), S. 53; Kormann (2018), S. 175.

182 Eigene Darstellung; in Anlehnung an Kormann (2018), S. $176 \mathrm{ff}$. 
Die Familienverfassung ist - analog zu einem Vertrag, wenngleich die Familienverfassung gerade eben kein Vertrag im rechtlichen Sinne (»Vertrag ohne Schwert? « $)^{183}$ sein möchte, wie sich noch im Laufe dieser Arbeit zeigen wird ${ }^{184}$ Ausdruck der Willensbildung. Oft werden Verträge, dies kennt man auch im privaten Alltag, erst dann relevant, wenn man sich uneinig ist. Neben den innerhalb einer Familiengesellschaft typischen, rechtlich bindenden Vertragsdokumenten existiert eine Verfassungswirklichkeit ${ }^{185}$, welche die weichen Themen regelt: über die Zeit entstandene Wertvorstellungen, Sitten, Bräuche, Gewohnheiten und gesellschaftliche Erwartung. Eine Verfassungswirklichkeit arbeitet verschriftlicht mit Soll-Vorschriften, allgemeinen Erwartungen oder auch Wünschen.

\subsubsection{Die Familienstrategie als Wegbereiter der Familienverfassung}

"Plane das Schwierige da, wo es noch leicht ist! Tue das Große da, wo es noch klein ist! Alles Schwere auf Erden beginnt stets als Leichtes. Alles Große auf Erden beginnt stets als Kleines."

(Laotse)

»Scheitert ein Familienunternehmen, so scheitert es oft nicht am Markt - sondern an der Familie. ${ }^{186}$ Wenngleich die exogenen Faktoren des Marktes auf ein Familienunternehmen und dessen Erfolg sicherlich nicht $\mathrm{zu}$ vernachlässigen sind, spielt auch die Familie eine tragende Rolle. In Unternehmerfamilien waren die Vorstellungen früher hinsichtlich Familienzugehörigkeit, Formen der ehelichen Gemeinschaft oder bei Fragen in Sachen Nachfolgeregelung (Sohn vor Tochter, Älterer vor Jüngerer) recht uniform. Heutzutage trifft man häufig auf eine deutlich freiere Gestaltung des Familienverbundes. Darauf zu vertrauen, dass sich die Familie schon "irgendwie arrangieren und verstehen« wird, genügt nun nicht mehr. ${ }^{187}$ In jeder Unternehmerfamilie sollte rechtzeitig Klarheit darüber herrschen, wie die Zukunft aussehen soll. Je größer eine Familie wird, desto wichtiger wird beispielsweise auch die Frage, welches Familienmitglied auf welcher Grundlage welche Rolle ${ }^{188}$ übernehmen kann (so es denn überhaupt möchte) und damit einhergehend, welchen Betrag es leisten kann. Möchte man den Fortbestand des Unternehmens über viele Generationen in Familienhand

183 Vgl. Fleischer (2017a).

184 Vgl. Abschn. 2.4.

185 Vgl. Abschn. 2.4.1.

186 Hauck (2012b); vgl. hierzu auch Vernekohl (2007), S. 33.

187 Vgl. Rüsen/Groth (2012), S. 25.

188 Vgl. Abbildung 2. 
sichern, ist es unumgänglich, für einen innerfamiliären »Orientierungs- und Handlungskanon ${ }^{189}{ }^{18}$ zu sorgen. ${ }^{190}$ Die Familie darf sich nun nicht mehr nur mit dem gemeinsamen Unternehmen befassen, sondern auch mit sich selbst. ${ }^{191}$

Liegt ein gemeinsames Werteverständnis vor, sind die Rollen innerhalb der Familie zur Zufriedenheit aller geklärt und verfolgt die Familie ein gemeinsames Ziel, um nur einige Punkte zu nennen, so ist die Wahrscheinlichkeit zu scheitern tendenziell geringer. Oftmals entspricht dies in Unternehmerfamilien jedoch eher dem Wunsch als der Wirklichkeit - und so ist der Spagat zwischen Unternehmen, Familie und Eigentum kein leichter. Besondere Ereignisse mit weitreichender Konsequenz - wie beispielsweise die Regelung der Unternehmensnachfolge - können die Unternehmerfamilie dann bisweilen auf eine echte Belastungsprobe stellen. ${ }^{192}$

Hier kommt die Familienstrategie ${ }^{193}$ in Spiel. Analog zum Strategiebegriff im Unternehmenskontext zielt die Familienstrategie darauf an, ein »mittel- und langfristiges Zukunftsbild ${ }^{194} \mathrm{zu}$ entwickeln. Unter besonderem Einsatz der Familienmitglieder ${ }^{195}$ verständigt man sich auf eine gemeinsame Grundüberzeugung in Bezug auf Werte, Ziele und Rollen. Dies stärkt das gemeinsam verfolgte Interesse und sichert dadurch die Handlungs- und Entscheidungsfähigkeit der Familie. Darüber hinaus bildet die Familienstrategie das Fundament für eine Vertrauensbasis, die das Gefühl der Zusammengehörigkeit stärken und weiterentwickeln soll. ${ }^{196}$ "Eine gute Familienstrategie baut auf den bestehenden Verhältnissen auf, bezieht alle Beteiligten in die Entwicklung mit ein, liefert Antworten auf alle Kernfragen, ist handlungsorientiert und konkret, wird in der Familie gelebt, passt sich regelmäßig an die dynamischen Entwicklungen der Familie an und gibt klare Orientierung über Generationen. ${ }^{197}$ Es gibt unzählige Beispiele von durchdachten Familienstrategien in großen Familienunternehmen. $^{198}$

189 Rüsen/Groth (2012), S. 26.

190 Vgl. Baus (2017), S. 54; so im Ergebnis auch Kormann (2017), S. 468.

191 Vgl. Rüsen/Groth (2012), S. 26.

192 Vgl. Hauck (2012b).

193 Vgl. zur Verwendung des Begriffs Baus (2017); Carlock/Ward (2001); Plate et al. (2011); Schlippe/Groth/Plate (2011). May (2017b) spricht in diesem Zusammenhang auch von "Inhaberstrategie«. Im deutschsprachigen Raum wird er als einer der wesentlichen Bestandteile von Family Business Governance bzw. Family Governance angesehen, Schlippe/ Groth/Rüsen (2017), S. 30.

194 Schlippe/Groth/Rüsen (2017), S. 30.

195 Vgl. Vernekohl (2007), S. 33.

196 Vgl. Hauck (2012b); Vernekohl (2007), S. 33; Wolf/Paul/Zipse (2009), S. 152.

197 Zenner/Megerle (2014), S. 29.

198 Vgl. Simon/Wimmer/Groth (2017). 
Wird die Familie zu Recht als besondere Stärke eines jeden Familienunternehmens angesehen, so ist sie dennoch gleichzeitig auch dessen Achillesferse. ${ }^{199}$ Die Familienstrategie sucht die Unternehmerfamilie mit einem "Gerüst von Institutionen und Regeln $\aleph^{200} \mathrm{zu}$ stabilisieren, die dann in schriftlicher Form Niederschlag in der Familienverfassung finden. Sie hat damit das Ziel, als Grundlage für die Erstellung einer Familienverfassung zu dienen, um einer möglichen Entfremdung entgegenzutreten und das Konfliktpotenzial innerhalb der Familie zu minimieren. ${ }^{201}$

Die Mitglieder der Familien müssen sich schließlich selbst Regeln geben, die unmissverständliche Aussagen darüber treffen, wer bspw. unter welchen Voraussetzungen im Unternehmen tätig werden und sich beteiligen darf, ob das Unternehmen ggf. auch durch ein Fremdmanagement geführt oder das Führungsgremium des Unternehmens ganz oder teilweise aus den Reihen der Familienmitglieder besetzt werden soll, wie eine Verteilung der Unternehmensanteile erfolgen soll oder wie der Spagat zwischen Ausschüttungsverlangen und Investitionen im Unternehmen gelingen kann. ${ }^{202}$ Auch diese vorgenannte Aufzählung an zu klärenden Punkten ist keineswegs abschließend, sondern lediglich exemplarisch. Vielmehr gibt es zahlreiche weitere Themen, die ebenfalls einer Klärung im Vorfeld bedürfen. So sehr man an Antworten auf die gängigen Problemstellungen arbeiten mag, liegt allem auch die vorherige Entwicklung eines Zielbildes für die Unternehmerfamilie und das Familienunternehmen zu Grunde.

Das Rad muss dabei oftmals nicht neu erfunden werden. Jedoch sollte man davon Abstand nehmen, eine andere Familienverfassung blind zu kopieren, handelt es sich doch bei jeder um ein individuelles Werk der jeweiligen Unternehmerfamilie. ${ }^{203}$

Eine Familienstrategie darf aber auch nicht überbewertet werden. Wenn in diesem Kontext von Einheit der Familie die Rede ist, kann dies dahingehend missverstanden werden, dass hier ausschließlich ein harmonisches Miteinander den gewünschten Zielzustand beschreibt. Dies wäre zwar in jedem Falle vorzugswürdig und wünschenswert, doch die Familienstrategie strebt vielmehr eine funktionierende Unternehmerfamilie an, in der Meinungsunterschiede konstruktiv beigelegt werden. Somit zielt diese Einheit eher auf ein gemeinsames

199 Vgl. Halter (2009), S. 83; Vernekohl (2007), S. 33; Hauck (2012b).

200 Baus (2017), S. 43.

201 Vgl. Vernekohl (2007), S. 33; Holler (2018), S. 555; Hueck (2017), S. 20; Koeberle-Schmid/ Schween (2012), S. 321 f.; in diese Richtung auch Gläßer (2014), S. $237 \mathrm{ff.}$

202 Vgl. Vernekohl (2007), S. 33.

203 Zur Einzigartigkeit einer jeden Familienverfassung vgl. Fleischer (2016), S. 1515; Uffmann (2015), S. 2447f. Auf die Einzigartigkeit jeder Familienverfassung hinweisend Born (2014), Rn. 48. 
Interesse ab: das Familienunternehmen und -vermögen $\mathrm{zu}$ bewahren und mehren. $^{204}$

\subsubsection{Praktische Umsetzung einer Familienstrategie}

Die Ausgangslage ist häufig recht ähnlich: Konflikte führen zur inneren Lähmung und Handlungsunfähigkeit innerhalb der Unternehmerfamilie und begründen damit den Auslöser, sich Gedanken hinsichtlich einer Familienstrategie zu machen. ${ }^{205}$ Eine Familienstrategie und damit auch eine Familienverfassung sollte besser frühzeitig entwickelt werden, solange sich alle Familienmitglieder untereinander gut verstehen und noch miteinander sprechen (können). ${ }^{206}$ Doch auch aus einer konfliktären Situation heraus kann eine Familienstrategie sinnvoll sein. Ziel ist es dann primär, die Handlungsfähigkeit der Unternehmerfamilie wieder herzustellen. Hier sorgt sicherlich auch der externe Blick eines Beraters $^{207}$, der bei der Erstellung der Familienstrategie in aller Regel involviert ist, für die notwendige objektive Sichtweise. Die Familienstrategie selbst sucht eine holistische Sichtweise der Verhältnisse zu vermitteln und geht dabei bewusst weg von einzelnen Protagonisten und unlösbar erscheinenden Sachproblemen. Vielmehr beleuchtet sie systematisch die vorhandenen und nicht vorhandenen Strukturen, die für den Erhalt von Unternehmen und Vermögen erforderlich sind. Hierbei zielt die Familienstrategie insbesondere darauf ab, die einzelnen Erwartungshaltungen der Familienmitglieder in Bezug auf Werte, Rollenverteilung und Zielbild zu vereinen, Akzeptanz und Berücksichtigung von Regeln und verbindlich festgelegten Institutionen im Rahmen der aufzustellenden Familienverfassung zu schaffen. Sie löst sich damit bewusst von der Ebene der Sachthemen, die oft Auslöser der Handlungsunfähigkeit und -blockade innerhalb der Familie sind. ${ }^{208}$ Die Familienstrategie ignoriert aber keinesfalls die Gefühle der Familienmitglieder, betont jedoch mit dem Familienvermögen ihren "materiellen Bezugspunkt ${ }^{209}$. Hier wird ein gemeinsames Interesse in den Vordergrund gestellt, an welchem jedem Familienmitglied gelegen sein sollte, zumal dessen Vermögen bzw. Lebensstandard sehr häufig durch die erwirtschafteten Erträge des Unternehmens finanziert wird und sich die Familienmitglieder somit dem

204 Baus (2017), S. $69 \mathrm{f}$.

205 Vgl. Baus (2017), S. 45.

206 Vgl. Wolf/Paul/Zipse (2009), S. 152; Hauck (2012b), S. 1; Felden/Hack (2014), S. 326.

207 Vgl. Kormann (2017), S. 24, der in diesem Kontext die Arbeiten von Beratern für aufschlussreich empfindet, zumal die "Berichte anwaltlicher Berater über ihre Mandate in Aufsichtsräten und Beiräten einen Zugang zum 'wahren Leben` in diesen Institutionen« geben. Vgl. dazu auch Sigle (2013); Hennerkes/Kirchdörfer (2015), S. 362 ff.

208 Vgl. Baus (2017), S. 72.

209 Baus (2017), S. 46. 
gemeinsamen Unternehmen gegenüber emotional verpflichtet fühlen. ${ }^{210}$ Über ein gemeinsames Interesse, das hinter den Emotionen (hoffentlich) zu finden ist, lässt sich weitaus einfacher eine sachliche Verständigung herbeiführen. ${ }^{211}$

Eine Familienstrategie, die man sicherlich auch als »Prozess der Verfassungsgebung « ${ }^{212}$ sehen kann, erfolgt in mehreren Schritten. Begonnen wird mit einer Bestandsaufnahme, gefolgt von einer Richtungsentscheidung im Sinne einer Exit- oder einer Loyalitätslösung, die als Grundlage für die zu entwickelnde Familienverfassung dient. Auf dieser lässt sich dann die Family Governance aufbauen. $^{213}$

\subsection{Die »Inventur« der Familie}

$\mathrm{Zu}$ Beginn geht es zunächst darum, herauszufinden, welches Ziel die Familie verfolgt, was sie eint, aber auch, was sie möglicherweise trennt. ${ }^{214}$ Die Bestandsaufnahme besteht darin, alle schriftlichen Dokumente zu analysieren, die einerseits Aufschluss über das innerfamiliäre Verhältnis und andererseits über das Verhältnis zwischen Familie und Unternehmen geben können. Hier werden Eheund Erbverträge, Gesellschaftsverträge, Familienstammbäume, Poolverträge, Geschäftsordnungen, Organigramme etc. und nicht zuletzt auch Selbstauskünfte der einzelnen Familienmitglieder verwertet. Aber auch Dokumente, die grundsätzlich vorhanden sein sollten, tatsächlich aber nicht verfügbar sind, dienen als Indikator dafür, von welchen Themenfeldern besonderes Konfliktpotenzial ausgehen könnte und welche planerischen Defizite vorliegen. ${ }^{215}$ Es geht bei diesem Prozess grundsätzlich nicht nur darum, für zu erwartende oder bereits latent vorhandene Problemstellungen einen wirksamen Ordnungsrahmen zu schaffen, sondern auch für zu diesem Zeitpunkt der Erarbeitung noch nicht absehbare Ereignisse. ${ }^{216}$ So können beispielsweise nicht vorhandene Güterstandsvereinbarungen zwischen Ehegatten oder fehlende Ausschüttungsregeln im Ernstfall zu negativen Konsequenzen für Familie und Unternehmen führen, heißt es doch im Volksmund: »Bei guten Verträgen verträgt man sich gut « ${ }^{217} \cdot{ }^{218}$

Neben schriftlichen Vereinbarungen geben Organisationsdiagramme wichtige Hinweise darauf, wie stark einzelne Familienmitglieder - auch operativ - mit dem Unternehmen verflochten sind und welche Positionen sie bekleiden. Der

210 Vgl. Rüsen (2017b), S. $151 \mathrm{f}$.

211 Vgl. Baus (2017), S. 47.

212 Kormann (2018), S. 185.

213 Vgl. Abbildung 6.

214 Vgl. Hauck (2012b), S. 2.

215 Vgl. Baus (2017), S. 74.

216 Vgl. Kormann (2018), S. 185.

217 LeMar (2014), S. 209.

218 Vgl. Baus (2017), S. 74. 
Familienstammbaum hilft dabei für eine in die Zukunft gerichtete Perspektive, mit welchen Familienmitgliedern möglicherweise - in welcher Rolle auch immer - noch zu rechnen ist. All diese Informationen bilden die Grundlage für die Erstellung einer sinnvollen Familienstrategie.

\begin{tabular}{|c|}
\hline Bestandsaufnahme \\
\hline $\begin{array}{ll}\text { - } & \text { Tradition } \\
\text { - } & \text { Werte } \\
\text { - Ziele } \\
\text { - } & \text { Rollen } \\
\text { - } & \text { Family first - Business first }\end{array}$ \\
\hline
\end{tabular}

\begin{tabular}{|c|c|}
\hline \multicolumn{2}{|c|}{ Richtungsentscheidung } \\
\hline Exit-Lösungen & Loyalitäts-Lösungen \\
\hline $\begin{array}{l}\text { - Trennen } \\
\text { - Verkauf } \\
\text { - Stiftung } \\
\text { Vereinfachen } \\
\text { - Thronfolger-Lösung } \\
\text { - Realteilung } \\
\text { Family-Buy-out }\end{array}$ & $\begin{array}{l}\text { Operative Führung } \\
\text { - } \quad \text { Fremdmanagement } \\
\text { - } \quad \text { Familienmitglieder } \\
\text { - } \quad \text { Familienmitglied und } \\
\quad \text { Fremdmanager }\end{array}$ \\
\hline \multicolumn{2}{|c|}{ 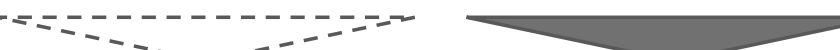 } \\
\hline \multicolumn{2}{|c|}{ Familienverfassung } \\
\hline
\end{tabular}

\section{Family Governance}

Abbildung 6: Der Weg zur Familienstrategie ${ }^{219}$

Die Bestandsaufnahme erfolgt im Rahmen eines Arbeitstreffens, zu welchem sich die Familienmitglieder einfinden, um über die gemeinsame strategische

219 Erweiterte Darstellung in Anlehnung an Baus (2017), S. 75. 
Ausrichtung zu sprechen. ${ }^{220}$ Der Grad der bereits erfolgten Entfremdung und Zersplitterung innerhalb der Familie bestimmt den Teilnehmerkreis. Entscheidet man sich für eine Familienstrategie aus einer bereits emotions- und konfliktgeladenen Situation heraus, empfiehlt sich zumindest die Anwesenheit aller Gesellschafter als Mindestanforderung. ${ }^{221}$

Ratsamer ist allerdings, das Arbeitstreffen in einem erweiterten Kreis durchzuführen, in welchem neben den Gesellschaftern auch deren Ehepartner und erwachsene Kinder, also möglichst alle Familienmitglieder, teilnehmen. ${ }^{222}$ Eingedenk der Familientradition ist das erklärte Ziel dieses Treffens, sich über das gemeinsame zukünftige Miteinander zu verständigen. Wieviel "Zusammenarbeit» ist möglich? Darüber entscheidet auch das vorhandene Maß an Verbundenheit in Bezug auf (gelebte) Werte, Zielbilder und Rollenverständnisse. »Family first « oder »business first «? Jedes Familienmitglied mag dazu eine eigene Meinung vertreten. Gerade wenn eine Familienstrategie aus einer Konfliktsituation heraus entsteht, werden einigen Familienmitgliedern diese Gemeinsamkeiten zunächst nicht klar erscheinen; doch gerade hierin liegt auch der Zweck der Zusammenkunft: die Familie (erneut) zusammenzubringen und die gemeinsamen Werte und Vorstellungen, die alle miteinander verbinden, wieder zu finden bzw. im Zweifel neu zu definieren. ${ }^{223}$

\subsection{Die Richtungsentscheidung}

Die Ergebnisse der Bestandsaufnahme entscheiden über die weitere Entwicklung. Je größer die Entfremdung und Spannung innerhalb der Familie, desto länger kann die Wiederentdeckung der gemeinsamen Werte, dessen, was einen verbindet, dauern. Die passende Entscheidung hängt nicht zuletzt von Kooperationswillen und -fähigkeit der Unternehmerfamilie ab. ${ }^{224}$ Reicht der kleinste gemeinsame Nenner jedoch nicht für ein Mindestmaß an Kooperation, wird man kaum über eine »Loyalitäts-Lösung" sprechen, sondern eher die »Exit-Lösung" ins Auge fassen müssen. BAus (2017) greift hier auf zwei Begrifflichkeiten der Organisationssoziologie zurück, die von dem US-amerikanischen Soziologen und Volkswirt Albert O. Hirschman als grundlegende Reaktionsmöglichkeiten auf den Leistungsabfall oder Krisensituationen in Organisationen verwendet werden. Bei Hirschman (1970) finden sich im Original die Begriffe »Exit», "Voice« und »Loyalty ${ }^{225}$. Mit »Exit« und »Loyalty« sind beide Extremzustände

220 So auch im Ergebnis Felden/Hack (2014), S. 327.

221 Vgl. Baus (2017), S. 48.

222 So auch Kormann (2018), S. 187.

223 Vgl. Baus (2017), S. $47 \mathrm{f}$.

224 Vgl. Hauck (2012b), S. 2.

225 Hirschman (1970). 
gemeint, derer sich auch BAUs (2017) bedient. ${ }^{226}$ Sie lässt jedoch die Option "Voice« außer Betracht. Hirschman (1970) verweist in seinen Ausführungen darauf, dass ein Kunde einer Marke, eines Produktes, eines Unternehmens etc. stets die Wahl hat zwischen zwei Lösungen: entweder sich gänzlich abzuwenden (Exit) oder zuzuwenden (Loyalty). Hirschman erweitert die Möglichkeiten um die Option "Voice«, womit er der Stimme des Kunden Gewicht verleiht. Dieser hat durchaus die Möglichkeit, sich zu beschweren und somit eine Änderung herbeizuführen, um die Exit-Option zu vermeiden. ${ }^{227}$ Dies auf die Situation einer Unternehmerfamilie übertragen, kann die »Voice«-Option auch als ein Versuch der Veränderung gesehen werden. Nichts anderes versucht die Familienstrategie. Indem man sich (wieder) der gemeinsamen Werte bewusst wird und das alle (und hoffentlich jeden) verbindende Element - nämlich das gemeinsame Familienunternehmen - sieht, besteht durchaus die Möglichkeit, jene, die an eine ExitOption gedacht haben, wieder einzufangen und zu einer Loyalitäts-Lösung zu bewegen. Ist allerdings die Möglichkeit einer Zusammenarbeit beschränkt bzw. nicht mehr vorhanden, kann eine Exit-Lösung befreiende Wirkung haben, indem sie die Verbindung zwischen Familienmitgliedern zum Unternehmen trennt und damit möglicherweise die Beziehung innerhalb der Familie wieder stärkt. Mögliche Varianten einer Exit-Option sind der Abbildung $6 \mathrm{zu}$ entnehmen: Diese reichen vom kompletten Verkauf bis hin zu einer klassischen Thronfolger-Lösung $^{228}$, die sich in der Vergangenheit bewährt hat. ${ }^{229}$

Besteht die Bereitschaft zur Zusammenarbeit in der Familie, kann eine Loyalitäts-Lösung angedacht werden. Dies ist immer dann der Fall, wenn sich die Familienmitglieder zumindest auf den kleinsten gemeinsamen Nenner einigen konnten und man sich so wieder als Einheit begreift. Oberstes Ziel bei einer Loyalitäts-Lösung muss sein, diese (wiedergewonnene) Einheit zu pflegen und zu bewahren. Dieses Ziel verfolgt dann auch die etablierte Family Governance mittels klarer Strukturen. ${ }^{230}$

Am Ende der Familienstrategie steht die Verschriftlichung dessen, was (hoffentlich) gemeinsam erarbeitet wurde. Insbesondere, wenn der Entwurf der Familienverfassung nicht von der Familie selbst geschrieben wurde (was in jedem Fall vorzugswürdig erscheint), sollte die Unternehmerfamilie diesen dann selbst

226 Vgl. Baus (2017), S. 76.

227 Vgl. Hirschman (1970), S. 30 f.

228 Unter Thronfolger-Lösung versteht man, dass das Unternehmen von einem designierten Nachfolger geführt wird, vgl. nur Neuvians (2011), S. 49 Rn. 228. Die anderen Gesellschafter erhalten i.d.R. eine entsprechende Abfindung.

229 Vgl. Baus (2017), S. 76; Graf (2005), S. 48. In diesem Zusammenhang sei auch auf die Höfeordnung verwiesen. Für weiterführende Informationen zum Erbhof vgl. Kalss/Probst (2013a), S. $41 \mathrm{ff}$.

230 Vgl. Baus (2017), S. 76. Vgl. zu Family Governance auch Abschn. 2.2.5. 
finalisieren, um sicherzustellen, dass die Sprache auch der Familie entspricht und somit das Ergebnis als stärker bindend wahrgenommen wird. ${ }^{231}$

Ist eine Familienverfassung im Falle einer Loyalitäts-Lösung grundsätzlich notwendig, kann sie aber auch bei einer Exit-Lösung hilfreich sein. So ist denkbar, dass eine Familie trotz eines Verkaufs des Unternehmens danach gemeinsam investiert bleiben und dabei auf funktionierende Strukturen vertrauen möchte. ${ }^{232}$

\subsubsection{Die Familienverfassung und ihre Stellung im System der »Family Governance«}

"In einer friedlichen Familie kommt das Glück von selbst.» (Chinesisches Sprichwort)

Was macht eine erfolgreiche Family Governance aus? Kann es hierauf überhaupt eine universelle Antwort geben, ist doch jede Familie so verschieden, wie sie nur sein kann? ${ }^{233}$ Schon deswegen kann es nicht ein richtiges oder falsches FamilyGovernance-System geben, das für jede Unternehmerfamilie gleichsam die beste Lösung bietet. Nach einer begrifflichen Einordnung der Family Governance in den Kontext sollen nachfolgend nun einige in der Literatur vertretene Modelle kritisch gegenübergestellt und analysiert werden, um somit zu einer Bewertung der Stellung der Familienverfassung in diesem System zu kommen.

\subsubsection{Von der Corporate Governance zur Family Governance}

Governance als Begriff wurde durch Ökonomen geprägt. ${ }^{234}$ Ganz allgemein versteht man darunter alle "Formen und Mechanismen der Koordinierung zwischen mehr oder weniger autonomen Akteuren, deren Handlungen interdependent sind, sich also wechselseitig beeinträchtigen oder unterstützen. « $^{235}$

Während man unter Corporate Governance grundsätzlich die Führung und Kontrolle eines Unternehmens mit dem klaren Ziel, langfristig den Erfolg des Unternehmens sichern zu wollen, versteht ${ }^{236}$, kommt bei einem Familienunternehmen ein weiterer Wunsch hinzu: der langfristige Verbleib des Unternehmens

231 Vgl. Le Breton-Miller/Miller/Steier (2004), S. 1169 ff.; Felden/Hack (2014), S. 326.

232 Vgl. Baus (2017), S. 77.

233 Vgl. dazu auch Born (2014), Rn. 48; Uffmann (2015), S. 2447; Kirchdörfer/Breyer (2014), S. 17; Meyer (2007), S. 22; Schween et al. (2011), S. 12:»[...] so farbig und variantenreich wie die Familien und ihre Unternehmen selbst."

234 Vgl. Behrens (1998), S. 491.

235 Benz et al. (2007), S. 9.

236 Vgl. nur Hausch (2004), S. 1. 
im Eigentum der Familie ${ }^{237}$. Die dafür notwendige Sicherung des Familienzusammenhalts gelingt allerdings nur durch klare Strukturen und Regeln. ${ }^{238}$ Die Family Governance ergänzt sozusagen die Corporate Governance. ${ }^{239}$ Allerdings fällt es durchaus schwer, die verschiedenen Themen immer trennscharf einem der beiden Bereiche zuzuordnen. ${ }^{240}$ Beispielhaft sei genannt, dass Fragestellungen der Unternehmensnachfolge durchaus beide Bereiche betreffen können. ${ }^{241}$

\subsubsection{Family Governance: eine Begriffsdefinition}

Die Familienverfassung ist im Kontext einer »spezifischen, auf das jeweilige Familienunternehmen zugeschnittenen `Governance-Struktur zu sehen. ${ }^{242}$ Obwohl der Begriff »Family Governance» im Schrifttum mittlerweile vielfach Verwendung findet ${ }^{243}$, scheint bis heute keine einheitliche Definition zu existieren. ${ }^{244}$ Es finden sich vielmehr verschiedene Versuche. So definiert ULRICH (2010) Family Governance als »Leitung und Organisation von Unternehmerfamilien ${ }^{245}$. BAUs (2017) versteht darunter ein »Regelungsgefüge«, bestehend aus »Strukturen und Regeln «, um so das »konstruktive Zusammenspiel mit Gesellschaftern und Unternehmen« zu ermöglichen ${ }^{246}$. Bei Felden/HACK (2014) »definiert [die Family Governance] auf strategischer Ebene die für das Unternehmen notwendigen Gremien, auf operativer Ebene die entsprechenden Instrumente. ${ }^{247}$ Bei KoEBERLE-SCHMID (2008) ist Family Governance als »Organisation zur Sicherung des Zusammenhalts der Familie« zu verstehen ${ }^{248}$. ANGUs (2005) versucht sich dem Kompositum Family Governance zu nähern, indem sie die beiden Begriffe separat untersucht. Zum einen analysiert sie die definitorische Ausprägung des Begriffs Familie und kommt zu dem Ergebnis, dass dieser weiter gefasst werden muss, will heißen, nicht nur

237 Vgl. Mietzner/Tyrell (2012), S. 108.

238 Vgl. Koeberle-Schmid (2008), S. 12 Rn. 78 m. w. N.

239 So beispielsweise Kirchdörfer/Lorz (2011), S. $97 \mathrm{ff}$.

240 Vgl. Kormann (2018), S. 220 f.; Lange (2013), S. 44f., der die Begriffe zwar auch unterscheidet aber gleichwohl annimmt, dass beide Bereiche in vielen Punkten inhaltlich zusammengehören.

241 So auch Hueck (2017), S. 42, der auf Grund der nicht vorhandenen Trennschärfe nur von Governance in Familienunternehmen spricht.

242 Kirchdörfer/Lorz (2011), S. 98.

243 Vgl. exemplarisch Angus (2005); f. Ulrich (2010), S. 717; Baus (2017); Fabis (2007); Felden/ Hack (2014); Koeberle-Schmid (2008), S. 12; Suáre/Santana-Martín (2004), S. 146; Gersick et al. (1997), S. 237; Mustakallio (2002), S. 110; Aronoff/Ward (1996), S. 2; Gallo/KenyonRouvinez (2005), S. 45.

244 Zur gleichen Ansicht kommend Angus (2005), S. 7.

245 Ulrich (2010), S. 717.

246 Baus (2017), S. XXVI.

247 Felden/Hack (2014), S. 282.

248 Koeberle-Schmid (2008), S. 12; vgl. dazu auch Koeberle-Schmid (2008), S. 12 Rn. 84 m. w. N. 
solche Personen, die auf Grund von Blutsverwandtschaft oder Eheschließung zur Familie gehören, seien zu berücksichtigen. Um den Begriff Governance zu definieren, bedient sich ANGus (2005) der lexikalischen Bedeutung: Übersetzt heißt »to govern $\aleph^{249}$ regieren, leiten oder regeln. Natürlich repräsentieren Familien kaum das Oberhaupt eines Staates, insofern stößt eine wörtliche Übersetzung mit "regieren " an ihre Grenzen. Dennoch darf nicht verkannt werden, dass gerade in großen Familien einzelnen Mitgliedern die Befugnis übertragen wird, andere Familienmitglieder zu steuern und deren Verhalten innerhalb der Familien zu überwachen. Jedes Individuum ist sich der Tatsache bewusst, dass es in seiner persönlichen Lebensführung durch Politik und geltendes Gesetz in gewissem Maße eingeschränkt ist und dadurch das Verhalten des einzelnen beeinflusst wird. Parallelen dazu lassen sich auch bei Unternehmerfamilien finden. Hughes (2004) macht bereits auf den ersten Seiten seines Werkes deutlich, dass der Wunsch einer Familie, den Wohlstand über mehrere Generationen zu erhalten, einer etablierten und funktionsfähigen Family Governance bedarf. ${ }^{250}$

Der grundsätzliche Tenor aller Definitionsversuche scheint derselbe zu sein. Im Governance Kodex für Familienunternehmen findet sich eine Definitionsvariante für den Begriff »Family Governance«, welche die wesentlichen Kernelemente zu umfassen scheint:

»Organisation der Inhaberfamilie mit dem Ziel, das Zusammengehörigkeitsgefühl der Mitglieder der Inhaberfamilie und deren Identifikation mit dem Unternehmen zu stärken und langfristig zu festigen. « ${ }^{251}$

Es ist jedoch zu kurz gedacht, das System der Family Governance losgelöst zu betrachten und lediglich auf das System der Unternehmerfamilie zu begrenzen. In einer Unternehmerfamilie spielen mindestens immer beide Dimensionen, die Familie und das Unternehmen, eine Rolle, die es auch bei einer erfolgreichen Family Governance in der Praxis zu berücksichtigen gilt.

\subsubsection{Eine erfolgreiche Family Governance}

Es ist Aufgabe der Familie ein stabiles Umfeld zu schaffen, welches das Governance-System fördert. Darüber hinaus muss ein solches System flexibel genug sein; grundsätzlich sollte für alle Familienmitglieder die Möglichkeit bestehen, Teil dieses Systems zu werden, es zu verlassen, oder in begründeten Fällen ihre Einbindung für bestimmte Zeit »ruhen« zu lassen. Dass sich dies in der Praxis häufig nicht einfach realisieren lässt, sollte nachvollziehbar sein. Auch muss

249 Vgl. http://de.pons.com/\%C3\%BCbersetzung/englisch-deutsch/govern. Zuletzt geprüft am 01.03.2020.

250 Vgl. Hughes (2004), S. 3 ff.

251 Governance Kodex für Familienunternehmen (2015), S. 37. 
dieses System stets an unvermeidbare Veränderungen innerhalb einer Familie und deren Umgebung anpassbar sein. ${ }^{252}$

ANGus (2005) hat den Family-Governance-Rahmen in einer Pyramide dargestellt und in drei Bereiche unterteilt. ${ }^{253}$ Über allem Handeln stehen, und damit ganz oben in der Pyramide zu finden, Grundsätze. Hughes (2004) behauptet, dass das Ziel eines jeden Family-Governance-System sein sollte, das jeweils eigene Streben nach Glück eines jeden Familienmitglieds bestmöglich zu unterstützen. ${ }^{254}$ Nach LANsBERG (1999) sollte eine Familie ihren gemeinsamen Traum verfolgen, wobei der jeweils eigene Traum eines jeden einzelnen Familienmitglieds hintanstehen muss. ${ }^{255}$ Doch ganz gleich, welche Begrifflichkeit verwendet wird, ein Family-Governance-System muss auf allumfassenden Grundsätzen fußen. Minimal sollten die folgenden Fragen beantwortet werden:

- Welche sind unsere gemeinsamen Überzeugungen?

- Was unterscheidet uns von anderen Familien?

- Was ist unsere gemeinsame Vision?

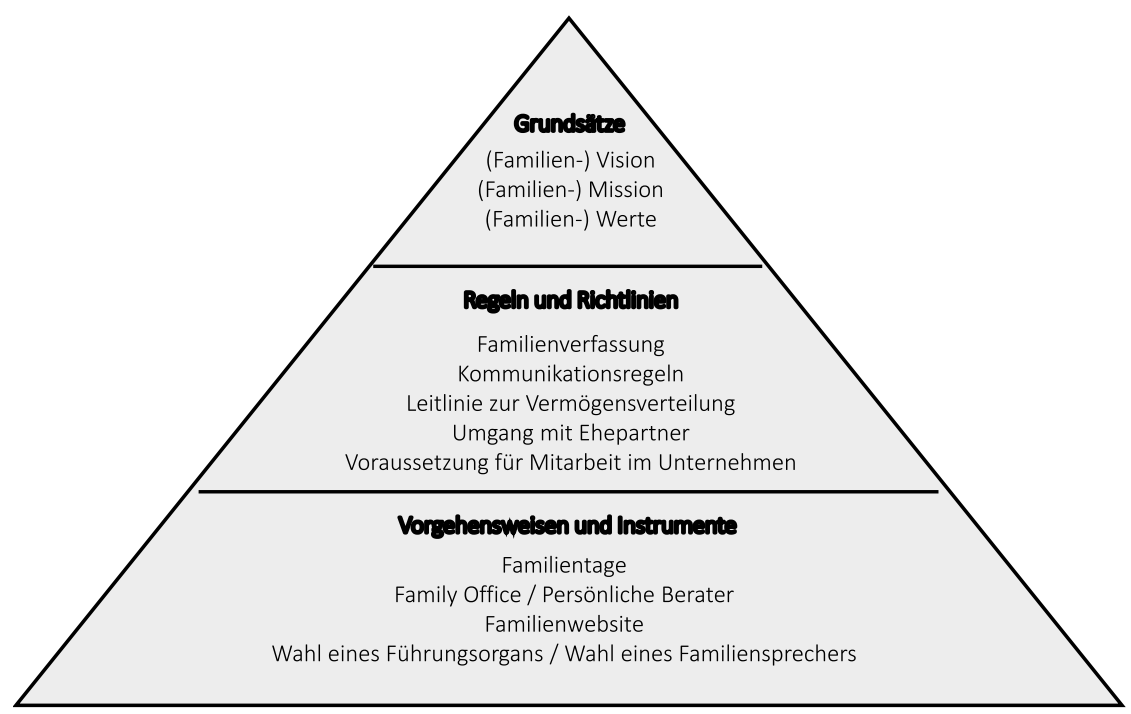

Abbildung 7: Die Family Governance Pyramide ${ }^{256}$

252 Vgl. Angus (2005), S. 8.

253 Vgl. Angus (2005), S. 8.

254 Vgl. Hughes (2004).

255 Vgl. Lansberg (1999).

256 Eigene Darstellung in Anlehnung an Angus (2005), S. 8. Die jeweils entsprechenden Beispiele sind nicht abschließend. 
Es gibt eine Reihe von Tools, die einer Familie helfen können, diese Grundsätze zu definieren. ${ }^{257}$ Diese Fragen werden auch im Prozess der Familienstrategie gestellt. $^{258}$

Auf der zweiten Stufe der Pyramide folgen allgemeine Regeln und Richtlinien, die sich auch auf bestimmte Handlungen innerhalb der Familie beziehen. Diese bedürfen in aller Regel der Schriftform.

Einige der wichtigsten Themenfelder, die diese Regeln abdecken sollten, sind:

- Kommunikationsregeln

- Richtlinien zur Nachlassplanung

- Voraussetzungen für die Mitarbeit im Unternehmen

- Rollen der Ehepartner

- Verwendung des Familienvermögens

- Entscheidungen über Investitionen

- Erwartungen an die Ausbildung der Familienmitglieder

- philanthropische Aktivitäten.

Wenngleich nicht immer erforderlich, so bietet es sich doch bei der ein oder anderen Regel an (z.B. Entscheidungen über Investitionen, philanthropische Aktivitäten, Rollen der Ehepartner etc.), diese im Rahmen einer Familienverfassung niederzuschreiben. ${ }^{259}$ ANGUs (2005) sieht die Familienverfassung dabei auf der zweiten Stufe verortet. Dies wird dem Wesen einer Familienverfassung - zumindest wie man sie in Deutschland kennt - aber keinesfalls gerecht, umfasst sie doch in aller Regel die Grundsätze der ersten Stufe und wird auch durchaus konkret, wenn sie Vorgehensweisen und Instrumente der dritten Stufe enthält, beschreibt und gleichzeitig einführt, wie sich noch in dieser Arbeit zeigen wird. ${ }^{260}$

Die dritte und letzte Stufe der Pyramide beschreibt eben diese Vorgehensweisen sowie Instrumente. Diese leiten sich aus den Regeln und Richtlinien ab. In diesem Zusammenhang soll sich die Familie beispielsweise auch überlegen, ob sie nicht einen Familienrat einführen oder einen Familienmanager wählen möchte. ${ }^{261}$

Bei Felden/Hack (2014) wird die Family Governance im Rahmen der Family Business Governance erläutert. Das Zusammenwirken von Business Governance $^{262}$ und Family Governance in Familienunternehmen wird häufig auch als

257 Vgl. dazu auch Angus (2005), S. 9.

258 Vgl. Abschnitt 2.2.4.

259 Vgl. Angus (2005), S. 9 f.

260 Vgl. Abschn. 2.3.1.3.

261 Vgl. Angus (2005), S. 10; Baumgartner (2009), S. 191.

262 Unter Business Governance oder auch Corporate Governance versteht man die Führung und Kontrolle eines Unternehmens mit dem Ziel, den langfristigen Erfolg des Unternehmens sicherzustellen. In Familienunternehmen kommt ein weiteres Ziel hinzu: den langfristigen 
Family Business Governance bezeichnet. ${ }^{263}$ In Abbildung 8 findet man Parallelen zur Family Governance Pyramide ${ }^{264}$. Hier wird nach Gremien und Instrumenten unterschieden. Der fundamentale Unterschied findet sich in der Stellung der Familienverfassung. Diese symbolisiert hier das Fundament, also die Basis und damit Voraussetzung für eine erfolgreiche Family Governance. Mit dieser Stellung wird man dem Wesen der Familienverfassung, wie sie hierzulande gesehen wird, gerechter, zumal sie zum einen die hier beschriebenen Gremien und Instrumente aufgreift und im Kontext der Familie zu erläutern sucht, aber auch auf die übergeordneten Traditionen und Werte der Familie eingeht, in diesem $\mathrm{Zu}$ sammenhang die Rollen definiert und die Ziele der Familie festlegt.

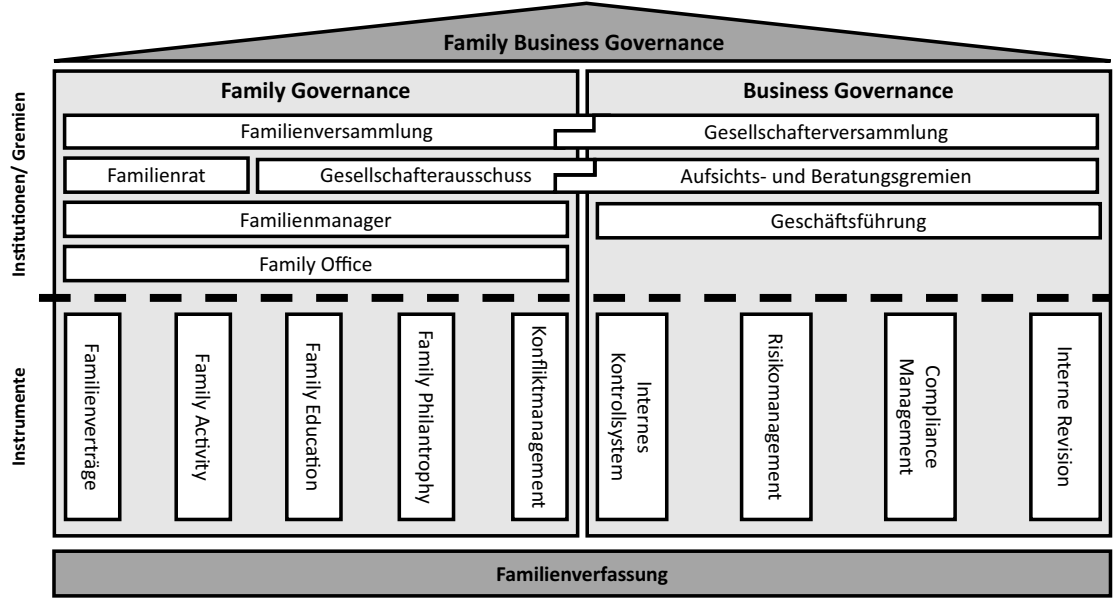

Abbildung 8: Die Family Business Governance ${ }^{265}$

Die Family Governance Pyramide (vgl. Abbildung 7) wird der Familienverfassung indes nicht gerecht, wenn diese auf der mittleren Ebene bei Regeln und Richtlinien positioniert wird. Abbildung 8 eignet sich somit dafür, die Stellung der Familienverfassung innerhalb der Family Governance zu verdeutlichen, so wie diese auch für die vorliegende Arbeit gesehen wird.

"Die Institutionen sind das organisatorische Innenleben einer Unternehmerfamilie. Sie bilden sozusagen die Hardware der Family Governance, während die in der Familienstrategie erarbeiteten Grundüberzeugungen und Regeln die Software darstellen. Die Institutionen sind das Gerüst, das die Familie gegen die Entfremdung stabilisiert. Sie

Verbleib des Unternehmens im Familieneigentum sicherzustellen. Vgl. http://www.kodexfuer-familienunternehmen.de/news. Zuletzt geprüft am 01.03.2020.

263 Vgl. Felden/Hack (2014), S. 282; Koeberle-Schmid (2008), S. 8ff.; Aronoff/Ward (1996); Koeberle-Schmid/Fahrion/Witt (2012), S. $476 \mathrm{f}$.

264 Vgl. Abbildung 7.

265 Felden/Hack (2014), S. 283. 
erzeugen das Bewusstsein eines gemeinsamen Interesses und das Verantwortungsgefühl, das die Familie im Ensemble der Funktionskreise im Familienunternehmen zu einem gestaltungsfähigen und berechenbaren Faktor macht. Die Institutionen stehen als zentraler Bestandteil der Familiencharta dafür, dass eine Family Governance tatsächlich in Gang gesetzt wird. ${ }^{266}$

Wie umfassend eine Family-Governance-Struktur ausgestaltet ist, hängt mitunter auch von dem Entwicklungsstadium des Familienunternehmens ab (vgl. Abbildung 9). Solange der Gründer eines Unternehmens (früher tendenziell eher patriarchalisch) die Geschicke lenkt, trifft er alle wesentlichen Entscheidungen (oft alleine). In diesem Fall ist er aber auch lediglich ihm gegenüber in der Verantwortung. Befindet sich das Unternehmen noch in der "Gründerphase» oder arbeiten ausschließlich wenige Familienmitglieder (und keine Fremdmanager) im Unternehmen, dann sind vermutlich nur am Rande etwaige umfassende Regelungen zur Family Governance angezeigt.

Auf der zweiten Stufe, der sog. Geschwistergesellschaft ${ }^{267}$, werden sich die Familien- und Gesellschafter- bzw. Unternehmensinteressen noch ähneln. Allerdings: Sobald weitere Familienmitglieder der nächsten Generation Anteile am Unternehmen erhalten, ist es vorbei mit der Eindeutigkeit, wer Entscheidungen zu treffen hat. Empirische Untersuchungen bestätigen, dass die Zahl der Gesellschafter, ob aktive oder passive, mit jedem Generationenwechsel in aller Regel zunimmt. ${ }^{268}$ Jeder Gesellschafter, also jedes Familienmitglied, das Anteile am Unternehmen hält, hat meist seine eigene Vorstellung darüber, welche Entwicklung er vom Unternehmen erwartet, wenngleich er diese Vorstellung nicht immer explizit kommunizieren mag; und so hat auch jeder Familiengesellschafter eine eigene Auffassung darüber, in welcher Weise er sich bei der Unternehmensentwicklung beteiligen möchte: mit einem aktiven Part als Mitglied der Unternehmensleitung, mit einem beobachtenden oder beratenden Part als Mitglied eines Aufsichtsgremiums oder gar lediglich als (passiver) Teilnehmer der jährlich stattfindenden Gesellschafterversammlung. Diese verschiedenen Formen einer möglichen Beteiligung bergen Konfliktpotenzial. So kann es bei mehreren Familiengesellschaftern schon um die Frage gehen, wer den Vorsitz der Geschäftsleitung übernimmt. Dieser ist gewöhnlich lediglich einer Person vorbehalten. ${ }^{269}$ Hinzu kommen also auf dieser zweiten Stufe erste Fragestellungen

266 Baus (2017), S. 121.

267 »Aus der Alleininhaberschaft des Gründers wird in der zweiten Generation in der Regel eine Geschwistergesellschaft, bei der die familiäre Inhaberschaft auf mehrere Geschwister aufgeteilt ist«; vgl. May (2012).

268 Vgl. Hack (2009), S. 1 ff.

269 Vgl. Wolf/Paul/Zipse (2009), S. $151 \mathrm{f}$. 
zur Wahrung der Familienharmonie und zur Streitvermeidung, die in der Gründungsphase noch unbeachtet bleiben konnten. ${ }^{270}$

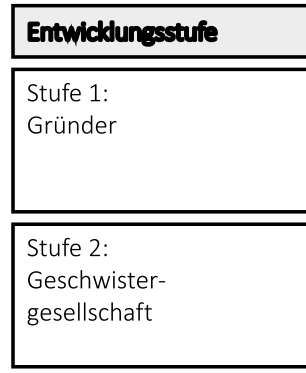

Stufe 3+4:

Vetterngesellschaft und

Familiendynastie

\section{Dominierende Themenstellungen}

- Übergabe der unternehmerischen Führung

- Nachfolge in den Gesellschaftsanteilen

Absicherung des Ehegatten

Erbschaftsteuerplanung

- Sicherung der Eigentümerstellung der Familien

- Bewahrung von Familienharmonie und Streitverhinderung

- Nachfolgeregelung

- Verwendung des Unternehmenskapitals

- Ausschüttung/Entnahme, Grad der Verschuldung

- Liquidität der Beteiligung

Familientradition und-kultur

Streitvermeidung und Streitlösung

Beteiligung und Rolle der Familie

Mission und Vision der Familie

Verbindung der Familie zum Geschäft

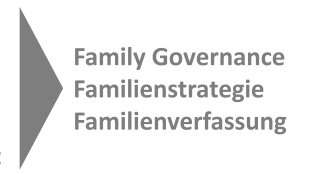

Abbildung 9: Eigentümerthemen in der Evolution des Familienunternehmens ${ }^{271}$

Die Familienstrategie erlangt auf der dritten bzw. vierten Stufe, die auch als Vetterngesellschaft ${ }^{272}$ bzw. Familiendynastie ${ }^{273}$ bezeichnet werden ${ }^{274}$, besondere Bedeutung. In zahlreichen Familienunternehmen beschränkt sich die Unternehmerfamilie in diesem Stadium auf eine eher begleitende, passive Rolle im Rahmen einer Aufsichtsfunktion. In diesem Stadium können sich die Familien-

270 Vgl. nur Baus (2017), S. $80 \mathrm{ff}$.

271 Kirchdörfer/Lorz (2011), S. 99. Je nachdem in welcher Phase des Lebenszyklus sich ein Unternehmen befindet und von welcher Generation es geführt oder gehalten wird, spricht man in der 1 . Generation von der »Gründergeneration «, in der zweiten Generation dann von einer "Geschwistergesellschaft» und in der dritten Generation von einer »Vetterngesellschaft« oder »Familiendynastie«.

272 Häufig auch als Vetternkonsortium bezeichnet. Es entsteht durch Erbschaft. »Es tritt als Eigentümerform des Familienunternehmens frühestens in der 2. Generation, i.d. R. aber erst ab der 3. Generation auf. [...] Vettern-Konsortien sind Unternehmen, an denen mehrere Gesellschafter, deren Abstammung sich auf eine zumindest 3 Generationen zurückliegende Ursprungsehe zurückführen läßt, beteiligt. In vielen Fällen stammen diese Gesellschafter zudem noch aus verschiedenen Generationen«, Klein (2010), S. 178.

273 Bei einer Familiendynastie teilen sich die Inhaberschaft des Unternehmens eine große Anzahl an Personen. Zu einer Familiendynastie wird ein Familienunternehmen meist erst einige Generationen nach der Vetterngesellschaft. Bekannte Beispiele dafür sind die Unternehmerfamilien Haniel und Heraeus.

274 Grundsätzlich zu den Evolutionsstufen im Familienunternehmen Ward (1991); vgl. dazu auch Baus (2017), S. 54 ff,.; Koeberle-Schmid/Fahrion/Witt (2012), S. 37; Kirchdörfer/Lorz (2011), S. 99; Papesch (2010), S. 19; Letmathe et al. (2008), S. 209; Gersick et al. (1997), S. 17; Alderson (2011), S. 73. 
und Gesellschafter- bzw. Unternehmensinteressen stark unterscheiden. Darüber hinaus muss der Gefahr einer wachsenden Entfremdung ob der zunehmenden Größe der Familie begegnet werden. ${ }^{275}$ In dieser Phase rücken Themen wie Familientradition und -kultur, Streitvermeidung und -lösung, die Frage nach der Beteiligung und Rolle der Familie, die Verbindung der Familie zum Unternehmen oder die Mission und Vision der Unternehmerfamilie deutlich stärker in den Vordergrund. Hieraus kann dann das Bedürfnis erwachsen, eine gemeinsame Familienstrategie zu entwickeln und diese in eine Familienverfassung zu gießen $^{276}$, die in den meisten Fällen von Unternehmerfamilien als identitätsstiftend wahrgenommen wird. ${ }^{277}$ Insbesondere in solchen Familienunternehmen, die sich mindestens bereits in der dritten Generation befinden, entsteht durch den familiären Kontext und die dadurch begründeten Interdependenzen ein deutlich höherer Komplexitätsgrad, als dies bei Familienunternehmen in der ersten Generation der Fall ist.

Diese Interaktionen, Beziehungen und Abhängigkeiten, die im Drei-KreisModell (vgl. Abbildung 2) dargestellt werden, können zu ernsthaften "Schnittstellenproblemen ${ }^{278}$ führen. Die Begrifflichkeit Corporate Governance wird auf Grund dieser verschiedenen Schnittstellen zur Unternehmerfamilie um die Dimension Familie erweitert und mit dem Anglizismus Family Governance bezeichnet - eine "selbst organisierte `Strukturierung einer Unternehmerfamilie. $\aleph^{279}$ Im Grunde genommen geht es bei der Entwicklung einer Familienstrategie oder der Erstellung einer Familienverfassung darum, die drei Dimensionen des Systems Familienunternehmen (nämlich Familie, Unternehmen und Eigentum/ Gesellschafterstellung) aufeinander abzustimmen. „Die Bändigung dieser drei Systeme durch die Etablierung einer leistungsfähigen Organisation mit Strukturen und festen Regeln [also die Family Governance] soll die Langfristigkeit und Nachhaltigkeit im Familienunternehmen sichern. ${ }^{280}$

275 Vgl. Baus (2017), S. XXIII; Lange (2005), S. 2588; Koeberle-Schmid/May (2011), S. 57; Schulz/Werz (2007a), S. 313; Binz/Mayer (2012), S. 201; Felden/Hack (2014), S. 22. Zum Identifikationsverlust durch Zersplitterung der Beteiligung, wenn in Folge von Generationswechseln die Anteile an immer mehr Erben fallen vgl. Scherer et al. (2012), Rn. 76.

276 Vgl. Reich/Bode (2018), S. 305: »Das Bedürfnis nach schriftlicher Fixierung eines gemeinsamen Wertekanons dürfte insbesondere in Familienunternehmen mit großem oder wachsendem Gesellschafterkreis ausgeprägt sein."

277 Vgl. Hueck (2017), S. 152; Kirchdörfer/Lorz (2011), S. 99; Kalss/Probst (2013c), S. 16; Felden/ Hack (2014), S. 325. Auf den emotionalen Aspekt der Familienverfassung hinweisend Schween et al. (2011), S. 20 f., 25.

278 Kirchdörfer/Lorz (2011), S. 99.

279 Rüsen (2017b), S. 188.

280 Kirchdörfer/Lorz (2011), S. 99f. 


\subsection{Die Familienverfassung in der Gestaltungspraxis}

\subsubsection{Aufbau einer Familienverfassung}

\subsubsection{Allgemeines}

So einzigartig wie jede Familie und jedes Familienunternehmen selbst, so unterschiedlich können auch die Inhalte und Regelungsbereiche einer Familienverfassung sein. ${ }^{281}$ Es kann daher auch kein Mustertext existieren, den man für eine Vielzahl von Unternehmerfamilien unreflektiert übernehmen und anwenden kann. ${ }^{282}$ Vielmehr sollte die Familienverfassung auf die jeweilige Familie abgestimmt sein. ${ }^{283} \mathrm{Da}$ es sich bei einer Familienverfassung um einen Art moralisch-psychologischen Vertrag handelt, der auf die besondere Situation, das besondere Verhältnis der Familienmitglieder zum Unternehmen und untereinander sowie auf ihre Struktur angepasst wird, gibt es keine verbindlichen Vorgaben über Aufbau und Inhalt. ${ }^{284}$

Auch wenn man sich über die verschiedenen möglichen Regelungsbereiche einer Familienverfassung tiefgehende Gedanken macht, so sollte man sich immer vor Augen halten, dass sie vornehmlich die Intention verfolgt, einen entscheidenden Beitrag zur langfristigen Überlebenssicherung des Familienunternehmens zu leisten und dessen wirtschaftlichen Wert kontinuierlich und nachhaltig zu steigern. ${ }^{285}$ Nicht zu vernachlässigen ist dabei der Familienzusammenhalt als entscheidendes Element und als Grundvoraussetzung für einen möglichst lang anhaltenden Erfolg. ${ }^{286}$

Bei Hennerkes/Kirchdörfer (2015) findet sich folgendes Zitat, bei dem es sich zweifelsohne um die Präambel einer Familienverfassung einer nicht näher benannten deutschen Unternehmerfamilie handelt:

,Wir haben großen Respekt vor der Arbeit und dem Einsatz der vorangegangenen Generationen. Wir wollen die uns mitgegebenen ideellen Werte bewahren und die finanziellen Werte vermehren. Wir stellen uns gerne dieser verantwortungsvollen und gleichzeitig erfüllenden Lebensaufgabe. Die nachfolgende Familienverfassung soll uns und den uns nachfolgenden Generationen helfen, diese Verbundenheit und die daraus resultierenden Chancen und Verpflichtungen gegenüber Unternehmen und Familie stets im Auge zu behalten und den Zusammenhalt innerhalb der gesamten Familien

281 Vgl. Born (2014), Rn. 48; Uffmann (2015), S. 2447; Kirchdörfer/Breyer (2014), S. 17; Meyer (2007), S. 22; Schween et al. (2011), S. 12: "[...] so farbig und variantenreich wie die Familien und ihre Unternehmen selbst."

282 Vgl. Born (2014), Rn. 48.

283 Vgl. Uffmann (2015), S. 2447.

284 Vgl. Felden/Hack (2014), S. 322; Arnhold/Rohner (2015), Rn. 85.

285 Vgl. Koeberle-Schmid/Witt/Fahrion (2010), S. 163.

286 Vgl. Ward (2005), S. 144ff.; Koeberle-Schmid/Schween/May (2011), S. 2499. 
nach innen zu festigen und nach außen zu dokumentieren. Wir wollen jetzt und für die Zukunft unternehmerisch eine gemeinsame Linie verfolgen, unser Familienunternehmen bewahren und dieses in der Familie zusammenhalten. Hierzu wollen wir mit Respekt und Fairness miteinander umgehen und in Geschlossenheit handeln. Unsere Familienverfassung ist im Sinne eines offenen und in der Generationenfolge fortzuschreibenden ethischen Leitbildes zu verstehen. Dies bedeutet, dass wir unsere Familienverfassung bei Bedarf um neue Prinzipien und Grundlagen erweitern und an geänderte Verhältnisse anpassen wollen. Das unternehmerische Werk von [...] kann nur erfolgreich fortgeführt werden, wenn sich die nachfolgenden Generationen - trotz Verschiedenheit der Persönlichkeiten, Ideen und Visionen - in langfristiger Denkweise, Kontinuität des Handelns, Unabhängigkeit ihrer Entscheidungen und im Bewusstsein der [...] - Traditionen den in dieser Familienverfassung zusammengefassten Regeln und Prinzipien unserer Familie unterordnen. ${ }^{287}$

Bei der vorstehenden, exemplarischen Einleitung einer Familienverfassung zeigt sich sehr deutlich, dass sowohl die Unternehmerfamilie als auch das Familienunternehmen von den positiven Wirkungen der Familienverfassung profitieren sollen. So soll eine Familienverfassung auch der Vielschichtigkeit von Familienunternehmen Rechnung tragen und mit ihrem Inhalt eine wertvolle Vorarbeit für die daraus ableitbare Family Governance wie auch für die Corporate Governance leisten. ${ }^{288}$

\subsubsection{Typische Regelungsinhalte und der Bezug zur »Familiness«}

Wie bereits erörtert, kann es nicht das Schema für die Ausgestaltung einer Familienverfassung geben. Vielmehr finden sich in der Literatur - die vornehmlich von der Beraterpraxis geprägt zu sein scheint ${ }^{289}$ - diverse Muster und Leitfäden.

Nachfolgend soll ein Überblick über die Inhalte und Regelungsbereiche gegeben werden, die sich im einschlägigen Schrifttum finden lassen.

287 Hennerkes/Kirchdörfer (2015), S. 66f.

288 Vgl. Felden/Hack (2014), S. 322.

289 Vgl. Gläßer (2014), S. 229; Uffmann (2015), S. 2446; Kormann (2018), S. 181. Die Ausführungen anwaltlicher Berater in diesem Themenkomplex als aufschlussreich empfindend Kormann (2017), S. 24. 


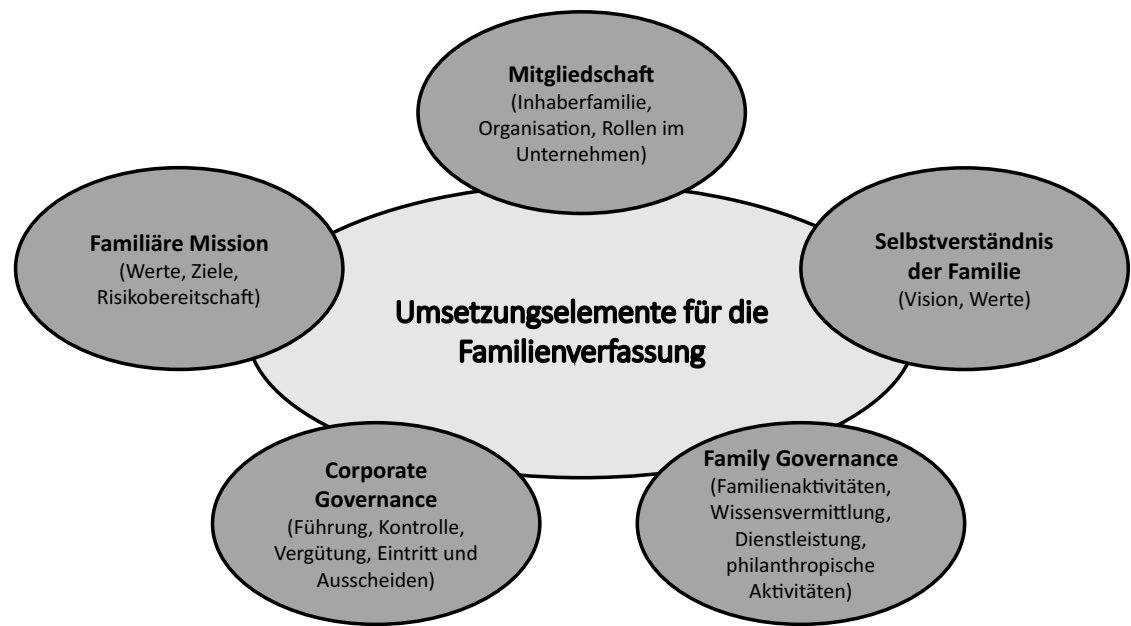

Abbildung 10: Gängige Umsetzungselemente einer Familienverfassung 290

Vergleicht man die existierenden Muster und Leitfäden ${ }^{291}$ zu Familienverfassungen, so wird deutlich, dass sich die darin üblicherweise behandelten Themen der Erkenntnisse aus der Wissenschaft $\mathrm{zu}$ »Familiness « und »sozialem Kapital « ${ }^{292}$ bedienen. ${ }^{293}$ Das Wirtschaftsmagazin »Newsweek« sprach bereits im Jahre 2004 recht plakativ vom "family factor« - dem Schlüssel ökonomischen Erfolges in Familienunternehmen. ${ }^{294}$ Der von HabBershon/Williams (1999) geprägte Begriff $»$ Familiness ${ }^{295}$ zielt auf die Operationalisierung des Familieneinflusses ${ }^{296}$ in Familienunternehmen ab, wobei sie dabei die Familie als einzigartige Ressource sehen, um einen strategischen Wettbewerbsvorteil zu erlangen. Diese besondere Ressource entsteht dabei durch die Interaktion von Familie, Famili-

290 Felden/Hack (2014), S. 328.

291 Vgl. Felden/Hack (2014), S. 322f.; Baus (2017), S. 110ff.; Schulz/Werz (2007a), S. 311 f.; Born (2014), Rn. 49; Holler (2016), Rn. 106; Koeberle-Schmid/Fahrion/Witt (2012), S. 487; Meyer (2007), S. 23; May (2009), S. 117; Gläßer (2014), S. 232f.; Kirchdörfer/Lorz (2011), S. 103; Kormann (2018), S. 181; Montemerlo/Ward (2011), S. $23 \mathrm{ff}$.; Angus (2005), S. $8 \mathrm{ff}$.

292 Allgemein zu soziales Kapital in Familienunternehmen vgl. Felden/Hack (2014), S. $73 \mathrm{ff} . \mathrm{Zu}$ den Auswirkungen des sozialen Kapitals auf die Unternehmensziele vgl. Cabrera-Suárez/ Déniz-Déniz/Martín-Santana (2015), S. 145 ff.; Zu den Auswirkungen von »Familiness« in Bezug auf den Erfolg in Familienunternehmen vgl. Tokarczyk et al. (2007), S. $17 \mathrm{ff}$.; ganz allgemein zu den strategischen Vorteilen von Familienunternehmen vgl. Habbershon/Williams (1999), S. $1 \mathrm{ff}$.

293 Uffmann (2015), S. 2447.

294 Vgl. Newsweek, April 2004, S. 44f.

295 Vgl. Habbershon/Williams (1999).

296 Vgl. Prym (2014), S. 24. 
enangehörigen und Unternehmen ${ }^{297}$, was gleichzeitig auch eine Dimension des sozialen Kapitals beschreibt ${ }^{298}$.

Welche Ressourcen tragen nun konkret zur Familiness bei, die damit auch Niederschlag in den Mustern und Leitfäden für Familienverfassungen finden? Zunächst ist hier ein Blick in die Vergangenheit angezeigt, zumal der historische Kontext des Familienunternehmens und die aus Familienwerten abgeleitete Unternehmenskultur eine wesentliche Rolle spielen ${ }^{299}$ - getreu dem Motto: Wer nicht weiß, woher er kommt, weiß auch nicht, wohin er geht ${ }^{300}$. Hierzu gehört auch der Leumund als Familienunternehmen; es genügt nicht, nur eines zu sein, ohne dass das Umfeld Kenntnis davon nimmt, wo doch gerade eine besonders lokale Verwurzelung auch zu diesen Ressourcen zählt. ${ }^{301}$ Für HaBbershoN/ Williams (1999) gehören auch die bei Familienunternehmen oftmals zu findenden andersartigen informellen und formellen Entscheidungsstrukturen und -prozesse sowie eine besondere Verbindung zwischen den Eltern und Kindern dazu.

In der Literatur lassen sich weitere Merkmale finden, wenngleich diese dort nicht unmittelbar in Zusammenhang mit Familiness gebracht werden. In Bezug auf deren Charakteristika lassen sich diese Merkmale dennoch in den Kontext der Familiness setzen und werden in den entsprechenden Mustern und Checklisten berücksichtigt. Hierzu zählen die Kultur (insb. Vertrauenskultur ${ }^{302}$ ) in Familienunternehmen als strategischer Vorteil ${ }^{303}$, eine in die Zukunft gerichtete Erwartungshaltung, Wertvorstellungen im Allgemeinen sowie der persönliche Einfluss der Unternehmerfamilie - nicht zuletzt auch auf Grund emotionaler Bindung an das Unternehmen. ${ }^{304}$ Darüber hinaus ist vielen Familienunternehmen eine Langfristorientierung zuzuschreiben, die den getroffenen Entscheidungen zum Wohle des Unternehmens und der Familie zu Grunde liegt. ${ }^{305}$

Stellt man auf die Zielfunktion eines Familienunternehmens ab, lässt sich feststellen, dass auch diese Charakteristika in Familienverfassungen Niederschlag finden. Dies bedeutet, dass Familienunternehmen in aller Regel nicht nur rein monetäre, objektive Ziele (z.B. Umsatz, Gewinn), sondern auch nicht-mo-

297 Vgl. Mertens (2009), S. 22.

298 Vgl. Felden/Hack (2014), S. 74f.

299 Vgl. Zahra/Hayton/Salvato (2004), S. 365.

300 Sinngemäß nach Montemerlo/Ward (2011), S. 23: »Knowing one’s history is crucial to preparing for the future«.

301 Vgl. Mertens (2009), S. 22.

302 Vgl. Schwass/Amann/Ward (2004), S. 261.

303 Vgl. Zahra/Hayton/Salvato (2004), S. 365; vgl. dazu auch Rogoff/Heck (2003), S. 559 ff.

304 Vgl. Cabrera-Suárez/Saá-Pérez/García-Almeida (2001), S. 37; Schwass/Amann/Ward (2004), S. 261; Baus (2017), S. 143.

305 Vgl. James Jr. (1999), S. 47. 
netäre, subjektive Ziele verfolgen. ${ }^{306}$ Dazu gehört u.a., sich einen guten Ruf als Familienunternehmen aufzubauen und zu bewahren, Familienmitgliedern die Möglichkeit einzuräumen, sich beruflich im Unternehmen zu betätigen ${ }^{307}$, sowie ein Umfeld zu schaffen, in dem persönliche Zufriedenheit und Selbstverwirklichung und somit ein adäquates Maß an Lebensqualität erreicht werden kann. ${ }^{308}$ Dieses Maß an Lebensqualität und Selbstverwirklichung erfordert nicht zuletzt auch eine gewisse finanzielle Unabhängigkeit der Unternehmerfamilie. ${ }^{309}$ Wenn nun den Regelungsinhalten einer solchen Familienverfassung mitunter die Sicherung des Unternehmenserfolges zugeschrieben wird ${ }^{310}$, so muss immer berücksichtigt werden, dass Erfolg in einem Familienunternehmen anders definiert wird, zumal sich diese Definition von einer rein objektiven, die vornehmlich auf den Gewinn abstellen mag, abzuheben sucht. ${ }^{311}$ Somit steht beispielsweise nicht nur die Steigerung des ökomischen Wertes im Vordergrund, sondern gleichzeitig auch die des emotionalen Wertes eines Familienunternehmens ${ }^{312}$; und ganz praktisch verstehen es nicht wenige Familien als Erfolg, das Unternehmen möglichst über viele Generationen in Familienhand zu bewahren. ${ }^{313}$

Der Bogen in einer Familienverfassung wird gespannt von der »Werte- über die Beziehungsebene zur Konfliktprävention und letztlich zur Bestandssicherung des Unternehmens «. ${ }^{314}$ Etwas konkreter findet sich in einer Familienverfassung das »Wer» (Wen sollen diese Regelungen betreffen?), das »Warum» (Was sind unsere Familienwerte und Glaubensfragen?), das »Was« (Was sind unsere Grundsätze in Bezug auf das Familienunternehmen?), das »Wie« (Wie soll unser Regelwerk in Bezug auf familiäre und geschäftliche Beziehungen aussehen?) und das »Wann« (Wann und wie muss die Familienverfassung auf Aktualität überprüft werden und welchen Prozess wollen wir für eine mögliche Anpassung zu Grunde legen?). ${ }^{315}$

Sich den »Corporate Governance Kodex" zum Vorbild nehmend, hat sich im Jahr 2004 eine Kommission aus Fachleuten und Familienunternehmern konstituiert, die den sogenannten »Governance Kodex für Familienunternehmen « ${ }^{316}$

306 Vgl. Mertens (2009), S. 22; dazu auch Chrisman/Chua/Litz (2003), S. 468; Chrisman/Chua/ Sharma (2003), S. 21; Dunn (1995), S. 21; Olson et al. (2003), S. $659 \mathrm{ff.}$

307 Vgl. Dunn (1995), S. 21.

308 Vgl. Stafford et al. (1999), S. 206.

309 Vgl. Olson et al. (2003), S. 644.

310 Vgl. Schulz/Werz (2007a), S. 311.

311 Vgl. Dunn (1995), S. 21.

312 Vgl. Zellweger (2017), S. 226f.

313 Vgl. Koeberle-Schmid/Fahrion/Witt (2012), S. 478; Koeberle-Schmid/Schween/May (2011), S. 2500; Koeberle-Schmid/May (2011), S. 56.

314 Gläßer (2014), S. 231.

315 Vgl. Montemerlo/Ward (2011), S. 26.

316 Siehe dazu unter http://www.kodex-fuer-familienunternehmen.de/; zuletzt geprüft am 01.03.2020. 
als Leitlinie für eine gewissenhafte und verantwortungsvolle Führung von Familienunternehmen erstellt haben. Im Jahr 2010 und zuletzt im Mai 2015 wurden diese Leitlinien einer Revision unterzogen und weiterentwickelt. Der Kodex gibt einen Überblick über wesentliche Themen, über die sich eine Unternehmerfamilie ihre eigenen Gedanken machen sollte. Er schließt in Kapitel 8 mit der klaren Empfehlung, die Bestandteile aus diesem Kodex individuell und gemeinsam, also in einer eigenen Familienverfassung, zu regeln. ${ }^{317}$

In Abbildung 11 findet sich exemplarisch ein typischer Aufbau einer Familienverfassung. Diese Übersicht soll gleichzeitig als Sammlung möglicher Regelungsinhalte dienen, was nicht zwingend bedeutet, dass in jeder Familienverfassung jeder der darin enthaltenen Punkte auch individuell geregelt werden muss. Eine solche Übersicht kann auch als Hilfestellung für die Unternehmerfamilie verstanden werden, zumal es ihr nicht selten schwer fällt, zwischen Inhaberperspektive und Unternehmensperspektive zu unterscheiden. ${ }^{318}$ So werden verschiedene Facetten aufgezeigt, zu denen sich die Unternehmerfamilie Gedanken machen kann.

Auch erhebt dieser Aufbau keinen Anspruch auf Vollständigkeit, sofern es eine solche überhaupt geben kann. Wie schon einleitend in Abschnitt 2.3.1.1 ausgeführt, können die Regelungsbereiche einer Familienverfassung genauso unterschiedlich sein wie jede Familie und jedes Familienunternehmen selbst.

\begin{tabular}{|l|l|}
\hline Präambel & - Vorwort \\
& - Definition des Geltungsbereichs \\
& - Konsensformel, Zweck und Reichweite \\
& - Festschreibung des Familienmottos \\
& - Selbstverpflichtung der Familienmitglieder \\
& - Erklärung zur rechtlichen Unverbindlichkeit des Doku- \\
& ments \\
\hline Werte/ & - Selbstverständnis der Unternehmerfamilie \\
Fundament & - Tragende/prägende Werte für die Familie \\
& - Tragende/prägende Werte für das/die Unternehmen ${ }^{319}$ \\
& - Ausmaß der Prägung des Unternehmens durch die Familie \\
\hline Mission & - Welchen Nutzen stiften wir als Unternehmerfamilie? \\
\hline Vision & - Was ist unser Traum als Unternehmerfamilie? \\
\hline
\end{tabular}

317 Vgl. Holler (2016), Rn. 101; siehe dazu auch Kapitel 8.1 unter http://www.kodex-fuer-famili enunternehmen.de/images/Downloads/Kodex_2015.pdf; zuletzt geprüft am 01.03.2020.

318 Vgl. May (2009), S. 115.

319 Inhaltlich findet man hier häufig die Verwurzelung in einer bestimmten Region, Wertschätzung gegenüber den eigenen Mitarbeitern, der Anspruch der Nachhaltigkeit der Unternehmensentwicklung und der Unternehmensprodukte oder auch vom Unternehmenszweck völlig unabhängige ideelle, förderungswürdige Aspekte. Vgl. hierzu auch Gläßer (2014), S. 232. 


\begin{tabular}{|l|l|}
\hline Ziele & - Ziele für Familie und Unternehmen (z. B. soziale und un- \\
& ternehmerische Ziele) \\
& - Ziele betreffend das Familienvermögen \\
& - Erwartungen an Wachstum, Rendite und Ausschüttung (ggf. \\
& unter Nennung von Zielpräferenzen und -prioritäten)
\end{tabular}

320 Hier wird typischerweise geklärt, wer zur Familie gehört und wie es sich mit Ehegatten, Lebenspartnern, Adoptivkindern, nicht-ehelichen Kindern, Schwiegerkindern etc. verhält; also wer zukünftig Teil dieser Familie werden kann und wer unter welchen Bedingungen ausscheidet.

321 Hier geht es insbesondere um Fragen der Übertrag- und Vererbbarkeit von Beteiligungen und wer Gesellschafter werden kann.

322 Sollen Familienmitglieder eine aktive Rolle im Unternehmen übernehmen und sich damit operativ einbringen - oder haben sie eine reine Eigentümer- und Gesellschafterrolle inne? Letztere könnte um eine Position im Aufsichtsrat/Beirat erweitert werden.

323 Hier muss die Frage gestellt werden, ob eine solche Mitarbeit grundsätzlich erwünscht ist. Wenn dem so ist, auf welchen Ebenen und in welcher Position und Funktion?

324 Soll das Unternehmen durch Familien- und/oder Fremdmanager geführt werden? Soll die operative Tätigkeit von Familienmitgliedern ermöglich werden, die selbst keine Gesellschafter sind?

325 Hier ist an sog. Familienverantwortliche, Familienmanager oder auch an Beiratsfunktionen zu denken. 


\begin{tabular}{|c|c|}
\hline $\begin{array}{l}\text { Vermögensrelevante } \\
\text { Regelungen }\end{array}$ & $\begin{array}{l}\text { - Entnahmepolitik } \\
\text { - Ausschüttungsmodus } \\
\text { - Gewinnverwendung }{ }^{326} \\
\text { - Sicherung und Gestaltung der Altersvorsorge von Famili- } \\
\text { enmitgliedern } \\
\text { - Reaktion auf etwaige finanzielle Schwierigkeiten des Un- } \\
\text { ternehmens } \\
\text { - Umgang mit dem Ausscheiden von Gesellschaftern }\end{array}$ \\
\hline Verfahrensregeln & $\begin{array}{l}\text { - Regeln für den Umgang miteinander und mit Dritten (intern } \\
\text { und in der Öffentlichkeit) } \\
\text { - Regeln zu Verantwortlichkeiten innerhalb der Unterneh- } \\
\text { merfamilie } \\
\text { - Regeln zu Konfliktmanagement } \\
\text { - }{ }^{328} \\
\text { - Regeln für Ehe-, Erb- und Schenkungsverträge } \\
\text { - Regeln für die Gesellschafterwechsel } \\
\text { - Regeln zur Informationspolitik nach innen und nach außen } \\
\text { - Regeln zur Anpassung und Besetzung von Ämtern } \\
\text { - Regeln zur Qualifikation potenzieller Nachfolgenden in Ei- } \\
\text { gentum und Management } \\
\text { - Bestimmungen für etwaige Änderungen/Anpassung der } \\
\text { Familienverfassung } \\
\text { - Kommunikationsregeln }\end{array}$ \\
\hline Schlussbemerkung & - Schlussbemerkung und Unterschriftenzeile \\
\hline Anhang & $\begin{array}{l}\text { - Familienstammbaum } \\
\text { - Grundlegende Dokumente, Protokolle, Motive etc. }\end{array}$ \\
\hline
\end{tabular}

Abbildung 11: Übersicht über gängige Inhalte einer Familienverfassung ${ }^{329}$

Nachdem der typische Aufbau einer Familienverfassung analysiert wurde, stellt sich die Frage, wie umfangreich eine solche Familienverfassung gestaltet werden soll. Auch hierauf wird es keine allgemeingültige Antwort geben. Der gesunde Menschenverstand lässt vermuten: "Nicht so viel wie möglich, sondern nur so viel wie nötig. « ${ }^{330}$ In der Literatur finden sich verschiedene Beispiele ausformulierter Familienverfassungen. ${ }^{331}$ Gleichzeitig finden sich immer auch Gegenstimmen, die die Mustertexte zu lang oder zu wenig ausführlich finden: So ist Kormann (2018) beispielsweise der Auffassung, dass das Muster von AndreaE

326 Hier können beispielsweise Grundsätze für gemeinsame Anlagestrategien, die Verwendung von Gewinnanteilen für soziale und karitative Zwecke oder die Gründung bzw. Ausstattung einer Stiftung festgelegt werden.

327 Auch in Bezug auf die Umsetzung und Durchführung der Family Governance-Regeln.

328 Zur Vermeidung und Lösung von Konflikten.

329 Eigene Darstellung in Anlehnung an Felden/Hack (2014), S. 323; Baus (2017), S. 137; Arnhold/Rohner (2015), Rn. 84; Fabis (2007), S. 362; Gläßer (2014), S. 232f.; Kormann (2018), S. 182; Meyer (2007), S. 23; Andreae (2007), S. 20; Schulz/Werz (2007a), S. 4; Kirchdörfer/Lorz (2011), S. 103; Montemerlo/Ward (2011), S. 23f.; Gersick et al. (1997).

330 Baus (2017), S. 114. In diesem Sinne auch Felden/Hack (2014), S. 290.

331 Vgl. exemplarisch Schulz/Werz (2007b), S. 353 ff.; Andreae (2007), S. $149 \mathrm{ff}$. 
$(2007)^{332}$ einen überzogenen Anspruch habe. KormanN (2018) sieht dieses Beispiel als »überfrachtet « an und geht davon aus, dass diese sehr ausführlichen und detaillierten Regelungen sehr bald bedingt durch die weitere Unternehmensentwicklung obsolet sein könnten und stellt somit eine längere Halbwertszeit in Frage. ${ }^{333}$ Insgesamt wird davor gewarnt, dass der in aller Regel hohe Abstraktionsgrad solcher Familienverfassungen die Gefahr birgt, "vollmundige Mission Statements ${ }^{334}$ abzugeben, denen es selbst an einem echten Bezug zu den tatsächlich in den Familienunternehmen vorzufindenden Prozessen und Problemen mangelt. ${ }^{335}$

\subsubsection{Institutionen, Instrumente und Verfahrensregeln}

Nachfolgend sollen einige ausgewählte Institutionen ${ }^{336}$, Instrumente und Verfahrensregeln einer erfolgreich etablierten Familienverfassung ausführlich beschrieben werden, zumal diese wichtige Bausteine bei der Frage der Absicherung der Regelbefolgung darstellen, wie sich im Rahmen der durchgeführten Interviews noch zeigen wird. ${ }^{337}$

\subsection{Der Familientag}

Der Familientag gleicht einem Familientreffen und kann damit auch als »Vollversammlung der Familienmitglieder ${ }^{338}$ bezeichnet werden. Die Idee zu Familientreffen in Unternehmerfamilien lieferte bereits vor 30 Jahren WARD (1987). ${ }^{339}$ Sein Vorschlag, dass die Mitglieder der Unternehmerfamilie in regelmäßigen Abständen ohne zwingend unternehmensrelevantem Anlass ${ }^{340}$ Zeit miteinander verbringen, ist so simpel wie wirksam. Dies begründet er damit, dass

»[e]njoying, activities together builds rapport, friendship, and ease of communication among family members. ${ }^{341}$

In adeligen Großfamilien wirken diese Familientage seit jeher als »Kitt « zwischen den einzelnen Stämmen. ${ }^{342}$ Diese Familientreffen sind aber nicht nur gesellige

\footnotetext{
332 Vgl. Andreae (2007), S. 149 f.

333 Kormann (2018), S. 181.

334 Bernhardt (2010), S. 18.

335 Vgl. Lorz/Kirchdörfer (2011), S. 103.

336 Als Begriff kann auch »Gremien« verwendet werden, vgl. Kieser (2004), S. 207.

337 Vgl. Abschnitt 4.1.

338 Baus (2017), S. 122; auch diese Bezeichnung verwendend Andreae (2007), S. 151.

339 Vgl. Ward (1987), S. $80 \mathrm{ff}$.

340 So auch Fabis (2007), S. 364.

341 Ward (2011), S. 158f.

342 Thunen (2015), S. 38.
} 
Veranstaltungen. Sie haben durchaus auch einen emotionalen Charakter. ${ }^{343}$ Sie sollen einen erheblichen Beitrag zur Sicherung des Zusammenhalts der Familie leisten $^{344}$ und damit die Sicherung der "Sprach- und Handlungsfähigkeit ${ }^{345}$ unterstützen. In manchen, weit verzweigten Familien können die Familientage auch dazu beitragen, sich besser kennenzulernen ${ }^{346}$, da insbesondere entfernt verwandte Familienmitglieder häufig nicht in ständigem Kontakt zueinander stehen. ${ }^{347}$ So dominieren in über 50 Prozent der Familienunternehmen die passiven Gesellschafter. ${ }^{348}$ Wurden früher persönliche Beziehungen innerhalb der Familie noch intensiver gepflegt, nimmt dies in der heutigen Generation durch die zunehmende Mobilität und eine geografische Trennung der Familienmitglieder beständig ab. ${ }^{349} \mathrm{Im}$ Wesentlichen geht es aber darum, wechselseitiges Vertrauen zu schaffen, das Zusammengehörigkeitsgefühl zu stärken und damit der Gefahr der zunehmenden Entfremdung entgegenzuwirken. ${ }^{350}$

"And, as family relationships continue, increasing interdependence and interactions produce greater levels of trust (based on shared norms and values), principles of reciprocity (obligations) and exchange among family members. « ${ }^{351}$

Darüber hinaus können und sollten Familientage dazu genutzt werden, über unternehmensbezogene Themen zu berichten und $\mathrm{zu}$ diskutieren. Hier kann beispielsweise die Geschäftsleitung einen kurzen Überblick über die Lage des Unternehmens und die strategische Ausrichtung geben. ${ }^{352}$ So haben alle Familienmitglieder die Möglichkeit, relevante Informationen zur Geschäftslage aus erster Hand $\mathrm{zu}$ erhalten, und es werden dadurch gleichzeitig Informationsasymmetrien vermieden. ${ }^{353}$ Gerade da an den Familientagen zumeist nicht nur Gesellschafter teilnehmen, sondern auch deren Ehepartner, Kinder, langjährige Lebenspartner ${ }^{354}$, können diese sich besser orientieren und damit die Entscheidungen der Gesellschafter einfacher nachvollziehen. ${ }^{355}$ Familientage kön-

343 Vgl. Baus (2017), S. 123; Thunen (2015), S. 46.

344 Vgl. Koeberle-Schmid (2008), S. 92.

345 Groth/Rüsen (2013), S. 100.

346 Vgl. Canessa et al. (2016), S. 103; so im Ergebnis auch Groth/Rüsen (2013), S. 100.

347 Vgl. Fabis (2007), S. 364; Baus (2017), S. 126.

348 Vgl. Stöhlker/Müller Tiberini (2005), S. 9. Eine gemeinsame Studie des Beratungsunternehmens Intes zusammen mit der der EBS (European Business School) sowie JP Morgan zeigt, dass in 53 Prozent der untersuchten Firmen die Mehrheit der Anteile bei passiven Gesellschaftern liegt, vgl. May/Redlefsen/Haller (2004).

349 Vgl. Kormann (2018), S. 283.

350 Vgl. Wiechers (2004), S. 242; Kormann (2018), S. 283; Baus (2017), S. 123; Fabis (2007), S. 364; Koeberle-Schmid (2008), S. 92.

351 Arregle et al. (2007), S. 77.

352 Vgl. Fabis (2007), S. 364.

353 Vgl. Ulrich (2011), S. 319.

354 Vgl. Groth/Rüsen (2013), S. 101; so im Ergebnis auch Kormann (2018), S. 282.

355 Vgl. Fabis (2007), S. 364. 
nen auch dazu dienen, konkrete Streitpunkte, die sich zu ernsthaften Konflikten verhärten können, anzusprechen und zu diskutieren. ${ }^{356}$ Indem Konflikte offen angesprochen und ausdiskutiert werden, kann von diesen auch ein positiver Effekt für Familie und Unternehmen ausgehen ${ }^{357}$ Nur wer sich kennt, kann sich vertrauen. Selbst wenn man in einer großen Familie für das eine oder andere Familienmitglied nur bedingt Sympathie hegen mag, so können die regelmäßigen Zusammenkünfte zumindest dazu führen, den anderen besser einschätzen zu können, was als Grundlage für gemeinsame Entscheidungen nicht irrelevant sein dürfte. ${ }^{358}$ Weiterhin werden Familientage dazu genutzt, die Familienverfassung zu überprüfen, ggf. anzupassen und zu genehmigen. ${ }^{359}$

Familientage sind »langfristig festgelegte, regelmäßig[e] $\aleph^{360}$, meist einmal ${ }^{361}$ - in manchen Familien bei Bedarf auch zweimal ${ }^{362}$ - jährlich ausgerichtete Zusammenkünfte von Familienmitgliedern, an denen möglichst alle Mitglieder der Unternehmerfamilie teilnehmen. ${ }^{363}$ Zumindest sollte eine möglichst hohe Präsenz der Familienmitglieder das Ziel sein. ${ }^{364}$ Es hat sich als zweckmäßig erwiesen, dass für ein solches Treffen ein fest geplantes Wochenende im Jahr vorgesehen wird $^{365}$ - am besten mit Anreise an einem Freitag.

Das Programm sollte sich klar vom Alltag abgrenzen. Es geht schließlich darum, eine Plattform zu schaffen, die Familie erleben zu können. ${ }^{366}$ Für BAus (2017) gehört zum Familientag ein strukturierter Ablauf mit Tagesordnung. Die inhaltliche Bandbreite umfasst: Information, Diskussion, Entscheidung über Sachfragen, Wahlen, Geselligkeit. ${ }^{367}$ Letztlich ist aber jeder Familientag individuell zu gestalten und an die Bedürfnisse der Familie anzupassen. ${ }^{368}$ Familientage bilden darüber hinaus eine geeignete Plattform, um auf die Geschichte der Unternehmerfamilie und ihr Unternehmen näher einzugehen. Diese Geschichten

356 Vgl. Baus (2017), S. 123; Canessa et al. (2016), S. 103.

357 Vgl. Kellermanns/Eddleston (2004), S. 221.

358 So auch Groth/Rüsen (2013), S. 100.

359 Vgl. Andreae (2007), S. 151.

360 Fabis (2007), S. 363; So auch Groth/Rüsen (2013), S. 101: »Familientage sollten immer an festen Wochenenden im Jahr durchgeführt warden [...].So können alle Beteiligten schon lange im Voraus planen, und eine Terminfindung entfällt, die zum einen mühsam ist und dann zwangsläufig zu Entscheidungen führt, in denen zugunsten einer Mehrheit andere ausgeschlossen werden."

361 Vgl. Groth/Rüsen (2013), S. 100; Ulrich (2011), S. 253; Kormann (2018) S. 282 f.; Andreae (2007), S. 151.

362 Vgl. Kormann (2018) S. $282 \mathrm{f}$.

363 Vgl. Wimmer/Groth/Simon (2004), S. $59 \mathrm{f}$.

364 Vgl. Kormann (2018) S. $282 \mathrm{f}$.

365 Vgl. Groth/Rüsen (2013), S. 101. So im Ergebnis auch Kormann (2018) S. $282 \mathrm{f}$.

366 Vgl. Kormann (2018) S. $282 \mathrm{f}$.

367 Vgl. Baus (2017), S. 124.

368 Vgl. Baus (2017), S. 124. 
und Gespräche zum gemeinsamen Hintergrund verstärken die Bindungswirkung. ${ }^{369}$

Bei diesen Familientreffen bilden sich (naturgemäß) Netzwerke heraus. Es sollte darauf geachtet werden, dass jeder in ein Netzwerk integriert ist. Ein sicherlich empfehlenswertes Netzwerk ist das der sogenannten nächsten Generation (»next generation network«). Diese Generation hat meist eigene Themen, die sie gemeinsam besprechen oder in Projekten realisieren kann. Für die Gesellschafter von morgen ist dieser Ort für gemeinsame Begegnungen besonders wichtig. Gemeinsame Reisen dieser "next generation« (beispielsweise zu ausländischen Niederlassungen des Unternehmens) können den Zusammenhalt in besonderer Weise fördern. ${ }^{370}$

Zweifelsfrei stärken Familientage das Bewusstsein der Familienmitglieder, Angehörige einer Eigentümerfamilie zu sein, und sie tragen gleichzeitig dazu bei, möglicherweise schon aus einer früheren Generation stammende Unstimmigkeiten, latente Konflikte oder auch nur Vorbehalte abzubauen oder diese gar beizulegen. ${ }^{371}$

\subsection{Der Familienrat}

Der Familienrat, auch »Familienrepräsentanz ${ }^{372}$, $»$ Family Council ${ }^{373}$, Familienmanager ${ }^{374}$ oder "Ausschuss der Familienversammlung ${ }^{375}$ genannt, ist ein Organ, das der $»$ Binnenorganisation $\aleph^{376}$ der Familie dient und sich damit vorrangig um alle Fragen und Belange der Familie kümmert und weniger um das Unternehmen. ${ }^{377}$ Dieses Familiengremium lässt sich vornehmlich bei Unternehmerfamilien mit einer größeren Anzahl an Gesellschaftern und Familienmitgliedern beobachten. ${ }^{378}$ Der Familienrat kann als Pendant zum Beirat bzw. Aufsichtsrat eines Unternehmens betrachtet werden. ${ }^{379}$

BiAnChi/Alderson (2012) definieren seine Rolle als

»[...] platform where the voices of family members of a family business can be heard. It is not a legal entity and has no fiduciary responsibilities; however, it is the governance

369 Vgl. Kormann (2017), S. 351.

370 So im Ergebnis auch Kormann (2017), S. 352.

371 Vgl. Fabis (2007), S. 364.

372 Koeberle-Schmid (2008), S. 15.

373 Kormann (2017), S. 504.

374 Canessa et al. (2016), S. 101.

375 Felden/Hack (2014), S. 300.

376 Rüsen/Groth (2012), S. 26.

377 Vgl. Redlefsen (2004), S. 78; Bianchi/Alderson (2012), S. 190; Kormann (2017), S. 505; Witt (2008).

378 Vgl. Rüsen (2017b), S. 293.

379 Vgl. Koeberle-Schmid (2008), S. 2; Kormann (2017), S. 476. 
structure of a family business wherein the emotional issues of a family business are addressed. ${ }^{380}$

Er ist damit zentraler Ansprechpartner für alle Belange der Familienmitglieder ${ }^{381}$ und gleichzeitig ein geeignetes Instrument, um die Family Governance erfolgreich umsetzen zu können. ${ }^{382}$ Wenngleich diese Institution nicht gesetzlich bedingt ist und sehr selten im Gesellschaftsvertrag namentliche Erwähnung findet, so ist deren Aufgabenspektrum zu konkretisieren, um mit diesem Gremium nicht in Konkurrenz zu einem anderen Aufsichtsgremium (z.B. Aufsichtsrat, Beirat) $\mathrm{zu}$ treten. ${ }^{383}$ Die Aufgaben des Familienrats umfassen die Organisation und Ausrichtung der Familientage ${ }^{384}$, die Integration neuer Familienmitglieder $^{385}$, die Kommunikation zwischen der Gesellschafterebene, den Familienmitgliedern und den anderen Governance-Institutionen ${ }^{386}$, die Organisation von Praktikantenstellen im Speziellen und die Mitarbeit von Familienmitgliedern im Unternehmen im Allgemeinen $^{387}$, die Durchführung von Maßnahmen des Familienrisikomanagements ${ }^{388}$, die Organisation der Gesellschafterkompetenz (Fortbildungsprogramme) ${ }^{389}$, sowie die Überwachung der Einhaltung der Familienverfassung ${ }^{390}$. Der Familienrat ist darüber hinaus die zentrale Anlaufstelle bei auftretenden Konflikten. ${ }^{391}$ Die vorgenannte Aufzählung ist keinesfalls abschließend und somit ist das Aufgabenspektrum des Familienrats stets an die individuellen Bedürfnisse der Unternehmerfamilie anzupassen. Der Nutzen eines solchen Familienrats - auch neben einem etablierten Beirat - wird in der Literatur bestätigt. ${ }^{392}$

Der Familienrat sollte so aufgestellt sein, dass er ein möglichst genaues Abbild aller Familienmitglieder repräsentiert. ${ }^{393}$ Familienfremde Personen eignen sich somit nicht als potenzielles Mitglied eines Familienrats. ${ }^{394}$ Es sind insbesondere solche Mitglieder geeignet, die innerhalb der Familie gut vernetzt sind und intensiven Kontakt zu vielen Familienmitgliedern pflegen. ${ }^{395}$ Darüber hinaus

380 Bianchi/Alderson (2012), S. 190.

381 Vgl. Baus (2017), S. 127; Schulz/Werz (2007b), S. 360.

382 Vgl. Stöhlker/Müller Tiberini (2005), S. 12.

383 Vgl. Kormann (2017), S. 505.

384 Vgl. Andreae (2007), S. 151; Canessa et al. (2016), S. 101.

385 Vgl. Stöhlker/Müller Tiberini (2005), S. 12; Canessa et al. (2016), S. 113.

386 Vgl. Kormann (2017), S. 505.

387 Vgl. Schulz/Werz (2007b), S. 360.

388 Vgl. Rüsen (2017b), S. 293.

389 Vgl. Canessa et al. (2016), S. 101; Rüsen (2017b), S. 295.

$390 \mathrm{Vgl}$. Schulz/Werz (2007b), S. 360.

391 Vgl. Rüsen (2017b), S. 293.

392 Vgl. Berent-Braun/Uhlaner (2012), S. 103 ff.; Kormann (2017), S. 505.

393 Vgl. Felden/Hack (2014), S. 296.

394 So im Ergebnis Uhlaner (2006), S. $125 \mathrm{ff}$.

395 Vgl. Felden/Hack (2014), S. 298. 
sollten sie sich in ihrer Qualifikation und Kompetenz bestenfalls ergänzen und eine klare Aufgabenverteilung vornehmen. ${ }^{396}$

Der Familienrat wird auf drei bis fünf Jahre gewählt. ${ }^{397}$ Die Wahl der Mitglieder obliegt der Familienversammlung, also dem Familientag. ${ }^{398}$ Die Anzahl der Mitglieder des Familienrats variiert und ist individuell in Abhängigkeit von der Größe der Familie zu bestimmen. Ist die Familie in Stämmen organisiert, sollte von jedem Stamm ein Familienmitglied entsandt werden. ${ }^{399}$ Sinnvoll erscheint es in der Praxis, wenn immer auch mindestens ein Familienmitglied der jüngeren Gesellschaftergeneration (»next generation«) vertreten ist. ${ }^{400}$

Im Vordergrund der Aufgaben steht insbesondere die dauerhafte Stärkung und nachhaltige Sicherung der Beziehungen untereinander und damit einhergehend auch die Bindung an das Familienunternehmen. ${ }^{401}$

\subsection{Der Familienmanager}

Der Familienmanager sollte nicht mit einem Mitglied der operativen Geschäftsführung verwechselt werden und auch nicht gleichzeitig Geschäftsführer sein $^{402}$; er steht vielmehr der Familie als Sprecher vor und fungiert als »Transmissionsriemen $\aleph^{403}$ zwischen den Eigentümern und der Unternehmensleitung. ${ }^{404}$ Ist die Unternehmerfamilie noch relativ klein (dies meint eine überschaubare Anzahl von Familienmitgliedern von etwa zehn bis 20$)^{405}$, mag die Installation eines Familienmanagers genügen. Der Familienmanager ersetzt dabei zumeist den Familienrat. ${ }^{406}$ Der Familienmanager operiert als Vertrauensperson und genießt in aller Regel bei allen Familienmitgliedern eine hohe Akzeptanz. ${ }^{407}$ Die Aufgaben sind mit denen des Familienrats vergleichbar.

\subsection{Family Education - die Kompetenzförderung der Gesellschafter}

Unter Family Education versteht man die Förderung der »Gesellschafterkompetenz ${ }^{408}$. Auf die Frage, über welche Kompetenzen ein verantwortungsbewusster Eigentümer verfügen sollte und wie er diese erwerben kann, gaben

396 Vgl. May (2017a), S. 586.

397 Vgl. Schulz/Werz (2007b), S. 360.

398 Vgl. Felden/Hack (2014), S. 298.

399 Vgl. Andreae (2007), S. 151.

400 Vgl. Noske (30.11.2017).

401 Vgl. Felden/Hack (2014), S. 301.

402 Vgl. Koeberle-Schmid/Fahrion/Witt (2012), S. 340.

403 Koeberle-Schmid/Fahrion/Witt (2012), S. 340.

404 Vgl. Nelton (2008), S. $1 \mathrm{ff}$.

405 Vgl. Koeberle-Schmid/Fahrion/Witt (2012), S. 339.

406 So auch Felden/Hack (2014), S. 304.

407 Vgl. May (2017a), S. 582.

408 Groth/Rüsen (2013), S. 101; Rüsen (2017a), S. 121; Kormann (2017), S. 506. 
ARONOFF/WARD (2011) als erste eine Antwort: »The best way to protect the future of the business and enhance its continuity in the family is to prepare the next generation of owners for their roles and responsibilities. ${ }^{409}$ So sind sie sich darüber einig, dass ein verantwortungsvoller Eigentümer nicht geboren wird. ${ }^{410}$ Vielmehr bedarf es entsprechender Programme, um sich zu einem »verantwortungsvollen und kompetenten Gesellschafter « entwickeln zu können. ${ }^{411}$ Die Übertragung des impliziten Wissens auf die nächste Generation ist von entscheidender Bedeutung. ${ }^{412}$

»...knowledge, since most of the times, it is >the taken-for-granted factor. « $^{413}$

Die »Kompetenz der Gesellschafter ist gefragt und gewünscht. ${ }^{414}$ Sie wird zunehmend wichtiger. ${ }^{415}$ Dies gelte insbesondere dann, "wenn im Generationenverlauf eine immer weitere Entfernung der Familienmitglieder vom Unternehmen droht und Kernkompetenzen für das Verständnis des Unternehmens nicht mehr am Küchentisch von jüngster Kindheit an vermittelt werden. «" ${ }^{416}$ Die Kompetenzen eines Gesellschafters unterscheiden sich von denen eines Unternehmenslenkers. So brauchen diese nicht selbst über Führungskompetenz zu verfügen, vielmehr müssen sie das Führungshandeln der Geschäftsleitung und dessen Auswirkungen verstehen. Auch muss ein Gesellschafter keine Unternehmensstrategie selbst entwickeln können, aber er muss in der Lage sein, diese entsprechend $\mathrm{zu}$ bewerten. ${ }^{417}$

»Es geht nicht darum, Künstler zu werden, sondern darum, Kunst von Kitsch unterscheiden zu können. ${ }^{418}$

Weiterhin muss er auch nicht zwingend nachvollziehen können, ob die von der Unternehmensleitung vorgeschlagene Strategie die beste Alternative darstellt, sondern er muss indes fähig sein zu beurteilen, ob der Strategievorschlag verantwortbar ${ }^{419}$ ist. ${ }^{420}$ Die Fortbildung der Familienmitglieder dient auch dazu, dass

409 Aronoff/Ward (2011), S. 50. Im Original in fetter Schriftstärke.

$410 \mathrm{Vgl}$. Aronoff/Ward (2011), S. 52: »Responsible ownership of a family business doesn't come naturally. It has to be learned."

411 Vgl. May et al. (2015), S. 14; Arnhold/Rohner (2015), Rn. 94; Felden/Hack (2014), S. 341.

412 Vgl. Papesch (2010), S. 146; Le Breton-Miller/Miller/Steier (2004), S. 307 f.; Trevinyo-Rodríguez/Tàpies (2006), S. 344.

413 Trevinyo-Rodríguez/Tàpies (2006), S. 343.

414 May (2017a), S. 581.

415 So im Ergebnis auch May et al. (2015), S. 17.

416 Breyer (2015), S. 153.

417 Vgl. Rüsen/Schlippe/Groth (2014), S. 106; Kormann (2018), S. 45.

418 Rüsen/Schlippe/Groth (2014), S. 105.

419 Eine Strategie wäre beispielsweise nicht verantwortbar, wenn dabei höhere Risiken eingegangen werden würden, als das von den Gesellschaftern akzeptierte Risikoniveau vorsieht.

420 Vgl. Kormann (2018), S. 45. 
sie den Bezug zum Unternehmen nicht verlieren und Kenntnis über ihre Rechte und Pflichten als Familienmitglied und Gesellschafter erlangen. ${ }^{421}$ Diese Wissensvermittlung ist insbesondere für solche Familienmitglieder von Bedeutung, die weder Mitglied der operativen Unternehmensführung noch in einem Aufsichtsgremium sind. ${ }^{422}$ Auch kann die Aufgabe der Gesellschafterkompetenz darin gesehen werden, Nachfolger auf ihre Funktion als Unternehmensverantwortliche vorzubereiten, indem ihnen sukzessive das notwendige Grundwissen (u. a. Traditionen, Werte und Ziele der Unternehmerfamilie ${ }^{423}$ ) vermittelt wird. ${ }^{424}$ So investieren Unternehmerfamilien mit Familienverfassung in 53 Prozent der Fälle in die Aus- und Weiterbildung ihrer Familienmitglieder und Gesellschafter, wohingegen Familienunternehmen ohne Familienverfassung lediglich in 17 Prozent der Fälle in Gesellschafterkompetenz investieren. ${ }^{425}$ Die Erfahrung zeigt: Je umfangreicher und besser alle Familienmitglieder regelmäßig über die Unternehmensbelange unterrichtet sind, desto größer sind deren Zufriedenheit und Vertrauen. Auch ist die Gefahr, den operativ aktiven Mitgliedern der Familie zu misstrauen, geringer. ${ }^{426}$ Also: gemeinsames Lernen zur Stärkung des $\mathrm{Zu}-$ sammenhalts und der emotionalen Bindung. ${ }^{427}$ Dies verringert gleichzeitig das Konfliktpotenzial. ${ }^{428}$ Mit systematischem Einsatz von Family Education kann so auch Familien inhärenten Schwächen begegnet werden (z. B. Nepotismus ${ }^{429}$ ). ${ }^{430}$

Verantwortlich für die Family Education können unterschiedliche Gremien sein: In kleineren Unternehmerfamilien mag dies der Familienmanager sein, in größeren vermutlich eher der Familienrat. ${ }^{431}$ Die Inhalte der Weiterbildungsmaßnahmen sollten sich stets am Adressatenkreis orientieren, wobei als Zielgruppe sowohl die operativ tätigen und nicht tätigen Gesellschafter, Nicht-Gesellschafter, Ehepartner, aber auch insbesondere deren Kinder angesprochen sind. ${ }^{432}$ Gerade die junge Nachfolgegeneration fordert diese Angebote heutzutage

421 Vgl. Koeberle-Schmid (2008), S. 91; Ward (1991), S. 227; Gersick et al. (1997), S. 237.

422 Vgl. Arnhold/Rohner (2015), Rn. 94.

423 Vgl. Felden/Hack (2014), S. 343. So im Ergebnis wohl auch Rüsen/Schlippe/Groth (2014), S. 107.

424 Vgl. Schulz/Werz (2007a), S. 312; Papesch (2010), S. 146.

425 Vgl. Koeberle-Schmid/Schween/May (2011), S. 2506.

426 Vgl. Arnhold/Rohner (2015), Rn. 94; Felden/Hack (2014), S. 341.

427 Vgl. Le Breton-Miller/Miller/Steier (2004), S. 305 ff.; May/Rieder (2008). Zur Abgrenzung zwischen Family Education und Familienaktivitäten vgl. die Übersicht Felden/Hack (2014), S. 342 .

428 Vgl. Shepherd/Haynie (2009), S. $1245 \mathrm{ff}$.

429 In diesem Kontext versteht man darunter die Besetzung von operativen Positionen mit Familienmitgliedern unabhängig von deren Kompetenz und Eignung.

430 Vgl. Bergfeld/Weber/Kraus (2009), S. 1; Felden/Hack (2014), S. 341; Lee/Lim/Lim (2003), S. $657 \mathrm{ff}$.

431 Vgl. Felden/Hack (2014), S. 341.

432 Vgl. Koeberle-Schmid/Fahrion/Witt (2012), S. $363 \mathrm{ff}$. 
bewusst ein. ${ }^{433}$ Im Rahmen von Praktika können junge Familienmitglieder auch einen ersten Einblick in das Innere des Unternehmens erhalten. ${ }^{434}$

Zumal sich bereits abzuzeichnen scheint, dass zukünftig immer mehr nichtoperative Gesellschafter über die Geschicke von Familienunternehmen entscheiden werden, wird der Gesellschafterkompetenz ein immer größer werdender Stellenwert beigemessen werden müssen. ${ }^{435}$

\subsection{Kommunikationsregeln und Konfliktmanagement}

Ein weiterer wichtiger Bestandteil einer erfolgreichen Family Governance bildet der Umgang mit Konfliktsituationen ${ }^{436}$ innerhalb der Familie und im Kontext von Geschäftsleitung und Familie. ${ }^{437}$ Im Rahmen der Umsetzung der Familienverfassung sollte also über ein Konfliktmanagement nachgedacht werden, ${ }^{438}$ damit Konflikte nicht eskalieren bzw. bereits eskalierte Konflikte beherrschbar werden. ${ }^{439}$ Dies umfasst auch die Einführung geeigneter Verfahrens- ${ }^{440}$ und Kommunikationsregeln im Familienkreis. ${ }^{441}$ Die häufig sehr engen und teils komplexen Beziehungen innerhalb einer Unternehmerfamilie bergen ein nicht unerhebliches Konfliktpotenzial. ${ }^{42}$ Teilweise sind Konflikte gar generationenübergreifend. ${ }^{443}$ So wundert es nicht, dass dieser Unternehmenstypus gar als »[...] fertile environments for conflict [...] «" ${ }^{44}$ betrachtet wird. Gerade emotional-destruktive Konfliktsituationen bergen ein großes Gefahrenpotenzial für das Familienunternehmen. ${ }^{45}$ Aufgabe der Family Governance sollte daher auch sein, innerfamiliäre Konflikte vom Unternehmen möglichst fernzuhalten. ${ }^{446}$

Nun lassen sich Konflikte trotz aller Vorkehrungen nicht gänzlich verhindern. ${ }^{447}$ Ein professionelles Konfliktmanagement und eine "funktionierende, dialoggeprägte Kommunikationskultur « ${ }^{448}$ dienen zum Erhalt des Familien-

433 Vgl. Prügl/Hauck (2015).

434 Vgl. Felden/Hack (2014), S. 343.

435 So auch Rüsen/Schlippe/Groth (2014), S. 107.

436 Allgemein zu Konfliktpotenzialen in Familienunternehmen vgl. Abschnitt 2.1.5.

437 Vgl. Krause (2012), S. 715.

438 Vgl. Oesterle (2007), S. 44; Kormann (2017), S. 476.

439 Vgl. Felden/Hack (2014), S. 334; (2015), Rn. 84.

440 Vgl. Schulz/Ruddat (2012), 312.

441 Vgl. Kormann (2018), S. 269; Koeberle-Schmid (2008), S. 88; Felden/Hack (2014), S. 334.

442 Vgl. Felden/Hack (2014), S. 334.

443 Vgl. Großmann (2014), S. 143. Schon früh sich mit der schädlichen Auswirkungen von Konflikten auf das Familienunternehmen beschäftigend Donnelly (1964).

444 Harvey/Evans (1994), S. 345.

445 Vgl. Koeberle-Schmid/May (2011), S. 55; Harvey/Evans (1994), S. 331 ff.; Schlippe/Kellermanns (2008), S. $40 \mathrm{ff}$.

446 Vgl. Governance Kodex für Familienunternehmen (2015), S. 33; dies aufgreifend Krause (2012), S. 715.

447 So im Ergebnis Weber (2009), S. 149; Vgl. dazu auch Felden/Hack (2014), S. 337.

448 Pirmanschegg (2016), S. 177. 
friedens. ${ }^{449}$ Häufig gibt es eine Vertrauensperson (z.B. Familienmanager) oder eine legitimierte Institution (z.B. Familienrat), die im Streitfall als Moderator schlichtet ${ }^{450}$ oder den Konflikt im Rahmen der definierten Vorgehensweise (im Zweifel unter Einschaltung eines neutralen Dritten, beispielsweise eines Mediators) beizulegen sucht. ${ }^{451}$ Ein unüberlegter und nicht professionalisierter Umgang mit Konflikten kann nicht nur den emotionalen Banden zwischen den Familienmitgliedern nachhaltig schaden, sondern auch die Existenz des Unternehmens bedrohen bis zerstören. ${ }^{452}$ Umso mehr verwundert es, dass in vielen Familienunternehmen einem professionellen Konfliktmanagement wenig Beachtung geschenkt wird. Nach einer Studie von PwC aus dem Jahre 2010 haben global betrachtet lediglich 30 Prozent der Familienunternehmen für einen Konflikternstfall vorgesorgt und ein institutionalisiertes Konfliktmanagement etabliert. ${ }^{453}$ Klar kommunizierte Spielregeln und Verhaltensweisen bei Konflikten stellen ein probates Mittel zur Prävention von Konflikten dar. ${ }^{454}$

Im Endeffekt können gegenseitiges Vertrauen sowie gemeinsam geteilte Ziele und gelebte Werte wahrscheinlich Konflikte vermindern. ${ }^{455}$ Unabhängig davon sollten im Rahmen der Family Governance klare Verhaltens- und Kommunikationsregeln aufgestellt werden, um präventiv das Konfliktpotenzial einzudämmen. Manche Unternehmerfamilien bedienen sich als Hilfestellung der »Konfliktmanagementordnung « der Deutschen Institution für Schiedsgerichtsbarkeit e.V. (DIS) ${ }^{456}$, welche beispielsweise zur Bereinigung eines Konflikts einen neutralen Dritten empfiehlt. ${ }^{457}$ Während Gerichtsverfahren auf Grund der mit ihnen einhergehenden negativen Begleiterscheinungen (hoher Zeit- und Kostenaufwand, Öffentlichkeit könnte Notiz nehmen, nachhaltig destruktiver Einfluss auf familiäre Beziehungen etc. ${ }^{458}$ ) möglichst vermieden werden sollten, könnte beispielsweise ein Mediationsverfahren ${ }^{459}$ als Maßnahme in die Familienverfassung aufgenommen werden. ${ }^{460}$

449 Vgl. Kidwell/Kellermanns/Eddleston (2012).

450 Vgl. Kormann (2017), S. 246, der die Moderation von Konflikten nicht bei einem "Gremium" sieht, sondern klarstellt, dass die Aufgabe vielmehr von einer Person (im Zweifel auch durch ein Team von zwei Personen) zu leisten ist.

451 Vgl. Rüsen (2016), S. 98; Rüsen (2017a), S. 123.

452 Vgl. Felden/Hack (2014), S. 334.

453 Vgl. Bartels/Schmid/Hofbauer (2010), S. 6.

454 Vgl. Kellerwessel (2003), S. $209 \mathrm{ff}$.

455 So im Ergebnis wohl auch Bertrand/Schoar (2006).

$456 \mathrm{Vgl}$. http://www.disarb.org/de/16/regeln/dis-konfliktmanagementordnung-10-kmo-id18; zuletzt geprüft am 01.03.2020.

457 Vgl. Felden/Hack (2014), S. 336.

458 Vgl. Felden/Hack (2014), S. $337 \mathrm{f}$.

459 Zur Aufnahme von Mediationsklauseln Wicke (2012), S. 481.

460 Grundsätzlich zu Mediationsverfahren in Familienunternehmen Prince (1990). 


\subsubsection{Geltungsdauer und Anpassungserfordernisse}

Betrachtet man eine Familienverfassung als eine Art Verfassung und damit als etwas Beständiges, grundsätzlich Unantastbares, so würde man davon ausgehen dürfen, dass mögliche Veränderungen bereits bei Entstehung berücksichtigt wurden und es sich somit um einen stabilen Rahmen handelt, der Ordnung und Struktur zu garantieren sucht. Doch auch jede Verfassung im klassischen Sinne unterliegt Veränderungen, indem auf sich verändernde Anforderungen - seien sie exogen oder endogen - reagiert wird. So muss auch eine Unternehmerfamilie auf Veränderungen - seien es Änderungen in der Familie, im Unternehmen oder im Marktumfeld ${ }^{461}$ - reagieren und entsprechende Anpassungen vornehmen. ${ }^{462}$

Die Familienverfassung ist also kein Dokument, das in Stein gemeißelt wird. Vielmehr muss sie kontinuierlich weiterentwickelt werden. Dies ist insbesondere dann der Fall, wenn die nächste Generation vor der Tür steht. Sodann sollten sich zunächst die Familienmitglieder der nächsten Generation untereinander finden und sich eine gemeinsame Vorstellung erarbeiten, um dann zusammen mit der Elterngeneration die bestehende Familienverfassung zu überprüfen und - falls notwendig - an die neue Situation entsprechend anzupassen. ${ }^{463}$ Außer dem Generationenübergang können weitere Ereignisse eine Überarbeitung der Familienverfassung rechtfertigen. Änderungen der Gesetzeslage oder aktuellen Rechtsprechung ${ }^{464}$ sowie reifende Erkenntnisse, wie Konflikte besser gelöst werden und die Gemeinschaft ihre verschiedenen Interessen besser aufeinander abstimmen kann, können eine solche Änderung rechtfertigen.

Die Meinungen in der Literatur hinsichtlich konkreter Vorgaben, wann eine Familienverfassung überarbeitet werden sollte, gehen auseinander. So gibt es Stimmen, die eine Familienverfassung eher auf Dauer angelegt sehen, welche aber gleichwohl einräumen, dass sie sich an veränderte Bedingungen anpassen muss. Diese sehen daher dieses Dokument für eine halbe Generation, also 15 Jahre, ausgelegt. ${ }^{465}$ Andere dagegen erachten eine Überprüfung alle fünf bis zehn Jahre ${ }^{466}$, oder gar alle drei bis fünf Jahre ${ }^{467}$ als sinnvoll. Es ist somit immer eine "Gratwanderung zwischen dynamischer Aktualisierung und der grundsätzlichen Unantastbarkeit«. ${ }^{468}$ In der heutigen Zeit verändern sich Markt, Ge-

461 Vgl. Kalss/Probst (2013a), S. 52f. So auch Koeberle-Schmid/Schween/May (2011), S. 2506.

462 Vgl. Kormann (2018), S. 187.

463 Vgl. Koeberle-Schmid/Fahrion/Witt (2012), S. 479; Kormann (2018), S. 187 f.

464 Hier sind vor allem Änderungen zum Familienrecht, Erbrecht oder Gesellschaftsrecht gemeint, die unmittelbare Auswirkungen auf die Gesellschafter haben können.

465 Vgl. Baus (2017), S. 136f.

466 Arnhold/Rohner (2015), Rn. 88.

467 Vgl. Kalss/Probst (2013c), S. 18.

468 Kormann (2018), S. 187. 
sellschaft und Familien immer schneller; es genügt somit nicht mehr, eine einmal erarbeitete Familienverfassung über Jahrzehnte unverändert beizubehalten. ${ }^{469}$

Die Wahrheit mag irgendwo dazwischen liegen. Sicherlich ist es geboten, die Familienverfassung alle fünf Jahre auf Aktualität zu prüfen, sollten nicht bereits davor Umstände eingetreten sein (beispielsweise die Vergrößerung des Gesellschafterkreises durch die Übertragung von Anteilen auf Kinder oder die Vergrößerung der Familie durch Heirat), die eine frühere Überarbeitung erforderlich gemacht haben.

Bei all diesen teils unterschiedlichen Meinungen in der Literatur vermisst man jedoch eine differenzierte Betrachtung in Bezug auf den Aufbau einer Familienverfassung. Nicht alle Bestandteile mögen derselben Halbwertszeit unterliegen. Beispielsweise ändern sich Werte und Selbstverständnis der Familie oft über Jahrzehnte nicht. Die Beständigkeit in dieser Hinsicht ist etwas, was Familienunternehmen auszeichnet. ${ }^{470}$ Auch die Vision, so sie denn in einer Familienverfassung enthalten ist, wird man kaum alle fünf oder zehn Jahre anpassen müssen. Hingegen mag die Mission Änderungen unterliegen. Bei den Zielen mag es beispielsweise dann zu Änderungen kommen, wenn eine nächste Generation in ein Alter kommt, das es den beteiligten Familienmitgliedern erlaubt, sich Gedanken hinsichtlich der unternehmerischen und familiären Ziele zu machen. Dabei mag es in zehn bis zwanzig Jahren durchaus zu Anpassungen kommen. Bei den etablierten Institutionen und Instrumenten sind grundsätzlich kaum Änderungen denkbar. Familientage wird man kaum abschaffen und der Familienrat hat sich in der Praxis bewährt. Vermögensrelevante Regelungen können sich auch im Laufe der Generationen durchaus ändern. Bei den Verfahrensregeln sind Anpassungen auch in kürzeren Abständen denkbar, insbesondere in der Anfangszeit, wenn die Familienverfassung gerade erst ins Leben gerufen wurde. So kann die Praxis im Laufe der Anwendungszeit neuere Erkenntnisse hervorbringen, auf Basis derer eine Anpassung der einen oder anderen Regel sinnvoll und wichtig erscheint, um den Umgang innerhalb der Familie zu »optimieren." Hier lohnt es sich sicherlich - gerade zu Beginn -, einmal jährlich im Rahmen des Familientages solche Änderungswünsche zu diskutieren und ggf. im Konsens zu beschließen.

469 Vgl. Koeberle-Schmid (2014).

470 So im Ergebnis auch Kaiser (2012), S. 5. 


\subsection{Die Bindungswirkung der Familienverfassung}

»Wenn man einem Menschen trauen kann, erübrigt sich ein Vertrag. Wenn man ihm nicht trauen kann, ist ein Vertrag nutzlos." (Jean Paul Getty)

Je nachdem, zu welchem Ergebnis man hinsichtlich einer Bindungswirkung der Familienverfassung gelangt und welche Antwort als Grundlage für diese Forschungsarbeit dienen soll, kann sich dies nicht unerheblich auf konkrete Maßnahmen zur Regelbefolgung auswirken.

Dieser Abschnitt soll daher den Stand der Literatur überblicksartig zusammenfassen und damit einhergehend die Grundlage für diese Arbeit vorgeben. Freilich kann und soll in dieser Arbeit keine ausführliche rechtliche Würdigung des Instruments erfolgen. Insbesondere schon deswegen nicht, da sich erst jüngst eine Arbeit zum ersten Mal recht ausführlich den rechtlichen Konturen dieses Instruments widmete. ${ }^{471}$

\subsubsection{Rechtliche oder moralische Bindung? Eine kursorische Übersicht}

Die rechtliche Einordnung der Familienverfassung ist nicht unumstritten und steckt noch in den Kinderschuhen. ${ }^{472}$ Dennoch findet sich eine Vielzahl an Meinungen in der einschlägigen Literatur. Die Frage nach der Bindungswirkung einer Familienverfassung wird im Schrifttum seither vornehmlich mit der Diskussion über den rechtlichen Charakter einer Familienverfassung verknüpft. ${ }^{473}$ Weit verbreitet ist die Auffassung, dass es sich bei einer Familienverfassung um eine reine Absichtserklärung handelt, die keine rechtlich bindende Wirkung entfalten soll ${ }^{474}$, sondern allenfalls moralisch bzw. emotional verpflichtend ist, und ihr damit vielmehr eine faktische Bindungskraft zugesprochen werden kann. ${ }^{475}$ In diesem Zusammenhang ist die Rede von einem "psychologischen Vertrag ${ }^{476}$, der per se $»$ niemals [einen] rechtsverbindlichen Charakter ${ }^{477}$ haben

471 Vgl. Hueck (2017).

472 Vgl. Born (2014), Rn. 50; Gläßer (2014), S. 240; Kirchdörfer/Breyer (2014), S. 20 f.; Uffmann (2015), S. 2450; Hueck (2017), S. 70; Reich/Bode (2018), S. 305.

473 Vgl. Gläßer (2014), S. 234.

474 Vgl. exemplarisch Kögel (2014), Rn. 11; Baus (2017), S. 109; Fabis (2007), S. 362; Hauck (2012a), S. 1 ; Koeberle-Schmid/Fahrion/Witt (2012), S. 479; Lange (2005), S. 147 m.w.N.

475 Vgl. Hauck (2012a); Gläßer/Kirchhoff/Wendenburg (2014), S. 50; Gläßer (2014), S. 234; Riedel (2012), 87; Kreklau (2013), S. 50; Felden/Hack (2014), S. 321.

476 Felden/Hack (2014), S. 322.

477 Iliou (2004), S. 164. 
kann. Die Familienverfassung beruhe vielmehr auf Selbstbindung. ${ }^{478}$ Insofern sei der Inhalt auch nicht wie in einem Vertrag durchsetzbar, also weder »einklagnoch vollstreckbar «. ${ }^{479}$ Mit einer Familienverfassung möchte eine Unternehmerfamilie eben gerade keine rechtlich verbindlichen Vorgaben treffen, sondern einen Konsens für familienrelevante (häufig moralische) Fragen finden und schriftlich fixieren. ${ }^{480}$ Daher wird sie an einer anderen Stelle als "moralischer Vertrag « ${ }^{481}$ bezeichnet. Auch der inzwischen bekannte »Governance Kodex für Familienunternehmen ${ }^{482}{ }^{4 e i c h n e t ~ k e i n ~ a n d e r e s ~ B i l d . ~ E r ~ z i e l t ~ d a r a u f ~ a b, ~ e i n e n ~}$ Verhaltenskodex - und meint damit auch das Grundgerüst für eine Familienverfassung - für verantwortungsvolle Führung von Familienunternehmen aufzustellen. Auch dieses Grundgerüst soll keinen rechtlich bindenden Charakter besitzen. ${ }^{43}$ Das lässt sich nicht zuletzt auch an Hand der Sprache seiner Formulierungen ableiten. So werden Formulierungen wie »soll» und »es wird empfohlen « verwendet. ${ }^{484}$

Versucht man die Familienverfassung im Kontext zwischen Verfassung und Verfassungswirklichkeit zu sehen, so wird schnell klar, dass sich in einer Verfassung gemeinhin aufeinander abgestimmte Regelungen in einer präzisen, häufig juristischen Sprache, die als Gebote und Verbote formuliert sind, finden. Dies trifft beispielsweise auf einen Gesellschaftsvertrag oder eine Unternehmenssatzung $\mathrm{zu}^{485}$. Daneben findet sich jedoch die Verfassungswirklichkeit aus über die Jahre gewachsenen Wertvorstellungen, Sitten, Bräuche, Gewohnheiten und gesellschaftlichen Erwartungen. Diese, oftmals noch nicht schriftlich fixierten, Normen können dann innerhalb der Familie präzisiert und gemeinsam im Rahmen einer juristisch nicht verbindlichen Familienverfassung niedergeschrieben werden. In dieser Verfassungswirklichkeit finden sich dann vornehmlich Soll-Vorschriften, formulierte Erwartungen und Wünsche sowie ein niedergeschriebenes Selbstverständnis der Familie. ${ }^{486}$

Allerdings könnte man die in einer Familienverfassung erarbeiteten Regeln auch als Handlungsorientierung für die Familie und damit gleichzeitig als

478 Vgl. Baus (2017), S. 134f.; darauf verweisend Holler (2016), Rn. 102; dies bezweifelnd Lange (2013), S. 43f.

479 Lange (2013), S. 44.

480 Vgl. Baumhauer et al. (2011), S. 117.

481 Felden/Hack (2014), S. 321.

482 Vgl. http://www.kodex-fuer-familienunternehmen.de. Der Vollständigkeit soll hier erwähnt werden, dass der GKFU im Schrifttum teilweise kritisch kommentiert wird; vgl. dazu Grottel et al. (2012), S. $153 \mathrm{ff} . \mathrm{m}$. w. N.; auch darauf verweisend Stengel (2014), Rn. 20; den DCGK und damit auch den GKFU komplett ablehnend Bernhardt (2010), S. 19.

483 Vgl. Stengel (2014), 20.

484 Vgl. Grottel et al. (2012), S. 153.

485 Vgl. Kormann (2018), S. 176. Vgl. dazu auch Arnhold/Rohner (2015), 55.

486 Vgl. Kormann (2018), S. 176. 
Grundlage werten, von welcher ausgehend die getroffenen Regelungen in einzelne, rechtlich bindende Verträge überführt werden. ${ }^{487}$ Auch wenn eine Familienverfassung weit überwiegend als rechtlich unverbindlich eingestuft wird, kann nach allgemeiner Auffassung nicht ausgeschlossen werden, dass die getroffenen Regelungen rechtliche Verbindlichkeit dadurch erlangen, dass sie in rechtlich bindende Dokumente (z.B. Gesellschaftsvertrag, Satzung etc.) übertragen werden. ${ }^{488}$ Eine Familienverfassung ist demnach Richtschnur sowie eine wichtige "Orientierungshilfe ${ }^{489}$ für die konkrete Ausgestaltung von Gesellschafts- ${ }^{490}$ und Erbverträgen, da nicht zuletzt Werte und klare Ziele bei der Erstellung von solchen Verträgen von Bedeutung sind. ${ }^{491}$ Gleichzeitig ist sie auch Grundlage für weitere Verträge zwischen den einzelnen Familienmitgliedern (z.B. Eheverträge, Schenkungsverträge etc.) oder zwischen Familienmitgliedern und dem Familienunternehmen (z.B. Anstellungsverträge, Beraterverträge etc.). ${ }^{492}$

Möchte man die Familienverfassung analog zu einer Normenhierarchie $« 493$ in einen Kontext bringen, so wird man feststellen: Während die einen die Familienverfassung als "höherrangiges Regelwerk «" ${ }^{494}$ ablehnen, sehen die anderen dieses Instrument auf einer »höheren Ebene ${ }^{495}$ verortet. Dies mag zunächst widersprüchlich erscheinen. Die Autoren eint jedoch, dass sie die Familienverfassung beispielsweise von einem juristisch bindenden Gesellschaftsvertrag unterscheiden. Dieser genieße rechtlich betrachtet Vorrang. ${ }^{496}$ Wenn von einer höheren Ebene die Rede ist, wird dabei entweder auf die "Wertvorstellungen, Gewohnheiten oder Erwartungen der Familie« abgestellt ${ }^{497}$ oder die Familienverfassung steht insofern über allen nachgeordneten Verträgen, als sich diese an ihr $\mathrm{zu}$ orientieren und mit ihr übereinzustimmen haben. ${ }^{498}$ So kommt auch Hueck (2017) in einer sehr differenzierten Betrachtung hinsichtlich der Ansiedlung der Familienverfassung innerhalb des rechtlichen Regelungsrahmens von Unternehmerfamilien zum Ergebnis, dass es »eher dem Willen der Betei-

487 Vgl. Felden/Hack (2014), S. 319.

488 Vgl. Felden/Hack (2014), S. 321; Kormann (2018), S. 190; Lange (2009), S. 148; Schulz/Werz (2007a), S. 311; Hueck (2017), S. 61; Reich/Bode (2018), S. 306.

489 Arnhold/Rohner (2015), 49.

490 Vgl. Schween et al. (2011), S. 12; Born (2014), Rn. 50; Koeberle-Schmid/Schween/May (2011), S. 2500; Arnhold/Rohner (2015), 49.

491 Vgl. Koeberle-Schmid/Schween/May (2011), S. 2499.

492 Vgl. Kögel (2014), Rn. 11; Arnhold/Rohner (2015), 49; Hueck (2017), S. 61.; Uffmann (2015), S. 2447.

493 Vgl. Bochmann (2013), S. 485.

494 Vgl. Bochmann (2013), S. 485.

495 Vgl. Kirchdörfer/Lorz (2011), S. 101; so auch Lange (2013), S. 39.

496 Vgl. Koeberle-Schmid/Fahrion/Witt (2012), S. 479; so auch Born (2014), Rn. 50.

497 Kirchdörfer/Lorz (2011), S. 101.

498 Vgl. Kalss/Probst (2013a), S. 56; Arnhold/Rohner (2015), 45. 
ligten $\mathrm{zu}$ entsprechen [scheint], die Familienverfassung insgesamt als ein Regelwerk anzusehen, das eine außerhalb der Gesellschaftsverhältnisses stehende Vereinbarung [...] darstellt. ${ }^{499}$

Insgesamt fällt negativ auf, dass sich das Schrifttum mehrheitlich nicht den einzelnen, konkret getroffenen Regelungen zuwendet, sondern die Familienverfassung stets in Summe als rechtlich verbindlich ablehnt. Jedoch lässt sich die Bindungswirkung einer Familienverfassung abstrakt überhaupt nicht beurteilen, sondern immer nur ausgehend vom jeweiligen Regelungsinhalt und dessen Wortlaut. $^{500}$

Es herrscht jedoch Einigkeit darüber, dass die Bindungswirkung keinesfalls soweit gehen kann, dass eine Einhaltungspflicht entsteht, obwohl man gerade durch die explizite Wahl rechtlicher Unverbindlichkeit diesen Umstand zu vermeiden suchte. ${ }^{501}$

Basierend auf dem auch in dieser Arbeit zu Grunde gelegten typischen Verständnis der Unternehmerfamilien und Familiengesellschafter "stellt die Familienverfassung eine rechtlich nicht [unmittelbar] durchsetzbare Vereinbarung sui generis dar. ${ }^{502}$

\subsubsection{Die Familienverfassung im Verhältnis zum Gesellschaftsvertrag}

Nachdem bereits darauf hingewiesen wurde, dass es sich bei der Familienverfassung und dem Gesellschaftsvertrag um zwei unterschiedliche Dokumente handelt, soll an dieser Stelle - nicht zuletzt auch der Vollständigkeit wegen überblicksartig die Familienverfassung vom Gesellschaftsvertrag abgegrenzt werden. Dies ist schon deswegen relevant, da bei der noch folgenden Erörterung der Rechtsverbindlichkeit auf die konkreten Wesensmerkmale der Familienverfassung im Kontext des rechtlichen Ordnungsrahmens eingegangen werden wird.

Die Familienverfassung adressiert in aller Regel ausschließlich Familienmitglieder. Ihr wird ein stabilisierendes Moment für die Familie zugeschrieben. Dies begründet sich durch den Entstehungsprozess, in welchem die Unternehmerfamilie die Familienverfassung in einer allgemein verständlichen und nicht technischen Sprache ohne Fachtermini verfasst und im (möglichst einstimmigen) Konsens beschließt sowie unterzeichnet. ${ }^{503}$ Aus diesem Prozess heraus leitet sich

499 Hueck (2017), S. 115.

500 Lediglich Hueck (2017), S. 129 ff. , 167 ff. widmet sich einer differenzierteren Betrachtung und stellt auf die einzelnen Kategorien der Regelungsinhalte ab.

501 Vgl. Knauff (2010), S. 493; Windbichler (2009), S. 32, 34; Hueck (2017), S. 198.

502 Hueck (2017), S. 196.

503 Vgl. Kalss/Probst (2013a), S. 48; Fabis (2007), S. 362. 
ihre rechtfertigende Wirkung $\mathrm{ab}$, alle Familienmitglieder $\mathrm{zu}$ binden und zur Einhaltung zu verpflichten. Sie bildet damit den Rahmen für zukünftige Entwicklungen innerhalb der Familie und des Familienunternehmens und sucht ein einheitliches Verständnis zu schaffen, was in den üblichen Verträgen ${ }^{504}$ konkret juristisch umgesetzt werden soll.

Eine Familienverfassung ist nur bedingt mit einem Gesellschaftsvertrag vergleichbar (Abbildung 12). Letzterer zeichnet sich durch eine abstrakt juristische Sprache aus und regelt die Rechtsbeziehungen und Beteiligungsverhältnisse der Gesellschafter in Bezug auf das Familienunternehmen. Ein stabilisierendes Moment, das die Familien zusammenhalten soll, so wie es der Familienverfassung zugeschrieben wird, sucht man beim Gesellschaftsvertrag vergeblich, ist es aber auch nicht sein eigentlicher Zweck. "Und in den seltensten Fällen ist er Ausdruck eines wirklichen gemeinsamen Interesses, viel häufiger ist er nicht mehr als der kleinste gemeinsame Nenner « ${ }^{505}{ }^{506}$

Den gemeinsamen Nenner und damit das gemeinsame Interesse der Unternehmerfamilie zu finden, ist Aufgabe der Familienverfassung. Während der Gesellschaftsvertrag die Lebenswirklichkeit auf Rechtsbeziehungen beschränkt, sucht die Familienverfassung die realen Verhältnisse innerhalb der Familie abzubilden. Dabei werden möglichst alle Familienmitglieder in den Gestaltungsprozess miteinbezogen; anders bei einer Gesellschafterversammlung, zu welcher grundsätzlich lediglich Gesellschafter eingeladen werden.

\begin{tabular}{|l|l|l|}
\hline & Familienverfassung & Gesellschaftsvertrag \\
\hline Adressat & Familienmitglieder & Gesellschafter \\
\hline Regelungsbereich & Familie und Unternehmen & $\begin{array}{l}\text { Gesellschafter und Unterneh- } \\
\text { men }\end{array}$ \\
\hline Sprache & $\begin{array}{l}\text { Allgemein verständlich, un- } \\
\text { technisch }\end{array}$ & $\begin{array}{l}\text { Abstrakt juristisch, technisch, } \\
\text { somit kaufmännisches und ju- } \\
\text { ristisches Verständnis nötig }\end{array}$ \\
\hline Bindungswirkung & Emotional bindend & Rechtlich verbindlich \\
\hline Verabschiedung & (Möglichst) einstimmig & Mehrheitsprinzip \\
\hline Regelungsgegenstand & $\begin{array}{l}\text { Rahmenregelung für das Ver- } \\
\text { hältnis der Familienmitglieder } \\
\text { untereinander und zur Gesell- } \\
\text { schaft }\end{array}$ & $\begin{array}{l}\text { Detaillierte Regelung der Ge- } \\
\text { sellschafter und Gesellschafts- } \\
\text { verhältnisse selbst }\end{array}$ \\
\hline Grundlage & $\begin{array}{l}\text { Für Gesellschaftsvertrag, Ehe- } \\
\text { und Erbvertrag, Testamente } \\
\text { und für Streitschlichtung }\end{array}$ & Für die Gesellschaft selbst \\
\hline
\end{tabular}

504 Hier sind im Wesentlichen Gesellschafts-, Ehe-, Erb- und Poolverträge gemeint.

505 Baus (2017), S. 134.

506 Vgl. Kirchdörfer/Lorz (2011), S. 102f.; Baus (2017), S. 108f. 
(Fortsetzung)

\begin{tabular}{|l|l|l|}
\hline & Familienverfassung & Gesellschaftsvertrag \\
\hline Beschlussfassung & Konsensprinzip & Mehrheitsprinzip \\
\hline
\end{tabular}

Abbildung 12: Unterschiede zwischen Familienverfassung und Gesellschaftsvertrag ${ }^{507}$

Bringt man die Familienverfassung in einen zeitlichen Kontext, so wird schnell klar, dass sie zum einen die Antwort auf eine zuvor entwickelte Familienstrategie gibt und zum anderen die Grundlage für (Gesellschafts-)Verträge - oder deren Überprüfung und Anpassung - darstellt und diesen somit grundsätzlich vorausgehen sollte. Aber auch ein gegenteiliges Szenario ist denkbar: Der betagte Unternehmenslenker und Familienpatriarch, der eine noch so wohl durchdachte und sinnvolle Überlegung in einem Gesellschaftsvertrag oder Erbvertrag umgesetzt haben mag, dem es aber an der Beteiligung der nachfolgenden Generation mangelt. Ein vermeintliches Diktum des Altvorderen kann im Nachhinein durch eine von der nachfolgenden Generation akzeptierte Familienstrategie "geheilt» und der in Rede stehende Vertrag - sei es ein Gesellschaftsvertrag, Erbvertrag etc. - legitimiert werden. ${ }^{508}$

Es wird in der Literatur davor gewarnt, Familienverfassungen vor allem mit solchen Regelungen zu »überfrachten«, die eigentlich allein im Gesellschaftsvertrag geregelt werden sollten ${ }^{509}$ - nicht zuletzt auch, um unnötiges Konfliktpotenzial zu vermeiden. In diesem Zusammenhang soll auch darauf hingewiesen werden, dass es zu vermeiden gilt, besondere Themen in beiden Regelungsstatuten - also sowohl im Gesellschaftsvertrag als auch innerhalb der Familienverfassung - abzubilden, zumal man dann Gefahr läuft, dass diese in widersprüchlicher Weise zueinanderstehen oder möglicherweise nicht kongruent geregelt wurden. ${ }^{510}$

Klar sollte sein, dass weder eine Familienverfassung noch besondere Kodizes für Familienunternehmen individuelle und auf die Bedürfnisse der Familien zugeschnittene Gesellschaftsverträge ersetzen können. Die primäre Intention einer Familienverfassung ist es, einen Leitfaden für ein harmonisches Zusammenleben $\mathrm{zu}$ erstellen und insbesondere damit ein individuelles Regelwerk $\mathrm{zu}$ begründen, wie mit innerfamiliären Konflikten umzugehen ist. ${ }^{511}$

507 Eigene Darstellung in Anlehnung an Baus (2017), S. 135; Kirchdörfer/Lorz (2011), S. 101; Andreae (2007), S. 18; jedoch ohne Anspruch auf Vollständigkeit.

508 Vgl. Baus (2017), S. 135.

509 Vgl. Kirchdörfer/Lorz (2011), S. 105 f.; Baus (2017), S. 135.

510 Vgl. Holler (2016), Rn. 106. Kritisch dazu Röthel/Schmidt (2013), S. 40.

511 Vgl. Kirchdörfer/Lorz (2011), S. 106; Koeberle-Schmid/Schween/May (2011), S. 2502. 


\title{
2.5 Der Legitimationsgedanke der Familienverfassung im Lichte privater Normsetzung und moralphilosophischer Ansätze
}

\author{
»Um immer gut sein zu können, \\ müssten die anderen glauben, \\ niemals straflos gegen uns böse sein zu dürfen." \\ François de La Rochefoucauld
}

Wie bereits ausgeführt, ist das Instrument der Familienverfassung noch sehr jung und mithin nicht ausreichend erforscht. Sich diesem aus theoretischer Sicht zu nähern, gestaltet sich ob der nicht vorhandenen wirtschaftswissenschaftlichen theoretischen Grundlage schwierig. Genauso wenig wie es die Familienverfassung gibt, soll nachfolgend der Versuch unternommen werden, sich diesem Instrument disziplinübergreifend zu nähern, um auf Basis dieser Erkenntnisse zusammen mit dem Ergebnis der Fallstudien entsprechende Maßnahmen für die Regelbefolgung ableiten zu können. ${ }^{512}$ Nur wenn eine Regel als legitim empfunden wird, besteht auch eine hohe Wahrscheinlichkeit, dass diese eingehalten wird. ${ }^{513}$ Nachdem eine Familienverfassung eben gerade nicht als unmittelbar juristisch bindend verstanden werden möchte, sondern lediglich als moralisch verpflichtend, kann man sich diesem Instrument einerseits ausgehend von moralphilosophischen Überlegungen annähern. Andererseits existiert neben dem positiven Recht eine private Ordnung, die auch und insbesondere unter dem Rubrum der Selbstregulierung zu finden ist und in Ansätzen der Intention einer Familienverfassung nahekommt.

Sicherlich könnte man sich diesem Instrument zusätzlich auch aus vertragstheoretischer Sicht nähern. Heranzuziehen wären beispielsweise die Überlegungen zur Transaktionskostentheorie ${ }^{514}$. Bei den sogenannten Ex-post-Transaktionskosten untersucht man insbesondere den Aufwand für Überwachung, Durchsetzung und nachträgliche Anpassung der vertraglich festgelegten Konditionen. ${ }^{515}$ Dieser theoretische Ansatz wäre insbesondere dann denkbar, wenn man in der Familienverfassung selbst eine Art Vertrag sieht. Dies setzt bei den Beteiligten allerdings einen Rechtsbindungswillen voraus, welcher in aller Regel nicht vorhanden ist. ${ }^{516}$ Daher wurde dieser theoretische Ansatz nicht weiter verfolgt. Nicht zuletzt würde er den Rahmen dieser Arbeit sprengen.

512 Vgl. Abschnitt 4.1.

513 So im Ergebnis wohl auch Leipold/Pies (2000), S. 339.

514 Vgl. zur Transaktionskostentheorie vor allem Williamson (1985), S. 41, 20 ff.; Ebers/Gotsch (1995), S. 209.

515 Vgl. Picot (1982), S. 270.

516 Vgl. Abschn. 2.4. 


\subsubsection{Private Normsetzung ${ }^{517}$ und Selbstregulierung}

Es ist heute unstreitig, dass soziale Ordnung nicht allein durch hoheitliche Planung gemeistert werden kann. Zweifelsfrei ist ein institutioneller Rahmen unabdingbar. Als wesentlicher Teil gehört hierzu das Recht. Auf dieser Basis können private Regelsetzer eine künstliche Ordnung schaffen, die das Zusammenleben von Gemeinschaften fördert.

Familienverfassungen gelten als ein selbstregulierendes internes Regelwerk ${ }^{518}$, das Antworten auf die wesentlichen Fragen des Zusammenspiels von Unternehmerfamilie und Familienunternehmen zu geben sucht. Wenngleich sie sich häufig einer an das Recht angelehnten Sprachform bedient, möchte eine Familienverfassung in seltenen Fällen Rechtsverbindlichkeit erzeugen. Losgelöst vom positiven Recht erlebt gerade das Phänomen der Selbstregulierung im Privatrecht in den letzten Jahren eine Art $»$ Renaissance ${ }^{519}$. Hier und da findet man Anhänger aber auch Gegner. ${ }^{520}$ Von einer Karriere des Konzepts gesellschaftlicher Selbststeuerung « ${ }^{521}$ bis hin $z u$ »Verdrängung des legalen Rechts ${ }^{522}$ ist die Rede. Die Familienverfassung als Steuerungsinstrument eigener Art bewegt sich durchaus im Forschungsfeld der Selbstregulierung durch Private. ${ }^{523}$ Insofern ist angezeigt, diese Sichtweise dahingehend näher zu beleuchten, ob sich Anhaltspunkte für Maßnahmen zur Sicherung der Regelkonformität bei Familienverfassungen ableiten lassen. Es soll dabei weniger eine rechtsdogmatische Einordnung und Bewertung erfolgen (was eine solche Arbeit auch nicht leisten kann und möchte), als vielmehr das Phänomen als solches skizziert und erfasst werden.

\subsubsection{Regeln schaffen Ordnung}

Regeln wohnt in der Tat ein Ordnungsfaktor inne, wobei Grundsätze aufgestellt werden müssen, mittels jener "regelgeleiteter Ordnung eine ökonomisch und ethisch verlässliche Basis verschafft werden kann. ${ }^{524}$ Der Legitimationsgedanke drückt diese Forderung sprachlich aus. ${ }^{525}$

517 Zum Begriffsstreit Norm versus Regel vgl. Abschnitt 2.5.1.1.2.

518 Vgl. Hueck (2017), S. 74.

519 Buck-Heeb/Dieckmann (2010), S. 2.

520 Während die einen am Sinn privater Selbstregulierung Zweifel hegen (vgl. Bizer (2001)), weckt es bei anderen die Neugier, was hinter der Expansion privater Rechtsproduktion steckt (vgl. Köndgen (2006), S. 478f.).

521 Schuppert/Bumke (2000), S. 79. Vgl. dazu auch Windmann (2008), S. $948 \mathrm{ff}$.

522 Mertens (1982), S. 29.

523 Vgl. Hueck (2017), S. 73. Grundlegend zu privater Ordnung und Selbstregulierung im Privatrecht Bachmann (2006) und Buck-Heeb/Dieckmann (2010).

524 Bachmann (2006), S. 26. Hervorh. im Original. 


\subsection{Die Ökonomik der Ordnung}

Wenn von Ordnung die Rede ist, mag einen dies in erster Linie an die Abwesenheit von Chaos erinnern. ${ }^{526}$ Unabhängig von der Vielzahl der Modelle sozialer Ordnung ${ }^{527}$ schwingt immer die These mit, dass menschliche Ordnung stets regelgeleitet sein muss. ${ }^{528}$

Die Ordnungsethik ${ }^{529}$ ist ein Modell sozialer Ordnung. Der Grundgedanke dieses Modells besteht in der Auffassung, dass sich »Kooperation« auf lange Sicht zum Vorteil aller Beteiligten auswirkt. ${ }^{530}$ Da ein einzelner aber nicht wissen kann, ob sich die anderen ebenfalls kooperativ verhalten oder zu seinem Nachteil lediglich von seiner Kooperationsbereitschaft profitieren wollen, wird er sich im Zweifel auch nicht kooperativ verhalten. Wenn alle dem gleichen Gedankengang verfallen, verhalten sich im Zweifel alle unkooperativ, obwohl sich die Kooperation für alle positiv hätte auswirken können. Dieses Problem kann man mit der Einführung von Institutionen lösen. ${ }^{531}$

Für BACHMANN (2006) zählen dazu »alle sozialen Mechanismen, die es ermöglichen, Kollektivhandlungsprobleme $\mathrm{zu}$ überwinden und $\mathrm{zu}$ einer sozial vorteilhaften Kooperation zu gelangen." Er sieht solche Mechanismen insbesondere in der "Einrichtung von Kommunikationswegen, vor allem aber im Generieren und Honorieren von Vertrauen [...]. ${ }^{532}$ Das Vertrauen ist insofern wichtig, als dass die Kooperationsbereitschaft erheblich steigt, wenn man darauf vertrauen darf, dass andere auch »mitmachen .

\subsection{Der Begriffsstreit Norm versus Regel}

Der Begriff der Norm sieht sich Kritik ausgesetzt. Sicherlich zu Recht, zumal er nicht eindeutig definiert ist. ${ }^{533}$ Die Bandbreite reicht von »sozialer Gewohnheit» bis hin zu »verbindlicher Regel ${ }^{534}$ In der Methodenlehre ist es bisweilen üblich

525 Vgl. Bachmann (2006), S. 26.

526 Vgl. Lembcke/Ritzi/Schaal (2012), S. 113; Bachmann (2006), S. 7; Bauman (2005), S. 16: "Ordnung ist, was nicht Chaos ist; Chaos ist, was nicht ordentlich ist: Ordnung und Chaos sind moderne Zwillinge." Hervorh. im Original.

$527 \mathrm{Zu}$ den diversen Modell sozialer Ordnung Bachmann (2006), S. $8 \mathrm{ff}$.

528 Vgl. Woll (1994), S. 179: „Ordnung kann auf vielfache Weise entstehen. [...] Ordnung strukturiert Komplexität. Aus diesem Grunde ist es wichtig, daß soziale Systeme regelgeleitet sind, damit sich Menschen darin zurecht finden.«

529 Analog verwendete Begriffe: »ökonomische Ethik«, »Ordnungsökonomik«.

530 Bachmann (2006), S. 15. Hervorh. im Original.

531 Die Institutionenökonomik ist ein Teil der Ordnungsökonomik, vgl. dazu nur Richter/ Furubotn (2010).

532 Bachmann (2006), S. 15f. Hervorh. im Original.

533 Vgl. Bachmann (2006), S. 20. Vgl. dazu auch Lautmann (1969), S. 98, der seinerzeit bereits 82 Normdefinitionen in der Literatur fand.

534 Raiser (2013), S. 173. 
geworden, zwischen "Regeln « und »Prinzipien ${ }^{535} \mathrm{zu}$ unterscheiden. ${ }^{536}$ Regeln drücken demnach einfache Anordnungen aus, während Prinzipien Werte verkörpern. ${ }^{537}$ Im Hinblick darauf, dass die Varianten privater Ordnung unter dem Begriff »Selbstregulierung« subsumiert werden ${ }^{538}$, bedarf es zunächst einer Eingrenzung der Begriffe, wie sie für die vorliegende Arbeit verwendet werden sollen. Soziologisch wird der Begriff »Norm» für rechtliche und vor allem für soziale Verhaltensmaßstäbe verwendet. ${ }^{539}$

"A norm is a social rule that does not depend on government for either promulgation or enforcement. ${ }^{540}$

Rechtssoziologisch definiert der Begriff Norm eher eine verbindliche Regel oder Verhaltensforderung bzw. Verhaltenserwartung. ${ }^{541}$

In der Rechtswissenschaft werden Normen partiell mit einer Rechtsnorm gleichgesetzt, wohingegen andere darunter eine eher generelle Regel verstehen, die heteronome Verbindlichkeit erzeugt, also ohne besondere Zustimmung der Regeladressaten. ${ }^{52}$ Das Schrifttum spricht die Empfehlung aus, den nicht eindeutigen Begriff "Norm» eher durch den neutraleren Term »Regel« auszutauschen. Soweit im Folgenden der Begriff »Norm» gebraucht wird, soll dieser synonym für den Begriff »Regel« und damit für alle »Sollenssätze ${ }^{543}$ stehen unabhängig davon, ob sie von den Adressaten selbst oder anderen aufgestellt wurden. ${ }^{544}$

Neben den im Bereich der privaten Ordnung gesetzten Regeln finden sich dort auch solche (oft ungeschriebene), die sich im Laufe der Zeit auf Grund privater Übung entwickelt haben. ${ }^{545}$ In diesem Kontext fällt in der Literatur auch der Begriff »spontane Ordnung ${ }^{546}{ }^{54}$ Dabei kann man beobachten, dass etwaige soziale Sanktionen, wie beispielsweise dass die Reputation Schaden nimmt, von den Beteiligten durchaus berücksichtigt und im Vorfeld akzeptiert werden, zumal die Auffassung vertreten wird, dass sich »effiziente Normen auch ohne staatlichen Einfluss auf Dauer von selbst ${ }^{547}$ herausbilden. Grundsätzlich aber gehören

535 Synonym wird häufig auch der Begriff »Grundsätze« verwendet.

536 Grundsätzlich dazu Esser (1956); Bachmann (2006), S. 19 m w. N.

537 Vgl. Bachmann (2006), S. 19.

538 Vgl. Bachmann (2006), S. 20.

539 Vgl. insbes. Raiser (2013), S. 177 ff. m w. N.

540 Posner et al. (1998), S. 369.

541 Vgl. Raiser (2013), S. 173.

542 Vgl. Kirchhof (1987), S. 64 ff., 84ff.; Bachmann (2006), S. 21.

543 Horn (2011), S. 6.

544 Vgl. dazu auch Kantorowicz (1957), S. 38f.

545 Vgl. Bachmann (2006), S. 23.

546 Vgl. dazu insbes. Posner et al. (1998); Posner/Rasmusen (2000), S. $369 f f$.

547 Bachmann (2006), S. $23 \mathrm{f}$. 
Sanktionen zum Normbegriff. In der Literatur finden sich viele Autoren, die die Sanktion als unverzichtbares Element des Normbegriffs ansehen. ${ }^{548}$

\subsection{Die Regelbeteiligten}

Untersucht man die Regelsetzung allgemein, sollte man sich auch der verschiedenen Gruppen bewusst sein. Es wird unterschieden zwischen Regelsetzer, Regeladressat und Regelbetroffener. Der Regelsetzer wird sich immer die Frage stellen, inwiefern die Regeladressaten die Regeln befolgen werden. Wohnt den Regeln lediglich ein Zweckmäßigkeitscharakter im Sinne von Empfehlungen inne, so wird der Einhaltungsgrad in erheblichem Maße davon abhängen, inwieweit diese Regeln zur Erreichung des jeweiligen Zwecks sachdienlich sind. So wird auch die Einschätzung des Regeladressaten aussehen, der sich vor der Einhaltung die Frage stellt, ob eine Regel dem intendierten Zweck dient. Einer präskriptiven Regel schenkt der Regeladressat Gehorsam, wenn er dazu gezwungen werden kann, wobei berücksichtigt werden sollte, dass der Zwang an sich lediglich als "vordergründige Stütze der Regelgeltung « ${ }^{549}$ betrachtet werden kann. ${ }^{550}$ Für den langfristigen Erfolg einer Regel ist entscheidend, ob sie für sich Legitimität beanspruchen kann. Die dritte Gruppe ist die der Regelbetroffenen. Betroffen kann auch jemand sein, wenn dieser durch die Regel lediglich faktisch begünstigt oder belastet wird. ${ }^{551}$

\subsubsection{Der Begriff der Selbstregulierung}

Konkrete Erscheinungsformen privater Ordnung finden sich heute vornehmlich unter dem Rubrum »Selbstregulierung«. In der Literatur findet sich der Begriff der Selbstregulierung ${ }^{552}$ im Zusammenhang mit unterschiedlichen Bereichen. ${ }^{53}$ Moderne Formen dieser Regelwerke treten u.a. unter dem Begriff "code of conduct $\aleph^{554}$ bzw. Verhaltenskodex auf ${ }^{555}$. Diese Kodizes wollen dabei weniger

548 Vgl. Popitz (1980), S. 21; Röhl (2006), S. $201 \mathrm{f}$.

549 Bachmann (2006), S. 25.

550 Vgl. hierzu auch Dreier/Paulson (2011), S. 80; Raiser (1999), S. 195; Raiser (2013), S. 103: "[D]er erwartete oder ausgeübte Zwang seitens des Rechtsstabs [bildet] keinesfalls immer das wahre Motiv für die Befolgung der Rechtsregeln [...]«.

551 Vgl. zur Drittwirkung auch Mestmäcker (1964), S. 441. In Anlehnung an G. Jellineks "normative Kraft des Faktischen."

552 Zur Abgrenzung des Begriffs Selbstregulierung zu anderen Begriffen vgl. Buck-Heeb/Dieckmann (2010), S. 21 m.w.N.

553 Erscheinungsformen der Selbstregulierung finden sich beispielsweise im Vereinsecht, Internetrecht, Gesellschafts-, Unternehmens-, Bank- und Kapitalmarktrecht sowie auch im Arbeitsrecht. Vgl. dazu nur Buck-Heeb/Dieckmann (2010), S. 8. Auch auf diese Tatsache hinweisend Roßkopf (1998), S. 37. Zum historischen Ursprung der Selbstregulierung vgl. Langhart (1993), S. 85 ff. m. w. N.; Jürgens (1980), S. 7 ff.; Wahlers (2011), S. 35 Fn 40 m. w. N.

554 Vgl. etwa Schmies (2003), S. 277 ff.; Bachmann (2006), S. 153. 
durch rechtliche Verbindlichkeit als vielmehr durch »sachliche Überzeugungskraft oder moralischen Appell « ${ }^{556}$ wirken. Trotz der zuvor genannten Renaissance dieses Phänomens mangelt es seither an einem einheitlichen und damit allgemein anerkannten Begriffsverständnis ${ }^{557}$ Der Begriff »Regel « steht in diesem Kontext für Ge- oder Verbote, losgelöst davon, ob sie von den Adressaten selbst oder von anderen erstellt wurden ${ }^{558}$ KANTOROwICZ (1957) definiert Regeln als »Bezeichnungen für Beziehungen, wie sie zwischen einem menschlichen Verhalten und einigen seiner möglichen Eigenschaften bestehen sollen. ${ }^{459}$ In Anlehnung an den soziologischen und ökonomischen Normbegriff umfasst eine Regel zwei Pflichten: einerseits sich in einer bestimmten, definierten Weise zu verhalten und andererseits im Falle eines Regelverstoßes sich einer Sanktion zu unterwerfen. ${ }^{560}$ Der Wortteil »selbst « meint dabei ein »Verhalten in eigener Verantwortung unabhängig von anderen [...] und hat demnach die Bedeutung eines von außen nicht beeinflussten Handelns (Autonomie). ${ }^{561}$ Also eine freie, von den Betroffenen selbst gestaltete Ordnung. ${ }^{562}$ Ein Konsens darüber, welche Art der Regelsetzung davon betroffen sein soll, fehlt. ${ }^{53}$

WAtTer/Dubs (2005) führen dazu sehr treffend, wenn auch allgemein gehalten, aus:

„Erfasst werden kann der Begriff `Selbstregulierung`am besten, wenn er im Gegensatz zur Gesetzgebung ${ }^{564}$ auf der Grundlage einer freiheitlich-demokratischen Verfassung verstanden wird [...]. Demgegenüber ist (normative) Selbstregulierung Rechtsetzung durch private Rechtssubjekte: Es geht [...] um das Konzept, dass ordnungsbedürftige Rechtsfragen von denjenigen [...] geregelt werden, [...] [die sich] in der Zukunft bewusst an die von ihnen selbst vorgegebenen Regeln halten [sollen]. Solche selbstgeschaffenen Regeln können auf der Grundlage von bloß konkludentem Verhalten, durch schriftliche oder mündliche Verträge und insbesondere durch Beschlüsse von privatrechtlichen Organisationen herbeigeführt werden ${ }^{565}$. In diesem Sinne ist mit Selbstregulierung gemeinhin >die Eigenbildung von Regeln anstelle des Erlasses staatlicher

555 Vgl. Buck-Heeb/Dieckmann (2010), S. 21; Bachmann (2006), S. 33.

556 Bachmann (2006), S. 34.

557 Vgl. Wahlers (2011), S. 35.

558 Vgl. Bachmann (2006), S. $20 \mathrm{ff}$.

559 Kantorowicz (1957), S. 38f.

560 Vgl. Kantorowicz (1957), S. 39.

561 Buck-Heeb/Dieckmann (2010), S. 19. Vgl. dazu auch Hoeren (1992), S. 5.

562 Vgl. Bachmann (2006), S. 27.

563 Vgl. Buck-Heeb/Dieckmann (2010), S. 12; Watter/Dubs (2005), S. 743. So im Ergebnis wohl auch Roßkopf (1998), S. 37 m.w.N.

564 Vgl. dazu auch Johnston (1980), S. 3: »self-regulation versus state regulation«.

565 Vgl. Watter/Dubs (2005), S. 743 Rn. 2: "In der reinen Form gilt das derart auf privatrechtlichem Weg gesetzte Recht denn auch nur für die der fraglichen Organisation zuzuordnenden Mitglieder (d.h. mit Austritt endet regelmäßig auch die Bindung an selbstgeschaffene Normen).» 
Vorschriften ${ }^{566}$ gemeint. Selbstregulierung geht aber insofern über die autonome ${ }^{567}$ Normenbildung hinaus, als eine effektive Ausprägung dieser Regelungsart nicht nur die Aufstellung von bindenden Regelungen, sondern auch die Durchsetzung der Regeln, v. a. über die Sanktion von Regelverstößen, erfassen muss. ${ }^{568}$

Die Selbstregulierung meint also allgemein die Aufstellung, Anwendung und Durchsetzung ${ }^{569}$ privater, d.h. selbst entworfener, nicht von Dritten auferlegter Regeln. ${ }^{570}$ Das dadurch entstehende Regelwerk sei »freiwillig und lediglich moralisch verpflichtend. ${ }^{571}$ Allgemein gesprochen sollen privat aufgestellte Regeln rechtlich unverbindlich sein, gerade wenn es ihnen an einem »staatlichen Geltungsbefehl ${ }^{572}$ mangelt. ${ }^{573}$

Dabei unterscheidet man die Selbstregulierung auf Basis externer und interner Regelsetzung. ${ }^{574}$ Die externe Regelsetzung meint jene Form der Regulierung durch Private, deren Geltungsanspruch sich nicht auf den Kreis der Regelsetzer beschränkt, und man dieser Form daher Allgemeingültigkeit zusprechen kann. ${ }^{575}$ Bei der internen Regelsetzung fallen Regelsetzer und Adressat grundsätzlich zusammen. Darunter versteht man eine Regelsetzung innerhalb einer geschlossenen Gemeinschaft bzw. Gruppe, wobei nicht zwingend die explizite Zustimmung der Mitglieder dieser Gemeinschaft erforderlich ist, solange sichergestellt ist, dass die Regelsetzer über eine Befugnis zur Regelsetzung verfügen, was bedeutet, dass sie die Zustimmung aller Adressaten, und damit der Mitglieder der Gemeinschaft, genießen. ${ }^{576}$ In diesem Kontext ist durchaus auch eine Anerkennung schon bestehender Regeln bei Eintritt in die Gemeinschaft denkbar. ${ }^{577}$

566 Nobel (1998), S. 122.

567 Auf ein gewisses Maß an Autonomie hinweisend Roßkopf (1998), S. 38; Ogus (2004), S. $109 \mathrm{ff}$.

568 Watter/Dubs (2005), S. 743.

569 Vgl. Buck-Heeb/Dieckmann (2010), S. 24.

570 Vgl. Nobel (1998), S. 124.

571 Roßkopf (1998), S. 37. Vgl. zur Freiwilligkeit und rechtlichen Unverbindlichkeit auch Wahlers (2011), S. 95; Bachmann (2006), S. 37.

572 Buck-Heeb/Dieckmann (2010), S. 42.

573 Vgl. Augsberg (2003), S. 278.

574 Vgl. Hueck (2017), S. 74. Zur Unterscheidung am Beispiel gesellschaftsrechtlicher Regulierung Buck-Heeb/Dieckmann (2010), S. $90 \mathrm{ff}$.

575 Vgl. Buck-Heeb/Dieckmann (2010), S. 23; Hueck (2017), S. 74. Vgl. weiterführend dazu auch Weiß (2012).

576 Vgl. Bachmann (2006), S. 403.

577 Vgl. Hueck (2017), S. 74. 


\subsubsection{Beurteilung der Selbstregulierung}

Das Regelungskonzept der Selbstregulierung geht mit Vor- und Nachteilen einher, wenngleich man feststellen muss, dass das Schrifttum in Deutschland der Selbstregulierung gegenüber eher kritisch eingestellt ist. ${ }^{578}$ Besondere Skepsis bringt beispielsweise SABRowsKy (1978) zum Ausdruck, da für ihn Selbstregulierung geradezu förmlich mit Unwirksamkeit gleichgesetzt werden könnte. ${ }^{579}$ Buck-Heeb/Dieckmann (2010) sehen in der Selbstregulierung dagegen ein durchaus mächtiges Instrument, da es zu kurz gedacht sei, wenn man »die Bedeutung der Selbstregulierung darauf beschränken will, dass der Inhalt von privaten Kodizes zum Entstehen rechtsethischer Maßstäbe und entsprechenden allgemeingültigen Verhaltensregeln beiträgt.. ${ }^{580}$ Bei der Beurteilung von Vorund Nachteilen der Selbstregulierung stellt sich jedoch die Frage nach einem »Besser« oder »Schlechter« privater Regelsetzung nicht immer, zumal die Entscheidung dafür oder dagegen häufig auf »Gebräuchlichkeit« oder »Mentalitäten und Gewohnheiten der Akteure ${ }{ }^{81}$ beruht. Vor- und Nachteile lassen sich dabei zwar grundsätzlich verallgemeinern, es kommt jedoch immer auf den Einzelfall und die Interessenlage der jeweiligen Akteure an, die den Erfolg einer Selbstregulierungsmaßnahme danach unterschiedlich einschätzen mögen. ${ }^{582}$

Es folgt nun eine nicht abschließende Aufzählung und Erläuterung einschlägiger Vor- und Nachteile der Selbstregulierung, ohne jedoch hier bereits konkret auf den Bezugsrahmen der Familienverfassung eingehen zu wollen. ${ }^{583}$

\subsection{Vorteile der Selbstregulierung}

a) Die Flexibilität und Geschwindigkeit

Ein wichtiger Vorteil der privaten Selbstregulierung wird in der Flexibilität und Geschwindigkeit gesehen. ${ }^{584}$ Diese positive Facette gilt bereits für den Prozess der Regelfindung. ${ }^{585}$ Staatliche Verfahren werden dabei nicht selten als schwerfällig bezeichnet. ${ }^{56}$ Die Regelsetzung auf Basis der Selbstregulierung nimmt weniger Zeit und Aufwand in Anspruch. ${ }^{587}$ Dies hat auch zur Folge, dass private Rege-

578 Vgl. Buck-Heeb/Dieckmann (2010), S. 217.

579 Vgl. Sabrowsky (1978), S. 22.

580 Buck-Heeb/Dieckmann (2010), S. 219.

581 Luttermann (2003), Rn. 53.

582 Vgl. Thoma (2008), S. $70 \mathrm{ff}$, , $74 \mathrm{f}$.

583 Umfassender und allgemein weiterführend Buck-Heeb/Dieckmann (2010), S. $217 \mathrm{ff}$.

584 Vgl. Watter/Dubs (2005), S. 748; Buck-Heeb/Dieckmann (2010), S. 220; Buck-Heeb/Dieckmann (2010), S. 220 Fn. 20 m. w. N.

585 Vgl. Buck-Heeb/Dieckmann (2010), S. 220.

586 Vgl. Breidenbach (1997), S. 137 m. w. N.

587 Vgl. Lehmann (2006), S. 123; Lehmann (2006), S. $126 f$. 
lungen einfacher und schneller bei Bedarf an sich verändernde Verhältnisse angepasst werden können. ${ }^{588}$ Dies mag insbesondere dann zutreffen, wenn sich die Akteure über die Änderung schnell einig sind. Es sollte allerdings nicht darüber hinweggetäuscht werden, dass auch private Regelungen im Kreis der Akteure zu Meinungsverschiedenheiten führen können und somit eine Änderung durchaus auch langwierig sein kann. ${ }^{589}$ Oder eine grundsätzliche Änderung hat nach Vorgabe der Akteure im Rahmen eines vereinbarten Verfahrens zu erfolgen, das eine gewisse Zeit in Anspruch nehmen kann. Darüber hinaus kommt erschwerend hinzu, dass manche Regelsetzer institutionalisiert sind und lediglich wenige Male im Jahre zusammenfinden, um solche Regeländerungen zu diskutieren und zu beschließen, was die zuvor angesprochenen Vorteile der Flexibilität und Geschwindigkeit relativieren kann. ${ }^{590}$

\section{b) Die Akzeptanz und Zustimmung}

Ein weiterer zu nennender Vorteil privater Selbstregulierung kann in der höheren Akzeptanz der aufgestellten Vorschriften durch die Regeladressaten bestehen. Damit einhergehen kann eine höhere Wahrscheinlichkeit der freiwilligen Regelbefolgung aus Überzeugung, zumal die Regelsetzung nicht einseitig ausgearbeitet und damit den Regeladressaten nicht - wie bei einer gesetzlichen Regelung beispielsweise üblich - aufgezwungen wird. ${ }^{591}$ WATTER/DuBs (2005) führen, von der Selbstregulierung überzeugt, dazu aus:

"Da die [...] Regel selbst gestaltet wurde, ist die Akzeptanz [...] bei den Regelungsadressaten verbessert, was sich sowohl präventiv bei der Regelbefolgung als auch bei der Duldung von wegen Verletzung ausgesprochenen Sanktionen auswirken sollte. ${ }^{592}$

So finden sich in der Literatur auch entsprechende Hinweise, dass gerade der Aspekt der Akzeptanz (es wird in diesem Kontext auch von "Normloyalität «"593 gesprochen) als das wichtigste Kriterium zur Beurteilung des Prozesses der Selbstregulierung sei. ${ }^{594}$ Im Prozess der Regelfindung wirkt sich positiv aus, dass die verschiedenen Interessen der Akteure berücksichtigt werden und somit eine verhältnismäßige Regel auf Basis eines gemeinsamen Nenners entsteht. ${ }^{595}$ Die Akzeptanz und damit einhergehend die Einhaltung der privaten Regeln soll auf

588 Vgl. Gelhausen/Hönsch (2003), S. 367 ff.; Voegeli-Wenzl, S. 815.

589 Darauf hinweisend Buck-Heeb/Dieckmann (2010), S. 220.

590 Vgl. Breidenbach (1997), S. 137; Buck-Heeb/Dieckmann (2010), S. 221.

591 Vgl. Bachmann (2006), S. 54; Nobel (1998), S. 120; Watter/Dubs (2005), S. 748; Lehmann (2006), S. 125f.; Werder/Talaulicar (2009), S. 689ff.; Bangert (2004), S. 79f.; Frenz (2001), S. 68.

592 Watter/Dubs (2005), S. 748.

593 Thoma (2008), S. 72. Vgl. auch Bangert (2004), S. 80.

594 Vgl. nur Buck-Heeb/Dieckmann (2010), S. 290.

595 Vgl. Achleitner (1995), S. 60 f. 
Grundlage der persönlichen Identifikation mit dem Regelinhalt erfolgen ${ }^{596}$ und nicht nur aus Furcht vor möglichen Sanktionen bei Nichteinhaltung. ${ }^{597}$

Die Legitimation einer Regel lässt sich nicht nur aus der Akzeptanz, also aus der Zustimmung der Regeladressaten, ableiten, sondern auch aus dem sogenannten Gemeinwohl ${ }^{598}$. BACHMANN (2006) führt dazu aus:

")Zustimmung^ legitimiert eine Regel, indem die von ihr Betroffenen sich mit ihr einverstanden erklären, `Gemeinwohl rechtfertigt ihre Geltung, wenn sie sich trotz fehlender Zustimmung aller Betroffenen auf lange Sicht als für sie vorteilhaft erweist. ${ }^{599}$

Lässt sich eine größtmögliche Akzeptanz des Regelinhalts erzielen, so kann weitgehend auf die Sanktionierung eines Regelverstoßes verzichtet werden. In diesem Zusammenhang lässt sich feststellen, dass der Sanktionierungsbedarf bei zunehmender Akzeptanz von privat aufgestellten Regeln abnimmt. Eine solche Akzeptanz bedarf jedoch einer intensiven Kooperation im Vorfeld. Eine enge Zusammenarbeit zwischen Regelsetzer und Regeladressaten führt zu effizienten privaten Regelungen. So kann sichergestellt werden, dass die privat aufgestellten Regeln das Interesse der Regeladressaten abbilden und sie aus diesem Grund freiwillig befolgen. ${ }^{600}$ Problematisch für die Akzeptanz privater Regelsetzung kann allerdings werden, wenn die Regeladressaten nicht angemessen an der Erstellung privater Bestimmungen beteiligt und in diese einbezogen werden. ${ }^{601}$ In diesem Kontext findet sich im Schrifttum auch der Hinweis, dass private Regeln den Adressaten oftmals besser bekannt und damit eher vertraut erscheinen als staatliche Normen. ${ }^{602}$

\subsection{Nachteile der Selbstregulierung}

Die Selbstregulierung hat unbestritten auch Nachteile gegenüber staatlicher Regulierung ${ }^{603}$.

596 Vgl. Lehmann (2006), S. 125 f.

597 Vgl. Christiansen (2000), S. 125.

598 Wenn hier von Gemeinwohl die Rede ist, so bezieht sich dieses vornehmlich auf ein „Gruppenwohl«, zumal private Regeln häufig einen abgeschlossenen Adressatenkreis betreffen. Vgl. dazu auch Bachmann (2006), S. 206.

599 Vgl. Bachmann (2006), S. 179.

600 Vgl. Buck-Heeb/Dieckmann (2010), S. 224.

601 Vgl. Buchholz (2004), S. 80 f.

602 Vgl. Ruch (2004), S. 449.

603 Dies feststellend Bachmann (2006), S. 54. 


\section{a) Die Unverbindlichkeit der Regeln}

Die Freiwilligkeit und damit auch die Unverbindlichkeit der privat geschaffenen Regeln wird nicht selten als Nachteil der Selbstregulierung gesehen. ${ }^{604}$ BUCKНеeв/Dieckmann (2010) mahnen an, dass die »Freiwilligkeit nicht zwangsläufig mit Sanktionslosigkeit gleichgesetzt werden« kann. »Zu kurz gegriffen ist daher die Aussage, Selbstregulierung lebe von einer freiwilligen Befolgung der aufgestellten Vorschriften, da häufig eine [...] soziale Sanktion fehle. ${ }^{605}$ Unverbindlichkeit wird man etwa dort ablehnen, wo sich Regeladressaten durch eine Erklärung der Anerkennung dem selbst geschaffenen Regeln unterworfen haben. ${ }^{606}$ Basierend auf den Akzeptanz- und Umsetzungsproblemen finden sich in der Literatur entsprechende Hinweise, die Anforderungen an eine Selbstkontrolle möglichst gering $\mathrm{zu}$ gestalten. Es mangle an einer umfassenden Einhaltung der im selbstregulativen Akt gegebenen Regeln, wenn hinreichende ökonomische Anreize für ein regelkonformes Verhalten fehlen. ${ }^{607}$ Die Wahrscheinlichkeit einer Regelkonformität lässt sich durch - wie auch immer geartete - präventive Kontrollen verstärken. ${ }^{608}$ Damit können Regelverstöße aufgedeckt und sanktioniert werden.

Wenn Unverbindlichkeit als Manko der Selbstregulierung gerügt wird, so ist dies zu pauschal. Sicherlich mag es an einer allgemeinen Verbindlichkeit fehlen, doch auf Grund eines gemeinsam verabschiedeten Regelwerkes sind zumindest die beteiligten Parteien von einer gewissen Verbindlichkeit betroffen. Die Unverbindlichkeit greift auf alle Fälle dann, wenn Dritte "automatisch « an das Regelwerk gebunden sein sollen. Doch auch so besteht ein Zwang zur Bindung grundsätzlich nicht. Vor diesem Hintergrund mag man es durchaus als Vorteil sehen, wenn die Regeladressaten (möglichst langfristig) auch einen mittelbaren oder gar unmittelbaren persönlichen Nutzen in der Einhaltung der selbst auferlegten Regeln sehen. Der persönliche Nutzen kann mannigfaltig sein. Er reicht von direkten und indirekten ökonomischen Vorteilen ${ }^{609}$ bis hin zur Vermeidung innerer Sanktionen im Sinne von Scham und Enttäuschung ob der Abweichung von den selbst auferlegten Regeln.

Buck-HeEb/DieckmanN (2010) führen grundsätzlich zu dem Vorwurf der Unverbindlichkeit aus:

$604 \mathrm{Vgl}$. Wiegand/Wichtermann (2000), S. 35.

605 Buck-Heeb/Dieckmann (2010), S. 229f.; vgl. dazu auch Augsberg (2003), S. 283.

606 Thiele (1966), S. 14ff.; Bachmann (2006), S. 173: »Privat gesetzte Regeln [...] werden grundsätzlich nur dann verbindlich, wenn der, an den sich die Regel richtet, sich damit einverstanden erklärt."

607 So wohl auch Hopt, S. 398.

608 So im Ergebnis auch Gilch/Pelz (2008), S. 134ff.

609 Vgl. Buck-Heeb/Dieckmann (2010), S. 230. 
»Abgesehen davon bezieht sich diese Argumentation im Grund auf das generelle Fehlen einer rechtlich verbindlichen Sanktionsordnung. Schließlich kann beim Vollzug und der Vollstreckung von Regelwerken der Selbstregulierung, bei denen sich die Privaten nicht rechtlicher Gestaltungsmittel (z. B. Vertrag, Satzung) bedienen, um die Adressaten zu verpflichten, nicht auf die staatliche Zwangsvollstreckung zurückgegriffen werden. Zudem mangelt es gewöhnlich an einem entsprechenden Rechtsschutz, wie etwa der Möglichkeit, eine Streitigkeit einer unbefangenen Instanz zur Entscheidung zu unterbreiten. ${ }^{610}$

\section{b) Das Durchsetzungsdefizit}

Der am häufigsten erhobene Vorwurf ist sicherlich die ungenügende Durchsetzbarkeit selbstregulatorischer Vorschriften sowie die mangelnde interne und externe Überwachung der Regelkonformität. ${ }^{611}$ Die Selbstregulierung sieht sich daher häufig der Kritik ausgesetzt, dass sich die private Regelsetzung zu sehr auf freiwillige Befolgung verlässt und dabei zu wenig auf Aufsicht und nötigenfalls Sanktion setzt. ${ }^{612}$ Rossкop F (1998) führt dazu aus:

»In Systemen der reinen freiwilligen Selbstkontrolle sind Sanktionen für Verstöße, falls solche vorgesehen sind, mangels rechtlicher Verbindlichkeit nicht zwangsweise vollstreckbar, außerdem sind zur Verfügung gestellte Sanktionen häufig bereits ihrer Natur nach stark eingeschränkt: oft sehen Selbstregulierungssysteme nur die beiden Extreme einer schlichten Verwarnung oder eines äußerst schwerwiegenden Ausschlusses aus der jeweiligen Organisation vor $[\ldots] . \star^{613}$

Die bei Rosskopf (1998) erwähnte "schlichte Verwarnung « bezieht sich auf die Ausführungen von RIDER (1978), welcher in diesem Sanktionsmechanismus der Missbilligung eine deutlich stärkere Wirkung sieht als in jedem anderen rechtlichen Sanktionsinstrument:

"In real terms the sanctions of disapproval and damaged reputations which lie in the field of self-regulation are far greater than any legal sanction. « ${ }^{614}$

610 Buck-Heeb/Dieckmann (2010), S. 231. Vgl. dazu auch Watter/Dubs (2005), S. 748.

611 Vgl. Roßkopf (1998), S. 47; Ulmer/Niemeier (1975), S. 554; Frenz (2001), S. 59; Bachmann (2006), S. 54; Thoma (2008), S. 76. Dies trifft selbstredend lediglich für die klassischen Fälle der Selbstregulierung zu, die keine Rechtspflichten begründen.

612 Vgl. Bachmann (2006), S. 54; Buck-Heeb/Dieckmann (2010), S. 234; Schmidt-Preuß (1997), S. 219.

613 Roßkopf (1998), S. 47.

614 Rider (1978), S. 339. Auch Puppis (2004) argumentiert ähnlich:»(Selbst-)Regulierung muss, wie ausgeführt, neben der Regelsetzung auch die Durchsetzung der Regeln und die Sanktionierung von Regelverstößen beinhalten. Die fehlende Sanktionierung ist einer der Hauptkritikpunkte an Selbstregulierung. Um erfolgreich zu sein, scheinen wirksame Sanktionsmechanismen unabdingbar. Hierbei darf allerdings interner Tadel durch Berufskollegen nicht unterschätzt werden, dessen Wirkung weit einschneidender sein mag als die Verhängung von Strafen«, vgl. Puppis (2004), S. 72. 
Mittelschwere Verstöße könnten allerdings nicht situationsadäquat geahndet werden, was der Glaubwürdigkeit des Systems schade. ${ }^{615}$ Buck-HeEb/DieckMANN (2010) stellen fest, dass auch eine "gesetzliche Regelung nur serfolgreich ist, wenn entweder eine allgemeine Akzeptanz der Vorschriften gegeben ist oder die Regelsetzung durch einen funktionierenden Sanktionsmechanismus flankiert wird. ${ }^{616}$ Bei der Selbstregulierung haben Private bei einem Regelverstoß nur dann eine Durchsetzungsmöglichkeit, wenn bereits bei der Regelsetzung eine solche vorgesehen wurde. ${ }^{617}$

\section{c) Die mangelnde Legitimation}

Ein weiterer vermeintlicher Nachteil der Selbstregulierung kommt in der ihr vorgehaltenen mangelnden Legitimation zum Ausdruck, dies meint die fehlende demokratische Legitimation und Kontrolle. ${ }^{618}$ Diesem Vorwurf kann nur bedingt gefolgt werden. ${ }^{619}$ So besteht für Private grundsätzlich die Möglichkeit, sich selbst Vorschriften zu geben, sofern diese auf Einstimmigkeit fußen. Darüber hinaus darf nicht verkannt werden, dass der Begriff der Legitimität außerhalb des juristischen Kontexts nicht zwingend auf eine demokratische Legitimation referenziert, sondern vielmehr auf eine Anerkennung durch die Regeladressaten. So kann festgehalten werden, dass die Unterstützung durch die Regeladressaten und allein ihr Glaube an die Rechtmäßigkeit entscheidend ist für die Legitimation privater Vorschriften. ${ }^{620}$

Buck-Heев/Dieckmann (2010) fassen die Legitimität privater Selbstregulierung treffend zusammen:

"Sofern private Regimes von den Adressaten als legitim erachtet würden, könne ein größerer Erfolg erwartet werden. Legitim sollen die in einer Bestimmung erhaltenen Verhaltenserwartungen werden, wenn sie verallgemeinerungsfähige Interessen zum Ausdruck bringen können. $\aleph^{621}$

Soziologisch betrachtet gelten Regeln dann als legitim, wenn sie von den Regeladressaten akzeptiert oder grundsätzlich hingenommen werden. ${ }^{62}$

615 Vgl. Roßkopf (1998), S. 47. Vgl dazu auch Ayres/Braithwaite (1994), S. 35 ff., die die Existenz eines abgestuften Sanktionsmechanismus für jegliches Kontrollsystem als essentiell ansehen (»enforcement pyramid«).

616 Buck-Heeb/Dieckmann (2010), S. 234.

617 Vgl. Buck-Heeb/Dieckmann (2010), S. 234.

618 Vgl. Watter/Dubs (2005), S. 748; Peters (2006), S. 148ff.; Buck-Heeb/Dieckmann (2010), S. 237.

619 Zur Legitimation privater Selbstregulierung vgl. nur Buck-Heeb/Dieckmann (2010), S. $257 \mathrm{ff}$.

$620 \mathrm{Vgl}$. Bachmann (2006), S. 104: So ist "grundsätzlich der Konsens der von einer privaten Regel Betroffenen das maßgebliche Kriterium« für deren Legitimität. Hervorh. im Original.

621 Buck-Heeb/Dieckmann (2010), S. 237. Vgl. dazu auch Biedermann (2007), S. 85 f.

622 Vgl. Bachmann (2006), S. 225f. 


\subsection{Kritische Bewertung der Vor- und Nachteile}

Insgesamt lässt sich feststellen, dass der Verwendung der Begriffe »Vorteil« und »Nachteil« in Bezug auf die Bewertung der Selbstregulierung in der Literatur meist eine gewisse Form der Ambivalenz innewohnt. Eine abstrakte Abwägung erscheint kaum möglich. ${ }^{623}$ Nicht zuletzt ist es immer auch eine Frage der Perspektive: Was aus staatlicher Sicht als Nachteil gesehen werden könnte, mag aus Sicht der privaten Akteure jedoch durchaus als Vorteil geschätzt werden. Nimmt man somit eine Bewertung vor, kann eine solche nach objektiven Kriterien immer nur unter Angabe der Bezugsebene erfolgen. ${ }^{624}$ Eine empirische Untersuchung der als Vor- oder Nachteil identifizierten Faktoren scheint bislang zu fehlen. ${ }^{62}$ Auch fehlen empirische Befunde, ob sich die Erwartungen an eine Selbstregulierung erfüllt haben und welche Chancen und Risiken mit den verschiedenen Möglichkeiten der Gestaltung privatautonomer Selbstregulierung einhergehen. ${ }^{626}$ Die spärlich vorhandenen empirischen Untersuchungen gelangen zu der Erkenntnis, dass die selbstregulativen Mechanismen "funktionieren ${ }^{627}$ "Selbstregulierung hat also nicht nur Vorteile [...]. Kritik jedenfalls an manchen Erscheinungsformen der Selbstregulierung ist deshalb begründet. Sie allein deshalb aber als Regulierungsform ganz abzulehnen, scheint angesichts der aufgezählten positiven Aspekte nicht gerechtfertigt. " $^{628}$ Gerade da das Funktionieren im Wesentlichen auf der freiwilligen Mitwirkung der Regeladressaten basiert, muss mangels rechtlicher Durchsetzbarkeit insbesondere an das »Gewissen [...] der Beteiligten appelliert« werden. ${ }^{629}$

\subsubsection{Regelbefolgung durch höhere Akzeptanz}

Wenn die aufgestellten Regeln von den Regeladressaten nicht befolgt werden, wird die eigentliche Intention ad absurdum geführt. Es ist vor diesem Hintergrund von besonderer Bedeutung zu verstehen, welche Faktoren die Akzeptanz der selbst geschaffenen Regeln sicherstellen oder gar erhöhen können.

623 Vgl. Bachmann (2006), S. 34.

624 So im Ergebnis wohl auch Buck-Heeb/Dieckmann (2010), S. 239.

625 Darauf hinweisend Buck-Heeb/Dieckmann (2010), S. 239.

626 Vgl. Puppis/Donges (2004), S. 12.

627 Vgl. Knebel et al. (1999), S. $330 \mathrm{ff}, 373 \mathrm{ff}$.

628 Roßkopf (1998), S. 51. Vgl. dazu auch Ogus (2004), S. 109.

629 Roßkopf (1998), S. 49. 


\subsection{Sachnähe zur Förderung der Akzeptanz}

Grundsätzlich wird der Sachnähe derer, die Regeln aufstellen, in Bezug auf die Akzeptanz des Regelwerks eine besondere Rolle zugewiesen. Die Sachnähe erhöht die Bereitschaft zur freiwilligen Regelbefolgung. ${ }^{630}$

\subsection{4.2 Verbindlichkeit zur Förderung der Akzeptanz}

Es ist unstreitig, dass Selbstregulierung nur dann erfolgreich sein kann, wenn die selbst geschaffenen Regeln von den Adressaten auch in der Praxis befolgt werden. Ein regelkonformes Verhalten bedarf eines entsprechenden Anreizes. Vornehmlich wird ein solcher besonders in der Akzeptanz der Regel(n) gesehen. ${ }^{631}$ Es kann festgestellt werden: Je höher die Akzeptanz der Regeln, desto weniger wirkt sich deren Unverbindlichkeit negativ auf deren Einhaltung aus. Soziale Ordnungen können daher langfristig nur stabil gehalten werden, wenn grundsätzlich damit gerechnet werden kann, dass die aufgestellten Regeln von den Regeladressaten akzeptiert und damit eingehalten werden. ${ }^{632}$ Entsprechende Anstrengungen sind also zu unternehmen, um die Akzeptanz zu fördern. ${ }^{633}$ Dafür bedarf es bei den Regeladressaten einer moralischen Zustimmung zu den Zielen der Regeln, oder die Regeln müssen von einer echten moralischen Überzeugung getragen werden, wenngleich die Bestimmungen für das Individuum im Einzelfall ungünstig sein mögen. ${ }^{634}$ LUHMANN (1987) greift in diesem Kontext auch auf das Konzept "Legitimation durch Verfahren $"{ }^{635}$ zurück. Die zu Grunde gelegte Vorstellung basiert darauf, dass eine verfahrensmäßige Teilhabe (Partizipation) gut sei und daher geboten. ${ }^{636}$ Weitere positive Aspekte zur Einhaltung der Regeln sind ein erkennbarer Eigennutz oder ein ökonomischer Anreiz. ${ }^{637}$ Auch genießen jene Regeln eine hohe Akzeptanz, die mit nur geringen Einbußen in Bezug auf die eigenen Interessen einhergehen. ${ }^{638}$ Umgekehrt gewinnt die drohende Sanktionierung bei Regelverstoß an Bedeutung, sofern die Akzeptanz der Regeln eher niedrig ist. ${ }^{639} \mathrm{Zu}$ verkennen ist auch nicht der mittelbare, soziale Druck zur Regelbefolgung, der von der Wahrnehmung des Umfeldes bei Regel-

630 Wahlers (2011), S. 139f.

631 Vgl. Achleitner (1995), S. 60f.; Breidenbach (1997), S. 132.

632 Vgl. Luhmann (1987), S. $259 \mathrm{ff}$.

633 Vgl. Wahlers (2011), S. 140.

634 Vgl. Magen (2007), S. 280.

635 Raiser (2013), S. 132.

636 Vgl. Bachmann (2006), S. 162, 192. Bachmann (2006), S. 193: »Soziologisch gesehen kann Teilhabe damit zu verbesserter Akzeptanz von Entscheidungen (und so gesehen zu deren Legitimität) beitragen."

637 Vgl. Ekardt (2005), S. 219; Buck-Heeb/Dieckmann (2010), S. 290. So im Ergebnis wohl auch Hoeren (1995), S. 15.

638 Vgl. Hopt, S. 398; Roßkopf (1998), S. 48.

639 So im Ergebnis auch Wüstemann/Bischof/Koch (2008), S. 17; Bangert (2004); Lukes (1974), S. $327 \mathrm{ff}$. 
missachtung ausgeht. Dem mag entgegengehalten werden, dass die Befolgung dann nur bedingt freiwillig erfolgt und sie somit nicht auf die Akzeptanz der Regel zurückgeführt werden kann. Entscheidend ist jedoch, dass die Regeln überhaupt eingehalten werden. ${ }^{640}$

\subsection{4.3 Durchsetzbarkeit zur Förderung der Akzeptanz}

Die auf Freiwilligkeit basierende private Selbstregulierung sieht sich der Kritik der Durchsetzbarkeit ausgesetzt. ${ }^{641}$ Die Kritik an der Durchsetzbarkeit wird vornehmlich auf mangelnde und ungenügende Sanktionsmechanismen zurückgeführt. ${ }^{62}$ Dieser Kritik kann nur bedingt gefolgt werden. Richtig ist sicherlich, dass etablierte Sanktionssysteme die Durchsetzbarkeit außerrechtlicher Normen erhöhen. Allerdings genießt die Normbefolgung bei privater Selbstregulierung eine weitaus größere Bedeutung als deren Durchsetzbarkeit. ${ }^{643}$

Es lassen sich drei wesentliche motivationale Gründe für die Einhaltung von Normen feststellen: Identifikation, Akzeptanz (Internalisierung) und Sanktionsorientierung. ${ }^{64}$

\section{a) Die Identifikation mit dem sozialen Umfeld}

Der erste Grund, die Identifikation, bringt zum Ausdruck, dass ein Regeladressat eine Norm befolgt, da er sich am Verhalten seiner Bezugsgruppe oder einzelner Vorbilder orientiert. ${ }^{645}$ Normen werden nicht auf Grund der mit ihnen verbundenen sozialen Werte befolgt, sondern da den Regeladressaten die Beziehung zu ihrem Umfeld, zu ihrer Bezugsgruppe oder zu den Führungspersonen wichtig ist und sie diese aufrechterhalten wollen (aus Angst vor dem Verlust sozialer Beziehungen, dem Verlust von Status oder anderer sozialer Sanktionen). ${ }^{646} \mathrm{Ob}$ und wie erfolgreich die Identifikation in Bezug auf die Regelbefolgung ist, hängt wesentlich vom jeweiligen Interesse der Person ab. Das Regelwerk als solches wird zwar nicht akzeptiert, jedoch auf Grund der Orientierung am Umfeld befolgt. Sanktionsmechanismen könnten diese Befolgung noch flankieren. ${ }^{647}$

640 Vgl. Wahlers (2011), S. 140.

641 Vgl. Wahlers (2011), S. 141.

642 Vgl. Wahlers (2011), S. 141 f.; Rider (1978), S. 339; Roßkopf (1998), S. 47; Ayres/Braithwaite (1994), S. $35 \mathrm{ff}$; Damrau (2003), S. $81 \mathrm{f}$.

643 Vgl. Wahlers (2011), S. 142.

644 Vgl. Pospisil (1987), S. 256ff.; Rehbinder (2014), 115f. Vgl. dazu auch Galbraith (1967), S. $130 \mathrm{ff}$.

645 Ehrlich (1989) sprach schon von der Wirkung der Normen durch Suggestion, vgl. Ehrlich (1989), S. 77. Vgl. dazu auch Geiger/Rehbinder (1987), S. $40 \mathrm{ff.}$

646 Vgl. Kelman (1980), S. 201f.; Rehbinder (2014), Rn. 116; Wahlers (2011), S. 142; Hovland/ Janis (1959), S. 144f.

647 Vgl. Wahlers (2011), S. 142. 


\section{b) Die Internalisierung als Form der inneren Akzeptanz}

Die Internalisierung repräsentiert einen zweiten Beweggrund. Die Regelbefolgung erfolgt auf Grund einer intrapersonellen Akzeptanz (»moral orientation «). ${ }^{648}$ Der inneren Akzeptanz wird im Schrifttum sicherlich zu Recht der entscheidende Motivationsgrund zugeschrieben. ${ }^{649}$ Anders als bei der Sanktionsvermeidung und Identifikation erfolgt die Regelbefolgung bei der Internalisierung nicht auf Grund von externen Einflüssen und unabhängig von der Einhaltung durch andere, sondern auf Grund intrinsischer Motivation und eigener Überzeugung von der Sinnhaftigkeit und Richtigkeit (»Vertretbarkeit ${ }^{650}$ ) der Regelung, was bedeutet, dass die Regeladressaten an deren Legitimität glauben und darin einen Grund zur Befolgung der Regeln sehen. ${ }^{61}$ Legitimität kann dabei zwei Auslöser haben: Entweder indem der Regeladressat die Regel auf Grund von persönlicher Überzeugung als geboten erachtet, oder weil die Norm von einem als legitim angesehenen Regelsetzer aufgestellt wurde. ${ }^{62}$ Der Homo sociologicus bewegt sich damit an der Schnittstelle zwischen gesellschaftlich erzwungener Rolle und seinen persönlichen Bedürfnissen ${ }^{653}$ :

»The former [homo oeconomicus] is 'pulled $₫$ by the prospect of future rewards, whereas the latter [homo sociologicus] is spushed from behind by quasi-inertial forces. $\aleph^{654}$

Das soziologische Konzept der Akzeptanz geht zurück auf Weber (1972), der eine Ordnung im soziologischen Sinne als "geltend« definierte, sobald sie von den Adressaten als »legitim» empfunden wurde. ${ }^{655}$

Ursächlich für die Internalisierung ist die Verinnerlichung von Werten und die Etablierung eines eigenen Wertegerüsts, das von externen Einflüssen (z. B. durch soziale Einflüsse) beeinflusst wird. ${ }^{656}$ Dies kann bereits in frühen Jahren der Jugend durch Einflussfaktoren im persönlichen Umfeld erfolgen oder dadurch, dass die Notwendigkeit und der Nutzen der Regeleinhaltung für die Gesellschaft, das Umfeld insgesamt eingesehen wird. ${ }^{657}$

648 Vgl. Pospisil (1987), S. 257; Rehbinder (2014), Rn. 116.

649 Vgl. Rehbinder (2014), Rn. 116; Hart (2011), S. 41; Feldges (1987), S. 153; Luhmann (1983), S. $27 \mathrm{f}$.

650 Rehbinder (2014), Rn. 116.

651 Vgl. Hillmann (1994), S. 615.

652 Vgl. Damrau (2003), S. 89.

653 Vgl. Damrau (2003), S. 89.

654 Elster (1989), S. 99.

655 Vgl. Weber (1972), S. 16.

656 Vgl. Raven (1965), S. 371f.; Wahlers (2011), S. 143.

657 Vgl. Damrau (2003), S. 89; Sagel-Grande (1990), S. 28. 
c) Die Sanktionsorientierung zur Vermeidung negativer Konsequenzen

Fehlt einer Regel eine immanente "Anreizkompatibilität « ${ }^{658}$, so muss eine solche durch eine wirksame Sanktionsdrohung kompensiert werden. Somit erfolgt hier die Einhaltung von Regeln, um Sanktionen zu vermeiden. Sanktionen können dabei das Verhalten eines Akteurs beeinflussen. Sanktionen werden als »Maßnahmen der reaktiven Kontrolle ${ }^{659}$ bezeichnet. Sie wirken entweder positiv (im Sinne einer Belohnung) oder negativ (durch Zufügung eines Übels). ${ }^{60}$ Der Regeladressat verhält sich somit dann verhaltenskonform, wenn »der erwartete Nutzen aus der Normbefolgung höher ist als der erwartete Nutzen aus dem Normbruch ${ }^{661}$ oder - im positiven Fall - die Belohnung einen Anreiz für ein verhaltenskonformes Handeln darstellt. ${ }^{662}$ Die Regel wird dabei weder aus persönlicher Überzeugung noch auf Grund von Akzeptanz befolgt. Der Regeladressat fügt sich der Überwachung und Kontrolle. ${ }^{663}$

Welcher motivationale Mechanismus im konkreten Einzelfall die Befolgung der Regel bewirkt, hängt stark vom Inhalt der Norm und der jeweiligen Persönlichkeitsstruktur des Regeladressaten ab. ${ }^{64}$ Es kommt am Ende aber sowohl auf die Art der Sanktion wie auch auf die Wahrscheinlichkeit ihrer Verhängung und damit auf eine effektive Überwachung an. ${ }^{665}$

\subsection{Kontrolle als Voraussetzung einer effektiven Durchsetzung}

Für eine verlässliche Durchsetzung der Regeln bedarf es eines funktionierenden Kontrollmechanismus. ${ }^{666}$ Es wird im Rahmen der effektiven Selbstregulierung immer wieder die Frage aufgeworfen, ob nicht eine »staatliche Letztaufsicht ${ }^{667}$ $\mathrm{zu}$ fordern ist. Insbesondere wird dort, wo die Regelsetzer und der Adressatenkreis nicht identisch sind, über eine mögliche Einbeziehung Dritter als Kontrollinstanz diskutiert. ${ }^{668}$ Dies führt zu der Diskussion, ob dann überhaupt noch von Selbstkontrolle gesprochen werden kann, wenn »Fremde« über die Einhaltung der selbst auferlegten Regeln wachen. ${ }^{669}$ Fraglich ist, ob eine Fremdkontrolle dabei nicht das Prinzip der Selbstregulierung ad absurdum führt. Selbstregu-

658 Damrau (2003), S. 91.

659 Wahlers (2011), S. 142.

660 Vgl. Meier (2016), S. $225 \mathrm{f}$.

661 Damrau (2003), S. 87.

662 Vgl. Pospisil (1987), S. 256; Rehbinder (2014), Rd. 115; Wahlers (2011), S. 142.

663 Vgl. Pospisil (1987), S. 256; Wahlers (2011), S. 142; Buck-Heeb/Dieckmann (2010), S. 290. Zur negativen Wirkung von Kontrollen vgl. Lüdemann (2007), S. 26.

664 Vgl. dazu auch Tyler (2006).

665 Vgl. Damrau (2003), S. 91.

666 Vgl. Damrau (2003), S. $94 \mathrm{ff}$.

667 Buck-Heeb/Dieckmann (2010), S. 291. Vgl. dazu auch Hopt, S. 398.

668 Vgl. Buck-Heeb/Dieckmann (2010), S. 291.

669 Vgl. Sabrowsky (1978), S. 179. 
lierung geht gemeinhin einher mit Freiwilligkeit; dies bedeutet, dass die Regeln nicht nur freiwillig befolgt werden, sondern auch den Aussprüchen einer Kontrollinstanz freiwillig und ohne jedweden Zwang Folge geleistet wird. ${ }^{670}$ Die Mitwirkung des Einzelnen ist mitentscheidend für den Erfolg der Selbstregulierung (so z.B. die Bereitschaft zur Auskunft gegenüber der Kontrollinstanz). Zur Aufdeckung und Aufklärung eines Regelverstoßes bedarf es immer der Mitwirkung der Beteiligten. ${ }^{671}$

\subsubsection{Sanktionsmechanismen}

\subsection{Freiwilligkeit und Sanktionen}

Wenngleich Regeln als Folge privatautonomer Entstehung für gewöhnlich auf Freiwilligkeit basieren, ist dies nicht gleichzusetzen mit der Tatsache, dass jede Sanktion fehlen muss. ${ }^{672}$ Sicherlich sind rechtlich verbindliche Regeln nötigenfalls auch mit staatlichem Zwang vollstreckbar, doch auch grundsätzlich unverbindliche Normen können durchgesetzt werden. Dies ist dann der Fall, wenn sich »die Unverbindlichkeit von privaten Vorschriften durch deren Anerkennung in eine partielle Verbindlichkeit umwandelt. In diesem Fall ist eine Regeldurchsetzung zumeist durch eigene Sanktionsmechanismen des privaten Selbstregulierungswerks vorgesehen [...]. ${ }^{673} \mathrm{Hinzu}$ komme eine mittelbare Sanktionierung durch den Markt oder die Gemeinschaft.

Die Verfahren bei Regelverstößen werden als "unvollkommen « bezeichnet, sie hätten »informellen Charakter« und seien »unterschiedlich formlos« ausgestaltet. Die Sanktionen seien »eher zurückhaltend « und man würde tendenziell auf den "gesellschaftlichen Verruf « als Konsequenz eines Verstoßes abstellen. ${ }^{674}$ So gibt es aber auch Regelwerke, in denen direkt Sanktionen bei Regelverstößen vorgesehen sind. Existiert ein eigenes Verfahren bei Regelverstößen, so wird von einer »Prozedualisierung der Selbstregulierung" gesprochen. ${ }^{675}$ Bei den Sanktionsmöglichkeiten selbst wird grundsätzlich zwischen autonomen, gesetzesunterstützten und gesetzlichen Methoden unterschieden. Besonders schwach ausgeprägt ist der Selbstregulierungsakt, wenn weder private noch gesetzgeberische Maßnahmen bei einem Regelbruch vorgesehen sind. Dies trifft vornehmlich für Kodizes zu. In diesen Fällen bleibt allein der Markt als Regulativ und es wird auf

670 Vgl. Lehmann (2006), S. 125.

671 Vgl. Roßkopf (1998), S. 48; Buck-Heeb/Dieckmann (2010), S. 291.

672 Vgl. Buck-Heeb/Dieckmann (2010), S. 229.

673 Buck-Heeb/Dieckmann (2010), S. 292.

674 Buck-Heeb/Dieckmann (2010), S. 292. Vgl. dazu auch die Ausführungen von Sabrowsky (1978), S. 176f.

675 Buck-Heeb/Dieckmann (2010), S. 292; vgl. dazu auch Hoeren (1995), S. 297. 
einen Reputationseffekt gesetzt. Enthält das private Regelwerk selbst konkrete Vorgaben zur Durchsetzung, werden diese als autonome Sanktionen bezeichnet. $^{676}$

\subsection{Sanktionsmöglichkeiten privater Selbstregulierung}

a) Die Wirksamkeit privatautonomer Regeldurchsetzung

Dass nicht nur der Staat alleine effektiv Vorschriften durchsetzen kann, beweisen verschiedene Formen der privaten Regeldurchsetzung, die teilweise sogar wirkungsvoller sind als das staatliche System. Beispielsweise kann der private Internetprovider selbst sehr schnell Regelverstöße sanktionieren, indem er seinen Kunden bei Verstößen gegen die geltenden Bestimmungen den Netzzugang sperrt. ${ }^{677}$ Welche Konsequenzen das für einen Nutzer heutzutage bedeutet, ist leicht nachvollziehbar. Als weiteres Beispiel führen Buck-HeEb/DieckmanN (2010) die Regeldurchsetzung im Internet an und verweisen auf die Internet Corporation of Assigned Names and Numbers (kurz: ICANN). Diese privatrechtliche Organisation ist für die Verwaltung der Internetadressen zuständig. Die ICANN entscheidet darüber, welche Internetadressen aufgenommen werden. So genießt sie auch eine Monopolstellung bei Streitigkeiten. Bei Domainstreitigkeiten entscheidet die ICANN im Rahmen des Verfahrens der alternativen Streitschlichtung, der sog. Uniform Domain Name Resolution Policy (kurz: UDRP). Sie kann daraufhin Domainnamen löschen oder in begründeten Fällen (z.B. Markenrechtsstreitigkeiten) die Domain auf einen neuen Inhaber übertragen. ${ }^{678}$

Eine weitere Form der privaten Regeldurchsetzung ist das Prinzip des »Naming and Shaming",

"[...] that is, a form of social control that uses negative publicity to threaten a social actor's reputation in order to promote change in the actor's behavior. ${ }^{679}$

Hierbei wird der Regelbruch öffentlich bekannt gemacht und der Regelverletzer namentlich benannt (naming). Dies kann in seinem jeweiligen Umfeld zu sozialen oder unerwünschten wirtschaftlichen Nachteilen führen (shaming). ${ }^{60}$

676 Vgl. Buck-Heeb/Dieckmann (2010), S. 292.

677 Vgl. Christiansen (2000), S. 125.

678 Vgl. Buck-Heeb/Dieckmann (2010), S. 293f.

679 Carroll (2016), S. 474.

680 Vgl. Bachmann (2006), S. 35; Magen (2007), S. 280; Heimann (2009), S. 255 f. Zu entsprechenden Beispielen vgl. Buck-Heeb/Dieckmann (2010), S. 294. 
Schon die Androhung einer öffentlichen Mahnung von Regelbrüchen kann ein probates Mittel sein, Normkonformität sicherzustellen. ${ }^{681}$

Neben der öffentlichen Rüge kann "cold shouldering « ${ }^{682}$ als zweite außerrechtliche Sanktionsmaßnahme angeführt werden. Dabei wirkt der Regelsetzer auf die sonstigen Regeladressaten so ein, dass sie Druck auf den Regelbrecher ausüben. ${ }^{683}$ Druck kann dadurch ausgeübt werden, dass Regelbrecher öfter kontrolliert werden oder man häufiger von ihnen Informationen anfordert. ${ }^{684}$

\section{b) Sanktionierung durch den Markt oder das Umfeld}

Bei der Selbstregulierung sollte der Markt als Sanktionsinstrument nicht unterschätzt werden. Die Einhaltung der Regeln mag positive Reaktionen des Marktes hervorrufen, wohingegen der Regelbruch negative auslösen kann. Unternehmen können im Falle negativer Publizität Schaden erleiden. An dieser Stelle sei lediglich auf einen sinkenden Börsenkurs als mögliche Auswirkung einer negativen Veröffentlichung hingewiesen. In diesem Zusammenhang wird auch gerne von "Publizitätswaffe ${ }^{685}$ gesprochen. ${ }^{686}$ Der DCGK erhält beispielsweise keinerlei Sanktionen. Er geht davon aus, dass der Markt die Einhaltung der darin enthaltenen Empfehlungen goutiert und jedwede Verfehlung entsprechend (beispielsweise durch Kursabschläge) sanktioniert. ${ }^{687}$ Im Falle des DCGK soll die vermutete Marktreaktion einen Anreiz für die Befolgung der Regeln erzeugen. ${ }^{68}$

\subsubsection{Soziologisch-moralphilosophische Annäherung an ein Chamäleon ${ }^{689}$}

Nachdem sich Abschnitt 2.5.1 mit den theoretischen Grundlagen der Selbstregulierung als konkrete Form der privaten Ordnung beschäftigte und in weiten Teilen sicherlich damit der Intention einer Familienverfassung nahekommt, soll sich dieses Kapitel nun dem moralischen Sollen und Müssen, der moralischen Verpflichtung und der damit einhergehenden möglichen Sanktionierung be-

681 Vgl. Roßkopf (1998), S. 47; Rider (1978), S. 339; Feldges (1987), S. 119f.; Schmidt-Preuß (1997), S. 187: Ein "Prangereffekt» sei ein nicht zu unterschätzender Steuerungsfaktor; Damrau (2003), S. 91.

682 Damrau (2003), S. 92.

683 Vgl. dazu auch Bittner (1992), S. 186.

684 Vgl. Bardach/Kagan (1982), S. 163f.; Damrau (2003), S. 92.

685 Thoma (1996), S. 1726, 1732.

686 Vgl. Buck-Heeb/Dieckmann (2010), S. 295.

687 Vgl. Augsberg (2003), S. 306f.; Bachmann (2002), S. 2137 ff.; Schüppen (2002), S. 1271.

688 Buck-Heeb/Dieckmann (2010), S. 295f. Zur »Belohnung« der Einhaltung der Kodex-Regeln vgl. Bernhardt (2008), S. 1690.

689 Gläßer (2014), S. 236 bezeichnet die Familienverfassung als Chamäleon und spielt darauf an, wie vielschichtig man dieses Instrument sehen kann: Als "zahnlose Absichtserklärung", »unzulässiges Disziplinierungsmittel« oder »integratives Steuerungsinstrument». 
schäftigen. Diese theoretischen Grundlagen sollen dazu beitragen, ein besseres Verständnis für eine grundsätzliche moralische Verpflichtung zu gewinnen, nachdem die Familienverfassung für sich gerade eine moralische Verbindlichkeit reklamiert. Diese theoretischen Exkurse sollen nicht zuletzt auch als Grundlage dafür dienen, geeignete Maßnahmen zur Regelbefolgung der Familienverfassungen abzuleiten bzw. die Ergebnisse aus den Fallstudien besser bewerten zu können. ${ }^{690}$

\subsubsection{Das Wesen der Moral}

Was ist unter Moral zu verstehen? Ist sie begründungsbedürftig und, wenn ja, wie kann sie begründet werden? Ist sie möglicherweise rational geboten? In einem vornehmlich formalen Verständnis wird unter Moral ein »Netz spezifischer Normen « verstanden. ${ }^{691}$ Die Intention dieser Regulierung ist "die Organisation des Zusammenlebens innerhalb einer Gemeinschaft. “ ${ }^{692}$ Die meisten moralischen Formen waren einst Regelungen für das Leben innerhalb eines Kollektivs. ${ }^{693}$ Indem sie darauf abzielen, die Beziehungen der Individuen zu regeln, adressieren sie alle Mitglieder dieser Gesellschaft. ${ }^{694}$ "[...] denn Moral ist nur dann Moral, wenn sie für alle Individuen gleichermaßen verbindlich ist. « ${ }^{695}$ Moralische Normen können auf verschiedene Weise verstärkt und etabliert werden. Der soziale Druck, den sie auszuüben vermögen, ist dabei nicht zu unterschätzen:

»[Z]unächst einmal sind moralische Normen in Praktiken informellen sozialen Drucks eingebettet. Zur Moral gehören typischerweise Sanktionen wie Lob und Tadel sowie emotionale Reaktionen wie Empörung und Schuldgefühle. Sie zielen auf das Anerkennungsbedürfnis der einzelnen, ihren Wunsch, zu einer Gesellschaft zu gehören und nicht von ihr - und sei es nur symbolisch - ausgeschlossen zu werden. ${ }^{696}$

SEeBAss (2002) kommt zu einem ähnlichen Schluss, wenn er schreibt:

"Ein Mensch, der geltenden sozialen Standards nicht entspricht und deshalb ausgegrenzt wird, wird darauf je nach Art und Umständen z.B. mit Gefühlen von Schuld, Scham, Schande, Erniedrigung, Niedergeschlagenheit, Selbstwertverlust oder Verzweiflung reagieren und diese Reaktionen später genauso antizipieren und seiner Willensbildung zugrunde legen wie den Vollzug der Ausgrenzung selbst. [...] Denn sind die Standards erst einmal `verinnerlicht $\iota$ und Teil der eigenen Person, [...] werden die

690 Vgl. Abschnitt 4.1.

691 Steinfath (2002), S. 71. Hervorh. im Original. So ähnlich auch bei Curcio (2008), S. 86.

692 Curcio (2008), S. 86.

693 Vgl. Copp (2001), S. 74ff.; Steinfath (2002), S. 71.

694 Vgl. Copp (2001), S. 124 ff.; Steinfath (2002), S. 71.

695 Horster (2004), S. 36. Auch darauf verweisend Curcio (2008), S. 80.

696 Steinfath (2002), S. 72. Hervorh. im Original. 
zunächst extern induzierten, sanktionierenden Gefühle zu internen Sanktionen, die bei der Willensbildung entweder als (z.B.) erwartbare `Gewissensbisse` antizipiert oder schon präsent wirksam werden als Begleiterscheinungen von Wünschen und Absichten, bei denen sich >das Gewissen regt «.« ${ }^{67}$

Gibbard (1992) und Tugendhat (1993) haben den Versuch unternommen, die Moral über »bestimmte negative emotionale Reaktionen ${ }^{698}$ zu definieren. ${ }^{699}$ Ihrer Definition nach handelt es sich dann um moralische Normen, wenn auf deren Verletzung Gefühle der Empörung oder Schuld folgen oder folgen sollten. Dieser Definition kann jedoch nur begrenzt gefolgt werden, engt es doch die negativen Reaktionen auf Verstöße erheblich ein. Darüber hinaus sind diese negativen Gefühle als Reaktion nicht ausschließlich auf moralische Fehltritte begrenzt. ${ }^{700}$ Dass die Moral an besondere Sanktionen gebunden ist, über welche sozialer Druck ausgeübt wird, fußt auf einer richtigen Einsicht, ist es doch so, dass die meisten moralischen Normen Bereiche betreffen, die für so wichtig erachtet werden, dass man deren Beachtung nicht persönlichen Präferenzen überlassen möchte und sollte. Moralische Normen können Handlungen einfordern, die nicht zwingend den eigenen Interessen entsprechen und deren Umsetzung somit nicht immer einfach fällt. Gefühle wie Empörung und Schuldgefühl können dabei eine motivationale Verstärkung auslösen. Die Einhaltung von moralischen Normen ist nicht nur etwas, was sich die Gemeinschaft von jedem einzelnen Mitglied wünscht, sondern von diesem fordert ${ }^{701} \cdot{ }^{702}$ Hält man etwas für moralisch richtig, erwartet man berechtigterweise dessen Einhaltung nicht nur von sich selbst, sondern von allen Mitgliedern einer Gemeinschaft. Eine Moral zu haben, bedeutet mehr, als ein persönliches Ideal zu besitzen. ${ }^{703}$ Moralische Normen sind Vorgaben, deren Einhaltung wir als verpflichtend ansehen fordern; mit ihnen geht eine Berechtigung einher, deren Einhaltung von anderen zu fordern. ${ }^{704}$ Moralische Forderungen gelten allerdings nur so lange, wie sie von der Gemeinschaft gewollt oder (von einem hinreichend großen Teil von ihr) getragen werden. ${ }^{705}$

697 Seebaß (2002), S. 159. Hervorh. im Original.

698 Steinfath (2002), S. 72.

699 Vgl. Gibbard (1992), S. 40 f.; Gibbard (1992), S. 47 ff.; Tugendhat (1993), S. 3. Ähnlich argumentiert auch Wallace (1996), S. $18 \mathrm{ff}$.

700 So kann ein Verstoß gegen ein persönliches Ideal durchaus auch zu Schuldgefühlen führen. Vgl. Steinfath (2002), S. 73 Fn. 5.

701 Vgl. Abschnitt 2.5.2.8.

702 Vgl. Steinfath (2002), S. 73.

703 Vgl. Strawson (1961), S. 1 ff.

704 Vgl. Steinfath (2002), S. 73.

705 Vgl. Steinfath (2002), S. 76. 
Der Moral kann auch eine soziale Funktion zugesprochen werden. ${ }^{706}$ Wie bei allen sozialen Institutionen stellt sich auch hier die Frage, inwieweit die Moral die Gemeinschaft besser oder überhaupt erst möglich macht. Wenn hier die Frage gestellt wird, ob die Moral das Leben »besser « macht, ist damit kein moralisches »besser« gemeint, sondern vielmehr ein »besser" im Sinne eines außermoralischen Nutzens: Ist das Leben erträglicher in einer moralisch verfassten Welt im Vergleich zu einer ohne Moral? Wird das »bonum commune» (Gemeinwohl) dadurch nachweislich gefördert? ${ }^{707}$ Die soziale Funktion der Moral besteht darin, dass sie das Leben in einer Gemeinschaft lebenswerter machen soll. ${ }^{708}$

\subsubsection{Vom moralischen Sollen und Müssen}

Es scheint so, als wohne moralischen Normen ein besonderer Verpflichtungscharakter inne. ${ }^{709}$ Wenn die Moral in bestimmten Situationen dem Einzelnen zumutet, dass er seine eigenen Interessen zu Gunsten der Interessen anderer oder der Allgemeinheit zurückstellt, so liegt es nahe, dafür gute Gründe zu erwarten. ${ }^{710}$ Sollensbegriffe gibt es dabei zahlreiche. «Von unverbindlichen Empfehlungen, [...], Bitten scheinen sie dadurch geschieden zu sein, daß sie verbindliche Forderungen und Verpflichtungen enthalten. Das Sollen hat [...] den Charakter des unabdingbaren, kategorischen Müssens, das nicht mehr unter Vorbehalt steht, d.h. nicht konditionalisiert oder relativiert ist. « ${ }^{711}$

Moralische Pflichten bieten gleichsam praktische Gründe, etwas Bestimmtes zu tun oder zu lassen. Woher rühren diese Gründe? Ist es so, dass sich die praktischen Gründe aus der moralischen Pflicht ergeben oder ist genau das Gegenteil der Fall und die moralische Pflicht ergibt sich aus Überlegungen der praktischen Gründe? ${ }^{712}$ Worauf sich dieser Verpflichtungscharakter zurückführen lässt, ist bis heute nicht unumstritten. Eine Erklärungsvariante versucht ihn mit einer speziellen Art »vernünftiger Rechtfertigung« zu begründen, eine andere führt ihn zurück auf eine »soziale Sanktioniertheit der Normen «. ${ }^{713}$

706 Vgl. Lind (2015), S. 25; Curcio (2008), S. 80.

707 Beobachtungen zu den verschiedenen Funktionen von »justice« und »beneficence» finden sich bei Smith (1976).

708 Vgl. Steinfath (2002), S. 77.

709 Vgl. Wolf (2013), S. 35; Stemmer (2002), S. 37.

710 Vgl. Steinfath (2002), S. 75.

711 Seebaß (2002), S. 180.

712 Vgl. Brandhorst (2013), S. 167.

713 Wolf (2013), S. 35. Als berühmter Vertreter der rein interessenorientierten und sanktionsbewehrten Normbefolgung lässt sich Peter Stemmer anführen, vgl. exemplarisch Stemmer (2008). Eine rein vernunftorientierte Moralauffassung vertritt Immanuel Kant und in seiner Folge Jürgen Habermas und Rainer Forst, vgl. dazu Kant (1924); Habermas (1992); Forst (2010). 
Die Moral selbst lässt sich dabei in (mindestens) drei Dimensionen untergliedern: Form, Inhalt und Motivation (Grund) ${ }^{714}$ Legt man das Prinzip der Normativität $\mathrm{zu}$ Grunde, besteht die Moral in Normen ${ }^{715}$, die einen vorrangigen Grund für eine Handlung ${ }^{716}$ liefern. Für ein Individuum bedeutet dies, dass die von einer Norm ausgehende Handlungserwartung befolgt werden sollte oder deren Befolgung gar verpflichtend ist. In diesem Kontext muss verstanden werden, worin der Sinn dieses Verpflichtetseins, dieses "praktischen Müssens « ${ }^{717}$, begründet ist und aus welchem Grund ein Individuum möglicherweise entgegen seiner eigenen Interessen handeln soll. ${ }^{718}$

In traditionellen Sinne ist die Moral häufig religiös begründet. Dann sind Normen von einer höheren, supranaturalen Instanz - und damit Autorität erlassen worden und begründen ihren Verpflichtungscharakter darin. Oft hat man die Vorstellung, der Pflichtbegriff sei ein Relikt einer theonomen Auffassung der Moral. ${ }^{719}$ Die Sorge vor einer Bestrafung und sich damit einhergehend negativen Konsequenzen ausgesetzt zu sehen, mag eine Erklärung für den Sinn des Verpflichtetseins liefern. »Verpflichtend ist ein Müssen, dessen Konstitution durch Sanktionen im Interesse der Betroffenen liegt, das heißt: für jeden der Betroffenen vernünftig ist. ${ }^{720}$ Verständlich ist jedoch auch, dass in aufgeklärten Zeiten, in denen der Glaube an supranaturale Autoritäten schwindet bzw. gar nicht mehr vorhanden ist, moralischen Normen und Verpflichtungen ihre Rechtfertigungsgrundlage entzogen wird. Die Normautorität wird dadurch grundsätzlich in Frage gestellt. ${ }^{721}$

Fraglich ist, ob und wie sich die moralische Verpflichtung retten lässt. Dies ist dann der Fall, wenn sich in einer Person eine Besonderheit oder ein besonderer Aspekt findet oder es unabhängig von dieser Person eine Autorität gibt, die ermächtigt ist, Normen aufzustellen und ggf. zu sanktionieren. Die erste Möglichkeit wird von Kant vertreten, die zweite von der sozialen Ansicht moralischer Verpflichtung. Kant unternimmt dabei den Versuch - auch in einer inzwischen aufgeklärten Welt -, ein besonderes moralisches Sollen über die reine Vernunft

714 Vgl. Schmidt (2002), S. 123: Von der Frage nach dem Inhalt moralischer Normen wird die nach der Art ihrer Verbindlichkeit bzw. ihrer Normativität, d. h. die Frage danach, inwiefern es Gründe gibt, sich den fraglichen Normen gemäß zu verhalten (oder, stärker, warum man diesen Normen folgen soll bzw. muß) meist unterschieden." Hevorh. im Original.

715 Vgl. Steinfath (2002), S. 71: »[...] Moral [lässt] sich als ein Netz spezifischer Normen charakterisieren $[\ldots] . \lll$

716 Eine Handlung kann durch aktives Tun oder auch durch ein Unterlassen begründet werden.

717 Stemmer (2002), S. 38.

$718 \mathrm{Vgl}$. Wolf (2013), S. $37 \mathrm{ff}$.

719 Vgl. Stemmer (2002), S. 37; Wolf (2013), S. 39f.

720 Stemmer (2002), S. 63.

721 Vgl. Wolf (2013), S. $39 \mathrm{f}$. 
zu begründen. ${ }^{722}$ Dieser Versuch muss scheitern, zumal genauso wenig alle Individuen einer säkularisierten Welt an die reine Vernunft wie an eine christliche Moral einer supranaturalen Instanz glauben müssen. Ungleich schwerer wiegen die evolutionstheoretischen und medizinischen Erkenntnisse, die für einen "empirisch fundierten Vernunftbegriff « ${ }^{723}$ sprechen. Die Kritik an der kantischen Sichtweise trifft auch auf alle anderen Versuche zu, die moralische Normativität aus einem nicht-empirischen Vernunftbegriff herleiten zu wollen. Wenn sich das moralische Sollen nun weder aus der reinen Vernunft noch von einer anderen, höheren Autorität ableiten lässt, kann es nur noch eine Erklärung geben; insbesondere, wenn das Sollen durch eine Art Normautorität begründet werden soll und aus dieser moralische Normen und Sanktionen hervorgehen: Diese Normautorität kann dann nur durch die anderen Gesellschaftsmitglieder verkörpert werden, die ihrerseits reziprok Normautorität und Ursprung von Sanktionen sind. ${ }^{724}$

Gerade in Bezug auf die "soziale Sanktioniertheit der Normen«sollte berücksichtigt werden: Möchte man soziales Sollen sanktionistisch begründen, dürfen nicht nur externe Sanktionen ${ }^{725}$ berücksichtigt werden. Bereits der in der Antike entwickelte Gedanke, dass Gefühle von Schuld und Scham konstitutiv für die Moral seien, ist im Ansatz bereits von Bentham (1970) und auch TugendHAT (1984) sanktionistisch gesehen worden. ${ }^{726}$

\subsubsection{Die Bedeutung von Sanktionen für die Normgeltung}

Unter Sanktionen, so führt SEEBASs (2002) aus, versteht man

»Maßnahmen, die für die von ihnen Betroffenen positive oder negative Effekte haben und von einer Kontrollinstanz absichtlich eingeführt werden, um die Konformität ihres Verhaltens mit bestimmten Standards prospektiv sicherzustellen oder zu fördern. « $^{727}$

Bei Sanktionen denkt man gemeinhin an negative Folgen als Konsequenz aus einer verbotenen Handlung. Es sind daher

"[...] nach einer üblichen Auffassung alle von den Mitgliedern einer Gemeinschaft beeinflussbaren Reaktionsmuster, die den Bruch bestimmter Regeln unvorteilhaft machen und damit die Wahrscheinlichkeit regelkonformen Verhaltens erhöhen. ${ }^{728}$

722 Vgl. Buddeberg/Vesper (2013), S. 41; Schneewind (2014), S. $484 \mathrm{ff}$.

723 Wolf (2013), S. 41.

724 Vgl. Wolf (2013), S. $41 \mathrm{f}$.

725 Vgl. zu externen Sanktionen auch Abschnitt 2.5.2.3.

726 Vgl. Bentham (1970), S. 47; Bentham (1970), S. 105f.; Tugendhat (1984), S. 73 ff.; Tugendhat (1984), S. 133f.; Tugendhat (1984), S. $140 \mathrm{f}$.

727 Seebaß (2002), S. 169.

728 Grundherr (2013), S. 54. 
Eine Sanktion ist also ganz allgemein eine als negativ empfundene Konsequenz, die von einer mit der nötigen Macht ausgestatteten Instanz (meist) in der Absicht verhängt wird, das (unmoralische) Verhalten (zukünftig positiv) zu beeinflussen. $^{729}$

Nach Stemmer (2000) ist ein "System von Sanktionen« ein »System von künstlich geschaffenen Nachteilen, die unweigerlich den treffen, der in bestimmter Weise handelt. ${ }^{730}$ Bei diesem System von Sanktionen ist es von zentraler Bedeutung, interne und externe moralische Sanktionen getrennt zu betrachten und sie dabei zu unterscheiden. Unter dem Begriff »sozialer Druck" werden alle externen moralischen Sanktionen subsumiert: Anerkennung entziehen, Kooperation verweigern, sozialen Druck aufbauen, Verfehlungen öffentlich anprangern ${ }^{731}$, aus der Gemeinschaft (wenngleich auch nur symbolisch) ausschließen.

„Der Betroffene kann auf einen Normbruch mit Verwunderung, Unverständnis, Verstimmung, Vorwürfen, Drohungen und schlimmstenfalls mit Zwangsmitteln oder mit dem Abbruch der Beziehung reagieren. ${ }^{732}$

Zur Wirksamkeit einer externen Sanktion bedarf es anderer Personen, die sich situationsbedingt adäquat verhalten müssen. ${ }^{733}$

Nach Peter Stemmer lautet die abstrakte Theorie für ein sanktionskonstituiertes moralisches Müssen ${ }^{734}$ :

»Das moralische Müssen [...] ist ein durch Sanktionen künstlich geschaffenes Müssen. ${ }^{735}$

Wenngleich dieser Satz sicherlich Interpretationsspielraum lässt, so lässt er doch zumindest folgende Minimalthese zu: Wenn jemand ausgehend von einer moralischen Betrachtung $\mathrm{X}$ tun soll, dann gibt es eine Sanktion, die es vorteilhaft erscheinen lässt, $\mathrm{X}$ auch zu tun. Diese Aussage kann kaum ohne Kritik bleiben. Demnach wäre jedes Handeln, das nicht zu einer Sanktion führen würde, moralisch nicht verwerflich. Diese Interpretation widerspricht jedoch erheblich der Erwartung, die man gemeinhin an eine moralische Regel stellt. STEMmer (2008) argumentiert daher, dass moralisches Handeln nicht ausschließlich auf Vermeidung von Sanktionen zurückführbar sein muss. Es kann durchaus auch andere Motive dafür geben:

729 Vgl. Anwander (2013), S. 252; Seebaß (2002), S. 169.

730 Stemmer (2000), S. 100.

731 Vgl. Bachmann (2006), S. 76.

732 Raiser (2013), S. $174 \mathrm{f}$.

733 Vgl. Grundherr (2013), S. 54; Leist (2000), S. 148; Stemmer (2002), S. 40.

734 Vgl. dazu auch Schmidt (2002), S. 145.

735 Stemmer (2000), S. 118. Vgl. dazu auch Stemmer (2002), S. 40. 
»Und man kann auch moralisch handeln, weil es die Norm so vorschreibt, ohne dass die moralischen Sanktionen dabei eine Rolle spielen. So wenn man moralisch handelt, weil man es für selbstverständlich hält, die moralischen Normen zu befolgen, oder weil man die Normen gut und vernünftig findet. In diesen Fällen spielt die Norm, aber nicht die Sanktion bei der Handlungswahl eine Rolle. Der eigentliche Kern der Norm, der Sanktionsmechanismus, kommt gar nicht ins Spiel. ${ }^{736}$

Diese anderen Motive treten insbesondere dann ein, wenn Menschen »begünstigende Vorabeinstellungen der Norm gegenüber mitbringen. ${ }^{737}$ Warum wir letztlich im Alltag eine Entscheidung treffen, hängt viel häufiger von Gewohnheiten (Sozialisation) und unbewussten Faktoren ab. ${ }^{738}$ Ein »aufgeklärter und reflektierter Akteur ${ }^{739}$, der moralische Normen auf Grund von moralischen Normen befolgt, wird dies nach STEMMER (2008) immer tun, um "moralischen Sanktionen zu entgehen. ${ }^{740}$ In Fällen, in denen »begünstigende Voreinstellungen« fehlen, müsse der »eigentliche Kern der Norm, der Sanktionsmechanismus» greifen. ${ }^{741}$

Eine Gegenposition zum sanktionistischen Ansatz findet sich bei HABERMAS (1992): "Sanktionen (wie sehr sie auch verinnerlicht sein mögen) sind für die Sollgeltung nicht konstitutiv, sie sind vielmehr symptomatisch für eine bereits wahrgenommene, also vorgängige Verletzung eines normativ geregelten Lebenszusammenhangs. ${ }^{742}$ Moralische Akteure sind auch ohne Verweis auf eventuelle Sanktionen stets bestrebt, moralische Regeln $\mathrm{zu}$ befolgen. Forst (2010) bringt es auf den Punkt, wenn er sagt: „Sanktionen konstituieren nicht die Geltung von Normen, sie setzen sie voraus. ${ }^{743}$ Vor diesem Hintergrund darf man ein »man soll« im Sinn von »es gibt eine gute begründete Norm, die X zu tun vorschreibt « verstehen. Forst (2010) begründet es so: "Denn sie [die Norm] >ist « ja durch ihre Geltung, und ob sie gerechtfertigt ist oder nicht, betrifft diese Geltung. ${ }^{744}$ Aus der Begründung der Norm wird also ihre Geltung abgeleitet und daraus wiederum das "Sein " der Norm, welche das moralische Sollen bestimmt. Die jeweilige Moraltheorie legt dabei fest, was unter einer guten Begründung verstanden wird. ${ }^{75} \mathrm{Ob}$ eine vernünftige Einsicht auch zu moralischem Handeln führt, ist jedoch fraglich. Schon Kant hatte Zweifel an dieser motivierenden Kraft:

736 Stemmer (2008), S. 321.

737 Stemmer (2008), S. 321.

738 Vgl. Grundherr (2013), S. 57.

739 Grundherr (2013), S. 57.

740 Stemmer (2008), S. 306.

741 Stemmer (2008), S. 321.

742 Habermas (1992), S. 144. Hervorh. im Original.

743 Forst (2010), S. 159.

744 Forst (2010), S. 160.

745 Vgl. Grundherr (2013), S. 58. 
»Urteilen kann der Verstand freilich, aber diesem Verstandesurteil eine Kraft zu geben, und daß es eine Triebfeder werde den Willen zu bewegen, die Handlung auszuüben, das ist der Stein der Weisen. ${ }^{746}$

Habermas (1992) geht vielmehr davon aus, dass wir als rationale Wesen gar nicht umhinkommen, die Kraft, die von einem besseren Grund auszugehen vermag, anzuerkennen und entsprechend danach $\mathrm{zu}$ handeln. Allerdings, so räumt er ein, handelt es sich dabei lediglich um eine "schwach motivierende Kraft « und somit nicht um eine "unmittelbar handlungsregulierende Kraft auBerhalb der Argumentationssituation ${ }^{7}{ }^{747}$

So muss Habermas einräumen, dass auch für ihn die motivierende Kraft des moralischen Sollens alleine nicht ausreicht, um sich gegen das Interesse am persönlichen Wohlergehen zu behaupten. ${ }^{748}$

Um den Konflikt zwischen rechtfertigungsbasierten und sanktionistischen Ansätzen des moralischen Sollens aufzulösen, bedarf es zweier Unterscheidungen: »[E]rstens, die Unterscheidung zwischen rationaler Notwendigkeit und motivationaler Nötigung, zweitens, die Unterscheidung zwischen einer internen und einer externen Perspektive auf die moralische Praxis. ${ }^{749}$

Bei Kant findet sich die Begrifflichkeit des "moralischen Sollens" nicht. Vielmehr spricht er von Verbindlichkeit. Der Verdacht liegt jedoch nahe, dass er damit dasselbe Phänomen zu beschreiben sucht. ${ }^{750}$ In der Erläuterung des kantischen Begriffs der Verbindlichkeit stecken wertvolle Hinweise für das moralische Sollen: »Verbindlichkeit ist die Notwendigkeit einer freien Handlung unter einem kategorischen Imperativ der Vernunft. ${ }^{751}$ Kant führt weiter aus, dass »Verbindlichkeit nicht bloß praktische Notwendigkeit [...], sondern auch Nötigung enthält $[\ldots] \ll^{752}$ - also einen motivationalen Zwang zur entsprechenden Handlung. Kant stellt somit direkt eine Verbindung zwischen beiden Elementen her. Daraus lässt sich ableiten, dass uns das, was wir als rational erforderlich einsehen, als "Vernunftwesen auch nötigt ${ }^{753}$. Wenn wir doch anders handeln, liegt es daran, dass wir eben keine reinen Vernunftwesen, sondern auch unseren persönlichen Neigungen unterworfen sind. Somit kann man zum Schluss kommen, dass Kant selbst darauf hinweist, dass das "moralische Sollen zwei voneinander trennbare Aspekte aufweist. ${ }^{754}$

746 Kant (1924), S. 54.

747 Habermas (1992), S. 135.

748 Vgl. ausführlich dazu Grundherr (2003), S. $64 \mathrm{ff}$.

749 Grundherr (2013), S. 59f.

750 Vgl. Grundherr (2013), S. 60.

751 Ludwig/Stark (1988), S. 85.

752 Kant/Weischedel (1786), S. 329.

753 Grundherr (2013), S. 60.

754 Grundherr (2013), S. 60. 


\subsubsection{Soziale Sanktioniertheit und moralische Verpflichtung}

Basierend auf dem Begründungsversuch, dass das moralische Sollen auf einer "psychosozialen Verankerung der Normen ${ }^{755}$ fußt, folgt daraus unmittelbar, dass jedweder Verstoß besondere Sanktionen und ein gewisses Maß an Empörung ob der Zuwiderhandlung hervorrufen muss. Es kann auch zu eigenen Schuldgefühlen und möglicherweise auch zu Selbstvorwürfen führen, sofern man selbst einen Verstoß begangen hat. ${ }^{756}$ Stellt man sich die Frage, was man eigentlich unter einer moralisch relevanten Situation versteht, kommen empirische Untersuchungen zum Ergebnis, dass es Personen dabei vornehmlich um das Wohl anderer geht, d.h. (bewusste) Verletzungen jeder Art zu vermeiden und insbesondere die Rechte anderer zu achten. ${ }^{757}$ Aber auch der psychosozialen Sanktioniertheit moralischer Normen wird Kritik entgegengebracht. Beispielsweise wird der Einwand erhoben, dass an solchen Stellen, wo die Gefahr, einen moralischen Fehltritt zu entdecken tendenziell eher gering ist, die soziale Sanktion fehlt. Darauf wird häufig mit der Internalisierung der Sanktion geantwortet, also eine Form intrapersoneller Sanktionierung in Form von Schuldgefühlen oder schlechtem Gewissen. Aber auch diese Begründung ist in sich nicht gänzlich schlüssig. Dieser Erklärungsversuch zeigt dann Schwächen, wenn »zeitlich weit entfernte Objekte der Moral« betroffen sind: Beispielsweise können Mitglieder kommender Generationen aus grundsätzlichen Erwägungen heraus nicht Ausgangspunkt von Sanktionen sein. Wenn die Frage der moralischen Motivation nicht geklärt werden kann, kann auch die Art der moralischen Verpflichtung nicht verstanden werden. ${ }^{758}$

Für Aristoteles steht eine moralische Handlung gleichsam wie jede andere Handlung immer im Zusammenhang mit dem (eigenen guten) Leben als Ganzes. Moralisch $\mathrm{zu}$ handeln bedeutet in diesem Kontext eine Einschränkung des eigenen guten Lebens aus Rücksichtnahme auf das Wohl anderer. Fraglich ist allerdings, ob ein System interner Sanktionen erfolgreich sein kann und moralische Prinzipien zu verankern vermag. Kann beispielsweise eine Person ihr Ziel lediglich unter Verletzung einer moralischen Norm erreichen, wird diese sich genau überlegen, was für sie schwerer wiegt: das Schuldgefühl, das im Zweifel nur für kurze Dauer anhält, oder die Aufgabe eines persönlichen Ziels. ${ }^{759}$

Bei Aristoteles findet sich ein weitaus sinnvolleres Bild der motivationalen Grundlage der Moral:

755 Wolf (2013), S. 42.

756 Vgl. Curcio (2008), S. 80; Wolf (2013), S. 42.

757 Vgl. Nado/Kelly/Stich (2009), S. 621 ff.; auch darauf verweisend Wolf (2013), S. 43.

758 Vgl. Wolf (2013), S. 43.

759 Vgl. Seebaß (2002), S. $177 \mathrm{f}$. 
»Was durch moralische Sozialisation erzeugt wird, sind primär Charakterdispositionen, bei guter Sozialisation Tugenden, die sicherstellen, dass Menschen durchgängig nach den Normen handeln, weil sie geeignete positive Handlungsmotive, die Ausrichtung auf ein Gut, internalisiert und in ihr Selbstbild aufgenommen haben. Das Schuldgefühl wäre dann nicht etwas Eigenes, was als Sanktion mit der Norm eingeführt wird, sondern es wäre die Kehrseite einer positiven Einstellung, deren Durchbrechung eine negative Selbstbewertung auslöst. Dass die moralische Sanktion so funktioniert, im Kontext von positiven Motiven, die in Tugenden internalisiert sind, und nicht als bloße negative Sanktion beim Übertreten einer Norm, scheint auch psychologisch verständlicher. ${ }^{760}$

Wenn von "positiven Motiven « die Rede ist, scheint dies primär »altruistische Gefühle ${ }^{761} \mathrm{zu}$ betreffen, wie man sie in verwandtschaftlichen und freundschaftlichen Beziehungen vorfindet, die meist zu mutuellen Erwartungen führen. Wird in einer solchen Beziehung von einer Bitte oder eine Aufforderung eines anderen abgewichen, kann der Abweichende sicherlich ein Schuldgefühl entwickeln, dass er eine (berechtigte) Erwartung nicht erfüllt hat. Bei dieser Reaktion wird man aber kaum von einer Sanktion auf Grund eines sozialen Drucks sprechen. Es ist eher die (logische) Konsequenz aus einer für die andere Person enttäuschenden Handlung im Rahmen eines bestehenden (verwandtschaftlichen oder freundschaftlichen) Beziehungsverhältnisses zwischen zwei Menschen. ${ }^{762}$

\subsubsection{Moral: Interesse rechtfertigt, Sanktion motiviert}

Geht man einen Schritt weiter, könnte man die These aufstellen, dass sich »moralisches Verhalten letztlich nur durch Interessen rechtfertigen und durch Sanktionen motivieren lässt. ${ }^{763}$

Die Unterscheidung der Rechtfertigung und Motivation ist eine grundsätzliche Überlegung, die angestellt werden muss, um eine zentrale Frage zu klären: Lässt sich Normativität - und damit auch Moral - ausschließlich mit der Zwangswirkung von Sanktionen begründen, die die Betroffenen aus persönlichem Interesse vermeiden wollen, oder lässt sich moralisches Handels auch anders herleiten ? $^{764}$ Handlungsgründe müssen dabei erklärt $u$ nd gerechtfertigt werden können, d.h. warum eine Person so gehandelt hat, und dass es auch rational war, so $\mathrm{zu}$ handeln, wie sie gehandelt hat; anders ausgedrückt: Ihr Handeln entsprach dem, wie sie hätte handeln sollen. ${ }^{765}$

An die Handlungsgründe werden zwei wesentliche Anforderungen gestellt:

760 Wolf (2013), S. 44.

761 Wolf (2013), S. 44.

762 Vgl. Seebaß (2002), S. 188; Wolf (2013), S. 44 f.

763 Gosepath (2013), S. 125.

764 Vgl. Gosepath (1999).

765 Vgl. Gosepath (2013), S. 125. 
»(1) eine motivationale Bedingung: eine (moralische) Überlegung oder Forderung, die beansprucht ein Grund zu sein, muss die betreffende Person motivieren können, und (2) eine normative Bedingung: Handlungsgründe müssen normativ sein oder rationale und nicht bloß eine kausale Notwendigkeit besitzen, das heißt, dass sie eine Handlung nicht nur kausal oder motivational erklären können müssen, sondern aus der ErstePerson-Perspektive auch als gute, rechtfertigende Gründe anerkannt werden müssen, sodass eine Handlung wider diese Gründe als irrational gelten würde. ${ }^{766}$

Hinsichtlich der Beziehung zwischen Rechtfertigung und Motivation herrscht keine Einigkeit. Die wunsch- bzw. sanktionsbasierte Theorie ist sicherlich die vorrangige. ${ }^{767}$ Auch hier muss die Frage gestellt werden: Welcher Zusammenhang, so es denn überhaupt einen gibt, besteht zwischen Normen und Sanktionen? Welche Bedeutung kann Zwang in einem durch Normen geleiteten Handeln haben? Sicherlich findet sich eine schnelle und einfache Antwort, wenn man sich vor Augen führt, was passiert, wenn eine Person sich nicht normkonform verhält. Bei allen Typen von Normen sind Sanktionen ${ }^{768}$, also negative Konsequenzen, maßgeblich beteiligt. Gemeinsam ist allen Typen, dass die Norm befolgt werden muss, sofern man Sanktionen vermeiden möchte. Die Befolgung der Norm kann aus rationalen Erwägungen oder auf Basis von Wünschen des Normadressaten heraus erklärt werden: Möchte man Sanktionen vermeiden, muss man entsprechend der Norm handeln; ein abweichendes Handeln wäre (rational betrachtet) dann unvernünftig und würde den eigenen Wünschen entgegenstehen. Demnach ist ein moralisches Zusammenleben lediglich auf der Grundlage von gemeinsam verbindlichen, also "vertragsanalog beschlossenen Sanktionen ${ }^{769}$ möglich.

Gegen das »Rechtfertigungsinstrument ${ }^{770}$ des interessenbasierten Kontraktualismus wird nicht selten zu Recht opponiert: Wunschbasierte Gründe als Rechtfertigung kann die reale Rechtfertigungspraxis nicht adäquat beschreiben. ${ }^{771}$ Das unter dem Begriff »belief-desire ${ }^{772}$ bekannte Modell hat eine nicht ausreichende Vorstellung vom Willen. Jedes Wollen einer Person basiert auf bestimmten Gründen. Die konkrete Handlung in einer bestimmten Situation fußt auf der Überlegung, was man sich in dieser Situation wünschen soll, weil es

766 Gosepath (2013), S. 126.

767 Das entscheidende Argument für die wunschbasierte Auffassung, so Gosepath, sei, dass, wenn Motivation vorhanden ist, auch ein Wunsch vorhanden sein muss; vgl. Gosepath (2013), S. 126 Fn. 2. Vgl. dazu auch Smith (1987).

768 Vgl. Gosepath (2013), S. 127:»Bei Verboten ist es Strafe oder sozialer Druck, bei Spielregeln der Ausschluss von der gemeinsamen Praxis, bei Klugheitsregeln das Nichterfüllen der Wünsche."

769 Gosepath (2013), S. 127.

770 Grundherr (2007), S. 193.

771 Vgl. Quinn (2003), S. $228 \mathrm{ff}$.

772 Ausführlich dazu Döring/Mayer (2002), S. $89 \mathrm{ff}$. 
für einen das Beste zu tun ist. Man darf dabei jedoch nicht vergessen, dass die Wünsche selbst auch begründet sein können. Die Theorie, dass das Handeln auf Grund einer Norm ausschließlich auf persönliche Interessen und damit einhergehend auch auf die Vermeidung von Sanktionen zurückzuführen ist, ist zu kurz gedacht, kann sie dabei dem - unter anderen insbesondere von Kant betonten - Unterschied zwischen rein normenkonformen und normengeleiteten, also tatsächlich durch Normen begründeten, Handeln nicht gerecht werden. Kant differenziert begrifflich und unterscheidet dabei zwei Aspekte des Sollens: das Sollen als rationale Folge eines "Begründungs- und Einsichtsprozess (rationale Notwendigkeit ${ }^{773}$ )« und das Sollen als «motivationale[r] Zwang ${ }^{774}$ (moralpsychologische Nötigung). « ${ }^{775}$

Der interessenbasierte Ansatz muss per se ablehnen, dass es überhaupt ein echtes, also ein "genuin normgeleitetes Handeln ${ }^{776}$ gibt oder geben kann. Es widerspricht aber dem gesunden Menschenverstand, dass wir als normative Wesen nicht (zumindest hin und wieder) unabhängig vom eigenen Vorteil und persönlichen Interessen in bestimmten Situationen so handeln, wie es in dieser Situation richtig ist. ${ }^{777}$

Um Handeln auf Basis von Normen definieren zu können, bedarf es der Untersuchung, wie Normen in Verbindung mit Sanktionsbewehrtheit begründet sind. Gosepath (2013) führt dazu aus:

»Es muss ein überzeugender, anerkennbarer Grund für das `Sollen` ihrer Befolgung angegeben werden können. Das Rätsel der Normativität ist also nicht, wie Normen unser Handeln beeinflussen, sondern ob diese Beeinflussung, über eine bloße kausale, etwa durch Zwang verbürgte Wirksamkeit hinaus, auch vernünftig oder begründet ist. ${ }^{778}$

\subsubsection{Moralische Pflicht und praktische Gründe}

Wie bereits erwähnt, gehen moralische Pflichten (also Formen eines moralischen Müssens ${ }^{779}$ ) einher mit praktischen Gründen, die eine Antwort darauf geben, ob ein bestimmtes Handeln zu tun oder zu unterlassen ist. Die Vermutung liegt also nahe, dass es einen notwendigen Zusammenhang zwischen moralischen Pflichten und praktischen Gründen geben muss. BRANDHORST (2013) nennt diesen

773 Vgl. dazu auch Busch (1979), S. 14.

774 Vgl. dazu auch Ihne (2010), S. 126.

775 Grundherr (2013), S. 53.

776 Gosepath (2013), S. 128.

777 Vgl. Gosepath (2013), S. 130.

778 Gosepath (2013), S. 130; Hervorh. im Original.

779 Vgl. Brandhorst (2013), S. 171. 
Zusammenhang den ${ }^{2}$ moralischen Rationalismus ${ }^{780}$. SHAFER-LANDAU (2009) führt dazu näher aus:

»Realism is sometimes contrasted with constructivism by invoking the claim that, for realists, moralty is mind-independent. [...] The way I would prefer to characterize the realist position is by reference to its endorsement of the stance-independece or moral reality. Realists believe that there are moral truths that obtain independently of any preferred perspective, in the sense that the moral standards that fix the moral facts are not made true by virtue of their ratification from within any given actual or hypothetical persepctive. That a person takes a particular attitude toward a putative moral standard is not what makes that standard correct. ${ }^{781}$

Für den moralischen Rationalisten ist dabei gleichgültig, wer diese Pflichten und Gründe hat und woher diese stammen. Die These besagt lediglich, dass diese miteinander einhergehen (müssen). ${ }^{782}$ Zwei Argumente sprechen für den moralischen Rationalismus: Das erste Argument spielt auf das Zusammenspiel von moralischer Pflicht und praktischen Gründen an und fragt, was eine moralische Pflicht wäre, wenn es keine moralischen Gründe gäbe, dieser Pflicht zu entsprechen. Ohne entsprechende Gründe wäre die Pflicht lediglich ein rein äußerlicher Zwang, der kaum unser eigenes Wollen zu erreichen vermag und es an einer vernünftigen Einsicht mangeln ließe. Sanktionen, oder bereits die Furcht vor dem Eintreten von persönlichen Nachteilen, können selbstredend als Grund dienen. Dieser Grund wird einem dann meist von anderen auferlegt. Moralische Pflichten sind gefühlt, aber keineswegs nur, äußerlich. Wir erleben moralische Pflichten viel intensiver, oft von innen heraus. Sie sind damit im empathischen Sinne verankert in unserem Wollen und unserer Einsicht und damit per se mit Gründen verbunden. Dem moralischen Rationalisten geht es aber nicht primär nur darum, dass die moralische Pflicht mit praktischen Gründen verknüpft ist (welche durchaus auch sanktionsbasiert sein können), sondern diese moralische Pflicht muss einhergehen mit ebenso moralischen Gründen. Das zweite Argument bezieht sich auf die moralische Praxis und stellt die Frage, inwieweit Sanktionen auf ein Fehlverhalten hin zu rechtfertigen sind, wenn der zu Sanktionierende keine moralischen Gründe hatte, entsprechend anders zu handeln. Wenn jemand beispielsweise moralisch verpflichtet ist, ein gegebenes Versprechen einzuhalten und er kommt diesem Versprechen nicht nach, wird man auf dieses Verhalten zumeist mit Missbilligung und (mehr oder minder deutlichen) Vorwürfen reagieren. Die moralische Praxis lässt dabei erwarten, dass ein Vergehen (also in diesem konkreten Verfall der Bruch eines Versprechens, das hätte

780 Brandhorst (2013), S. 167. Hervorh. im Original. Dieser Ausdruck findet in der einschlägigen Literatur Verwendung; vgl. dazu nur Shafer-Landau (2009), S. $13 \mathrm{ff}$.

781 Shafer-Landau (2009), S. 15. Hervorh. im Original.

782 Vgl. Brandhorst (2013), S. 167. 
eingehalten werden können und sollen) geahndet wird. Ein Verständnis dieses Zusammenhanges ist für den moralischen Rationalismus essenziell. BRANDHORST (2013) führt dazu aus:

»Der Zusammenhang zwischen moralischer Pflicht und Sanktion ist insofern sentlich`, als es zu unserer Praxis moralischer Pflicht gehört, Verletzungen der Pflicht zu ahnden. Der Zusammenhang von Pflicht und Sanktion ist aber nicht in einem strengen Sinn notwendig. Es kann stets vorkommen, dass eine Sanktion nicht verhängt wird, zum Beispiel, weil es gute Gründe gibt, die dagegen sprechen [...]. Wenn aber moralische Pflichten, wie wir sie kennen, im Kontext des menschlichen Lebens, wie wir es kennen, grundsätzlich nicht mehr sanktioniert werden würden, wären wir mehr als geneigt zu bezweifeln, dass es sich wirklich noch um moralische Pflichten handelt. Das zeigt, in welcher Weise der Begriff der moralischen Pflicht mit dem der Sanktion verknüpft ist. ${ }^{783}$

Würde bei Pflichtverstößen kein Schuld- oder Schamgefühl mehr entstehen, niemand mehr pflichtwidriges Verhalten kritisieren oder »richtiges« von "falschem» Handeln unterscheiden, so wäre die moralische Pflicht obsolet. BRANDHORST (2013) bringt es auf den Punkt, wenn er sagt, »[m]oralische Pflichten sind begrifflich mit Handlungsmustern verbunden, und nur im Kontext solcher Handlungsmuster hat es überhaupt Sinn, von moralischen Pflichten zu sprechen. $\mathrm{Zu}$ diesen Handlungsmustern gehören verschiedene Reaktionen auf Fehlverhalten, und dazu zählen Sanktionen. ${ }^{784}$ Nach STRAwson (2008) gehören Reaktionen auf moralische Verstöße zu einer Form des menschlichen Lebens und daher braucht es keine weitere, unabhängige Rechtfertigung. Eine solche sei ohnehin nicht möglich. $^{785}$

Pereboom (2001) stellt dagegen in Frage, dass wir überhaupt über die Art von »Willensfreiheit« verfügen, derer es bedarf, moralische Verantwortung zu übernehmen. Vor diesem Hintergrund sei jede Form von Sanktion - und dies würde auch auf Schuldzuweisungen und Vorwürfe zutreffen - unangemessen und damit unfair. Vielmehr sollten moralische Verfehlungen durch Ermahnung und Belehrung geahndet werden. ${ }^{786}$ Dieser Argumentation kann nur bedingt gefolgt werden, sind doch Ermahnungen und Belehrungen im weiteren Sinne durchaus auch als Form von Sanktionen zu verstehen.

783 Brandhorst (2013), S. 176; Hervorh. im Original. Vgl. dazu auch Wittgenstein (1989), § 143242.

784 Brandhorst (2013), S. 177.

785 Vgl. Strawson (2008), S. 1 ff., welcher in seinem Kapitel »Freedom and Resentment« weitere Ausführung dazu macht. Das Thema entwickelte er ausgehend von Strawson (1985), Kap. 2 und Strawson (1992), Kap. 10.

786 Vgl. Pereboom (2001); Willaschek (2013), S. 204. 


\subsubsection{Intersubjektive Sanktionen: Moralbegründung nach Adam Smith}

Adam Smith gilt heutzutage insbesondere als Ökonom; sein Werk "Wealth of Nations ${ }^{787}$ wird vielfach als Geburtsurkunde der modernen Ökonomie gesehen. ${ }^{788}$ Was dabei häufig übersehen wird, ist die Tatsache, dass Smith auch - oder vielleicht sogar allem voran - Moralphilosoph war. ${ }^{789}$ Mit seinem philosophischen Hauptwerk »Theory of Moral Sentiments (Theorie der ethischen Gefühle), das in sechs Auflagen erschien und somit seinen Stellenwert erahnen lässt, legte er den Grundstein für die Ausbildung einer Moralphilosophie, welche auf der Idee der "sympathy ${ }^{790}$ und der Solidargemeinschaft fußt. Smith vertritt dabei eine Theorie zur Begründung von Normen auf Grundlage eines nicht-dualistischen Ansatzes, bei dem Gründe und Sanktionen sehr eng verknüpft sind, ohne jedoch die Ebene der Gründe gänzlich aufzugeben. Um Smiths Theorie der Moralbegründung zu verstehen, bedarf es auch hier des Verständnisses einer Unterscheidung zwischen externen und internen Sanktionen. ${ }^{791}$ Die externen Sanktionen lassen sich dabei weiter unterscheiden nach formellen und informellen Sanktionen. Während formelle Sanktionen offizielle Institutionen verhängen (z. B. Geldzahlungen oder Gefängnisstrafe), werden informelle Sanktionen von Mitgliedern einer Gemeinschaft untereinander verhängt, beispielsweise durch Lob und Kritik oder Beendigung des Kontakts und damit möglicherweise einhergehend der Ausschluss aus einer Gemeinschaft. ${ }^{792}$ Eine Kombination aus informellen externen und internen Sanktionen ist für Smith für die Entstehung moralischer Normen Voraussetzung. Er geht dabei davon aus, dass informelle Sanktionen zur Bewahrung moralischer Normen ausreichend sind - zumindest innerhalb kleinerer sozialer Gemeinschaften. Für ein Verständnis der Umsetzung solcher Sanktionen bedarf es der Berücksichtigung eines wesentlichen Elements seiner Theorie: Die soziale Konstituiertheit des Menschen. ${ }^{793}$ GrIswOLD (1999) führt dazu aus, dass wir für Smith überhaupt kein moralisches Ich außerhalb einer Gemeinschaft haben und haben können: ${ }^{794}$

787 Vgl. Smith (1976). Der volle Titel des 1776 erstmals erschienenen Werkes lautet »An Inquiry into the Nature and Causes of the Wealth of Nations".

788 Vgl. Dumont (1991), S. 121.

789 Ross (2010), Kap. 23.

790 Adam Smith verwendet den Begriff »sympathy«, welcher sich nur schwer durch einen einzelnen Begriff ins Deutsche übersetzen lässt und insbesondere nicht durch den Begriff "Sympathie» treffend umschrieben wäre. Bei Smith muss "sympathy» als Mitfühlen mit jeglichen Leidenschaften anderer (»our fellow-feeling with any passion whatever«) verstanden werden; vgl. Smith (1790), S. $26 \mathrm{ff}$; Smith (2011), S. 9. Vgl. dazu auch Herzog (2013), S. 210 Rn. 5.

791 Vgl. Abschnitt 2.5.2.3.

792 Vgl. Raiser (2013), S. $171 \mathrm{f}$.

793 Vgl. Herzog (2013), S. $211 \mathrm{f}$.

794 Vgl. Griswold (1999), S. 105. 
»It is this that man, who can subsist only in society, was fitted by nature to that situation for which he was made. All the members of human society stand in need of each others assistance, and are likewise exposed to mutual injuries. Where the necessary assistance is reciprocally afforded from love, from gratitude, from friendship, and esteem, the society flourishes and is happy. All the different members of it are bound together by the agreeable bands of love and affection, and are, as it were, drawn to one common centre of mutual good offices. ${ }^{795}$

Hier kommt der Begriff "sympathy« als eine Art unsichtbares Band zwischen Menschen zum Tragen. Smith selbst definiert diesen Begriff als »our fellowfeeling with any passion whatever. ${ }^{796} \mathrm{Ihm}$ geht es dabei vornehmlich darum, dass Menschen - oft auch ganz spontan - Gefühle teilen können. Er meint damit aber weniger die Wahrnehmung der Gefühle des Gegenübers, sondern vielmehr die Wahrnehmung der Situation als solche, in der sich die Personen befinden. ${ }^{797}$ Durch »sympathy« entsteht bereits vor jeder Kommunikation eine Art der Verbundenheit zwischen Menschen. Im empathischen Sinne geht es nicht um eine "Ansteckung « durch Gefühle, sondern um die Vorstellungskraft (»imagination «, "fancy ${ }^{798}$, sich in die Situation des anderen hineinzuversetzen. Dadurch ist auch die Möglichkeit geboten, sich kritisch in Bezug auf das Verhalten des anderen zu distanzieren. ${ }^{799}$ HaAkonssen (1981) betont, dass im Sinne des "sympathy«-Begriffs bei Smith die Möglichkeit besteht, sich selbst zu fragen, wie man in der einen oder anderen Situation reagiert hätte. Seine Moraltheorie könne damit an dieser kritischen Lücke ansetzen. ${ }^{800}$ Smith geht noch einen Schritt weiter und stellt die These auf, dass Menschen instinktiv die "sympathy" ihrer Mitmenschen suchen, weil sie schlichtweg angenehm sei: »[...] nothing pleases us more than to observe in other men a fellow-feeling with all the emotions of our own breast; nor are we ever so much shocked as by the appearance of the contrary. $\varkappa^{801}$ Daraus ergibt sich, dass Menschen ein grundsätzliches Interesse daran haben, wie es ihren Mitmenschen geht, und an der Beibehaltung der Gesellschaft, in welcher dies als wichtig empfunden wird. ${ }^{802}$ Wie jemand ein anderes Individuum sieht, ist stets mit Wertungen verbunden. In Bezug auf dessen Verhalten mit Billigung oder Missbilligung. ${ }^{803}$

795 Smith (2011), S. 82.

796 Smith (2011), S. 9.

797 Vgl. Smith (2011), S. $11 \mathrm{f}$.

798 Vgl. Smith (2011), S. 8: «By the imagination we place ourselves in his situation, we conceive ourselves enduring all the same torments, [...].«»That this is the source of our fellow-feeling for the misery of others, that it is by changing places in fancy with the sufferer, that [...]."

799 Vgl. Herzog (2013), S. 213f.

800 Vgl. Haakonssen (1981), S. 46; Herzog (2013), S. 214.

801 Smith (2011), S. 13.

802 Vgl. Herzog (2013), S. 214.

803 Vgl. Smith (2011), S. 102 f. 
Indem »sympathy« zwangsläufig Gefühle zwischen Menschen überträgt, entstehen mutuelle Erwartungen und Ansprüche zwischen diesen. Dadurch stellt man sich zwangsläufig die Frage, wie man in einer Situation selbst reagieren mag, in die man sich durch »sympathy« hineinversetzt fühlt: Würde man selbst ähnlich oder gänzlich anders reagieren? Teilt man die Gefühle des anderen? Smith sieht darin einen ersten Maßstab für die Bewertung des Verhaltens anderer: ${ }^{804}$

"[I]f we consider all the different passions of human nature, we shall find that they are regarded as decent or indecent, just in proportion as mankind are more or less disposed to sympathize with them. ${ }^{805}$

Das Verhalten kann demgemäß entweder als angemessen (»decent«) oder unangemessen (»indecent «) empfunden werden. Da das Individuum in der Lage ist, wahrzunehmen, wie andere Menschen auf seine eigenen Gefühle und sein Verhalten reagieren, wird es diese zu steuern suchen, um die "sympathy" seines Gegenübers zu empfangen. Auf diesem Mechanismus beruht für Smith auch die Einhaltung moralischer Regeln. Werden diese Regeln verletzt, wird einer Person "sympathy« entzogen. Dieser Entzug wird grundsätzlich als unangenehm empfunden und so wird man dies immer zu vermeiden suchen. Dies mag man als eine sanfte Sanktion verstehen; für Smith repräsentiert sie aber genau das unsichtbare Band, das Gemeinschaften verbindet und verbunden hält. ${ }^{806}$ Nach Smith ist es das größte Glück eines Menschen, geliebt zu werden: "What so great happiness as to be beloved, and to know that we deserve to be beloved? What so great misery as to be hated, and to know that we deserve to be hated? ${ }^{807}$ Da man Glück nicht ohne Einhaltung moralischer Normen erfährt, ist ein besonderer Anreiz zur Befolgung gegeben. $^{808}$

\subsubsection{Die Forderungstheorie als weiterer Erklärungsversuch moralischer Verpflichtungen}

AnWANDER (2013) regt an, eine Art Forderungstheorie aufzustellen, um moralische Verpflichtungen besser verstehen zu können. Die Kernidee besteht darin, Verpflichtungen auf Forderungen zurückzuführen. Damit geht es nicht mehr nur explizit um Gründe, die ein Akteur dafür hat, eine Handlung auszuführen. Vielmehr wird der Blick auf die Verhaltenserwartungen anderer gelenkt, die diese an ihn haben.

804 Vgl. Herzog (2013), S. 215.

805 Smith (2011), S. 26.

806 Vgl. Herzog (2013), S. 215.

807 Smith (2011), S. 104.

808 Vgl. Herzog (2013), S. 216. 
Aus soziologischer Sichtweise nimmt jede Erwartung den Charakter einer Forderung ein, sobald derjenige, der etwas erwartet, ein persönliches Interesse an der erwarteten Handlung seines Gegenübers hat. ${ }^{809}$

Moralisches Sollen hängt somit nicht alleine mit den Gründen des Handelnden zusammen. Diese Forderungen sollen als "normativer Anspruch» - gleichgültig, ob bewusst artikuliert oder stillschweigend erwartet - darauf verstanden werden, dass eine Person in einer bestimmten, erwarteten Weise handelt. Es liegt in der Natur der Sache, dass nicht Erwartungen, die irgendjemand hegt, oder Forderungen, die irgendwer stellt, Ausgangspunkt für eine Verpflichtung sein können. ${ }^{810}$ Gerade wenn wir Moral als ein System von Forderungen an einzelne Individuen begreifen, werden diejenigen, von denen etwas gefordert wird, die Frage stellen, "mit welchem Recht dies geschieht und warum sie dem Geforderten genügen sollen. ${ }^{811}$ Vielmehr müssen diese Ansprüche dann auch legitim sein, was bedeutet, dass nur Forderungen von einer Person erhoben werden können, welche die Autorität dazu hat oder welcher man die Autorität - aus welchem Grund auch immer - in einer Gemeinschaft zubilligt. Verpflichtungen allgemein fußen auf autoritativen Forderungen. Eine moralische Verpflichtung geht hingegen zurück auf eine distinkte moralische Autorität. ${ }^{812}$ Es gibt verschiedene Optionen, wer diese moralische Autorität verkörpert. Im traditionalistischen Sinne ist sicherlich Gott, oder irgendeine andere supranationale Instanz, eine Option. ${ }^{813}$ Einleuchtender ist aber vielmehr, dass diese moralische Autorität allen Mitgliedern einer moralischen Gemeinschaft zugebilligt wird. Das moralische Sollen, also letztlich das konkrete Tun oder Unterlassen einer Handlung, ergibt sich auf Grundlage der Forderungen, die jedes Individuum einer moralischen Gemeinschaft im Namen aller an jeden zu richten befugt ist. ${ }^{814}$ Stellt man die Frage nach den Gründen, so liefern legitime Forderungen einerseits den Adressaten Handlungsgründe und andererseits den Adressaten die Grundlage für reaktive Handlungen (bei Missachtung) ${ }^{815}$ Nach der Sanktionstheorie sind moralische Pflichten keine Privatangelegenheit eines Individuums, sondern sie zeigen vornehmlich, warum andere Druck auf dieses Individuum ausüben dürfen. Im Rahmen der Forderungstheorie ist die moralische Verpflichtung als interpersonal zu betrachten, zumal hier jedes Sollen auf autori-

809 Vgl. Raiser (2013), S. 175.

810 Vgl. Anwander (2013), S. $277 \mathrm{f}$.

811 Steinfath (2002), S. 74.

812 Vgl. Anwander (2013), S. 278.

813 Vgl. Wolf (2013), S. $39 \mathrm{f}$.

814 Legitime Forderungen sind damit kein besonderes Privileg. Die Autorität fußt basiert qua Mitgliedshaft in der moralischen Gemeinschaft und rechtfertigt damit Forderungen erheben können.

815 Vgl. Anwander (2013), S. 280. 
tativen Beziehungen fußt, in welchen wir als moralisch Handelnde zueinanderstehen. Allem voran bedarf es eines Verständnisses der moralischen Verpflichtung dahingehend, was andere (berechtigterweise) von einem fordern können. Dann ist man ihnen gegenüber auch für sein Handeln verantwortlich. Somit ist auch nachvollziehbar, wenn diese mit Unmut und Entrüstung auf das Fehlverhalten reagieren, da eine Geringschätzung ihrer Autorität vorausging. ${ }^{816}$ Der Forderungstheorie mag eine gewisse Überlegenheit innewohnen:

»Der simple Rationalismus reduziert moralisches Sollen ausschließlich auf Gründe des Akteurs; die Sanktionstheorie reduziert moralisches Sollen ausschließlich auf Gründe für Reakteure. ${ }^{817}$ Indem sie moralisches Sollen auf legitime Forderungen zurückführt, versteht dagegen die Forderungstheorie moralische Verpflichtung als für beide Seiten normativ: Zum einen wird uns als Reakteuren gesagt, was wir von anderen fordern können und welche Reaktionen für den Fall angebracht sind, dass jemand diesen Forderungen nicht nachkommt. Zum anderen wird uns als Akteuren gesagt, was wir zu tun Grund haben, insofern eine legitime Forderung an uns besteht, so zu handeln. $\aleph^{818}$

Insofern zeigt die Forderungstheorie einen Weg auf, um die Schwachstellen des Rationalismus und der Sanktionstheorie zu eliminieren und die Einwände der Befürworter der jeweiligen Theorie zu entkräften.

\subsubsection{Ohne Ermächtigung keine Autorität}

Die Forderungstheorie sucht einen Weg aufzuzeigen, um den Rationalismus und die Sanktionstheorie zusammenzuführen und ihre Schwachstellen zu beseitigen. Forderungen können dabei allerdings nur von Personen erhoben werden, die die Autorität dazu haben oder welchen man die Autorität in einer Gemeinschaft zubilligt. ${ }^{819}$ Wenngleich Peter Stemmer ausschließlich ein sanktionskonstituiertes moralisches Müssen vertritt ${ }^{820}$, so findet sich auch bei ihm die Frage nach der Ermächtigung:

»Wenn die Mitglieder der moralischen Gemeinschaft sich nicht gegenseitig ermächtigen, gibt es den Unterschied zwischen dem Müssen, bei dem die, die nötigen, dazu ermächtigt sind [...] nicht. ॥ $^{821}$

816 Vgl. Anwander (2013), S. 280.

817 Bezogen auf moralphilosophische Diskussionen lassen sich den Positionen von simplem Rationalismus und Sanktionstheorie die beiden konkurrierenden buck-passing Projekte zu deontischen Eigenschaften zuordnen Für weiterführende Literatur dazu vgl. Anwander (2013), S. 281 Rn. 44.

818 Anwander (2013), S. 281.

819 Vgl. Abschnitt 2.5.2.8.

820 Vgl. Wittwer (2010), S. $133 \mathrm{f}$.

821 Stemmer (2002), S. 57. 
Peter Stemmer erinnert dabei an das verpflichtende moralische Müssen, das sich von einem erpresserischen Müssen folglich unterscheiden soll:

»Es gibt den Unterschied zwischen dem Müssen, das zu setzen, sich rationale Individuen gegenseitig ermächtigen würden, wenn sie aus einem vormoralischen Zustand heraus eine Moral erst hervorbringen müssten, und dem Müssen, das zu setzen, sich rational Individuen nicht ermächtigen würden, wenn sie eine Moral erst erfinden müssten. ${ }^{822}$

Hier wird nicht nach einer faktischen Ermächtigung unterschieden, sondern vielmehr nach einer hypothetischen. STEMmer (2002) erklärt dies mit einem vormoralischen Naturzustand, in welchem zu leben als unangenehm empfunden wird und es die Individuen daher als notwendig ansehen, ein System von Normen zu schaffen, das dann bestimmte Handlungen gebietet und andere verbietet. Ein solches System sei für jeden Einzelnen von Vorteil. Wenngleich man durch ein solches System einem Müssen unterliegt, so überwiegt doch der Vorteil, dass alle anderen Individuen der Gemeinschaft demselben Müssen unterliegen. Um eine solche moralische Ordnung zu schaffen, bedarf es eines Übereinkommens, um bestimmte Handlungen zu "gemussten « Handlungen zu machen. Dies erfordert, dass man jede Abweichung davon mit Sanktionen, also negativen Konsequenzen, belegt. Die Einführung eines Systems von Sanktionen hilft, dass solche Handlungen unterlassen werden, deren Ausführung man verhindern möchte; hingegen werden die präferierten Handlungen dadurch begünstigt. Auf Grund der drohenden Sanktionen werden die nicht gewünschten Handlungen zu solchen Handlungen, die man rationalerweise nicht tun kann bzw. tun muss. Sanktionen werden von den Mitgliedern der Gemeinschaft verhängt, die das moralisch bindende Normensystem haben entstehen lassen. Bei Urheber und Adressaten dieser moralischen Ordnung handelt es sich um dieselben Personen, die gleichzeitig die Autorität und damit Ermächtigung verkörpern:

"Jeder einzelne von ihnen stellt sich durch die Aufrichtung der Moral unter ein `Muss`, und er tut dies, indem er die Gemeinschaft bzw. alle ihre Mitglieder autorisiert, ihn selbst im Falle eines moralwidrigen Verhaltens zu sanktionieren. Dieser - gegenseitige Ermächtigungsakt ist der Grundbaustein, aus dem die moralische Ordnung erbaut ist. ${ }^{823}$

822 Stemmer (2002), S. 59f.

823 Stemmer (2002), S. 60. 
Open-Access-Publikation im Sinne der CC-Lizenz BY 4.0

(c) 2020, Vandenhoeck \& Ruprecht GmbH \& Co. KG, Göttingen ISBN Print: 9783847111719 - ISBN E-Lib: 9783737011716 


\section{Darstellung der Fallstudien}

Die Vorstellungen der Fallstudien werden in drei Abschnitte unterteilt. Zu Beginn soll ein Überblick darüber gegeben werden, wie es zu der Erstellung einer Familienverfassung in der jeweiligen Unternehmerfamilie kam. Welche Gründe spielten dafür eine Rolle? Wie lief der Erstellungsprozess ab? Wie und ab welchem Alter erhalten Familienmitglieder ein Exemplar? Die Hintergründe dazu können wichtige Anhaltspunkte dafür liefern, wie konfliktanfällig die jeweilige Familie ist. Als nächstes sollen die mit der Familienverfassung ins Leben gerufenen Institutionen und Instrumente der Unternehmerfamilie vorgestellt werden. Diesen Einrichtungen kann ein präventiver und reaktiver Charakter in Bezug auf Regelverstöße zugeschrieben werden, wie sich noch im Laufe der Analyse zeigen wird. Im dritten und letzten Abschnitt werden schließlich die Regelbefolgung und mögliche Abweichungen untersucht. Wie stellt die jeweilige Unternehmerfamilie sicher, dass die Regeln eingehalten werden? Wie wird mit Regelverletzungen umgegangen? Wie schafft man moralische Verbindlichkeit innerhalb der Familie? Die Ausführungen dazu bilden sozusagen den zentralen Schwerpunkt der Fallrekonstruktionen.

Die Fallstudien basieren auf den persönlich geführten Interviews (Primärdaten) als auch auf den Sekundärdaten (d.h. schriftliche Versionen der Familienverfassung, Begleitschreiben, Informationsraster, Verfahrensanweisungen für den Familienrat usw.). An dieser Stelle soll nochmals darauf hingewiesen werden, dass die Fallstudien zur Wahrnehmung der den Teilnehmern zugesicherten Anonymität entsprechend an den Stellen angepasst wurden, die Rückschlüsse auf deren Identität zugelassen hätten. Im Wesentlichen wurden die Namen der Unternehmerfamilien sowie der Interviewpartner anonymisiert. Auch wurde hinsichtlich des Geschlechts der Gesprächspartner nicht immer unterschieden. Wenn von einem Gesellschafter die Rede ist, könnte es sich bei dem Interviewpartner in der Realität auch um eine Gesellschafterin gehandelt haben. Da das Geschlecht bei dieser Arbeit keine signifikante Rolle spielt, wird auf eine Unterscheidung zu Gunsten der Anonymität der Fallstudienteilnehmer verzichtet. Überdies wird grundsätzlich darauf geachtet, dass die bestimmenden Merkmale 
der Fallstudien erhalten bleiben. Wenngleich die Fallstudien hier in leicht entfremdeter Version widergegeben werden, sollte jedoch berücksichtigt werden, dass die Analyse und Interpretation auf Grundlage der tatsächlichen Gegebenheiten und unter Zuhilfenahme aller verfügbaren Informationen erfolgte.

\title{
3.1 Fallstudie 1: Unternehmerfamilie Asbrecht (A)
}

\author{
Umsatzkategorie: 5 \\ Anzahl der Familienmitglieder ${ }^{824}: 18$ \\ Generationen: 3
}

Interviewpartner: Gesellschafter, Mitglied des Familienrats (A-1), 3. Gen.

Gesellschafter, ehemaliger Vors. d. AR (A-2), 1. Gen.

Auswertbare Unterlagen: Begleitschreiben »Gedanken zum Familien-Kodex»

\subsubsection{Entstehung und Erstellung der Familienverfassung}

Anton Asbrecht (A-2) hat als Unternehmer viele Jahrzehnte das Unternehmen geprägt und zu dem gemacht, was es heute ist: ein Weltmarktführer auf seinem Gebiet. Irgendwann trieb ihn die Frage um, welcher Maßnahmen es bedarf, um das Gesamtgebilde zu erhalten:

"Als Unternehmer, der sowas aufgebaut hat, geht man immer mit dem Gedanken um, wie bewahre ich das Gesamtgebilde, wie gebe ich es weiter und wie sorge ich dafür - das ist menschlich -, dass es in meinem Sinne weitergeht. ${ }^{825}$

Diese Überlegung war die Geburtsstunde der Familienverfassung. Die Idee entsprang diesem Wunsch, die Zukunft geregelt zu wissen. Anton Asbrecht weiß um seine natürliche Autorität, die er innerhalb der Familie genießt. Die Bewährungsprobe sieht er vielmehr nach seinem Ableben:

»[...] das ist jetzt ein blödes Wort, aber ich sag es trotzdem, ich bin ja sozusagen der Patriarch des Ganzen, die Bewährung kommt eigentlich nach mir. Solange ich da bin, ist eine natürliche Autorität da; der hat das gemacht, das muss man doch respektieren. ${ }^{826}$

Die erste Version der Familienverfassung entstand auf Grund seiner Initiative - sie wurde diktiert, zurückgezogen in den Bergen. Seine Intention war die Erstellung einer »moralisch wirksame[n]« Verfassung, die der Familie sagt,

824 Die Anzahl der Familienmitglieder basiert auch hier auf der Definition von Familie nach der jeweiligen Familienverfassung. Die »Familienmitglieder« können daher in manchen Fällen auf Gesellschafter beschränkt sein. In vielen Fällen jedoch werden auch Nicht-Gesellschafter (Ehefrauen, Kinder etc.) zum Kreis der Familienmitglieder gezählt.

825 Interview A-2 (08.11.2017), S. 1.

826 Interview A-2 (08.11.2017), S. 11. 
»[...] was wir gemeinsam machen wollen und wo die Maximen sind, nach denen wir uns zu richten haben. $"{ }^{827}$ Dieser Entwurf wurde dann seinen Kindern mit der Bitte überreicht, ihn durchzulesen und ggf. Ergänzungen, Korrekturen oder Ähnliches anzumelden. Sein heute 25-jähriger Enkel Alexander Asbrecht (A-1) erinnert sich an keine grundsätzlichen Widerstände in der 2. Generation gegen die Einführung einer Familienverfassung. Er ist sich jedoch sicher, dass die Familienverfassung nicht existieren würde, wenn es kein gemeinsames Unternehmen gäbe:

»Es wurden Dinge diskutiert oder vielleicht auch kontrovers diskutiert. Größtenteils bestand aber Einigkeit. [...] Da kann ich mich nicht daran erinnern, dass es da heftige Widerstände gegeben hätte. [...] Ich glaube, wenn es diese Firma nicht gäbe, würde der Kodex so in der Form nicht existieren. ${ }^{828}$

Die Familienverfassung existiert bei Familie Asbrecht nun seit über 15 Jahren. Im Jahre 2006 wurde sie überarbeitet. Die Exemplare sind in Leder gebunden und das Dokument wurde von allen Familienmitgliedern unterzeichnet. Mit Vollendung des 15. Lebensjahres, also zum 16. Geburtstag, bekommen Familienmitglieder die Familienverfassung feierlich überreicht und unterzeichnen diese dann. Zusätzlich erhalten diese Familienmitglieder auch den unter der Überschrift »Gedanken zum Familien-Kodex « verfassten zweiseitigen Begleitbrief von Anton Asbrecht, in welchem er zum Ausdruck bringt, dass ihm die Familienverfassung ein »Herzensanliegen« ist. Er weist darin darauf hin, dass die Familienverfassung "kein rechtlich bindender Vertrag" ist, sondern »mehr Gewicht als ein solcher [hat], weil [sie] moralische Kategorien festschreibt. Verträge können geändert oder ganz aufgehoben werden, ethische Grundsätze bleiben.« Er schließt dieses Schreiben mit der Hoffnung, dass die Familienverfassung nicht nur gelesen wird, sondern der Inhalt »selbstverständlicher Teil« des Handelns wird: »Die Lebenshaltung und der Beitrag Eurer Vorfahren sollten Euch alle dazu verpflichten. « Anton Asbrecht möchte mit diesem Schreiben, sicherlich auch für die Zeit nach seinem Ableben, die nachfolgenden Generationen daran erinnern, dass die Privilegien der Familienmitglieder auch Verantwortung für das Ganze bedeuten. "Nur so bleiben Wohlstand und Frieden « in der Familie erhalten. ${ }^{829}$

Für Anton Asbrecht wurde mit der Familienverfassung nicht etwas völlig Neues geschaffen, was vorher nicht bereits existiert hätte. Der Umgang in der Familie beschreibt er als "gut» und »funktionierend». Die Familienverfassung wurde daher nicht geschrieben um »etwas zu korrigieren, sondern zu destillieren

827 Interview A-2 (08.11.2017), S. 1.

828 Interview A-1 (12.10.2017), S. 2.

829 Entnommen aus dem Begleitschreiben "Gedanken zum Familien-Kodex«, das Familienmitgliedern, die 16 Jahre alt werden, bei der Übergabe der Familienverfassung überreicht wird. 
und zu fixieren.« Mit der Familienverfassung wurde »nur eine Gegebenheit und Befindlichkeit versucht zu erfassen, in Worte zu fassen und festzuschreiben. ${ }^{830}$

\subsubsection{Inhalt, Institutionen und Alltag}

Auf etwa 30 Seiten werden verschiedene Themen behandelt. Dabei werden Fragen zur Herkunft, zum gemeinsamen Umgang miteinander, zum Benehmen in der Öffentlichkeit, zur Rolle der Familie, zu einem möglichen (beruflichen) Einstieg in das Unternehmen, zum Umgang mit Firmenanteilen oder zur Rolle der Stiftungen beantwortet, um lediglich einige Aspekte herauszugreifen.

Die Familienverfassung als schriftliche Leitlinie scheint im Alltag lediglich eine marginale Rolle zu spielen, der Inhalt jedoch ist allgegenwärtig. Es wurden die "gemeinsame Grundüberzeugung, die Ziele und Maxime der Familien fixiert. ${ }^{831}$ Anton Asbrecht schrieb im Vorwort, dass »[d]ies kein Testament oder eine Anweisung aus der Feder des Familienältesten« sei, er ist vielmehr davon überzeugt, dass

»Voraussetzung für den Erhalt des Ansehens unserer Familie und unseres Vermögens ist $[\ldots]$ auch die Pflege der engen familiären Verbindungen, der Gesellschafter und ihrer Angehörigen. ${ }^{832}$

Sein Enkel Alexander bestätigt, dass die Inhalte der gelebten Wirklichkeit entsprechen und für ihn nach der ersten Lektüre nichts Neues waren:

»Alles, was ich da drin gelesen habe, war für mich eigentlich nur eine Bestätigung dessen, was man mir bisher in meinem Leben vermittelt hatte, was Familie und Firma und die Verbindung betrifft. ${ }^{833}$

Alexander Asbrecht ist zwar davon überzeugt, dass das Dokument als solches bereits heute einen wichtigen Stellenwert in der Familie einnimmt; die besondere Relevanz dieses Dokuments sieht er jedoch eher in der Zukunft verortet. Er scheint auf die Zeit nach dem Ableben seines Großvaters anzuspielen, auch vor dem Hintergrund, dass es zehn Enkelkinder gibt und die Familie in der 3. Generation somit zu einer beachtlichen Größe anwachsen wird.

Mit der Familienverfassung wurden der Familientag und der Familienrat als Institution ins Leben gerufen.

Seit etwa 15 Jahren findet einmal jährlich ein Familientag statt, der der »Förderung des Erhalts innerhalb der Familie, sowie der Vermittlung von Wis-

830 Interview A-2 (08.11.2017), S. 2.

831 Interview A-2 (08.11.2017), S. 2.

832 Interview A-2 (08.11.2017), S. 2.

833 Interview A-1 (12.10.2017), S. 2. 
sen, Werten und Zielen dient ${ }^{834}$ und die Teilnahme daran für alle Familienmitglieder verpflichtend ist. Er dauert von Freitagabend bis Sonntagmittag und findet in geraden Jahren am Hauptsitz des Unternehmens und in ungeraden Jahren im Land einer ausländischen Tochtergesellschaft statt. Sanktionen bei Fernbleiben sind nicht vorgesehen: "Also es ist nicht gesagt, wer zum Familientag nicht kommt, da wird seine Dividende, wenn er denn eine hat, gekürzt. ${ }^{835}$ Alexander Asbrecht erinnerte sich, dass etwa bei jedem zweiten Familientag in irgendeiner Form die Familienverfassung thematisiert wird, auch wenn dies nicht ein fester Tagesordnungspunkt ist.

Mit der Einführung der Familienverfassung wurde auch ein Familienrat etabliert, der aus drei bis fünf Familienmitgliedern, die mindestens 25 Jahre alt sind, besteht. Die Mitglieder werden auf drei Jahre gewählt. Es soll zumindest aus jeder Generation ein Vertreter in den Familienrat entsandt werden. Aufgabe des Familienrats ist es, unter anderem mit darüber zu befinden, wenn ein Familienmitglied im Unternehmen eine operative Position bekleiden möchte oder auch bei familiären Streitigkeiten einzuwirken.

Ein weiterer wichtiger Punkt der Familienverfassung ist der Hinweis auf die Stiftung und die der Stiftung zu Grunde liegenden Intention. Die Stiftung soll auch als »Auffangbecken« für Gesellschafter fungieren, die »mit der wirtschaftlichen Geschichte nichts zu tun haben« wollen: ${ }^{836}$

"[...] dass das Ausscheiden nicht zu einem Buchwert, [...] sondern dass der Verkehrswert zu Grunde zu legen ist. Dass aber die Zahlung sich über acht Jahre erstrecken muss oder kann und dass ein Abschlag von 25 Prozent auf den Verkehrswert hinzunehmen ist. Den kann man mindern, den Abschlag, wenn man Geld, das man erlöst, in die Stiftung gibt. Und dann kann man in der Stiftung einen Kuratoriumssitz kriegen. ${ }^{837}$

Insgesamt ist $\mathrm{zu}$ beobachten, dass sich die 3. Generation verstärkt einbringt und auch Vorschläge für Veränderungen der Familienverfassung diskutiert und durchsetzt - auch solche, die eine vorhandene Regelung eher noch verschärfen.

Die wichtige Wirkung des familiären Zusammenhalts scheint auch insbesondere von der dritten Generation erkannt worden zu sein. So wurde bei einem Familientag der Wunsch geäußert, »[...] wir, die dritte Generation wollen aber Dinge zusammen machen. Wir [...] wollen uns treffen, nur wir in der dritten Generation und wir möchten dann eine Firma besuchen. ${ }^{838}$

834 Interview A-2 (08.11.2017), S. 3.

835 Interview A-2 (08.11.2017), S. 9.

836 Interview A-2 (08.11.2017), S. 5.

837 Interview A-2 (08.11.2017), S. 5.

838 Interview A-2 (08.11.2017), S. 10. 


\subsubsection{Regelbefolgung, -brüche und moralische Verbindlichkeit}

Die geführten Interviews vermittelten den Eindruck, dass die Familienverfassung gelebt wird. Zu gravierenden Regelverstößen sei es bisher nicht gekommen:

»Wir haben bisher Glück gehabt, dass es zu solchen Fällen bisher nicht gekommen ist.

[...] Ich habe den Eindruck, dass sich alle über das, was da in der Familienverfassung steht, einig sind und sich da wirklich bemühen, danach zu leben, sich daran zu halten. ${ }^{839}$

Präventive und reaktive Maßnahmen zur Regelbefolgung - zumindest aus Sicht der interviewten Personen - scheinen nicht viele zu existieren:

"Also wir haben sehr wenig getan oder sagen sehr wenig zu Sanktionen. Im Grunde gar nichts [...]. Wir setzen voraus, das wird akzeptiert. Und wenn es nicht akzeptiert wird, dann muss sich die Familie [...] zusammensetzen und muss sagen, Leute, das stimmt aber nicht mehr. ${ }^{840}$

Auf Grund der Tatsache, dass man die Familienverfassung explizit als moralisches Dokument betrachtet, sieht man offenbar nicht die Notwendigkeit oder auch nicht die Möglichkeit, entsprechende Sanktionen zu etablieren, die über eine klassische Sanktion, die beispielsweise aus einer Verletzung des Gesellschaftsvertrags resultieren kann, hinausgeht. Während Anton Asbrecht sehr stark darauf zu vertrauen scheint, dass die Familienverfassung Kraft ihrer moralischen Bindungswirkung von allen eingehalten wird, ist Alexander Asbrecht eher skeptischer, indem er sagt: »[W]enn man realistisch ist [...], dann muss klar sein, dass man damit rechnen muss, dass es irgendwann zu Regelbrüchen vielleicht auch kommt, und dass man sich da auch darauf vorbereiten muss. ${ }^{841}$

Im Familienrat wird eine Institution gesehen, die eine schlichtende Funktion übernehmen soll, wenn es zu einem gravierenden Regelbruch innerhalb der Familie kommt. Bei einfachen Fehltritten wird man in der Familie zunächst versuchen, dies bilateral zu lösen. Erst wenn es sich um ein gravierendes und dauerhaftes Problem handelt, würde der Familienrat tätig werden. Alexander Asbrecht ist davon überzeugt, dass schwerwiegende Verstöße auch bei einem Familientag angesprochen werden könnten. Zumindest gab es in der Vergangenheit hin und wieder allgemeinere Anmerkungen seitens Anton Asbrecht, wenn ihm Abweichungen auffielen.

Anton Asbrecht sieht in der Familienverfassung einen "moralischen Überbau, der nicht einklagbar ist ${ }^{842}$ Das wesentliche Anliegen der Familienverfassung

839 Interview A-1 (12.10.2017), S. 7.

840 Interview A-2 (08.11.2017), S. 9.

841 Interview A-1 (12.10.2017), S. 5.

842 Interview A-2 (08.11.2017), S. 9. 
besteht seiner Auffassung darin, die »Verhaltensweise vor dem Fehlverhalten zu beeinflussen. ${ }^{843}$ Damit wird in der Familienverfassung selbst bereits eine inkorporierte präventive Wirkung vermutet.

Es wird derzeit, insbesondere durch Anton Asbrecht darauf gehofft, »[d]ass sich jeder eingedenk ist, ich bin Teil eines Ganzen, wo ich Rücksichten zu nehmen habe. Ich glaube, dass das auch von unseren Enkeln [...] respektiert wird. Die sehen das so, glaube ich. ${ }^{844}$

Über eine reine verbale Maßregelung hinaus, die auch mal vor allen Familienmitgliedern am Familientag passieren kann, scheinen keine harten Sanktionen etabliert zu sein. Vielmehr wird gehofft, dass die moralische Verbindlichkeit, die von der Familienverfassung ausgehen soll, ihre Wirkung entfaltet.

Moralische Verbindlichkeit in der Familie kann gelingen, wenn die Familie selbst eine Vorbildfunktion übernimmt und das gewünschte Verhalten vorlebt:

"[...] ich glaube, das kann in Familien gelingen, wenn man von klein auf mit den Kindern über diese Dinge spricht und wenn man ein bestimmtes Verhalten lebt und vorlebt. Und das Vorbild ist die wichtigste Erziehungsfunktion. Oder das wichtigste Erziehungsmittel. $\aleph^{845}$

\subsection{Fallstudie 2: Unternehmerfamilie Brunai (B)}

\section{Umsatzkategorie: 3 \\ Anzahl der Familienmitglieder: ca. 35 \\ Generationen: 5}

Interviewpartner: Gesellschafterin, Mitgl. d. Familienrats, eingeh. (B-1), 3. Gen. Gesellschafter, Geschäftsf., Mitgl. d. Familienrats (B-2), 4. Gen.

Gesellschafter, Mitgl. d. Familienrats, Vors. d. AR (B-3), 3. Gen. Auswertbare Unterlagen: Familienverfassung, Informationsraster, Geschäftsordnung des Familienrats, Förderkatalog der Gesellschafterkompetenz, Anmerkungen zum Informationsraster

\subsubsection{Entstehung und Erstellung der Familienverfassung}

Die Anfänge des Unternehmens als OHG begannen Ende des 19. Jahrhunderts. Wie früher gemeinhin üblich, wurden lediglich die männlichen Nachkommen zu Gesellschaftern und führten das Unternehmen. So auch in diesem Fall bis zur dritten Generation. Berthold Brunai (B-3) hat zusammen mit seinen Geschwistern die Vererbungsregel geändert, indem sie alle Kinder gleich behandelten. So

843 Interview A-2 (08. 11.2017), S. 14.

844 Interview A-2 (08. 11.2017), S. 14.

845 Interview A-2 (08.11.2017), S. 8. 
wuchs die Zahl der Gesellschafter von seinerzeit drei plötzlich auf 18 an, indem neben den Kindern auch die Ehefrauen zu Gesellschaftern wurden. Im Laufe der 90er-Jahre des 20. Jahrhunderts wurde die OHG in eine Kapitalgesellschaft überführt - sicherlich nicht zuletzt auch aus Haftungsgründen und der Tatsache, dass es innerhalb der 3. Generation Meinungsverschiedenheiten in Bezug auf die Ausrichtung der Unternehmensgruppe gab. Berthold Brunai erinnert sich, "[...] so [ging] es nicht weiter. Und wir verzehrten auch unsere Harmonie. Das war sowieso grenzwertig. ${ }^{846}$ Man wollte mit der Kapitalgesellschaft zeitgleich auch klare Zuordnungen und Verantwortlichkeiten einführen. In der OHG hatte zwar jeder seinen Bereich, doch niemand das "letzte Wort ${ }^{847}$. Neben der Tatsache, dass sich die Gesellschafterzahl plötzlich versechsfachte, wollte man dem Umstand Rechnung tragen, dass nun auch eine 4. Generation Gesellschafter wurde, die noch weiter vom Unternehmen entfernt waren:

"So und damit hat sich aufgedrängt, [...] wir sind plötzlich eine große Gesellschafterfamilie und man kann eben nicht mehr alle Entscheidungen treffen am Konferenztisch, [...] was bedeutet das denn eigentlich, dass eine vierte Generation Gesellschafter geworden ist, wie man diese an das Unternehmen heranführen und auch die Rolle der Gesellschafter. ${ }^{848}$

Plötzlich musste man sich auch als Unternehmerfamilie "managen « ${ }^{849}$. Die Familienverfassung war das Ergebnis einer langen Diskussion zwischen den Mitgliedern der 3. Generation und auch der immer mehr involvierten 4. Generation. Man nahm für die erste Version der Familienverfassung Anleihe bei einer großen Unternehmerfamilie und hatte deren Juristen als Unterstützung. Als Anfang des 21. Jahrhunderts auch die Familienstämme aufgelöst wurden, wurde die Familienverfassung unterzeichnet und eine Familiengesellschaft gegründet. Als sich die Gesellschafter der Familien im Jahre 2011 auf geteilte Werte und Ziele verständigten, was in eine Familienstrategie mündete, wurden daraufhin der Gesellschaftsvertrag und die bis dahin unveränderte Familienverfassung entsprechend angepasst.

Der Impuls, also der Auslöser zur Erstellung einer Familienverfassung, war klar:

»[W]ir brauchen eine Art Agens, wie wir eigentlich hier miteinander umgehen wollen, wie wir unsere Kinder erziehen wollen, wie wir unser Verhältnis zum Unternehmen strukturieren usw. ${ }^{850}$

846 Interview B-3 (09.11.2017), S. 3.

847 Interview B-3 (09.11.2017), S. 3.

848 Interview B-2 (06.11.2017), S. 1.

849 Interview B-2 (06.11.2017), S. 2.

850 Interview B-2 (06.11.2017), S. 3. 
Die Notwendigkeit für eine solche Familienverfassung wurde von allen Familienmitgliedern erkannt. Widerstände gab es keine, vielmehr gab es »unterschiedliche Level an Engagement, aber auch kein Nicht-Engagement. ${ }^{851}$ Auch der Prozess der Überarbeitung im Jahre 2011 wurde insofern als hilfreich wahrgenommen, als dass die den Prozess begleitende Beraterin durch gezielte Fragen unterschiedliche Perspektiven unter den Familienmitgliedern zu Tage förderte, woraus man entsprechenden Handlungsbedarf ableiten konnte. Dabei sind auch »Unklarheiten im Gesellschaftsvertrag entdeckt worden [...], und damit haben [sie] auch den Gesellschaftsvertrag angepasst. ${ }^{852}$ Auch gab es wichtige Impulse, beispielsweise "was gerade auch die Schulung der jungen Gesellschafter betrifft. ${ }^{853}$

Zum Prozess selbst hat Benjamin Brunai (B-2) eine klare Meinung:

"Das ist eigentlich fast schon eine Binse. Aber darauf kommt es an. Das Resultat ist ja ganz nett und es gibt auch so Aspekte aus unseren Statuten, die man so im Dialog miteinander hin und wieder verwendet. $\aleph^{854}$

Es sind sich alle interviewten Familienmitglieder darüber einig, dass die Familienverfassung nicht rechtlich verbindlich ist. Wenngleich Benjamin Brunai sie gerne etwas $»$ justiziabler « ${ }^{855}$ hätte, gibt er gleichzeitig $\mathrm{zu}$, dass die Familienverfassung insbesondere auch solche Dinge regeln soll, die »wir nicht im Gesellschaftsvertrag haben wollen. Einfach, weil wir sie zu persönlich finden. ${ }^{856}$

\subsubsection{Inhalt, Institutionen und Alltag}

Die 15 Seiten umfassende Familienverfassung geht neben einer Vorbemerkung in Form eines historischen Exkurses auf das Thema »Gemeinschaft « ein, was in Ansätzen mit der Definition des Familienbegriffs in anderen Familienverfassungen vergleichbar ist. Sie führt aus, dass das Unternehmen im Besitz der Familie bleiben soll und welche Maßnahmen nach Meinung der Familie dafür erforderlich sind. Neben dem klaren Bekenntnis zur Qualifizierung des Nachwuchses (Gesellschafterkompetenz) werden Vorgaben für eine Betätigung im Unternehmen getroffen. Enthalten ist auch der Hinweis, dass die in der Familienverfassung enthaltenen Prinzipien vom Familienrat in konkrete Handlungsregeln übersetzt und im Kommunikationsraster der Gemeinschaft festgehalten

851 Interview B-2 (06.11.2017), S. 4.

852 Interview B-3 (09.11.2017), S. 16.

853 Interview B-1 (17.10.2017), S. 1.

854 Interview B-2 (06.11.2017), S. 5.

855 Interview B-2 (06.11.2017), S. 5.

856 Interview B-2 (06.11.2017), S. 5. 
werden. Eine klare Regelung wird darin auch für einen möglichen Regelverstoß und zum Ausschluss aus der Gemeinschaft getroffen.

Der Familienrat besteht aus mindestens drei Mitgliedern, grundsätzlich aus fünf. Nichtgesellschafter können ebenfalls in den Familienrat gewählt werden. Sie werden auf fünf Jahre gewählt und können aus wichtigem Grund durch Gesellschafterbeschluss mit 75 Prozent Mehrheit der Stimmen abberufen werden. Der Familienrat ist - tendenziell eher außergewöhnlich - bei Familie Brunai im Gesellschaftsvertrag verankert und übernimmt damit auch die Aufgabe der Überwachung der Geschäftsführung, was bedeutet, dass er auch den Aufsichtsrat wählt. Der Familienrat, der in seiner inneren Ordnung einen Vorsitzenden und Stellvertreter wählt, ist für die Familienmitglieder mittlerweile auch vermögensbildend tätig (z.B. Gewährung von Darlehen an Gesellschafter). Es ist klar geregelt, zu welchen Handlungen es der Zustimmung des Familienrats bedarf. Darüber hinaus ist ihm aufgegeben, interessierten Familienmitgliedern die Möglichkeit zu eröffnen, ihre berufliche Laufbahn innerhalb der Unternehmensgruppe zu gestalten.

Der Familienrat wacht über die Einhaltung der Familienverfassung und wird bei Verstößen aktiv. Wie jüngere Gesellschafter gefördert und an das Unternehmen herangeführt werden sollen, ist Teil der Arbeit des Familienrats. Dafür wurde ein "Förder-Katalog " erstellt, eine Übersicht über verschiedene Schulungsmaßnahmen, welche von der Unterstützung bei der Berufs-/Ausbildungswahl über Kurse zu Sozialkompetenz und sozialem Engagement bis hin zu unternehmerischen Grundkenntnissen zum Erhalt des Unternehmens reichen.

Britta Brunai (B-1), selbst Mitglied des Familienrats, betonte, wie wichtig es für den Familienrat ist, die Junggesellschafter zu fördern:

"Das ist jetzt unser aller Bestreben. Im Familienrat haben wir das auch ganz klar geregelt, dass wir Schulungen für die Junggesellschafter machen. Also wir haben einmal im Jahr ein Junggesellschafterseminar [...]. Also von Bilanzen über [...] das Unternehmen an sich. Also da werden einfach alle Themen [...] wirklich auch in Eigenarbeit erarbeitet. ${ }^{857}$

Der Familienrat hat darüber hinaus für die Familiengemeinschaft ein »Informationsraster « erarbeitet, das Auskunft darüber gibt, in welchem Umfang und in welcher Regelmäßigkeit Gesellschafter, Familienmitglieder, Geschäftsleitung des Unternehmens sowie Familienrat miteinander kommunizieren. »Auf diese Weise wird allen Beteiligten klar, auf welche Informationen sie Anspruch haben, wer an welchen Entscheidungen beteiligt ist und für wen dies nicht gilt. ${ }^{858}$ Das erleichtert nicht nur die Arbeitsteilung, sondern soll Streit über Ungeklärtes ver-

857 Interview B-1 (17.10.2017), S. 3.

858 Schriftliche Anmerkungen des Familienrats zum Informationsraster. 
meiden. Die Unternehmerfamilie Brunai ist davon überzeugt, dass erst in einer geordneten Art und Weise die Regeln der Zusammenarbeit und des Zusammenlebens weiterentwickelt werden können. Jeder Gesellschafter kann auf Wunsch an einer Sitzung des Familienrats teilnehmen oder ein persönliches Anliegen dem Familienrat vorbringen und um Klärung bitten.

Die Familienmitglieder geben weitreichende Entscheidungsbefugnisse an den Familienrat ab:

"[...] ich würde es einfach noch breiter fassen und sagen, der Familienrat ist überhaupt die Institution, dass wir als Familie entscheidungsfähig sind. Wir haben uns einfach eine Form gegeben. ${ }^{859}$

Das umfangreiche Aufgabengebiet des Familienrats erinnert in Ansätzen an die eines Family Office.

Ein jährlich stattfindender Familientag führt die Familienmitglieder an wechselnde Unternehmensstandorte in ganz Europa.

Obwohl die Familienverfassung in ihrer jetzigen, sehr detaillierten Form nun vor über sechs Jahren verabschiedet worden ist, ist sie für Britta Brunai, obwohl sie aktiv im Familienrat mitwirkt, noch kein Dokument, das wirklich so lebt, wie es in ihren Augen sein sollte:

»Es ist noch nicht wirklich so in unseren Köpfen und im Alltag drin, dass wir das als lebendig betrachten. ${ }^{860}$

Die Unternehmerfamilie Brunai scheint die Familienverfassung allerdings auch sehr ernst zu nehmen. Auch wenn nicht alle Regeln detailgenau im Kopf präsent sind, so wird sie jedenfalls gelebt: »[...] und diese Prozesse halten wir ein. ${ }^{861}$ Abweichungen werden in Maßen durchaus toleriert; man hat sich den Aspekt der "spielerischen Offenheit ${ }^{862}$ auf die Fahnen und auch in die Familienverfassung geschrieben. Diesen "Joker ${ }^{863}$ zieht man gerade in Situationen, in denen sich Familienmitglieder wegen eines eher unwichtigen Details zu verkeilen drohen:

"Das einzige, was wir glaube ich machen, wir flaggen die Abweichung als Abweichung aus. Also wir sagen nicht, wir kehren es untern Tisch oder sagen nicht, da wollen wir jetzt nicht darüber reden. Wir sagen, da haben wir uns bewusst entschieden. Wir haben zwar Regeln aber wir machen eine Ausnahme. Wenn es anfangen würde, dass die Leute sich jetzt ständig auf die Ausnahmen berufen würden, dann würden wir wahrscheinlich strenger die Regeln durchsetzen. ${ }^{864}$

859 Interview B-2 (06.11.2017), S. 12.

860 Interview B-1 (17.10.2017), S. 13.

861 Interview B-2 (06.11.2017), S. 9.

862 Interview B-2 (06.11.2017), S. 5.

863 Interview B-2 (06.11.2017), S. 7.

864 Interview B-2 (06.11.2017), S. 8. 
Im Alltag wird die Familienverfassung weniger als ein "symbolisch[es], sondern [mehr als] ein praktisches Dokument ${ }^{865}$ verstanden:

»Aber Gesellschaftsvertrag und Statuten ${ }^{866}$ werden immer wieder angefragt. Wir diskutieren irgendetwas und dann sagen wir plötzlich, Moment mal, wie steht das denn, wie ist das alles geregelt. ${ }^{867}$

In den Interviews wurde deutlich, dass die Familie durch ihre Informationspolitik und den vielen etablierten Maßnahmen eine starke Bindung innerhalb der Familie schaffen konnte:

»[...] und weil wir tatsächlich eine starke Bindung haben, durch die Seminare, durch die Jahresversammlung, Hauptversammlung; und durch den Ausflug. Und durch die ständigen Kontakte und Berichte, die wir ständig schicken. Ich schicke immer einen Quartalsbericht über den Geschäftsverlauf. Oder manchmal berichte ich auch über Fragen, die wir gerade im Familienrat erörtern, die ganz interessant sind. Wir arbeiten wahnsinnig intensiv über Informationen. Ein Geflecht von Informationen, persönlichen Kontakten, bilateralen Kontakten. ${ }^{868}$

\subsubsection{Regelbefolgung, -brüche und moralische Verbindlichkeit}

Insgesamt lässt sich feststellen, dass es in der Familie seit Bestehen der Familienverfassung keine gravierenden Regelverstöße gab. Die erste Version der Familienverfassung hat vielmehr dazu beigetragen, dass sich die "plötzliche» Großfamilie besser kennenlernte und so das Zusammengehörigkeitsgefühl gestärkt wurde:

»Das hat auch was damit zu tun, dass wir uns alle recht schlecht kannten. Also dann sind meine Cousine zweiten Grades und mein Cousin zweiten Grades jetzt Gesellschafter geworden, wir hatten [davor] eigentlich wenig Anlass, uns miteinander auseinanderzusetzen. ${ }^{869}$

Als präventives und "regulatives ${ }^{870}$ Instrument wird die Familienverfassung selbst (»Ja, feiner ausgedrückt glaube ich, ist es ein rekursiver Zusammenhang; einerseits schreiben sie was auf, und andererseits wirkt das Aufgeschriebene dann auch wieder. ${ }^{871}$ ) gesehen, die zu den konfliktanfälligen Themen entsprechende, von allen akzeptierten Regeln enthält. Diese Regeln werden dann ein-

865 Interview B-2 (06.11.2017), S. 9.

866 Familie Brunai hat für ihre Familienverfassung den Begriff »Familienstatuten« gewählt.

867 Interview B-3 (09.11.2017), S. 9.

868 Interview B-3 (09.11.2017), S. 23.

869 Interview B-2 (06.11.2017), S. 1.

870 Interview B-2 (06.11.2017), S. 6.

871 Interview B-2 (06.11.2017), S. 6. 
gehalten, wenn sie die typischen Fälle abdecken und von allen verstanden werden:

»[...] Statuten sind dazu da, Konflikte zu vermeiden, [...] was machen denn Statuten. Statuten bauen im Vorfeld eine Kasuistik auf, wie man mit bestimmten Situationen umgeht und gute Statuten decken halt sehr viele Fälle ab. Was sind denn die typischen Konfliktfälle in Familienunternehmen? Es sind halt in der Regel die Frage der Teilnahme. Teilnahme an Informationen, Teilnahme an Entscheidungen, Teilnahme an Zuwendungen, also finanzieller Art. Ich glaube, dass die Konfliktlinien laufen sehr stark an der Frage, was steht mir zu und was steht mir nicht zu und was bin ich schuldig und was bin ich nicht schuldig. Und daran zerknallen glaube ich die meisten Familien. Und gute Statuten regeln das und unsere Statuten regeln das halt sehr ausführlich [...]. Ich glaube [...], dass wir Dutzende von Entscheidungen getroffen haben, in den letzten Jahren, die sofort ein mega Konfliktpotenzial gehabt hätten, wenn wir die Statuten nicht gehabt hätten. Ganz klar, immer die Frage, wer darf hier mitarbeiten? Wer darf hier nicht mitarbeiten? ${ }^{872}$

Ein weiteres präventives Instrument ist der Familienrat:

»[...] dass wir die Statuten festgeschrieben haben und sie jedem in die Hand drücken, der 18 wird, und generell als Familienrat eine Instanz darstellen, das ist vielleicht schon ein wichtiger Faktor. Wenn der Familienrat [...] einen gewissen Respekt hat, dann ist das eine Instanz, die auch im Grunde eine präventive Maßnahme ist [...]. Also sprich, wenn man diesen Familienrat gar nicht kennt, gar nicht weiß, was der so tut, weil die sich gar nie melden, und wenn man dann auch gar keine große Angst oder keinen Respekt vor dieser Instanz hat, dann wird man sich auch an keine Regel halten wollen. Also ich denke so eine Instanz braucht es schon. [...] Und wir haben uns natürlich auch im Laufe der Jahre Respekt verschafft, weil wir auch liefern. Wir informieren viel. Wir zeigen Präsenz bei allem, wo wir sie auch zeigen müssen. ${ }^{873}$

Der Familienrat kümmert sich um die persönlichen Belange der Gesellschafter, steht ihnen mit Rat und Tat zur Seite: Wenn abzusehen ist, dass sich ein Gesellschafter verschulden könnte, bietet der Familienrat Unterstützung an. Lässt sich ein Gesellschafter scheiden, steht er mit gutem Rat oder der Empfehlung eines Rechtsanwalts zur Seite. Für Benjamin Brunai sind auch die eingeführten »Mikrokündigungen« ein Teil der Prävention:

»Gesellschafter können bei uns Mikrokündigungen machen. Das heißt, sie können Minianteile kündigen. Wir erlauben Gesellschaftern ohne Zustimmung der anderen Gesellschaftern Anteile zu kündigen im Promille-Bereich. Das hat den riesen Vorteil, dass Gesellschafter sich nicht erleben in dieser hand-cuffed Situation. Bei uns ist es eigentlich so, der Verkauf von Anteilen ist bei uns sehr schwer, weil man Vorkaufsrechte hat und Fristen [...] und es dauert alles immer ewig. Und das empfinden viele Gesellschafter, oder könnten es als Gängelei empfinden [...]. Und deswegen sagen wir, wir

872 Interview B-2 (06.11.2017), S. 8 f.

873 Interview B-1 (17.10.2017), S. 14. 
haben so eine ganz lockere Möglichkeit, hat noch nie ein Gesellschafter genutzt aber jeder Gesellschafter weiß, dass er es könnte, dass er sagen kann, ich kann hier ganz ein bisschen den Hahn aufmachen und mir ein bisschen Geld rausholen. ${ }^{874}$

Das Kommunikationsraster stellt sicher, dass alle Familienmitglieder mit den ihnen zustehenden Informationen versorgt werden und sie damit stets im Bilde darüber sind, welche Informationen ihnen zustehen. So lassen sich klassische Befindlichkeiten im Keim ersticken.

Die professionalisierte Gesellschafterkompetenz sorgt dafür, dass auch die jungen Familienmitglieder entsprechend eingebunden werden und möglichst früh mit dem Familienunternehmen in Berührung kommen; sei es über historische Erzählungen zu den Anfängen oder über Fortbildungsveranstaltungen, die sie gezielt auf eine verantwortungsbewusste Rolle als Gesellschafter vorbereitet:

"[...] vor allem es dann auch geschafft hat, die jungen, die jüngeren Gesellschafter mit ins Boot zu holen, [...] das ist alles Regelwerk, Papier, aber wichtig ist uns vor allem die Schulung der Gesellschafter. Dass jeder auch weiß, worum es geht, das Unternehmen kennenlernt, die ganzen Unternehmensstrukturen parat hat, auch die Ausrichtung, die Unternehmensphilosophie mitträgt. « ${ }^{875}$

Sobald ein Familienmitglied das 18. Lebensjahr erreicht hat, wird es vom Vorsitzenden des Familienrats zu einer Sitzung eingeladen. In dieser Sitzung wird er über die Familiengemeinschaft und die Inhalte der Familienverfassung unterrichtet und gebeten, diese sodann zu unterzeichnen.

Die Einhaltung der Familienverfassung überwacht der Familienrat und dieser ist auch bei Verstößen erster Ansprechpartner.

»Der Familienrat soll bei einem einfachen Verstoß eines Mitglieds dieses zum Gespräch bitten und ermahnen. Bei wiederholtem einfachen oder im Falle eines schwerwiegenden Verstoßes soll der Familienrat zu einer Versammlung der Gemeinschaft [...] einladen. [...] und den Verstoß auf die Tagesordnung zu setzen. Wird auf dieser Versammlung ein Verstoß eines Mitglieds gegen die Prinzipien dieser Statuten zum Tagesordnungspunkt gemacht, kann jedes Mitglied der Gemeinschaft im Zuge derer eine Abstimmung über den Ausschluss des Mitglieds fordern, [...] für den eine Mehrheit von 75 Prozent Voraussetzung ist. ${ }^{876}$

Mit dem Ausschluss aus der Familiengemeinschaft bleiben der Person fortan alle Privilegien, die die Familienverfassung enthält, versagt. Auch sieht der Gesellschaftsvertrag der Familienkommanditgesellschaft vor, dass ein Ausscheiden aus der Gemeinschaft auch ein Ausscheiden als Gesellschafter mit sich zieht. Inso-

874 Interview B-2 (06.11.2017), S. 13.

875 Interview B-1 (17.10.2017), S. 2.

876 Auszug aus der Familienverfassung der Familie Brunai zu Punkt »Verstoß und Ausschluss«. 
fern ist nach einem Ausschluss aus der Gemeinschaft gemäß Gesellschaftsvertrag auch eine Abstimmung der Familienkommanditgesellschaft abzuhalten.

Ob diese Vorgehensweise einer richterlichen Prüfung standhält, soll an dieser Stelle dahingestellt bleiben. Die abschreckende Wirkung dieser Ultima Ratio bleibt und die Familie möchte sich diesen Schritt für wirklich gravierende Verstöße vorbehalten:

"Ob die juristisch standhält, das weiß ich nicht. Ist, glaube [ich], aber auch nicht so wichtig. ${ }^{877}$

In der Vergangenheit kam es durchaus schon vor, dass sich ein Junggesellschafter bei einem der Familientage im Ausland unangemessen verhielt. Dieser wurde daraufhin vor den Familienrat vorgeladen und musste Rechenschaft über sein Verhalten ablegen. Dies zeigte zwar grundsätzlich seine Wirkung, doch wirklich eingefangen konnte diese Person nicht werden. Sein häufiges Fehlen an den Familientagen unterstreicht sein Desinteresse. Britta Brunai ist davon überzeugt:

"[d]as ist natürlich auch immer eine charakterliche Frage und auch eine Erziehungsfrage. Ich will uns jetzt nicht als gutes Beispiel hinstellen, aber wir haben unsere Kinder zum Beispiel nicht in die Lage versetzt, dass sie die Ausschüttungen abgreifen. Die haben überhaupt gar keinen Zugang zu ihren, obwohl sie das rechtlich natürlich dürften. Aber da ist bei uns eine ganz klare Regelung, dass sie auf eigene Füße kommen müssen, dass sie ihren Beruf ergreifen müssen. ${ }^{878}$

Moralische Verbindlichkeit wird nach Ansicht von Britta Brunai »bis zu einem gewissen Alter über die Eltern « geschaffen. Erziehung ist für sie das "A und O. « ${ }^{879}$ Danach müsse das der Familienrat übernehmen:

»Also das sehen wir einfach in unserer Familie, dass die Erziehung über die Eltern eine ganz wichtige Rolle spielt. Aber halt nur bis zu einem gewissen Alter und ab da müssen dann wir im Familienrat das dann übernehmen, weil ab einem gewissen Alter ist das dann kontraproduktiv, was die Eltern sagen. [...] Also das muss man schon auch relativieren. Aber Erziehung ist schon eine ganz essenzielle Sache. Ja. ${ }^{880}$

Benjamin Brunai sieht die moralische Verbindlichkeit etwas differenzierter, wenn er sagt:

»Ich glaube zunächst mal, schafft man die ja nicht. Ich würde wieder sagen, das ist, glaube ich, ein Weber'scher Begriff vom regulativen Ideal. Ich glaube, ich kann ein regulatives Ideal schaffen. So sollen wir sein und dann ist das, wie soll ich sagen, dann hat das eine gewisse Attraktion zu sagen, so agieren wir. ${ }^{881}$

877 Interview B-2 (06.11.2017), S. 6.

878 Interview B-1 (17.10.2017), S. 9.

879 Interview B-1 (17.10.2017), S. 16.

880 Interview B-1 (17.10.2017), S. 16.

881 Interview B-2 (06.11.2017), S. 11. 
Es wird dabei darauf angespielt, dass die in der Familienverfassung getroffenen Regeln für die betroffenen Familienmitglieder attraktiv erscheinen und nachvollziehbar sein müssen. Moralische Akzeptanz setzt dann voraus, dass man den Nutzen in der Einhaltung auch erkennen kann und die Regeln als vernünftig bewertet.

\subsection{Fallstudie 3: Unternehmerfamilie Calari (C)}

Umsatzkategorie: 1
Anzahl der Familienmitglieder: 12

Generationen: 4

Interviewpartner: Gesellschafter, Vors. d. Verwaltungsrats (C-1), 2. Gen.

Gesellschafter, Assistent der GL (C-2), 3. Gen.

Auswertbare Unterlagen: Keine

\subsubsection{Entstehung und Erstellung der Familienverfassung}

Das Unternehmen, das Mitte des 20. Jahrhunderts in Deutschland gegründet wurde, besteht aus zwei Gesellschafterfamilien, wobei eine der Familien im benachbarten, europäischen Ausland lebt. Während die Übergabe von der ersten auf die zweite Generation "noch ganz informell ${ }^{882}$ ablief, war für den Vater von Constantin Calari (C-2) und seinen Onkel Clemens Calari (C-1) klar, »dass man das irgendwie regeln muss ${ }^{883}$, wenn nun die dritte Generation größer wird und vielleicht die ersten Vertreter dieser Generation ins Unternehmen kommen:

"Ja, es war etwas zwischen meinem Bruder und mir. Das schwelte schon die ganze

Zeit. Wir wollten gerne das, was bei uns durch meinen Vater gegründet worden ist, in irgendeiner Art und Weise nachvollziehbarer und für nachfolgende Generationen greifbarer gestalten. ${ }^{884}$

Es gab kein initiales Moment, das Auslöser für die Erstellung der Familienverfassung war; vielmehr war es ein schleichender Prozess. Mit Unterstützung durch zwei Berater (um die rechtlichen Implikationen beider Länder berücksichtigen zu können) wurden die beiden Familien durch den Prozess geführt. Die beiden Familien trafen sich über ein Jahr verteilt an etwa sechs bis sieben Wochenenden für jeweils eineinhalb Tage. An diesen Treffen nahmen bis auf die Mutter von Clemens Calari (aus Altersgründen) alle Familienmitglieder teil. Die vierte Generation existierte zum damaligen Zeitpunkt noch nicht:

882 Interview C-2 (10.11.2017), S. 1.

883 Interview C-2 (10.11.2017), S. 1.

884 Interview C-1 (02.11.2017), S. 1. 
»Das war uns auch wirklich wichtig. Das hat auch gut funktioniert. Und das war auch bei uns wirklich die Basis dann für die Akzeptanz der Familiencharta und der Inhalte, weil eben nicht in kleinen Grüppchen, kleinen Kreisen Sachen ausbaldowert wurden, die hinterher alle zu akzeptieren haben, sondern weil jeder an jeder Stelle, wo er wollte, seine Meinung einbringen und dazu sagen durfte. ${ }^{885}$

Über diesen Prozess sind die beiden Familien auch »nochmal zusammengewachsen. ${ }^{886}$ Während dieses Prozesses haben sich alle, vom »16-/17-Jährigen bis zum knapp 60-Jährigen, oder knapp über 60-Jährigen, [...] alle entsprechend mit ihrem Erfahrungshintergrund und ihrer Perspektive eingebracht. ${ }^{887}$ Widerstand in Bezug auf die Erstellung einer Familienverfassung gab es keinen, auch wurde die "grundsätzliche Sinnhaftigkeit $[\ldots]$ nie in Frage ${ }^{888}$ gestellt. Constantin Calari stellt fest,

»[...] dass die Charta dann zwar ein wichtiges und schönes Ergebnis war, aber eigentlich ja der Weg fast schon als Ziel beschrieben werden konnte. ${ }^{889}$

Hauptanliegen war, »dass das Unternehmen im Familienbesitz bleibt. ${ }^{890}$ Beide Interviewpartner gaben an, dass in der Familienverfassung das festgeschrieben wurde, was seit Gründergeneration ohnehin gelebt wird. Clemens Calari bezeichnete den Inhalt gar als "Summe gelebter Selbstverständlichkeiten « ${ }^{891}$ Constantin Calari ergänzte: »Natürlich angereichert [...] durch die Perspektiven [...] durch eine dritte Generation, die natürlich manche Aspekte anders sieht. ${ }^{892}$

Die Moderation wurde als hilfreich und notwendig empfunden; einerseits um wichtige Aspekte, die sich in der Praxis bewährt haben (»allein schon zum Beispiel eine Definition der Familie, enger Familienkreis, weiter Familienkreis. Wie ist es mit Adoptivkindern ${ }^{893}$ ), zu berücksichtigen und andererseits kann ein nicht zur Familie gehörender Dritter bei kontroversen Diskussionen als Moderator eingreifen und die Gruppe aus einer möglichen Sackgasse herausmanövrieren.

Auch wenn über einzelne Formulierungen teilweise heftig diskutiert wurde, wird das Ergebnis als rund empfunden. Es seien ja nun "Spielregeln« entstanden, »die wir uns [...] im Konsensverfahren ${ }^{894}$ gegeben haben.

885 Interview C-2 (10.11.2017), S. 2.

886 Interview C-2 (10.11.2017), S. 3.

887 Interview C-2 (10.11.2017), S. 3.

888 Interview C-2 (10.11.2017), S. 3.

889 Interview C-2 (10.11.2017), S. $3 \mathrm{f}$.

890 Interview C-1 (02.11.2017), S. 3.

891 Interview C-1 (02.11.2017), S. 1.

892 Interview C-2 (10.11.2017), S. 4.

893 Interview C-1 (02.11.2017), S. 2.

894 Interview C-2 (10.11.2017), S. 21. 


\subsubsection{Inhalt, Institutionen und Alltag}

Die 23 Seiten umfassende Familienverfassung fußt auf dem Grundgedanken, das Unternehmen als Familienunternehmen zu erhalten:

»Und zwar am liebsten als familiengeführtes Familienunternehmen und als zweibeste Lösung, als fremdgeführtes Familienunternehmen und erst als allerletzte Möglichkeit der Verkauf. Ich glaube der Grundgedanke in Kombination mit den Werten, die wir da reingeschrieben haben, die das Miteinander mit uns definiert, das ist so der Kern. ${ }^{895}$

$\mathrm{Zu}$ den konkreten Inhalten gaben die beiden Interviewpartner nur wenig Auskunft. Es beschränkte sich auf einige Informationen: Das Dokument definiert und systematisiert u. a. den Begriff der Familie und sieht Maßnahmen bei groben Regelverstößen vor, die einen Ausschluss als Gesellschafter vermuten lassen. Auch beinhaltet sie Verfahrensvorschläge zur Kommunikation im Konfliktfall. Mit der Familienverfassung wurde ein "Familienverantwortlicher $"{ }^{896}$ eingeführt, der die Koordination der Familienangelegenheiten übernimmt. Darüber hinaus gibt es einen "Familiendelegierten« - als Bindeglied zwischen Unternehmen und Familie. Er »sollte, muss aber nicht unbedingt, aus einem Führungsgremium des Unternehmens kommen. ${ }^{897}$

Die Familienverfassung definiert auch einen Familientag, der nun zweimal im Jahr stattfindet:

»[...] auch mit dem zweimal im Jahr, mit dem Treffen, das hatten wir vorher nicht. Das ist ein sehr positiver Effekt. Weil wir durch die räumliche Trennung dann doch ein bisschen gehandicapt sind. ${ }^{898}$

Die Formulierung der Familienverfassung basiert vornehmlich auf Sollenssätzen:

»Wenn Sie so fragen, dann eher mit `soll - also mit `muss überhaupt nicht. Es ist mehr, dass wir `wollen`, wir sind `überzeugt, dass`, wir `sind der Auffassung « usw. « ${ }^{899}$

Das Regelwerk wird nicht als starr empfunden, sondern als etwas, das »lebt. ${ }^{900}$ Die Interviewpartner ließen keine Zweifel dran aufkommen, dass die Inhalte im Alltag gelebt werden. Sie haben »einen großen Einfluss auf den Alltag. ${ }^{901}$ Dies

895 Interview C-1 (02.11.2017), S. 4.

896 Der Familienverantwortlich ist vergleichbar mit dem Familienmanager, vgl. dazu Abschnitt 2.3.1.3.3.

897 Interview C-1 (02.11.2017), S. 8.

898 Interview C-1 (02.11.2017), S. 9.

899 Interview C-2 (10.11.2017), S. 15.

900 Interview C-1 (02.11.2017), S. 3.

901 Interview C-2 (10.11.2017), S. 7. 
wurde damit begründet, dass die Familienverfassung »im Prinzip die Niederschrift des täglich Gelebten « ${ }^{902}$ ist:

»Sie wird gelebt, automatisch, aber nicht, weil man sie sich dauernd unter das Kopfkissen legt oder nachgucken müsste, nach dem Motto, was muss ich denn jetzt eigentlich machen. In normalen, wichtigen Dingen brauchen wir da nicht reinzuschauen. Bei manchen Formalia dann schon. ${ }^{903}$

Die Mitarbeiter des Unternehmens wurden darüber unterrichtet, dass sich die Familie eine Familienverfassung gegeben hat:

»[F]ür die Mitarbeiter war das durchaus wichtig, dass es so etwas gibt. Sie haben das interessant gefunden [...]. Weil das unserer Meinung nach auch eine gewisse Sicherheit geben kann. Der Mitarbeiterschaft. Weil einfach die Nachfolge damit geregelt ist, kann man sagen. ${ }^{904}$

Die Regeln sind in der Weise zu befolgen, wie sie aktuell in der Familienverfassung festgeschrieben sind. Abweichungen davon werden - zumindest nach dem Wortlaut der Familienverfassung - nicht gestattet:

»Weil wir schreiben ja nicht da was rein, damit man hinterher davon abweicht. [...]. Wir schreiben was rein und daran wollen wir uns halten, sonst brauchen wir es nicht reinzuschreiben. « $^{905}$

Um dies sicherzustellen, wurde bei der Erstellung teilweise auch über einzelne konkrete Formulierungen diskutiert, wie diese denn ganz präzise zu verstehen seien. Das war Familie Calari besonders wichtig, um so ein gemeinsames Verständnis davon zu haben, eine gemeinsame Formulierung - um diese dann im Nachgang nicht interpretieren zu müssen.

Möchte man von den Regelungen abweichen, muss die Familienverfassung entsprechend angepasst werden. Die Möglichkeit ist durchaus vorgesehen, dass bei Bedarf eine Anpassung erfolgen kann. So möchte man vermeiden, dass es »Einzellösungen gibt. ${ }^{906}$

\subsubsection{Regelbefolgung, -brüche und moralische Verbindlichkeit}

Da die Familienverfassung erst seit wenigen Jahren im Einsatz ist, gab es noch keine Bewährungsprobe. Doch auch so hat es den Anschein, dass die Inhalte von den Familienmitgliedern eingehalten werden, da mit der Familienverfassung

902 Interview C-1 (02.11.2017), S. 4.

903 Interview C-1 (02.11.2017), S. 4.

904 Interview C-2 (10.11.2017), S. 8.

905 Interview C-2 (10.11.2017), S. 10.

906 Interview C-1 (02.11.2017), S. 5. 
lediglich etwas verschriftlich wurde, was ohnehin tradiert und gelebt wird. In diesem gemeinsamen Prozess wird auch eine präventive Maßnahme zur Regelbefolgung gesehen:

"Aber ich möchte auch hier eigentlich wieder auf den Prozess abheben. Ich glaube das präventive, dieses präventive Moment, das Sie ansprechen. Bei uns bestand dieses darin, dass wir es zusammen erarbeitet haben. « $^{907}$

Unabhängig davon hat die Familie durchaus auch für den Fall vorgesorgt, dass sich jemand nicht an die Vereinbarung hält. Für Clemens Calari ist ein Regelbruch,

»[w]enn man es nicht einhält. Das klingt jetzt ein bisschen flapsig. In der Tat jetzt, wenn man zum Beispiel gegen die Werte verstößt, wenn man offensichtlich gewisse Interessen vor andere stellt, was eigentlich anders vorgesehen ist. Das wäre ein Regelbruch und da geht es gar nicht so sehr ums Sanktionieren. Wir würden es besprechen und dann unter uns klären. ${ }^{908}$ "Ich bin, und das ist jetzt meine persönliche Meinung, und das ist jetzt nicht das, was die Charta aussagt, ich bin nicht für Sanktionieren von Fehlverhalten, sondern für Abstellen. Wenn Sie grundsätzlich Gutes beim anderen unterstellen, nicht zuletzt deswegen haben wir Werte in der Charta drin, dann kann ja ein Fehlverhalten nicht böswillig, sondern nur versehentlich sein, dann ist die Sanktionierung nicht der richtige Ansatz, sondern das >Darauf-aufmerksam-Machen und abstellen. ${ }^{909}$

Bei einem Regelverstoß wird, so die Ansicht von Constantin Calari, "gegen gelebte Werte, gegen Kultur « ${ }^{910}$, verstoßen. Das würde die Familie als Ganzes nicht tolerieren können:

»Da werden nicht zwei irgendwie in Clinch geraten, da wird wahrscheinlich die ganze Familie sagen: ^Hey du einer, der du da jetzt gerade dich eben nicht konform benommen hast, überleg dir das doch mal, was sollte das gerade.< Und natürlich gibt es Verhaltensweisen, die sind nicht strafbewehrt und es gibt Verhaltensweisen, auf die folgen dann Sanktionen. Das ist auch klar. ${ }^{911}$

Auf einen Regelverstoß folgt eine entsprechende "Konsequenz « ${ }^{912}$ - von Sanktion würde man in dem Fall gar nicht unbedingt sprechen wollen. Es sei keine Strafe, schließlich seien die Maßnahmen im Vorfeld allen hineinreichend bekannt; man hatte sich darauf im Kollektiv geeinigt und die Maßnahmen »als fair anerkannt. ${ }^{913}$ Bei den Maßnahmen geht es letztlich darum, "das Familienunter-

907 Interview C-2 (10.11.2017), S. 12f.

908 Interview C-1 (02.11.2017), S. $9 \mathrm{f}$.

909 Interview C-1 (02.11.2017), S. 12.

910 Interview C-2 (10.11.2017), S. 15.

911 Interview C-2 (10.11.2017), S. 15.

912 Interview C-2 (10.11.2017), S. 19.

913 Interview C-2 (10.11.2017), S. 20. 
nehmen auch zu schützen. ${ }^{914}$ Einen Strafcharakter möchte man in der Familienverfassung nicht verankert sehen. Constantin Calari beschreibt dies sehr deutlich:

"Jemand, der sich nicht an die Spielregeln hält, kann einfach nicht mitspielen, aber deswegen geben wir dem auch nicht noch eins drüber hinterher als Strafe. Sondern wer sich nicht an die Regeln hält, spielt dann einfach nicht mehr mit. Punkt. Das mag der dann persönlich, subjektiv als Strafe empfinden, das kann schon sein. Aber so ist es nicht gemeint. [...] Es gibt Spielregeln, daran hält man sich, wunderbar, hält man sich daran nicht, kann man nicht mitspielen. ${ }^{915}$

Da die Familienverfassung in Einklang mit dem Gesellschaftsvertrag steht (»Es gibt natürlich dann diverse darüber hinaus gehende Verträge, die das Ganze rechtlich absichern ${ }^{916}$ ), ist dieser Aussage zu entnehmen, dass offenbar für den Fall eines Querulanten (»Und, wie man so schön sagt, in den besten Familien, irgendwann gibt es halt einen Querulanten ${ }^{917}$ ), der nicht mehr einzufangen ist, eine Trennung aus dem Gesellschafterkreis vorgesehen wurde. Diese Person nicht mehr zum Familientag einzuladen, mit ihm aber bei der nächsten Gesellschafterversammlung an einem Tisch zu sitzen, wäre für Constantin Calari undenkbar. Maßnahmen bei Verstößen hält er dennoch für sinnvoll:

"Ja ganz allgemein, aber wirklich dann abstrahiert dann von der Charta und von der Familie: Wenn Sie jetzt mich persönlich fragen. Ja, natürlich, aber auch eher wieder in dem Sinne des vorher Gesagten. Jemand der sich nicht an Gesetze hält, der gehört natürlich in gewisser Weise sanktioniert. Aber auch nur schon deswegen, weil natürlich andere sehen müssen, ich halte mich daran, mir passiert nichts, halte ich mich nicht dran, passiert mir eben sowas wie dem, der sich auch nicht daran gehalten hat. Sonst funktioniert es nicht. ${ }^{918}$

Für Constantin Calari ist zudem wichtig, dass man im Erstellungsprozess der Familienverfassung über alles spricht und nicht gewisse Aspekte totschweigt. Hierzu gehört für ihn auch darüber zu sprechen, was passieren soll, wenn sich jemand nicht regelkonform verhält.

Unabhängig von dem sicherlich letzten Schritt, sich von jemandem aus dem Gesellschafterkreis zu trennen, sieht die Familienverfassung bei Auseinandersetzungen entsprechende Maßnahmen vor. Den Interviews war zu entnehmen, dass Empfehlungen zur Kommunikation im Streitfall festgeschrieben wurden. Darüber hinaus würde die Familie immer eine Mediation dort vorziehen, wo sich eine andere, öffentliche Auseinandersetzung vermeiden lässt:

914 Interview C-2 (10.11.2017), S. 20.

915 Interview C-2 (10.11.2017), S. 20.

916 Interview C-1 (02.11.2017), S. 9.

917 Interview C-2 (10.11.2017), S. 20.

918 Interview C-2 (10.11.2017), S. 23. 
"Schmutzige Wäsche wird intern und nicht extern gewaschen. ${ }^{919}$

Die Familienverfassung, das »höchste Werk oder die höchste Flugebene ${ }^{920}$, wird als etwas "Moralisches ${ }^{921}$ verstanden und

»[...] die Moral bindet einen, sich daran zu halten. Es gibt natürlich dann diverse darüber hinaus gehende Verträge, die das Ganze rechtlich absichern. ${ }^{922}$

Moralische Verbindlichkeit wird einerseits dadurch geschaffen, „dass alle mitarbeiten durften. Wer das unterschrieben hat, für den muss es eine moralische Bindung haben. ${ }^{923}$ Andererseits wird für Clemens Calari moralische Verbindlichkeit »durch Erziehung « ${ }^{924}$ erreicht:

»Moralische Verbindlichkeit ist eine Frage der Kinderstube. ${ }^{925}$

Constantin Calari ergänzte diese Aussage von seinem Onkel:

»Erziehung. Aufwachsen in der Familie. Ist sicherlich das Hauptinstrument, was aber nicht garantiert, dass es auch funktioniert am Ende. Aber ich kann auch für mich sagen, die Erziehung muss das leisten, sich die Vor- und Nachteile, die eine Zugehörigkeit zu einer Unternehmerfamilie bedeutet und mit sich bringt, klarzumachen. Den Kindern auch die Entscheidung freizulassen, inwiefern sie ins Unternehmen reinkommen möchten oder nicht, aber letztlich klar zu machen, zur Unternehmerfamilie gehören sie einfach dazu. [...] So, und dann wird über Erziehung hoffentlich bei Menschen, die eben diese Erarbeitung nicht mitgemacht haben, sichergestellt werden, dass sie sich damit - ich glaube, das ist das richtige Wort an der Stelle - identifizieren können. Da kann man ihnen nicht sagen: lies das und identifizier' dich. ${ }^{926}$

\subsection{Fallstudie 4: Unternehmerfamilie Deuther (D)}

\section{Umsatzkategorie: 1 Anzahl der Familienmitglieder: 6 \\ Generationen: 4}

Interviewpartner: Gesellschafterin, Mitglied d. GL, eingeh. (D-1), 2. Gen.

Gesellschafter, Mitglied d. GL (D-2), 3. Gen. Auswertbare Unterlagen: Businessfassung der Familienverfassung

919 Interview C-1 (02.11.2017), S. 11.

920 Interview C-2 (10.11.2017), S. 25.

921 Interview C-1 (02.11.2017), S. 9.

922 Interview C-1 (02.11.2017), S. 9.

923 Interview C-2 (10.11.2017), S. 14.

924 Interview C-1 (02.11.2017), S. 10.

925 Interview C-1 (02.11.2017), S. 10.

926 Interview C-2 (10.11.2017), S. 13. 


\subsubsection{Entstehung und Erstellung der Familienverfassung}

In dem in den 50er-Jahren des 20. Jahrhunderts gegründeten Unternehmen befinden sich heute bereits auch Vertreter der 3. Generation in der Geschäftsführung. Daniel Deuther (D-2), Vertreter der 3. Generation und auch Geschäftsführer des Unternehmens, berichtete, dass er ursprünglich mit seinem Vater vereinbart hatte, nur eine kurze Übergangszeit zusammen mit ihm in der Geschäftsführung arbeiten zu wollen, da sie festgestellt hatten, dass sie sich privat gut verstanden, "aber geschäftlich konnte es durchaus kriseln. ${ }^{927}$ Seinem Studium hatte er es zu verdanken, dass er eine Referentin kennenlernte, die über Familienstrategie referierte. Nachdem Daniel Deuther zwischenzeitlich klar geworden war, dass er in die Fußstapfen seines Vaters treten wollte, der nun auch den Wunsch hegte, in Rente zu gehen, hatte er eingesehen, dass er nicht umhinkommen würde, einige Zeit gemeinsam mit ihm $\mathrm{zu}$ arbeiten, um den Geschäftsbetrieb besser zu verstehen. Vor dem Hintergrund, dass dadurch bereits Auseinandersetzungen programmiert waren, schlug er seinem Vater vor, alles dafür tun zu wollen, dass es dann »doch ein reibungsloser Übergang “" ${ }^{928}$ wird. Es sollte dann auch die Referentin aus seinem Studium werden, die der Familie Deuther half, eine Familienstrategie und eine Familienverfassung zu erstellen. Konkreter Auslöser war also die Nachfolgeplanung im Hause Deuther, da die Familie neben Daniel Deuther noch drei weitere Kinder hat.

Der Prozess selbst dauerte mehrere Wochenenden, an denen sich die Familie traf. Die Unterstützung durch die Beraterin dabei war für Doris Deuther (D-1) elementar:

"Das hätten wir alleine nicht hingekriegt. Das war schon deswegen so interessant, weil sie eigentlich so Fragen gestellt hat, die eigentlich für uns klar waren. Und dann kam auf einmal eine Antwort, wo wir dann dachten, ah, da habe ich jetzt gar nicht damit gerechnet. ${ }^{929}$

An dem Prozess waren alle Familienmitglieder beteiligt. Auch die 1. Generation, die zu diesem Zeitpunkt bereits ihre Anteile übertragen hatte, folgte mit großem Interesse dem Prozess. Als die Familie an einem Zeitstrahl saß, der festlegen sollte, ab wann welches Kind ins Unternehmen einsteigen möchte, stieg Unmut bei einer der Töchter von Familie Deuther auf, die sich seinerzeit noch inmitten ihres Studiums befand. Bei ihr gab es keine grundsätzlichen Widerstände gegen die Einführung einer Familienverfassung, aber zum damaligen Zeitpunkt konnte und wollte sie noch keine klare Auskunft darüber geben, ob und wann sie in das Familienunternehmen einsteigen möchte. Sie fühlte sich gedrängt:

927 Interview D-2 (06.11.2017), S. 1.

928 Interview D-2 (06.11.2017), S. 1.

929 Interview D-1 (06.11.2017), S. 2. 
»Dann sagt sie snein, ich kann das nicht und ich will das nicht und ich möchte mich nicht so festlegen . Und das war dann auch für uns wichtig zu hören und zu wissen, dass sie eigentlich noch ganz frei entscheiden möchte, was zum damaligen Zeitpunkt auch absolut richtig war. So mitten im Studium. ${ }^{930}$

Aus der Retrospektive betrachtet zieht Daniel Deuther eine positive Bilanz:

\begin{abstract}
»Also vom Prozess her und vom Ergebnis her würde ich sagen sehr positiv. Im Prozess steckend, also gerade bei der Erarbeitung natürlich nicht immer angenehm; weil da werden ja, und das ist ja auch das Ziel, dass wirklich alle Themen auf den Tisch gepackt werden. Gerade auch die unangenehmen. Und dann wird es natürlich auch sehr emotional. Von super Stimmung bis Krisenstimmung hatten wir an diesen Wochenenden wirklich alles durchlebt. Ich sage aber, lieber von Vornherein auch Dinge klarstellen, auch wenn sie vielleicht enttäuschend sind und weh tun, als wenn man später in der Praxis feststellt, ich bin unter einer ganz anderen Voraussetzung hier reingekommen. ${ }^{931}$
\end{abstract}

Die Familienverfassung wurde am Ende des Prozesses von allen Familienmitgliedern unterzeichnet, die zu dem Zeitpunkt bereits 16 Jahre alt waren, und ist zum Zeitpunkt des Interviews, Ende 2017, etwa drei Jahre im Einsatz.

\title{
3.4.2 Inhalte, Institutionen und Alltag
}

Die 63 Seiten (hierzu zählen in der privaten Version auch zahlreiche Anhänge) umfassende Familienverfassung enthält Ausführungen zu den Themenkomplexen Werte, Ziele, Rollen und Institutionen. Die Werte der Familie (z.B. Liebe, Vertrauen, Zusammenhalt, Aufrichtigkeit etc.) werden den Werten im Unternehmen (Unabhängigkeit, Vorsichtigkeitsprinzip, Nachhaltigkeit etc.) gegenübergestellt. Bei den Zielen wurde gleich verfahren. Die Ziele der Familie (z.B. Erhalt des Familienfriedens, Integration von Ehe- und Lebenspartnern, Raum für die Familie etc.) stehen den Zielen und Visionen für das Unternehmen (Erhalt des Unternehmens als Familienunternehmen, nachhaltiges Wachstum, Erhalt der Handlungs- und Entscheidungsfähigkeit etc.) gegenüber. In der Sektion »Rollen« finden sich Ausführungen zum Gesellschafterdasein (z.B. Beteiligungsnachfolge, Ehe- und Erbverträge, Ausscheiden, Informationspflichten etc.), zur Geschäftsführung (Zahl der Geschäftsführer sowie der Familienmitglieder in der Geschäftsführung), grundsätzlich zur Mitarbeit von Familienmitgliedern im und Tätigkeiten für das Unternehmen sowie zu Ehe- und Lebenspartnern (Ehepartnern ist es demnach nicht gestattet, Anteile zu halten oder im Unternehmen operativ tätig zu werden).

930 Interview D-1 (06.11.2017), S. 3.

931 Interview D-2 (06.11.2017), S. 2. 
Mit der Familienverfassung wurden Institutionen ins Leben gerufen. Einmal jährlich findet verpflichtend für grundsätzlich alle Familienmitglieder ein Familientag statt, der »dem Erhalt und der Weiterentwicklung der familiären Einheit wie der konstruktiven Zusammenarbeit, außerdem der Heranführung der nachwachsenden Generation und der Vermittlung von Kenntnissen über das Unternehmen ${ }^{932}$ dienen soll. Teilnehmen dürfen alle Gesellschafter mit ihren Ehe- bzw. Lebenspartnern und den Kindern, wobei nur Lebenspartner von Familienmitgliedern, die mindestens 26 Jahre alt sind, teilnehmen dürfen, solange die beiden schon mindestens drei Jahre in eheähnlicher Gemeinschaft leben. Bei einem gemeinsamen Kind ist eine sofortige Teilnahme erlaubt. Der Familientag wird bei Familie Deuther auch dafür genutzt, die Familienverfassung auf Aktualität zu prüfen oder besondere Vorkommnisse, beispielsweise Regelbrüche, zu besprechen:

"Also wir hatten schon die Situation, wo man meiner Meinung nach davon abgewichen ist, wo ich dann gemeint habe, dann nehmt den Punkt mit zum nächsten Familientag. Meiner Meinung [nach] ist der Familientag halt dafür da, um auch die Familienverfassung wieder zu betrachten und zu schauen, ist [sie] noch relevant oder muss man [sie] irgendwie anpassen. ${ }^{933}$

Die Beraterin, die Familie Deuther dabei unterstützte, die Familienstrategie zu erarbeiten und die Familienverfassung zu erstellen, wird zu jedem Familientag als Moderatorin eingeladen (»[...] da es bei uns dann oftmals emotionale Diskussionen sind, haben wir gesagt, ist immer besser mit Moderator. ${ }^{934}$ ). Der Familientag ist für Daniel Deuther besonders wichtig, auch schon allein um die Situation für später zu üben, wenn die Familie dann einmal stark gewachsen sein wird:

»[Der] Familientag besteht bei uns, um die Gesellschafter abzuholen. Alle Gesellschafter, auch solche, die nicht im Unternehmen tätig sind. Damit sie quasi up to date sind, was im Unternehmen passiert und um sie ans Unternehmen zu binden und aber auch mit denen dann am zweiten Tag losgelöst von Firmenthemen irgendwas zu unternehmen, was irgendwie Spaß macht und einen als Familien zusammenschweißt. Jetzt wir sind aber ein Familienstamm, das heißt, man braucht es noch gar nicht. [...] Trotzdem haben wir gesagt, jetzt ist die Zeit, es zu üben. Weil später, wenn es dann verschiedenen Familienstämme sind, dann ist es umso schwieriger, wenn man es vorher nicht geübt hat. ${ }^{935}$

Neben dem Familientag sieht die Familienverfassung als Institution einen "Hüter der Charta» vor, welcher "Änderungen und deren Einarbeitung in

932 Familienverfassung der Familie Deuther, S. 13.

933 Interview D-2 (06.11.2017), S. 5.

934 Interview D-2 (06.11.2017), S. 6.

935 Interview D-2 (06.11.2017), S. 5 f. 
sämtliche Exemplare der Charta ${ }^{936}$ veranlasst. Er sorgt dafür, dass jedes Familienmitglied eine aktuelle Fassung erhält und führt ein Register darüber.

Jedes junge Familienmitglied erhält zu seinem 16. Geburtstag ein Exemplar der Familienverfassung von seinen Eltern überreicht und ausführlich erläutert. Künftige Ehepartner erhalten ein Exemplar zur Verlobung. Lebenspartner mit der Teilnahme am Familientag.

Die Familienverfassung erhält in ihrer Schlussbemerkung die Möglichkeit, sie bei Bedarf an neue Erfordernisse anzupassen. Grundsätzlich ist dafür der Familientag vorgesehen.

Im Anhang befinden sich unter anderem auch »Regeln für den Umgang miteinander «, die die Kommunikation innerhalb der Familie regeln und auch Regeln für den Umgang miteinander im Konfliktfall vorsehen. Hier ist beispielsweise die »48-Stunden-Regel« vermerkt: »Andererseits sprechen wir Vorfälle, die uns geärgert haben, zeitnah an (»48-Stunden-Regel«).« ${ }^{937}$

Die Familienverfassung nimmt im Alltag - zumindest im Unterbewusstsein eine wichtige Rolle ein. Die gemeinsame Erarbeitung ist dabei der Schlüssel, der ein »besseres Zusammenarbeiten « ${ }^{938}$ förderte:

»Innerhalb der Familie, was ich gerade eben gesagt hatte, dadurch, dass wir es erarbeitet haben, bestimmt es das Miteinander. ${ }^{939}$

\subsubsection{Regelbefolgung, -brüche und moralische Verbindlichkeit}

Da die Familienverfassung erst seit wenigen Jahren verabschiedet ist, kam es bislang zu keinen gravierenden Regelbrüchen. Doris Deuther erinnert sich jedoch, dass im Alltag in entsprechenden Situationen durchaus Bezug auf die Familienverfassung genommen wird:

"Also, bis jetzt hat es noch nicht zu Regelbrüchen geführt. Wir versuchen schon uns da dran zu halten und irgendeiner, wenn irgendwas nicht so richtig läuft, sagt dann immer, aber hier steht es drin. [...] Wenn zwei sich gerade streiten, wie es aktuell ist, dann habe ich gerade jetzt gesagt, aber hier steht drin, jetzt innerhalb von 48 Stunden sollte man das geklärt haben. ${ }^{940}$

Bei einem Regelbruch würde man zunächst versuchen, es selbst zu lösen. Im Zweifel würde man die Situation - sofern es sich zeitlich aufschieben lässt - am

936 Familienverfassung der Familie Deuther, S. 14.

937 Familienverfassung der Familie Deuther, S. 18.

938 Interview D-2 (06.11.2017), S. 3.

939 Interview D-2 (06.11.2017), S. 4.

940 Interview D-1 (06.11.2017), S. 8. 
Familientag mit der Beraterin besprechen und um Hilfe bei der Problemlösung bitten. Die Beraterin fungiert dann als Mediatorin.

Ein Regelbruch wäre für Doris Deuther ganz klar ein "Vertrauensbruch ${ }^{941}$, insbesondere vor dem Hintergrund, dass die Familienverfassung auf »Liebe und Vertrauen ${ }^{942}$ basiert.

Über gravierende Regelbrüche und den richtigen Umgang mit diesen hat man seither nicht nachgedacht, würde aber in jedem Fall eine außergerichtliche Lösung präferieren:

»Da haben wir kein Werkzeug und da kann ich Ihnen jetzt auch gar nicht sagen, wie wir da jetzt uns verhalten würden. Ja. Interessant aber, das ist echt interessant. Wir haben da so eine rosa Brille auf, dass man da denkt, also so richtig Brutales macht keiner. [...] Also so lange wie möglich außergerichtlich. Klar. ${ }^{943}$

Für gravierende Regelbrüche, die sich unternehmensgefährdend auswirken können, sei der Gesellschaftsvertrag einschlägig, der bei grob fahrlässigem Verhalten auch einen Ausschluss vorsieht:

"Also, Regelbrüche, die sanktioniert werden müssen bzw. die unternehmensgefährdend sind, tatsächlich auch große Auswirkungen auf das Unternehmensgeschäft haben, ich meine, solche Themen sind im Gesellschaftsvertrag verankert, da ist es dann auch rechtlich bindend, das hat dann auch Konsequenzen. $"{ }^{44}$

In der Familienverfassung seien hingegen eher die weichen Themen geregelt. Verstößt nun jemand gegen Werte, beispielsweise gegen den Wert der Bodenständigkeit, ist man sich nicht sicher, ob dies gleich sanktioniert werden muss oder man da nicht besser ein "verständnisvolles Gespräch « ${ }^{945}$ führt und sagt: »[W]ir hatten uns doch damals darauf verständigt, dass wir so und so innerhalb der Familie nach außen wirken wollen, da passt jetzt irgendwie dein neuer Ferrari nicht dazu. ${ }^{946}$

Bei einem groben Verstoß in Bezug auf die Familienverfassung würde Daniel Deuther unterscheiden, ob dieser »böswillig, mutwillig « ${ }^{947}$ erfolgte oder eher aus Unachtsamkeit oder vielleicht auch wiederholt. Sanktionen seien nicht wirklich Teil der Familienkultur:

»... Sanktionen sind nicht wirklich Teil der Familienkultur, also da muss schon extrem viel passieren, dass wirklich ... also Sanktionen als Gespräch, klar, immer, da spricht auch überhaupt nichts dagegen, egal, ob mutwillig oder nicht, aber wirklich, dass man

941 Interview D-1 (06.11.2017), S. 16.

942 Interview D-1 (06.11.2017), S. 16.

943 Interview D-1 (06.11.2017), S. 16.

944 Interview D-2 (06.11.2017), S. 10.

945 Interview D-2 (06.11.2017), S. 10.

946 Interview D-2 (06.11.2017), S. 11.

947 Interview D-2 (06.11.2017), S. 12. 
so weit geht, dass es tatsächlich den Familienfrieden zerrütten kann, da muss schon sehr viel passieren. ${ }^{948}$

Bei entsprechenden Auseinandersetzungen greift man gerne auch auf einen Mediator zurück, ein früheres Beiratsmitglied des Unternehmens:

»Und das ist dann so derjenige, der Mediator, wenn es dann mal richtig gekracht hat und man jetzt nicht auf den Familientag warten möchte, [...] oder zwischen zwei Familienmitgliedern, wenn es mal wirklich ziemlich gekracht hat, dann trifft man sich mit dem. ${ }^{949}$

Moralische Verbindlichkeit wird bei Familie Deuther durch den Umgang miteinander geschaffen:

"Wie haben wir es geschafft? Indem noch keiner den anderen richtig schlecht behandelt hat. Also, dass einfach so ein Vertrauen da ist; okay, wir streiten uns, und klar, da kracht es auch mal. Aber es ist nicht so, dass jetzt so ein Vertrauensbruch auf Dauer da ist. [...] Oder irgendwie so, dass es da kein Zurück gibt. Das hatten wir noch nie. « $^{950}$

Daniel Deuther fasst es etwas weiter:

"Ich glaube, es ist wichtig, dass alle Familienmitglieder wissen, warum man diese Familiencharta erstellt hat bzw. was in Familien passiert, wenn solche Themen nicht geregelt sind. [...] Wenn man so argumentiert und alle wissen, dass mit so einer Familiencharta das Risiko miniert wird, glaube ich, dass man dann auch eher als Familienmitglied gewillt ist, sich an diese Regeln zu halten. ${ }^{951}$

Als präventive Maßnahme zur Einhaltung der Regeln wird bereits die Art und Weise der Erstellung einer Familienverfassung gesehen. Darüber hinaus die Tatsache, sich immer wieder mit den Inhalten und den Regeln zu beschäftigen. Der Familientag sei dafür mit entscheidend:

»Ich glaube, schon die Art und Weise der Erstellung stellt schon mal präventiv sicher, dass sich auch daran gehalten wird. Weil es waren alle beteiligt, es wurden alle gehört und jeder hat seine Unterschrift darunter gesetzt. Allein dadurch hat man sich irgendwie von sich aus committed. [...] Ich glaube, später ist es dann tatsächlich der Fall, zu sagen, sich so viel wie möglich mit dem Thema beschäftigen, immer wieder die Leute hören, anpassen, so dass jeder für sich das quasi als Regelwerk sieht, was er auch selbst akzeptiert, weil durchsetzen werden wir es nicht können, auch nicht wollen. Der Familientag ist da, glaube ich, echt der Schlüssel « ${ }^{952}$

948 Interview D-2 (06.11.2017), S. 12.

949 Interview D-2 (06.11.2017), S. 14.

950 Interview D-1 (06.11.2017), S. 18.

951 Interview D-2 (06.11.2017), S. 9.

952 Interview D-2 (06.11.2017), S. 9. 


\subsection{Fallstudie 5: Unternehmerfamilie Eichmyller und Erhardt (E)}

Umsatzkategorie: 4

Anzahl der Familienmitglieder: ca. 100

Generationen: 5

Interviewpartner: Gesellschafterin, Mitglied d. Familienrats (E-1), 4. Gen. Gesellschafter, Ehem. Vors. d. Verwaltungsrats (E-2), 3. Gen.

Auswertbare Unterlagen: Keine

\subsubsection{Entstehung und Erstellung der Familienverfassung}

Die zu Beginn des 20. Jahrhunderts gegründete Unternehmensgruppe gehört zwei nicht blutsverwandten Gesellschafterfamilien: Familie Eichmyller und Familie Erhardt. Dass sich die beiden Unternehmerfamilien für eine Familienverfassung entschieden haben, hatte mehrere Gründe. Zum einen war es die zunehmende Anzahl an Gesellschaftern.

»[...] die Quantität der Gesellschafter wird auch größer und dadurch auch die Diversität innerhalb der Gesellschafter. [...] Vor vier bis fünf Jahren gab es auch nochmals einen Ruck, das war auch erbschaftsteuerbedingt, dass man relativ viele neuen Gesellschafter 'plötzlich` willkommen heißen durfte. ${ }^{953}$

Ein weiterer Auslöser wurde im Generationenwechsel gesehen. Enno Erhardt (E-2) kannte als Enkel die beiden Gründer noch persönlich. Die Entfernung zu den Gründern werde jedoch immer größer. Inzwischen gehe es schon in Richtung Ur-Ur-Enkel. Früher kannte man sich noch persönlich, es war alles sehr ländlich geprägt. Teilweise seien sie zusammen in die Schule gegangen. Heutzutage ist die Familie weit verzweigt, und dies nicht nur in Deutschland:

"... bei uns ist ja die Tradition, dass es eben immer eine große Nähe zum Unternehmen, auch zu dem Vermächtnis der Gründer usw. gibt. Also diese Identifikation. Natürlich mit dieser beschriebenen Entwicklung gerät die in Gefahr, das ist ja logisch. Abstand Menge. ${ }^{954}$

Enno Erhardt, Familiensprecher der Familie Erhardt und langjähriger Vorsitzender des Verwaltungsrats, machte sich irgendwann mit seinem Kollegen aus der Familie Eichmyller auch Gedanken über die Zeit danach:

»Und dann kam natürlich auch der Gedanke auf. Achtung. Irgendwann sind wir ja nicht mehr da. Die `Binde- und Klebewirkung`, die wir haben, auch die Autorität, die wir

953 Interview E-2 (22.11.2017), S. 1.

954 Interview E-2 (22.11.2017), S. 1. 
persönlich noch durch unseren Hintergrund ausstrahlen, irgendwann ist sie nicht mehr da. ${ }^{955}$

Man war sich in der Familie darüber einig, dass man etwas benötigt, das die »Kohäsion der Gesellschafter fördert « und mit einhergehend auch die »Identifikation der zunehmenden Anzahl der Gesellschafter mit dem Unternehmen« herstellt und erhält. ${ }^{956}$

»[...] und dann war irgendwie ganz klar, dass wir irgendwas brauchen, was so die Werte und die Gedanken der Gründer damals für die nächsten Generationen einmal festhält, was vielleicht auch eine geordnete Zusammenarbeit und Gesellschaftersein definiert $[\ldots] .{ }^{457}$

Für Enno Erhardt spielte noch ein weiterer Grund eine Rolle. Seiner Auffassung nach steigert eine Familienverfassung die Attraktivität als Arbeitgeber:

»Wir wollen ja als Familienunternehmen attraktiv sein. Das müssen wir auch, aus Gründen des Arbeitsmarktes, Kampf um die Talente. Und der Gedanke war natürlich auch der, dass man sagt, wenn wir eine Familienverfassung haben, das ist ein Pfund, mit dem wir wuchern sollten. [...] Die Business-Version, da gehen wir sehr großzügig damit um. Also wir haben die zum Beispiel dem Bürgermeister übergeben, dem örtlichen Bürgermeister. Ist ja auch wichtig, ganz formal haben wir die Charta übergeben und dann auch alle leitenden Mitarbeiter bekommen und haben Zugriffe auf die BusinessVersion der Charta [...]. ॥ $^{98}$

Beide Familien haben sich dafür entschieden, den Weg der Erstellung zusammen mit einem Berater zu gehen. Zu einem Kick-off-Meeting wurden alle Familienmitglieder - auch die Nicht-Gesellschafter - eingeladen:

"[...] denn eine Erkenntnis aus dieser Beratung war auch, dass man nicht nur die Gesellschafter, sondern auch die Partner der Gesellschafter miteinbezieht. Die nennen das Familienverbund. [...] der Hintergrund ist aber auch nachvollziehbar, weil natürlich Partner, also Ehepartner oder auch sagen wir mal Lebensgefährten, die jetzt gemeinsame Kinder haben, gemeinsamen Hausstand, die haben Einfluss auf ihre Partner, die Gesellschafter sind. « $^{959}$

Man hat sich dann im Kollektiv auf die Themenblöcke verständigt und den großen Familienkreis in Gruppen aufgeteilt, die die einzelnen Themenblöcke bearbeiten sollten. Themen und Ergebnisse wurden dann in einem sehr systematischen Prozess zusammengeführt. Der Prozess dauerte in Summe etwa zwei Jahre. Man wollte bewusst möglichst alle Familienmitglieder einbinden:

955 Interview E-2 (22.11.2017), S. 2.

956 Interview E-2 (22.11.2017), S. 2.

957 Interview E-1 (21.11.2017), S. 1.

958 Interview E-2 (22.11.2017), S. 2 f.

959 Interview E-2 (22.11.2017), S. 6. 
»Wenn wir das von oben verordnen und dann runterspiegeln, das ist nicht ausreichend. Also wir wollten das bottom up machen $\left[\ldots . . .^{960}\right.$

Schwierigkeiten hatte man dabei, die Familienmitglieder einzubinden, die nicht in Deutschland lebten. Man stellte insbesondere fest, dass es generell dadurch eine Informationslücke gab, die man im Zuge der Erstellung behoben hat: Eine Gesellschafterplattform wurde aufgesetzt.

Insgesamt hat der Prozess dazu beitragen, dass die beiden, nicht blutsverwandten Familien sich nun als eine Großfamilie sehen. Emilia Eichmyller (E-1) denkt gerne zurück an die Zeit der Erstellung:

»Ich glaube, wenn ich jetzt zurückblickend sage, 2010, wenn man da so das erste Mal dabei ist, aus meinem persönlichen Empfinden heraus, hat man viele mit Sie und Nachname angesprochen, gerade von der anderen Familienseite, wohingegen das jetzt ein per Du und beim Vornamen ist. $" ~ "{ }^{961}$ - Es ist viel menschlicher geworden. Also davor, es ist, glaube ich das, was man oft mit so zwei Stämmen hat, dass man sagt >die und ‘wir`, und das ist wirklich gar nicht mehr spürbar. ${ }^{962}$

Wirklichen Widerstand in den beiden Großfamilien gegen die Erstellung einer Familienverfassung gab es nicht. Es gab vereinzelt ein paar kritische Stimmen, die die Sinnhaftigkeit einer solchen Familienverfassung zu Beginn anzweifelten. Beim Prozess selbst haben dann aber alle »ausnahmslos ${ }^{963}$ mitgewirkt. Die Familienverfassung wurde nach Fertigstellung 2013 von allen Mitgliedern des Familienverbunds in einem affirmatorischen Akt unterzeichnet.

\subsubsection{Inhalt, Institutionen und Alltag}

Die 13 Seiten umfassende Familienverfassung folgt dem bekannten Dreiklang: »Werte, Ziele, Rollen«. Sie beginnt damit, Werte des Familienverbundes zu definieren (z. B. Vertrauen, Freiraum für Unternehmertum, Fairness usw.). Es werden Ziele für den Familienverbund beschrieben; neben anderen, dass man Familienunternehmen bleiben möchte:

"[...] mit der wichtigste Satz in der Verfassung ist natürlich der, dass wir ein Familienunternehmen bleiben wollen. Dass wir keine Anteile verkaufen wollen. Wir können nicht verhindern, dass einer Anteile verkauft. Das können wir nicht verhindern. Ich kann jetzt nicht sagen, Moment einmal, du kannst keine, du hast hier erklärt, dass. [...] Also das ist eine moralische Keule, natürlich spielen gruppendynamische Prozesse

960 Interview E-2 (22.11.2017), S. 4.

961 Interview E-1 (21.11.2017), S. 3.

962 Interview E-1 (21.11.2017), S. 6.

963 Interview E-2 (22.11.2017), S. 7. 
dabei auch eine Rolle, wenn jemand ausbüchst und dann alle anderen gegen ihn sind, da kommt man ja ganz schön ins Schwitzen. Das spielt dann schon auch eine Rolle. ${ }^{964}$

In der jüngeren Generation war man sich einig, dass man nicht mehr nur vom Unternehmen leben möchte, auch da sich die Anteile mit den Generationen so stark verteilen, dass das gar nicht mehr möglich ist. Es wurden Werte und Ziele für die einzelnen Unternehmen festgelegt (»Makroziele, das sind ja keine detaillierten Ziele, [...] ${ }^{965}$ ), die man als Familie den Unternehmen geben möchte. Das beinhaltet auch, welches Risiko man als Unternehmerfamilie für die Unternehmen akzeptieren möchte:

»Wir haben natürlich ein bisschen eine sehr gewachsene traditionelle Kultur. Da gibt es dann so Sätze wie, `man soll immer nur so weit ins Wasser gehen, dass man seine Füße noch sehen kann`. Das ist so ein typischer Spruch bei uns. ${ }^{966}$

Ein weiterer wichtiger Baustein sind auch die Ausführungen zu den »Rollen«: wie man als Familie mit den Unternehmen zusammenarbeiten möchte, wer unter welchen Voraussetzungen welche Rolle in einem der Unternehmen einnehmen kann. Dann werden die Institutionen erläutert, die mit der Familienverfassung ins Leben gerufen wurden:

»Ja, ich glaube, dieses klare Regelwerk an Institutionen und wie die Zusammenarbeit zwischen den Gesellschaftern und dem Unternehmen aussieht, das waren so die Knackpunkte, wo man wirklich gemerkt hat, hier passiert etwas. ${ }^{967}$

Die Familienverfassung regelt klar, welches Familienmitglied wann und wo teilnehmen darf:

"[...] also gerade, wenn es darum geht, wer nimmt an den Gesellschafterversammlungen teil, dass es klar ist, da gab es dann oft die Frage, ab wann ist denn ein Lebenspartner, Lebensabschnittsgefährte, wenn man nicht verheiratet ist zum Beispiel, dann berechtigt, da auch teilzunehmen an Versammlungen [...].. ${ }^{968}$

Auch ist in der Familienverfassung ein Verfahren festgelegt worden, wie der Verwaltungsrat zu besetzen ist. Gerade bei diesem Thema wird die Familienverfassung immer mal wieder konsultiert.

Dem Unternehmen wurde eine abgespeckte Business-Version der Familienverfassung ausgehändigt, was für Emilia Eichmyller wichtig ist:

»Das ist auch etwas, was im Unternehmen kommuniziert wurde, und dem Unternehmen wurde eine abgespeckte Form der Charta übergeben, sodass Transparenz herrscht,

964 Interview E-2 (22.11.2017), S. 8.

965 Interview E-2 (22.11.2017), S. 8.

966 Interview E-2 (22.11.2017), S. 9.

967 Interview E-1 (21.11.2017), S. 4.

968 Interview E-1 (21.11.2017), S. 6. 
was wir als Gesellschaftergruppe machen. Dass die es nicht losgelöst vom Unternehmen machen, sondern dass das da zusammenhängt. ${ }^{969}$

Als Institution wurde der Familientag ins Leben gerufen, der zu Beginn zweimal im Jahr stattfinden sollte. Zwischenzeitlich hat man jedoch festgestellt, dass dies nicht praktikabel ist. Die Familientage waren immer an die Gesellschafterversammlungen gekoppelt. Die Familienverfassung wurde dahingehend geändert, dass der Familientag nun nur noch einmal jährlich stattfindet. Der Familientag wird als besonders wichtige Institution gesehen:

"Das war natürlich genau das Medium, um sozusagen diese Kohäsion der Gesellschaft zu fördern. ${ }^{970}-$ »Weil, da ist eine Bombenstimmung, und die Gesellschafter, kreuz und quer, unterhalten sich und lachen viel und machen Programme zusammen und so weiter und sofort. Und ich sage mal, da entsteht natürlich so ein richtiges `Oberfamiliengefühl aller Gesellschafter und dieses Familiengefühl hat ja eine Kohäsionswirkung. Das soll sie auch haben, das ist ja kein Zufall, das ist eine Absicht. Und, wissen Sie, je enger und verbundener diese Familie, dieser Familienverbund ist, desto schwerer wird es, aus dem auszubrechen. ${ }^{971}$

Obwohl es gern gesehen wird, dass alle Mitglieder des Familienverbundes an den Familientagen teilnehmen, gibt es einzelne Personen, deren Präsenz eher gering ausfällt, sei es aus räumlichen Gründen (»[...] ich meine, man ist jetzt in London und kann vielleicht nicht extra rüberfliegen, weil es sich zeitlich jetzt nicht einrichten lässt. ${ }^{972}$ ) oder aus ganz persönlichen:

»Ein Beispiel wäre meine Schwester, die [...] das Ganze versteht und da auch emotional sehr verbunden ist, aber auf der anderen Seite zu der finanziellen Seite des Gesellschafterdaseins eine ganz gespaltene Haltung hat. Für sie ist das eher fast befremdlich, würde ich jetzt mal sagen. Für sie ist es dann so, dass sie natürlich an einem Tag der Gesellschafterversammlung dann vielleicht eher am Nachmittag dazu stößt, wenn es gegen Ende geht und dann am Familientag trotzdem dabei ist oder dem Ganzen auch fernbleibt und lieber ein langes Wochenende in die Natur geht. ${ }{ }^{973}$

Emilia Eichmyller, selbst Mitglied des Familienrats, sieht es allerdings realistisch und ist davon überzeugt, dass man diese wenigen Personen nicht mehr umstimmen kann. Entscheidend ist für sie, ein attraktives Programm für alle Generationen anzubieten und

"[...] unser Ziel ist es, die nochmal jüngere Generation, also die, die jetzt im Kindesalter sind, $[\ldots]$ in einer Weise zu motivieren, dass das toll ist, wenn man sich [...] sieht, und

969 Interview E-1 (21.11.2017), S. 5.

970 Interview E-2 (22.11.2017), S. 7.

971 Interview E-2 (22.11.2017), S. 15.

972 Interview E-1 (21.11.2017), S. 11.

973 Interview E-1 (21.11.2017), S. $11 \mathrm{f}$. 
man immer in den Kletterpark geht und Aktivitäten zusammen macht, dass man sich dann gut kennt $[\ldots] \cdot \aleph^{974}$

Neben dem Familientag wurde der Familienrat ins Leben gerufen. Dieser wird alle vier Jahre neu gewählt und besteht aus acht Mitgliedern. Der Familientag ist derzeit noch paritätisch mit jeweils vier Personen pro Familie besetzt. Erste Überlegungen gehen allerdings schon dahin, dass man die paritätische Besetzung abschafft. Der Familienrat wird als »Organ der Familie ${ }^{975}$ gesehen und dabei klar vom Verwaltungsrat differenziert. Der Verwaltungsrat ist das Bindeglied für die Familien zum Unternehmen. Der Familienrat hingegen kümmert sich ausschließlich um den Familienverbund. Er hat die Aufgabe, den Zusammenhalt zu fördern, Versammlungen wie den Familientag zu organisieren oder sich auch um solche Familienmitglieder zu kümmern, die ein Praktikum in einem der Unternehmen machen wollen. Die Mitglieder des Verwaltungsrats können nicht gleichzeitig Mitglied im Familienrat sein. Hier wird klar getrennt. Allerdings dürfen (und sollen) die Verwaltungsratsmitglieder jederzeit den Sitzungen des Familienrats als Gäste beiwohnen. Der Familienrat wurde in verschiedene Ressorts aufgeteilt. Wenn nun Mitglieder des Familienverbundes in einzelnen Ressorts mitarbeiten möchten, so ist dies jederzeit möglich, auch ohne Mitglied des Familienrats zu sein. Der Familienrat entscheidet dann darüber.

Im Familienrat wurde ein "Hüter der Charta» benannt, der insbesondere sicherstellen soll, dass notwendige Änderungen auch umgesetzt werden. Grundsätzlich wird die Familienverfassung ohnehin alle vier Jahre auf Aktualität überprüft und ggf. angepasst. Eventuelle Änderungen müssen dann allerdings vom gesamtem Familienverbund verabschiedet werden.

Die Familienverfassung hat den Alltag verändert. Gerade die jüngeren Generationen wachsen ohne Stammesdenken auf. Für sie gibt es nur eine Großfamilie.

\subsubsection{Regelbefolgung, -brüche und moralische Verbindlichkeit}

Die Familie versucht offen zu sein, beispielsweise dadurch, dass niemand «verpflichtend ${ }^{976}$ an einem Familientag teilnehmen muss. Vornehmlich sind es ohnehin Vertreter der älteren Generation. Die Arbeit des Familienrats wird dabei als besonders wichtig empfunden, um bei der jüngeren Generation gleich zu Beginn präventiv etwas zu ändern:

974 Interview E-1 (21.11.2017), S. 12.

975 Interview E-1 (21.11.2017), S. 8.

976 Interview E-1 (21.11.2017), S. 10. 
$»[\ldots]$ ich finde, in der jüngeren Generation fällt auf, gerade auch durch die Arbeit der letzten Jahre, ist es eigentlich relativ gering, also ich sehe das eher so in der Altersklasse vielleicht so von 50 bis 60 . Wie wir damit umgehen? Ich glaube, wir versuchen offen $\mathrm{zu}$ sein. Wir sagen, niemand muss verpflichtend da sein. Denn lieber sind wir in dem Kreis zusammen, der gerne da ist, der sich gerne zusammen austauscht [...] Natürlich ist es schade um jeden, der die Möglichkeit nicht in Anspruch nimmt; auf der anderen Seite sehen wir die aber auch nicht als allzu große Gefahr, da wir in den zwei Pools [...] jeweils mit einer Stimme entscheiden und solange wir die Mehrheit davon überzeugen können, dass es wert ist, sich einzubringen und da einen aktiven Teil zu haben, solange sehen wir das, glaube ich, nicht als Problem an. Deswegen ist die Arbeit des Familienrats umso wichtiger, um genau diese Einstellung in den künftigen Generationen zu verhindern. ${ }^{977}$

Für Enno Erhardt ist es wichtig, dass die Erkenntnis gereift ist, auch die Partner der Gesellschafter, also den kompletten Familienverbund, miteinzubeziehen, da auch diese einen nicht unerheblichen Einfluss auf die Gesellschafter haben können:

»Und mit denen muss man auch rechnen. Es kann ja auch mal Situationen geben, einer stirbt und er hat das Sorgerecht und ähnliche Dinge. ${ }^{978}$

So stellt man sicher, dass auch sie hinter den Zielen und Werten der Familienverfassung stehen und nicht durch einen gefühlten Ausschluss aus der Gemeinschaft Dinge verhindern und blockieren.

Grundsätzlich sieht Enno Erhardt die "gruppendynamische Disziplinierung “ ${ }^{979}$, die besonders im Familientag zum Ausdruck kommt, als wichtiges präventives Instrument. "Je enger und verbundener diese Familie, dieser Familienverbund ist, desto schwerer wird es, aus dem auszubrechen. ${ }^{980}$

Die Tatsache, dass man die Inhalte der Familienverfassung - zumindest in einer Business-Fassung - publik gemacht hat, erhöht natürlich auch den Druck auf die Familie, sich regelkonform zu verhalten, zumal nun auch Außenstehende die Werte und Verhaltensregeln der Familien kennen.

Die Familienverfassung sieht keine Sanktionsinstrumente vor. Ein Gesellschafterausschluss oder ein Ausschluss aus dem Familienverbund ist nicht expressis verbis vorgesehen. Emilia Eichmyller ist davon überzeugt, dass ein Eingreifen in Konfliktsituationen über die jeweiligen Familienvertreter im Verwaltungsrat erfolgen würde.

"Also sicherlich haben die vier Vertreter der Gesellschafter im Verwaltungsrat eine besondere Aufgabe und Autorität. Also wenn da jetzt einer steuerlich ganz merkwürdige Sachen machen würde und in irgendwelchen komischen Dingen auftauchen

977 Interview E-1 (21.11.2017), S. 10.

978 Interview E-2 (22.11.2017), S. 6.

979 Interview E-2 (22.11.2017), S. 8.

980 Interview E-2 (22.11.2017), S. 15. 
würde, wer würde dann mit denen sprechen? Zunächst einmal, wenn es einer von den Eichmyllers wäre, die beiden Eichmyller-Vertreter, wenn es ein Erhardt ist, die beiden Erhardt-Vertreter [...]." ${ }^{981}$

Die Familienvertreter gelten sozusagen als Autoritäten der jeweiligen Familie und werden von den Gesellschaftern auch als solche anerkannt.

Sollten sich gewisse Szenarien häufen, so kann Emilia Eichmyller sich schon sehr gut vorstellen, dass man das Thema als Diskussionspunkt, ohne konkret werden zu wollen, bei einem Familientag aufnehmen würde. Strittige Themen würde man bevorzugt immer in der Familie klären. Eine gerichtliche Auseinandersetzung sei eher unvorstellbar. Vielmehr würde man jemanden, der permanent negativ in Erscheinung tritt, ermutigen, seine Anteile zu verkaufen:

»Ich kann mir das auch schwer vorstellen. Der wird dann so unter moralischen Druck kommen, er würde eigentlich von seiner ganzen Familie geächtet werden. Das muss er dann auch erst einmal aushalten. ${ }^{982}$

Ganz grundsätzlich ist Enno Erhardt wichtig, dass Fehlverhalten sofort adressiert werden. Es gelte in der Familie der Grundsatz: "Miteinander reden und nicht übereinander reden. ${ }^{983}$

Auf die Frage, wie man innerhalb der Familie ein Gefühl von moralischer Verbindlichkeit schaffen kann, wird Enno Erhardt sehr deutlich. Die Vorbildfunktion spiele eine entscheidende Rolle.

»Durch Vorbild - einerseits. [...] Vorbild spielt eine große Rolle, das heißt das Vorleben. Natürlich auch der Bezug zu den Gründern und ihren Werten. Also das Vermächtnis der Gründer. Und beide haben ja sehr hohe Werte gehabt [...]. Deswegen haben wir auch dieses Buch herausgebracht. Es hat jeder das Buch über die ganze Lebensgeschichte und Werdegang der beiden Unternehmerpersönlichkeiten. Dann gibt es eine [...] Stiftung [...] und Sie sehen auch, überall taucht immer mal wieder ein Bild von den Altvorderen auf usw. [...] es wird auch bei uns gepflegt. [...]. Das Vorleben, [...] geht natürlich auch in die nächste Generation. Das tue ich [...] eben andere auch, wissen Sie, das hat ja auch eine gewisse Sogwirkung. Diese moralische Kraft, die von den Gründern ausgeht, die dann in der nächsten Generation weitergelebt und -getragen wird; und das strahlt ja weiter, $[\ldots] . \aleph^{984}$

981 Interview E-2 (22.11.2017), S. 19.

982 Interview E-2 (22.11.2017), S. 21.

983 Interview E-2 (22.11.2017), S. 22.

984 Interview E-2 (22.11.2017), S. $16 f$. 


\subsection{Fallstudie 6: Unternehmerfamilie Feyner (F)}

Umsatzkategorie: 3

Anzahl der Familienmitglieder: ca. 25

Generationen: 5

Interviewpartner: Gesellschafterin, Beirat, operative Leitungsfunktion (F-1), 4. Gen.

Gesellschafter, Vors. d. GL (F-2), 3. Gen.

Auswertbare Unterlagen: Familienverfassung

\subsubsection{Entstehung und Erstellung der Familienverfassung}

Die Wurzeln des Unternehmens lassen sich zurückverfolgen bis zum Ende des 19. Jahrhunderts. Doch erst der Vater von Ferdinand Feyner (F-2) machte das Unternehmen zu dem, was es heute ist. Die Idee, der Familie eine Familienverfassung zu geben, entstammt dem expliziten Wunsch des Vaters von Ferdinand Feyner, der »sein Werk auf Ewigkeit [...] erhalten sehen möchte und seine 51 Prozent in eine Stiftung gegeben hat. ${ }^{985}$ Der Vater habe gewisse Vorstellungen vom Leben, auch davon, wie die Familienmitglieder sich zu verhalten haben. Ferdinand Feyner sieht die Familienverfassung als eine "Art Vermächtnis ${ }^{986}$. Er vermutet, dass die Erbauseinandersetzungen nach dem Tod seines Großvaters für seinen Vater einen bleibenden Eindruck hinterlassen haben, der keineswegs positiv konnotiert ist:

"Wahrscheinlich mit ein Grund, warum er es in die Stiftung reingetan hat, um solchen Streitigkeiten und den Erbauseinandersetzungen vorzubeugen. ${ }^{987}$

Die Familienverfassung wird als aufgezwungen empfunden (»Sie sehen, das ist schon ein bisschen von oben nach unten gekommen. ${ }^{988}$ ):

"[...] also ich bin mir nicht sicher, ob es irgendeinen Grund dafür gab, [...] irgendwann wurden wir damit konfrontiert, dass es so etwas gibt, die wurde uns dann auch vorgestellt, war dann so. ${ }^{989}$

Aus der Wahrnehmung der Enkeltochter, Friederike Feyner (F-1), die damals noch relativ weit weg vom Unternehmen war, gab es gegen die Einführung der Familienverfassung an sich keine Widerstände in der Familie:

985 Interview F-2 (27.11.2017), S. 1.

986 Interview F-2 (27.11.2017), S. 6.

987 Interview F-2 (27.11.2017), S. 31

988 Interview F-2 (27.11.2017), S. 5.

989 Interview F-1 (24.11.2017), S. 1. 
"Also in meiner Wahrnehmung gab es da keine Widerstände. Es wurde eher, wie soll ich sagen, positiv aufgefasst. Wir geben uns selbst eine Familiencharta. Das war irgendwie so etwas Tolles. ${ }^{990}$

Bei der Erstellung wurde die Familie nur bedingt eingebunden:

"Aber ich glaube, wenn da jemand etwas geschrieben hat, dann war es meine Mutter. [...] Die Themen hat man schon ein wenig diskutiert, aber das war teilweise [Name des Beraters] und mein Vater. ${ }^{991}$

Ferdinand Feyner sollte ursprünglich das Unternehmen erben. Heutzutage hält er zusammen mit seinen beiden Schwester 49 Prozent der Anteile, sein Vater 51 Prozent. Im Zuge der Übertragung der 51 Prozent in eine Familienstiftung wurde die Familienverfassung erstellt. Ferdinand Feyner ist sein Unmut über die Entwicklung anzumerken:

»Nein, man war nicht begeistert. Ich war nicht begeistert, weil man mir ja gesagt hat: Du wirst mal die Firma übernehmen. ${ }^{992}$

Zum Zweck der Erstellung findet man entsprechende Ausführungen in der Familienverfassung:

»Zweck [...] ist die Festlegung der tragenden Prinzipien der Familie Feyner für deren unternehmerisches Verhaltens. Die Familiencharta dient der Fixierung grundlegender Werte für die Familie, für das Unternehmen und für das Zusammenspiel zwischen Familie und Unternehmen. In diesem Sinne bezweckt die Familiencharta die Verhinderung, erforderlichenfalls die Schlichtung, von Interessensgegensätzen zwischen Familie und Unternehmen bzw. zwischen den verschiedenen Interessen der Familienmitglieder. Die Mitglieder der Familie Feyner haben sich darauf verständigt, dass ihr Familienunternehmen nach Möglichkeit in der/den Familie(n) Feyner erhalten bleiben soll $[\ldots] \cdot{ }^{993}$

Die Familienverfassung wurde ungefähr im Jahre 2012 verabschiedet und von allen Familienmitgliedern unterzeichnet.

\subsubsection{Inhalt, Institutionen und Alltag}

Die 13 Seiten umfassende Familienverfassung folgt vom Aufbau der bekannten Triade »Werte - Ziele - Rollen«. In der vorgeschalteten Präambel wird neben dem Zweck der Familienverfassung das Konsensprinzip eingeführt, wonach es der Einstimmigkeit bedarf, um die Familienverfassung zu ändern. Weiterhin

990 Interview F-1 (24.11.2017), S. 2.

991 Interview F-2 (27.11.2017), S. 3 f.

992 Interview F-2 (27.11.2017), S. 5.

993 Familienverfassung der Familie Feyner, S. 5. 
wird ausgeführt, dass die Familienverfassung »im Sinne eines ethischen Prinzips verpflichtend « sei. Es sei eine Absichtserklärung, an die sich die Familie Feyner halten wolle, »ohne, dass hieraus rechtlich einklagbare Verpflichtungen oder rechtlich einklagbare Schadensersatzansprüche oder sonstige Ansprüche entstehen können. ${ }^{994}$

Das Kapitel »Werte« beginnt mit einer Definition des Begriffs "Familie«. Ehepartner und Lebensgefährten von Gesellschaftern sind nach Definition der Familienverfassung keine »Familienmitglieder».

"[...] da haben sie immer Angst, da lässt sich jemand scheiden und dann wird das Unternehmen auseinandergerissen. Sie kennen doch die ganzen Schauergeschichten $\left[\ldots . . . .^{995}\right.$

Als Werte der Familie wurden »Eintracht, Fairness, Ehrlichkeit und Wahrhaftigkeit, soziale Verantwortung, verantwortungsvoller Umgang mit Vermögenswerten und Sparsamkeit « ${ }^{996}$ niedergeschrieben.

Nach den Werten für das Unternehmen und dem Einfluss der Familie auf das Unternehmen folgt das Kapitel »Ziele», welches sich unterteilt in "Ziele der Familie» ("gemeinsames Handeln fördern«, "nach Konsens streben «"7) und "Ziele des Unternehmens«. Die Familienverfassung stellt Erwartungen an die »Eigenkapitalverzinsung und -quote« sowie an die »Umsatzrentabilität«. Unter dem Punkt "Erwartungen an Ausschüttung" wird klargestellt, dass die Unternehmensinteressen im Zweifel vor die Individualinteressen zu stellen sind, will heißen, von den Familienmitgliedern wird erwartet, dass sie ihren »Lebensunterhalt außerhalb der Gewinnausschüttungen $"{ }^{998}$ verdienen:

»Die Handlungsanweisungen sind klar. Also es gibt zum Beispiel, man soll selbständig seinen Lebensunterhalt verdienen und nicht [...] von der Firma Feyner herunterleben. Das kommt da deutlich heraus. Das ist aber ein Punkt, das wird von einigen so nicht gemacht. Aber ich denke, da muss sich jeder selbst fragen, was will er, wie will er sein Leben gestalten und ja, dann ist auch, wie gesagt, wo kein Richter, da kein Henker. Es gibt bisher keinen, der sagt, aber du, guck mal, dass du auf eigene Füße kommst und nicht hier am Tropf von Feyner hängst. ${ }^{999}$

Der letzte Punkt der Familienverfassung trägt die Überschrift »Rollen«. Hier werden Regelungen zur »Mitarbeit von Familienmitgliedern« im Unternehmen getroffen. Grundsätzlich können Familienmitglieder demnach im Unternehmen eine Position bekleiden, wenn diese "mindestens ebenso gut für diese

994 Familienverfassung der Familie Feyner, S. 4.

995 Interview F-2 (27. 11.2017), S. 13.

996 Familienverfassung der Familie Feyner, S. 6 f.

997 Familienverfassung der Familie Feyner, S. 8.

998 Familienverfassung der Familie Feyner, S. 10.

999 Interview F-1 (24.11.2017), S. 4. 
Stelle qualifiziert sind wie fremde, nicht der Familie zugehörige, potenzielle Mitarbeiter. ${ }^{1000}$ Tendenziell sollten Familienmitglieder nicht unterhalb der Geschäftsleitungsebene im Unternehmen beschäftigt werden. Ausnahmen davon bedürfen nach Wortlaut der Familienverfassung der Zustimmung des Beirats und sind im Einzelfall zu begründen. Familienmitglieder, die eine Geschäftsführerposition im Unternehmen einnehmen wollen, sollten zuvor in einem anderen Unternehmen mindestens fünf Jahre gearbeitet und gezeigt haben, dass sie für die angestrebte Tätigkeit ausreichend qualifiziert sind:

»Ich bin jetzt hier im Unternehmen. Ich habe knapp die fünf Jahre geschafft, aber ich war jetzt nicht in einer wirklichen, ich bin jetzt kein Geschäftsführer, ich bin jetzt nicht in einer Geschäftsführerposition, trotzdem. « ${ }^{1001}$

Weiterhin regelt die Familienverfassung, dass das Unternehmen von einem Fremdmanagement geführt werden darf, wenn keine ebenso qualifizierten Mitglieder in der Familie zu finden sind. Der Bereich »Rollen« führt überdies einen "Verantwortlichen der Familie» und einen "Verantwortlichen im Unternehmen« ein. Der Familienverantwortliche kümmert sich um die Familie und um das Verhältnis zwischen Familie und Unternehmen. Hierzu gehört, jährlich zu einem Familientag einzuladen, auf welchem »Probleme innerhalb der Familie bzw. zwischen Familie und Unternehmen besprochen werden und auf welchem der für das Unternehmen Verantwortliche über den Stand und die Ziele des Unternehmens « berichtet. ${ }^{1002}$

"Einmal im Jahr, also es ist ein Wochenende, Anreise Freitag, Abreise Sonntag und eigentlich immer so der gleiche Ablauf. Also immer auch am gleichen Wochenende. ${ }^{1003}$

Zum Familientag können jederzeit auch Dritte, insbesondere leitende Angestellte aus dem Unternehmen, hinzugezogen werden.

Der "Verantwortliche im Unternehmen« soll - muss aber nicht - ein geschäftsführendes Familienmitglied sein. Dieser berichtet mindestens einmal jährlich auf dem Familientag über alle wichtigen Vorgänge im Unternehmen und über die Strategie des Unternehmens.

Friederike Feyner beschreibt die Familientage als eine wichtige Institution, die grundsätzlich gerne von allen besucht wird:

"[...] das wird bei uns schon sehr positiv aufgenommen dieses Ganze, es ist nicht so, dass es rähh - es ist eine Muss-Veranstaltung, ich habe keinen Bock auf die Familier. Bei uns in der Familie, man freut sich darauf, auch wenn es ein paar Nörgler gibt, die dann auch kommen. Und wenn sie dann da sind, dann ist auch immer gute Stimmung. [...]

1000 Familienverfassung der Familie Feyner, S. 11.

1001 Interview F-1 (24.11.2017), S. 5.

1002 Familienverfassung der Familie Feyner, S. 12.

1003 Interview F-1 (24. 11.2017), S. 14. 
Der Familientag ist eigentlich vorwiegend dazu da, uns alle zusammenzubringen und uns eben diesen Wert `Familienunternehmen` klar zu machen, weil es ist eben, wir werden immer größer, das sieht man jetzt, also sobald mal meine Generation anfängt mit richtig Nachwuchs zu bringen, dann läuft das auseinander. Und wenn man da eben sowas nicht täte, dann kommt man auch immer viel weiter weg vom Unternehmen, [...] das wird uns auch gesagt, dass der Familientag dafür da ist, das Bewusstsein fürs Familienunternehmen und die Wichtigkeit und Wertigkeit von dem Familienunternehmen darzustellen. ${ }^{1004}$

Die Ehepartner der Gesellschafter sind zu den Familientagen auch eingeladen. Den Familienmitgliedern ist bewusst, dass es eine Familienverfassung gibt. Das habe man im Kopf, aber keinesfalls kenne man den Wortlaut auswendig:

"Ja, also man hat die Familiencharta im Kopf, aber nicht auswendig. Bei mir war es immer so, ok, mit dem Hintergrund, dass ich vielleicht mal ins Unternehmen gehe, da hat mich zum Beispiel interessiert, was sind da die Regelungen, ab wann kann ich hier einsteigen und was sind auch Voraussetzungen. $\aleph^{1005}$

Gerade bei den ersten Familientagen wurde die Familienverfassung immer wieder thematisiert, um die Familienmitglieder damit vertraut zu machen. Dies war dem Umstand geschuldet, dass die Familienangehörigen in den Erstellungsprozess nicht eingebunden waren:

"Also da hat einfach der Vater seinen Willen durchgesetzt.« ${ }^{1006}$

In den Interviews wurde deutlich, dass diese Familienverfassung vom Senior den Familienmitgliedern quasi aufoktroyiert wurde. Solange der Senior lebt, wird er als Patriarch das letzte Wort haben. Die Familienverfassung wird ihre Wirkung erst nach seinem Tod voll entfalten können, sofern sich alle Familienmitglieder auf sie einlassen:

"[...] ich weiß nicht, ob ich für meine Generation sprechen kann; wir wissen, dass es etwas gibt, aber es ist halt im Hinterkopf, aber wie gesagt, wir kennen den Wortlaut nicht, wir wissen, dass es für unsere Großeltern wichtig ist, also ist es auch irgendwie für uns wichtig. ${ }^{1007}$

\subsubsection{Regelbefolgung, -brüche und moralische Verbindlichkeit}

Seit der Einführung der Familienverfassung gab es keine größeren Verwerfungen innerhalb der Familie:

1004 Interview F-1 (24.11.2017), S. 15.

1005 Interview F-1 (24.11.2017), S. 5.

1006 Interview F-2 (27.11.2017), S. 2.

1007 Interview F-1 (24.11.2017), S. 11. 
»Sie, die Charta, ist ja noch zu jung eigentlich und es hat jetzt noch keine größeren Verwerfungen gegeben seither. ${ }^{1008}$

»[...] bisher hat sich ja mehr oder weniger jeder daran gehalten. « ${ }^{1009}$

Konkrete Maßnahmen bei Regelverstößen sind in der Familienverfassung nicht verankert. Ferdinand Feyner ist davon überzeugt, dass die Regelbefolgung grundsätzlich daher rührt, dass die Regeln sinnvoll erscheinen. Darüber hinaus gab der Berater seines Vaters bei der Einführung einschlägige Negativbeispiele, die wohl abschreckende Wirkung hatten:

»Weil es relativ sinnvoll ist. Es ist in sich sinnvoll. Also [Name des Beraters] hat das ausführlich erklärt an Hand von Beispielen. Also anonym, aber aus seiner Praxis, da gibt es sicher genügend. $\aleph^{1010}$

Nun gab es aber auch bereits kleinere Abweichungen von den Regeln der Familienverfassung - zumindest in den Augen der Interviewten. Es sind neben Friederike Feyner zwei weitere Vertreter der 4. Generation operativ im Unternehmen tätig, die offenbar beide weniger als fünf Jahre in einem Unternehmen verbracht haben. Streng genommen sieht die Familienverfassung die Fünf-Jahres-Regel lediglich für solche Personen vor, die eine Geschäftsleitungsfunktion übernehmen. Offenbar interpretieren die Familienmitglieder diese Regel anders, was zu Unverständnis führte. Ferdinand Feyner hat dafür aber eine sehr simple Erklärung:

»Man hat es einfach gemacht. Ich habe ein Stück Papier in der Mappe drin, da steht drin, wie die Firma Feyner funktioniert. Die Firma Feyner funktioniert so, wie wenn mein Vater der Chef aller ist, also 100 Prozent hätte. Es passiert prinzipiell das, was ihm gefällt. ${ }^{1011}$

Auf Grund der Tatsache, dass die Einstellung von Friederike Feyner nicht offiziell im Familienkreis besprochen wurde, sorgte dies auch bei ihrem Bruder für Unmut, was Auswirkungen dahingehend hat, dass er die gesamte Familienverfassung anzweifelt:

"Mein Bruder hat mich darauf angesprochen und hat gesagt, du, das ist doch aber eigentlich anders, [...] er ist dann immer so, ja, die Familiencharta ... hält sich ja eh keiner dran, hätten wir ja überhaupt gar nicht machen brauchen. Das ist immer so. Hat aber keine größeren Wellen geschlagen. Also mehr als seinen Unmut geäußert hat er dann nicht $[\ldots] . \ll^{1012}$

1008 Interview F-2 (27.11.2017), S. 17.

1009 Interview F-2 (27.11.2017), S. 22.

1010 Interview F-2 (27.11.2017), S. $22 \mathrm{f}$.

1011 Interview F-2 (27.11.2017), S. 24.

1012 Interview F-1 (24.11.2017), S. 18. 
Insgesamt schreckt man in der Familie Feyner vor gerichtlichen Maßnahmen gänzlich zurück. Man versucht interne Auseinandersetzungen im Gespräch zu lösen. Ferdinand Feyner sieht in einer zu strengen Regelbefolgung aber auch ein Risiko:

»Also, wenn man irgendjemanden dauernd ärgert, dann bricht jede Familie irgendwann auseinander. ${ }^{1013}$

Als präventive Maßnahme zur Regelbefolgung sieht Frederike Feyner die Tatsache, dass man das Werk der Familie ausführlich erläuterte und die Gesellschafter entsprechend weiterbildet:

»Also, ich glaube, die erste Maßnahme [...] war erst einmal, dieses Regelwerk [...] uns bekannt zu machen. [...] Weil ohne Wissen, dass es da etwas gibt, keine Chance. Das zweite ist [...], [z]um einen vielleicht zu schauen, dass wir eine gewisse Ausbildung bekommen oder genießen, um eben selbständig zu sein. Ich sage mal, da wird uns schon, ist aber bei keinem bisher erfolgt, es würde uns sicherlich Unterstützung angeboten werden, auch finanziell. Mein Großvater, das sagt er immer, er hat ja diese Stiftung, wo er seine Anteile eingebracht hat, da wird gesagt, dass die auch gerade für die Mitglieder und die Gesellschafter da sind, um sich weiterzubilden. $\aleph^{1014}$

Als weitere positive Institution wird der Familientag und die Familienbeauftragte wahrgenommen:

»[...] Verhinderung von Interessensgegensätzen, das ist natürlich auch der Familientag, der tut da schon so auch vermitteln. Und wir haben ja mittlerweile auch eine Familienbeauftragte und das ist schon so, wenn man irgendwie so das Gefühl hat, es passt etwas nicht, dann kann man sich dann immer an die Familienbeauftragte wenden und die versucht dann auch [...]...1015

Für Friederike Feyner ist es auch wichtig, immer wieder die Sinnhaftigkeit der Familienverfassung zu betonen und bei den Familientagen $\mathrm{zu}$ thematisieren:

»Ja, immer wieder ins Gedächtnis rufen. Wenn man das jetzt [...] am ersten Familientag einfach vorgestellt [hätte] und seither nie wieder erwähnt. Dann wäre das auch weg. ${ }^{1016}$

Ferdinand Feyner würde es begrüßen, wenn auch Verlobte oder Lebensgefährten mit zu den Familientagen eingeladen werden würden:

»Also da wäre ich jetzt großzügiger, weil die Vereinzelung der Menschen [...] man sollte einfach auch offen gegenüber den Freunden des Hauses sein. ${ }^{1017}$

1013 Interview F-2 (27.11.2017), S. 25.

1014 Interview F-1 (24.11.2017), S. 12.

1015 Interview F-1 (24.11.2017), S. 13.

1016 Interview F-1 (24.11.2017), S. 16.

1017 Interview F-2 (27.11.2017), S. 23. 
Moralische Verbindlichkeit wird nach Aussage von Friederike Feyner durch "Vorleben« geschaffen. Entscheidend sei auch, »immer wieder die Wertigkeit heraus[zu]stellen. ${ }^{1018}$ Hierfür werden die Familientage genutzt.

\subsection{Fallstudie 7: Unternehmerfamilie Gönner (G)}

Umsatzkategorie: 2

Anzahl der Familienmitglieder: 5

Generationen: 7

Interviewpartner: Gesellschafter, Vors. d. GL (G-1), 5. Gen.

Auswertbare Unterlagen: Keine

\subsubsection{Entstehung und Erstellung der Familienverfassung}

Das Unternehmen nahm seinen Anfang um die Mitte des 19. Jahrhunderts. Etwa Mitte des 20. Jahrhunderts wurde Gustav Gönner (G-1) als Vertreter der 5. Generation alleiniger geschäftsführender Gesellschafter. Der Unternehmenserfolg heute ist maßgeblich auf seine Verdienste zurückzuführen. Ausschlaggebender Grund für die Erstellung einer Familienverfassung war der Moment, als er Anteile an seine Kinder übertrug. Als einziger Gesellschafter musste er sich nie mit anderen hinsichtlich irgendwelcher Entscheidungen abstimmen. Als dann die Kinder erste Anteile erhielten, merkte er schon, dass es nun eine andere Situation sein würde:

"Und nachdem unsere vier Kinder dann schon beteiligt waren, mit einem minimalen Betrag zwar, hat man natürlich schon gesehen, dass sie es so einfach nicht mehr haben werden. ${ }^{1019}$

Diese Erkenntnisse flossen in die Erstellung der Familienverfassung mit ein. Bei der Erstellung ließ man sich von einem Berater unterstützen. Dass die Familienverfassung später vom Rechtsanwalt des Hauses begleitet werden sollte, lag daran, dass ein anderer Berater eine etwas andere Vorstellung von der Ausgestaltung der Familienverfassung hatte. Man wollte weniger ein "psychologisch" gefasstes Regelwerk, vielmehr hat man sich auf ein »Mindestmaß an Inhalten« verständigt. ${ }^{1020}$

1018 Interview F-1 (24.11.2017), S. 16.

1019 Interview G-1 (04.12.2017), S. 4.

1020 Interview G-1 (04.12.2017), S. 1. 
Zusammen mit dem Berater hat man die Wünsche, Vorstellungen und Zielsetzungen diskutiert, "was unbedingt aufgenommen werden sollte, und haben das eigentlich visionär in die Zukunft geworfen ${ }^{1021}$ :

»Wenn die Kinder alle verheiratet sind und dann wieder Kinder haben, dann ändert sich ja manchmal die Sichtweise auch. Das haben wir ihm also praktisch als Gedankenentwurf auf den Tisch gelegt. Er hat es in Worte gefasst und wir haben uns dann zwar immer noch getroffen und haben den letzten Schliff noch gegeben $[\ldots]$. « $^{1022}$

Widerstände gegen die Erstellung gab es in der Familie keine, allerdings sei dies auch vor der Anteilsübertragung gewesen:

»Da wäre es also ganz schlecht gewesen, wenn jemand eine andere Meinung geäußert hätte [lacht]. «1023

Dennoch wurde im Vorfeld kontrovers diskutiert, insbesondere über den Punkt, wer zukünftig Gesellschafter werden darf:

„Da gab es also dann Meinungsverschiedenheiten. Ob zum Beispiel adoptierte Kinder auch in diesen Kreis aufgenommen werden. Das war aber eigentlich der einzige Punkt. ${ }^{1024}$

Die Familienverfassung wurde nach Fertigstellung im Jahre 2014 von allen Gesellschaftern unterzeichnet und war zum Zeitpunkt des Interviews seit etwa drei Jahren im Einsatz.

\subsubsection{Inhalt, Institutionen und Alltag}

Das etwa 20 Seiten umfassende Regelwerk hat auch gelebte Handlungsgrundsätze der Familie verschriftlicht. Der Kreis der »Familie« umfasst nach der Definition der Familienverfassung lediglich Gesellschafter. Gesellschafter können nur leibliche Abkömmlinge werden. Ehepartner sind außen vor.

Mit der Familienverfassung wurden Familientage ins Leben gerufen. Mit diesen Familientagen möchte man insbesondere heranwachsende Kinder möglichst frühzeitig einbinden und jenen Gesellschaftern, die nicht im Unternehmen operativ tätig sind, die Möglichkeit geben, abgeholt zu werden und sie gleichzeitig stärker an das Unternehmen zu binden:

"Also wir haben jetzt in unserem Gesellschafterkreis eine Gesellschafterin, die ist

Ärztin, und die ist natürlich nicht so beheimatet in Fragen der Firmenpolitik, wie es die

1021 Interview G-1 (04.12.2017), S. 2.

1022 Interview G-1 (04.12.2017), S. 2.

1023 Interview G-1 (04.12.2017), S. 2.

1024 Interview G-1 (04.12.2017), S. 2. 
anderen sind, die jeden Tag mitarbeiten. Da ist es aber natürlich ganz gut, wenn man sowas macht und natürlich für die heranwachsenden Kinder, um das Interesse zu wecken und sie möglichst irgendwo interessiert zu halten, dass sie nicht irgendwann in die Lage kommen zu sagen, ja schön, das gibt es eine Firma Gönner; die wirft mir Dividende $\mathrm{ab}$ und wieso habe ich nun ein halbes Prozent weniger bekommen als im Vorjahr? Was haben sie da nun falsch gemacht? Das wollten wir nicht haben und das ist eigentlich der Hauptzweck der Familiencharta. Dass die Anbindung funktioniert. ${ }^{1025}$

Die Familientage finden einmal jährlich statt. Die Familie hat sich das Ziel gesetzt, im Rahmen der Familientage zukünftig auch ausländische Niederlassungen zu besuchen, was momentan mit Kleinkindern allerdings nicht praktikabel ist. Vielmehr ist es so, dass die Familientage derzeit eher einer Gesellschafterversammlung gleichen, bei der die Bilanz besprochen wird und eventuelle Fragen beantwortet werden. Lediglich ein Kind ist nicht operativ im Unternehmen tätig und die 7. Generation ist noch viel zu jung. An den Familientagen dürfen allerdings auch Familienfremde teilnehmen:

"Sie müssen verheiratet sein oder fünf Jahre in einer eheähnlichen Gemeinschaft leben. ${ }^{1026}$

Weitere Institutionen sind derzeit nicht etabliert. Sobald die Familie größer wird und sich die Zahl der Gesellschafter durch die vier Kinder vergrößert, ist auch eine Art Familienrat vorgesehen, in welchen jeder Familienstamm dann einen Vertreter entsenden soll.

Das Regelwerk wird im Alltag als »Spielregeln $«{ }^{1027}$ wahrgenommen. Da dieses allerdings noch relativ jung ist, hat es jeder noch im Kopf. Gustav Gönner ist nicht restlos überzeugt von der Sinnhaftigkeit einer Familienverfassung. Die Präsenz einer Familienverfassung würde erst dann sichtbar, wenn es irgendwo Schwierigkeiten gäbe. Das sei wie mit einem guten Vertrag. Der liege »eigentlich in der Schublade und sollte tunlichst dort bleiben. ${ }^{1028}$

"Also ich stehe ihr nicht negativ, das ist vielleicht das falsche Wort, gegenüber. Ich denke eigentlich mehr, wenn es wirklich so weit kommt, dass sie die Familiencharta dann rausziehen müssen und dann zu einem der Gesellschafter sagen müssen, das geht hier nicht so, wir haben uns dazu verpflichtet. Und deswegen ist dein Benehmen in dem Fall oder deine Tätigkeit unmöglich. Dann denke ich, da rettetet es die Familiencharta auch nicht. Ganz einfach. Weil dann ist ja schon irgendwo ein gewisser Vertrauensbruch wahrscheinlich da, sonst würde jeder Gesellschafter irgendwo kompromissbereit sein und man würde versuchen, einen Kompromiss zu erreichen, aber wenn es mal so weit

1025 Interview G-1 (04.12.2017), S. 5.

1026 Interview G-1 (04.12.2017), S. 21.

1027 Interview G-1 (04.12.2017), S. 4.

1028 Interview G-1 (04.12.2017), S. 4. 
kommt, dann denke ich, dann gibt es schon richtig Knatsch. Und dann habe ich eine Familiencharta, die nicht bindend ist. ${ }^{1029}$

Die Familienverfassung, die als lebendiges Dokument verstanden wird, kann jederzeit, wenn Veränderungen dies notwendig machen, angepasst werden. Einen festen Turnus zur Überprüfung wurde nicht vereinbart. Wenn alle Familienmitglieder einverstanden sind, wird das Regelwerk an die neue Situation angepasst.

\subsubsection{Regelbefolgung, -brüche und moralische Verbindlichkeit}

Bislang gab es "nichts Eklatantes, was der Charta widersprochen hätte. ${ }^{1030}$ Präventive Maßnahmen zur Regelbefolgung seien nicht vorgesehen. Gustav Gönner ist davon überzeugt, dass schon jeder aus "Eigennutz « ${ }^{1031}$ aufpasst und sich an die Regeln hält. Man habe solche Maßnahmen auch nicht als notwendig erkannt:

"Es sind eigentlich auch Regeln aufgestellt für Dinge, die Sie gar nicht präventiv verhindern können. Also, gut, das müsste alles irgendwo dann schriftlich Niederschlag finden. Oder Notarniederschlag, und dann geht das nicht. ${ }^{1032}$

Allerdings wurden die Familientage als Maßnahme verstanden, um die nichtoperativen Gesellschafter stärker an die Firma zu binden.

Eine geeignete Reaktion auf einen Regelverstoß bemisst sich nach Gustav Gönner an der Schwere des Verstoßes. Er würde immer versuchen, schon die ersten Anzeichen im Keim zu ersticken und die betreffende Person darauf hinzuweisen. Bei schwereren Verstößen würde er die Familie zusammenrufen und die Vorgehensweise besprechen - dies aber möglichst nicht am Familientag.

Gustav Gönner schreckt aber auch vor einer härteren Gangart nicht zurück. Sollte sich ein Familienmitglied einen gravierenden Verstoß im Rahmen des Geschäftslebens leisten, so würde er auch arbeitsrechtliche Konsequenzen in Erwägung ziehen:

»Hätte ich aber übrigens auch kein Problem, wenn sich einer irgendwo hier in der Firma nicht so korrekt verhält, würde er auch eine Abmahnung erhalten. Ein Familienmitglied. Problemlos. ${ }^{1033}$

1029 Interview G-1 (04.12.2017), S. 7.

1030 Interview G-1 (04.12.2017), S. 3.

1031 Interview G-1 (04.12.2017), S. 11.

1032 Interview G-1 (04.12.2017), S. 11.

1033 Interview G-1 (04.12.2017), S. 15. 
Im Rahmen der Erstellung der Familienverfassung hat man sich beispielsweise die Frage gestellt, wann die Grenze des Erträglichen beispielsweise in Bezug auf den Wert »Respekt « erreicht ist, und kam relativ schnell zum Ergebnis, man wolle die Gesellschafter als fremde Dritte betrachten und die gleichen Toleranzgrenzen und Maßnahmen heranziehen, wie man bei einem fremden Dritten anwenden würde.

In der Familienverfassung sei auch die Variante geregelt, dass jemand seine Anteile verlieren kann:

„Gegen eine gewisse Gebühr können es dann die anderen, oder müssen sie es, abkaufen. ${ }^{1034}$

Juristische Maßnahmen werden zwar nicht präferiert, zurückschrecken würde Gustav Gönner allerdings auch davor nicht:

»Ja, gut, man kann gerichtliche Maßnahmen ja nicht verhindern, wenn eine Partei da hinspringt. [...] aber ich wäre durchaus, [...] ich würde auf dem Standpunkt stehen, wenn es gar nicht anders geht und es kann innerhalb der Familie nicht geregelt werden, dann wäre mir immer noch ein neutraler Dritter oder das Urteil eines neutralen Dritten lieber, bevor sich alle zerfleischen. $\aleph^{1035}$

Ein Mediationsverfahren wird eher kritisch gesehen. Ein Mediator müsse beide Seiten gut kennen. Ein als Mediator ausgebildetes Beiratsmitglied beispielsweise, das alle Familienmitglieder kennt, würde in dem Fall aber auch nicht in Frage kommen, da es zwangsläufig Sympathien für die eine oder andere Partei hege:

»[...] er kennt die Gesellschafter schon länger und er hat schon immer gewisse $\mathrm{Zu}-$ neigung zum einen oder zum anderen, ich glaube, das lässt sich nicht verheimlichen. ${ }^{1036}$

Grundsätzlich ist Gustav Gönner davon überzeugt, möglichst frühzeitig einzugreifen, wenn sich Verwerfungen abzeichnen (»Ist ja auch richtig so. Man sagt ja, wehret den Anfängen. ${ }^{1037}$ ).

Es sei dabei immer wichtig, "einen klaren Kurs $«^{1038} \mathrm{zu}$ fahren. Beispielsweise hat man bei der Bestellung eines Geschäftsführers aus dem Kreise der Kinder darauf bestanden, dass er ein Vorstellungsgespräch führt, einen Abriss über seinen Lebenslauf gibt und seine speziellen Tätigkeiten und Leidenschaften vorstellt, da man dies auch von einem fremden Dritten, der als Geschäftsführer berufen wird, verlangen würde:

1034 Interview G-1 (04.12.2017), S. 16.

1035 Interview G-1 (04.12.2017), S. 16.

1036 Interview G-1 (04.12.2017), S. 17.

1037 Interview G-1 (04.12.2017), S. 20.

1038 Interview G-1 (04. 12.2017), S. 20. 
"Und er war dagegen, er hat sich natürlich gewehrt und gesagt: Moment mal, ihr kennt mich doch alle und was soll das. Nein, das wollen wir eigentlich sehen, wie du dich da anstellst, und das wurde dann auch so gemacht. ${ }^{1039}$

Moralische Verbindlichkeit erreicht man nach Auffassung von Gustav Gönner »durch die Erziehung«. Die »Vorbildfunktion« sei dabei entscheidend. ${ }^{1040}$

\subsection{Fallstudie 8: Unternehmerfamilie Hirter $(\mathrm{H})$}

Umsatzkategorie: 1

Anzahl der Familienmitglieder: ca. 50

Generationen: 5

Interviewpartner: Gesellschafterin (H-1), 4. Gen.

Gesellschafter (H-2), 5. Gen.

Auswertbare Unterlagen: Familienverfassung

\subsubsection{Entstehung und Erstellung der Familienverfassung}

Das zu Beginn des 20. Jahrhunderts gegründete Unternehmen wird heute von einem Vertreter der 4. Generation geführt. Die beiden Interviewpartner sind nicht operativ im Unternehmen tätig. Als sich die 3. Generation langsam aus dem Unternehmensalltag zurückzog und das Zepter an die 4. Generation übergab, stellte man fest, dass die Familie bereits weit verzweigt war und viele Familienmitglieder "relativ weit weg stehen vom Unternehmen. ${ }^{1041}$ Vor dem Hintergrund, dass sich auch die Sichtweise von Generation zu Generation änderte, stellte man sich nun die Frage:

"[...] wie gehen wir damit um. Wie wollen wir uns auch aufstellen und organisieren? Und es gab natürlich auch einige Streitpunkte, die das dann zugespitzt haben, wo wir gemerkt haben, hoppla, jetzt stehen wir an einem Punkt, wo wir nicht recht wissen, wie es jetzt weitergeht. ${ }^{1042}$

Diese Konflikte beschreibt Henrik Hirter (H-2), Vertreter der 5. Generation, als heftig (»Da hat man sich teilweise richtig verkracht ${ }^{1043}$ ). Darin ging es um die Besetzung des Beirats oder den Eintritt eines Familienmitglieds in die Geschäftsführung. Man war sich in der Familie einig, dass man diese Szenarien nicht wiederholt sehen möchte; für die Erarbeitung einer Familienstrategie, mit

1039 Interview G-1 (04.12.2017), S. 22.

1040 Interview G-1 (04.12.2017), S. 14.

1041 Interview H-1 (05. 12.2017), S. 1.

1042 Interview H-1 (05.12.2017), S. 1.

1043 Interview H-2 (05. 12.2017), S. 1. 
dem Ziel, eine Familienverfassung zu erstellen, hat man sich dann externe Unterstützung geholt. Aus Sicht von Henrik Hirter ging es dabei auch darum,

$»[\ldots]$ die Kommunikation und die gemeinsame Arbeit in der Gesellschafterfamilie zu organisieren. Und zwar in so einer Form, dass es eben nicht, oder in so einer Form, dass, wenn es zu Konflikten kommt, die dann auch systematisch und, sage ich mal, möglichst zielführend und möglichst wenig emotional sozusagen behandelt werden. ${ }^{1044}$

Die Vergangenheit hat gezeigt, dass die Kommunikationskultur im Argen lag (»Da wurde dann in Grüppchen kommuniziert, da wurden schlimme E-Mails rumgeschickt, da wurde angerufen und sich schlimm beschimpft irgendwie. $\left.{ }^{1045}\right)$.

Mit der Familienverfassung sollte eine neue Ära anbrechen. Die 5. Generation soll frei von diesen Konflikten der Vergangenheit sein. Verankert wurde in der Familienverfassung auch, das Stammesdenken abzuschaffen. Seither wurde in drei Stämmen gedacht, was auch gesellschaftsvertraglich wohl noch so geregelt ist und im Zuge der Verabschiedung der Familienverfassung durch eine Änderung der Gesellschaftsverträge gelöst werden soll.

Der Prozess der Erstellung der Familienverfassung dauerte mehrere Jahre. Im Dezember 2017 lag eine unterschriftsreife Version vor. Nach Anpassung des Gesellschaftsvertrags soll die Familienverfassung von allen Familienmitgliedern unterzeichnet werden -nach aktuellem Stand werden dieser Aufforderung zur Unterschrift einige wenige Familienmitglieder aus persönlichen Gründen nicht nachkommen. Wichtig war es der Familie, berichtete Helen Hirter (H-1), dass alle Gesellschafter eingebunden wurden. Durch den relativ großen Kreis und die Tatsache, dass man davor noch nie über irgendwelche Themen ausführlich gesprochen hatte, gab es größeren Diskussionsbedarf:

»[...] zum einen, dass man auch mal gucken muss. Wie gesagt, wir haben da vorher noch nie miteinander darüber gesprochen. Was hat man für Vorstellungen mit dem Unternehmen, wie möchte man es weitergeben, was ist man bereit, auch zu investieren an Zeit und Energie und dann aber auf der anderen Seite, dass es tatsächlich über Streitereien so weit war, dass man eben kaum mehr miteinander am Tisch gesessen hat. ${ }^{1046}$

In diesem Prozess hat man versucht, sich einen Rahmen zu geben, wie man (wieder) miteinander kommunizieren kann. Die Verletzungen der Vergangenheit sind jedoch noch nicht ganz verheilt, diese waren offenbar zu tiefgreifend und selbst wenn die 4. Generation keine optimale Ebene mehr finden sollte, so wollte man zumindest für die 5. Generation die Rahmenbedingungen schaffen,

1044 Interview H-2 (05. 12.2017), S. 2.

1045 Interview H-2 (05. 12.2017), S. 2.

1046 Interview H-1 (05. 12.2017), S. 3. 
dass es nicht erneut zu solchen Vorkommnissen kommt. Einige wenige Mitglieder haben sich aus dem Prozess der Erstellung herausgezogen. Es war ihnen zu emotional. Insgesamt hat der Erstellungsprozess jedoch bereits seine positive Wirkung gezeigt, wie Henrik Hirter resümiert:

»Also ich kenne es vorher vor allem aus Erzählungen und da waren die Erzählungen immer eher negativ, also, boah, man hat keine Lust, schon wieder auf die Gesellschafterversammlung und dann gibt es wieder nur Streit. Das war relativ negativ besetzt. [...]. Und seit wir uns damit beschäftigen, hat sich das eher ins Neutrale bis Positive gewendet. ${ }^{1047}$

Vermutlich auf Grund der Erfahrungen aus der Vergangenheit hat man die Familienverfassung so gestaltet, dass die »Mitgliedschaft» freiwillig erfolgt. Mit anderen Worten: Die Familienverfassung führt einen Familienverbund ein, dem man angehören kann, den man aber auch wieder verlassen kann.

\subsubsection{Inhalt, Institutionen und Alltag}

Die (mit Anhang) 23 Seiten umfassende Familienverfassung folgt im Wesentlichen der Struktur "Werte - Ziele - Leitlinien - Rollen - Institutionen." In der Präambel des Regelwerks wird das Bekenntnis zum Familienunternehmen und der Wunsch nach dessen langfristigem Verbleib in der Unternehmerfamilie manifestiert. Auch spricht die Familienverfassung davon, dass sie die Nachfolgeplanung für die 5. Generation einleiten möchte, um so die positive Entwicklung des Unternehmens dauerhaft zu sichern. Der Aufzählung der gemeinsamen Werte (z.B. Verlässlichkeit, Toleranz, Verantwortungsbewusstsein, usw.) folgt eine Definition für den Begriff »Gesellschafterfamilie«:

»Zur Gesellschafterfamilie gehören alle Gesellschafter [...], die ehemaligen und potentiellen Gesellschafter/innen (d.h. die Gesellschafter/innen, die ihre Anteile bereits vererbt/verschenkt haben und die anteilsberechtigten Erben der aktuellen Gesellschafter/innen) sowie deren Ehe und Lebens-partner, sofern diese die Verfassung unterzeichnet haben. Nachkommen von Gesellschaftern sind bis zum 18. Geburtstag automatisch Mitglieder der Gesellschafterfamilie. Danach verbleiben sie in der Gesellschafterfamilie, sofern sie die Verfassung unterzeichnet haben. ${ }^{1048}$

Die Familienverfassung erinnert die Mitglieder der Gesellschafterfamilie an einen respektvollen Umgang untereinander. Die Eigeninteressen seien »hinter das gemeinschaftliche Interesse der Gesellschafterfamilie zu stellen ${ }^{1049}$. Als Organ der Gesellschafterfamilie soll ein Familienrat gewählt werden.

1047 Interview H-2 (05. 12.2017), S. 10.

1048 Familienverfassung der Familie Hirter, S. 4.

1049 Familienverfassung der Familie Hirter, S. 4. 
Innerhalb der »Leitlinien für die Arbeit der Gesellschafterfamilie» wird das Thema »Meinungsbildung" thematisiert. Man ist sich der Meinungspluralität in dieser Familie bewusst. Dennoch soll man Konsensentscheidungen anstreben. Ist kein Konsens möglich, werden Entscheidungen mit einfacher Mehrheit getroffen, um handlungsfähig zu bleiben. Jedes Mitglied der Gesellschafterfamilie hat dabei eine Stimme. Persistierende Meinungsverschiedenheiten werden zur Erarbeitung von Kompromissvorschlägen dem Familienrat übertragen. Für den Umgang miteinander und für Diskussionen wurden allgemeingültige Kommunikationsregeln formuliert (z.B. Gefühle, Gedanken und Wahrnehmungen offen äußern, ausreden lassen, Killerphrasen unterdrücken, kritische Themen offen ansprechen, Frontenbildung vermeiden usw.).

Ein weiterer Punkt beschäftigt sich mit dem »Konflikt- und Krisenmanagement in der Gesellschafterfamilie«. Die Familie möchte ein mehrstufiges Konflikt-management einrichten. Zunächst wird der Konflikt dem Familienrat vorgetragen. Erzielt der Familienrat keine befriedigende Lösung, kann er einen von allen Konfliktparteien akzeptierten Moderator hinzuziehen. Wenn auch diese Maßnahme zu keinem zufriedenstellenden Ergebnis führen sollte, setzt sich der Familienrat zu einem späteren Zeitpunkt erneut mit dieser Problematik auseinander. Diese Vorgehensweise mag auf den ersten Blick kurios anmuten, scheint aber in dieser Familie ein wirkungsvoller Ansatz zu sein:

»Ja, die klingt auf den ersten Blick, kann ich total nachvollziehen, dass sie merkwürdig klingt. [...]. Aber witzigerweise hat genau dieses Verfahren in der Vergangenheit auch schon zum Erfolg geführt, ohne dass wir es da schon irgendwie so festgeschrieben hatten. Man konnte sich nicht einigen, man saß zusammen und konnte sich nicht einigen und dann hat man sich vier Monate später nochmal zusammengesetzt und dann hat man sich geeinigt, obwohl sich die Ausgangslage nicht geändert hat. Also ich halte das, auch wenn es absurd klingt für Außenstehende, für unsere Familie im Moment für kein schlechtes Instrument. «1050

Der Punkt »Organisation der Arbeit in der Gesellschafterfamilie» gibt einen Überblick über die Institutionen und Gremien. Nachdem die Besetzung des Beirats in der Vergangenheit bereits zu diesen heftigen Diskussionen führte, wird nun genau geregelt, wie der Beirat besetzt werden soll, wer welche Vorschlagsrechte und Stimmrechte bei einer Abstimmung hat.

Als weitere, wichtige Institution soll ein Familienrat etabliert werden. Dieser »kümmert sich einerseits um sämtliche Belange der Gesellschafterfamilie. Andererseits fungiert er als Repräsentant der Gesellschafterfamilie gegenüber der Öffentlichkeit. ${ }^{1051}$ Der Familienrat besteht aus maximal vier Mitgliedern und wird für eine Amtsdauer von drei Jahren gewählt. Jeder aus der Gesellschafter-

1050 Interview H-2 (05. 12.2017), S. 16.

1051 Familienverfassung der Familie Hirter, S. 9. 
familie kann sich zur Wahl aufstellen. Es wird der explizite Wunsch geäußert, dass ein Mitglied im Beirat vertreten sein soll und ein weiteres Mitglied sollte aus den Reihen der 5. Generation stammen. Die Arbeit im Familienrat wird entsprechend vergütet.

Das Aufgabenspektrum des Familienrats ist breit angelegt. Zum einen organisiert er den Zusammenhalt der Gesellschafterfamilie, andererseits repräsentiert er die Familie gegenüber dem Beirat und dem Unternehmen. Er soll für die Kohäsion der Mitglieder untereinander sorgen und die Bindung der Familie an das Unternehmen stärken.

Ein »Familienmanagementsystem» sorgt für einen Überblick über die Termine des Familienkalenders. Einmal jährlich findet beispielsweise ein Familientag statt, bei dem auch die Teilnahme von Ehe- und Lebenspartnern erwartet wird.

Auch dem »Aufbau von Gesellschafterkompetenz« ist ein Kapitel gewidmet. Man hat verstanden, dass die "professionelle Ausübung der Gesellschafterfunktion ein hohes Maß an Kompetenz« verlangt. »Durch das GKE-Programm sollen eine emotionale Bindung an das Unternehmen entwickelt, Verständnis für die Rolle eines Familiengesellschafters geweckt, Kommunikationsfähigkeit erworben, betriebswirtschaftliche Grundkenntnisse sowie der Erwerb von Gremienkompetenz vermittelt werden. ${ }^{1052}$ Auch hierfür ist der Familienrat verantwortlich. Darüber hinaus werden an ein bis zwei Tagen pro Jahr spezielle Programme für die Altersgruppen »NextGen (15 bis 30 Jahre)« und »Oldies (>30 Jahre)« angeboten.

Auch wenn vermutlich nicht alle Gesellschafter nun die Familienverfassung unterschreiben werden, da sie sich derzeit emotional noch nicht im Stande sehen, wollen die übrigen Familienmitglieder die "Mitgliedschaft» so attraktiv wie möglich gestalten. Helen Hirter kann dies nachvollziehen:

"Ja, ich meine, es ist natürlich schon, wenn man das unterschreibt, eine enge Zusammenarbeit auch, und man gibt Verantwortlichkeiten ab an den Familienrat. Und wenn ich das gerade nicht kann, dann kann ich es auch nicht unterschreiben. ${ }^{1053}$

\subsubsection{Regelbefolgung, -brüche und moralische Verbindlichkeit}

Auf Grund der Vorgeschichte ist sich die Familie darüber einig, dass man das Thema »Regelbrüche« nicht ausblenden darf. Es wird sich vielmehr zeigen, ob alle Gesellschafter die Familienverfassung unterschreiben und damit Mitglied in diesem Familienverbund werden. Der Familienrat wacht über die Einhaltung der

1052 Familienverfassung der Familie Hirter, S. 10.

1053 Interview H-1 (05. 12.2017), S. 10. 
Regeln. Wenn beispielsweise jemand Informationen an Dritte preisgibt, über die er Stillschweigen zu wahren hat, wäre das für Helen Hirter ein klarer Regelverstoß gegen die Familienverfassung. Der Punkt »Konflikt- und Krisenmanagement in der Gesellschafterfamilie» der Familienverfassung sieht ein klar definiertes Prozedere im Falle eines wiederholten Regelbruchs vor:

"Hält sich ein Mitglied der Gesellschafterfamilie wiederholt nicht an die vereinbarten Regeln, sucht der Familienrat ein klärendes Gespräch. Führt das Gespräch letztlich zu keinem positiven Ergebnis, entscheidet der Familienrat über einen möglichen Austritt des betroffenen Familienmitglieds aus der Familienverfassung. Der Familienrat stellt hierzu einen Antrag auf Austritt des betroffenen Mitglieds aus der Verfassung. Der Antrag gilt als angenommen, wenn 75 Prozent der stimmberechtigten Mitglieder der Gesellschafterfamilie (ohne das betroffene Familienmitglied) dem zustimmen. Stimmberechtigt sind alle Familienmitglieder, welche die Verfassung unterschrieben haben. ${ }^{1054}$

Für Helen Hirter sind auch die Maßnahmen bei einem Regelbruch wichtig, da man sich als Familienverbund moralisch verpflichtet fühlt, sich an diese Regeln zu halten. Wenn nun eine Person permanent absichtlich dagegen verstößt, wird auch irgendwann den anderen Familienmitgliedern der Grund genommen, sich an die Regeln zu halten:

"[...] wenn man jetzt Wert darauf legt, dass man sich da daran hält an diese Regeln, und es gibt tatsächlich jemand, der hält sich nicht daran. Wie soll ich dann [damit umgehen]; mit meinen anderen Mitgliedern dieser Familienverfassung möchte ich das ja leben und wenn jetzt jemand nicht [mitspielt]. >Der andere macht es ja auch nicht $\longleftarrow .{ }^{1055}$

Auch der freiwillige Austritt aus der Familienverfassung ist vorgesehen und dann dem Familienrat schriftlich mitzuteilen. Ein Austritt bedeutet allerdings die Rückgabe sämtlicher Rechte und Pflichten gegenüber der Gesellschafterfamilie:

Diese Freiwilligkeit kann als präventive Maßnahme verstanden werden. Jeder, der der Familienverfassung beitritt, tut dies freiwillig. Er ist auch nicht auf immer gebunden, sondern kann jederzeit frei entscheiden, ob er sich von seinem Gesellschafterdasein verabschieden möchte.

Grundsätzlich, so bestätigt Henrik Hirter, habe die Familie eher ein »Harmoniebedürfnis. « ${ }^{1056}$ Bis man einen Ausschluss von jemandem erwirken würde, müsste es schon weit kommen. Man versuche, wo möglich, die Entscheidungen im Konsens zu treffen. Der Familienrat sei die entscheidende Institution, die im Konfliktfall und bei Regelverstößen tätig werden müsse.

1054 Familienverfassung der Familie Hirter, S. 7.

1055 Interview H-1 (05. 12.2017), S. 15.

1056 Interview H-2 (05. 12.2017), S. 18. 
Die Familienverfassung wird als moralisch verbindlich wahrgenommen. Die Familie ist sich einig darüber, dass sie »juristisch keine Geltung « ${ }^{1057}$ entfalten soll. Moralische Verbindlichkeit erlangt sie schon allein dadurch, "weil alle daran mitgewirkt haben. ${ }^{1058}$ In Bezug auf die nachfolgende Generation, die bei der Erstellung nicht mitgewirkt hat, würde man zunächst die Einhaltung einfordern und ihr in Aussicht stellen, bei der nächsten Überarbeitung aktiv gestalten zu können. Helen Hirter sieht einen wesentlichen Punkt darin, dass moralische Verbindlichkeit vorgelebt werden muss und

$»[\ldots]$ dann muss ich es auch meinen Kindern vermitteln. $\aleph^{1059}$

1057 Interview H-2 (05. 12.2017), S. 3.

1058 Interview H-2 (05.12.2017), S. 13.

1059 Interview H-1 (05.12.2017), S. 14. 
Open-Access-Publikation im Sinne der CC-Lizenz BY 4.0

(c) 2020, Vandenhoeck \& Ruprecht GmbH \& Co. KG, Göttingen ISBN Print: 9783847111719 - ISBN E-Lib: 9783737011716 


\section{$4 \quad$ Analyse und Diskussion der Ergebnisse}

\subsection{Wesentliche Ergebnisse der fallübergreifenden Auswertung}

In den folgenden Abschnitten soll nun beschrieben werden, welche konkreten Rückschlüsse sich aus der fallvergleichenden Kontrastierung ableiten lassen. Wie stellen Unternehmerfamilien sicher oder wie erhöhen sie zumindest die Wahrscheinlichkeit dafür, dass die Regeln, die sie sich selbst gegeben haben, von den Familienmitgliedern eingehalten werden? Welche präventiven Maßnahmen sollen die Regelbefolgung gewährleisten? Was sind Regelbrüche und wie reagiert man darauf? Wenn Familienverfassungen moralisch verbindlich sein wollen wie schafft man moralische Verbindlichkeit in einer (Unternehmer-)Familie? Welche Gemeinsamkeiten lassen sich zwischen den Fallstudien feststellen und welche Unterschiede gibt es? Die aus dem gesammelten Interviewmaterial extrahierten Ergebnisse werden eingehend in einer Form diskutiert, die gleichzeitig bereits ausführlich die Forschungsfrage beantworten soll; dabei wird - wo immer sinnvoll und möglich - Bezug auf die theoretischen Vorüberlegungen aus Kapitel 2 genommen. Zuvor sollen jedoch einige Anmerkungen und Beobachtungen allgemeiner Natur erfolgen.

\subsubsection{Allgemeine Vorbemerkungen}

Die erste allgemeine Vorbemerkung zielt auf den Hintergrund zur Erstellung einer Familienverfassung ab. Theodor Heuss sagte einst: "Nur wer weiß, woher er kommt, weiß, wohin er geht.« Wenngleich dieses Thema noch ausführlicher im nächsten Abschnitt dargestellt wird, soll an dieser Stelle der Versuch einer Rechtfertigung unternommen werden. Erst wenn man versteht, aus welchen Gründen sich Unternehmerfamilien für die Erstellung einer Familienverfassung entschieden haben, kann man über entsprechend wirksame Maßnahmen nachdenken, die die Regelbefolgung sicherstellen können oder zumindest deren 
Wahrscheinlichkeit erhöhen. Aus diesem Grund wurde dieser Frage in der Darstellung der einzelnen Fallstudien besondere Aufmerksamkeit geschenkt.

Da die meisten Familienverfassungen der untersuchten Unternehmerfamilien erst seit wenigen Jahren im Einsatz sind, gab es bei den entsprechenden Familien seither keine eklatanten Verwerfungen. Dies könnte natürlich einerseits dafür sprechen, dass die Familienverfassung als konfliktpräventives Instrument bereits ihre Wirkung zeigte, andererseits könnte die Bewährungsprobe der Familienverfassung in diesen Unternehmerfamilien erst noch ausstehen; vielleicht auch insbesondere dann, wenn es zu einem Generationenübergang kommt.

Als weitere Besonderheit fiel in den Gesprächen auf, dass man über präventive Maßnahmen zur Regelbefolgung bis zum Zeitpunkt der Interviews oftmals nicht explizit nachgedacht hatte. Maßnahmen, die durchaus als solche dienlich sein können, werden häufig von den Familienmitgliedern nicht als solche erkannt. In diesem Zusammenhang kann auch festgestellt werden, dass der Sanktionierung von Regelverstößen in der Vergangenheit sehr wenig Aufmerksamkeit geschenkt wurde. Man vertraut vielmehr darauf, dass sich alle Familienmitglieder an die Regeln halten. Insbesondere wurde bei den Unternehmerfamilien über Sanktionsmechanismen nachgedacht, die die Familienverfassung aus einer konfliktären Situation heraus erstellten oder wo es bereits in der Vergangenheit zu entsprechenden Verwerfungen kam, die nicht selten auch den Fortbestand des Familienunternehmens gefährden können:

»Es ist in der Praxis immer so, dass diejenigen, die einen Konflikt haben, so etwas [Regeln zum Umgang mit Verstößen] definieren wollen. Andere Familien, die keinen Konflikt haben, scheuen sich eher davor. Ich glaube, es ist wichtig, sich trotzdem im Vorfeld damit auseinander zu setzen. Es gehört einfach als Bestandteil dazu. ${ }^{1060}$

Die letzte Beobachtung, die bei den geführten Gesprächen angestellt werden konnte, ist die Tatsache, dass bei den Gesprächspartnern Einigkeit drüber herrschte, dass die Familienverfassung moralisch verbindlich ist und sich daraus keine unmittelbar rechtlichen Ansprüche ableiten lassen. Den Grundstein für moralische Verbindlichkeit innerhalb einer Familie lasse sich - auch darüber herrschte ein gemeinsames Verständnis - bereits im Kindesalter durch eine entsprechende Erziehung legen. Die Vorbildfunktion spiele dabei eine genauso große Rolle.

An dieser Stelle soll auch darauf hingewiesen werden, dass die identifizierten Maßnahmen nicht uneingeschränkt für jede Unternehmerfamilie gleich praktikabel sein können. Jede positive Wirkung einer Maßnahme in Bezug auf die Regelbefolgung mag auch negative Auswirkungen auf andere Bereiche haben. Es muss somit immer auf den individuellen Einzelfall abgestellt werden. Auch eine

1060 Interview Alexander Koeberle-Schmid (30.11.2017), S. 7. 
Bewertung der Effizienz der einzelnen Maßnahmen soll an dieser Stelle nicht erfolgen, sie würde den Rahmen dieser Arbeit sprengen.

\subsubsection{Der Impetus für die Erstellung}

Um den Auslöser für Konflikte und Regelbrüche besser einordnen zu können, ist es unerlässlich zu verstehen, was die Intentionen und Hintergründe sind, wenn sich eine Unternehmerfamilie für eine Familienverfassung entscheidet.

Alle Fallstudien haben gemeinsam, dass sie mit der Erstellung einer Familienverfassung den Verbleib des Unternehmens in Familienbesitz sicherstellen wollen:

»Und zwar am liebsten als familiengeführtes Familienunternehmen und als zweitbeste Lösung als fremdgeführtes Familienunternehmen und erst als allerletzte Möglichkeit der Verkauf. Ich glaube, der Grundgedanke in Kombination mit den Werten, die wir da reingeschrieben haben, die das Miteinander mit uns definiert, das ist so der Kern. ${ }^{1061}$

Wird die Familienverfassung dabei von einer Generation initiiert, die das Unternehmen entweder gegründet hat oder auf deren Leistungen sich das Wachstum und der Erfolg des Unternehmens maßgeblich zurückführen lassen, so wird mit der Einführung des Regelwerks versucht, ein Lebenswerk zu erhalten. In Ausnahmefällen versuchen Familienpatriarchen, über dieses Instrument der jüngeren Generation ihre persönlichen Vorstellungen aufzuzwingen.

Während die Übergabe eines Familienunternehmens von der ersten auf die zweite, in einigen Fällen sicherlich auch noch von der zweiten auf die dritte Generation, häufig informell und eher problemlos abzulaufen scheint, gestaltet es sich in den weiteren Generationen schwieriger. Insbesondere die Zahl der Gesellschafter nimmt mit jedem Generationenwechsel in aller Regel zu. ${ }^{1062}$ Darüber hinaus gab es vor einigen Jahren erbschaftsteuerbedingt in einigen Unternehmen eine plötzliche Zunahme von Gesellschaftern, da man vorzeitig Anteile an Kinder und Enkel übertrug. So wird dies auch häufig als Grund dafür genannt, dass man sich plötzlich Gedanken machte, wie man zukünftig in diesem großen (Gesellschafter-)Kreis miteinander umgehen möchte:

"[...] deshalb sollte sich die Familie mit steigender Anzahl an Gesellschaftern auf Grund der unterschiedlichen Interessen Gedanken dazu machen, wie sie ein gemeinsames Verständnis hinsichtlich der strategischen Ausrichtung des Unternehmens erreicht. Und dazu dient eine Familienverfassung. Ein anderer Aspekt ist, wenn es gerade tätige und nicht-tätige Gesellschafter im Unternehmen gibt. Da unterscheiden sich die

1061 Interview C-1 (02.11.2017), S. 4.

1062 Vgl. Hack (2009). 
Interessen dann deutlich. Auch hier kann eine Familienverfassung helfen, sich über eine gemeinsame Ausrichtung einvernehmlich zu verständigen. « ${ }^{1063}$

Nicht nur stellt man Überlegungen zur Nachfolgeplanung an, sondern auch darüber, wie die Familienmitglieder weiterhin an das Unternehmen gebunden werden können. Mit zunehmender Anzahl an Gesellschaftern ist zumindest heutzutage die Gefahr groß, dass sich einzelne oder mehrere Familienmitglieder räumlich weit entfernt vom Unternehmen niederlassen und dort ihren eigenen Lebensentwurf verwirklichen. Der persönliche Kontakt zu den Familienmitgliedern wird schwieriger und nimmt dadurch nicht selten ab:

»So und damit hat sich aufgedrängt, [...] wir sind plötzlich eine große Gesellschafterfamilie und man kann eben nicht mehr alle Entscheidungen treffen am Konferenztisch, [...] was bedeutet das denn eigentlich, dass eine vierte Generation Gesellschafter geworden ist, wie [kann] man diese an das Unternehmen heranführen und auch die Rolle der Gesellschafter. [...] Das hat auch was damit zu tun, dass wir uns alle recht schlecht kannten, also [...] wir hatten eigentlich wenig Anlässe, uns miteinander auseinanderzusetzen. «1064

In diesen vorgenannten Fällen wird von der Familienverfassung erwartet, dass sie Ordnung bringt. ${ }^{1065}$ Sie soll für ein "geordnetes Verfahren für Entscheidungen« sorgen und "Planungssicherheit schaffen. «1066

Mit zunehmender Zahl an Gesellschaftern drängt sich irgendwann zwangsläufig die Frage auf, welches Familienmitglied auf welcher Grundlage eine operative (Führungs-)Funktion im Unternehmen oder eine aktive Rolle in einem der Aufsichtsgremien bekleiden kann. Solange die Gründergeneration noch lebt, die zudem nicht selten einen patriarchischen Stil pflegt, werden solche Entscheidungen häufig von dieser eigenmächtig und unbegründet getroffen. Von den anderen Familienmitgliedern werden diese Entscheidungen zwar meist nicht akzeptiert, immerhin aber stillschweigend toleriert - aus Respekt vor den Altvorderen und deren Leistungen. Nachdem die Generation der Patriarchen langsam ausstirbt und nicht zuletzt die Rolle des Patriarchen in Familienunternehmen ausgedient zu haben scheint, bedarf es auch hierbei einer transparenten Vorgehensweise. Ein wichtiger Themenkomplex beschäftigt sich daher in den meisten Familienverfassungen mit der Rollenfrage. Hierbei geht es nicht zuletzt auch darum, die Handlungsfähigkeit zu sichern. ${ }^{1067}$

Auch gibt es Beispiele, in denen eine Familienverfassung aus einer konfliktären Situation heraus entsteht oder eine unschöne Konfliktsituation in der

1063 Interview Alexander Koeberle-Schmid (30.11.2017), S. 1.

1064 Interview B-2 (06.11.2017), S. 1.

1065 Vgl. Abschnitt 2.2.3.

1066 Hueck (2017), S. 19.

1067 Vgl. Abschnitt 2.2.3. 
Vergangenheit mit ausschlaggebend dafür ist, dass sich eine Unternehmerfamilie veranlasst fühlt, eine Familienverfassung zu erstellen. Diese Regelwerke fallen meist detaillierter aus. Man vereinbart beispielsweise eine Kommunikationskultur oder legt konkrete Regeln fest, wie im Konfliktfall zu verfahren ist. In diesen Fällen dient die Familienverfassung zuvorderst der Konfliktbefriedung bzw. -vermeidung ${ }^{1068}$ :

"Aus meiner Sicht will man damit [...] die Kommunikation und die gemeinsame Arbeit in der Gesellschafterfamilie organisieren. Und zwar in so einer Form, dass es eben nicht, oder in so einer Form, dass, wenn es zu Konflikten kommt, die dann auch systematisch und, sage ich mal, möglichst zielführend und möglichst wenig emotional [...] behandelt werden. ${ }^{1069}$

Mehrere Unternehmerfamilien gaben in den Gesprächen an, eine spezielle »Business-Version« der Familienverfassung an ihre leitenden Angestellten oder sogar allen Mitarbeiten und anderen Stakeholdern (z. B. Banker, Bürgermeister usw.) auszuhändigen. Eine Familienverfassung soll damit die Attraktivität des Familienunternehmens insgesamt steigern:

"Wir wollen ja als Familienunternehmen attraktiv sein. [...]. Die Business-Version, da gehen wir sehr großzügig damit um. Also wir haben die zum Beispiel dem Bürgermeister übergeben [...] und dann auch alle leitenden Mitarbeiter [...] haben Zugriffe auf die Business-Version der Charta [...]. ${ }^{1070}$

"[F]ür die Mitarbeiter war das durchaus wichtig, dass es so etwas gibt. Sie haben das interessant gefunden [...]. Weil das unserer Meinung nach auch eine gewisse Sicherheit geben kann. Der Mitarbeiterschaft. Weil einfach die Nachfolge damit geregelt ist, kann man sagen. ${ }^{1071}$

Die Familienverfassung übernimmt in diesem Moment eine Vertrauensfunktion. ${ }^{1072}$ Das Systemvertrauen fungiert als Pendant zum persönlichen Vertrauen gegenüber Menschen.

Einen weiteren, interessanten Grund liefert das Interview mit E-2. Während es in der einen Unternehmerfamilie der Gründer, der Patriarch, ist, der als zentrale Integrations- oder Identifikationsfigur ${ }^{1073}$ dient, welche die Familie zusammenhält, findet sich in anderen Familien eine ähnlich exponierte Persönlichkeit, sozusagen ein primus inter pares. Wenn diese Integrations- und

1068 Vgl. Abschnitt 2.2.3.

1069 Interview $\mathrm{H}-2$ (05. 12.2017), S. 2.

1070 Interview E-2 (22.11.2017), S. 2 f.

1071 Interview C-2 (10.11.2017), S. 8.

1072 Vgl. Abschnitt 2.2.3.

1073 Jene Variante der personenbezogenen Identifikation wird in der Literatur auch als "classical identification « bezeichnet; vgl. dazu Kelman (1961), S. 57 ff.; Ashforth/Mael (1989). 
Identifikationsfiguren mit ihrer natürlichen Autorität verschwinden, stellt sich die Frage, wer oder was an deren Stelle tritt:

»Und dann kam natürlich auch der Gedanke auf. Achtung. Irgendwann sind wir ja nicht mehr da. Die `Binde- und Klebewirkung`, die wir haben, auch die Autorität, die wir persönlich noch durch unseren Hintergrund ausstrahlen, irgendwann ist sie nicht mehr da. ${ }^{1074}$

An deren Stelle kann eine Familienverfassung treten, die mit ihren Prozessen und Institutionen bzw. Instrumenten für diese »Binde- und Klebewirkung» sorgen soll. Die Familienverfassung nimmt an dieser Stelle eine Identifikationsfunktion ein.

Die in Abschnitt 2.2.3 dargestellten Aufgaben und Funktionen einer Familienverfassung müssen folglich um die Identifikationsfunktion ergänzt werden.

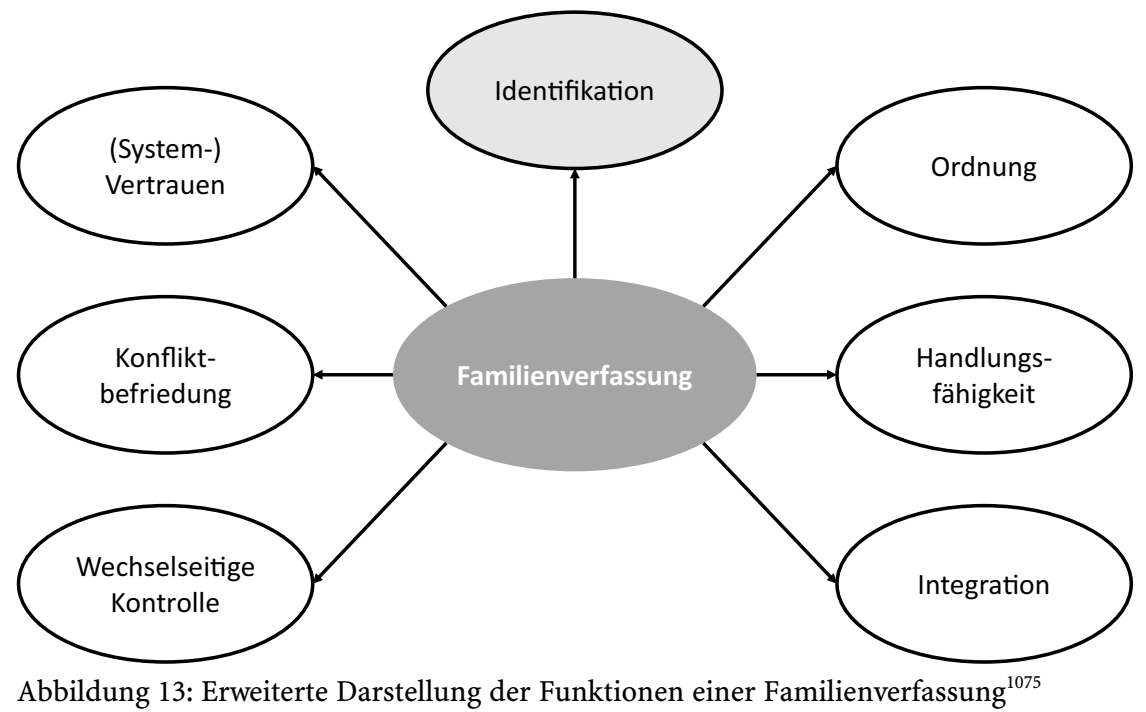

\subsubsection{Vorsorge ist besser als Nachsorge: die präventiven Maßnahmen zur Sicherung der Regelbefolgung}

Bei Maßnahmen zur Sicherstellung der Regelkonformität muss man grundsätzlich zwischen präventiven und reaktiven Maßnahmen unterscheiden. Nachfolgender Abschnitt widmet sich zunächst den präventiven Maßnahmen.

1074 Interview E-2 (22.11.2017), S. 2.

1075 Erweiterte Darstellung; in Anlehnung an Abbildung 5. 


\subsubsection{Die Ausgangslage entscheidet}

Einen gemeinsamen Nenner und damit das gemeinsame Interesse der Unternehmerfamilie zu finden, ist neben anderen Aspekten auch die Aufgabe einer Familienverfassung. Otto Fürst von Bismarck sagte einst: "Nichts ist besser geeignet, die Verschmelzung der widerstrebenden Elemente zu fördern, als gemeinsame Arbeit an gemeinsamen Aufgaben." Mit einer Familienverfassung wird die Strategie verfolgt, ein gemeinsames Verantwortungsbewusstsein innerhalb der Familie zu schaffen. Doch auch wenn etwas gemeinsam erschaffen wurde (wobei dabei stets fraglich ist, wie groß der aktive Part eines jeden Einzelnen war), garantiert ein solcher Akt nicht zwingend, dass man sich auch an die dabei aufgestellten Regeln hält.

Soll mit der Familienverfassung ein Familienrat installiert werden, an den die Mitglieder der Familie gewisse Kompetenzen abgeben sollen, kann dies nur gelingen, wenn man den Familienmitgliedern, die in den Familienrat gewählt werden, vorbehaltlos vertraut. Hindert aber ein latenter Konflikt daran, wird man sich dem Ganzen entziehen und im Zweifel nicht einmal die Familienverfassung mittragen können. Hier ist es entscheidend, einen gemeinsamen »Generalkurs« festzulegen und idealerweise für eine Vielzahl möglicher Eventualitäten bereits eine Vorgehensweise vorzubereiten. ${ }^{1076}$ Im Rahmen der einer Familienverfassung vorgelagerten strategischen Überlegung (die man unter dem Begriff Familienstrategie ${ }^{1077}$ kennt) muss die Unternehmerfamilie zunächst ein klares, gemeinsames Bekenntnis für die Richtungsentscheidung abgeben: sei es eine Exit-Strategie mit dem klaren Wunsch, das Unternehmen zu veräußern, oder sei es eine Loyalitätslösung mit dem klaren Bekenntnis zum gemeinsamen Unternehmen und dessen Verbleib im Familienbesitz. ${ }^{1078}$ Die Interviews haben gezeigt, dass es dafür sehr hilfreich ist, einen Experten für Familienverfassungen als Moderator zu verpflichten, um diesen Prozess zu begleiten:

"Das hätten wir alleine nicht hingekriegt. Das war schon deswegen so interessant, weil sie eigentlich so Fragen gestellt hat, die eigentlich für uns klar waren. Und dann kam auf einmal eine Antwort, wo wir dann dachten, ah, da habe ich jetzt gar nicht damit gerechnet. ${ }^{1079}$

Der Vorteil, diesen Prozess zusammen mit einem erfahrenen Experten zu gehen, liegt insbesondere darin, dass dieser auf Grund seiner Erfahrung viele Fallstricke bereits kennt:

1076 Vgl. Abschnitt 2.4.2.

1077 Vgl. Abschnitt 2.2.4.

1078 Vgl. Abschnitt 2.2.4.1.2.

1079 Interview D-1 (06.11.2017), S. 2. 
»Es sind halt in der Regel die Frage der Teilnahme. Teilnahme an Informationen, Teilnahme an Entscheidungen, Teilnahme an Zuwendungen, also finanzieller Art. Ich glaube, die Konfliktlinien laufen sehr stark an der Frage, was steht mir zu und was steht mir nicht zu und was bin ich schuldig und was bin ich nicht schuldig. Und daran zerknallen, glaube ich, die meisten Familien. Und gute Statuten regeln das [...]. $\ll^{1080}$

Ein spezialisierter Berater weist die Unternehmerfamilie auch auf Themen, Regelungen oder sonstige entscheidende Punkte hin, die sich in der Praxis bereits bewährt haben und somit die Wahrscheinlichkeit der Regelbefolgung erhöhen:

»[...] denn eine Erkenntnis aus dieser Beratung war auch, dass man nicht nur die Gesellschafter, sondern auch die Partner der Gesellschafter miteinbezieht. [...] der Hintergrund ist aber auch nachvollziehbar, weil natürlich Partner, also Ehepartner oder auch sagen wir mal Lebensgefährten, die jetzt gemeinsame Kinder haben, gemeinsamen Hausstand, die haben Einfluss auf ihre Partner, die Gesellschafter sind. ${ }^{1081}$

Wenngleich die Familienverfassung grundsätzlich in einem Stadium entwickelt werden sollte, in dem sich alle Familienmitglieder untereinander gut verstehen und noch miteinander sprechen können, kommt der umgekehrte Fall, dass sie aus einer konfliktären Situation heraus entsteht, in der Realität häufig genug vor. ${ }^{1082}$ In diesen Fällen ist es jedoch von entscheidender Bedeutung, diese (teils auch nur latent vorhandenen) Konflikte beizulegen, bevor eine gemeinsame Familienverfassung erstellt wird:

»Deswegen ist es aus meinem Blickwinkel immer wichtig, zu Beginn zu schauen, wie ist die Konstellation in der Familie, wie ist die aktuelle Situation des Unternehmens, welche Konflikte, welche Koalitionen gibt es in der Familie und auch zu schauen, was sind wirklich die Themen, die gerade zu besprechen sind, die relevant sind - für heute und auch für die Zukunft. Da mag es durchaus manchmal der Fall sein, dass man erst ein heißes Eisen anfassen und das lösen muss, bevor man dann überhaupt in die Erstellung einer Familienverfassung einsteigen kann. ${ }^{1083}$

Auch dafür eignet sich ein neutraler Dritter als Moderator, der dafür sorgen kann, dass die Diskussionen wertschätzend und möglichst emotionsfrei ablaufen. Gerade Unternehmerfamilie H, die den Prozess aus einer eher konfliktären Situation heraus gestartet hat, empfand die Begleitung durch den Experten als notwendig und wichtig:

»Ja, das muss man auch üben. Am Anfang fällt man sehr schnell wieder in alte Muster zurück. Da ist dann natürlich jemand, der von außen kommt und sagt, jetzt versuchen wir wieder auf eine sachliche Ebene zurückzukommen.«1084

1080 Interview B-2 (06.11.2017), S. 8.

1081 Interview E-2 (22.11.2017), S. 6.

1082 Vgl. Abschnitt 2.2.4.1.

1083 Interview Alexander Koeberle-Schmid (30.11.2017), S. 2.

1084 Interview H-1 (05. 12.2017), S. 4. 
Oft liegen die ursprünglichen Sachverhalte, die Auslöser für einen Konflikt waren, sogar Generationen zurück. Diese Konflikte wurden dann von Generation zu Generation "vererbt «. ${ }^{1085}$ Interessant und zugleich positiv erschien, dass im Falle der Unternehmerfamilie $\mathrm{H}$ die Generation der Junggesellschafter (»next generation«) gar kein Verständnis für die Konflikte aus der Vergangenheit hat:

"Und wenn ich so mit meinen Cousinen und Cousins und meinem Bruder aus der 5. Generation spreche, also das sind die, die schon ein entsprechendes Alter haben, [...] [d]a ist [...] überhaupt kein Verständnis für diese alten Konflikte da. Das ist schon mal gut. ${ }^{1086}$

Doch völlig unabhängig davon, wann ein Konflikt entstanden ist und woher er rührte, die Bewältigung der Vergangenheit und der gemeinsame Blick nach vorne als solide Ausgangsbasis für die Familienverfassung kann entscheidend dazu beitragen, dass das Regelwerk von allen Familienmitgliedern eingehalten wird; immerhin soll es das Fundament für eine Vertrauensbasis bilden, die das Gefühl der Zusammengehörigkeit stärkt und weiterentwickelt. ${ }^{1087}$

\subsubsection{Betroffene zu Beteiligten machen}

In den Fallstudien wurde deutlich, dass der Prozess der Erstellung einer Familienverfassung von besonderer Bedeutung ist:

An diesem Prozess sollten im Idealfall alle Familienmitglieder mitwirken ${ }^{1088}$, die nach deren Verabschiedung auch von der Familienverfassung betroffen sein werden:

"[...] es gibt unterschiedliche Formen, wie die Verfassung erarbeitet werden kann. Dafür gibt es kein Patentrezept. [...]. Ein wichtiger Grundsatz ist der: Eine Familienverfassung wird in einem Prozess erarbeitet und deswegen ist es wichtig, entsprechend die Familie einzubinden und sich nicht eine Familienverfassung aus dem Internet herunterzuladen oder von einem befreundeten Familienunternehmen schicken zu lassen und dann zu kopieren. Denn der Wert einer Familienfassung liegt nicht nur in dem Dokument, sondern in dem Erarbeitungsprozess, durch den sich die Familienmitglieder noch intensiver kennenlernen und sie die Motive der anderen besser verstehen; das ist ein ganz zentraler Bestandteil einer Familienverfassung. « ${ }^{1089}$

1085 Vgl. Rüsen (2017b), S. 138.

1086 Interview $\mathrm{H}-2$ (05.12.2017), S. 8.

1087 Vgl. Vernekohl (2007), S. 33; Wolf/Paul/Zipse (2009), S. 152; Hauck (2012b).

1088 Vgl. Reich/Bode (2018), S. 309: »Besonders bedeutsam [...] ist aber auch der gemeinsame Prozess ihrer Erarbeitung, da die Familienmitglieder viel übereinander und das Familienunternehmen erfahren. Das stärkt [...] den Zusammenhalt der Familie und die Identifikation mit dem Unternehmen."

1089 Interview Alexander Koeberle-Schmid (30.11.2017), S. 2. 
Gleichlautende Empfehlungen finden sich auch im Schrifttum:

"Waren es früher die Patriarchen aus Hoch- oder Geldadel, welche die entsprechenden Regeln einseitig dekretierten, so erfolgt deren Festlegung heute in einem gemeinsamen Prozess aller Familienmitglieder. Dabei ist der Erstellungsprozess nach der Selbsteinschätzung der Familienmitglieder und den Beobachtungen ihrer Berater mindestens ebenso bedeutsam wie das Ergebnis. ${ }^{1090}$

In einigen Fällen umfasst die Familienverfassung lediglich Gesellschafter, in vielen anderen jedoch auch die Ehepartner, Kinder oder langjährige Lebensgefährten - denn auch diese Familienmitglieder können in positiver oder negativer Weise auf die Gesellschafter einwirken.

Auch wenn die Beteiligung aller ein mühsamer Prozess bedeuten kann, da sich eine gewisse Meinungspluralität meist nicht vermeiden lässt, so ist die Akzeptanz und Bindungswirkung hinterher deutlich höher:

"Wenn ich gemeinsam eine Regel definiert habe, habe ich eine höhere moralische Bindung. « ${ }^{1091}$

Schon auf Grund der daraus resultierenden moralischen Bindungswirkung sollte eine Familienverfassung im Rahmen eines »affirmative[n] Akt[s] « ${ }^{1092}$ nach Fertigstellung von allen unterzeichnet werden. ${ }^{1093}$ In den untersuchten Fallstudien ließ sich bestätigen, dass alle Familienverfassungen von Mitgliedern der Unternehmerfamilien unterzeichnet wurden, wenngleich in solchen Fällen, in denen eine Familienverfassung aus einer konfliktären Situation heraus entsteht, das eine oder andere Familienmitglied durchaus seine Unterschrift verweigerte.

Bei besonders großen Unternehmerfamilien, deren Familienkreis schnell 50, 100 oder mehr Familienmitglieder zählt, kann es möglicherweise sinnvoll sein, die Erarbeitung der Themen in Gruppen aufzuteilen. Während an einem Kickoff-Meeting alle Familienmitglieder teilnehmen, sollten die Arbeitsgruppen dann vom Familienkreis gewählt werden, um sicherzustellen, dass die Personen die Interessen der anderen offiziell vertreten dürfen. Wählt jede Gruppe einen Sprecher, können sich die Sprecher in regelmäßigen Abständen über den Fortgang der Arbeit austauschen und am Ende die Ergebnisse konsolidieren.

Sieht man in der Familienverfassung ganz allgemein einen Akt der Selbstregulierung, so lässt sich feststellen, dass die Wahrscheinlichkeit der Akzeptanz der aufgestellten Regeln, die Normloyalität, durch die Regeladressaten dann höher ist, wenn die Regeln nicht oktroyiert werden, sondern selbst gestaltet

1090 Fleischer (2017b), S. 1206f. So im Ergebnis auch May/Bartels (2017), S. 93, 102, 113; Hueck (2017), S. 12.

1091 Interview Alexander Koeberle-Schmid (30.11.2017), S. 6.

1092 Fabis (2007), S. 362.

1093 Vgl. Baus (2017), S. 134; Brockhoff et al. (2012), S. 479; Brückner et al. (2010), S. 248; Schlippe/Groth/Rüsen (2017), S. 32. 
wurden. Nur wenn eine Regel als legitim empfunden wird, besteht auch eine hohe Wahrscheinlichkeit, dass diese von den Regeladressaten eingehalten wird. ${ }^{1094}$ Durch die Beteiligung der Regeladressaten können deren Interessen berücksichtigt werden und es entstehen verhältnismäßige Regeln auf Basis eines gemeinsamen Nenners. Bei diesem Prozess kann die intrapersonelle Akzeptanz gefördert werden. Es ist unstrittig, dass die Regelbefolgung bestenfalls auf Grund von intrinsischer Motivation und eigener Überzeugung von der Sinnhaftigkeit und Richtigkeit der Regeln erfolgen sollte. ${ }^{1095}$ Problematisch für die Akzeptanz der Regelsetzung kann es werden, wenn die Regeladressaten nicht angemessen an der Erstellung beteiligt und einbezogen werden. ${ }^{1096}$ In den Fallstudien finden sich auch entsprechende Beispiele dafür. Bei Unternehmerfamilie $\mathrm{F}^{1097}$ wird die Familienverfassung beispielsweise zwar grundsätzlich akzeptiert, weil sie sinnvoll erscheint, doch man merkte in den Gesprächen deutlich, dass hier jemand seinen Willen durchgesetzt und die Familienverfassung etabliert hat, ohne die Familie vollumfänglich einzubeziehen. In diesem Fall wird die Familienverfassung im Alltag nicht in dem Maße gelebt, wie dies in anderen Familien der Fall ist.

\subsubsection{3 (Moralische) Verbindlichkeit fördert Akzeptanz}

Unzweifelhaft können im Rahmen von Selbstregulierung erstellte Kodizes nur dann erfolgreich sein, wenn die selbst geschaffenen Regeln von den Adressaten in der Praxis auch befolgt werden. Ein regelkonformes Verhalten bedarf eines entsprechenden Anreizes. Ein solcher Anreiz kann in der Akzeptanz der Regeln gesehen werden. Es ist belegt, dass, je höher die Akzeptanz einer Regel ist, desto weniger sich deren Unverbindlichkeit negativ auf deren Einhaltung auswirkt. Folglich sind entsprechende Anstrengungen zu unternehmen, um die Akzeptanz einer Familienverfassung zu fördern. Das Schrifttum führt dazu aus, dass es dafür der moralischen Zustimmung zu den Zielen der Regeln durch die Regeladressaten bedarf. Oder die Regeln müssen von einer echten moralischen Überzeugung getragen werden. ${ }^{1098}$ LUHMANN (1987) greift dabei auf das Konzept "Legitimation durch Verfahren ${ }^{1099}$ zurück. Die Vorstellung basiert darauf, dass eine Partizipation gut sei und daher geboten ist. Bereits in Abschnitt 4.1.3.2 wird ausgeführt, wie wichtig es ist, die Familienmitglieder in den Prozess der Regelsetzung einzubeziehen. Ein weiterer Anreiz wird darin gesehen, dass die Regeln

1094 So im Ergebnis wohl auch Leipold/Pies (2000), S. 339.

1095 Vgl. Abschnitt 2.5.1.4.3 b).

1096 Vgl. Abschnitt 2.5.1.3.1 b).

1097 Vgl. Abschnitt 3.6.

1098 Vgl. Abschnitt 2.5.1.4.2; Luhmann (1987).

1099 Raiser (2013), S. 132. 
einen erkennbaren Nutzen bieten und dadurch für die Regeladressaten sinnvoll erscheinen.

B-2 sieht in guten Gründen durchaus eine regulative Kraft:

„Genau, gute Gründe haben ja eine gewisse regulative Kraft unter vernünftigen Leuten $[\ldots] . \aleph^{1100}$

Ein Nutzen kann dadurch begründet werden, dass es der Unternehmerfamilie darum geht, den Verbleib des Familienunternehmens im Besitz der Familie zu sichern, was auch auf Basis der ausgewerteten Fallstudien als eine der Hauptintentionen genannt werden kann. Die Familienverfassung wird in diesem $\mathrm{Zu}$ sammenhang als sinnvolles Instrument zur Konfliktprävention wahrgenommen:

»[Die Familienverfassung soll] die Kommunikation und die gemeinsame Arbeit in der Gesellschafterfamilie organisieren. Und zwar in so einer Form, dass es eben nicht, oder in so einer Form, dass, wenn es zu Konflikten kommt, die dann auch systematisch und, sage ich mal, möglichst zielführend und möglichst wenig emotional sozusagen behandelt werden. ${ }^{1101}$

Fraglich ist auch, wie man eine moralische Zustimmung, die eine moralische Verbindlichkeit voraussetzt, innerhalb einer Familie erreichen kann. ${ }^{1102}$ Die Antworten der Interviewpartner im Rahmen der Fallstudien waren alle sehr ähnlich und lassen sich auf zwei wesentliche Merkmale konzentrieren. Zum einen sei es eine Frage der Erziehung. Hier kann bereits bei Kindern der Grundstein gelegt werden.

»Handlungsweisen und Wertvorstellungen der Unternehmerfamilie prägen die Identität der Nachkommen bereits in jungen Jahren, wobei sich Teile des persönlichen Lernprozesses meist unbewusst vollziehen. Aufgrund dieser engen Wechselbeziehung zwischen Unternehmen und Familie ist es sinnvoll, bereits früh - aber auf spielerische Art und Weise - mit der >Ausbildung ` der Abkömmlinge anzufangen. Anders als für die jungen Erwachsenen, für die mittlerweile Vorträge, Workshops, Literatur und (Nachfolge-)Beratung organisiert werden, gibt es für diese Altersgruppe allerdings nur wenige Angebote. ${ }^{1103}$

So rät auch Koeberle-Schmid zu einer frühen Einbindung der Kinder:

»Eine frühe Einbindung der Kinder ist immer gut, da die nächste Generation einen Bezug zum Unternehmen aufbauen muss. Damit kann man nicht früh genug beginnen. Also zum Beispiel Schnitzeljagd über das Firmengelände oder die Fahrt mit der Betriebsfeuerwehr auf dem Firmengelände ${ }^{1104}$

1100 Interview B-2 (06.11.2017), S. 12.

1101 Interview H-2 (05. 12.2017), S. 2.

1102 Vgl. Abschnitt 2.5.1.4.2.

1103 Felden/Hack (2014), S. 343.

1104 Interview Alexander Koeberle-Schmid (30.11.2017), S. 4. 
In diesem Kontext ist es sinnvoll, sich die bei Aristoteles zu findende motivationale Grundlage der Moral anzusehen:

„Was durch moralische Sozialisation erzeugt wird, sind primär Charakterdispositionen,
bei guter Sozialisation Tugenden, die sicherstellen, dass Menschen durchgängig nach
den Normen handeln, weil sie geeignete positive Handlungsmotive, die Ausrichtung auf
ein Gut, internalisiert und in ihr Selbstbild aufgenommen haben. Das Schuldgefühl
wäre dann nicht etwas Eigenes, was als Sanktion mit der Norm eingeführt wird, sondern
es wäre die Kehrseite einer positiven Einstellung, deren Durchbrechung eine negative
Selbstbewertung auslöst. Dass die moralische Sanktion so funktioniert, im Kontext von
positiven Motiven, die in Tugenden internalisiert sind, und nicht als bloße negative
Sanktion beim Übertreten einer Norm, scheint auch psychologisch verständlicher. "1105

Neben der Internalisierung dient die Identifikation als ein motivationaler Grund für die Einhaltung einer Regel. ${ }^{1106}$ Die Identifikation als Grund bringt zum Ausdruck, dass ein Regeladressat eine Regel befolgt, weil er sich am Verhalten seiner Bezugsgruppe oder einzelnen Vorbildern orientiert (Identifikation mit dem sozialen Umfeld). ${ }^{1107}$ Kinder sehen oft in den Eltern diese Vorbildfunktion und identifizieren sich mit ihnen. Darüber hinaus kann jedes Familienmitglied durch eine Vorbildfunktion zu dieser moralischen Verbindlichkeit beitragen. Indem man die Einhaltung der Regeln bewusst vorlebt, werden alle anderen implizit auch aufgefordert, ihr Verhalten entsprechend anzupassen:

"[...] ich glaube, das kann in Familien gelingen, wenn man von klein auf mit den Kindern über diese Dinge spricht und wenn man ein bestimmtes Verhalten lebt und vorlebt. Und das Vorbild ist die wichtigste Erziehungsfunktion. Oder das wichtigste Erziehungsmittel. ${ }^{1108}$

Moralphilosophisch lässt sich diese Art der Verbindlichkeit auf Basis der Moralbegründung nach Adam Smith erläutern, welcher die unsichtbaren Bande zwischen Menschen als »sympathy« bezeichnet. ${ }^{1109}$

Da der Entzug von »sympathy« grundsätzlich als unangenehm empfunden wird - und dieses negative Gefühl in verwandtschaftlichen Beziehungen sicherlich noch deutlich stärker ausgeprägt sein wird -, ist der implizite Druck, sich regelkonform $\mathrm{zu}$ verhalten, in familiären Beziehungen dermaßen hoch, dass jeder Verstoß mit Verachtung oder gar Ausschluss aus der Gesellschaft geahndet werden könnte:

1105 Wolf (2013), S. 44.

1106 Vgl. Abschnitt 2.5.1.4.3.

1107 Vgl. Abschnitt 2.5.1.4.3 a).

1108 Interview A-2 (08.11.2017), S. 8.

1109 Vgl. Abschnitt 2.5.2.7. 
»Der wird dann so unter moralischen Druck kommen, er würde eigentlich von seiner ganzen Familie geächtet werden. Das muss er dann auch erst einmal aushalten. ${ }^{1110}$

\subsubsection{Inkonsistenz vermeiden: die Anpassung des Gesellschaftsvertrags}

Wie bereits ausgeführt, handelt es sich bei einer Familienverfassung und einem Gesellschaftsvertrag um zwei unterschiedliche Dokumente. Die Familienverfassung kann als Wegbereiter des Gesellschaftsvertrags gesehen werden, was nicht darüber hinwegtäuschen soll, dass existierende Familienunternehmen bereits einen Gesellschaftsvertrag haben. Mit »Wegbereiter« ist vielmehr der Umstand gemeint, dass im Nachgang an die Erstellung der Familienverfassung der Gesellschaftsvertrag ggf. angepasst werden sollte ${ }^{1111}$. In den Fallstudien finden sich einige Beispiele, wo nach Fertigstellung der Familienverfassung der Gesellschaftsvertrag entsprechend geändert wurde. Zwar sind die meisten Formulierungen einer Familienverfassung eher sehr allgemein gehalten, doch manche Familienverfassungen werden in Teilen sehr konkret. Diese Formulierungen könnten dann in Konflikt zu dem Wortlaut des Gesellschaftsvertrags stehen. Dies insbesondere dann, wenn in der Familienverfassung sehr eindeutige Vorgaben im Hinblick auf das Unternehmen und die Gesellschafterstellung gemacht werden. Sieht eine Familienverfassung beispielsweise als Sanktionsmechanismus den Ausschluss eines Gesellschafters vor (an dieser Stelle soll zunächst unbeachtet bleiben, dass eine solche Regelung ohnehin rechtlich zweifelhaft ist ${ }^{1112}$ ) und ist dies nicht Bestandteil des Gesellschaftsvertrags, sollte dieser dringend nach Fertigstellung der Familienverfassung angepasst werden. Eine Klausel, die den Ausschluss eines Gesellschafters bei groben Verstößen vorsieht, ist durchaus in vielen Gesellschaftsverträgen zu finden, wenngleich die Regelungen je nach Rechtsform des Unternehmens variieren mögen. ${ }^{1113}$ Sieht eine Familienverfassung also beispielsweise den Ausschluss eines Gesellschafters vor und diese Maßnahmen finden sich nicht rechtswirksam im Gesellschaftsvertrag verankert, mag es für den einen oder anderen Gesellschafter, um diese Tatsache wissend, einen unnötigen Grund liefern, sich nicht an die gemeinsamen Regeln zu halten, zumal die eigentlich abschreckende Konsequenz ohnehin rechtlich nicht durchgesetzt werden könnte.

Ohne an dieser Stelle zu sehr auf rechtliche Erfordernisse eingehen zu wollen, wird dringend empfohlen, den Gesellschaftsvertrag im Nachgang an die Fami-

1110 Interview E-2 (22.11.2017), S. 21.

1111 Vgl. Abschnitt 2.4.2.

1112 Vgl. Abschnitt 4.1.4.3.4.4.

1113 Je nach Rechtsform sind diese Regelungen anders zu gestalten. Bei Hueck (2017) finden sich einige Ausführungen dazu. 
lienverfassung auf entsprechenden Anpassungsbedarf durch einen Rechtsberater prüfen zu lassen. ${ }^{1114}$

\subsubsection{Die Business-Fassung als Garant für Regelkonformität?!}

»Was nützt es Dir, keinen Mitwisser zu haben, da Du ein Gewissen hat? « ${ }^{1115}$ Mit diesem Zitat soll die Überlegung eingeführt werden, die Inhalte der Familienverfassung einem größeren Kreis zugänglich zu machen. Nicht nur wird in der Literatur empfohlen, neben der eigentlichen Version der Familienverfassung für die Familie auch eine Business-Version zu erstellen. ${ }^{1116}$ Dieser Vorschlag findet auch bereits in der Praxis häufig Anwendung. So ließ sich diese Idee auch durch die Fallstudien bestätigen:

"Das ist auch etwas, was im Unternehmen kommuniziert wurde, und dem Unternehmen wurde eine abgespeckte Form der Charta übergeben, so dass Transparenz herrscht, was wir als Gesellschaftergruppe machen. Dass die es nicht losgelöst vom Unternehmen machen, sondern dass das da zusammenhängt. ${ }^{1117}$

Nun variiert es von Unternehmen zu Unternehmen, welcher Personenkreis in den Genuss einer solchen Business-Fassung kommt. So kann dieser Personenkreis bei Fremdmanagern der Geschäftsführung und leitenden Angestellten anfangen, über die komplette Belegschaft mit Zugriff über das Intranet bis hin zu externen Stakeholdern wie Banker oder Bürgermeister.

Auch wenn bei solchen Überlegungen vornehmlich die Vertrauensfunktion der Familienverfassung im Vordergrund steht ${ }^{1118}$, so hat dieses Vorgehen einen nicht ganz unwichtigen Nebeneffekt. Indem man die Inhalte der Familienfassung einem weiteren Kreis - der nicht Familie ist - zugänglich macht, erhöht sich automatisch der Druck auf die Familienmitglieder, sich regelkonform zu verhalten.

»Wenn das Dokument selbst Dritten zugänglich gemacht werden soll [...], sollte [es] gleichzeitig einen Ausdruck von Festigkeit und Selbstbewusstsein vermitteln. [...] was wiederum der verpflichtenden Kraft des Dokuments zugutekommt. «1119

1114 Vgl. Lorz/Sarholz (2014), S. 11:»Gleiches gilt, wenn die einzelnen Dokumente nicht richtig aufeinander abgestimmt sind, wenn also z. B. eine Familiencharta gemeinsam entwickelt wurde, die hierin getroffenen, nicht bindenden Festlegungen jedoch von den bindenden Vorgaben des Gesellschaftsvertrags abweichen. Auch dann ist der Keim eines Streits gelegt und die gute Absicht verkehrt sich leicht in ihr Gegenteil."

1115 Dieser Ausspruch wird Lucius Annaeus Seneca zugeschrieben.

1116 Vgl. Baus (2017), S. $116 \mathrm{ff}$.

1117 Interview E-1 (21.11.2017), S. 5.

1118 Vgl. Abschnitt 2.2.3.

1119 Baus (2017), S. 110. 
Unterstützt wird die Regelkonformität dann, wenn der Regeladressat einen (mittelbaren oder unmittelbaren) persönlichen Nutzen in der Einhaltung erkennt. Einen persönlichen Nutzen kann der Regeladressat dabei auch in der Vermeidung innerer Sanktionen im Sinne von Scham und Enttäuschung ob der Abweichung von den selbst auferlegten Regeln sehen. ${ }^{1120}$ Das Gefühl der Scham mag sich dann noch verstärken, wenn der Regelbruch auch Dritten (Nicht-Familienmitgliedern) zur Kenntnis kommt. Die Gefahr ist umso größer, je größer der Kreis derer ist, die Zugang zu einer Business-Version der Familienverfassung haben. Ein Regelbrecher fürchtet dann eine Sanktionierung durch das Umfeld. ${ }^{1121}$ In Ansätzen kann dieser Mechanismus mit der Wirkweise des DCGK erklärt werden. Eine echte Sanktionierung bei Verstößen gegen den DCGK gibt es nicht. Man geht vielmehr davon aus, dass der Markt, das Umfeld die Einhaltung goutiert oder jedwede Verfehlung entsprechend sanktioniert. ${ }^{1122}$

Gibt man als Unternehmerfamilie also eine Business-Version der Familienverfassung heraus, gewährt man Dritten einen Einblick in ein Regelwerk, dessen Einhaltung sie dann auch von den Familienmitgliedern erwarten können. Daraufhin fühlt sich jedes Familienmitglied, moralisch betrachtet, diesen Dritten gegenüber auch für sein Handeln verantwortlich und muss damit rechnen, dass diese auf ein Fehlverhalten mit Unmut und Entrüstung reagieren. Diese Überlegung erhöht zwangsläufig den Druck auf die Familienmitglieder, sich regelkonform zu verhalten.

\subsubsection{Wissen schafft Vertrauen und fördert Akzeptanz}

Im Zuge der Einführung einer Familienverfassung wird häufig auch über »Family Education « nachgedacht. ${ }^{1123}$ Es geht dabei um die gezielte Förderung der Gesellschafterkompetenz:

"The best way to protect the future of the business and enhance its continuity in the family is to prepare the next generation of owners for their roles and responsibilities. " 1124

Man ist sich einig, dass es entsprechender Programme bedarf, um sich zu einem verantwortungsvollen und kompetenten Gesellschafter entwickeln zu können. Eine im Jahr 2013 von PwC und dem WIFU durchgeführte Studie zum Thema Gesellschafterkompetenz mit 263 Familienunternehmen führte zu der Erkenntnis, dass knapp 70 Prozent der befragten Familienunternehmer der Mei-

1120 Vgl. Abschnitt 2.5.1.3.2 a).

1121 Vgl. Abschnitt 2.5.1.5.2 b).

1122 Vgl. Bachmann (2002), S. $2137 \mathrm{ff}$.

1123 Vgl. Abschnitt 2.3.1.3.4.

1124 Aronoff/Ward (2011), S. 50. Im Original in fetter Schriftstärke. 
nung sind, dass die Entwicklung von Gesellschafterkompetenz Konflikte in der Gesellschafterfamilie verhindern kann. Deutlich über 80 Prozent der befragten Unternehmen sind darüber hinaus der Auffassung, dass sie den Zusammenhalt und die Bindung der Gesellschafterfamilie fördert und für eine gemeinsame Willensbildung sorgen kann.

Bei kleinen Unternehmerfamilien, bei denen noch dazu die Mehrzahl der Familienmitglieder operativ im Unternehmen tätig ist, mag dies nicht zwingend erforderlich sein. Es ist jedoch leicht nachvollziehbar, dass insbesondere Unternehmerfamilien mit einem größeren und weit verzweigten Gesellschafterkreis über die Einführung von Gesellschafterkompetenz-Programmen nachdenken sollten. Insbesondere zeichnet sich schon länger ab, dass zukünftig immer mehr nicht-operative Gesellschafter über die Geschicke von Familienunternehmen entscheiden werden ${ }^{1125}$. Gerade über solche Programme kann man auch bei jüngeren Gesellschaftern der "next generation« eine stärkere emotionale Bindung an das Unternehmen erreichen. ${ }^{1126}$ Greift man hierbei auf die bereits entwickelten Programme etablierter Dienstleister zurück, geht dies mit dem positiven Nebeneffekt einher, dass die eigenen Kinder so in Kontakt mit dem Nachwuchs aus anderen Unternehmerfamilien kommen und ihr Netzwerk erweitern können. ${ }^{1127}$

Gerade auch das Interview mit B-2 zeigte, dass man

"[...] vor allem es dann auch geschafft hat, die jungen, die jüngeren Gesellschafter mit ins Boot zu holen, [...] das ist alles Regelwerk, Papier, aber wichtig ist uns vor allem die Schulung der Gesellschafter. Dass jeder auch weiß, worum es geht, das Unternehmen kennenlernt., die ganzen Unternehmensstrukturen parat hat, auch die Ausrichtung, die Unternehmensphilosophie mitträgt. «1128

Je mehr die Familienmitglieder, die jüngeren und älteren Gesellschafter, mit Informationen zum Unternehmen versorgt werden und sich selbst Wissen im Rahmen der Kompetenzprogramme aneignen, desto stärker ist die Beziehung und emotionale Bindung zum Unternehmen und damit auch zur Familie. Diese Bindung erschwert wiederum eine Abkehr von dem die Familie verbindenden Element: das Familienunternehmen. Je stärker diese Beziehung und emotionale Bindung zum Unternehmen und zur Familie ist, desto höher ist die Wahrscheinlichkeit, dass der Zweck der Familienverfassung erkannt wird und die Regeln auch eingehalten werden. Die Sachnähe erhöht die Bereitschaft zur freiwilligen Regelbefolgung. ${ }^{1129}$ Auch eignet sich die Einführung einer Gesell-

1125 Vgl. Rüsen/Schlippe/Groth (2014), S. 107.

1126 Vgl. dazu die Ausführungen zur Unternehmerfamilie H, Abschnitt 3.8.

1127 Auch darauf hinweisend Breyer (2015), S. 153.

1128 Interview B-1 (17.10.2017), S. 2.

1129 Vgl. Abschnitt 2.5.1.4.1. 
schafterplattform um hier zentral alle relevanten Informationen für Gesellschafter bereitzustellen. So können sich auch solche Familienmitglieder jederzeit informieren, die möglicherweise räumlich sehr weit entfernt wohnen.

\subsubsection{Die Zwangswirkung des Gesellschafterdaseins relativieren}

Die geführten Interviews mit Vertretern von Unternehmerfamilien gaben teilweise Hinweise darauf, dass einzelne Familienmitglieder das Gesellschafterdasein als Zwang oder teilweise auch als befremdlich empfinden:

»Ein Beispiel wäre meine Schwester, die [...] das Ganze versteht und da auch emotional sehr verbunden ist, aber auf der anderen Seite zu der finanziellen Seite des Gesellschafterdaseins eine ganz gespaltene Haltung hat. Für sie ist das eher fast befremdlich, würde ich jetzt mal sagen. «1130

Sicherlich ist es in den meisten Familienunternehmen der Fall, dass es nicht gerne gesehen wird, wenn Gesellschafter ihre Anteile verkaufen und austreten wollen. So kann dieses Szenario in einem Unternehmen - je nach prozentualem Anteil des jeweiligen Gesellschafters - durchaus zu einer Liquiditätsbelastung führen. Doch mitunter ist es auch so, dass diese "Mitgliedschaft« als Zwang empfunden wird, was dem gemeinsamen Ziel der Unternehmerfamilie, welches sie auch mit einer Familienverfassung zum Ausdruck bringt, eher abträglich sein kann. Das Gesellschafterdasein mag einerseits mit jährlichen Ausschüttungen durchaus angenehme Vorteile bieten, andererseits ist es auch mit Verpflichtungen verbunden:

"Wir stellen in Krisen Kapital zur Verfügung usw. Das ist sozusagen die andere Seite. Das ist kein bedingungsloser Freifahrtschein. ${ }^{1131}$

Vor diesem Hintergrund wird der Austritt häufig erschwert, geht es doch um die Überlebensfähigkeit des Unternehmens. Die Ausstiegsklauseln sind meist so konzipiert, dass ein Ausscheiden tendenziell eher uninteressant erscheint: lange Auszahlungsfristen, Abschläge vom Unternehmenswert usw. ${ }^{1132}$

Es bedarf sicherlich keiner besonderen Erläuterung, dass ein Gesellschafter, der mit seinem Gesellschafterdasein hadert, sich möglicherweise nicht immer uneingeschränkt regelkonform verhalten wird. Das die Familie eigentlich verbindende Element wird dann als Belastung empfunden.

Es soll an dieser Stelle keinesfalls die Regelung, dass Individualinteressen grundsätzlich hinter dem Gemeinschaftsinteresse zurückzustellen sind, in Frage gestellt werden, die sich so in vielen Familienverfassungen findet, doch darf

1130 Interview E-1 (21.11.2017), S. $11 \mathrm{f}$.

1131 Interview H-2 (05. 12.2017), S. 6.

1132 Vgl. dazu beispielhaft Kormann/Schmeing (2016), S. $14 \mathrm{ff}$. 
gleichzeitig auch nicht verkannt werden, dass unzufriedene Gesellschafter eher geneigt sind, sich abzuwenden und tendenziell häufiger Regelbrüche zu begehen.

Es soll nun wahrlich keine Empfehlung dafür ausgesprochen werden, die Ausstiegsoptionen für Gesellschafter zu erleichtern, es soll lediglich bedacht werden, wie damit umzugehen ist. Unternehmerfamilie $\mathrm{H}$ hat dazu eine klare Meinung:

"Wer rausgehen will, kann rausgehen. Das war ein großer Schritt emotional in der Familie. [...] Aber im Endeffekt haben wir schon auch gemeinsam diskutiert, dass wir sagen, wenn jemand jetzt eigentlich das Gefühl hat, mir fehlt die Bindung zum Unternehmen oder das belastet mich, ich möchte einfach nicht mehr, dass das dann auch ok ist, dass diese Person rausgeht. Und wir haben das mal durchgespielt tatsächlich, also es gibt eine Person im Gesellschafterkreis, die noch einen großen Anteil hat, ein Viertel ungefähr, alle anderen haben sich schon viel mehr verteilt. Da bin ich dann bei drei Prozent, zwei Prozent, vier Prozent, da ist das nicht so wild. Aber selbst diese 25 Prozent und das haben wir mal durchgespielt, würde das Unternehmen - sofern es normal läuft - auch verkraften. « ${ }^{1133}$

Gerade bei größeren Unternehmerfamilien werden die Anteile, die ein Gesellschafter hält, mit jeder Generation tendenziell kleiner, so dass man überlegen sollte, ob man das Ausscheiden eines Gesellschafters grundsätzlich nicht als Hochverrat wertet, sondern bei Beibehaltung entsprechender Hürden gestattet und darüber auch im Rahmen der Erstellung der Familienverfassung spricht.

Ein weiterer interessanter Aspekt zu dieser Diskussion steuerte das Gespräch mit Unternehmerfamilie B bei. Sie gestattet ihren Gesellschaftern sogenannte Mikrokündigungen bei Kapitalbedarf, die als Präventionsinstrument gesehen werden:

»Ich sag Ihnen auch noch einen Aspekt von Prävention [...]. Gesellschafter können bei uns Mikrokündigungen machen. Das heißt, sie können Minianteile kündigen. Wir erlauben Gesellschaftern ohne Zustimmung der anderen Gesellschaftern Anteile zu kündigen im Promille-Bereich. Das hat den Riesenvorteil, dass Gesellschafter sich nicht erleben in dieser hand-cuffed Situation. Bei uns ist [...] der Verkauf von Anteilen [...] sehr schwer, weil man Vorkaufsrechte hat und Fristen hat und, und, und, es dauert alles immer ewig. Und das empfinden viele Gesellschafter, oder könnten es als Gängelei empfinden [...] Und deswegen sagen wir, wir haben so eine ganz lockere Möglichkeit, hat noch nie ein Gesellschafter genutzt, aber jeder Gesellschafter weiß, dass er es könnte, dass er sagen kann, ich kann hier ganz ein bisschen den Hahn aufmachen und mir ein bisschen Geld rausholen. Sie verlieren dann eben Anteile, die wachsen den anderen zu verhältnismäßig und die Gesellschaft wird dann halt gedrained ein Stück weit an der Stelle, aber damit haben wir eine Riesenprävention. Eine ganz wichtige

1133 Interview H-2 (05.12.2017), S. 5 f. 
Prävention von Konflikten, dass Leute einfach wissen, ich bin hier nicht gefangen auf Gedeih und Verderb und muss hier immer hard ball spielen, wenn ich Geld brauche. ${ }^{1134}$

Indem die Zwangswirkung des Gesellschafterdaseins relativiert wird, sinkt die Gefahr, dass ein Gesellschafter ausbricht und sich nicht regelkonform verhält.

\subsubsection{Vertrauen ist gut, Kontrolle ist besser}

Es besteht kein Zweifel daran, dass sich die Wahrscheinlichkeit einer Regelkonformität durch - wie auch immer geartete - präventive Kontrollen verstärken lässt. ${ }^{1135}$ Geht man von einer psychosozialen Verankerung von Normen ${ }^{1136}$ aus, folgt daraus unmittelbar, dass jeder Regelverstoß gewisse Sanktionen (seien es interne oder externe) und ein gewisses Maß an Empörung hervorrufen muss. Es kann zu internen Sanktionen im Sinne von Schuldgefühlen und möglicherweise auch zu Selbstvorwürfen führen. Allerdings wird der psychosozialen Sanktioniertheit moralischer Normen berechtigte Kritik entgegengebracht. So wird der Einwand erhoben, dass an solchen Stellen, wo die Gefahr eher gering ist, einen Fehltritt zu entdecken, die soziale Sanktion fehlt. ${ }^{1137}$ So wundert es nicht, dass für eine effektive Durchsetzung von Regeln ein funktionierender Kontrollmechanismus empfohlen wird. ${ }^{1138}$

Der im Rahmen einer Familienverfassung etablierte Familienrat (eher bei größeren Familien zu finden) oder der Familienmanager/ Familienbeauftragte (eher bei kleineren Familien zu finden) kann eine solche Kontrollinstanz repräsentieren. ${ }^{1139}$ Nun ist es sicherlich so, dass der Familienrat in sehr seltenen Fällen ausschließlich als Kontrollinstanz etabliert wird. Vielmehr wird aber von seinen gewählten Vertretern erwartet, dass sie in ständigem Kontakt mit den Familienmitgliedern stehen und somit einen intensiveren Einblick bekommen, um Verfehlungen zu entdecken und entsprechend anzusprechen:

"[...] und generell als Familienrat eine Instanz darstellen, das ist vielleicht schon ein wichtiger Faktor. Wenn der Familienrat innerhalb einer Gesellschafterrunde einen gewissen Respekt hat, dann ist das eine Instanz, die auch im Grunde eine präventive Maßnahme ist, wenn man so sagt, so sehen will. Also sprich, wenn man diesen Familienrat gar nicht kennt, gar nicht weiß, was der so tut, weil die sich gar nie melden, und wenn man dann auch gar keine große Angst, oder keinen Respekt vor dieser Instanz hat, dann wird man sich auch an keine Regel halten wollen. Also ich denke, so eine Instanz

1134 Interview B-2 (06.11.2017), S. 13.

1135 So im Ergebnis auch Gilch/Pelz (2008), S. $134 \mathrm{ff}$.

1136 Vgl. Wolf (2013), S. 42.

1137 Vgl. Abschnitt 2.5.2.4.

1138 Vgl. Damrau (2003), S. 94ff. Allgemein zur Kontrolle als Voraussetzung einer effektiven Regeldurchsetzung Abschnitt 2.5.1.4.4.

1139 Vgl. Fabis (2009), S. 18; Schulz/Werz (2007b), S. 360. Vgl. dazu auch Abschnitt 2.3.1.3.2. 
braucht es schon. [...] Und wir haben uns natürlich auch im Laufe der Jahre Respekt verschafft, weil wir auch liefern. Wir informieren viel. Wir zeigen Präsenz bei allem, wo wir auch zeigen müssen. $\aleph^{1140}$

Neben dem Familienrat kann diese Kontrollfunktion natürlich auch vom Familienbeauftragten/Familienmanager ausgehen ${ }^{1141}$. Bei Unternehmerfamilie D und E wurde ein sogenannter "Hüter der Charta" nominiert. Dieser Begriff könnte falsche Assoziationen auslösen. Damit ist nicht die Kontrollinstanz gemeint, sondern die dafür nominierte Person soll Sorge dafür tragen, dass alle Familienmitglieder immer die aktuelle Version der Familienfassung haben; sie behält die Übersicht über die vorzunehmenden Änderungen und veranlasst deren Einarbeitung in sämtliche Exemplare.

\subsubsection{Blut ist dicker als Wasser: Stärkung innerfamiliärer Kohäsion}

Emotionale Bindung, ein starker Zusammenhalt und damit verbunden eine hohe Identifikation mit der Familie und dem gemeinsamen Unternehmen können vieles bewirken: Konflikten vorbeugen oder der zunehmenden Entfremdung präventiv begegnen. ${ }^{1142}$ Die Fallstudien haben unverkennbar gezeigt, dass die Unternehmerfamilien davon ausgehen, dass die innerfamiliäre Kohäsion einer der wichtigsten Schlüssel in der präventiven Vermeidung von Regelbrüchen und Abweichungen ist:

"[...] und dieses Familiengefühl hat ja eine Kohäsionswirkung. Das soll sie auch haben, das ist ja kein Zufall, das ist eine Absicht. Und [...] je enger und verbundener diese Familie, dieser Familienverbund ist, desto schwerer wird es, aus dem auszubrechen. ${ }^{1143}$

Doch auch Konflikte können den Familienfrieden erheblich stören und zu irrationalen Handlungen führen. Nicht ohne Grund wird im Streit der größte Wertvernichter in Familienunternehmen gesehen. ${ }^{1144}$ Wenn in Unternehmer-familien nicht mehr miteinander gesprochen wird, ein »wir gegen die« zum Alltag gehört und die Familienzweige nicht mehr konstruktiv, sondern destruktiv arbeiten, dann ist sicherlich leicht nachvollziehbar, dass man nicht davon ausgehen sollte, dass die ehemals getroffenen Vereinbarungen eines rechtlich nicht verbindlichen Regelwerks immer zwingend eingehalten werden.

1140 Interview B-1 (17.10.2017), S. 14.

1141 Vgl. Abschnitt 2.3.1.3.3.

1142 So auch Hueck (2017), S. 54.

1143 Interview E-2 (22.11.2017), S. 15.

1144 Nicht zuletzt aus diesem Grund findet sich zwischenzeitlich zahlreiche Literatur zu diesem Thema. Vgl. beispielhaft Wedemann (2013); Rüsen (2017b); Kirchdörfer/Sarholz (2012); Großmann (2014); Fabis (2007). 
Es soll nachfolgend aufgezeigt werden, mit welchen konkreten Maßnahmen im Rahmen der Familienverfassung die innerfamiliäre Kohäsion gestärkt, die Konfliktanfälligkeit verringert und damit die Wahrscheinlichkeit der Regelbefolgung tendenziell erhöht werden kann.

\subsection{Ein gemeinsamer Erstellungsprozess schweißt zusammen}

In Abschnitt 4.1.3.1 wurde bereits näher darauf eigegangen, dass die Ausgangslage mit darüber entscheidet, wie wahrscheinlich ein regelkonformes Verhalten der Familienmitglieder sein wird. Es ging dabei vornehmlich darum, dass man gemeinsam den Generalkurs festlegt und möglichst mit Hilfe eines Beraters als Moderator und Experte sicherstellt, dass die in der Praxis erprobten Inhalte berücksichtigt werden. Zudem wurde darauf hingewiesen, dass eventuell vorhandene Konflikte vor der Erstellung einer Familienverfassung beigelegt werden sollten, um mit einer positiven Ausgangsbasis die Ära mit Familienverfassung einzuleiten.

Dieser Erstellungsprozess soll allerdings an dieser Stelle erneut thematisiert werden. Allerdings nun vor dem Hintergrund, welche Auswirkungen dieser auf den Zusammenhalt der Familie haben kann. FABIs (2009) führt dazu aus:

»Die befriedigende Wirkung der Familienverfassung [...] wird zudem durch die gemeinschaftliche Erarbeitung bedingt. Indem der gesamte, auch der nur mittelbar betroffene, Personenkreis eingeladen ist, [...] erhält die Familienverfassung den Charakter eines gemeinschaftlichen Regelwerkes, in dem alle Beteiligten ihren Beitrag wiederfinden können. Diese gemeinschaftliche Basis schafft eine viel höhere Akzeptanz [...]. Die Beteiligten sehen sich damit einer [...] moralisch wirkenden Hemmschwelle gegenüber, gegen dieses gemeinsame Regelwerk zu verstoßen. ${ }^{1145}$

Auch die Interviewpartner unterstrichen die Wichtigkeit des Erstellungsprozesses - zumindest diejenigen, bei denen die Erstellung unter Beteiligung der Familienmitglieder erfolgte. Gerade auch bei Familien mit einer großen Anzahl an Mitgliedern kennt man sich oftmals gar nicht gut. Der Erstellungsprozess kann dann auch dazu dienen, alle Familienmitglieder besser kennenzulernen.

E-1 hat diesen Prozess noch heute in bester Erinnerung:

»Ich glaube, wenn ich jetzt zurückblickend sage, 2010, wenn man da so das erste Mal dabei ist, aus meinem persönlichen Empfinden heraus, hat man viele mit Sie und Nachname angesprochen, gerade von der anderen Familienseite, wohingegen das jetzt ein per Du und beim Vornamen ist. ${ }^{1146} »$ Es ist viel menschlicher geworden. Also davor, es ist glaube ich das, was man oft mit so zwei Stämmen hat, dass man sagt `die` und `wir «, und das ist wirklich gar nicht mehr spürbar. ${ }^{1147}$

1145 Fabis (2009), S. 16f.

1146 Interview E-1 (21.11.2017), S. 3.

1147 Interview E-1 (21.11.2017), S. 6. 
Darüber hinaus wird der Zusammenhalt der Familie dadurch gefördert, dass man - vielleicht sogar zum ersten Mal - im Detail über die Ausrichtung eines Elements spricht, das die Familie verbindet und im Zuge dessen eventuell bestehende Konflikte aus der Welt schafft:

»Ich glaube, der Prozess ist das Entscheidende, auch weil man ja als Familie nicht gewohnt ist, zu entscheiden, und nicht gewohnt ist, sich gegenseitig zu befragen, was ist denn deine Haltung hier und dazu. Als Familie IST man ja eigentlich nur. Ich glaube nicht, dass man sozusagen laufend sich gegenseitig vermessen muss. Und ich glaube, dass daran viele Familien scheitern, dass sie plötzlich sozusagen aus diesem Mal erschreckend kalten, mal wohnlich warmen Zusammenleben heraus gezwungen sind, gemeinsam Entscheidungen zu treffen, und dann ganz wenig voneinander wissen: über die innere Haltung, die inneren Erwartungen, über die Ansprüche. So, und das Arbeiten an einer Charta, der Prozess, und für die Charta sage ich jetzt Charta, Familienstrategie, Gesellschaftsvertrag, Informationsraster und was es da so alles gibt, lernt man vor allem über den anderen, wie argumentiert der, wie tickt der, was ist ihm wichtig, wie kann man ihn auch wieder besänftigen usw. Dieser Prozess ist das Entscheidende. Das Papier ist nur ein Rudiment, das davon übrig bleibt. « $^{1148}$

Die gemeinsame Erarbeitung der Familienstrategie und der Familienverfassung führt die Familienmitglieder zusammen und stärkt entschieden das Zusammengehörigkeitsgefühl. Dies trägt in nicht unerheblichem Maße dazu bei, dass es dem Individuum später schwerer fällt, aus dieser Gemeinschaft auszubrechen und sich nicht regelkonform zu verhalten.

Doch wie verhält es sich mit jungen Familienmitgliedern der zukünftigen Gesellschaftergeneration? Diese sind ob ihres Alters nicht in den Erstellungsprozess eingebunden oder im Zweifel noch gar nicht geboren, bekommen die Familienverfassung meist erst ab einem Alter von 16 oder 18 (feierlich) überreicht und sollen diese dann unterzeichnen. Die Wirkung des gemeinsamen Erstellungsprozesses haben sie nicht erfahren können.

BACHMANN (2006) führt dazu aus, dass im Rahmen einer Selbstregulierung nicht zwingend die explizite Zustimmung der Mitglieder dieser Gemeinschaft erforderlich ist, solange sichergestellt ist, dass die Regelsetzer über eine Befugnis zur Regelsetzung verfügen, was bedeutet, dass sie die Zustimmung aller Adressaten, und damit der Mitglieder der Gemeinschaft, genießen. ${ }^{1149}$ In diesem Zusammenhang sei für HUEcK (2017) die Anerkennung schon bestehender Regeln bei Eintritt in die Gemeinschaft denkbar. ${ }^{1150}$ Dieser Denkansatz kann in der Praxis allerdings nicht voll überzeugen. Freilich wird man den Erstellungsprozess nicht immer wiederholen, sobald ein Familienmitglied das entsprechende Alter erreicht hat, in welchem es die Familienverfassung grundsätzlich unterzeichnen

1148 Interview B-2 (06.11.2017), S. 5.

1149 Bachmann (2006), S. 403.

1150 Hueck (2017), S. 74. 
darf. Daher empfiehlt sich in der Praxis, die Inhalte ausführlich vorzustellen (diese Aufgabe können die Eltern oder der Familienrat übernehmen), sie immer wieder $\mathrm{zu}$ thematisieren, sodass sie alle Familienmitglieder sukzessive verinnerlichen. Hierfür eignen sich auch die Familientage:

$»[\ldots]$ im Zuge der Familientage wollen wir immer wieder auch in die Charta reinschauen. Jetzt ist sie relativ schlank im Vergleich zu anderen vielleicht, aber das wir uns portionsweise pro Familientag einen Abschnitt vornehmen. ${ }^{1151}$

Den »neuen« Familienmitgliedern kann man darüber hinaus die Möglichkeit einräumen, im Rahmen der nächsten Überarbeitung aktiv mitwirken zu dürfen. Die Familienverfassung ist ohnehin ein Dokument, das lebt und regelmäßiger Überprüfung bedarf. ${ }^{1152}$ Zunächst wird man jedoch bei diesen jungen Familienmitgliedern um Vertrauen werben, dass sich diejenigen, die in den Erstellungsprozess eingebunden waren, zum Wohle der gesamten Familie auf die jetzige Fassung verständigt haben:

»Also ich würde jetzt im Hinblick auf die neue Generation, also die nicht mitgewirkt hat, würde ich sagen, man kann sozusagen, so eine moralische Verbindlichkeit erst mal einfordern mit der Perspektive, hört zu, wenn wir dieses Ding überarbeiten, dann sitzt ihr mit am Tisch und könnt Euch da einbringen. Aber erst mal ist sie jetzt so wie sie ist, die Verfassung, und da haben sich viele Leute aus der Familie den Kopf zerbrochen und sich darauf geeinigt [...]. Also mit dieser Perspektive würde ich das versuchen. ${ }^{1153}$

\subsection{Die gemeinsame Geschichte verbindet}

Einige Fallstudien haben gezeigt, dass die gemeinsame Geschichte und Identität als verbindendes Element eingesetzt wird, um die familiäre Kohäsion zu stärken. Insbesondere möchte man damit die jüngere Gesellschaftergeneration erreichen, die oftmals nicht mehr den/die Gründer oder die Familienmitglieder, die das Unternehmen maßgeblich geprägt haben, persönlich kannten.

A-2 als Vertreter der ersten Generation, der das Unternehmen maßgeblich über viele Jahrzehnte geprägt und zu dem gemacht hat, was es heute ist, hat einen Brief $^{1154}$ verfasst, welcher jedem Familienmitglied zum 16. Geburtstag überreicht wird, wenn es die Familienverfassung unterzeichnen darf:

»Ich habe auch einen Brief geschrieben. Wo ich geschrieben habe, so kriegt das moralische Qualität und lest es durch und nehmt es ernst. Und, wissen Sie, [...] man muss sich als, das ist jetzt ein blödes Wort, aber ich sag es trotzdem, ich bin ja sozusagen der

1151 Interview C-2 (10.11.2017), S. 17.

1152 Vgl. Abschnitt 2.3.1.4.

1153 Interview H-2 (05.12.2017), S. 14.

1154 Eine Sammlung fiktiver Vermächtnisse für die nächste Generation findet sich in EQUAStiftung (2017). 
Patriarch des Ganzen, die Bewährung kommt eigentlich nach mir. Solange ich da bin, ist eine natürliche Autorität da [...]. 1155 $^{115}$

Bei Unternehmerfamilie B wird B-3, der die Geschichte des Unternehmens maßgeblich mitgeprägt hat, im Rahmen der Gesellschafterkompetenz eingeladen, über die Historie der Familie und des Unternehmens zu berichten:

»[...] weil wir jetzt für die jüngeren, also die Gesellschafter, die jetzt 18 werden und die vorbereitet werden, auf ihre Gesellschafterrolle über Seminare, die wir veranstalten. Das sind mehrtägige Seminare. Die werden außerhalb der Firma irgendwo. [...] dann kochen die da und dann kriegen die Aufgaben, die müssen selber Referate halten usw. Das Ganze wird proaktiv gemacht. [...] Und dann gibt es auch Bilanz und [...] ich wurde jetzt aufgerufen für die Geschichte, wo kommen wir eigentlich her, wer sind die Ahnen, wie ist das Unternehmerische in der Familientradition, $\left[\ldots . . \ll^{1156}\right.$

E-2 der Unternehmerfamilie E weiß um die »Binde- und Klebewirkung « ${ }^{1157}$, die von einzelnen Familienmitgliedern qua ihrer Autorität ausgehen kann. Doch auch E-2 als Vertreter der 3. Generation, der die Unternehmensgründer noch persönlich kannte, ist sich dessen bewusst, dass die Personen, die die Gründer noch kannten, irgendwann nicht mehr da sein werden. In der Familie hat man versucht, dieses verbindende Element der gemeinsamen Geschichte in einem Buch festzuhalten:

"[...] und dass diese Werte auch sehr stark von den Gründern beeinflusst wurden, also dass [...] deren Vermächtnis bis heute irgendwo sicher sehr abstrakt mitschwingt. Und wir tun ja auch einiges, um sozusagen, die Gründer am Leben, lebhaft zu halten. [...] Deswegen haben wir auch dieses Buch herausgebracht. [Name des 1. Gründers] und [Name des 2. Gründers]. Es war mit einer der Gründe. Es hat jeder das Buch über die ganze Lebensgeschichte und den Werdegang der beiden Unternehmerpersönlichkeiten. [...] Sie sehen auch, überall taucht immer mal wieder ein Bild von den Altvorderen auf usw. Das $[. .$.$] wird auch bei uns gepflegt. { }^{1158}$

Die generationenübergreifende Überlieferung solcher Geschichten kann dabei unterstützen, bestimmte Säulen der gemeinsamen Zusammenarbeit dauerhaft im kollektiven Gedächtnis zu manifestieren und dadurch die Beziehung der Familienmitglieder untereinander zu stabilisieren. Die Geschichten dienen dabei in gewissem Maße als "Vehikel«, durch welches die positive Beziehung und gegenseitige Wertschätzung der Gründergeneration an ihre jeweiligen Nachfahren weitergegeben werden: ${ }^{159}$

1155 Interview A-2 (08.11.2017), S. $10 \mathrm{f}$.

1156 Interview B-3 (09.11.2017), S. 11.

1157 Interview E-2 (22.11.2017), S. 2.

1158 Interview E-2 (22.11.2017), S. 17.

1159 Zur Bedeutung des Geschichtenerzählens in Familienunternehmen Schlippe/Groth (2007), S. 29ff.; Zwack (2011). 
»In den Geschichten wird dabei das Erfahrungswissen geeigneter Koordinierungsformen von Familie, Gesellschafter und Unternehmer überliefert. In der Regel haben sie den Zweck, Zentripetalkräfte aufzubauen, um die Zentrifugalkräfte, die von Generation zu Generation zunehmen, auszubalancieren. «1160

Geschichten können die Bindung und Identität verstärken und damit gleichzeitig für Kohärenz und Zusammenhalt sorgen. ${ }^{1161}$

\subsection{Bindung durch Kommunikation: der Familientag}

Regelmäßige Familientage oder Familientreffen ${ }^{1162}$ bieten die Möglichkeit, dass alle Mitglieder der Unternehmerfamilie zusammenfinden.

KLETT (2007) beschreibt die Situation treffend und mit einem leicht kritischen Unterton:

"Denn alle Unternehmensfamilien, die damit anfangen, sich um sich selbst zu bemühen, haben zunächst nur die Hoffnung, dass die Nähe irgendwann wirklich kommt, die man anfänglich etwas aufgesetzt und verlegen inszeniert. Das Familienfest ist gleichsam das Thermometer, mit dem sich messen lässt, wie warm man miteinander geworden ist. Denn hier kann sich die Familie kaum mit Unternehmensthemen von sich selbst ablenken. $\aleph^{1163}$

Obwohl Familienverfassungen meist sehr individuell auf die jeweilige Unternehmerfamilie zugeschnitten sind, findet sich eine Institution in so gut wie jedem durchdachten Regelwerk: der Familientag. Dies ließ sich auch in den Fallstudien bestätigen. Ausnahmslos jede Unternehmerfamilie hat mit der Familienverfassung einen Familientag ins Leben gerufen und hält diesen für besonders wichtig:

"[...] das wird bei uns schon sehr positiv aufgenommen dieses Ganze, es ist nicht so, dass es rähh - es ist eine Muss-Veranstaltung, ich habe keinen Bock auf die Familier. Bei uns in der Familie, man freut sich darauf, auch wenn es ein paar Nörgler gibt, die dann auch kommen. Und wenn sie dann da sind, dann ist auch immer gute Stimmung. [...] Der Familientag ist eigentlich vorwiegend dazu da, uns alle zusammenzubringen und uns eben diesen Wert `Familienunternehmen` klar zu machen, weil es ist eben, wir werden immer größer, das sieht man jetzt, also sobald mal meine Generation anfängt mit richtig Nachwuchs zu bringen, dann läuft das auseinander. Und wenn man da eben sowas nicht täte, dann kommt man auch immer viel weiter weg vom Unternehmen, [...] das wird uns auch gesagt, dass der Familientag dafür da ist, das Bewusstsein fürs Familienunternehmen und die Wichtigkeit und Wertigkeit von dem Familienunternehmen darzustellen. ${ }^{1164}$

1160 Schlippe/Groth (2007), S. 33.

1161 Vgl. Schlippe/Groth (2007), S. 35.

1162 Vgl. Abschnitt 2.3.1.3.1.

1163 Klett (2007), S. 18.

1164 Interview F-1 (24. 11.2017), S. 15. 
Mit den Familientagen werden heute verloren gegangene »kommunikative Selbstverständlichkeiten " aus früheren Zeiten immerhin in Ansätzen wieder belebt. ${ }^{1165}$ Insbesondere große Unternehmerfamilien bestehen häufig aus verschiedenen Familienzweigen, die sich im Laufe der Generationen auf der ganzen Welt verteilen, was zur geografischen Trennung führt und einen engen emotionalen Kontakt gefährden kann. ${ }^{166}$ Die Gefahr der Entfremdung wächst dadurch erheblich. ${ }^{1167}$

Die Familientage, die bei vielen Unternehmerfamilien an einem fest vereinbarten Tag im Jahr stattfinden und meist freitags beginnen und sonntags enden, sind ein wichtiges Instrument dafür, den Zusammenhalt innerhalb der Unternehmerfamilie zu fördern; gerade, da meist alle Familienmitglieder, also »mit Kind und Kegel « ${ }^{1168}$, teilnehmen dürfen (also nicht nur die operativen Familienmitglieder, die sich im Zweifel ohnehin jeden Tag im Unternehmen sehen) und das Rahmenprogramm auch für ein emotionales, verbindendes Erlebnis sorgt:

»Weil da ist eine Bombenstimmung und die Gesellschafter und kreuz und quer und unterhalten sich und lachen viel und machen Programme zusammen und so weiter und so fort. Und ich sage mal, da entsteht natürlich so ein richtiges ১Oberfamiliengefühl aller Gesellschafter und dieses Familiengefühl hat ja eine Kohäsionswirkung, das soll sie auch haben, das ist ja kein Zufall, das ist eine Absicht. [...]. [J]e enger und verbundener diese Familie, dieser Familienverbund ist, desto schwerer wird es, aus dem auszubrechen. $\ll^{1169}$

Da an den Familientagen neben den Gesellschaftern auch die Ehepartner und Kinder teilnehmen, sollte neben dem geselligen Zusammentreffen die Gelegenheit auch dahingehend genutzt werden, Sachverhalte mit Unternehmensbezug zu erörtern. So kann sichergestellt werden, dass die Familienangehörigen über die Ausrichtung des Unternehmens im Bilde sind und Entscheidungen, die die Gesellschafter treffen, besser nachvollziehen können. So können entsprechende Informationen dafür sorgen, dass Angehörige der Familie eher Verständnis für Entscheidungen aufbringen, die sich möglicherweise temporär negativ auf sie auswirken: beispielsweise eine reduzierte Ausschüttung, um notwendige Investitionen tätigen zu können. ${ }^{1170}$ So kann man unnötigen Disharmonien, die zu Konflikten führen können, frühzeitig begegnen.

Im Rahmen der Familientage kann auch der jüngeren (zukünftigen) Gesellschaftergeneration eine Plattform geboten werden, sich sukzessive mit dem

1165 Kormann (2018), S. 283.

1166 Vgl. Kormann (2018), S. 283.

1167 Vgl. Reich/Bode (2018), S. 306.

1168 Vgl. Hess (2006), S. $39 \mathrm{ff}$.

1169 Interview E-2 (22.11.2017), S. 15.

1170 Vgl. Fabis (2009), S. 19. 
Unternehmen vertraut $\mathrm{zu}$ machen und die wichtige Bindung aufzubauen. Auch eignen sich spezielle Programmpunkte für die jüngere Generation, um ein Netzwerk der Junggesellschafter entstehen zu lassen und zu fördern:

»[...] unser Ziel ist es, die nochmal jüngere Generation, also die, die jetzt im Kindesalter sind [...] in einer Weise zu motivieren, dass das toll ist, wenn man sich [...] sieht, und man immer in den Kletterpark geht und Aktivitäten zusammen macht, dass man sich dann gut kennt $[\ldots] .{ }^{1171}$

So plädiert auch Kormann (2018) dafür, dass sich in jedem Familienkreis ein Netzwerk der nächsten Generation (»next generation network») herausbilden sollte. »Es umfasst die Familienmitglieder im heranwachsenden Alter, die mit der Unbefangenheit der Jugend Kontakt miteinander knüpfen, die gleiche Sprache sprechen und schon vorbereitend ihre Zusammenarbeit an der Planung eigener Projekte (Reisen oder was auch immer) erproben können. Für die Entwicklung solcher Netzwerke ist es günstig, einen gemeinsamen Ort der Begegnung zu schaffen. Insbesondere gemeinsame Reisen schaffen einen solchen gemeinsamen $>$ Raum « ${ }^{1172}$ :

»[...] sehr interessant, die Tochter unseres Sohnes [...] hat sich zu Wort gemeldet. Die dritte Generation, die zehn Enkel sind zwischen 25 und [...] acht. Also die 16-Jährige/ 17-Jährige meldet sich zu Wort, bei dem letzten Familientag, bei der Aussprache, und sagt, wir die dritte Generation wollen aber Dinge zusammen machen. Wir möchten zum Beispiel, wir wollen uns treffen, nur wir in der dritten Generation und wir möchten dann eine Firma besuchen oder großzügig wie sie ist, [...] sie schlug eine Asienreise vor. Das haben wir dann ein bisschen übertrieben gefunden (lacht). Aber die wollen jetzt also unter sich jetzt mal treffen und über die Firma und über ihre Zukunft und über, also wollen sich mit dem Kodex befassen. ${ }^{1173}$

Gemeinschaftliche Identifikation mit dem Unternehmen und den anderen Mitgliedern der Unternehmerfamilie ist ein wichtiger Faktor dabei, das Gefühl der Zusammengehörigkeit zu stärken, Konflikten präventiv zu begegnen und damit die Einhaltung der Regeln positiv zu beeinflussen. Familientage sollten daher als Pflichttermin im Kalender eines jeden Familienmitglieds eingetragen sein. Empfohlen wird auch dafür immer dasselbe Wochenende eines Jahres zu reservieren.

1171 Interview E-1 (21.11.2017), S. 12.

1172 Kormann (2018), S. 283.

1173 Interview A-2 (08.11.2017), S. 10. 


\subsection{Kommunikationsregeln: das Was und Wie der Kommunikation}

$$
\begin{aligned}
& \text { "Jetzt, wo Kommunikation einfacher wäre denn je, } \\
& \text { haben wir uns nichts mehr zu sagen. "1174 }
\end{aligned}
$$

(Bernhard Steiner)

Im vorherigen Abschnitt wurde im Rahmen der Familientage gezeigt, dass Kommunikation grundsätzlich ein verbindendes Element besitzt. Dies soll aber nicht darüber hinwegtäuschen, dass Kommunikation neben konstruktiven Zügen auch destruktive aufweisen kann. Kommunikation kann integrierend und verbindend wirken, sie kann aber auch Menschen ausschließen und bei den Betroffenen für negative Emotionen sorgen, was eine Abkehr von der gemeinsamen Sache zur Folge haben kann. In beiden Fällen spielen die damit ausgelösten Gefühle und Emotionen eine entscheidende Rolle:

»Gefühle und Emotionen haben [...] integrierende Funktion, indem sie auf Gemeinschaftlichkeit (communio) verweisen und durch diese Modalisierung Erwartungsstrukturen stabilisieren. Gefühle und Emotionen integrieren Sozialsysteme als Gemeinschaften. Dies hat eine starke Bindung an Personen zur Folge. [...] Gefühle und Emotionen haben neben dieser positiven Gemeinschaft stiftenden Bedeutung auch destruktive, desintegrierende und ausgrenzende Folgen. ${ }^{1175}$

Einzelne Fallstudien haben gezeigt, dass das »Was« und »Wie« der Kommunikation helfen kann, Konflikten vorzubeugen und Familienmitglieder zu integrieren und mitzunehmen, was sich in der Konsequenz positiv auf die Wahrscheinlichkeit der Regelbefolgung auswirken kann.

Auslöser für Unternehmerfamilie H, mit dem Prozess einer Familienverfassung zu beginnen, war unter anderem, „die Kommunikation und die gemeinsame Arbeit in der Gesellschafterfamilie zu organisieren. ${ }^{1176}$ Die Lehren aus der Vergangenheit während der Erstellung im Hinterkopf, war man darauf bedacht, sich »eine Ebene zu geben, auf der wir miteinander kommunizieren können. « ${ }^{1177}$ Destruktive Kommunikation hat in der Familie zu tiefgreifenden Verletzungen bei einzelnen Familienmitgliedern geführt. Vor diesem Hintergrund hat man klare Kommunikationsregeln eingeführt, die die Zusammenarbeit erheblich verbessern sollen:

"Also es gibt zum Beispiel eine sehr detaillierte Liste über Kommunikationsregeln. Das vielleicht auch vor dem Hintergrund, den ich vorher geschildert habe. «1178

1174 Steiner (2009), S. 96.

1175 Muraitis (2016), S. 59.

1176 Interview H-2 (05. 12.2017), S. 2.

1177 Interview H-1 (05. 12.2017), S. 3.

1178 Interview H-2 (05. 12.2017), S. 3. 
Die Kommunikationsregeln verbieten beispielsweise, »kritische Themen eigenständig per E-Mail zu diskutieren bzw. über das Medium E-Mail weiterzuverbreiten «, vielmehr soll die »Diskussion kritischer Themen [...] vom Familienrat organisiert« werden. Jeder kann »eine Diskussion zu einem `heiklen Punkt temporär beenden und auf einen geeigneteren Zeitpunkt vertagen.« Die Kommunikation soll insgesamt von Fairness geprägt sein und jedem die Möglichkeit einräumen, seine »Gefühle, Gedanken und Wahrnehmungen « zu äußern. ${ }^{1179}$

Nachdem nun die Art, das Wie, der Kommunikation thematisiert wurde (und hier muss jede Familie ihren Modus Operandi finden), soll nun der Inhalt, das Was, diskutiert werden. Unternehmerfamilie $\mathrm{H}$ hat dazu unter dem Punkt »Informationsfluss in der Gesellschafterfamilie» entsprechende Hinweise zusammengestellt. Unternehmerfamilie B hat dafür vom Familienrat ein »Informationsraster « entwickeln lassen. Die Intention ist in beiden Familien dieselbe. Ein Informationsblatt der Unternehmerfamilie B mit "Anmerkungen zum Informationsraster« fasst diese Intention sehr treffend zusammen:

»Wir haben euch auf der Gesellschafterversammlung [...] ein Informationsraster vorgestellt, in dem festgehalten ist, in welchem Umfang und in welcher Regelmäßigkeit Gesellschafter, Mitglieder der Familiengemeinschaft B, Mitarbeiter der [Unternehmensname], Vorstand, Aufsichtsrat und Familienrat miteinander kommunizieren. Die Erfahrung vieler langlebiger Familienunternehmen lehrt, dass man derlei Regeln mit sehr viel Sorgfalt formulieren und sie dann auch mit großem Ernst leben sollte. Auf diese Weise wird allen Beteiligten klar, auf welche Informationen sie Anspruch haben, wer an welchen Entscheidungen beteiligt ist und für wen dies nicht gilt. Das erleichtert die Arbeitsteilung, vermeidet Streit über Ungeklärtes und erlaubt auch erst, in einer geordneten Art und Weise die Regeln der Zusammenarbeit und des Zusammenlebens weiterzuentwickeln. ${ }^{1180}$

Indem die Regeln der Kommunikation festgelegt werden und darüber hinaus für alle beteiligten Familienmitglieder Klarheit darüber besteht, wer mit wem zu welchen Themen kommuniziert und auf welche Informationen sie einen Anspruch haben, ist ein wesentlicher Punkt der Spielregeln des gemeinsamen Miteinanders geklärt. Dies stärkt den familiären Zusammenhalt und trägt mit dazu bei, dass sich alle an die vereinbarten Spielregeln halten.

1179 Familienverfassung der Familie H, S. 6.

1180 Anmerkungen zum Informationsraster der Unternehmerfamilie B vom 12. Januar 2012, S. 1. 


\subsection{Die Koordinatoren des familiären Zusammenhalts: der Familienrat}

Der Familienrat oder Familienmanager (meist bei relativ kleinen Unternehmerfamilien anzutreffen) als Institution der Familienverfassung kümmert sich vornehmlich um die Belange der Unternehmerfamilie. ${ }^{1181}$ Man könnte ihn auch als Koordinator des familiären Zusammenhalts bezeichnen:

"[...] ich würde es einfach noch breiter fassen und sagen, der Familienrat ist überhaupt die Institution, dass wir als Familie entscheidungsfähig sind. Wir haben uns einfach eine Form gegeben. ${ }^{1182}$

Zumal der Familienrat mit Mitgliedern aus der Unternehmerfamilie (meist nicht nur Gesellschafter) besetzt wird, kann es gelingen, die Interessen der Gesamtfamilie zu bündeln. Der Familienrat als Instanz sorgt in mehrerlei Hinsicht für den Zusammenhalt der Unternehmerfamilie und beugt präventiv durch seine Arbeit Konflikten vor. ${ }^{1183}$

Mit den vielfältigen Aufgaben, die der Familienrat übernimmt, stärkt er den Zusammenhalt der Unternehmerfamilie. Dazu gehören die Organisation der Familientage, die Integration neuer Familienmitglieder, die Kommunikation zwischen den Familienmitgliedern und die Organisation der Mitarbeit von Familienmitgliedern im Unternehmen. Darüber hinaus kümmert er sich um die Junggesellschafter mittels Familienkompetenzprogrammen:

»Im Familienrat haben wir das auch ganz klar geregelt, dass wir Schulungen für die Junggesellschafter machen. Also wir haben einmal im Jahr ein Junggesellschafterseminar [...]. Also von Bilanzen über [...] das Unternehmen an sich. Also da werden einfach alle Themen [...] wirklich auch in Eigenarbeit erarbeitet. ${ }^{1184}$

Durch die Weiterbildung der jungen Familienmitglieder kann für Stabilität in der Familie gesorgt werden. ${ }^{1185}$ In manchen Fällen sieht der Familienrat seine Arbeit auch in der Erziehung der Junggesellschafter. So kann einer Entfremdung vorgebeugt werden:

"[...] dass die Erziehung über die Eltern eine ganz wichtige Rolle spielt. Aber halt nur bis zu einem gewissen Alter und ab da müssen dann wir im Familienrat das dann übernehmen, weil ab einem gewissen Alter ist das dann kontraproduktiv, was die Eltern sagen. ${ }^{1186}$

Dass der Familienrat eine umfassende und wichtige Organisationsfunktion innerhalb der Familie einnimmt, zeigt sich auch dadurch, dass er in manchen

1181 Vgl. Abschnitt 2.3.1.3.2.

1182 Interview B-2 (06.11.2017), S. 12.

1183 Vgl. Koeberle-Schmid (2008), S. 90 f.

1184 Interview B-1 (17.10.2017), S. 3.

1185 Vgl. Koeberle-Schmid (2008), S. 90.

1186 Interview B-1 (17.10.2017), S. 16. 
Familien die Mitglieder bei der Regelung von Eheverträgen ${ }^{1187}$ etc. berät, um das Risiko für das Unternehmen und dadurch die Konfliktgefahr zu minimieren.

Ein Beispiel soll zeigen, wie wichtig diese grundsätzliche Organisationsfunktion sein kann. Meist ist in einer Familienverfassung im Kapitel »Rollen « genau festgelegt, welches Familienmitglied unter welchen Voraussetzungen eine operative Funktion im Unternehmen wahrnehmen kann. Der Familienrat ist dabei Ansprechpartner für alle Familienmitglieder, die im Unternehmen mitarbeiten möchten (unabhängig davon ob als Praktikant, Werkstudent, in einer Führungsfunktion oder als Mitglied der Geschäftsleitung). Er sollte allerdings gleichzeitig auch für Transparenz innerhalb der Familie sorgen, wenn ein Familienmitglied eine neue Funktion im Unternehmen wahrnimmt, um Unmut und Spekulationen vorzubeugen und den Familienfrieden dadurch nicht unnötig zu gefährden.

Beispielsweise hat F-1 eine operative Führungsfunktion im Unternehmen übernommen. Ihr Bruder war aber der Ansicht, dass sie die Voraussetzung, die in der Familienverfassung schriftlich verankert wurde, nicht erfüllt.

So kann ein Regelbruch schnell als »Verrat am Ganzen ${ }^{1188}$ gewertet werden. Auch ihr Onkel, F-2, vermutete hinter ihrer Einstellung eher die Anweisung seines Vaters im Hintergrund, der als Patriarch noch die Geschicke des Unternehmens zu lenken scheint:

"Und ich habe zum Personalchef gesagt, das ist doch kein Zufall, dass jetzt plötzlich [F-1] eine Führungsrolle erhalten hat. [Ich vermute,] da hat irgendwer Anweisung von meinem Vater bekommen oder von meiner Mutter. ${ }^{1189}$

Auf Grund der Tatsache, dass die Einstellung von F-2 vermutlich nicht offiziell kommuniziert wurde und sich der Familienrat (im konkreten Fallbeispiel der Familienverantwortliche als Bindeglied zwischen Familie und Unternehmen) nicht ausreichend um diesen Fall gekümmert hat, sorgt dies unnötigerweise für Spekulationen und Unmut, die darin enden können, dass man die Familienverfassung als Ganzes anzweifelt und sich dann möglicherweise selbst nicht mehr an die Regeln hält. Dies kann im schlimmsten Fall eine nicht gewünschte Kettenreaktion im Familienkreis auslösen. Im konkreten Beispiel noch dazu zu Unrecht, da die Familienverfassung diesen Fall eindeutig regelt. Zunächst können Familienmitglieder demnach "grundsätzlich auf verschiedenen Ebenen im

1187 Hierbei geht es insbesondere darum, dass der Ehepartner eines Gesellschafters im Scheidungsfall keine Geschäftsanteile des Unternehmens erhält. Dazu wird ein Ausschluss des Zugewinnausgleichs im Ehevertrag vereinbart. Verzichten Gesellschafter bei EheschlieBung auf einen entsprechenden Ehevertrag, kann dies ein erhebliches finanzielles Risiko für das Unternehmen und damit für die gesamte Unternehmerfamilie im Scheidungsfall bedeuten.

1188 Rüsen (2017a), S. 121.

1189 Interview F-2 (27.11.2017), S. 14. 
Unternehmen mitarbeiten«. Mitglieder der Familien sollten »tendenziell eher nicht unterhalb der Geschäftsführungsebene [...] beschäftigt werden."Somit ist es für F-2 durchaus möglich, eine »einfache« operative Leitungsfunktion zu bekleiden. Die Familienverfassung sieht vor, dass "Ausnahmen [...] nur mit Zustimmung des Beirats [...] erfolgen und im Einzelfall $\mathrm{zu}$ begründen ${ }^{1190}$ sind. Dies ist mutmaßlich nicht erfolgt. F-2 sah sich mit dem Vorwurf durch ihren Bruder konfrontiert, dass sie nicht ganz fünf Jahre in einem anderen Unternehmen tätig war, bevor sie nun als Führungskraft im Familienunternehmen einstieg. Die Familienverfassung sieht allerdings lediglich für Familienmitglieder, die eine Geschäftsführungsposition im Unternehmen einnehmen wollen, eine mindestens fünfjährige Tätigkeit in einem anderen Unternehmen vor.

Dieses Beispiel zeigt, wie wichtig die administrierende und informierende Funktion eines Familienrats sein kann. Indem er alle Familienmitglieder in regelmäßigen Abständen über wichtige Dinge informiert und den Kontakt hält, bindet er alle ein. Dies gibt den Familienmitgliedern das Gefühl, informiert und Teil der Unternehmerfamilie zu sein, wenngleich sie möglicherweise auch geografisch weit entfernt vom Unternehmen leben mögen:

"[...] und weil wir tatsächlich eine starke Bindung haben, ...] durch die ständigen Kontakte und Berichte, die wir ständig schicken. Ich schicke immer einen Quartalsbericht über den Geschäftsverlauf. Oder manchmal berichte ich auch über Fragen, die wir gerade im Familienrat erörtern, die ganz interessant sind. Wir arbeiten wahnsinnig intensiv über Informationen. Ein Geflecht von Informationen, persönlichen Kontakten, bilateralen Kontakten. ${ }^{1191}$

Um dieser Aufgabe entsprechend gerecht werden zu können, hängt die (optimale) Anzahl an Mitgliedern im Familienrat grundsätzlich von der Größe der Gesamtfamilie ab. Wie bereits erörtert, kann bei einem sehr kleinen Familienkreis ein Familienbeauftragter genügen, bei größeren Unternehmerfamilien wird das Gremium schnell drei, fünf oder auch mehr Mitglieder umfassen. Die Anzahl hat dabei einen wesentlichen Einfluss auf den Erfüllungsgrad ihrer Aufgabe, den Familienzusammenhalt zu sichern und zu fördern. ${ }^{1192}$ Mehr Mitglieder können zu stärkeren Beziehungen und Interaktionen führen. ${ }^{1193}$ Daraus resultiert zwangsläufig, dass die Aufgaben intensiver erfüllt werden können und die Beziehungen stabiler sind. Gleichzeitig können mehr Familienmitglieder erreicht werden: ${ }^{1194}$

1190 Familienverfassung der Familie Feyner, S. $10 \mathrm{f}$.

1191 Interview B-3 (09.11.2017), S. 23.

1192 Vgl. Koeberle-Schmid/Fahrion/Witt (2012), S. 165.

1193 Vgl. Bubolz (2001), S. $129 \mathrm{ff}$.

1194 Vgl. Koeberle-Schmid (2008), S. 90. 
"And, as family relationships continue, increasing interdependence and interactions produce greater levels of trust (based on shared norms and values), principles of reciprocity (obligations) and exchange among family members. ${ }^{1195}$

LANSBerg (1999) empfiehlt, dass jedes Mitglied zu etwa zehn bis 15 Familienmitgliedern Kontakt halten sollte. ${ }^{1196}$ So kann eine »informelle Kommunikationskultur ${ }^{1197}$ geschaffen werden und der Auf- und Ausbau von vertrauensvollen Beziehungen wird gleichzeitig verbessert.

\subsubsection{Präventive Wirkung von Sanktionen}

"Die Strafe zu fürchten ist der beste Weg, ihr zu entgehen."

(Chinesisches Sprichwort)

Wenngleich man unter einer Sanktion grundsätzlich die Antwort auf ein Fehlverhalten erwartet und die reaktiven Maßnahmen erst in Abschnitt 4.1.4 näher erläutert werden, kann Sanktionen grundsätzlich auch eine präventive Wirkung zugeschrieben werden:

»Naja, da gibt es. Okay, dann ist zumindest die Angst davor, präventive Wirkung ist die Angst davor. ${ }^{1198}$

Bei Unternehmerfamilie B findet sich in der Familienverfassung eine mehrstufige Vorgehensweise, wie mit Regelabweichungen zu verfahren ist. Als Ultima Ratio wird auch ein möglicher Ausschluss eines Gesellschafters diskutiert. $\mathrm{Ob}$ diese Regel juristisch Bestand hat, dessen ist sich B-2 nicht sicher. Er glaubt aber vielmehr an die abschreckende Wirkung, dass die Familie überhaupt über einen solchen Schritt bei der Erstellung nachgedacht und diesen auch gemeinsam festgeschrieben hat:

"Es gibt aber auch Sanktionsmöglichkeiten. Unsere Familienstatuten [...] enthalten so ein mehrstufiges Modell, dass sich Gesellschafter, die sich halt nicht beteiligen, die werden dann vorgeladen, und [wir] versuchen das eher, sozial zu regulieren und am Endpunkt kann womöglich eine Kündigung stehen, ja, der Anteile. Ob die juristisch standhält, das weiß ich nicht. Ist glaube [ich] aber auch nicht so wichtig. «1199

1195 Arregle et al. (2007), S. 77.

1196 Vgl. Lansberg (1999), S. 303: "For larger families, I usually recommend that each council member reports to a constituency of ten to fifteen family members, including people in other branches as well as in his or her own."

1197 Felden/Hack (2014), S. 298.

1198 Interview B-2 (06.11.2017), S. 10.

1199 Interview B-2 (06.11.2017), S. 6. 
Im Rahmen der Selbstregulierung spricht man hier von einer Sanktionsorientierung zur Vermeidung negativer Konsequenzen. Sanktionen können das Verhalten eines Akteurs entsprechend beeinflussen. ${ }^{1200}$

Für GRUndHERR (2013) sind Sanktionen daher »[...] beeinflussbare Reaktionsmuster, die den Bruch bestimmter Regeln unvorteilhaft machen und damit die Wahrscheinlichkeit regelkonformen Verhaltens erhöhen. « ${ }^{1201}$

Auf Grund der motivationalen Wirkung von Sanktionen sollten entsprechende Sanktionsmechanismen in die Familienverfassung aufgenommen werden. Allein von der Tatsache, dass man sich als Familie auf einen konkreten Mechanismus einigt und dieser damit jedem bekannt ist, kann eine präventive Wirkung ausgehen, die die Wahrscheinlichkeit der Regelbefolgung erhöhen kann:

"Die auf diese Weise in der Familienverfassung konkret vor Augen geführte Folge eines Pflichtenverstoßes mag im Einzelfall die außerrechtliche Durchsetzbarkeit der Familienverfassung tatsächlich erhöhen und damit präventiv wirken. ${ }^{1202}$

\subsubsection{Wo kein Maß ist, ist keine Vergeltung?! Die reaktiven Maßnahmen zur Sicherung der Regelbefolgung}

Nachdem im vorherigen Abschnitt präventive Maßnahmen - vornehmlich aus den Fallstudien abgeleitet - aufgezeigt wurden, die die Wahrscheinlichkeit der Regelbefolgung erhöhen können, sollen nun mögliche Reaktionen auf Regelverstöße diskutiert werden. Zuvor soll ein kurzer Überblick über allgemeine Erkenntnisse gegeben und erläutert werden, warum Regelverstöße auch innerhalb einer (Unternehmer-)Familie nicht sanktionslos bleiben dürfen.

\subsubsection{Allgemeine Erkenntnisse}

So sehr die Interviewpartner von der Sinnhaftigkeit ihrer Familienverfassung insgesamt überzeugt sind (alles andere wäre auch eine traurige Erkenntnis gewesen), waren die Reaktionen auf die Frage, wie die Unternehmerfamilien mit Regelverstößen umgehen, sehr verschieden. Die Antworten reichten von »Es gibt keine schriftliche Verankerung von Maßnahmen. Und Maßnahmen bei Regelverstößen gibt es auch nicht « ${ }^{1203}$ über IIch würde auch dort gar nicht unbedingt von Sanktion sprechen, sondern von Konsequenz. Dann passieren halt gewisse

1200 Vgl. Abschnitt 2.5.1.4.3 c).

1201 Grundherr (2013), S. 54.

1202 Hueck (2017), S. 322.

1203 Interview F-1 (24.11.2017), S. 17. 
Sachen [...].. ${ }^{1204}$ und $» J a$, das hatte ich ja schon gesagt, dass wir das noch nicht so ganz ausformuliert haben, uns aber vorgenommen haben, das noch zu machen. Also wir erkennen, dass unser Sanktionspotenzial noch ein bisschen klein ist « ${ }^{1205}$ bis hin zu »Ja, also als tatsächliche wirklich harte Maßnahme gibt es lediglich eine Stufe. Das ist dann die Endstufe. Dann fange ich mal von hinten an. Man wird ausgeschlossen aus der Verfassung ${ }^{1206}$. Davor gibt es verschiedene Treppenstufen, wo man versucht, das zu vermeiden. $\aleph^{1207}$

Ein differenziertes Sanktionsarsenal hatten jedoch nur sehr wenige Unternehmerfamilien. Das mag vielleicht insbesondere daran liegen, dass man die harten Sanktionen, wie beispielsweise den Ausschluss eines Gesellschafters, im Gesellschaftsvertrag verortet sieht:

"Also, Regelbrüche, die sanktioniert werden müssen, bzw. die unternehmensgefährdend sind, tatsächlich auch große Auswirkungen auf das Unternehmensgeschäft haben, ich meine, solche Themen sind im Gesellschaftsvertrag verankert, da ist es dann auch rechtlich bindend, das hat dann auch Konsequenzen. $\aleph^{1208}$

Bei anderen mag es das Vertrauen darauf sein, dass die Familie zusammenhält und man sich ohnehin nicht vorstellen kann, dass es zu gravierenden Regelbrüchen kommen wird ${ }^{1209}$, zumal es in der Vergangenheit bisher auch keine gab:

"Was uns im Moment etwas hemmt, ist der Umstand, dass wir am Horizont einen solchen Konflikt noch nicht sehen, der uns zu einer Sanktion bringen könnte, weil wir, erstmal weil wir nicht so wahnsinnig viele sind und weil wir tatsächlich eine starke Bindung haben, durch die Seminare, durch die Jahresversammlung, Hauptversammlung und durch den Ausflug. « ${ }^{1210}$

Andere wiederum mögen ganz grundsätzlich Schwierigkeiten haben mit der Vorstellung von Sanktionen, zumal sie mit der Familienverfassung eine lediglich moralische Verbindlichkeit assoziieren, die eher "soft facts" thematisiert. Auf die Frage, was passieren würde, wenn einer nicht zum eigentlich verbindlichen $\mathrm{Fa}-$ milientag erscheint, antwortete einer der Interviewpartner:

»Kann man nichts machen. Nein. Sanktionen, nach denen Sie sehr fragen, sind überhaupt nicht wesentlich. ${ }^{1211}$

1204 Interview C-2 (10.11.2017), S. 19.

1205 Interview B-3 (09.11.2017), S. 22.

1206 Bei Unternehmerfamilie H ist als letzte Konsequenz der Ausschluss aus der Familienverfassung bei Regelverstößen vorgesehen, was bedeutet, dass das verstoßene Familienmitglied zukünftig nicht mehr in den Genuss der Vorteile kommt.

1207 Interview H-2 (05.12.2017), S. 15.

1208 Interview D-2 (06.11.2017), S. 10.

1209 Dies auch in der Praxis feststellend Rüsen (2016), S. 96.

1210 Interview B-3 (09.11.2017), S. 23.

1211 Interview A-2 (08.11.2017), S. 3. 
So findet sich auch in der Literatur ein ähnliches Bild, wenn SCHLIPPE/GROTH/ RüSEN (2017) ausführen:

"Wir erleben es immer wieder, dass sich Unternehmerfamilien wenig Gedanken darüber machen, was passiert, wenn ein Familienmitglied der Verfassung zwar mit unterzeichnet, sich dann aber nicht an die Inhalte gebunden fühlt und zum Beispiel ein Auftreten in der Öffentlichkeit pflegt, welches mit den Werthaltungen der Verfassung bzw. der Familie nicht kompatibel ist. ${ }^{1212}$

So sehr alle Interviewpartner darauf hoffen mögen, dass es zu keinen gravierenden Regelabweichungen kommen wird, geben viele direkt oder indirekt zu, dass sie dafür keine geeigneten Maßnahmen kennen und etabliert haben. Um im Fall der Fälle als Familie nicht überfordert zu sein, sollten etwaige Szenarien im Vorfeld durchdacht werden - bei der Erstellung der Familienverfassung:

"Aber von Anfang an war es [...] zu Zeiten meines Mannes, dass man sich die schlimmsten Szenarien im Vorfeld ausgemalt hat. Das habe ich noch mitbekommen, da bin ich praktisch in die letzte Phase reingekommen. Dass man sich wirklich die - im Spaß natürlich - allerschlimmsten Szenarien ausgemalt hat und gesagt hat, was machen wir, wenn das oder jenes jetzt passiert. Also man hat schon darüber geredet. Man hat natürlich auch Sanktionen mit eingeschlossen. ${ }^{1213}$

\subsubsection{Sanktionen innerhalb der Familie. Wirklich? Immer?}

Unreflektiert mag man bei einer Sanktion gleich eine drakonische Strafe erwarten. Doch was ist eine Sanktion? Eine Sanktion ist zunächst ganz allgemein eine als negativ empfunden Konsequenz, die von einer mit der nötigen Macht ausgestatten Instanz (meist) in der Absicht verhängt wird, das (unmoralische) Verhalten (zukünftig positiv) zu beeinflussen. ${ }^{1214}$ Karl Deutsch hat einst Macht als die »erwartbare Fähigkeit, Sanktionen zu verhängen« definiert. Doch um welche Sanktionen kann es sich handeln? Eine Sanktion kann in diesem Kontext beispielsweise eine bilaterale verbale Maßregelung, ein öffentliches Anprangern der Verfehlung oder gar einen kompletten Ausschluss aus einer Gemeinschaft bedeuten. Die Bandbreite ist groß.

Schließlich stellt sich die Frage, ob Sanktionen im familiären Kontext überhaupt angebracht und notwendig sind. Um diese Frage beantworten zu können, sollte man sich vor Augen führen, welches Szenario eintritt, wenn man ohne jegliche Konsequenz Regeln brechen kann. STESsL (2014) findet dazu - zumindest für Compliance-Systeme im Unternehmenskontext und damit übertragbar - klare Worte:

1212 Schlippe/Groth/Rüsen (2017), S. 268.

1213 Interview B-1 (17. 10.2017), S. 23.

1214 Vgl. Anwander (2013), S. 252; Seebaß (2002), S. 169. 
»Empirische Studien belegen, dass die Wahrscheinlichkeit für Regelverletzungen steigt, wenn Unternehmensangehörige die Wahrnehmung haben, dass man unentdeckt und unbestraft non-compliant sein kann. ${ }^{1215}$

Die geführten Interviews haben gezeigt, dass das Thema Sanktion innerhalb der Familien kein Tabuthema zu sein scheint. Jede Familie geht allerdings anders damit um. Während die eine eher harmoniebedürftig ist, ist es für eine andere selbstverständlich, Verstöße anzusprechen und nötigenfalls zu sanktionieren:

»[...] wer sich nicht an die Regeln hält, spielt dann einfach nicht mehr mit. Punkt. Das mag der dann persönlich, subjektiv als Strafe empfinden, das kann schon sein. Aber so ist es nicht gemeint. Es ist nicht so gemeint, dass wir als Familie hinterher noch jemand explizit bestrafen wollen. Es gibt Spielregeln, daran hält man sich, wunderbar, hält man sich daran nicht, kann man nicht mitspielen. ${ }^{1216}$

Doch was tun, wenn es zu mehrfachen oder gar vorsätzlichen Zuwiderhandlungen gegenüber den Inhalten der Familienverfassung kommt? Denkbare Regelabweichungen gibt es viele. Das mag ein Junggesellschafter sein, der sich nicht so ganz an das in der Verfassung verankerte angemessene und bescheidene Verhalten in der Öffentlichkeit hält, indem er sich einen auffälligen roten Sportwagen leistet und dem exzessiven Alkoholkonsum nicht abgeneigt ist; das könnte auch ein patriarchisch angehauchter Mehrheitsgesellschafter und Vertreter der ersten Generation sein, der seinen Lieblingsenkel in eine verantwortungsvolle Position im Unternehmen hievt, ohne den in der Familienverfassung verankerten mindestens fünfjährigen Hintergrund im Rahmen einer Führungsfunktion in einem anderen Unternehmen vorweisen zu können; oder ein Mitglied des Familienrats, das bewusst die vereinbarten Kommunikations- und Informationsregeln missachtet und einzelne Familienmitglieder möglicherweise aus persönlichen Befindlichkeiten heraus nicht an den ihnen gemäß der Familienverfassung zustehenden Informationen teilhaben lässt.

Würde man diese vorgenannten exemplarischen Verfehlungen unkommentiert lassen und nicht innerhalb der Familie thematisieren, wäre auch anderen Familienmitgliedern Tür und Tor geöffnet, gleichfalls nach eigenem Gutdünken zu verfahren und im Zweifel immer den letzten Vorfall zu zitieren, welcher doch auch sanktionslos toleriert wurde. Vor diesem Hintergrund sollte leicht nachvollziehbar sein, weshalb es Konsequenzen bei Nichteinhaltung bedarf. Regelabweichungen sollten daher stets thematisiert werden, wenn man von seinen Familienmitgliedern grundsätzlich ein regelkonformes Verhalten erwartet. Um im Ernstfall nicht erst darüber diskutieren zu müssen, welche Reaktion nun die richtige auf einen konkreten Regelverstoß ist, sollte man innerhalb der Familie

1215 Steßl (2014), S. 20.

1216 Interview C-2 (10.11.2017), S. 20. 
bereits bei Erstellung der Verfassung darüber diskutieren und sich auf eine entsprechende Vorgehensweise verständigen, die festlegt, was passieren soll, wenn es zu einem Regelverstoß kommt. Mit Familienmitgliedern, die nicht am Erstellungsprozess beteiligt waren und die fertige Familienverfassung zu einem späteren Zeitpunkt unterzeichnen (da sie erst dann das entsprechende Alter erreichen oder über eine Eheschließung Teil der Unternehmerfamilie wurden), sollte bei der Unterzeichnung ein ausführliches Gespräch geführt werden, in welchem die Ideen und Hintergründe hinter den Regeln dargelegt werden. In diesem Gespräch sollte dann auch thematisiert werden, welche Sanktionsmechanismen es gibt und warum diese als notwendig erachtet werden. ${ }^{1217}$

Im Vorfeld definierte und damit allen Familienmitgliedern hinreichend bekannte Maßnahmen haben sich in mehrerlei Hinsicht als nützlich erwiesen. Die präventive Wirkung wurde bereits in Abschnitt 4.1.3.10. thematisiert. Im Falle des Regelverstoßes ist damit die Vorgehensweise bereits definiert und der Regelbrecher empfindet im besten Fall die Konsequenz nicht als (ungerechtfertigte) Strafe:

»Das weiß aber ja jeder vorher. Das ist also keine Strafe im Sinne von `Du Böser`, dann greift halt einer der Mechanismen. [...]. Da ist was festgeschrieben, im Moment von allen miterarbeitet, von allen unterschrieben, und jeder weiß es, das ist nicht so schwierig zu verstehen hier in der Charta, dass man hinterher sagen kann, habe ich nicht gewusst. Wir sorgen auch dafür, dass die Inhalte, wie gesagt, durchaus präsent sind, und dann greift eben das, was wir dort zusammen festgeschrieben haben. $\aleph^{1218}$

$\mathrm{Zu}$ prüfen wird im Einzelfall dann immer sein, ob die verhängte Sanktion zu der gewünschten Verhaltensänderung führt. Sanktionen scheitern jedoch zunächst einmal grundsätzlich nicht daran, dass es ihnen nicht gelingt, eine beabsichtigte Verhaltensänderung zu erreichen, sondern allenfalls dann, wenn die Berufung auf die zugrundeliegende Regel nicht gelingt und die Sanktion dann zu Recht als ungerechtfertigte Strafe betrachtet wird.

Nachdem klar geworden sein sollte, dass es auch im familiären Umfeld entsprechender Sanktionen bei Regelverstößen im Kontext der Familienverfassung bedarf, stellt sich abschließend noch die Frage, ob dies ausnahmslos und immer der Fall sein muss. Hierauf kann es keine pauschale Antwort geben. Wenn sich die Familie beispielsweise in der Familienverfassung die Möglichkeit einräumt, mit einer gewissen Anzahl an Stimmen von einzelnen Regelungen abweichen zu dürfen, so wäre eine Abweichung vor diesem Hintergrund kein Regelbruch, sondern regelkonform. So wäre auch denkbar, dass der Familienrat über die Mitarbeit von Familienmitgliedern im Unternehmen entscheidet, wenn diese nicht ganz die vorgegebenen Kriterien erfüllen. Solange dies im Konsens ge-

1217 So auch Rüsen (2016), S. 98.

1218 Interview C-2 (10.11.2017), S. 19. 
schieht und im Kreise der Familie thematisiert wird, ist dagegen grundsätzlich nichts einzuwenden. Wichtig bei einer Abweichung ist, dass diese als solche nicht verheimlicht wird. So wird dies auch in der Familie von B-2 praktiziert.

Ganz grundsätzlich kann festgestellt werden: Sind in der Familienverfassung keine Abweichungen vorgesehen und ist die Familie bei einer Regelabweichung der Auffassung, dass es sich dabei um einen klaren Regelbruch handelt, müssen Konsequenzen greifen, die hoffentlich bereits im Vorfeld besprochen und schriftlich fixiert wurden.

\subsubsection{Konkrete Maßnahmen zum Umgang mit Regelbrüchen}

Für den Umgang mit Regelbrüchen kann es kein Patentrezept geben. Auf Basis der geführten Interviews eignet sich vermutlich ein gestuftes Verfahren. ${ }^{1219}$

\subsection{Die Thematisierung des Regelbruchs}

Zunächst ist es wichtig, dass innerhalb der Familie ein Konsens herrscht, dass Regelverstöße thematisiert werden können und sollen. Nachdem man sich im Rahmen der Familienverfassung auf gemeinsame Spielregeln verständigt hat, muss es zur Selbstverständlichkeit werden, dass jede »Schummelei« oder Abweichung angesprochen wird. Vermutlich macht es einen Unterschied, ob ein Regelbruch mit voller Absicht und wissentlich geschah, oder ob es sich um ein Versehen handelte. Hier muss jede Familie ihren eigenen Weg finden, wie sie damit umgehen will. Während die einen ein Auge zudrücken werden und das Thema bilateral in einem Nebensatz ansprechen, wird es in einer anderen Familie durch den Familienrat zur Sprache gebracht werden. Beispielsweise gibt es bei einer Unternehmerfamilie eine sogenannte 48-Stunden-Regel. Vorfälle, über die man sich geärgert hat, müssen innerhalb von 48 Stunden angesprochen und thematisiert werden.

B-2 berichtete von einer auch in der Familienverfassung schriftlich verankerten »spielerischen Offenheit«, die man sich auferlegt hat:

»Naja, ich glaube, wir haben eben diesen genannten Joker, die spielerische Offenheit. Das ist so, dass man mal nicht so spießig sein soll. [...] ich gebe Ihnen jetzt mal so ein Beispiel. Wir [...] möchten eigentlich so Vermögensangelegenheiten, das heißt auch Darlehenskonten und Auszahlung von Kontokorrentkonten von Kommanditisten [...] außerhalb des Unternehmens hier regeln. Wir sind dabei, eigentlich systematisch das zu trennen. Dass auch nicht unsere Buchhaltung hier die Familienbuchhaltung mitmacht. Was natürlich vor 20 Jahren ganz anders war. [...] Und doch sehen wir, dass es da eine alte Generation gibt, die ist das halt gewohnt. Und auch wenn wir uns die Regeln

1219 Eine ähnliche Vorgehensweise empfehlend Rüsen (2016); Rüsen (2017a); Rüsen (2017a), S. $123 \mathrm{ff}$. 
auferlegt haben, wir wollen diese Trennung, sagen wir, müssen wir jetzt meiner Tante, die irgendwie auf die 80 zugeht und nicht mehr ganz beweglich ist, jetzt sagen, ne, ne, ne das darfst du aber nicht, jetzt wird dir das berechnet und so weiter. [...]. Und da gibt es so ein paar Beispiele, wo wir sagen, komm', lass auch mal fünf gerade sein. ${ }^{1220}$

Erwähnt werden muss an dieser Stelle allerdings, dass in der Unternehmerfamilie $\mathrm{B}$ die Abweichungen als solche auch thematisiert werden.

Ganz unabhängig davon, wie man letztlich mit Abweichungen verfährt, dürfen diese auf keinen Fall tabuisiert werden und sind als solche klar zu benennen. Darüber hinaus bedarf es bestenfalls der Regelung, welche Person oder welches Gremium ermächtigt wird, einen Regelbruch anzusprechen. Eine bilaterale beiläufige Ansprache kann in manchen Fällen auch eher kontraproduktiv sein. Man stelle sich die Situation vor, in welcher der Vater seinen pubertierenden Sohn tadelt, bei einem Besuch einer ausländischen Tochtergesellschaft im Rahmen des Familientags nachts in aller Öffentlichkeit über die Stränge geschlagen und dadurch dem Ansehen und dem Namen der Familie erheblich geschadet zu haben. Ob dies allein die gewünschte Wirkung erzielt, darf bezweifelt werden. Selbstverständlich ist es Aufgabe der Eltern, mit ihrem Kind darüber zu sprechen. Allerdings sollte hier auch die entsprechende Instanz, sei es der Familienbeauftragte oder der Familienrat, ein Gespräch mit dem Regelbrecher führen. So bekommt dies einen anderen Charakter und wird auch vom Regelbrecher selbst anders wahrgenommen.

"Also wir haben einen Brief geschrieben, vom Familienratsvorsitzenden unterschrieben und sie eingeladen in den Familienrat und die mussten dann auch erscheinen und sind aber dann nur von einer Person, nicht vom ganzen Familienrat, nur von einer Person belehrt worden. ${ }^{1221}$ - "Aber das war dann schon auch richtig, dass wir uns da dann deutlich als Instanz, die darauf achtet, positioniert haben. Das war für uns dann schon auch der erste Verstoß, den wir vielleicht auch ein bisschen mehr behandelt haben, weil wir auch ein Zeichen setzen wollten. Wir wollten zeigen, es ist jetzt nicht etwas wirklich Schlimmes passiert, aber es ist kein gutes Verhalten gewesen. Wenn ihr euch nicht richtig verhaltet, müsst ihr damit rechnen, dass wir uns euch zur Brust nehmen und das ist verstanden worden. ${ }^{1222}$

\section{1.4.3.2 Die Aufklärung des Sachverhalts als Chance}

Wie bereits thematisiert, ereignet sich ein Regelbruch im besten Fall aus Unkenntnis oder Unachtsamkeit. Ein ausführliches Gespräch kann daher für Klarheit sorgen, sei es in der Auslegung der Regel oder dadurch, dass diese der entsprechenden Person zukünftig präsent(er) sein wird. Solche Gespräche können auch die Chance bieten, Regelungslücken oder Unklarheiten aufzude-

1220 Interview B-2 (06.11.2017), S. 7.

1221 Interview B-1 (17.10.2017), S. 8.

1222 Interview B-1 (17.10.2017), S. 16. 
cken, um in die nächste Überarbeitung der Familienverfassung einzufließen. Darüber hinaus ist es für einen Regelbrecher meist unangenehm, Rechenschaft für sein Verhalten ablegen zu müssen. Besteht der Familienrat aus mehreren Mitgliedern, ist bei jedem Einzelfall konkret zu überlegen, ob das gesamte Gremium das Gespräch führen soll oder welches Mitglied diesen Part übernimmt. In manchen Fällen mag es hilfreich sein, dass ein Vertreter derselben Generation das Gespräch führt, in anderen Fällen eher nicht (ein Vertreter der ersten Generation wird tendenziell Schwierigkeiten damit haben, wenn er von einem Vertreter der dritten Generation verbal gerügt wird).

In einem solchen klärenden Gespräch, in welchem der Regelbrecher Rechenschaft über sein Verhalten ablegen muss, kann herausgefunden werden, ob der Verstoß vorsätzlich oder versehentlich erfolgte. Dann wird es zur Aufgabe des Gespräches, die Gründe zu eruieren. Möglicherweise kann man sich dann auf Abhilfemaßnahmen einigen, oder das Thema wird aufgenommen und bei einem nächsten Familientag thematisiert:

"Wenn Sie grundsätzlich Gutes beim anderen unterstellen, nicht zuletzt deswegen haben wir Werte in der Charta drin, dann kann ja ein Fehlverhalten nicht böswillig, sondern nur versehentlich sein, dann ist die Sanktionierung nicht der richtige Ansatz,

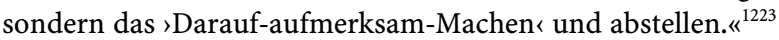

\subsection{Verwarnung und Bekanntgabe: die gelbe Karte}

Handelt es sich um eine erstmalige absichtliche Verfehlung, entscheidet die Schwere des Regelbruchs darüber, ob darauf mit einer einfachen Verwarnung ("gelbe Karte«) reagiert wird oder ob das Familienmitglied eingeladen wird, die Familienverfassung mit einem Vertreter des Familienrats oder dem Familienbeauftragten erneut zu besprechen (»Nachsitzen«). Im Sinne der Transparenz und der Prävention sollte das Ergebnis, ohne auf die Einzelheiten eingehen zu müssen, publik gemacht werden. So demonstriert die Familie nach innen Stärke und es zeugt gleichzeitig von einem funktionierenden Ordnungsrahmen. Bei einem erstmaligen Verstoß wird man noch über keine anderen Sanktionen nachdenken (müssen). Tritt das Familienmitglied zukünftig nicht mehr in Erscheinung, kann der Vorfall damit als erledigt betrachtet werden.

\subsection{Konsequenzen bei wiederholtem Vergehen}

Tritt ein bereits verwarntes Familienmitglied erneut negativ in Erscheinung, bedarf es entsprechender Konsequenzen, um die Kredibilität der Familienverfassung und den Familienfrieden nicht unnötig zu gefährden. Der Familienrat (oder der Familienbeauftragte) als koordinierende Instanz der Familie hat sich

1223 Interview C-1 (02.11.2017), S. 12. 
auch mit einer weiteren Verfehlung zu befassen. Hier können grundsätzlich drei Reaktionsmöglichkeiten unterschieden werden.

\subsection{Die familien-öffentliche Aussprache: die rote Karte}

Hat die erste Verwarnung »im kleinen Kreis« kein Erfolg gezeigt, kann sich der Familienrat intern beraten und eine familien-öffentliche Aussprache anregen. Dies kann im Rahmen eines Familientags erfolgen, sofern ein zeitlicher Aufschub der Diskussion des Vergehens möglich scheint; in kritischen Fällen wird die Familie zu einer Sondersitzung einberufen. Wenn diese Sitzung kurzfristig erfolgt, werden vermutlich nicht - wie bei einem im Voraus geplanten Familientag - alle Familienmitglieder persönlich teilnehmen können. Allerdings sollte zumindest eine kritische Masse anwesend sein. Allein die Tatsache, dass zu einer Sondersitzung eingeladen wird und damit der gesamte Familienkreis Kenntnis davon nimmt, steigt der soziale Druck auf den Regelbrecher erheblich. Die Befragung und damit die Leitung des Gespräches sollte in diesem Fall das Familienmitglied mit der größten Autorität übernehmen. Im besten Fall ist dieses Familienmitglied gleichzeitig Mitglied des Familienrats (in den Fallstudien war dies meist der Fall). Die Aussprache im Familienkreis kann als unangenehm empfunden werden und gleicht einem öffentlichen "Anprangern ${ }^{1224}$ des Verstoßes, was per se als Sanktionsmaßnahme gesehen werden kann. ${ }^{1225}$

»Das würde man am Familientag zur Sprache bringen. [...] Wenn es ein krasser Fall wäre, würde der Familienrat schon aktiv werden und sich mit dem Thema befassen und die Familie anschreiben [...]. ${ }^{1226}$

Das regelbrechende Familienmitglied erhält im Rahmen dieser Aussprache eine letzte Verwarnung - die rote Karte. Dem Familienmitglied wird unmissverständlich klar gemacht, dass der nächste Verstoß zu deutlicheren Sanktionen führen wird und muss - im Sinne aller, die sich regelkonform verhalten und dies damit auch von allen anderen in einer familiären Gemeinschaft erwarten dürfen.

\subsection{Das Mediationsverfahren}

Sieht man sich als Familie außer Stande, das regelbrechende Familienmitglied selbst wieder einzufangen oder kam es in Folge des Regelbruchs möglicherweise zu einem innerfamiliären Konflikt, eignet sich in Familienunternehmen grundsätzlich das Mediationsverfahren. ${ }^{1227}$ Die Fallstudien haben deutlich gemacht, dass man Konflikte, Unstimmigkeiten und innerfamiliäre Spannungen nach Möglichkeit immer intern zu regeln sucht:

1224 Bachmann (2006), S. 76.

1225 Vgl. Abschnitt 2.5.2.3.

1226 Interview A-2 (08.11.2017), S. 12.

1227 Zum Mediationsverfahren in Familienunternehmen Neuvians (2011). 
»Schmutzige Wäsche wird intern und nicht extern gewaschen. [...] Ja, eine Mediation ist durchaus ein Weg, den wir vorziehen $[\ldots] .{ }^{1228}$

Bestenfalls kennt und vertraut die Familie bereits einem Mediator, der möglicherweise in einem der Aufsichtsgremien des Unternehmers sitzt oder saß:

»Weil da gibt es neben der Frau [Name der Beraterin] nochmal jemand. Der war früher in unserem Beirat als es noch einen Beirat gab. Und das ist dann so derjenige, der Mediator, wenn es dann mal richtig gekracht hat und man jetzt nicht auf den Familientag warten möchte, keine Ahnung, oder zwischen zwei Familienmitgliedern, wenn es mal wirklich ziemlich gekracht hat, dann trifft man sich mit dem. ${ }^{1229}$

An dieser Stelle soll nicht verheimlicht werden, dass das Mediationsverfahren in einem Interview auch kritisch gesehen wurde. Dieser Interviewpartner setzt für ein erfolgreiches Mediationsverfahren voraus, dass der Mediator die Personen(en) gut kennt, beispielsweise über eine Beiratsfunktion. So läge dann aber immer die Schwierigkeit darin, dass dieser Sympathien für die ein oder andere Partei hege und dies seine sachliche Urteilskraft trübe:

$»[\ldots]$ wenn mir ein guter [Mediator] einfallen würde. Das ist gar nicht so einfach. [...] Die müssen dann schon beide Parteien gut kennen. [...] aber da ist natürlich immer das Problem, wenn jemand im Beirat sitzt, dann sieht er ja die Gesellschafter eigentlich, oder er kennt die Gesellschafter schon länger und er hat schon immer gewisse Zuneigung zum einen oder zum anderen, ich glaube das lässt sich nicht verheimlichen. [...] Und dann wird es wieder aus der Sachlichkeit rausgerissen und geht bisschen in den emotionalen Bereich. Und ich glaube, gerade hierfür ist es schädlich. [...] dann wird dann immer der andere, zu dem er nicht die Neigung hat, das Empfinden haben, dass er übers $\mathrm{Ohr}$ gehauen wird. ${ }^{1230}$

So sehr diese grundsätzlichen Zweifel berechtigt sein mögen, sollte man allerdings bei einem entsprechend ausgebildeten Mediator erwarten dürfen, dass er die Situation sachlich, neutral und emotionsfrei bewertet. Insofern eignet sich ein Mediationsverfahren durchaus dazu, das regelbrechende Familienmitglied wieder einzufangen.

Ohne im Detail auf Mediationsverfahren eingehen zu wollen, soll an dieser Stelle die systemische Mediation Erwähnung finden, die sich im Umfeld von Familienunternehmen langsam zu etablieren scheint. Zumindest erfährt diese seit 2006 eine institutionelle Förderung durch die Gründung der Deutschen Gesellschaft für systemische Mediation e.V. (DGSYM). ${ }^{1231}$ Diese Ansätze beruhen auf einem konstruktivistischen und systemtheoretischen Gerüst. ${ }^{1232}$ Dieses

1228 Interview C-1 (02.11.2017), S. 11.

1229 Interview D-2 (06.11.2017), S. 14.

1230 Interview G-1 (04.12.2017), S. $16 \mathrm{f}$.

1231 Vgl. Neuvians (2011), S. 172.

1232 Vgl. Gruber (2008), S. 71 ff.; Duss-Von Werdt (2008), S. 25 ff. 
Gerüst zieht ein systemisches Konfliktverständnis und damit eine systemische Grundhaltung nach sich. ${ }^{1233}$ Die Besonderheit bei dieser Mediationstechnik besteht darin, dass sie sich nicht nur auf den aktuellen Konflikt (»Aktualkonflikt $^{1234}$ ) konzentriert, sondern auch das Konfliktmuster und dessen Umfeld einbezieht:

»Wer den Blick auf Mediation systemisch einstellt, konstruiert sie als System von Teilnehmenden, deren wechselseitiger Umgang und wechselseitiges Handeln als zusammenhängendes Ganzes gesehen wird. « ${ }^{1235}$

Ein wesentlicher Unterschied zur nicht-systemischen Mediation wird in der Stellung des Mediators gesehen. Während dieser in der nicht-systemischen Mediation stets als "Herr des Verfahrens" gesehen wird, können seine "Interventionen « im Rahmen der systemischen Mediation lediglich »Impulse « sein. ${ }^{1236}$ Neuvians (2011) führt dazu treffend aus, dass die systemische Mediation

»die selbstreferenzielle Weiterentwicklung der Konfliktbeteiligten und des vorhandenen Klientensystems [anregt] und neue Problemlösungsmöglichkeiten eröffnet [...]. Es wird anerkannt, dass eine direkte und willkürliche Einflussnahme auf psychische und soziale Systeme ausgeschlossen ist, denn selbstreferenzielle Einheiten weisen einen Operationsmodus auf, der sie einer zugriffssicheren Außensteuerung entzieht. [...] Die systemische Mediatorin wird lediglich als Prozessbegleiterin und nicht als Wissensexpertin verstanden. Sie erhöht die Komplexität, sodass sich die Konfliktbeteiligten [...] das herausnehmen können, was sie zu ihrer Weiterentwicklung brauchen. Die Selbstveränderung liegt dann in der Verantwortung der Konfliktbeteiligten. Die systemische Mediatorin hält sich in Hinblick auf Rat- und Vorschläge zurück und konzentriert sich auf die Unterstützung dieser Selbstveränderung. [...] Die Anregung zur Lösungsfindung stellt dabei jedoch nur ein Grundelement [...] dar. Ein weiteres Element kann auch die vergangenheitsbezogene Analyse der Probleme sein, um die kommunikativen, konstruktivistischen und intrapsychischen Zusammenhänge besser zu verstehen. Insofern bietet die Phasenstruktur der Mediation und die damit verbundene Interessenerörterung die Gelegenheit, auch die Symptome, ihre Vernetzung und die Strukturen des Klientensystems zu berücksichtigen. Die Beziehungsebene findet dabei prinzipiell ihre Berücksichtigung. ${ }^{1237}$

So gibt es in der Praxis durchaus auch Beispiele von Familienverfassungen, in denen auf konkrete Mechanismen zur Streitbelegung, und damit auch auf Mediationsverfahren, referenziert wird. ${ }^{1238}$

1233 Vgl. Neuvians (2011), S. 172.

1234 Neuvians (2011), S. 173.

1235 Duss-Von Werdt (2008), S. 10.

1236 Neuvians (2011), S. 174.

1237 Neuvians (2011), S. 176f.

1238 Vgl. Reich/Bode (2018), S. 306. 


\subsection{Der (temporäre) Ausschluss}

Ist man der Auffassung, dass bei einem bereits verwarnten Familienmitglied Worte nicht mehr helfen, müssen Taten folgen und entsprechende Sanktionen verhängt werden. Nachvollziehbarerweise tun sich damit viele Unternehmerfamilien sehr schwer:

"Also im Familienrat wird offen darüber gesprochen, aber wenn es dann zum Schwure kommt, tut sich schon jeder dann auch schwer. ${ }^{1239}$

Entsprechende Maßnahmen führen zu einem Ausschluss aus der Gemeinschaft und von Privilegien. Unternehmerfamilie $\mathrm{H}$ sieht expressis verbis einen Ausschluss aus der Familienverfassung vor. Die Familienverfassung scheint hier synonym für die Unternehmerfamilie zu stehen:

»Hält sich ein Mitglied [...] wiederholt nicht an die vereinbarten Regeln, sucht der Familienrat ein klärendes Gespräch. Führt das Gespräch letztendlich zu keinem positiven Ergebnis, entscheidet der Familienrat über einen möglichen Austritt des betroffenen Familienmitglieds aus der Familienverfassung. ${ }^{1240}$

Huєck (2017) bezeichnet dieses Vorgehen als »einen (außerrechtlichen) Ausschluss aus dem Adressatenkreis der Familienverfassung. « ${ }^{1241}$

Über den Ausschluss kann je nach Ausgestaltung der Familienverfassung der Familienrat alleine entscheiden oder dieser stellt einen Antrag auf Austritt des regelbrechenden Mitglieds:

»Der Familienrat stellt hierzu einen Antrag auf Austritt des betroffenen Mitglieds [...]. Der Antrag gilt als angenommen, wenn 75 Prozent der stimmberechtigten Mitglieder [der Familie nach Definition der Familienverfassung] dem zustimmen. Stimmberechtigt sind alle Familienmitglieder, welche die Verfassung unterschrieben haben. ${ }^{1242}$

Das regelbrechende Familienmitglied wird daraufhin als Konsequenz des Ausschlusses nicht mehr zum Familientag und anderen Feierlichkeiten eingeladen und muss allen sozialen, gemeinschaftlichen Aktivitäten fernbleiben. Damit einhergehend bleibt ihm dann meist auch die Teilnahme an Weiterbildungsveranstaltungen im Rahmen der bei vielen Unternehmerfamilien institutionalisierten Gesellschafterkompetenz verwehrt. Darüber hinaus kann das Familienmitglied auch nicht mehr auf die organisatorischen Dienste des Familienrats zurückgreifen, die in einigen Unternehmerfamilien fast schon Family-OfficeAusmaße annehmen. RüsEN (2017a) gibt in diesem Kontext zu Recht zu bedenken, dass darauf zu achten ist, mit welchen Finanzmitteln die Aktivitäten des Familienrats (in manchen Familienverfassungen erhalten die Mitglieder des

1239 Interview B-1 (17.10.2017), S. 22.

1240 Familienverfassung der Unternehmerfamilie H, S. 7.

1241 Hueck (2017), S. 322.

1242 Familienverfassung der Unternehmerfamilie H, S. 7. 
Familienrats für ihre Tätigkeit eine entsprechende Aufwandsentschädigung) oder die durchgeführten Weiterbildungsveranstaltungen und Familienreisen im Rahmen der Familientage finanziert werden. »Sollten hier auch Finanzmittel der regelbrechenden Person zum Einsatz kommen, ist zur Vermeidung von juristischen Auseinandersetzungen eine alternative Finanzierungsform durch die verbleibenden Familienmitglieder zu wählen. « ${ }^{1243}$

Durch die aussetzende Teilnahme an den Familientagen verwirkt das regelbrechende Familienmitglied die Chance, an familieninternen Entscheidungen mitzuwirken. An dieser Stelle soll vorsorglich darauf hingewiesen werden, dass die vorgenannten Maßnahmen die Rechte als möglicher Gesellschafter (sofern es sich um ein Familienmitglied mit Gesellschafterstatus handelt) nicht beeinträchtigen können. Dies wäre rechtlich unzulässig. Insofern ist das regelbrechende Familienmitglied selbstverständlich zu Gesellschafterversammlungen einzuladen und hat weiterhin sein Stimmrecht in Bezug auf unternehmerische Entscheidungen. Vor diesem Hintergrund ist genau zu prüfen, ob diese Maßnahmen erfolgversprechend sein können. HuEck (2017) gibt hier zu bedenken, dass sich

»die Unternehmerfamilie bei der Durchsetzung primär auf sozialer Ebene wirkenden Sanktionen der Gefahr aussetzen [würde], dass sich betreffende Familienmitglieder emotional noch weiter von der Familie entfernen. Die Chance, dass sich die Familienmitglieder - etwa im Rahmen von Familienveranstaltungen - wieder annähern, könnte so vergeben werden. Dies ist vor allem deshalb kritisch, da sie weiterhin als Gesellschafter miteinander verbunden und unter Umständen aufeinander angewiesen sind. ${ }^{1244}$ "

Vor diesem Hintergrund sollte ein Ausschluss temporär erfolgen und stets explizit die Möglichkeit einer Reintegration vorsehen. Während des Ausschlusses sollte daher ein Familienmitglied (das über eine natürliche Autorität verfügt) beispielsweise ein Mitglied des Familienrats - in (engem) Kontakt mit der ausgeschlossenen Person bleiben. Wird ein temporärer Ausschluss erwirkt, sollte dies immer mit der Hoffnung verbunden sein, dass die entsprechende Person zur Vernunft kommt und Einsicht ob ihres Fehlverhaltens zeigt. Hierbei kann helfen, sich die Vorzüge der Mitgliedschaft in der Gemeinschaft der Unternehmerfamilie vor Augen zu führen.

Nach einem im Vorfeld zeitlich begrenzten Ausschluss sollte der Familienrat oder der Familienverantwortliche ein klärendes Gespräch führen und mit dem regelbrechenden Mitglied einen geeigneten Weg für die »Reintegration« vereinbaren. In diesem Gespräch sollte vorsorglich darauf hingewiesen werden, dass im Falle weiterer Verstöße über eine dauerhafte Trennung gesprochen werden muss. In jedem Fall muss die Familie hier ihre individuelle Vorgehensweise finden. Eine

1243 Rüsen (2017a), S. 124 Fn. 8.

1244 Hueck (2017), S. 322. 
persönliche Entschuldigung im Rahmen eines nächsten Familientags verbunden mit dem Versprechen, sich zukünftig an die gemeinsam geschaffenen Regeln zu halten, ist sicherlich angemessen. Von den anderen Familienmitgliedern sollte man allerdings dann auch erwarten dürfen, dass man der regelbrechenden Person vergibt und ihr gegenüber nicht bei jeder Gelegenheit den Regelbruch thematisiert. Nur so ist eine effektive Rückkehr in die Gemeinschaft möglich, woran allen gelegen sein sollte.

\section{1.4.3.4.4 Der Gesellschafteraustritt als Ultima Ratio}

Inwieweit ein (mehrfacher oder schwerer) Verstoß gegen die Regeln der Familienverfassung einen wichtigen Grund darstellt, der zum Ausschluss des betreffenden Gesellschafters aus der Gesellschaft führen kann, ist strittig und er wird bei einer rechtlich unverbindlich gestalteten Familienverfassung meist abgelehnt. ${ }^{1245}$ Insofern bleibt lediglich die Möglichkeit, mit dem regelbrechenden Familienmitglied in entsprechend ernste und intensive Gespräche einzusteigen und das Mitglied dazu zu bewegen, einem Austritt aus der Gesellschaft zuzustimmen.

Da es dazu der Zustimmung des Mitglieds bedarf, bietet sich auch hierfür an, über den Beginn dieser Gespräche abzustimmen. In der Praxis hat sich gezeigt, dass es dafür einer Mehrheit von 75 Prozent bis 80 Prozent der stimmberechtigten Familienmitglieder bedarf:

»Bei wiederholtem einfachen oder im Falle eines schwerwiegenden Verstoßes soll der Familienrat zu einer Versammlung der Gemeinschaft [...] einladen [...] und den Verstoß auf die Tagesordnung setzen. Wird auf dieser Versammlung ein Verstoß eines Mitglieds gegen die Prinzipien dieser Statuten zum Tagesordnungspunkt gemacht, kann jedes Mitglied der Gemeinschaft im Zuge derer eine Abstimmung über den Ausschluss des Mitglieds fordern. [...] muss die Gemeinschaft dann über den Ausschluss abstimmen, für den eine Mehrheit von 75 Prozent Voraussetzung ist. « ${ }^{1246}$

Diese Abstimmung erhöht den sozialen Druck auf das Familienmitglied und sollte die sich daran anschließenden Gespräche etwas vereinfachen. Dabei geht man davon aus, dass bei der regelbrechenden Person nur noch wenig Interesse an der Eigentümerstellung besteht. In den Gesprächen wird man somit eine Lösung finden müssen, wie eine nachhaltige Trennung von dem Familienmitglied aussehen kann. Für diesen Fall sollte auch im Vorfeld im Familienkreis Klarheit darüber herrschen, wie das gebundene Vermögen verteilt werden soll. Verfügt ein scheidender Gesellschafter über große Anteile am Unternehmen, kann eine Trennung das Unternehmen in finanzielle Schwierigkeiten bringen, wenn sich keine Familienmitglieder finden, die die Anteile übernehmen können

1245 Vgl.Hueck (2017), S. 320.

1246 Familienverfassung der Unternehmerfamilie B, S. $11 \mathrm{f}$. 
und wollen. Insofern sei hier dringend angeraten, auch diesen Fall im Vorfeld genau zu durchdenken.

Wenn ein Familienmitglied und Gesellschafter auf Grund seines Verhaltens nicht mehr erkennen lässt, dass er am familiären Zusammenhalt und an dem alle verbindenden Element, dem Familienunternehmen, Interesse hat, ist es allemal besser, sich dauerhaft zu trennen, als die ständig latente Gefahr von konfliktären Auseinandersetzungen hinzunehmen und damit das Unternehmen möglicherweise in seiner Existenz zu gefährden. ${ }^{1247}$

\subsection{Das Maßnahmenraster zur Regelbefolgung}

Die in den vorherigen zwei Abschnitten gewonnenen Erkenntnisse präventiver und reaktiver Maßnahmen zur Regelbefolgung werden nachfolgend überblicksartig in einem Maßnahmenraster (Abbildung 14) dargestellt.

1247 So auch Rüsen (2017a), S. 124. 

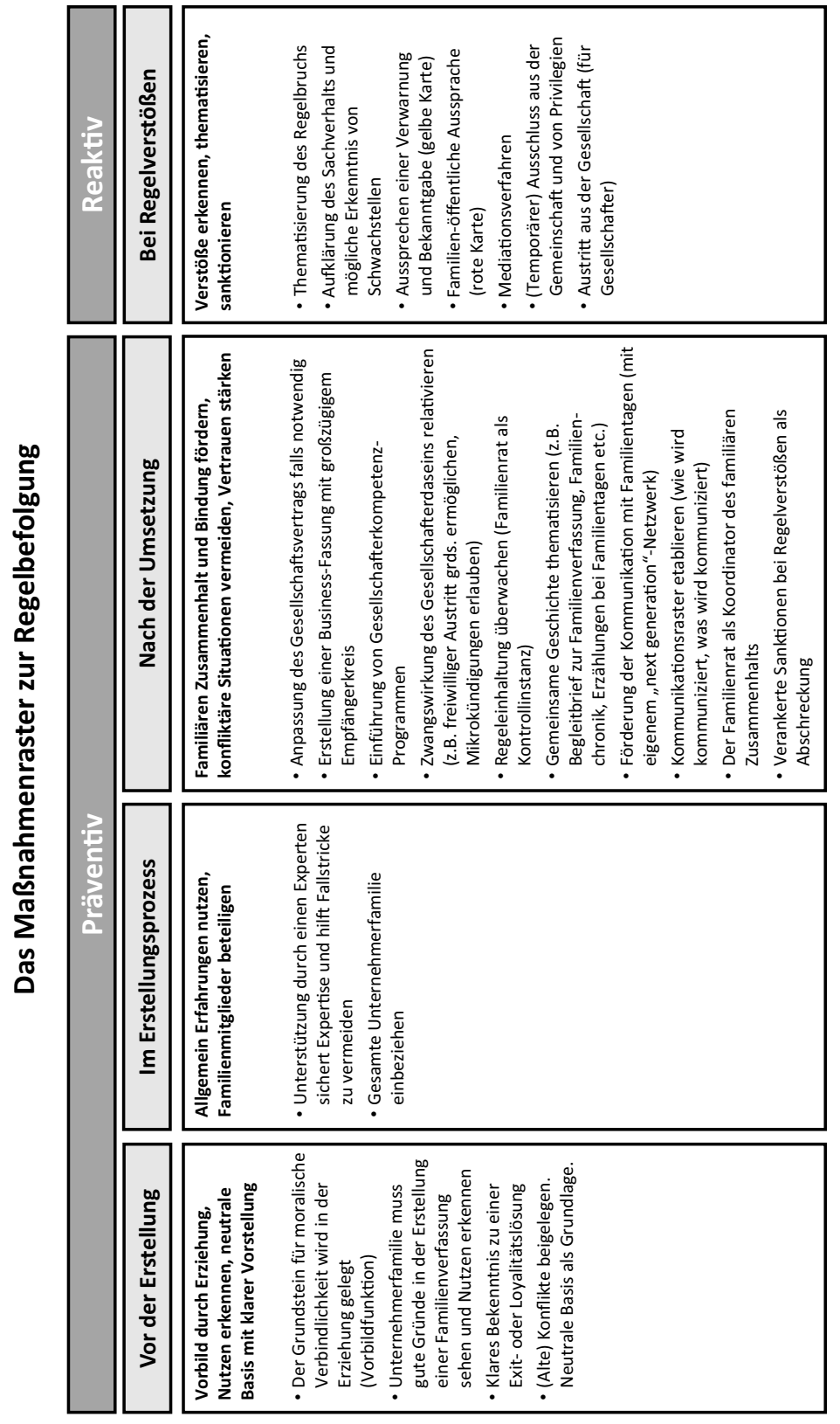

Abbildung 14: Maßnahmenraster zur Regelbefolgung ${ }^{1248}$

1248 Eigene Darstellung. 


\section{$5 \quad$ Zusammenfassung und Ausblick}

\subsection{Thesenartige Zusammenfassung der wichtigsten Handlungsempfehlungen}

Nachdem die positive Wirkung einer Familienverfassung als konfliktpräventives Instrument in der Praxis kaum bezweifelt wird, sollten sich Unternehmerfamilien der möglichen präventiven und reaktiven Maßnahmen zur Regelbefolgung bewusst sein. Besondere Beachtung sollte dabei vornehmlich (aber nicht ausschließlich) den präventiven Maßnahmen geschenkt werden.

Unternehmerfamilien sollten bei der Erarbeitung und Erstellung einer Familienfassung die folgenden Handlungsempfehlungen beachten, die auf Grund der empirischen Untersuchung in dieser Arbeit als wichtig für die Praxis angesehen werden, um die Wahrscheinlichkeit der Regelbefolgung zu erhöhen:

- Die Familienverfassung möchte als moralisch bindendes Regelwerk verstanden werden. Der Grundstein für das Verständnis und die Akzeptanz einer moralischen Verbindlichkeit wird bereits in der Erziehung gelegt. Handlungsweisen und Wertvorstellungen der Unternehmerfamilie prägen die Identität der Nachkommen bereits in jungen Jahren. Die Vorbildfunktion der Eltern spielt dabei eine erhebliche Rolle.

- Bevor mit der Erstellung einer Familienverfassung begonnen wird, sollten alte Konfliktherde beseitigt werden, sonst können sie immer wieder aufflammen. Auch sollte sich die Unternehmerfamilie im Vorfeld klar für oder gegen eine Loyalitätslösung (den Fortbestand des Unternehmens über Generationen sichern) entscheiden, zumal dieses klare Bekenntnis gleichzeitig als guter Grund für die Erstellung einer Familienverfassung dient und somit der Nutzen allen Beteiligten klar wird.

- In den Erstellungsprozess sollten alle Familienmitglieder (die dann auch von der Familienverfassung als solche erfasst werden) eingebunden werden. Dies fördert die Akzeptanz der Regeln und bringt Familienmitglieder (teilweise überhaupt erst dadurch) näher zusammen. Wenn der Prozess durch einen 
erfahrenen Berater begleitet wird, kann sichergestellt werden, dass wertvolle Erfahrungen aus der Praxis einfließen sowie Regelungen und Maßnahmen, die sich als hilfreich herauskristallisiert haben, nicht vergessen werden.

- Mit der Familienverfassung sollten sich in der Praxis bewährte Institutionen und Instrumente wie der Familientag, der Familienrat, die Gesellschafterkompetenz, ein Kommunikationsraster usw. eingeführt werden. Ein jährlich stattfindender Familientag fördert den familiären Zusammenhalt und bindet geografisch entfernte Familienmitglieder ein. Der Familienrat dient als Schnittstelle und Ansprechpartner in allen Belangen, überwacht die Einhaltung der Regeln, organisiert die Familientage und hält (engen) Kontakt zu allen Mitgliedern. Mit der Förderung der Gesellschafterkompetenz wird erreicht, dass die Familienmitglieder sich wichtiges Wissen aneignen, was die Beziehung und emotionale Bindung zum Unternehmen und damit auch zur Familie stärken kann. Ein Kommunikationsraster, das klar regelt, wer welche Informationen erhält, wer auf welche Information einen Anspruch hat und wie in der Familie kommuniziert wird, vermeidet Unverständnis, unnötigen Groll und wirkt auf die Familienmitglieder integrierend.

- Eine frühzeitige Einbindung der so genannten "next generation« mittels spezieller Veranstaltungen baut frühzeitig die emotionale Bindung der Gesellschafter von morgen auf.

- Über Maßnahmen bei Regelverstößen sollte zwingend bereits bei der Erstellung der Familienverfassung diskutiert werden. Solche Maßnahmen, die im Konsens im Regelwerk explizit verankert werden, sind den Familienmitgliedern präsent(er) und wirken durch ihre abschreckende Wirkung präventiv.

- Jedwede Abweichung von den gemeinsam erstellten Regeln - selbst in Ausnahmefällen im Konsens beschlossene - sollten immer als solche kommuniziert werden. Ein Sanktionsmechanismus für den Umgang mit Regelverstößen sollte mehrstufig aufgebaut sein. Der Sachverhalt, der zu einem Regelbruch führte, sollte in allen Fällen thematisiert, geklärt und abgestellt werden. Die Antwort auf einen Regelbruch kann (je nach Grad und Schwere) eine einfache Verwarnung (gelbe Karte), eine familienöffentliche Aussprache, ein Mediationsverfahren, ein (temporärer) Ausschluss aus der Gemeinschaft und von Privilegien oder ein intensives Gespräch mit dem Ziel einer dauerhaften Trennung im Falle eines Gesellschafters sein.

\subsection{Limitationen}

Wie bereits im Ablauf dieser Forschungsarbeit herausgestellt wurde, stellt die Familienverfassung ein von der Wissenschaft im Rahmen des Forschungsfeldes Familienunternehmen noch weitgehend unerforschtes Instrument dar. Nach- 
dem deren positive Wirkung in der Praxis nicht bezweifelt wird, hat sich die vorliegende Arbeit dieser Forschungslücke angenommen und erste vorläufige Erkenntnisse darüber gewonnen, wie die Wahrscheinlichkeit erhöht werden kann, dass das im Rahmen einer Familienverfassung erstellte Regelwerk von möglichst allen Familienmitgliedern eingehalten wird.

Eine erste Limitation der vorliegenden Forschungsarbeit ergibt sich nachvollziehbarerweise aus der bewussten Wahl einer induktiven Forschungsstrategie. Da die Schlüsse aus einer begrenzten Anzahl an Fallstudien gezogen wurden, kann naturgemäß nicht von einer statistischen Repräsentanz der hier gewonnenen Erkenntnisse für alle deutschen Unternehmerfamilien ausgegangen werden.

Eine weitere Limitation bestand darin, dass viele Familienverfassungen der Fallstudien zum Zeitpunkt der Interviews erst seit Kurzem (wenige Jahre) im Einsatz waren, was sich insbesondere darauf auswirkte, dass es bis zu diesem Zeitpunkt zu keinen gravierenden Regelbrüchen gekommen war. Im (gutgläubigen) Vertrauen auf die Wirksamkeit ihrer Familienverfassung hatten die Interviewpartner Schwierigkeiten, sich überhaupt folgenschwere Regelbrüche vorzustellen.

Darüber hinaus besteht eine Limitation in den dargestellten Maßnahmen selbst. Da keine Unternehmerfamilie der anderen gleicht, eignen sich nicht alle Maßnahmen in gleichem Maße zwingend für jede Familie und können unterschiedlich effizient sein. Eine entsprechende Vorgehensweise muss stets für jede Unternehmerfamilie individuell abgewogen werden. So soll an dieser Stelle nicht verschwiegen werden, dass jede Maßnahme, die sich grundsätzlich positiv auf die Wahrscheinlichkeit der Regelbefolgung auswirken kann, gleichzeitig mit Nachteilen in anderen Bereichen einhergehen könnte. Die Voraussetzungen, die Effizienz sowie die damit verbundenen Vor- und Nachteile der Maßnahmen darzustellen, hätte den Rahmen dieser Arbeit gesprengt. 
Open-Access-Publikation im Sinne der CC-Lizenz BY 4.0

(c) 2020, Vandenhoeck \& Ruprecht GmbH \& Co. KG, Göttingen ISBN Print: 9783847111719 - ISBN E-Lib: 9783737011716 


\section{$6 \quad$ Literatur}

Achenbach, Christoph (2015): Der Generationenübergang in mittelständischen Familienunternehmen. Die fachlichen und emotionalen Erfolgsfaktoren. 1. Aufl., Düsseldorf. Achleitner, Ann-Kristin (1995): Die Normierung der Rechnungslegung. Eine vergleichende Untersuchung unterschiedlicher institutioneller Ausgestaltungen des nationalen und internationalen Standardsetzungsprozesses, Zürich, Winterthur.

Albach, Horst (1989): Generationswechsel und Unternehmenskontinuität - Chancen, Risiken, Maßnahmen. Eine empirische Untersuchung bei Mittel- und Großunternehmen, Gütersloh.

Albers, Sönke (Hrsg.) (2009): Methodik der empirischen Forschung. 3., überarb. und erw. Aufl., Wiesbaden.

Alderson, Keanon J. (2011): Understanding The Family Business, New York.

Alheit, Peter (1999): »Grounded Theory«: Ein alternativer methodologischer Rahmen für qualitative Forschungsprozesse. Unveröffentlichtes Manuskript, Göttingen.

Ammer, Jakob (2017): Die Organisation der Unternehmerfamilie in Stämmen. Strategien und Maßnahmen zur Prävention von intergruppalen Konflikten in Stammesorganisationen. 1. Auflage, Göttingen, Niedersachsen.

Andreae, Caspar v. (2007): Familienunternehmen und Publikumsgesellschaft: Führungsstrukturen, Strategien und betriebliche Funktionen im Vergleich, Wiesbaden.

Angus, Patricia M. (2005): The Family Governance Pyramid: From Principles to Practice, in: The Journal of Wealth Management 2005, S. 7-13.

Anwander, Norbert (2013): Die halbe Wahrheit der Sanktionstheorie nebst einem Ausblick auf die andere Hälfte, in: Buddeberg, E./Vesper, A. (Hrsg.): Moral und Sanktion. Eine Kontroverse über die Autorität moralischer Normen, Frankfurt, S. 249-290.

Arndt, Dominik E. (2011): Sinn und Unsinn von Soft Law. Prolegomena zur Zukunft eines indeterminierten Paradigmas. 1. Aufl., Baden-Baden.

Arnhold, Peter/Rohner, Marcus H. (2015): $\$ 3$ Compliance und Business Governance bei Personengesellschaften, in: Gummert, H. (Hrsg.): Münchener Anwalts-Handbuch Personengesellschaftsrecht, München, Rn. 1-100.

Aronoff, Craig E./Ward, John L. (1996): Family business governance. Maximizing family and business potential. 3, New York.

Aronoff, Craig E./Ward, John L. (2011): Family Business Ownership. How to Be an Effective Shareholder, New York. 
Arregle, Jean-Luc/Hitt, Michael A./Sirmon, David G./Very, Philippe (2007): The Development of Organizational Social Capital. Attributes of Family Firms, in: J Management Studies 44, 1, S. 73-95.

Ashforth, Blake E./Mael, Fred (1989): Social Identity Theory and the Organization, in: The Academy of Management Review 14, 1, S. 20-39.

Astrachan, Joseph H./Klein, Sabine B./Smyrinos, Kosmas X. (2002): The F-PEC Scale of Family Influence. A Proposal for Solving the Family Business Definition Problem1, in: Family Business Review 15, 1, S. 45-58.

Astrachan, Joseph H./McMillan, Kristi S. (2003): Conflict and communication in the family business, Marietta, GA.

Atteslander, Peter (2010): Methoden der empirischen Sozialforschung. 13., neu bearb. und erw. Aufl., Berlin.

Augsberg, Steffen (2003): Rechtsetzung zwischen Staat und Gesellschaft. Möglichkeiten differenzierter Steuerung des Kapitalmarktes, Berlin.

Aulehner, Josef (2011): Grundrechte und Gesetzgebung, Tübingen.

Ayres, Ian/Braithwaite, John (1994): Responsive Regulation, in: J Legal Studies Education 12, 2, S. 345-353.

Bachmann, Gregor (2002): Der »Deutsche Corporate Governance Kodex«: Rechtswirkung und Haftungsrisiken, in: WM 2002, S. 2137-2143.

Bachmann, Gregor (2006): Private Ordnung. Grundlagen ziviler Regelsetzung, Tübingen.

Baecker, Dirk (Hrsg.) (2003): Organisation und Management. Aufsätze. 4. Aufl., Frankfurt am Main.

Baecker, Dirk (2003): Tabus in Familienunternehmen, in: Baecker, D. (Hrsg.): Organisation und Management. Aufsätze. 4. Aufl., Frankfurt am Main, S. 123-133.

Bangert, Steffen (2004): Die rechtliche Organisation von Durchsetzungssystemen zur Kontrolle ordnungsmäßiger Rechnungslegung, Frankfurt am Main [u.a.].

Bardach, Eugene/Kagan, Robert E. (1982): Going by the book. The problem of regulatory unreasonableness, Philadelphoa.

Barnes, Louis B./Hershon, Simon A. (1976): Transferring Power in the Family Business, in: Harvard Business Review 54, 4, S. 105-114.

Barrédy, Céline (2016): Typology of interactions and data content in qualitative family case study research, in: Randersen, K., et al. (Hrsg.): Family entrepreneurship. Rethinking the research agenda, Abingdon, Oxon, S. 279-301.

Barry, Bernard (1989): The Development of Organization Structure in the Family Firm, in: Family Business Review 2, 3, S. 293-315.

Bartels, Peter/Schmid, Peter/Hofbauer, Karl (2010): Fels in der Brandung? Studie über Familienunternehmen 2010/11.

Bärwaldt, Roman/Prinz, Ulrich (Hrsg.) (2014): Beck'sches Handbuch der Personengesellschaften. Gesellschaftsrecht - Steuerrecht. 4. [bearb.] Aufl., München.

Basedow, Jürgen/Drobnig, Ulrich (Hrsg.) (1998): Festschrift für Ulrich Drobnig zum siebzigsten Geburtstag, Tübingen.

Bauman, Zygmunt (2005): Moderne und Ambivalenz. Das Ende der Eindeutigkeit. Neuausgabe, Hamburg.

Baumgarth, Carsten/Eisend, Martin/Evanschitzky, Heiner (2009): Empirische Mastertechniken: Eine anwendungsorientierte Einführung für die Marketing- und Managementforschung, Wiesbaden. 
Baumgartner, Bernhard (2009): Familienunternehmen und Zukunftsgestaltung: Schlüsselfaktoren zur erfolgreichen Unternehmensnachfolge, Wiesbaden.

Baumhauer, Jon/Böninger, Christoph/Prügl, Reinhard/Schlippe, Arist v. (2011): Das Management eines großen Gesellschafterkreises, in: FuS Zeitschrift für Familienunternehmer und Stiftungen Nr. 3/2011, S. 113-120.

Baur, Nina/Blasius, Jörg (Hrsg.) (2014): Handbuch Methoden der empirischen Sozialforschung, Wiesbaden.

Baus, Kirsten (2017): Die Familienstrategie. Wie Familien ihr Unternehmen über Generationen sichern. 5., überarb. und erw. Aufl, Wiesbaden.

Baus, Kirsten/Kögel, Rainer (2006): Vertrauen statt Misstrauen, Stuttgart.

Behrens, Peter (1998): Corporate Governance, in: Basedow, J./Drobnig, U. (Hrsg.): Festschrift für Ulrich Drobnig zum siebzigsten Geburtstag, Tübingen, S. 491-506.

Bentham, Jeremy (1970): An introduction to the principles of morals and legislation, London.

Benz, Arthur/Lütz, Susanne/Schimank, Uwe, et al. (2007): Handbuch Governance. Theoretische Grundlagen und empirische Anwendungsfelder. 1. Aufl, Wiesbaden.

Berent-Braun, Marta M./Uhlaner, Lorraine (2012): Family governance practices and teambuilding:. paradox of the enterprising family, in: Small Business Economics 38, 1, S. 103-119.

Bergfeld, Marc-Michael H./Weber, Felix-Michael/Kraus, Sascha (2009): Innovationsverhalten und Performance in Familienunternehmen. Durch generationenübergreifende Innovation zum Großunternehmen, in: ZfKE - Zeitschrift für KMU und Entrepreneurship 57, 1, S. 1-26.

Bernhardt, Wolfgang (2008): Sechs Jahre Deutscher Corporate Governance Kodex -. Eine Erfolgsgeschichte?, in: BB Nr. 32/2008, S. 1682-1692.

Bernhardt, Wolfgang (2010): Corporate Governance Kodex für Familienunternehmen? Eine Widerrede. Universität Leipzig, Working Paper, No. 87.

Bertrand, Marianne/Schoar, Antoinette (2006): The Role of Family in Family Firms, in: Journal of Economic Perspectives 20, 2, S. 73-96.

Bianchi, Carmen/Alderson, Keanon J. (2012): Do Family Councils Really Work? The Need for Empirical Study, in: Carsrud, A. L./Brännback, M. (Hrsg.): Understanding family business. Undiscovered approaches, unique perspectives, and neglected topics. 1. ed., New York, NY [u. a.], S. 185-202.

Biedermann, Reinhard (2007): Sozialstandards durch private Governance. Zwei-StufenKooperation in der globalen Spielzeugbranche. 1. Aufl., Baden-Baden.

Binder, Andreas (2014): Innovation in erfolgreichen Familienunternehmen: Untersuchung der frühen Phase von Innovationen in der chemischen Industrie.

Binz, Mark K./Mayer, Gerd (2012): Anteilsvinkulierung bei Familienunternehmen, in: NZG Nr. 6/2012, S. 201-212.

Bittner, Claudia (1992): Die EG-Übernahmerichtlinie aus englischer Sicht, in: RIW 1992, S. $182-189$.

Bizer, Johann (2001): Editorial: Wozu Selbstregulierung in Deutschland, in: DuD Nr. 3/ 2001.

Bochmann, Christian (2013): Gründungssymposium des Notarrechtlichen Zentrums Familienunternehmen an der Bucerius Law School, in: DNotZ Nr. 7/2013, S. 484-487. 
Boettcher, Erik (Hrsg.) (1994): Die Einheit der Gesellschaftswissenschaften. Studien im Grenzbereich der Wirtschafts- und Sozialwissenschaften, Tübingen.

Bogner, Alexander/Littig, Beate/Menz, Wolfgang (Hrsg.) (2009): Experteninterviews. Theorie, Methoden, Anwendungsfelder, Wiesbaden.

Bogner, Alexander/Littig, Beate/Menz, Wolfgang (2014): Interviews mit Experten. Eine praxisorientierte Einführung, Wiesbaden.

Bogner, Alexander/Menz, Wolfgang (2009): Das theoriegenerierende Experteninterview. Erkenntnisinteresse, Wissensformen, Interaktion, in: Bogner, A./Littig, B./Menz, W. (Hrsg.): Experteninterviews. Theorie, Methoden, Anwendungsfelder, Wiesbaden, S. 6198.

Bohnsack, Ralf (2013): Rekonstruktive Sozialforschung: Einführung in Methodologie und Praxis qualitativer Forschung, Wiesbaden.

Born, Manfred/Ghassemi-Tabar, Nima/Gehle, Burkhard (Hrsg.) (2016): Münchener Handbuch des Gesellschaftsrechts. Band 7: Gesellschaftsrechtliche Streitigkeiten. 5, München.

Born, Werner (2014): Familienverfassung. Stand 10/2014, in: DWS-Institut (Hrsg.): Handbuch Familienunternehmen. Gesellschafts-, Steuer-, Güter- und Erbrecht mit Mustersammlung, Köln, Rn. $47 \mathrm{ff}$.

Bortz, Jürgen/Döring, Nicola (2006): Forschungsmethoden und Evaluation. Für Humanund Sozialwissenschaftler. 4., überarbeitete Auflage, Berlin, Heidelberg.

Brandhorst, Mario (2013): Moralische Pflicht und praktische Gründe, in: Buddeberg, E./ Vesper, A. (Hrsg.): Moral und Sanktion. Eine Kontroverse über die Autorität moralischer Normen, Frankfurt, S. 167-196.

Breidenbach, Karin (1997): Normensetzung für die Rechnungslegung, Wiesbaden.

Breidenstein, Georg/Hirschauer, Stefan/Kalthoff, Herbert, et al. (2013): Ethnografie: Die Praxis der Feldforschung, Stuttgart.

Breuer, Franz/Mruck, Katja/Roth, Wolff-Michael (2002): Subjectivity and Reflexivity: An Introduction, in: Forum Qualitative Sozialforschung / Forum Qualitative Social Rese$\operatorname{arch} 3,3,1-10$.

Breuer, Franz/Muckel, Petra/Dieris, Barbara (2017): Reflexive Grounded Theory, Wiesbaden.

Breyer, Michael (2015): Family Governance. Die Organisation der Familie, in: FuS Zeitschrift für Familienunternehmer und Stiftungen Nr. 4/2015, S. 151-154.

Brockhoff, Klaus/Eiben, Jan/Fahrion, Hans-Jürgen, et al. (2012): Family Business Governance. Erfolgreiche Führung von Familienunternehmen. 2., völlig. neu bearb. und wesentlich erw. Aufl, Berlin.

Brosius, Hans-Bernd/Koschel, Friederike/Haas, Alexander (2009): Methoden der empirischen Kommunikationsforschung. Eine Einführung. 5th ed, Wiesbaden.

Brückner, Daniel/Eigelshoven, Jörg/Heinz, Carsten, et al. (2010): Family Office Management als (Bank-)Dienstleistung für vermögende Privatkunden. 2. [vollst. überarb.] Aufl., Heidelberg.

Brüsemeister, Thomas (2008): Qualitative Forschung. Ein Überblick. 2., überarb. Aufl., Wiesbaden.

Bryant, Antony/Charmaz, Kathy (Hrsg.) (2010): SAGE Handbook of Grounded Theory. Paperback Edition, London.

Buber, Renate/Holzmüller, Hartmut H. (2009): Qualitative Marktforschung: Konzepte Methoden - Analysen, Wiesbaden. 
Bubolz, Margaret M. (2001): Family as source, user, and builder of social capital, in: The Journal of Socio-Economics 30, 2, S. 129-131.

Buchholz, Carsten (2004): Ein neues DRSC? Zur Weiterführung der Zuständigkeiten des DRSC als privatverbandliches Gremium für den handelsrechtlichen Einzelabschluss, Frankfurt am Main, Berlin, Bern, Bruxelles, New York, Oxford, Wien.

Buck-Heeb, Petra/Dieckmann, Andreas (2010): Selbstregulierung im Privatrecht, Tübingen.

Buddeberg, Eva/Vesper, Achim (Hrsg.) (2013): Moral und Sanktion. Eine Kontroverse über die Autorität moralischer Normen, Frankfurt.

Busch, Werner (1979): Die Entstehung der kritischen Rechtsphilosophie Kants: 1762-1780, Berlin.

Busche, Hübertus/Schmitt, Anton (Hrsg.) (2010): Kant als Bezugspunkt philosophischen Denkens: Festschrift für Peter Baumanns zum 75. Geburtstag, Würzburg.

Cabrera-Suárez, María K./Déniz-Déniz, María/Martín-Santana, Josefa D. (2015): Family Social Capital, Trust within the TMT, and the Establishment of Corporate Goals Related to Nonfamily Stakeholders, in: Family Business Review 28, 2, S. 145-162.

Cabrera-Suárez, María K./Saá-Pérez, Petra de/García-Almeida, Desiderio (2001): The Succession Process from a Resource- and Knowledge-Based View of the Family Firm, in: Family Business Review 14, 1, S. 37-46.

Calder, Grant H. (1961): The peculiar problems of a family business, in: Business Horizons 4, 3, S. 93-102.

Calliess, Gralf-Peter/Mahlmann, Matthias (2002): Der Staat der Zukunft: Vorträge der 9. Tagung des Jungen Forum Rechtsphilosophie in der IVR, 27.-29. April 2001 an der Freien Universität Berlin, Havertown, PA.

Canessa, Boris/Escher, Jens/Koeberle-Schmid, Alexander, et al. (2016): Das Family Office. Ein Praxisleitfaden, Wiesbaden.

Carlock, Randel S./Ward, John L. (2001): Strategic Planning for the Family Business, London.

Carroll, Craig E. (2016): The SAGE Encyclopedia of Corporate Reputation, CA.

Carsrud, Alan L./Brännback, Malin (Hrsg.) (2012): Understanding family business. Undiscovered approaches, unique perspectives, and neglected topics. 1. ed., New York, NY [u.a.].

Caspary, Simon (2018): Das Familienunternehmen als Sozialisationskontext für Unternehmerkinder, Wiesbaden.

Cassell, Catherine/Symon, Gillian (Hrsg.) (1994): Qualitative methods in organizational research. A practical guide, London.

Charmaz, Kathy (2014): Constructing grounded theory. 2nd ed., Los Angeles.

Chrisman, James J./Chua, Jess H./Kellermanns, Franz W./Matherne III, Curtis F./Debicki, Bart J. (2008): Management Journals as Venues for Publication of Family Business Research, in: Entrepreneurship Theory and Practice 32, 5, S. 927-934.

Chrisman, James J./Chua, Jess H./Litz, Reginald (2003): A unified systems perspective of family firm performance: an extension and integration, in: Journal of Business Venturing 18, 4, S. 467-472.

Chrisman, James J./Chua, Jess H./Sharma, Pramodita (2003): Current Trends and Future Directions in Family Business Management Studies: Toward a Theory of the Family Firm, in: Coleman White Paper series 2003. 
Christiansen, Per (2000): "Selbstregulierung, regulatorischer Wettbewerb und staatliche Intervention im Internet«, in: Multimedia und Recht Nr. 03/2000, S. 123-129.

Churchill, Gilbert A. (1991): Marketing research. Methodological foundations. 5. ed., Chicago.

Cole, Tim (2015): Unternehmen 2020: Das Internet war erst der Anfang. 1. Aufl., Berlin. Copp, David (2001): Morality, Normativity, and Society.

Corbetta, Guido (1998): Le imprese familiari. Caratteri originali, varietà e condizioni di sviluppo, Milano.

Corbetta, Guido/Montemerlo, Daniela (2000): Family protocols: our experience in Italy, in: The Family Business Network Newsletter 26.

Corbetta, Guido/Salvato, Carlo (2012): Strategies for longevity in family firms. A European perspective, Basingstoke.

Craig, Justin B./Moores, Ken/Howorth, Carole/Poutziouris, Panikkos (2009): Family business research at a tipping point threshold, in: Journal of Management \& Organization 15, 03, S. 282-293.

Craig, Justin B./Salvato, Carlo (2012): The Distinctiveness, Design, and Direction of Family Business Research, in: Family Business Review 25, 1, S. 109-116.

Curcio, Gian-Paolo (2008): Verantwortungsmotivation zwischen Moralität und Gerechtigkeit, Münster.

Damrau, Jan (2003): Selbstregulierung im Kapitalmarktrecht. Eine rechtsökonomische Analyse der Normsetzung der deutschen Börsen und ihrer Träger, Berlin.

Dauner-Lieb, Barbara/Freudenberg, Götz/Werner, Götz W. (Hrsg.) (2014): Familienunternehmen im Fokus von Wirtschaft und Wissenschaft. Festschrift für Mark K. Binz, München.

Davis, Peter S./Harveston, Paula D. (2001): The Phenomenon of Substantive Conflict in the Family Firm. A Cross-Generational Study, in: Journal of Small Business Management 39,1, S. $14-30$.

Denzin, Norman K. (1989): The Research Act. A theoretical introduction to sociological methods. 3, Prentice Hall.

Detjen, Joachim (2009): Die Werteordnung des Grundgesetzes, Wiesbaden.

Dey, Ian (2010): Grounding Categories, in: Bryant, A./Charmaz, K. (Hrsg.): SAGE Handbook of Grounded Theory. Paperback Edition, London, S. 167-190.

Diekmann, Andreas (2007): Empirische Sozialforschung: Grundlagen, Methoden, Anwendungen, Hamburg.

Dittmar, Norbert (2009): Transkription. Ein Leitfaden mit Aufgaben für Studenten, Forscher und Laien. 3. Aufl., Wiesbaden.

Donnelly, Robert G. (1964): The family business, in: Harvard Business Review 42, S. 93-105.

Döring, Sabine A./Mayer, Verena (2002): Die Moralität der Gefühle, Wiesbaden.

Dreier, Ralf/Paulson, Stanley L. (2011): Gustav Radbruch - Rechtsphilosophie. Studienausgabe. 2., überarbeitete Auflage 2003, Heidelberg.

Dresing, Thorsten/Pehl, Thorsten (2015): Praxisbuch Transkription. Regelsysteme, Software und praktische Anleitungen für qualitative ForscherInnen. 5. Aufl., Marburg.

Dumont, Louis (1991): Individualismus. Zur Ideologie der Moderne, Frankfurt/Main, New York, Paris.

Dunn, Barbara (1995): Success Themes in Scottish Family Enterprises. Philosophies and Practices Through the Generations, in: Family Business Review 8, 1, S. 17-28. 
Duss-Von Werdt, Joseph (2008): Einführung in Mediation. 1. Aufl., Heidelberg.

DWS-Institut (Hrsg.) (2014): Handbuch Familienunternehmen. Gesellschafts-, Steuer-, Güter- und Erbrecht mit Mustersammlung, Köln.

Dyer, William G. (2003): The Family: The Missing Variable in Organizational Research, in: Ent. Theory \& Pract 27, 4, S. 401-416.

Ebel, Karin (2014): Warum und worüber Gesellschafter am meisten streiten, in: DaunerLieb, B./Freudenberg, G./Werner, G. W. (Hrsg.): Familienunternehmen im Fokus von Wirtschaft und Wissenschaft. Festschrift für Mark K. Binz, München, S. 171-176.

Ebers, Mark/Gotsch, Wilfried (1995): Institutionenökonomische Theorien der Organisation, in: Kieser, A./Kubicek, H. (Hrsg.): Organisationstheorien. 2. überarb. Aufl., Stuttgart, S. 185-235.

Eddleston, Kimberly A./Kellermanns, Franz W. (2007): Destructive and productive family relationships. A stewardship theory perspective, in: Journal of Business Venturing 22, 4, S. 545-565.

Ehrlich, Eugen (1989): Grundlegung der Soziologie des Rechts. 4. Aufl., Berlin.

Einsele, Dorothee (2015): $\$ \$ 125-132$, in: Säcker, F.-J./Rebmann, K. (Hrsg.): Münchener Kommentar zum Bürgerlichen Gesetzbuch. 7. Auflage, München.

Eisenhardt, Kathleen M. (1989): Building Theories from Case Study Research, in: The Academy of Management Review 14, 4, S. 532-550.

Ekardt, Felix (2005): Information, Verfahren, Selbstregulierung, Flexibilisierung. Instrumente eines effektiven Umweltrechts?, in: NuR 27, 4, S. 215-222.

Ellinger, Andrea D. (2005): Case Study Research Methods, in: Swanson, R. A./Holton III, E. F. (Hrsg.): Research in Organizations. Foundations and methods of inquiry, California, S. 327-350.

Elster, Jon (1989): Social Norms and Economic Theory, in: Journal of Economic Perspective 4, 3, S. 99-117.

Engel, Christoph/Englerth, Markus/Lüdemann, Jörn (Hrsg.) (2007): Recht und Verhalten. Beiträge zu Behavioral Law and Economics, Tübingen.

EQUA Stiftung (Hrsg.) (2017): Familienunternehmen der Zukunft. Fiktive Vermächtnisse für die nächste Generation. Ergebnisse des ersten EQUA-ThinkTanks in Neumühle (Franken), Bonn.

Esser, Josef (1956): Grundsatz und Norm in der richterlichen Fortbildung des Privatrechts, Tübingen.

Ezzy, Douglas (2002): Qualitative analysis. Practice and innovation, Crows Nest, N.S.W.

Fabis, Felix G. (2007): Instrumentarien zur Vermeidung und Lösung von Gesellschafterkonflikten in Familienunternehmen, in: OSC Organisationsberatung Supervision Coaching 14, 4, S. 354-365.

Fabis, Felix G. (2009): Konflikte im Familienunternehmen. Instrumente zur Vermeidung und Lösung. 1. Aufl., Lohmar, Köln.

Felden, Birgit/Hack, Andreas (2014): Management von Familienunternehmen. Besonderheiten - Handlungsfelder - Instrumente, Wiesbaden.

Feldges, Joachim (1987): Konsumentenschutz durch private Normen, Zürich.

Fleischer, Holger (2016): Das Rätsel Familienverfassung. Realbefund - Regelungsnatur Rechtswirkungen, in: ZIP - Zeitschrift für Wirtschaftsrecht 37, 32, S. 1509-1519.

Fleischer, Holger (2017a): Die Familienverfassung. Vertrag ohne Schwert? Forschungsbericht 2017, Hamburg. 
Fleischer, Holger (2017b): Familiengesellschaften und Familienverfassungen. Eine historisch-vergleichende Standortbestimmung, in: Neue Zeitschrift für Gesellschaftsrecht (NZG) 31, S. 1201-1210.

Fleming, Quentin J. (2000): Keep the family baggage out of the family business. Avoiding the seven deadly sins that destroy family businesses, New York, N.Y.

Flick, Uwe (2011): Triangulation. Eine Einführung. 3., aktualisierte Aufl., Wiesbaden.

Flick, Uwe (2014): Gütekriterien qualitativer Sozialforschung, in: Baur, N./Blasius, J. (Hrsg.): Handbuch Methoden der empirischen Sozialforschung, Wiesbaden, S. 411-424.

Flick, Uwe (2016): Qualitative Sozialforschung. Eine Einführung. Originalausgabe, vollständig überarbeitete und erweiterte Neuausgabe, 7. Auflage, Reinbek bei Hamburg.

Flick, Uwe/Kardorff, Ernst v./Keupp, Heiner, et al. (Hrsg.) (2012): Handbuch qualitative Sozialforschung. Grundlagen, Konzepte, Methoden und Anwendungen. 3., neu ausgestattete Auflage, Weinheim.

Flick, Uwe/Kardorff, Ernst v./Steinke, Ines (Hrsg.) (2015): Qualitative Forschung. Ein Handbuch. 11. Aufl., Reinbek bei Hamburg.

Forst, Rainer (2010): Kritik und Antwort. Zu Peter Stemmer: Normativität. Die Reise nach Phantasia, in: Deutsche Zeitschrift für Philosophie 58, 1, S. 157-161.

Frank, Hermann/Kessler, Alexander/Nosé, Lavinia/Suchy, Daniela (2011): Conflicts in family firms. state of the art and perspectives for future research, in: Journal of Family Business Management 1, 2, S. 130-153.

Frenz, Walter (2001): Selbstverpflichtungen der Wirtschaft, Tübingen.

Friebertshäuser, Barbara (1997): Interviewtechniken - ein Überblick, in: Friebertshäuser, B./Prengel, A. (Hrsg.): Handbuch Qualitative Forschungsmethoden in der Erziehungswissenschaft, Weinheim, Basel, S. 371-395.

Friebertshäuser, Barbara/Prengel, Annedore (Hrsg.) (1997): Handbuch Qualitative Forschungsmethoden in der Erziehungswissenschaft, Weinheim, Basel.

Friese, Susanne (o.J.): Computergestützte Analyse qualitative Daten, Universität Hannover. http://www.quarc.de/fileadmin/downloads/CUQDAS\%20und\%20Medienfor schung.pdf. Zuletzt geprüft am 24.06.2019.

Fritz, Wolfgang (1992): Marktorientierte Unternehmensführung und Unternehmenserfolg. Grundlagen und Ergebnisse einer empirischen Untersuchung, Stuttgart.

Froschauer, Ulrike/Lueger, Manfred (2003): Das qualitative Interview. Zur Praxis interpretativer Analyse sozialer Systeme. 1. Aufl., Tübingen.

Froschauer, Ulrike/Lueger, Manfred (2009): Interpretative Sozialforschung: Der Prozess. 1. Aufl., Stuttgart.

Galbraith, John K. (1967): The new industrial state. 1st Princeton ed., Princeton, N.J.

Gallo, Miguel A. (2000): Family protocol, in: The Family Business Network Newsletter 26.

Gallo, Miguel A./Kenyon-Rouvinez, Denise (2005): The Importance of Family and Business Governance, in: Kenyon-Rouvinez, D./Ward, J. L. (Hrsg.): Family business. Key issues, Houndmills, Basingstoke, Hampshire, New York, S. 45-57.

Gallo, Miguel A./Ward, John L. (1991): Protocolo Familiar. Note Técnica de la division di Investigacion del IESE, Barcelona.

Garz, Detlef/Kraimer, Klaus (Hrsg.) (1991): Qualitativ-empirische Sozialforschung. Konzepte, Methoden, Analysen, Wiesbaden.

Gasteiger-Klicpera, Barbara/Julius, Henri/Klicpera, Christian, et al. (2008): Sonderpädagogik der sozialen und emotionalen Entwicklung, Göttlingen. 
Geiger, Theodor/Rehbinder, Manfred (1987): Vorstudien zu einer Soziologie des Rechts. 4. Aufl., Berlin.

Gelhausen, Hans F./Hönsch, Henning (2003): Folgen der Änderung des Deutschen Corporate Governance Kodex für die Entsprechenserklärung, in: AG 2003, S. 367-371.

Gersick, Kelin E./Davis, John A./McCollom Hampton, Marion, et al. (1997): Generation to Generation: Life Cycles of the Family Business, Watertown, Massachusetts.

Gibbard, Allan (1992): Wise Choices, Apt Feelings: A Theory of Normative Judgment, Cambridge, Mass.

Gibbs, Graham R./Friese, Susanne/Mangabeira, Wilma C. (2002): Technikeinsatz im qualitativen Forschungsprozess. Einführung zu FQS Band 3(2), in: Forum Qualitative Sozialforschung / Forum Qualitative Social Research 3, 2.

Gilch, Andreas/Pelz, Christian (2008): Compliance-Klauseln gut gemeint aber unwirksam?, in: CCZ 1, 4, S. 131-136.

Glaser, Barney G./Strauss, Anselm L. (1967): The discovery of grounded theory. Strategies for qualitative research, New Brunswick [u. a.].

Glaser, Barney G./Strauss, Anselm L./Paul, Axel T. (2008): Grounded theory. Strategien qualitativer Forschung. 1. Nachdr. 2008 der 2., korrig. Aufl., 2005, Bern [u. a.].

Gläser, Jochen/Laudel, Grit (2010): Experteninterviews und qualitative Inhaltsanalyse. Als Instrumente rekonstruierender Untersuchungen. 4. Aufl, Wiesbaden.

Gläßer, Ulla (2014): Die Familienverfassung - zahnlose Absichtserklärung, unzulässiges Disziplinierungsmittel oder integratives Steuerungsinstrument? Annäherung an ein Chamäleon, in: Dauner-Lieb, B./Freudenberg, G./Werner, G. W. (Hrsg.): Familienunternehmen im Fokus von Wirtschaft und Wissenschaft. Festschrift für Mark K. Binz, München, S. 228-240.

Gläßer, Ulla/Kirchhoff, Lars/Wendenburg, Felix (2014): Konfliktmanagement in der Wirtschaft. Ansätze, Modelle, Systeme. 1. Aufl., Baden-Baden.

Goette, Wulf/Habersack, Mathias (Hrsg.) (2003): Münchener Kommentar zum Aktiengesetz. 2. Auflage, München.

Gosepath, Stefan (Hrsg.) (1999): Motive, Gründe, Zwecke. Theorien praktischer Rationalität, Frankfurt am Main.

Gosepath, Stefan (1999): Praktische Rationalität. Eine Problemübersicht, in: Gosepath, S. (Hrsg.): Motive, Gründe, Zwecke. Theorien praktischer Rationalität, Frankfurt am Main.

Gosepath, Stefan (2013): Moral aus Interesse und Sanktion? Anmerkungen zu Michael von Grundherr, Michael Kühler und Frank Brosow, in: Buddeberg, E./Vesper, A. (Hrsg.): Moral und Sanktion. Eine Kontroverse über die Autorität moralischer Normen, Frankfurt, S. 125-134.

Governance Kodex für Familienunternehmen (2015): Leitlinien für die verantwortungsvolle Führung von Familienunternehmen und Unternehmerfamilien. http://www.kodex-fuerfamilienunternehmen.de/images/Downloads/Kodex_2015.pdf. Zuletzt geprüft am 24.06. 2019.

Graf, Manuel (2005): Emotionale Aspekte der Unternehmensübergabe von Familienunternehmen, Hamburg.

Grathoff, Richard/Sprondel, Walter M. (Hrsg.) (1979): Alfred Schütz und die Idee des Alltags in den Sozialwissenschaften, Stuttgart. 
Griswold, Charles L. (1999): Adam Smith and the virtues of enlightenment, Cambridge [etc.].

Großmann, Steffen (2014): Konflikte und Krisen in Familienunternehmen. Eine Untersuchung der Wechselwirkungen zwischen Konflikten in Familie und Unternehmen und dem Untergang von Familienunternehmen, Osnabrück.

Groth, Torsten (2011): Gute Lösungen von Generation zu Generation: Langlebige Familienunternehmen, in: Schlippe, A. v./Nischak, A./El Hachimi, M. (Hrsg.): Familienunternehmen verstehen. Gründer, Gesellschafter und Generationen. 2. Aufl., Göttingen, S. $30-41$.

Groth, Torsten/Koenen, Leonie (2009): Das Management der Gesellschafter und die Funktion von Familienverfassungen, Witten.

Groth, Torsten/Rüsen, Tom A. (2013): Der Familientag, in: private wealth Nr. 3/2013, S. 100101.

Groth, Torsten/Vater, Gudrun (2009): Die Familie im Familienunternehmen, in: Rüsen, T. A./Schlippe, A. v./Groth, T. (Hrsg.): Familienunternehmen. Exploration einer Unternehmensform. 1. Aufl., Lohmar, Köln, S. 73-92.

Grottel, Bernd/Kieser, Matthias/Helfmann, Laura/Rau, Bernd/Kettenring, Tim (2012): Governance Kodex für Familienunternehmen. Kritische Analyse und Stand der Umsetzung, in: Zeitschrift für Corporate Governance (ZCG) 4, S. 153-157.

Gruber, Michaela (2008): Systemische Mediation, in: Zeitschrift für Konfliktmanagement Nr. 3/2008, S. 71-73.

Grundherr, Michael v. (2003): Kants Ethik in modernen Gesellschaften, Münster [u.a.].

Grundherr, Michael v. (2007): Moral aus Interesse. Metaethik der Vertragstheorie (Ideen \& Argumente), Berlin.

Grundherr, Michael v. (2013): Notwendigkeit und Nötigung: Konzeptionelle und moralpsychologische Gründe für eine hybride Theorie des moralischen Sollens, in: Buddeberg, E./Vesper, A. (Hrsg.): Moral und Sanktion. Eine Kontroverse über die Autorität moralischer Normen, Frankfurt, S. 53-74.

Grunwald, Guido/Hempelmann, Bernd (2012): Angewandte Marktforschung: Eine praxisorientierte Einführung, Berlin.

Gummert, Hans (Hrsg.) (2015): Münchener Anwalts-Handbuch Personengesellschaftsrecht, München.

Haakonssen, Knud (1981): The science of a legislator. The natural jurisprudence of David Hume and Adam Smith, Cambridge.

Habbershon, Timothy G./Williams, Mary L. (1999): A Resource-Based Framework for Assessing the Strategic Advantages of Family Firms, in: Family Business Review 12, 1, S. $1-25$.

Habbershon, Timothy G./Williams, Mary L./MacMillan, Ian C. (2003): A unified systems perspective of family firm performance, in: Journal of Business Venturing 18, 4, S. 451465.

Habermas, Jürgen (1992): Erläuterungen zur Diskursethik, in: Habermas, J. (Hrsg.): Erläuterungen zur Diskursethik. 6. Auflage, Frankfurt am Main, S. 119-226.

Habermas, Jürgen (Hrsg.) (1992): Erläuterungen zur Diskursethik. 6. Auflage, Frankfurt am Main. 
Hack, Andreas (2009): Sind Familienunternehmen anders? Eine kritische Bestandsaufnahme des aktuellen Forschungsstands, in: Zeitschrift für Betriebswirtschaft, ZfBSpecial Issue Nr. 2/2009, S. 1-29.

Hack, Andreas/Meyer, Jennifer (2012): Gründer für eine spezielle Governance von Familienunternehmen, in: Koeberle-Schmid, A./Fahrion, H.-J./Witt, P. (Hrsg.): Family Business Governance. Erfolgreiche Führung von Familienunternehmen, Berlin, S. 5977.

Häder, Michael (2015): Empirische Sozialforschung. Eine Einführung. 3. Aufl., Wiesbaden.

Halter, Frank A. (2009): Familienunternehmen im Nachfolgeprozess: die Emotionen des Unternehmers, Siegburg.

Hammann, Peter/Erichson, Bernd (1994): Marktforschung. 54 Tabellen. 5. Aufl., Stuttgart. Hammann, Peter/Erichson, Bernd (2000): Marktforschung. Grundwissen der Ökonomik. Betriebswirtschaftslehre. 4., überarb. und erw. Aufl., Stuttgart.

Handler, Wendy C. (1994): Succession in Family Business. A Review of the Research, in: Family Business Review 7, 2, S. 133-157.

Handy, Charles B. (1995): The Age of Paradox, Brighton, Massachusetts.

Harms, Henrik (2014): Review of Family Business Definitions. Cluster Approach and Implications of Heterogeneous Application for Family Business Research, in: IJFS 2, 3, S. $280-314$.

Harris, Dawn/Martinez, Jon I./Ward, John L. (1994): Is Strategy Different for the FamilyOwned Business?, in: Family Business Review 7, 2, S. 159-174.

Hart, Herbert L. (2011): Der Begriff des Rechts. 1. Aufl., Berlin.

Harvey, Michael/Evans, Rodney E. (1994): Family business and multiple levels of conflict, in: Family Business Review 7, 4, S. 331-348.

Hauck, Jana (2012a): Die Familiencharta, Friedrichshafener Institut für Familienunternehmen (FIF), pFIFig. https://www.zu.de/institute/fif/assets/pdf/pfifig/04_2012_pFI Fig_Familiencharta_FIF.pdf. Zuletzt geprüft am 24.06.2019.

Hauck, Jana (2012b): Die Familienstrategie, Friedrichshafener Institut für Familienunternehmen (FIF), pFIFig. https://www.zu.de/institute/fif/assets/pdf/pfifig/01_2012_ pFIFig_Familienstrategie_FIF.pdf. Zuletzt geprüft am 24.06.2019.

Haunschild, L./Wolter, H.-J. (2010): Volkswirtschaftliche Bedeutung von Familien- und Frauenunternehmen, Bonn.

Hausch, Kerstein T. (2004): Corporate Governance im deutschen Mittelstand:. Veränderungen externer Rahmenbedingungen und interner Elemente, Wiesbaden.

Heck, R./Hoy, F./Poutziouris, P. Z. (2008): Emerging paths of family entrepreneurship research, in: Journal of Small Business Management 46, S. 317-330.

Hefermehl, Wolfgang (Hrsg.) (1974): Festschrift für Harry Westermann zum 65. Geburtstag, Karlsruhe.

Heimann, Felix (2009): Der Pressekodex im Spannungsfeld zwischen Medienrecht und Medienethik, Frankfurt am Main.

Heinze, Thomas (2016): Qualitative Sozialforschung. Einführung, Methodologie und Forschungspraxis. Reprint 2016, Berlin, Boston, Berlin, Boston.

Helferich, Cornelia (2014): Leitfaden- und Experteninterviews, in: Baur, N./Blasius, J. (Hrsg.): Handbuch Methoden der empirischen Sozialforschung, Wiesbaden, S. 559-574.

Helfferich, Cornelia (2011): Die Qualität qualitativer Daten. Manual für die Durchführung qualitativer Interviews. 4. Aufl., Wiesbaden. 
Hennerkes, Brun-Hagen (1998): Familienunternehmen sichern und optimieren, Frankfurt am Main.

Hennerkes, Brun-Hagen (2004): Die Familie und ihr Unternehmen:. Strategie, Liquidität, Kontrolle, Frankfurt am Main.

Hennerkes, Brun-Hagen/Berlin, Malte/Berlin, Tilo (2007): Die Familie und ihr Unternehmen in Österreich. Strategie, Kontrolle, Nachfolge, Vermögenssicherung. 1. Aufl, München.

Hennerkes, Brun-Hagen/Kirchdörfer, Rainer (2015): Die Familie und ihr Unternehmen. Strategie, Liquidität, Kontrolle. 2. Aufl., Frankfurt/Main.

Herb, K./Brandt, R. (2012): Jean-Jacques Rousseau: Vom Gesellschaftsvertrag: oder Prinzipien des Staatsrechts, Berlin.

Hertz, Rosanna/Imber, Jonathan (Hrsg.) (1995): Studying Elites Using Qualitative Methods, 2455 Teller Road, Thousand Oaks California 91320 United States.

Herzog, Lisa (2013): Intersubjektive Sanktionen als moralische Gründe. Nicht-dualistische Moralbegründung am Beispiel Adam Smiths, in: Buddeberg, E./Vesper, A. (Hrsg.): Moral und Sanktion. Eine Kontroverse über die Autorität moralischer Normen, Frankfurt, S. 209-236.

Hess, Edward D. (2006): The successful family business. A proactive plan for managing the family and the business, Westport, CT.

Hiebl, Martin R. W. (2012): Die Rolle des Chief Financial Officer in großen Familienunternehmen, Berlin.

Hienerth, Claudia (Hrsg.) (2009): Wissenschaftliches Arbeiten kompakt. Bachelor- und Masterarbeiten erstellen, Wien.

Hillmann, Karl-Heinz (1994): Wörterbuch der Soziologie. 4. überarbeitete und ergänzte Aufl, Stuttgart.

Hinterhuber, Hans H./Rechenauer, Ottmar/Stumpf, Monika (1994): Die mittelständische Familienunternehmung. Die Integration der beiden Subsysteme Familie und Unternehmung in den 90er Jahren, Frankfurt am Main, New York.

Hirschman, Albert O. (1970): Exit, voice and loyality. Responses to the decline in firms, organizations and State, Cambridge.

Hoeren, Thomas (1992): Das neue Verfahren für die Schlichtung von Kundenbeschwerden im deutschen Bankgewerbe - Grundzüge und Rechtsprobleme, in: NJW Nr. 43/1992, S. 2727-2732.

Hoeren, Thomas (1995): Selbstregulierung im Banken- und Versicherungsrecht, Karlsruhe.

Hofelich, Markus (2013): »Die Familienverfassung kommt häufig ins Spiel, wenn ein Unternehmer seine Nachfolgeplanung startet«, in: Unternehmer Edition Nr. 1/2013, S. 5051.

Hoffmann-Riem, Christa (1980): Die Sozialforschung einer interpretativen Soziologie. Der Datengewinn, in: Kölner Zeitschrift für Soziologie und Sozialpsychologie 32, 2, S. 339372.

Holler, Lorenz (2016): $\$ 75$ Grundlagen des Rechts der Familiengesellschaften und der gesellschaftsrechtlichen Streitigkeiten in Familiengesellschaften, in: Born, M./Ghassemi-Tabar, N./Gehle, B. (Hrsg.): Münchener Handbuch des Gesellschaftsrechts. Band 7: Gesellschaftsrechtliche Streitigkeiten. 5, München, 1-271. 
Holler, Lorenz (2018): Die Familienverfassung im Recht der Familienunternehmen. Eine (gesellschafts-)rechtliche Einordnung aus Sicht der Gestaltungspraxis, in: ZIP - Zeitschrift für Wirtschaftsrecht 39, 12, S. 553-564.

Hollstein, Bettina/Straus, Florian (2006): Qualitative Netzwerkanalyse: Konzepte, Methoden, Anwendungen, Wiesbaden.

Holton, Judith A. (2010): The Coding process and its challenges, in: Bryant, A./Charmaz, K. (Hrsg.): SAGE Handbook of Grounded Theory. Paperback Edition, London, S. 265-290.

Hopf, Christel (1978): Pseudo-exploration - Thoughts on the techniques of qualitative interviews in social research, in: Zeitschrift für Soziologie 7, 2, S. 97-115.

Hopt, Klaus J.: Europäisches und deutsches Übernahmerecht, in: ZHR 1997, 161, S. 368420.

Hopt, Klaus J./Kämmerer Jörn A./Veil, Rüdiger (Hrsg.) (2008): Kapitalmarktgesetzgebung im europäischen Binnenmarkt, Tübingen.

Horn, Norbert (2011): Einführung in die Rechtswissenschaft und Rechtsphilosophie. 5. Aufl., Karlsruhe.

Horster, Detlef (2004): Was soll ich tun? Moral im 21. Jahrhundert. 1. Aufl., Leipzig.

Hovland, Carl I./Janis, Irving L. (1959): Personality and Persuasibility. Yale Studies in Attitude and Communication, New Haven, CT, US.

Hueck, Tobias (2017): Die Familienverfassung - Rechtliche Konturen eines Instruments der Governance in Familienunternehmen, Tübingen.

Hughes, James E. (2004): Family Wealth: Keeping It in the Family. How Family Members and Their Advisers Preserve Human, Intellectual, and Financial Assets for Generations, Hoboken, New Jersey.

Hussy, Walter/Schreier, Margrit/Echterhoff, Gerald (2013): Forschungsmethoden in Psychologie und Sozialwissenschaften für Bachelor.

Hüttner, Manfred/Schwarting, Ulf (2002): Grundzüge der Marktforschung. 7., überarb. Aufl., München [u.a.].

Ichihara, Kiichi/Takamiya, Susumu (2013): Die japanische Unternehmung: Strukturwandlungen in einer wachsenden Wirtschaft, Wiesbaden.

Ihne, Hartmut (2010): Moralische Vernunft und ökonomische Rationalität, in: Busche, H./ Schmitt, A. (Hrsg.): Kant als Bezugspunkt philosophischen Denkens: Festschrift für Peter Baumanns zum 75. Geburtstag, Würzburg, S. 119-132.

Iliou, Christopher D. (2004): Die Nutzung von Corporate Governance in mittelständischen Familienunternehmen, Berlin.

James Jr., Harvey (1999): Owner as Manager, Extended Horizons and the Family Firm, in: International Journal of the Economics of Business 6, 1, S. 41-55.

Jaskiewicz, Peter/Gonzalez, Victor M./Menendez, Susana/Schiereck, Dirk (2005): LongRun IPO Performance Analysis of German and Spanish Family-Owned Businesses, in: Family Business Review 18, 3, S. 179-202.

Jendritzky, Tatjana (2007): Die korporative Gruppenbildung als Organisationsmodell für Familienunternehmen. 1. Auflage, Baden-Baden.

Johnston, Alexander (1980): The city take-over code, New York.

Jürgens, Ulrich (1980): Selbstregulierung des Kapitals. Erfahrungen aus der Kartellbewegung in Deutschland um die Jahrhundertwende zum Verhältnis von Politik und Ökonomie, Frankfurt/Main.

Kaiser, Joachim (2012): Generationenwechsel im Familienunternehmen, München. 
Kaiser, Robert (2014): Qualitative Experteninterviews. Konzeptionelle Grundlagen und praktische Durchführung, Wiesbaden.

Kalss, Susanne (2014): Regelungen helfen, Gesellschafterkonflikte auszutragen oder nicht entstehen zu lassen, in: Dauner-Lieb, B./Freudenberg, G./Werner, G. W. (Hrsg.): Familienunternehmen im Fokus von Wirtschaft und Wissenschaft. Festschrift für Mark K. Binz, München, S. 343-357.

Kalss, Susanne/Probst, Stephan (2013a): Familienunternehmen. Gesellschafts- und zivilrechtliche Fragen; [Handbuch], Wien.

Kalss, Susanne/Probst, Stephan (2013b): Familienunternehmen - eine erste Vermessung, in: Der Gesellschafter - Zeitschrift für Gesellschafts- und Unternehmensrecht 42, 3, S. 115-123.

Kalss, Susanne/Probst, Stephan (2013c): Familienunternehmen - Familienverfassung, in: Kathrein Stiftungsletter 1, S. 14-18.

Kant, Immanuel (1924): Eine Vorlesung über Ethik, Berlin.

Kant, Immanuel./Weischedel, Wilhelm (1786): Schriften zur Ethik und Religionsphilosophie, Berlin.

Kantorowicz, Alfred (1957): Der Begriff des Rechts, Osnabrück.

Kardorff, Ernst v. (2012): Qualitative Sozialforschung: Versuch einer Standortbestimmung, in: Flick, U., et al. (Hrsg.): Handbuch qualitative Sozialforschung. Grundlagen, Konzepte, Methoden und Anwendungen. 3., neu ausgestattete Auflage, Weinheim, S. 3-8.

Kaschuba, W. (2012): Einführung in die Europäische Ethnologie, München.

Kaya, Maria (2009): Verfahren der Datenerhebung, in: Albers, S. (Hrsg.): Methodik der empirischen Forschung. 3., überarb. und erw. Aufl., Wiesbaden, S. 49-64.

Kelle, Udo (2010): The Development of Categories: Different Approaches in Grounded Theory, in: Bryant, A./Charmaz, K. (Hrsg.): SAGE Handbook of Grounded Theory. Paperback Edition, London, S. 191-213.

Kelle, Udo/Kluge, Susann (2010): Vom Einzelfall zum Typus. Fallvergleich und Fallkontrastierung in der qualitativen Sozialforschung. 2., überarbeitete Aufl., Wiesbaden.

Kellermanns, Franz W./Eddleston, Kimberly A. (2004): Feuding Families. When Conflict Does a Family Firm Good, in: Ent. Theory \& Pract 28, 3, S. 209-228.

Kellerwessel, Wulf (2003): Normenbegründung in der analytischen Ethik, Würzburg.

Kelman, Herbert C. (1961): Process of opinion, in: Public Opinion Quarterly 25, S. 57-78.

Kelman, Herbert C. (1980): International Behavior. A Social-Psychological Analysis, U.S.

Kenyon-Rouvinez, Denise/Ward, John L. (Hrsg.) (2005): Family business. Key issues, Houndmills, Basingstoke, Hampshire, New York.

Kidwell, Roland E./Kellermanns, Franz W./Eddleston, Kimberly A. (2012): Harmony, Justice, Confusion, and Conflict in Family Firms. Implications for Ethical Climate and the »Fredo Effect«, in: Journal of Business Ethics 106, 4, S. 503-517.

Kieser, Alfred (2004): Organisation, in: Kieser, A./Oechsler, W. (Hrsg.): Unternehmungspolitik. 2., überarb. und aktualisierte Aufl., Stuttgart, S. 174-231.

Kieser, Alfred/Kubicek, Herbert (Hrsg.) (1995): Organisationstheorien. 2. überarb. Aufl., Stuttgart.

Kieser, Alfred/Oechsler, Walter (Hrsg.) (2004): Unternehmungspolitik. 2., überarb. und aktualisierte Aufl., Stuttgart.

King, Nigel (1994): The qualitative research interview, in: Cassell, C./Symon, G. (Hrsg.): Qualitative methods in organizational research. A practical guide, London, S. 15-36. 
Kirchdörfer, Rainer/Breyer, Michael (2014): Family Business Governance im Familienunternehmen als Instrument der Streitprävention, in: FuS Sonderheft Nr. 13-25/2014.

Kirchdörfer, Rainer/Lorz, Rainer (2011): Corporate Governance in Familienunternehmen, Familienverfassungen und Schnittstellen zum Gesellschaftsvertrag, in: FuS Zeitschrift für Familienunternehmer und Stiftungen Nr. 3/2011, S. 97-106.

Kirchdörfer, Rainer/Lorz, Rainer/Wiedemann, Andreas, et al. (Hrsg.) (2009): Familienunternehmen in Recht, Wirtschaft, Politik und Gesellschaft. Festschrift für Brun-Hagen Hennerkes zum 70. Geburtstag, München.

Kirchdörfer, Rainer/Sarholz, Olivia (2012): Vermeidung und Lösung von Konflikten in Familienunternehmen. Der Generationenwechsel als besondere Herausforderung, in: Konfliktdynamik 1, 4, S. 300-308.

Kirchhof, Ferdinand (1987): Private Rechtsetzung, Berlin.

Kirchhof, Paul/Nieskens, Hans (Hrsg.) (2008): Festschrift für Wolfram Reiß. Zum 65. Geburtstag, Köln, Germany.

Kirsch, Werner (1974): Betriebswirtschaftslehre: Systeme, Entscheidungen, Methoden, Wiesbaden.

Kleemann, Frank/Krähnke, Uwe/Matuschek, Ingo (2009): Interpretative Sozialforschung: Eine Einführung in die Praxis des Interpretierens, Wiesbaden.

Klein, Sabine (1991): Der Einfluss von Werten auf die Gestaltung von Organisationen, Berlin.

Klein, Sabine B. (2010): Familienunternehmen. Theoretische und empirische Grundlagen. 3. Aufl., Lohmar, Köln.

Kleindiek, Detlef/Oehler, Wolfgang (Hrsg.) (2000): Die Zukunft des deutschen Bilanzrechts im Zeichen internationaler Rechnungslegung und privater Standardsetzung. [Vorträge und Diskussionsberichte des interdisziplinären Symposions »Die Zukunft des Deutschen Bilanzrechts«, Universität Bielefeld am 25. und 26. Juni 1999], Köln.

Klett, David (2005): Zwischen Kompetenz und Herkunft - zwischen Gleichheit und Selektion. Paradoxe Anforderungen an Familienunternehmen und ihre Unternehmensfamilien. 1. Aufl., Heidelberg.

Klett, David J. (2007): Familie qua Unternehmen. Wie sich Großfamilien an den Zumutungen eines Betriebs stärken können, in: Kontext - Zeitschrift für systemische Therapie und Familientherapie 38, 1, S. 6-25.

Kleve, Heiko (2017): Das Tetralemma der Unternehmerfamilie, in: systeme 31, 2, S. 224243.

Kluge, Susann (1999): Empirisch begründete Typenbildung. Zur Konstruktion von Typen und Typologien in der qualitativen Sozialforschung, Düsseldorf.

Knauff, Matthias (2010): Der Regelungsverbund. Recht und Soft Law im Mehrebenensystem, Tübingen.

Knebel, Jürgen/Michael, Gerhard/Wicke, Lutz, et al. (1999): Selbstverpflichtungen und normersetzende Umweltverträge als Instrumente des Umweltschutzes. Forschungsbericht 29618081; UBA-FB 98-123, Berlin.

Koeberle-Schmid, Alexander (2008): Family Business Governance. Aufsichtsgremium und Familienrepräsentanz. 1. Aufl., Wiesbaden.

Koeberle-Schmid, Alexander (2014): Führung in Familienunternehmen im Wandel, in: BB 19, 4, Die erste Seite. 
Koeberle-Schmid, Alexander (22.03.2017): Familienunternehmen im Clinch. Warum eine Familienverfassung für Firmen wichtig ist, in: Manager Magazin Online, 22.03.2017. http://www.manager-magazin.de/finanzen/artikel/familienunternehmen-familienver fassung-hilft-bei-streit-und-nachfolge-a-1139938.html. Zuletzt geprüft am 24.06.2019.

Koeberle-Schmid, Alexander/Fahrion, Hans-Jürgen/Witt, Peter (Hrsg.) (2012): Family Business Governance. Erfolgreiche Führung von Familienunternehmen, Berlin.

Koeberle-Schmid, Alexander/May, Peter (2011): Governance in Familienunternehmen. Führung und Kontrolle situationsadäquat regeln, in: ZRFC Nr. 2/2011, S. 54-61.

Koeberle-Schmid, Alexander/Schween, Karsten (2012): Familienverfassungen individuell erarbeiten. Hinweise für Unternehmerfamilien vor dem Hintergrund der Konfliktprävention, in: Konfliktdynamik Nr. 4/2012, S. 320-327.

Koeberle-Schmid, Alexander/Schween, Karsten/May, Peter (2011): Governance Kodex für Familienunternehmen in der Praxis - Ergebnisse einer Studie über Familienverfassungen, in: BB 66, 41, S. 2499-2506.

Koeberle-Schmid, Alexander/Witt, Peter/Fahrion, Hans-Jürgen (2010): Gestaltung der Governance im Familieunternehmen, Gremien und Instrumente der Business und Family Governance, in: Zeitschrift für Corporate Governance (ZCG) 2010, S. 161-169.

Kögel, Rainer (2014): $\$ 40$ Die Nachfolge in Unternehmen und Gesellschaftsanteile, in: Scherer, S. (Hrsg.): Münchener Anwaltshandbuch Erbrecht. 4., überarb. Aufl., München, 1-155.

Kohli, Martin (1978): »Offenes« und »geschlossenes« Interview: Neue Argumente zu einer alten Kontroverse, in: Soziale Welt 29, 1, S. 1-25.

Kollmer-von Oheimb-Loup, Gert/Wischermann, Clemens (Hrsg.) (2008): Unternehmernachfolge in Geschichte und Gegenwart. (Stuttgarter historische Studien zur Landesund Wirtschaftsgeschichte), Ostfildern.

Köndgen, Johannes (2006): Privatisierung des Rechts. Private Governance zwischen Deregulierung und Rekonstitutionalisierung, in: Archiv fuer die civilistische Praxis 206, 2, S. 477-525.

König, Eckard/Zedler, Peter (Hrsg.) (2002): Qualitative Forschung. Grundlagen und Methoden. 2éme éd, Weinheim und Basel.

König, René (1962): Das Interview. Formen, Technik, Auswertung. 3. Aufl., Köln.

Kormann, Hermut (2011): Zusammenhalt der Unternehmerfamilie. Verträge, Vermögensmanagement, Kommunikation, Berlin.

Kormann, Hermut (2017): Governance des Familienunternehmens, Wiesbaden.

Kormann, Hermut (2018): Zusammenhalt der Unternehmerfamilie. Verträge, Vermögensmanagement, Kommunikation. 2., vollst. überarb. u. erw. Auflage, Wiesbaden.

Kormann, Hermut/Schmeing, Thomas (2016): Der Ausstieg von Gesellschaftern aus Familienunternehmen, in: FuS Zeitschrift für Familienunternehmer und Stiftungen Nr. 1/ 2016, S. 13-19.

Kotter, John P. (1997): Matsushita Leadership. Lessons from the 20th century's most remarkable entrepreneur, New York.

Kötz, Heinz (2012): Vertragsrecht. 2., überarbeitete und aktualisierte Auflage, Tübingen. KPMG AG Wirtschaftsprüfungsgesellschaft (Hrsg.) (2014): Das wirksame ComplianceManagement-System. Ausgestaltung und Implementierung in Unternehmen, Herne.

Kraimer, Klaus (2002): Einzelfallstudien, in: König, E./Zedler, P. (Hrsg.): Qualitative Forschung. Grundlagen und Methoden. 2éme éd, Weinheim und Basel, S. 213-232. 
Krause, Nils (2012): Corporate und Family Governance in Familienunternehmen als Teil des Risikomanagements, in: BB Nr. 12/2012, S. 714-716.

Kreklau, Jan (2013): Die Familienverfassung kommt häufig ins Spiel, wenn ein Unternehmer seine Nachfolgeplanung startet, in: Unternehmer Edition Nr. 1/2013, S. 50-51.

Kreyenberg, Jutta (2004): Handbuch Konfliktmanagement. Konfliktdiagnose, -definition und -analyse; Konfliktebenen, Konflikt- und Führungsstile; Interventions- und Lösungsstrategien, Beherrschung der Folgen, Berlin.

Krieger, Gerd/Lutter, Marcus/Schmidt, Karsten, et al. (Hrsg.) (2013): Festschrift für Michael Hoffmann-Becking zum 70. Geburtstag, München.

Krippendorff, Klaus (1980): Validity in Content Analysis, in: Mochmann, E. (Hrsg.): Computerstrategien für die Kommunikationsanalyse, Frankfurt.

Krüger, Dirk/Parchmann, Ilka/Schecker, Horst (Hrsg.) (2014): Methoden in der naturwissenschaftsdidaktischen Forschung, Berlin, Heidelberg.

Krüger, Dirk/Riemeier, Tanja (2014): Die qualitative Inhaltsanalyse. Eine Methode zur Auswertung von Interviews, in: Krüger, D./Parchmann, I./Schecker, H. (Hrsg.): Methoden in der naturwissenschaftsdidaktischen Forschung, Berlin, Heidelberg, S. 133145.

Kruse, Jan (2011): Reader: Einführung in die Qualitative Interviewforschung. 2., überarb. u. erg. Aufl., Freiburg i.Br.

Kuckartz, Udo (2010): Einführung in die computergestützte Analyse qualitativer Daten, Wiesbaden.

Kuckartz, Udo/Dresing, Thorsten/Rädiker, Stefan, et al. (2008): Qualitative Evaluation. Der Einstieg in die Praxis. 2. aktual. Auflage, Wiesbaden.

Kuckartz, Udo/Rädiker, Stefan (2014): Datenaufbereitung und Datenbereinigung in der qualitativen Sozialforschung, in: Baur, N./Blasius, J. (Hrsg.): Handbuch Methoden der empirischen Sozialforschung, Wiesbaden, S. 383-396.

Kühl, Stefan/Strodtholz, Petra/Taffertshofer, Andreas (Hrsg.) (2009): Handbuch Methoden der Organisationsforschung. Quantitative und Qualitative Methoden. 1. Aufl., Wiesbaden.

Kühl, Stefan/Strodtholz, Petra/Taffertshofer, Andreas (2009): Qualitative und quantitative Methoden der Organisationsforschung - ein Überblick, in: Kühl, S./Strodtholz, P./ Taffertshofer, A. (Hrsg.): Handbuch Methoden der Organisationsforschung. Quantitative und Qualitative Methoden. 1. Aufl., Wiesbaden, S. 13-31.

Küsters, Ivonne (2009): Narrative Interviews, Wiesbaden.

Küsters, Ivonne (2014): Narratives Interview, in: Baur, N./Blasius, J. (Hrsg.): Handbuch Methoden der empirischen Sozialforschung, Wiesbaden, S. 575-580.

Kutz, Rudolf (2015): Empirische Sozialforschung. Darstellung von Wissenschaftstheorie, Forschungsansätzen, Forschungsspraxis und Qualität der Forschung, München.

La Porta, Rafael/Lopez-de-Silanes, Florencio/Shleifer, Andrei (1999): Corporate Ownership around the World, in: The Journal of Finance 54, 2, S. 471-517.

Lamnek, Siegfried (1995): Qualitative Sozialforschung. Band 2: Methoden und Techniken. 3., korr. Aufl., München [u.a.].

Lamnek, Siegfried (2010): Qualitative Sozialforschung. Lehrbuch. 5. Aufl., Weinheim [u.a.].

Lange, Knut W. (2005): Corporate Governance in Familienunternehmen, in: BB 2005, S. 2585-2590. 
Lange, Knut W. (2009): Kodex und Familienverfassung als Mittel der Corporate Governance in Familienunternehmen, in: Kirchdörfer, R., et al. (Hrsg.): Familienunternehmen in Recht, Wirtschaft, Politik und Gesellschaft. Festschrift für Brun-Hagen Hennerkes zum 70. Geburtstag, München, S. 135-150.

Lange, Knut W. (2013): Die Familiencharta und das Familienunternehmen - Bedeutung und Schnittstellen zum Gesellschaftsvertrag, in: Röthel, A./Schmidt, K. (Hrsg.): Die Verträge der Familienunternehmer, Hamburg, S. 33-46.

Langer, Inghard (1985): Das persönliche Gespräch als Weg in der psychologischen Forschung, in: Zeitschrift für personenzentrierte Psychologie und Psychotherapie 4, 4, S. $447-457$.

Langhart, Albrecht (1993): Rahmengesetz und Selbstregulierung. Kritische Betrachtungen zur vorgeschlagenen Struktur eines Bundesgesetzes über die Börsen und den Effektenhandel unter Berücksichtigung des amerikanischen und englischen Börsenrechts, Zürich.

Lansberg, Ivan (1999): Succeeding generations. Realizing the dream of families in business, Boston.

Lansberg, Ivan/Perrow, Edith L./Rogolsky, Sharon (1988): Family business as an emerging field, in: Family Business Review 1, 1.

Lautmann, Rüdige (1969): Wert und Norm. Begriffsanalysen für die Soziologie, Opladen.

Le Breton-Miller, Isabelle/Miller, Danny (2009): Agency vs. Stewardship in Public Family Firms. A Social Embeddedness Reconciliation, in: Entrepreneurship Theory and Practice 33, 6, S. 1169-1191.

Le Breton-Miller, Isabelle/Miller, Danny/Steier, Lloyd P. (2004): Toward an Integrative Model of Effective FOB Succession, in: Ent. Theory \& Pract 28, 4, S. 305-328.

Lee, Khai S./Lim, Guan H./Lim, Wei S. (2003): Family Business Succession: Appropriation Risk and Choice of Successor, in: The Academy of Management Review 28, 4, S. 657666.

Lee, Raymond M. (1993): Doing research on sensitive topics. Reprinted., London [u. a.]. Lehmann, Alexandra (2006): Werbeselbstkontrolle in Italien und Deutschland. Vor- und Nachteile der Systeme freiwilliger Selbstregulierung im Vergleich, in: GRUR Int 2006, S. 123-129.

Leipold, Helmut/Pies, Ingo (2000): Ordnungstheorie und Ordnungspolitik. Konzeptionen und Entwicklungsperspektiven, Stuttgart.

Leist, Anton (2000): Die gute Handlung: Eine Einführung in die Ethik, Berlin.

Leist, Anton (Hrsg.) (2002): Moral als Vertrag? Beiträge zum moralischen Kontraktualismus, Berlin.

LeMar, Bernd (2014): Generations- und Führungswechsel im Familienunternehmen. Mit Gefühl und Kalkül den Wandel gestalten. 2., vollständig überarbeitete und erweiterte Auflage, Wiesbaden.

Lembcke, Oliver W./Ritzi, Claudia/Schaal, Gary S. (2012): Zeitgenössische Demokratietheorie:. Band 1: Normative Demokratietheorien, Wiesbaden.

Letmathe, Peter/Welter, Friederike/Kathan, Daniel, et al. (2008): Management kleiner und mittlerer Unternehmen. Stand und Perspektiven der KMU-Forschung, Wiesbaden.

Levinson, Harry (1971): Conflicts that plague family businesses, in: Harvard Business Review 49, 1, S. 90-98. 
Liebhold, Renate/Trinczek, Rainer (2009): Experteninterviews, in: Kühl, S./Strodtholz, P./ Taffertshofer, A. (Hrsg.): Handbuch Methoden der Organisationsforschung. Quantitative und Qualitative Methoden. 1. Aufl., Wiesbaden, S. 32-56.

Lind, Georg (2015): Moral ist lehrbar! Wie man moralisch-demokratische Fähigkeiten fördern und damit Gewalt, Betrug und Macht mindern kann, Berlin.

Litz, Reginald A. (1995): The Family Business: Toward Definitional Clarity, in: Family Business Review 8, 2, S. 71-81.

Lofland, John/Snow, David A./Anderson, Leon, et al. (2006): Analyzing social settings. A guide to qualitative observation and analysis. 4. ed., Belmont, Calif. [u.a.].

Lorz, Rainer/Kirchdörfer, Rainer (2011): Unternehmensnachfolge. Rechtliche und steuerliche Gestaltungen. 2. Aufl., München.

Lorz, Rainer/Sarholz, Olivia (2014): Streitdynamik und Konfliktsituation, in: FuS Sonderheft 2014, S. 3-12.

Losee, John/Hoering, Walter (1977): Wissenschaftstheorie. Eine historische Einführung, München.

Lüdders, Lisa (2017): Qualitative Methoden und Methodenmix: Ein Handbuch für Studium und Berufspraxis, Bremen.

Lüdemann, Jörn (2007): Die Grenzen des homo oeconomicus und die Rechtswissenschaft, in: Engel, C./Englerth, M./Lüdemann, J. (Hrsg.): Recht und Verhalten. Beiträge zu Behavioral Law and Economics, Tübingen, S. 7-59.

Ludwig, Bernd/Stark, Werner (1988): Kants Rechtslehre, Hamburg.

Lueger, Manfred/Frank, Hermann (Hrsg.) (2015): Zukunftssicherung für Familienunternehmen. Good Practice Fallanalysen zur Family Governance; eine Studie des Forschungsinstituts für Familienunternehmen an der WU. 1. Aufl., Wien.

Lueger, Manfred/Süss-Reyes, Julia (2015): Family Governance als Strukturierung der Familie. Lehren aus den Fallanalysen, in: Lueger, M./Frank, H. (Hrsg.): Zukunftssicherung für Familienunternehmen. Good Practice Fallanalysen zur Family Governance; eine Studie des Forschungsinstituts für Familienunternehmen an der WU. 1. Aufl., Wien, S. 199-233.

Luhmann, Niklas (1983): Legitimation durch Verfahren. 9. Aufl., Frankfurt am Main.

Luhmann, Niklas (1984): Soziale Systeme. Grundriss einer allgemeinen Theorie, Frankfurt am Main.

Luhmann, Niklas (1987): Rechtssoziologie. 3. Aufl, Opladen.

Luhmann, Niklas (2005): Sozialsystem Familie, in: Luhmann, N. (Hrsg.): Soziologische Aufklärung 5. Konstruktivistische Perspektiven. 3. Aufl., Opladen, S. 189-209.

Luhmann, Niklas (Hrsg.) (2005): Soziologische Aufklärung 5. Konstruktivistische Perspektiven. 3. Aufl., Opladen.

Luhmann, Niklas (2010): Organisation und Entscheidung, Wiesbaden.

Lührs, John C. (2013): Strategische Unternehmensführung bei hoher Marktturbulenz: Entwicklung eines Systematisierungsmodells am Beispiel von Netzwerkbranchen, Wiesbaden.

Lukes, Rudolf (1974): Erstreckung der Vereinesgewalt auf Nichtmitglieder durch Rechtsgeschäft, in: Hefermehl, W. (Hrsg.): Festschrift für Harry Westermann zum 65. Geburtstag, Karlsruhe, S. 325-435. 
Luttermann, Claus (2003): \$243. a) Ansatz: Freiheit und Privatrechtsordnung, in: Goette, W./Habersack, M. (Hrsg.): Münchener Kommentar zum Aktiengesetz. 2. Auflage, München, 52-53.

Magen, Stefan (2007): Fairness, Eigennutz und die Rolle des Rechts. Eine Analyse auf Grundlage der Verhaltensökonomik, in: Engel, C./Englerth, M./Lüdemann, J. (Hrsg.): Recht und Verhalten. Beiträge zu Behavioral Law and Economics, Tübingen, S. 261-360. Mann, Thomas (1901): Buddenbrooks. Verfall einer Familie, Berlin.

Margelisch, Claude-Alain/Winzeler, Christoph (Hrsg.) (1998): Freiheit und Ordnung im Kapitalmarkrecht. Festgabe für Jean-Paul Chapuis, Zürich.

Marquardt, Valentin (2007): Datenerhebungstechniken im Vergleich - Befragung, Beobachtung, Inhaltsanalyse, München.

Martin, Paul (2006): Humanwissenschaftliche Zugänge. Approaches to the social sciences, Berlin.

Massis, Alfredo d./Kotlar, Josip (2014): The case study method in family business research. Guidelines for qualitative scholarship, in: Journal of Family Business Strategy 5, 1, S. 1529.

Massis, Alfredo d./Sharma, Pramodita/Chua, Jess H./Chrisman, James J./Kotlar, Josip (2012): State-of-the-art of family business research, in: Massis, A.d., et al. (Hrsg.): Family Business Studies. An Annotated Bibliography. annotaed edition, Cheltenham, S. 10-46.

Massis, Alfredo d./Sharma Pramodita/Chua Jess H., et al. (Hrsg.) (2012): Family Business Studies. An Annotated Bibliography. annotaed edition, Cheltenham.

Mastronardi, Philippe A./Taubert, Denis (Hrsg.) (2006): Staats- und Verfassungstheorie im Spannungsfeld der Disziplinen. Tagung [der Schweizerischen Vereinigung für Rechtsund Sozialphilosophie, Universität St. Gallen,] 12./13. November 2004, Stuttgart.

May, Karin (2017a): Der Familienmanager. Eigenschaften, Fähigkeiten und Aufgaben, in: May, P./Bartels, P. (Hrsg.): Governance im Familienunternehmen. Das Handbuch für die erfolgreiche Führung von Familienunternehmen und Unternehmerfamilien, Köln, S. 581-592.

May, Peter (Hrsg.) (2008): Das INTES-Handbuch Familienunternehmen. 1. Aufl., Bonn.

May, Peter (2008): Familienunternehmen: nicht nur anders, sondern besser - Grundzüge einer Managementtheorie für Familienunternehmen, in: Rödl, C./Rödl, B. (Hrsg.): Internationale Familienunternehmen. Recht, Steuern, Bilanzierung, Finanzierung, Nachfolge, Strategien [Festschrift für Bernd Rödl zum 65. Geburtstag], München, S. $417-429$.

May, Peter (2009): Familienunternehmen erfolgreich führen - Von der Inhaber-Strategie zur Unternehmens-Strategie, in: ZfB Zeitschrift für Betriebswirtschaft - Special Issue Nr. 2/2009, S. 113-126.

May, Peter (2012): Erfolgsmodell Familienunternehmen. Das Strategie-Buch, Hamburg. May, Peter (2017b): Die Inhaberstrategie im Familienunternehmen:. Eine Anleitung, Hamburg.

May, Peter/Bartels, Peter (Hrsg.) (2017): Governance im Familienunternehmen. Das Handbuch für die erfolgreiche Führung von Familienunternehmen und Unternehmerfamilien, Köln. 
May, Peter/Bartels, Peter/Müller, Christina, et al. (2015): Family Governance in Familienunternehmen. Eine Untersuchung zu Einsatz und Wirkung von Family-GovernanceMechanismen in Familienunternehmen.

May, Peter/Redlefsen, Matthias/Haller, Martin J. (2004): Nicht aktive Gesellschafter in Familienunternehmen. Ergebnisse und Handlungsempfehlungen.

May, Peter/Rieder, Gerold (2008): Family Education - Ein Eckpfeiler vom Good Governance im Familienunternehmen, in: May, P. (Hrsg.): Das INTES-Handbuch Familienunternehmen. 1. Aufl., Bonn, S. 394-402.

Mayer, Horst O. (2013): Interview und schriftliche Befragung: Grundlagen und Methoden empirischer Sozialforschung, Berlin.

Mayntz, Renate/Holm, Kurt/Hübner, Peter (1978): Einführung in die Methoden der empirischen Soziologie. 5. Aufl., Opladen.

Mayring, Philipp (2002): Einführung in die qualitative Sozialforschung. Eine Anleitung zu qualitativem Denken. 5., überarb. und neu ausgestattete Aufl., Weinheim [u.a.].

Mayring, Philipp (2007): Generalisierung in qualitativer Forschung, in: Forum Qualitative Sozialforschung / Forum Qualitative Social Research 8, 3.

Mayring, Philipp (2010): Qualitative Inhaltsanalyse. Grundlagen und Techniken. 11. Aufl., Weinheim [u.a.].

McClain, Linda C. (2006): Family Constitutions and the (New) Constitution of the Family, in: Fordham Law Review 73, S. 833-881.

Meier, Bernd-Dieter (2016): Kriminologie. 5. Auflage, München.

Melin, Leif/Nordqvist, Mattias/Sharma, Pramodita (Hrsg.) (2014): The SAGE Handbook of Family Business, London [u. a.].

Merriam, Sharan B. (2009): Qualitative research. A guide to design and implementation, San Francisco.

Mertens, Christian (2009): Herausforderungen für Familienunternehmen im Zeitverlauf: eine empirische Analyse am Beispiel von Nachfolge und Internationalisierung, Siegburg.

Mertens, Hans-Joachim (1982): Leges praeter legem, in: AG 1982, S. 29-41.

Mestmäcker, Ernst-Joachim (1964): Über die normative Kraft privatrechtlicher Verträge, in: Juristenzeitung (JZ) 19, 14, S. 441-446.

Meuser, Michael/Nagel, Ulrike (1991): ExpertInneninterviews - vielfach erprobt, wenig bedacht. (1991) ExpertInneninterviews - vielfach erprobt, wenig bedacht:, in: Garz, D./ Kraimer, K. (Hrsg.): Qualitativ-empirische Sozialforschung. Konzepte, Methoden, Analysen, Wiesbaden, S. 441-471.

Meuser, Michael/Nagel, Ulrike (1997): Das Experteninterview - Wissenssoziologische Voraussetzungen und methodische Durchführung, in: Friebertshäuser, B./Prengel, A. (Hrsg.): Handbuch Qualitative Forschungsmethoden in der Erziehungswissenschaft, Weinheim, Basel, S. 481-491.

Meuser, Michael/Nagel, Ulrike (2009): Das Experteninterview. Konzeptionelle Grundlagen und methodische Anlage, in: Pickel, S., et al. (Hrsg.): Methoden der vergleichenden Politik- und Sozialwissenschaft. Neue Entwicklungen und Anwendungen, Wiesbaden, S. $465-479$.

Mey, Günter/Mruck, Katja (2010): Grounded-Theory-Methodologie, in: Mey, G./Mruck, K. (Hrsg.): Handbuch Qualitative Forschung in der Psychologie, Wiesbaden, S. 614-626. 
Mey, Günter/Mruck, Katja (Hrsg.) (2010): Handbuch Qualitative Forschung in der Psychologie, Wiesbaden.

Mey, Günter/Mruck, Katja (2011): Qualitative Interviews, in: Naderer, G./Balzer, E. (Hrsg.): Qualitative Marktforschung in Theorie und Praxis. Grundlagen, Methoden, Anwendungen. 2., überarb. Aufl, Wiesbaden, S. 257-288.

Meyer, Anna (2007): Unternehmerfamilie und Familienunternehmen erfolgreich führen. Unternehmertum fördern, Führungskultur entwickeln, Konflikte konstruktiv lösen/ by Anna Meyer, Wiesbaden.

Meyer, Jörn-Axel (2003): Die Fallstudie in der betriebswirtschaftlichen Forschung und Lehre, in: WIST 32, 8, S. 475-480.

Mietzner, Mark/Tyrell, Marcel (2012): Das Verhalten von Familienunternehmen gegenüber ihren Stakeholdern. Erste Ergebnisse aus der Forschung einer auf den deutschen Kapitalmarkt bezogenenen Untersuchung, in: FuS Zeitschrift für Familienunternehmer und Stiftungen Nr. 3/2012, S. 108-113.

Miles, Matthew B./Huberman, A. M./Saldana, Johnny (2014): Qualitative data analysis. A methods sourcebook. Edition 3, London.

Miller, Mark/Deecke, Jan/Burfeind, Arne, et al. (2013): Familienunternehmer heute: Herausforderungen, Strategien, Erfahrungen, Wiesbaden.

Misoch, Sabina (2015): Qualitative interviews, Berlin.

Mochmann, Ekkehard (Hrsg.) (1980): Computerstrategien für die Kommunikationsanalyse, Frankfurt.

Möllers, Thomas M. J. (Hrsg.) (2009): Geltung und Faktizität von Standards. 1. Aufl., Baden-Baden.

Montemerlo, Daniela/Ward, John L. (2005): The family constitution. Agreements to secure and perpetuate your family and your business, Georgia, GA.

Montemerlo, Daniela/Ward, John L. (2011): The family constitution. Agreements to secure and perpetuate your family and your business, New York.

Morikawa, Hidemasa (1995): Zaibatsu. The rise and fall of family enterprise groups in Japan. 3. print, Tokyo.

Muraitis, Audris A. (2016): Emotionen in Familienunternehmen: Eine kommunikationstheoretische Fallstudie über das Scheitern eines Joint Ventures, Osnabrück.

Mustakallio, Mikko A. (2002): Contractual and relational governance in family firms. Effects on strategic decision-making quality and firm performance, Espoo.

Mutter, Christoph (2011): Vermögensmanagement für Familienunternehmer. Rechtsgestaltung, Steueroptimierung, Vermögensverwaltung. 2. Aufl (Online-ausg.), Stuttgart.

Naderer, Gabriele (2011): Auswertung \& Analyse qualitativer Daten, in: Naderer, G./Balzer, E. (Hrsg.): Qualitative Marktforschung in Theorie und Praxis. Grundlagen, Methoden, Anwendungen. 2., überarb. Aufl, Wiesbaden, S. 405-436.

Naderer, Gabriele/Balzer, Eva (Hrsg.) (2011): Qualitative Marktforschung in Theorie und Praxis. Grundlagen, Methoden, Anwendungen. 2., überarb. Aufl, Wiesbaden.

Nado, Jennifer/Kelly, Daniel/Stich, Stephan (2009): Moral Judgement, in: Symons, J./Calvo, P. (Hrsg.): The Routledge companion to philosophy of psychology, London [England], S. 621-633.

Naumes, William/Naumes, Margaret J. (2011): The Art and Craft of Case Writing, London. Nelton, Sharon (2008): Leading the family, in: Family Business 2008, S. 1-3. 
Neubauer, Franz-Friedrich/Lank, Alden G. (1998): The family business. Its governance for sustainability, Houndmills, Basingstoke, Hampshire.

Neuvians, Nicola (2011): Mediation in Familienunternehmen. Chancen und Grenzen des Verfahrens in der Konfliktdynamik, Wiesbaden.

Niebert, Kai/Gropengießer, Harald (2014): Leitfadengestützte Interviews, in: Krüger, D./ Parchmann, I./Schecker, H. (Hrsg.): Methoden in der naturwissenschaftsdidaktischen Forschung, Berlin, Heidelberg, S. 121-132.

Noack, Ulrich (1994): Gesellschaftervereinbarungen bei Kapitalgesellschaften, Tübingen.

Nobel, Peter (1998): Selbstregulierung, in: Margelisch, C.-A./Winzeler, C. (Hrsg.): Freiheit und Ordnung im Kapitalmarkrecht. Festgabe für Jean-Paul Chapuis, Zürich, S. 119-134.

Nohl, Arnd-Michael (2012): Interview und dokumentarische Methode. Anleitungen für die Forschungspraxis. 4. Aufl. 2012, Wiesbaden.

Nosé, Lavinia/Korunka, C./Frank, Hermann/Suchy, Daniela (2013): Familienklima und Konflikte in Familienunternehmen. Eine empirische Analyse ihrer Erfolgswirkungen, in: ZfKE - Zeitschrift für KMU und Entrepreneurship 61, 1-2, S. 55-81.

Noske, Nikola (30.11.2017): Ohne Fassung, in: Süddeutsche Zeitung, 30.11.2017. http:// www.sueddeutsche.de/wirtschaft/familienunternehmen-ohne-fassung-1.3773079. Zuletzt geprüft am 24.06.2019.

Oesterle, Michael-Jörg (2007): Corporate Governance für Familienunternehmen, in: Zeitschrift für Management (ZfM) 2, 1, S. 28-59.

Ogus, Anthony (2004): Regulation. Legal form and economic theory. Re-issue without revisions, Oxford.

O'Hara, William T. (Hrsg.) (2004): Centuries of success. Lessons from the world's most enduring family businesses, Avon (Massachusetts).

Olson, Patricia D./Zuiker, Virginia S./Danes, Sharon M./Stafford, Kathryn/Heck, Ramona K./Duncan, Karen A. (2003): The impact of the family and the business on family business sustainability, in: Journal of Business Venturing 18, 5, S. 639-666.

Osterhaus, Ingrid (2011): Autobiographisches Erzählen - Risiko oder Chance? Mögliche Wirkungen narrativer Explorationen auf die Erzählperson, Wien.

Papesch, Matthias (2010): Corporate Governance in Familienunternehmen. Eine Analyse zur Sicherung der Unternehmensnachfolge. 1. Auflage, Wiesbaden.

Patton, Michael Q. (2002): Qualitative research \& evaluation methods. 3, Saint Paul.

Peemöller, Volker H. (2008): Corporate Governance in Familienunternehmen. Notwendigkeit oder Modeerscheinung?, in: Kirchhof, P./Nieskens, H. (Hrsg.): Festschrift für Wolfram Reiß. Zum 65. Geburtstag, Köln, Germany, S. 721-738.

Pereboom, Derk (2001): Living without free will. [Online-Ausg.], Cambridge, U.K. [u.a.]. Peters, Anne (2006): Privatisierung, Globalisierung und die Resistenz des Verfassungsstaates, in: Mastronardi, P. A./Taubert, D. (Hrsg.): Staats- und Verfassungstheorie im Spannungsfeld der Disziplinen. Tagung [der Schweizerischen Vereinigung für Rechtsund Sozialphilosophie, Universität St. Gallen,] 12./13. November 2004, Stuttgart, S. 100159.

Pfannenschwarz, Armin (2006): Nachfolge und Nicht-Nachfolge in Familienunternehmen, Heidelberg, Neckar.

Phillips, Bernard S./Bodzenta, Erich (2013): Empirische Sozialforschung: Strategie und Taktik. 
Pickel, Susanne/Jahn, Detlef/Lauth, Hans-Joachim, et al. (Hrsg.) (2009): Methoden der vergleichenden Politik- und Sozialwissenschaft. Neue Entwicklungen und Anwendungen, Wiesbaden.

Picot, Arnold (1982): Transaktionskostenansatz in der Organisationstheorie. Stand der Diskussion und Aussagewert, in: Die Betriebswirtschaft 42, 4, S. 267-284.

Pieper, Torsten M./Smith, Anne D./Kudlats, Jerry/Astrachan, Joseph H. (2015): The Persistence of Multifamily Firms. Founder Imprinting, Simple Rules, and Monitoring Processes, in: Entrepreneurship Theory and Practice 39, 6, S. 1313-1337.

Pirmanschegg, Philipp (2016): Die Nachfolge in Familienunternehmen. Aus Konflikten lernen, Wiesbaden.

Plate, Markus (2009): Paradoxiemanagement mit dem Werte- und Entwicklungsquadrat, in: Rüsen, T. A./Schlippe, A. v./Groth, T. (Hrsg.): Familienunternehmen. Exploration einer Unternehmensform. 1. Aufl., Lohmar, Köln, S. 177-193.

Plate, Markus/Groth, Torsten (2009): Beratung von Familienunternehmen als permanentes Entfalten von Paradoxien, in: Rüsen, T. A./Schlippe, A. v./Groth, T. (Hrsg.): Familienunternehmen. Exploration einer Unternehmensform. 1. Aufl., Lohmar, Köln, S. 159175.

Plate, Markus/Groth, Torsten/Ackermann, Volker, et al. (Hrsg.) (2011): Grosse deutsche Familienunternehmen. Generationenfolge, Familienstrategie und Unternehmensentwicklung, Osnabrück.

Popitz, Heinrich (1980): Die normative Konstruktion von Gesellschaft, Tübingen.

Popper, Karl R. (1994): Logik der Forschung, in: Boettcher, E. (Hrsg.): Die Einheit der Gesellschaftswissenschaften. Studien im Grenzbereich der Wirtschafts- und Sozialwissenschaften, Tübingen.

Posner, Richard A./Cooter, Robert/Ellickson, Robert C., et al. (1998): Social norms, social meaning, and the economic analysis of law. Symposium issue of Journal of legal studies, v. 27, Chicago, Ill.

Posner, Richard A./Rasmusen, Eric B. (2000): Creating and Enforcing Norms, with Special Reference to Sanctions. Coase-Sandor Working Paper Series in Law and Economics.

Pospisil, Leopold (1987): Anthropologie des Rechts. Recht und Gesellschaft in archaischen und modernen Kulturen, München.

Poutziouris, Panikkos Z./Smyrnios, Kosmas X./Klein, Sabine B. (Hrsg.) (2008): Handbook of Research on Family Business, Cheltenham.

Prigge, Stefan/Braun, Maike/Kleefass, Jakob (18.12.2014): Familienverfassung als Bindeglied zwischen den Generationen.

Prince, Russ A. (1990): Family Business Mediation. A Conflict Resolution Model, in: Family Business Review 3, 3, S. 209-223.

Prügl, Reinhard/Hauck, Jana (2015): Deutschlands nächste Unternehmergeneration. Eine empirische Untersuchung der Werte, Einstellungen und Zukunftspläne, München.

Prym, Stephan D. (2014): Die Bedeutung von Sozialkapital beim Internationalisierungsprozess von Familienunternehmen, Siegburg.

Przyborski, Aglaja/Wohlrab-Sahr, Monika (2014): Qualitative Sozialforschung: Ein Arbeitsbuch, Berlin.

Puppis, Manuel (2004): Erfolgsbedingungen von Selbst- und Co-Regulierung, in: Puppis, M., et al. (Hrsg.): Selbstregulierung und Selbstorganisation. Unveröffentlichter Schlussbericht zuhanden des Bundesamtes für Kommunikation (BAKOM), Zürich, S. 69-73. 
Puppis, Manuel/Donges, Patrick (2004): Einführung, in: Puppis, M., et al. (Hrsg.): Selbstregulierung und Selbstorganisation. Unveröffentlichter Schlussbericht zuhanden des Bundesamtes für Kommunikation (BAKOM), Zürich, S. 9-18.

Puppis, Manuel/Künzler, Matthias/Schade, Edzard, et al. (Hrsg.) (2004): Selbstregulierung und Selbstorganisation. Unveröffentlichter Schlussbericht zuhanden des Bundesamtes für Kommunikation (BAKOM), Zürich.

Quinn, Warren (Hrsg.) (2003): Morality and action, Cambridge.

Quinn, Warren (2003): Putting Rationality in its Place, in: Quinn, W. (Hrsg.): Morality and action, Cambridge, S. 228-255.

Raiser, Thomas (1999): Das lebende Recht. Rechtssoziologie in Deutschland. 2., neubearb. und stark erw. Aufl., Baden-Baden.

Raiser, Thomas (2013): Grundlagen der Rechtssoziologie. Das lebende Recht. 6., überarb. Auflage, Tübingen.

Ramsethaler, Christina (2013): Was ist "Qualitative Inhaltsanalyse«, in: Schnell, M., et al. (Hrsg.): Der Patient am Lebensende. Eine Qualitative Inhaltsanalyse, Wiesbaden, S. 2342.

Randersen, Kathleen/Bettinelli, Cristina/Dossena, Giovanna, et al. (Hrsg.) (2016): Family entrepreneurship. Rethinking the research agenda, Abingdon, Oxon.

Raven, Bertram H. (1965): Social influence and power, in: Steiner, I. D./Fishbein, M. (Hrsg.): Current studies in social psychology, New York, S. 371-382.

Reay, Trish/Zhang, Zhen (2014): Qualitative Methods in Family Business Research, in: Melin, L./Nordqvist, M./Sharma, P. (Hrsg.): The SAGE Handbook of Family Business, London [u. a.], 29.

Redlefsen, Matthias (2004): Der Ausstieg von Gesellschaftern aus grossen Familienunternehmen. Eine praxisnahe Untersuchung der Corporate Governance-Faktoren. 1. Aufl., Wiesbaden.

Rehbinder, Manfred (2014): Rechtssoziologie. Ein Studienbuch. 8., neu bearb. Aufl., München.

Reich, Manfred/Bode, Christoph (2018): Familienverfassung für das Familienunternehmen, in: DStR Nr. 6/2018, S. 305-309.

Reichertz, Jo (2016): Qualitative und interpretative Sozialforschung: Eine Einladung.

Reuß, Karl F. (1955): Die Intensitätsstufen der Abreden und die Gentlemen-Agreements, in: AcP 154, 6, S. 485-526.

Richter, Rudolf/Furubotn, Eirik G. (2010): Neue Institutionenökonomik. 4., überarbeitete und erweiterte Auflage, Tübingen.

Rider, Barry A. (1978): Self-Regulation. The British Approach to Policing Conduct in the Securities Business with Particular Reference to the Role of the City Panel on Take-Overs and Mergers in the Regulation of Insider Trading, in: Journal of Comparative Corporate Law and Securities Regulation 1, S. 319-348.

Riedel, Christopher (2012): $\$ 22$ Familienstrategie - „Grundgesetz für die Familie«, in: Riedel, C. (Hrsg.): Praxishandbuch Unternehmensnachfolge. 1. Aufl., Bonn, 1-87.

Riedel, Christopher (Hrsg.) (2012): Praxishandbuch Unternehmensnachfolge. 1. Aufl., Bonn.

Riesenhuber, Felix (2009): Großzahlige empirische Forschung, in: Albers, S. (Hrsg.): Methodik der empirischen Forschung. 3., überarb. und erw. Aufl., Wiesbaden, S. 1-16. 
Roberts, Kathryn A./Wilson, Richard W. (2002): ICT and the Research Process: Issues Around the Compatibility of Technology with Qualitative Data Analysis, in: Forum Qualitative Sozialforschung / Forum Qualitative Social Research 3, 2.

Rodik, Petra/Primorac, Jaka (2015): To Use or Not to Use: Computer-Assisted Qualitative Data Analysis Software Usage among Early-Career Sociologists in Croatia, in: Forum Qualitative Sozialforschung / Forum Qualitative Social Research 16, 1.

Rödl, Christian (Hrsg.) (2008): Internationale Familienunternehmen. Recht, Steuern, Bilanzierung, Finanzierung, Nachfolge, Strategien; [Festschrift für Bernd Rödl zum 65. Geburtstag], München.

Rödl, Christian/Rödl, Bernd (Hrsg.) (2008): Internationale Familienunternehmen. Recht, Steuern, Bilanzierung, Finanzierung, Nachfolge, Strategien [Festschrift für Bernd Rödl zum 65. Geburtstag], München.

Rogoff, Edward G./Heck, Ramona (2003): Evolving research in entrepreneurship and family business: recognizing family as the oxygen that feeds the fire of entrepreneurship, in: Journal of Business Venturing 18, 5, S. 559-566.

Röhl, Klaus F. (2006): Rechtssoziologie. Ein Lehrbuch, Köln, München [u.a.].

Rohwetter, Marcus (19.05.2016): Ausgeprägte Streitkultur. Aldi, Tönnies, Müller, Dr. Oetker: Viele deutsche Familienunternehmen neigen zur Selbstbeschädigung, in: Zeit Online, 19.05.2016. https://www.zeit.de/2016/20/familienunternehmen-deutschland-al di-mueller-selbstbeschaedigung. Zuletzt geprüft am 24.06.2019.

Ross, Ian S. (2010): The life of Adam Smith. 2nd ed., Oxford, New York.

Roßkopf, Gabriele (1998): Selbstregulierung von Übernahmeangeboten in Grossbritannien, Berlin.

Röthel, Anne/Schmidt, Karsten (Hrsg.) (2013): Die Verträge der Familienunternehmer, Hamburg.

Ruch, Alexander (2004): Regulierungsfragen der Gentechnologie und der Internet, in: ZSR Nr. II/2004, S. 373-475.

Rüsen, Tom A. (Hrsg.) (2011): Familienunternehmen erfolgreich sanieren. Einfluss des Familienfaktors bei Restrukturierungen, Berlin.

Rüsen, Tom A. (2016): Family Compliance - der Umgang mit Regelbrüchen, in: private wealth Nr. 4/2016, S. 96-99.

Rüsen, Tom A. (2017a): Family Compliance als Bestandteil der Familienstrategie. Über den intelligenten Umgang mit Regelbrüchen in Unternehmerfamilien, in: FuS Zeitschrift für Familienunternehmer und Stiftungen 4, S. 120-125.

Rüsen, Tom A. (2017b): Krisen und Krisenmanagement in Familienunternehmen. Schwachstellen erkennen, Lösungen erarbeiten, Existenzbedrohungen meistern. 2. Aufl., Wiesbaden.

Rüsen, Tom A./Groth, Torsten (2012): Der Weg zur Familienstrategie, in: private wealth Nr. 2/2012, S. 26-27.

Rüsen, Tom A./Schlippe, Arist v. (Hrsg.) (2017): Dynamiken in Familie und Unternehmen. Sammelband 3 (Wittener Schriften zu Familienunternehmen), Göttingen.

Rüsen, Tom A./Schlippe, Arist v./Groth, Torsten (Hrsg.) (2009): Familienunternehmen. Exploration einer Unternehmensform. 1. Aufl., Lohmar, Köln.

Rüsen, Tom A./Schlippe, Arist v./Groth, Torsten (2014): Gesellschafterkompetenz in Familienunternehmen - Über gezielte Aus- und Weiterbildungsprogramme in Unter- 
nehmerfamilien, in: FuS Zeitschrift für Familienunternehmer und Stiftungen Nr. 3/ 2014, S. 101-108.

Sabrowsky, Klaus-Dieter (1978): Selbstregulierung im Wirtschafts- und Unternehmensrecht. Die niederländ. Fusionsverhaltensregeln im europäischen Vergleich. Mit Einf. v. Harm Peter Westermann, Bonn.

Säcker, Franz-Jürgen/Rebmann, Kurt (Hrsg.) (2015): Münchener Kommentar zum Bürgerlichen Gesetzbuch. 7. Auflage, München.

Sagel-Grande, Irene (1990): Der Zusammenhang zwischen Normakzeptanz, Normhandhabung und Normbefolgung, in: Zeitschrift für Rechtspolitik 23, 1, S. 26-29.

Sandraschitz, Irene (2007): Software zur qualitativen Auswertung von Text- und Videomaterial in der empirischen Marktforschung: Übersicht, systematischer Vergleich und praktische Analyse, Hamburg.

Schäfer, Thomas (2016): Die Rolle Angeheirateter in Familienunternehmen, Wiesbaden. Scherer, Stephan (Hrsg.) (2014): Münchener Anwaltshandbuch Erbrecht. 4., überarb. Aufl., München.

Scherer, Stephan/Wimmer, Rudolf/Blanc, Michael, et al. (2012): Familienunternehmen: Erfolgsstrategien zur Unternehmenssicherung, Frankfurt am Main.

Schlippe, Arist v. (2009a): Bewusst mit Risiken umgehen. Paradoxiemanagement in Familienunternehmen, in: Rüsen, T. A./Schlippe, A. v./Groth, T. (Hrsg.): Familienunternehmen. Exploration einer Unternehmensform. 1. Aufl., Lohmar, Köln, S. 47-71.

Schlippe, Arist v. (2009b): Zwischen Ökonomie und Psychologie. Konflikte in Familienunternehmen, in: Zeitschrift für Konfliktmanagement 12, 1, S. 17-21.

Schlippe, Arist v. (2011): Besonderheiten von Familienunternehmen und Unternehmerfamilien, in: Rüsen, T. A. (Hrsg.): Familienunternehmen erfolgreich sanieren. Einfluss des Familienfaktors bei Restrukturierungen, Berlin, S. 19-42.

Schlippe, Arist v./Frank, Hermann (2013): The Theory of Social Systems as a Framework for Understanding Family Businesses, in: Family Relations 62, 3, S. 384-398.

Schlippe, Arist v./Groth, Torsten (2007): The Power of Stories - Zur Funktion von Geschichten in Familienunternehmen, in: Kontext 38, 1, S. 26-47.

Schlippe, Arist v./Groth, Torsten/Plate, Markus (2011): Entscheidungsfähigkeit sicherstellen. Familienstrategie und Familienmanagement in Familienunternehmen, in: Plate, M., et al. (Hrsg.): Grosse deutsche Familienunternehmen. Generationenfolge, Familienstrategie und Unternehmensentwicklung, Osnabrück, S. 522-561.

Schlippe, Arist v./Groth, Torsten/Rüsen, Tom A. (2017): Die beiden Seiten der Unternehmerfamilie. Familienstrategie über Generationen: Auf dem Weg zu einer Theorie der Unternehmerfamilie. 1. Auflage, Göttingen.

Schlippe, Arist v./Kellermanns, Franz W. (2008): Emotionale Konflikte in Familienunternehmen, in: ZfKE - Zeitschrift für KMU und Entrepreneurship 56, 1-2, S. 40-58.

Schlippe, Arist v./Kellermanns, Franz W. (2017): Konflikte in Familien und Unternehmen erkennen, managen und vermeiden, in: Rüsen, T. A./Schlippe, A. v. (Hrsg.): Dynamiken in Familie und Unternehmen. Sammelband 3 (Wittener Schriften zu Familienunternehmen), Göttingen, S. 237-250.

Schlippe, Arist v./Nischak, Almute/El Hachimi, Mohammed (Hrsg.) (2011a): Familienunternehmen verstehen. Gründer, Gesellschafter und Generationen. 2. Aufl., Göttingen. 
Schlippe, Arist v./Nischak, Almute/El Hachimi, Mohammed (2011b): Familienunternehmen verstehen, in: Schlippe, A. v./Nischak, A./El Hachimi, M. (Hrsg.): Familienunternehmen verstehen. Gründer, Gesellschafter und Generationen. 2. Aufl., Göttingen, S. 19-29.

Schlippe, Arist v./Rüsen, Tom A./Groth, Torsten (Hrsg.) (2009): Beiträge zur Theorie des Familienunternehmens, Siegburg.

Schmidt, Thomas (2002): Hobbes' Ethik und hobbesianische Ethik. Zum Projekt einer vertragstheoretischen Begründung moralischer Verpflichtung, in: Leist, A. (Hrsg.): Moral als Vertrag? Beiträge zum moralischen Kontraktualismus, Berlin, S. 121-154.

Schmidt-Preuß, Matthias (1997): Verwaltung und Verwaltungsrecht zwischen gesellschaftlicher Selbstregulierung und staatlicher Steuerung, in: Veröffentlichungen der Vereinigung der Deutschen Staatsrechtslehrer Nr. 56/1997, S. 160-234.

Schmies, Christian (2003): Codes of Conduct in der Bankwirtschaft, Britisches Beispiel und europäische Weiterungen, in: ZBB Nr. 4/2003, S. 277-290.

Schneewind, Jerome B. (2014): The invention of autonomy. A history of modern moral philosophy, Cambridge.

Schnell, Martin/Schulz, Christian/Kolbe, Harald, et al. (Hrsg.) (2013): Der Patient am Lebensende. Eine Qualitative Inhaltsanalyse, Wiesbaden.

Schnell, Rainer/Hill, Paul B./Esser, Elke (2008): Methoden der empirischen Sozialforschung. 8., unveränd. Aufl., München [u.a.].

Schubert, Cornelius (2014): Gebrauchsgegenstände und technische Artefakte, in: Baur, N./ Blasius, J. (Hrsg.): Handbuch Methoden der empirischen Sozialforschung, Wiesbaden, S. 899-906.

Schulz, Marlen/Ruddat, Michael (2012): "Let's talk about sex!» Über die Eignung von Telefoninterviews in der qualitativen Sozialforschung, in: Forum Qualitative Sozialforschung / Forum Qualitative Social Research 13, 2, 1-41.

Schulz, Peter/Werz, Ralf S. (2007a): Die Familienverfassung (Teil 1). Ziele, Grundlagen, Inhalte, in: ErbStB 2007, S. 310-313.

Schulz, Peter/Werz, Ralf S. (2007b): Die Familienverfassung (Teil 2). Musterformulierung mit Erläuterung, in: ErbStB 2007, S. 353-360.

Schulze, Götz (2008): Die Naturalobligation. Rechtsfigur und Instrument des Rechtsverkehrs einst und heute - zugleich Grundlegung einer zivilrechtlichen Forderungslehre, Tübingen.

Schüppen, Matthias (2002): To comply or not to comply - that's the question! »Existenzfragen« des Transparenz- und Publizitätsgesetzes im magischen Dreieck kapitalmarktorientierter Unternehmensführung, in: ZIP - Zeitschrift für Wirtschaftsrecht Nr. 29/2002, S. 1269-1279.

Schuppert, Gunnar F./Bumke, Christian (2000): Verfassungsrechtliche Grenzen privater Standardsetzung. Vorüberlegungen zu einer Theorie der Wahl rechtlicher Regelungsformen (Regulatory Choice), in: Kleindiek, D./Oehler, W. (Hrsg.): Die Zukunft des deutschen Bilanzrechts im Zeichen internationaler Rechnungslegung und privater Standardsetzung. [Vorträge und Diskussionsberichte des interdisziplinären Symposions »Die Zukunft des Deutschen Bilanzrechts«, Universität Bielefeld am 25. und 26. Juni 1999], Köln, S. 71-125.

Schütze, Fritz (1976): Zur Hervorlockung und Analyse von Erzählungen thematisch relevanter Geschichten im Rahmen soziologischer Feldforschung dargestellt an einem 
Projekt zur Erforschung von kommunalen Machtstrukturen, in: Weymann, A. (Hrsg.): Kommunikative Sozialforschung. Alltagswissen und Alltagshandeln, Gemeindemachtforschung, Polizei, politische Erwachsenenbildung, München, S. 159-260.

Schütze, Fritz (1977): Die Technik des narrativen Interviews in Interaktionsfeldstudien. dargestellt an einem Projekt zur Erforschung von kommunalen Machtstrukturen, Bielefeld.

Schütze, Fritz (1983): Biographieforschung und narratives Interview, in: Neue Praxis 13 13, 3, S. 283-293.

Schütze, Fritz (1987): Das narrative Interview in Interaktionsfeldstudien, Hagen: Fernuniv. Schwarz, Sebastian H. (2005): Regulierung durch Corporate Governance Kodizes, Berlin. Schwass, Joachim/Amann, Wolfgang/Ward, John L. (2004): Die nächste Generation, in: Zeitschrift Führung und Organisation (ZFO) 73, 5, S. 260-265.

Schween, Karsten/Koeberle-Schmid, Alexander/Bartels, Peter, et al. (2011): Die Familienverfassung. Zukunftssicherung für Familienunternehmen; [Studie], Bonn.

Seebaß, Gottfried (2002): Die sanktionistische Theorie des Sollens, in: Leist, A. (Hrsg.): Moral als Vertrag? Beiträge zum moralischen Kontraktualismus, Berlin, S. 155-198.

Seidman, Irving (2013): Interviewing as Qualitative Research: A Guide for Researchers in Education and the Social Sciences, New York.

Shafer-Landau, Russ (2009): Moral realism. A defence. Repr, Oxford, UK.

Sharma, Pramodita/Chrisman, James J./Gersick, Kelin E. (2012): 25 Years of Family Business Review, in: Family Business Review 25, 1, S. 5-15.

Shepherd, Dean/Haynie, J. M. (2009): Family Business, Identity Conflict, and an Expedited Entrepreneurial Process. A Process of Resolving Identity Conflict, in: Entrepreneurship Theory and Practice 33, 6, S. 1245-1264.

Siebels, Jan-Folke/Knyphausen-Aufseß, Dodo z. (2012): A Review of Theory in Family Business Research: The Implications for Corporate Governance, in: International Journal of Management Reviews 14, 3, S. 280-304.

Sigle, Walter (2013): Beiräte im Familienunternehmen, in: Krieger, G., et al. (Hrsg.): Festschrift für Michael Hoffmann-Becking zum 70. Geburtstag, München, S. 1147-1162.

Simon, Fritz B. (2001): Geld oder Liebe - Familien und ihre Unternehmen, in: Familiendynamik Nr. 4/2001, S. 333-337.

Simon, Fritz B. (2008): Von Generation zu Generation. Unterschiedliche Entwicklungsschritte von Mehr-Generationen-Familienunternehmen, in: Kollmer-von OheimbLoup, G./Wischermann, C. (Hrsg.): Unternehmernachfolge in Geschichte und Gegenwart. (Stuttgarter historische Studien zur Landes- und Wirtschaftsgeschichte), Ostfildern, S. 13-38.

Simon, Fritz B. (Hrsg.) (2011a): Die Familie des Familienunternehmens. 3. Aufl., Heidelberg.

Simon, Fritz B. (2011b): Einleitung: Zwischen Gefühl und Geschäft. Familien und ihre Unternehmen, in: Simon, F. B. (Hrsg.): Die Familie des Familienunternehmens. 3. Aufl., Heidelberg, S. 7-15.

Simon, Fritz B. (2011c): Familien und Unternehmen, in: Simon, F. B. (Hrsg.): Die Familie des Familienunternehmens. 3. Aufl., Heidelberg, S. 17-34.

Simon, Fritz B. (2012): Einführung in die Theorie des Familienunternehmens, Heidelberg.

Simon, Fritz B./Wimmer, Rudolf/Groth, Torsten (2017): Mehr-Generationen-Familienunternehmen. Erfolgsgeheimnisse von Oetker, Merck, Haniel u.a. 3. Auflage, Heidelberg. 
Smith, Adam (1790): The Theory of Moral Sentiments. 6th edition.

Smith, Adam (1976): An Inquiry into the Nature and Causes of the Wealth of Nations. Reprint of 1790 London Edition.

Smith, Adam (2011): The theory of Moral Sentiments, Kapaau (T.H.).

Smith, Michael (1987): The Humean Theory of Motivation, in: Mind XCVI, 381, S. 36-61.

Smyrnios, Kosmas/Poutziouris, Panikkos/Goel, Sanjay (Hrsg.) (2006): Handbook of research on family business. 2nd ed., Cheltenham, Northampton.

Spiegelberger, Sebastian (2008): Die Familienverfassung - Gestaltung von Gesellschaftsverträgen, in: Rödl, C. (Hrsg.): Internationale Familienunternehmen. Recht, Steuern, Bilanzierung, Finanzierung, Nachfolge, Strategien; [Festschrift für Bernd Rödl zum 65. Geburtstag], München, S. 89-114.

Sprondel, Walter M. (1979): »Experte« und »Laie« zur Entwicklung von Typenbegriffen in der Wissenssoziologie, in: Grathoff, R./Sprondel, W. M. (Hrsg.): Alfred Schütz und die Idee des Alltags in den Sozialwissenschaften, Stuttgart, S. 140-154.

Stafford, Kathryn/Duncan, Karen A./Dane, Sharon/Winter, Mary (1999): A Research Model of Sustainable Family Businesses, in: Family Business Review 12, 3, S. 197-208.

Stamm, Isabell (2013): Unternehmerfamilien. Über den Einfluss des Unternehmens auf Lebenslauf, Generationenbeziehungen und soziale Identität, Leverkusen, Berlin.

Starck, Christian (1995): Der demokratische Verfassungsstaat: Gestalt, Grundlagen, Gefährdungen, Tübingen.

Steiner, Bernhard (2009): Nachtsicht: Ansichten und Behauptungen, Norderstedt.

Steiner, Ivan D./Fishbein, Martin (Hrsg.) (1965): Current studies in social psychology, New York.

Steinfath, Holmer (2002): Wir und Ich. Überlegungen zur Begründung moralischer Normen, in: Leist, A. (Hrsg.): Moral als Vertrag? Beiträge zum moralischen Kontraktualismus, Berlin, S. 71-96.

Stemmer, Peter (2000): Handeln zugunsten anderer. Eine moralphilosophische Untersuchung, Berlin, New York.

Stemmer, Peter (2002): Der Begriff der moralischen Pflicht, in: Leist, A. (Hrsg.): Moral als Vertrag? Beiträge zum moralischen Kontraktualismus, Berlin, S. 37-70.

Stemmer, Peter (2008): Normativität: eine ontologische Untersuchung, Berlin.

Stengel, Arndt (2014): $\$ 3$ Organisationsrecht, in: Bärwaldt, R./Prinz, U. (Hrsg.): Beck’sches Handbuch der Personengesellschaften. Gesellschaftsrecht - Steuerrecht. 4. [bearb.] Aufl., München, S. 148-274.

Steßl, Antonia (2014): Compliance-Kultur. Unterschätzt und zugleich unerlässlich!, in: KPMG AG Wirtschaftsprüfungsgesellschaft (Hrsg.): Das wirksame Compliance-Management-System. Ausgestaltung und Implementierung in Unternehmen, Herne, S. 1426.

Stierlin, Helm (2007): Gerechtigkeit in nahen Beziehungen. Systemisch-therapeutische Perspektiven. 2. Aufl., Heidelberg, Heidelberg.

Stigler, Hubert/Felbinger, Günter (2005): Der Interviewleitfaden im qualitativen Interview, in: Stigler, H./Reicher, H. (Hrsg.): Praxisbuch empirische Sozialforschung in den Erziehungs- und Bildungswissenschaften, Innsbruck, Wien, Bozen, S. 129-134.

Stigler, Hubert/Reicher, Hannelore (Hrsg.) (2005): Praxisbuch empirische Sozialforschung in den Erziehungs- und Bildungswissenschaften, Innsbruck, Wien, Bozen. 
Stöhlker, Fidel S./Müller Tiberini, Franziska (2005): Familienrat. Formelle Treffen im trauten Kreis, in: io new management 74, 1-2, S. 9-12.

Strauss, Anselm/Corbin, Juliet (2010): Grounded theory. Grundlagen qualitativer Sozialforschung. Unveränd. Nachdr. der letzten Aufl., Weinheim.

Strauss, Anselm L. (1991): Grundlagen qualitativer Sozialforschung. Datenanalyse und Theoriebildung in der empirischen und soziologischen Forschung, München.

Strawson, Peter F. (1961): Social Morality and Individual Ideal, in: Philosophy 36, 136, S. 117.

Strawson, Peter F. (1985): Skepticism and naturalism. Some varieties, New York.

Strawson, Peter F. (1992): Analysis and Metaphysics: An Introduction to Philosophy.

Strawson, Peter F. (2008): Freedom and resentment and other essays. [New ed.], London.

Strübing, Jörg (2009): Grounded Theory. Zur sozialtheoretischen und epistemologischen Fundierung des Verfahrens der empirisch begründeten Theoriebildung, Wiesbaden.

Suáre, K. C./Santana-Martín, D. J. (2004): Governance in Spanish family business, in: International Journal of Entrepreneurial Behavior \& Research 10, 1/2, S. 141-163.

Swanson, Richard A./Holton III, Elwood F. (Hrsg.) (2005): Research in Organizations. Foundations and methods of inquiry, California.

Symons, John/Calvo, Paco (Hrsg.) (2009): The Routledge companion to philosophy of psychology, London [England].

Tagiuri, Renato/Davis, John (1996): Bivalent Attributes of the Family Firm, in: Family Business Review 9, 2, S. 199-208.

Tänzler, Jan K. (2014): Corporate Governance und Corporate Social Responsibility im deutschen Mittelstand. Ein empirischer Vergleich mittelständischer Unternehmen mit unterschiedlichem Familieneinfluss, Lohmar, Köln.

Taylor, Steven J./Bogdan, Robert (1984): Introduction to quantitative research methods. The search for meaning. 2. ed, New York (etc.).

Thiele, Wolfgang (1966): Die Zustimmung in der Lehre vom Rechtsgeschäft. 1, Köln.

Thoma, Anselm C. (2008): Regulierte Selbstregulierung im Ordnungsverwaltungsrecht, Berlin.

Thoma, Georg F. (1996): Der neue Übernahmekodex der Börsensachverständigenkommission, in: ZIP - Zeitschrift für Wirtschaftsrecht Nr. 41/1996, S. 17251734.

Thomas, Robert J. (1995): Interviewing Important People in Big Companies, in: Hertz, R./ Imber, J. (Hrsg.): Studying Elites Using Qualitative Methods, 2455 Teller Road, Thousand Oaks California 91320 United States, S. 3-17.

Thunen, Sebastian v. (2015): Vermögenskontinuität in Adelsfamilien. Von Thronfolgern und Hausgesetzen, Stuttgart.

Tokarczyk, John/Hansen, Eric/Green, Mark/Down, Jon (2007): A Resource-Based View and Market Orientation Theory Examination of the Role of »Familiness « in Family Business Success, in: Family Business Review 10, 1, S. 17-31.

Trevinyo-Rodríguez, Rosa N./Tàpies, Josep (2006): Effective knowledge transfer in family firms, in: Smyrnios, K./Poutziouris, P./Goel, S. (Hrsg.): Handbook of research on family business. 2nd ed., Cheltenham, Northampton, S. 343-357.

Tugendhat, Ernst (1984): Probleme der Ethik, Stuttgart.

Tugendhat, Ernst (1993): Vorlesungen über Ethik. Erstausgabe, Frankkfurt am Main.

Tyler, Tom R. (2006): Why people obey the law, Princeton, NJ <etc.>. 
Uffmann, Katharina (2015): Family Business Governance - Rule-Making in the Shadow of Law and Love, in: ZIP - Zeitschrift für Wirtschaftsrecht 36, 51-52, S. 244-2451.

Uhlaner, Lorraine (2006): Business family as a team. Underlying forces for sustained competitive advantage, in: Smyrnios, K./Poutziouris, P./Goel, S. (Hrsg.): Handbook of research on family business. 2nd ed., Cheltenham, Northampton, S. 125-144.

Ulmer, Peter/Niemeier, W. G. (1975): Die freiwillige Selbstkontrolle in Wirtschaft und Presse. Erscheinungsformen und Strukturen - Rechtsfrage - Haftungsrisiken, in: WRP 1975, S. 549-560.

Ulrich, Patrick (2010): Bernhard Baumgartner: »Familienunternehmen und Zukunftgestaltung: Schlüsselfaktoren zur erfolgreichen Unternehmensnachfolge«, in: ZfB Zeitschrift für Betriebswirtschaft 80, 6, S. 717-720.

Ulrich, Patrick (2011): Corporate Governance in mittelständischen Familienunternehmen. Theoretische und empirische Analyse unter besonderer Berücksichtigung der Wechselwirkungen zwischen Corporate Governance und Controlling. 1., neue Ausg, Wiesbaden.

Vernekohl, Robert W. (2007): Familienstrategie und Nachfolgeplanung, in: BBP Betriebswirtschaft im Blickpunkt Nr. 2/2007, S. 33.

Villalonga, Belen/Amit, Raphael (2006): How do family ownership, control and management affect firm value?, in: Journal of Financial Economics 80, S. 385-417.

Voegeli-Wenzl, Julia: Internet Governance am Beispiel der Internet Corporation of Assigned Names and Numbers (ICANN), in: GRUR Int 2006, S. 807-816.

Vogd, Werner (2013): Polykontexturalität: Die Erforschung komplexer systemischer Zusammenhänge in Theorie und Praxis, in: Familiendynamik 38, 1, S. 32-41.

Wahlers, Christiane (2011): Private Selbstregulierung am Beispiel des Kapitalmarktrechts: Vorteile, Nachteile, Optimierung, Osnabrück.

Wallace, R. J. (1996): Responsibility and the Moral Sentiments. 1st Harvard University Press pbk. ed., Cambridge, Mass.

Ward, John L. (1987): Keeping the family business healthy. How to plan for continuing growth, profitability and family leadership, Marietta, Ga.

Ward, John L. (1991): Creating effective boards for private enterprises. Meeting the challenges of continuity and competition. 1st ed., San Francisco.

Ward, John L. (2001): Developing Effective Ownership in the Family-Controlled Business, in: DIRECTOR - Newsletter of the Institute of Corporate Directors Nr. 100/2001, S. 1-4.

Ward, John L. (2004): Perpetuating the family business;50 lessons learned from long lasting, successful families in business, Basingstoke.

Ward, John L. (2005): Unconventional Wisdom: Counterintuitive Insights for Family Business Success, West Sussex.

Ward, John L. (2011): Keeping the family business healthy. How to plan for continuing growth, profitability, and family leadership, New York.

Watter, Rolf/Dubs, Dieter (2005): Bedeutung und Zukunft der Selbstregulierung im Kapitalmarktrecht, in: Der Schweizer Treuhänder Nr. 10/2005, S. 743-750.

Watzlawick, Paul/Beavin, Janet H./Jackson, Don D. (2011): Menschliche Kommunikation. Formen, Störungen, Paradoxien. 12., unveränd. Aufl, Bern.

Weber, Hendrik (2009): Familienexterne Unternehmensnachfolge. Eine empirische Untersuchung über Akquisitionen von Familienunternehmen, Wiesbaden.

Weber, Max (1972): Wirtschaft und Gesellschaft, Tübingen. 
Wedemann, Frauke (2013): Gesellschafterkonflikte in geschlossenen Kapitalgesellschaften, Tübingen.

Weiß, Michael (2012): Hybride Regulierungsinstrumente. Eine Analyse rechtlicher, faktischer und extraterritorialer Wirkungen nationaler Corporate-Governance-Kodizes, Tübingen.

Werder, Axel v./Talaulicar, Till (2009): Die Akzeptanz der Empfehlungen und Anregungen des Deutschen Corporate Governance Kodex, in: DB 2009, S. 689-696.

Weymann, Ansgar (Hrsg.) (1976): Kommunikative Sozialforschung. Alltagswissen und Alltagshandeln, Gemeindemachtforschung, Polizei, politische Erwachsenenbildung, München.

White, Richard/Gunstone, Richard (2014): Probing Understanding, London.

Wicke, Hartmut (2012): Corporate Governance-Fragen in der Kautelarjurisprudenz kleiner und mittelgroßer Unternehmen, in: Zeitschrift für Unternehmens- und Gesellschaftsrecht $41,2-3$.

Wiechers, Ralph (2004): Die Unternehmerfamilie. Ein Risiko des Familienunternehmens? 1 , Norderstedt.

Wiechers, Ralph (2006): Familienmanagement zwischen Unternehmen und Familie. Zur Handhabung typischer Eigenarten von Unternehmensfamilien und Familienunternehmen. 1. Aufl., Heidelberg.

Wiechers, Ralph/Klett, David (2005): Die Unternehmerfamilie. Ein Risiko des Familienunternehmens?, in: Risknews Nr. 03/2005, S. 34-50.

Wiedemann, Peter M. (1986): Erzählte Wirklichkeit. Zur Theorie und Auswertung narrativer Interviews, Weinheim, München.

Wiegand, Wolfgang/Wichtermann, Jürg (2000): Die Standesregeln der Banken als »blosse« Auslegungshilfe- zur (Un-)Verbindlichkeit von Selbstregulierungen, in: recht 18, 1, S. 28-36.

Wieselhuber, Norbert/Lohner, Andreas M./Thum, Gustl F. (2007): Gestaltung und Führung von Familienunternehmen. 3. Aufl, Bonn.

Willaschek, Marcus (2013): Moral ohne Sanktion? Anmerkungen zu Julia Hermann und Mario Brandhorst, in: Buddeberg, E./Vesper, A. (Hrsg.): Moral und Sanktion. Eine Kontroverse über die Autorität moralischer Normen, Frankfurt, S. 197-208.

Williamson, Oliver E. (1985): The Economic Institutions of Capitalism. Firms, Markets, Relational Contracting.

Wimmer, Rudolf/Domayer, Ernst/Oswald, Margit, et al. (2018): Familienunternehmen Auslaufmodell oder Erfolgstyp? 3. Aufl., Wiesbaden.

Wimmer, Rudolf/Gebauer, Annette (2004): Nachfolge in Familienunternehmen, in: Zeitschrift Führung und Organisation (ZFO) 73, 5, S. 244-252.

Wimmer, Rudolf/Groth, Torsten/Simon, Fritz B. (2004): Erfolgsmuster von Mehrgenerationen-Familienunternehmen. Wittener Diskussionspapier Nr. 2, Witten.

Wimmer, Rudolf/Groth, Torsten/Simon, Fritz B. (2009): Erfolgsmuster von Mehr-Generationen-Familienunternehmen, in: Schlippe, A. v./Rüsen, T. A./Groth, T. (Hrsg.): Beiträge zur Theorie des Familienunternehmens, Siegburg, S. 95-171.

Windbichler, Christine (2009): Bindungswirkung von Standards im Bereich Corporate Governance, in: Möllers, T. M. J. (Hrsg.): Geltung und Faktizität von Standards. 1. Aufl., Baden-Baden, S. 19-34. 
Windmann, Jörg (2008): Privatrechtliche Kontrollmechanismen im Rahmen staatlicher Gewährleistungsverantwortung, in: DÖV 61, S. 948-954.

Winterhoff, Christian (2007): Verfassung - Verfassunggebung - Verfassungsänderung: zur Theorie der Verfassung und der Verfassungsrechtserzeugung, Tübingen.

Wirsching, Katharine T. (2017): Familieneinfluss und Unternehmenserfolg, Wiesbaden. Witt, Harald (2001): Forschungsstrategien bei quantitativer und qualitativer Sozialforschung, in: Forum Qualitative Sozialforschung / Forum Qualitative Social Research 2, 1.

Witt, Peter (2008): Corporate Governance in Familienunternehmen, in: ZfB Zeitschrift für Betriebswirtschaft - Special Issue 2, S. 1-19.

Wittgenstein, Ludwig (Hrsg.) (1989): Philosophische Untersuchungen. Tractatus logicophilosophicus / Tagebücher 1914-1916, Frankfurt.

Wittgenstein, Ludwig (1989): Wittgensteins Analysen des Regelbefolgens, in: Wittgenstein, L. (Hrsg.): Philosophische Untersuchungen. Tractatus logico-philosophicus / Tagebücher 1914-1916, Frankfurt, 143-242.

Wittwer, Héctor (2010): Ist es vernünftig, moralisch zu handeln?, Berlin.

Witzel, Andreas (1982): Verfahren der qualitativen Sozialforschung. Überblick und Alternativen, Frankfurt am Main [u.a.].

Witzel, Andreas (2000): Das problemzentrierte Interview, in: Forum Qualitative Sozialforschung / Forum: Qualitative Social Research, 1, 1, 1-22.

Wolf, Jochen/Paul, Herbert/Zipse, Thomas (2009): Erfolg im Mittelstand. Tipps für die Praxis. 1. Aufl., Wiesbaden.

Wolf, Katharina (2011): Internationalisierungsstrategien von deutschen Familienunternehmen. 1. Aufl., Lohmar.

Wolf, Ursula (2013): Vom moralischen Sollen, in: Buddeberg, E./Vesper, A. (Hrsg.): Moral und Sanktion. Eine Kontroverse über die Autorität moralischer Normen, Frankfurt, S. 35-52.

Woll, Helmut (1994): Menschenbilder in der Ökonomie, Berlin.

Wrona, Thomas (2005): Die Fallstudienanalyse als wissenschaftliche Forschungsmethode. ESCP-EAP Working Paper, Berlin.

Wüstemann, Jens/Bischof, Jannis/Koch, Christopher (2008): Regulierung durch Transparenz. Ökonomische Analysen, empirische Befunde und Empfehlungen für eine europäische Kapitalmarktregulierung, in: Hopt, K. J./Kämmerer Jörn A./Veil, R. (Hrsg.): Kapitalmarktgesetzgebung im europäischen Binnenmarkt, Tübingen, S. 1-18.

Yin, Robert K. (2009): Case study research: Design and methods. 4th ed., London.

Yin, Robert K. (2012): Applications of Case Study Research, London.

Yin, Robert K. (2013): Case study research: Design and methods. (Applied Social Research Methods). 5th. ed., London.

Zahra, Shaker A./Hayton, James C./Salvato, Carlo (2004): Entrepreneurship in Family vs. Non-Family Firms: A Resource-Based Analysis of the Effect of Organizational Culture, in: Ent. Theory \& Pract 28, 4, S. 363-381.

Zahra, Shaker A./Klein, Sabine B./Astrachan, Joseph H. (2008): Theory building and the survival of family firms -. three promising research directions, in: Poutziouris, P. Z.I Smyrnios, K. X./Klein, S. B. (Hrsg.): Handbook of Research on Family Business, Cheltenham, S. 614-618.

Zahra, Shaker A./Sharma, Pramodita (2004): Family Business Research: A Strategic Reflection, in: Family Business Review 17, 4. 
Zellweger, Thomas (2017): Managing the Family Business: Theory and Practice, Cheltenham Glos, UK.

Zenner, Thomas A./Megerle, Marcel (2014): Familienstrategie - Die Lösung für Familienunternehmer, in: GELD Magazin Nr. 4/2014, S. 28-29.

Zwack, Mirko (2011): Die Macht der Geschichten. Erzählungen als Form der Wertevermittlung in Familienunternehmen. 1. Aufl., Heidelberg. 


\section{Die WIFU-Schriftenreihe}

Herausgegeben von Tom A. Rüsen, Marcel Hülsbeck und Arist von Schlippe

Seit Mitte 2009 gibt das Wittener Institut für Familienunternehmen (WIFU) eine eigene Schriftenreihe zum Thema Familienunternehmen heraus. Seitdem sind insgesamt 26 umfassende, aber dennoch praxisnahe Bücher erschienen.

Das WIFU beschäftigt sich seit mehr als 20 Jahren mit diesem Thema und hat hierzu bereits zahllose Bücher und Zeitschriftenbeiträge herausgegeben. Da diese Unternehmensform ebenso spannend wie komplex ist, nimmt der Umfang der hierzu herausgegebenen Veröffentlichungen immer mehr zu. Die Schriftenreihe soll es ermöglichen, einzelne Themenkomplexe in dem erforderlichen und angemessenen Umfang zu veröffentlichen.

Das Wittener Institut für Familienunternehmen (WIFU) der Fakultät für Wirtschaft und Gesellschaft/ Department für Management und Unternehmertum der Universität Witten/Herdecke ist in Deutschland der Pionier und Wegweiser akademischer Forschung und Lehre zu den Besonderheiten von Familienunternehmen. Drei Forschungs- und Lehrbereiche - Betriebswirtschaftslehre, Psychologie/Soziologie und Rechtswissenschaften - bilden das wissenschaftliche Spiegelbild der Gestalt von Familienunternehmen. Dadurch hat sich das WIFU eine einzigartige Expertise im Bereich Familienunternehmen erarbeitet. Seit 2004 ermöglichen die Institutsträger, ein exklusiver Kreis von 75 Familienunternehmen, dass das WIFU auf Augenhöhe als Institut von Familienunternehmen für Familienunternehmen agieren kann. Mit derzeit 20 Professoren leistet das WIFU einen signifikanten Beitrag zur generationenübergreifenden Zukunftsfähigkeit von Familienunternehmen.

Das Leiten und Führen von Familienunternehmen stellt eine komplexe und mitunter auch paradoxe Herausforderung dar. Das Studienangebot der Universität Witten/Herdecke leistet hier wichtige Unterstützung: Im Bachelor- und Masterstudiengang »Management « kann der Schwerpunkt »Unternehmertum " gewählt werden. Der besondere Fokus liegt dabei auf dem operativen und strategischen Management von Familienunternehmen. So ist es dem WIFU möglich, seine Expertise an potenzielle Nachfolger, Fach- und Führungskräfte sowie Berater in Familienunternehmen weiterzugeben. Außerdem organisiert das WIFU in Zusammenarbeit mit dem Zentrum für Fort- und Weiterbildung der Universität Witten/Herdecke regelmäßig Workshops und Seminare für Gesellschafter, Nachfolger und Mitglieder aus Familienunternehmen.

\section{Zuletzt erschienene Bände dieser Reihe:}

Band 26: Otto W. Obermaier

Familienunternehmer als externe Beiräte

Empirische Untersuchung einer häufig gewählten Besetzung: Wie gut ist sie wirklich? 2019, 238 Seiten, gebunden, ISBN 978-3-8471-0994-5

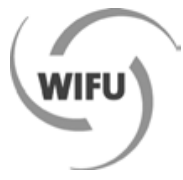

Band 25: Xing Ke

Succession and the Transfer of Social Capital in Chinese Family Businesses

Understanding Guanxi as a Resource - Cases, Examples

and Firm Owners in Their Own Words

2018, 247 Seiten, gebunden, ISBN 978-3-8471-0892-4

Band 24: Thomas Treiber

Pfade in die Krise

Der Einfluss des Faktors Familie auf die Genese und Dynamik

strategischer Pfadabhängigkeit in Familienunternehmen

2018, 456 Seiten, gebunden, ISBN 978-3-8471-0835-1

Vandenhoeck $\bullet$ Ruprecht Verlage

VER unipress

Leseproben und weitere Informationen unter www.vandenhoeck-ruprecht-verlage.com

E-Mail: info-unipress@v-r.de | Tel.: +49 (0)551 / 50 84-301 | Fax: +49 (0)551/ 50 84-333 